\title{
Domain Specific High-level Synthesis of Cryptographic Workloads
}

\author{
Von der Fakultät für Elektrotechnik und Informationstechnik \\ der Rheinisch-Westfälischen Technischen Hochschule Aachen \\ zur Erlangung des akademischen Grades \\ eines Doktors der Ingenieurwissenschaften \\ genehmigte Dissertation
}

\author{
vorgelegt von \\ Ayesha Khalid \\ aus Sargodha, Pakistan
}

Berichter: Universitätsprofessor Dr.-Ing. Anupam Chattopadhyay Universitätsprofessor Dr.-Ing. Gerd Ascheid Tag der mündlichen Prüfung: 17.02.2017

Diese Dissertation ist auf den Internetseiten der Hochschulbibliothek online verfügbar. 



\section{Acknowledgements}

As I write the acknowledgement of my dissertation, I cant help recollecting my experience of working as a research assistant at Institute for Communication Technologies and Embedded Systems (ICE) and how much it taught me as a person and as a researcher. I feel honored to have undertaken this journey.

This work would not have been possible without my advisor Prof. Anupam Chattopadhyay and I would like to express my sincere gratitude for his patience, support, motivation, valuable suggestions and technical guidance. My thesis co-supervisor Prof. Ascheid deserves a special thanks for his valuable feedback for my work. My list of gratitude extends to include Goutam Paul, Subhamoy Maitra and Sourav Sen Gupta who ignited several initial ideas captured in this work.

I am grateful to the rest of my thesis committee members who read over my thesis draft and made valuable suggestions for its improvement. This includes Prof. Heinen, who chaired my thesis defense oral exam and Prof. Mähönen who was the observer. Their insightful comments and encouragement incensed me to widen and improve my research from various perspectives.

Amongst my colleagues at ICE I would like to show my deep appreciation to Zheng Wang and Zoltán Rákossy for their undue assistance and support. I would like to thank my students Muhammad Hassan, Khawar Shahzad, Syed Imad, Faezeh Abediostad, Boishik Biswas, Deblin Bagchi, Rajat Sen, Ravi Prasanna, Tapas Jana for their significant technical contribution.

Last but not the least, I would like to thank my family and friends, especially my parents for their never ending love and trust. I am humbled by the consistent support of my husband Bilal, throughout the eventful journey of this thesis compilation in particular and my life in general.

Ayesha Khalid, May 2017 



\section{Abstract}

As the world is getting closer to the paradigm of Internet of Things, ensuring security for all the phenomenally increasing information exchange is becoming more challenging. The VLSI designer for security solutions must judiciously juggle various resource constraints by weighing multiple possible microarchitectures in the entire design space before reaching an optimal solution for an application in mind. Additionally, the inherently dynamic nature of cryptography leverages newer cryptographic functions and attacks to frequently surface, shortening further the time to market. With traditional VLSI design cycle requiring significant man hours and sufficient design expertise, reaching in-time design optimality is hardly possible.

Automation can expedite the VLSI design cycle. A rapid prototyping tool facilitating the automatic software and hardware implementation of cryptographic workloads is the major outcome of this thesis. The tool (called CRYKET) presents a language independent GUI based design capture, eliminating the steep learning curve often requried by traditional high-level synthesis tools. The cryptographic workloads were analyzed to identify a functionally complete set of constructive computation kernels as well as architectural sub-structures that are representative of a class of cryptography and their optimized hardware/software implementation. CRYKET allows fast design exploration by quick reconfiguration of design through a flexible API. A major technical challenge addressed by CRYKET is to seamlessly integrate these architectural substructures and operational functions into a working cipher model, without sacrificing the performance of the implementation, both of software and hardware platforms. Various symmetric key ciphers were successfully implemented and their performance was benchmarked to be on-par with their already-known hand-optimized HDL implementations.

Other than the known microarchitectural optimizations, optimization strategies, which are too algorithm specific to be undertaken automatically by CRYKET are discussed, for example, flexible cryptographic processors supporting multiple cryptographic functions and emerging cryptanalytic schemes. Moreover, memory bank reorganization in the context of designing efficient cryptographic cores, and the scalability of cryptographic functions on modern multi-core GPUs is taken up. Development of a cryptanalytic hardware solution for attacking SHA-1 is also undertaken. These case 
studies can serve as a guide for future design exploration of efficient cryptographic implementations.

\section{Keywords.}

Block cipher, stream cipher, hash function, feistel network cipher, Substitution Permutation Network (SPN), Addition Rotation Xor (ARX), lightweight cryptography, High Level Synthesis (HLS), rapid prototyping, eSTREAM, Application Specific Integrated Circuit (ASIC), Field Programmable Gate Array (FPGA) 


\section{Contents}

1 Introduction 1

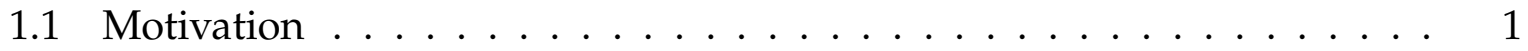

1.2 Contributions and Structure of this Thesis . . . . . . . . . . . . . . 2

2 Background 5

2.1 High Level Synthesis . . . . . . . . . . . . . . . . . 6

2.1 .1 Motivation ..................... 6

2.1.2 Generation through Automation . . . . . . . . . . . 7

2.1.3 Steps of High Level Synthesis . . . . . . . . . . . . . . . . . . 9

2.1.4 HLS: A Brief Retrospection . . . . . . . . . . . . . . . . 10

2.1.5 The Current Generation of HLS . . . . . . . . . . . . . . . . . . . 12

2.2 High Level Synthesis for Cryptographic Workloads . . . . . . . . . . . 14

2.3 ASIC Design Flow Setup . . . . . . . . . . . . . . . . . 15

2.3.1 The Standard Cell Digital Design Flow . . . . . . . . . . . . . 15

2.3 .2 ADL based Design Flow . . . . . . . . . . . . . . . . . 16

2.3 .3 Metrics . . . . . . . . . . . . . . . . . 17

2.4 Experimental Setup for CPU-GPGPUs Environment . . . . . . . . . . 18

2.5 Concluding Remarks . . . . . . . . . . . . . . . . . . . 18

3 Dwarfs of Cryptography 19

3.1 Berkeley Dwarfs for Parallel Computing . . . . . . . . . . . . . . . . 19

3.2 Cryptology Background . . . . . . . . . . . . . . . . 20

3.2.1 Block Ciphers . . . . . . . . . . . . . . . . . . . . 21

3.2.2 Stream Ciphers ...................... 21

3.2.3 Hash Functions . . . . . . . . . . . . . . . . 22 
3.3 Block Ciphers as Workhorses of Symmetric Key Cryptography _. . . . 23

3.3.1 Transformations Under Modes Of Operation . . . . . . . . . . . 24

3.3.2 Commonalities in the Computational Building Blocks of Symmetric Key Cryptography . . . . . . . . . . . . . . . . . . 25

3.4 Cipher Algorithmic Configuration Space . . . . . . . . . . . . . 26

3.4 .1 Block Ciphers . . . . . . . . . . . . . . . . . . . 27

3.4 .2 Stream Ciphers . . . . . . . . . . . . . . . . . . . 33

3.4 .3 Hash Functions . . . . . . . . . . . . . . . . . . . . . . . 39

3.5 Concluding Remarks . . . . . . . . . . . . . . . . . . . . . 41

4 High Level Synthesis for Symmetric Key Cryptography 43

4.1 CRYKET (CRYptographic Kernels Toolkit) . . . . . . . . . . . . . . 44

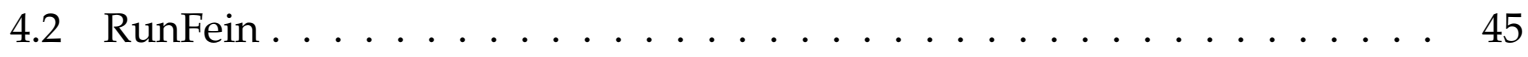

4.2.1 Design Specification Compilation . . . . . . . . . . . . . . . 45

4.2.2 Specification Validation and Formal Model Creation . . . . . . 46

4.2 .3 Software Generation Engine . . . . . . . . . . . . . . . 48

4.2.4 Hardware Generation Engine . . . . . . . . . . . . . 50

4.2.5 Results and Analysis: Software efficiency . . . . . . . . . . 55

4.2.6 Results and Analysis: Hardware efficiency . . . . . . . . . . 56

4.3 RunStream . . . . . . . . . . . . . . . . . . . . . . . . . 62

4.3.1 Design Specification Compilation . . . . . . . . . . . . . . . 62

4.3.2 Specification Validation and Formal Model Creation . . . . . . 64

4.3.3 Software Generation Engine . . . . . . . . . . . . . . . 66

4.3.4 Hardware Generation Engine . . . . . . . . . . . . . . . 67

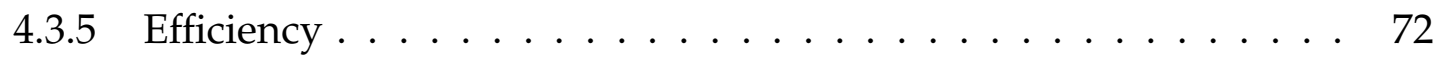

4.3.6 Comparison with Manual Implementations . . . . . . . . . . 74

4.4 Concluding Remarks . . . . . . . . . . . . . . . . . . . . . . . 79

5 Manual Optimizations for Efficient Designs $\quad 81$

5.1 Optimization Strategies . . . . . . . . . . . . . . . . . . 81

5.1.1 Memory Bank Structure Optimizations _ . . . . . . . . . . . 81

5.1.2 Unification of Multiple Cryptographic Proposals . . . . . . . . 81

5.2 Memory Bank Structure Optimizations _ . . . . . . . . . . . . 82 
5.2.1 Reviewing Known Techniques . . . . . . . . . . . . . . . . 82

5.2.2 Optimized Memory Utilization for HC-128 . . . . . . . . . . 83

5.2.3 Design Space Exploration of HC-128 Accelerator . . . . . . . . . 84

5.2.4 State Split Optimizations for HC-128 . . . . . . . . . . . . . 88

5.2 .5 Performance Evaluation . . . . . . . . . . . . . . . . . . 94

5.3 Integrated Implementation of Multiple Cryptographic Proposals . . . 97

5.3 .1 Motivation . . . . . . . . . . . . . . . . . . . . 97

5.3.2 Previous Work . . . . . . . . . . . . . . . . . . . . . . . 97

5.3.3 Contribution: HiPAcc-LTE-Integrated Accelerator for SNOW 3G

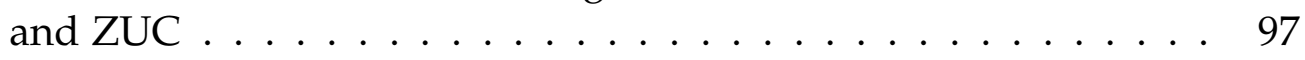

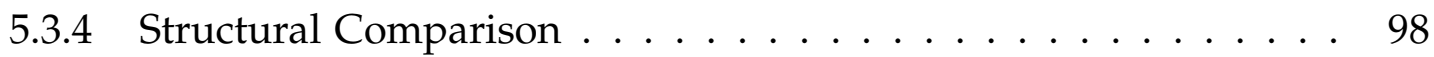

5.3.5 Integrating the Main LFSR . . . . . . . . . . . . . . . . . 99

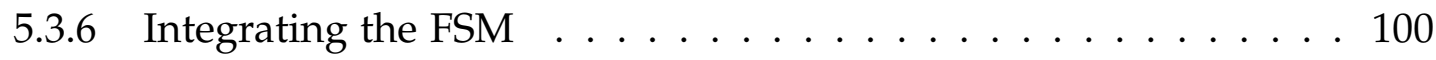

5.3.7 ASIC Implementation of HiPAcc-LTE . . . . . . . . . . . . 105

5.4 Concluding Remarks . . . . . . . . . . . . . . . . . . . . 114

6 Study of Flexibility $\quad 115$

6.1 Motivation . . . . . . . . . . . . . . . . . . . . . 115

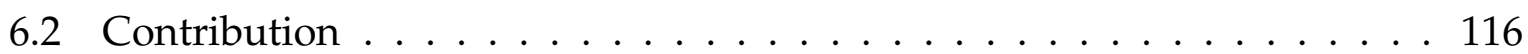

6.3 CoARX: A Coprocessor for ARX-based Cryptographic Algorithms . . . 116

6.3 .1 Related Work . . . . . . . . . . . . . . . . . . . . 117

6.3.2 Design Space Exploration . . . . . . . . . . . . . . 120

6.3.3 Mapping of the ARX Algorithms . . . . . . . . . . . . 123

6.3.4 Implementation and Benchmarking . . . . . . . . . . . . 127

6.3.5 Flexibility Study: Threefish-512 and Skein-512 Design Variants . 131

6.4 RC4-AccSuite: A Hardware Acceleration Suite for RC4-like Stream Ciphers . . . . . . . . . . . . . . . . . . . . . 132

6.4.1 RC4 Stream Cipher Algorithm . . . . . . . . . . . . . 132

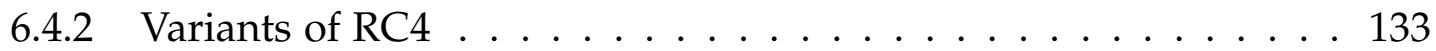

6.4 .3 Contribution . . . . . . . . . . . . . . . . . . . . . . . 134

6.4.4 High-level Architecture of RC4-AccSuite . . . . . . . . . . 135

6.4.5 Performance Enhancement by Memroy Replication Technique . . 136

6.4.6 Resource Economization in RC4-AccSuite . . . . . . . . . . . . 142 
6.4.7 Implementation and Benchmarking . . . . . . . . . . . . . . 147

6.5 Concluing Remarks . . . . . . . . . . . . . . . . . . . 152

7 Study of Scalability $\quad 153$

7.1 Motivation . . . . . . . . . . . . . . . . . 153

7.2 Major Contributions . . . . . . . . . . . . . . . . 154

7.3 CUDA Programming Model Overview . . . . . . . . . . . . . 154

7.3.1 Execution Model . . . . . . . . . . . . . . . . . . . 154

7.3 .2 Memory Model . . . . . . . . . . . . . . . 155

7.4 Block Ciphers Performance Acceleration on GPUs . . . . . . . . . . . . 155

7.5 Mapping Salsa20 Stream Cipher on GPUs . . . . . . . . . . . . . . . 156

7.5.1 Analyzing Parallelism Opportunities of Salsa20 . . . . . . . 156

7.5.2 Batch Processing Framework . . . . . . . . . . . . . . 157

7.5.3 Programming Recommendations . . . . . . . . . . . . . . 160

7.5 .4 Optimization for Salsa20 . . . . . . . . . . . . . . . . . . 160

7.5.5 Autotuning Framework for Performance Optimizations . . . . . 161

7.5.6 Results and Discussion . . . . . . . . . . . . . . . . 164

7.6 Mapping HC-128 Stream Cipher on GPUs . . . . . . . . . . . . . . . . 169

7.6.1 Limitations in Parallelization of HC Ciphers . . . . . . . . . . 169

7.6 .2 Optimization Strategies . . . . . . . . . . . . . . 171

7.6 .3 Experimental Results . . . . . . . . . . . . . . 176

7.7 Concluding Remarks . . . . . . . . . . . . . . . . . . . . 179

8 Efficient Cryptanalytic Hardware $\quad 181$

8.1 Introduction . . . . . . . . . . . . . . . 181

8.2 Background . . . . . . . . . . . . . . . . 182

8.2.1 Attacks Against SHA-1 . . . . . . . . . . . . . . . . . 182

8.2.2 Reported Hardware Attacks . . . . . . . . . . . . . . . . 183

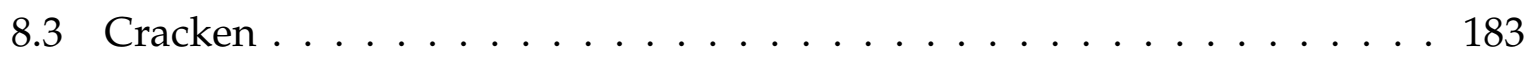

8.3.1 Attack Algorithm . . . . . . . . . . . . . . 183

8.3 .2 Cracken Architecture . . . . . . . . . . . . . . . . . . . 184

8.4 Performance Analysis and Comparisons . . . . . . . . . . . . . . . . 192

8.4 Synthesis Results . . . . . . . . . . . . . . . . . . . 192 
8.4.2 Physical Synthesis _. . . . . . . . . . . . . . . . . . . 193

8.4.3 Cost-performance Approximation with Memories . . . . . . . . . 194

8.4.4 Power Budget . . . . . . . . . . . . . . . . . . . . . . . . . 195

8.4.5 Mapping Cracken on FPGAs . . . . . . . . . . . . . . . 196

8.4.6 Comparison with Other Implementations . . . . . . . . . . . 196

8.5 Concluding Remarks . . . . . . . . . . . . . . . . . . . . . . 197

9 Conclusion and Future Work 199

9.1 Outlook . . . . . . . . . . . . . . . . . . . . . . 200

$\begin{array}{ll}\text { Appendix } & 201\end{array}$

A RunFein Generated AES-128 Code 201

$\begin{array}{ll}\text { B RunFein GUI Snapshots } & 205\end{array}$

C Description of Some ARX based Cryptographic Functions 207

C.1 HC-128 Stream Cipher [224] . . . . . . . . . . . . . . . . . . . . . . . 207

C.2 HC-256 Stream Cipher [223] . . . . . . . . . . . . . . . . . . . . . . . . 208

C.3 BLAKE Hash Functions Family [99] . . . . . . . . . . . . . . . 210

C.4 Skein Hash Functions Family [73] . . . . . . . . . . . . . . . . 213

C.5 Salsa20/r Stream Cipher [21] . . . . . . . . . . . . . . . . . . . . 215

C.6 ChaCha20/r Stream Cipher $[20] \ldots \ldots$. . . . . . . . . . . 217

D Overview of SNOW 3G and ZUC Stream Ciphers 219

D.1 SNOW 3G Stream Cipher [2] . . . . . . . . . . . . . . . . . . . . . . 219

D.2 ZUC Stream Cipher $[232] \ldots \ldots \ldots \ldots \ldots$

$\begin{array}{ll}\text { List of Figures } & 221\end{array}$

$\begin{array}{ll}\text { List of Tables } & 225\end{array}$

$\begin{array}{lr}\text { Bibliography } & 229\end{array}$ 


\section{Chapter 1}

\section{Introduction}

This era is witnessing a phenomenal increase in the amount and frequency of the information exchange. The imminent Internet of Things paradigm underpins an network of interconnected devices capable of information processing and dynamic communication with their environment. Ensuring security for all the increased information exchange has introduced new challenges. On one end of the performance-cost spectrum, the sheer magnitude of this increased communication requires network security services at sub-Gigabit speeds. This is viable due to the continuous downscaling of fabrication technology favoring better processing efficiencies for integrated circuits [154]. On the other end of the spectrum are the ubiquitous devices, with resource constraints far more harsh compared to the traditional cryptographic functions. In addition to the conventional constrains of a VLSI design, security and flexibility of the cryptographic function design may also be traded off for performance and cost. Hence the VLSI development of any security solution has a huge design space having many design points that may be interesting to specific application classes.

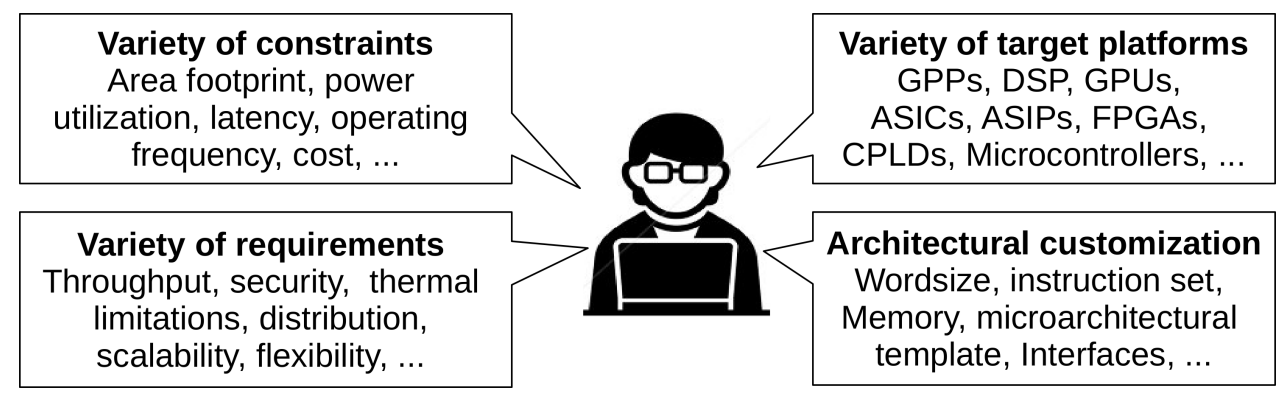

Figure 1.1: The crypto developer must juggle up conflicting requirements and constraints, exploiting platform dependent microarchitectures for an optimized solution

\subsection{Motivation}

The field of cryptography is highly dynamic where newer cryptographic proposals frequently surface. These proposals claim specific platform suitability, flexibility, levels of security or resistance against various cryptanalytic attacks, etc. Successful cryptanalysis, advances in computing devices and newer application avenues also welcome new cryptographic proposals for adoption. These fast evolving cryptographic functions pressure up the VLSI designers to come up with a best in class solutions within 
an ever shortening time to market window. Additionally, there is a design trend of having multi-component heterogeneous systems to address the conflicting requirements of increasingly complex systems. As depicted in Fig. 1.1, the crypto developer must also consider the platform dependent optimizations whenever possible. The system components may include General Purpose Processors (GPPs), Graphics Processing Units (GPUs), Application Specific Integrated Circuits (ASICs), Field Programmable Gate Arrays (FPGAs), all of which may require different expertise and optimization strategies. This indicates that a crypto developer should have the necessary expertise to juggle up often conflicting design requirements and constraints. Often the design cycle is repetitive and tedious as the designer must explore a huge design space of microarchitectural optimizations intelligently before reaching a desired solution.

Automation can expedite the VLSI design cycle, saving significant man hours to develop and verify the hardware design manually. Various Electronic Design Automation (EDA) tools have been proposed to analyze and refine the design specifications into a lower abstraction level in the VLSI design cycle. Starting from the highest level of abstraction, the algorithmic specifications are converted to digital hardware using High-level synthesis (HLS) tools. However, most of these require learning a new system specification language, making the learning curve steep. Additionally, since these tools do not focus on any specific application class, the optimizations undertaken by them remain generic and often suboptimal compared to the hand optimized HDL implementations.

\subsection{Contributions and Structure of this Thesis}

This work proposes a rapid prototyping tool (called CRYKET) to facilitate the automatic software and hardware implementation of cryptographic workloads. CRYKET stand for Cryptographic kernels toolkit. Compared to existing schemes, it has several advantages. Firstly this tool has a language independent interface and instead enables a sophisticated design capture via a GUI. This also eliminates the design quality dependence on the coding style of the programmer. Secondly since this tool deals with domain specific application class, i.e., symmetric key cryptographic functions, it lets the user pick a mircoarchitectural template specific to that domain of application. This limits the design space of possible structural optimizations to a class of applications and allows better in-cooperation. A fast design exploration by quick reconfiguration of design through a flexible API is possible.

A rapid prototyping tool facilitating the automatic software and hardware implementation of cryptographic workloads is the major outcome of this thesis. In the following, the chapter-wise breakdown of the thesis is given, together with the respective contributions and the respective publications.

Chapter 2 discusses the background. It sets up the motivation and presents a brief history of HLS tools along with a survey of currently available HLS tools and synopsis. A description of vendor tools used for development of various cryptographic implementations throughout this work are also summarized. 
Chapter 3 discusses and analyzes the cryptographic workloads to identify a functionally complete set of constructive computation kernels that are representative of symmetric key cryptography. Chapter 4 discusses the HLS tool flow for block ciphers and stream ciphers. Various design points in the scope of architectural customizations available to the CRYKET user are elaborated. A thorough benchmarking to compare against the hand crafted solutions is undertaken, where their performances are found on-par with hand-optimized HDL implementations. The results are published $[119,120,123]$.

Chapter 5 takes up two optimization techniques that are algorithm/ application specific and are not straight forward to be undertaken automatically by the CRYKET tool. Firstly, the memory bank structure optimizations are discussed in general and then applied to the HC-128 stream cipher implementation in particular (published in [124]). Secondly, the design of a unified VLSI core supporting multiple co-existing cipher functions is analyzed. The merger of SNOW 3G and ZUC stream ciphers of 3GPP LTE-Advanced security suite is taken up as a case study. The substantial area efficiency achieved henceforth is evaluated and compared to the state-of-the-art implementations (published in [92]).

Chapter 6 explores flexibility as a design feature for cryptographic processors. A crypto-coprocessor, named CoARX, supporting block ciphers, stream ciphers, hash functions etc. belonging to ARX based operations is undertaken. It is benchmarked to offer an excellent performance-flexibility trade-off, between the dedicated hardware implementations and general-purpose computers, along with the adaptability to resist generic cryptanalysis. The results were published in [198]. In the context of flexibility, an ASIP supporting an entire class of stream ciphers (RC4 and its prominent variants) is also taken up. Through aggressive resource reuse, the ASIP bagged substantial area economization, compared to individual cores, along with the performance-security flexibility [122].

Chapter 7 evaluates the scalability of various cryptographic functions on modern multi-core GPGPUs. A throughput efficient mapping of two prominent eSTREAM [65] finalists is taken up on NVIDIA GPUs, i.e., Salsa20 and HC-128. One of these implementations remains the highest performing academic result published so far $[118,121]$.

Chapter 8 takes up the design and implementation of a processor to efficiently realize a near collision attack on SHA-1 cipher, that is the most widely used hash function being used in TLS/SSL for internet security. The cost-time estimates of the processor to execute the attack are provided both for FPGA based system and as an ASIC. Previously reported SHA-1 attacks based on ASICs and cloud computing platforms are also compiled and benchmarked for reference.

Chapter 9 concludes this thesis and provides an outlook for the future. 


\section{Chapter 2}

\section{Background}

The growth in the consumer market for digital devices goes hand in hand with the demand for enabling more comprehensive functionality, higher performance and lower power budgets from these devices. The mutually conflicting requirements of these increasingly complex systems, coupled with the continuous downscaling of fabrication technology [154], has welcomed the design trend of multi-component heterogeneous systems [230]. Security is one of the increasingly important application required for embedded devices. Unlike the provision/support of a service, security is considered critical enough to be taken now as a design metric for a system, along with other performance metrics such as cost, performance etc [134]. In keeping with the trend of heterogeneity of embedded systems, the computational burden for security applications is often isolated from the central computing resource of a system and levied instead on a separate computing entity dedicated to security applications [9]. Consequently, all major semiconductor IP core vendors offer security services IPs.

The world of cryptography is dynamic where newer cryptographic proposals, their optimized implementations and cryptanalytic attacks are frequently proposed.

1. New Cryptanalysis: Successful cryptanalytic attempts not only render the further use of the attacked ciphers vulnerable but also invite their replacements (or modification). One such example is the replacement of A/5-1 and A/5-2 stream ciphers in GSM encryption, after some weaknesses were pointed out in them [15], by A/5-3 (or KASUMI) block cipher [86].

2. New Machines: Advances in computing machines influence the new cryptographic schemes so as to improve their throughput performance. In a parallel trend, latest GPPs have extended instruction set for enhanced performance for cryptographic schemes. For the selection of Rijndael as AES winner and Keccak as SHA-3 winner, their computational efficiency as hardware and software implementations was taken up as a decisive factor [164,171]. Also, the eSTREAM competition categorized the candidate cryptographic proposals into being software friendly or hardware friendly [65]. Moreover, the development of custom solutions for cryptanalytic attacks enables even the brute force attack for small key sized proposals today, DES can be broken in less than a day by the COPACOBANA machine [181].

3. New Applications: The imminent ubiquitous computing era has initiated lightweight cryptography, which unlike the traditional cryptography requires harsh cost constraints along with reasonable security requirements [113]. Consequently, a lot of research is not focused on development of lightweight kernel for cryptography. 
The active participation of an increasing cryptology community is evident by the growing number of candidate proposals submitted to various cryptographic competitions held over the last few decades. Table 2.1 summaries the salient features of cryptographic competitions arranged, chronologically. Out of these, NESSIE and CRYPTREC evaluated both Public key and private key ciphers, while the other competitions focused on one type of cipher functions. The influence of cryptanalysis is evident from the fact that ESTREAM was initialized as all the 6 stream cipher candidate proposals of NESSIE were attacked. CAESAR is a still running competition.

Table 2.1: Summary of various cryptographic competitions

\begin{tabular}{|c|c|c|c|c|c|}
\hline Competition & Category & Organization & Time span & Proposals & Winners \\
\cline { 1 - 4 } AES [164] & Block Ciphers & NIST & $1997-2000$ & 15 & Rijndael \\
\hline NESSIE [161] & Broader & Panel of Experts & $2000-2003$ & 42 & 18 \\
\cline { 4 - 7 } & Evaluations & Japanese Govt. & $2000-2003$ & - & 29 \\
\hline CRYPTREC [47] & Stream Ciphers & ECRYPT & $2004-2008$ & 35 & 7 winners \\
\hline eSTREAM [65] & Hash Function & NIST & $2007-2012$ & 59 & Keccak \\
\hline SHA-3 [197] & AE & ECRYPT & $2012-2017$ & 58 & - \\
\hline CAESAR [35] & Password Hashes & Panel of Experts & $2013-2015$ & 24 & Argon2 \\
\hline PHC [176] & \multicolumn{5}{l}{}
\end{tabular}

\subsection{High Level Synthesis}

\subsubsection{Motivation}

The VLSI design of a system is an involved task requiring diverse skills. Once the algorithmic specifications are in place, the floating point, sequential software implementation of the design is coded by a system designer or a software engineer. This floating-point implementation of the application is converted to a fixed point implementation after necessary dynamic range and precision considerations. Next the hardware designer takes up this untimed implementation and develops its digital architecture as shown in Fig. 2.2 (left). Keeping in mind the system constraints, a microarchitecture is designed and implemented in HDL. After successful verification through gate level simulation of the digital design, logic synthesis is carried out to get the gate level netlist with the help of EDA tools. Logic synthesis converts the RTL description into elementary Boolean operations from an available cell library (in a particular technology). Physical synthesis converts the design into an exact physical layout, it further optimizes the resource bindings on the basis of updated timing estimates that take the wire loads into accurate values due to physical floor-planning considerations. The physical synthesis and chip layout in the VLSI design is generally offloaded to foundry vendors. 


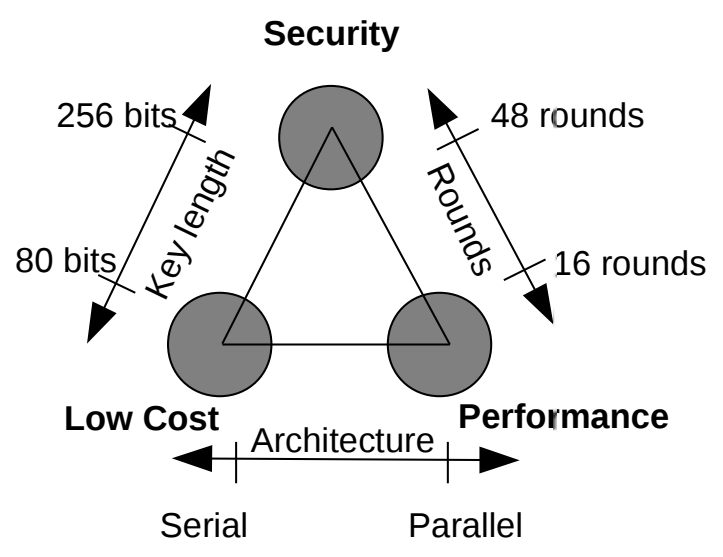

Figure 2.1: Design trade-offs for lightweight cryptography

Traditionally, the VLSI design is performed by experts of two separate domains as they require expertise in software tools and hardware microarchitectural description, respectively. Consequently, a lack of proper specifications exchange between these groups may lead to incorrect understanding of the system, leading to expensive late re-design cycles. Moreover, the traditional VLSI development methods are firstly, time consuming requiring manual steps such as architectural design, handwritten RTL, simulation, verification, debug and secondly, repetitive as non conformity to cost-performance budget after synthesis would require to re-architect the design at RTL level again.

With large number of design options available, these issues become even more challenging. Fig. 2.1 shows the design space for the lightweight cryptography. Numerous available design options must be weighed against each other before reaching an optimal point in the entire design space. With a tedious and error-prone design manual methodology, this is hardly possible.

\subsubsection{Generation through Automation}

To overcome the complexity of VLSI design automation is the solution. The system is modeled at a high abstraction level of the design process. This model can serve as an executable specification for early design space exploration. The abstract modeling at a high abstraction level of the design process High Level Synthesis (HLS) or behavioral synthesis techniques bridge the gap between algorithm developers and hardware designers by supplying a platform for co-development. Consequently, design exploration is accelerated and time-to-market is shortened.

The HLS techniques are carried out either through High Level Languages (HLLs) or through Architecture Description Languages (ADLs).

- Algorithm description through a High Level Language (HLL): HLS through a HLL requires sequential specification of the system by the user in a higher abstraction level language. The HLS tool transforms this untimed design spec- 

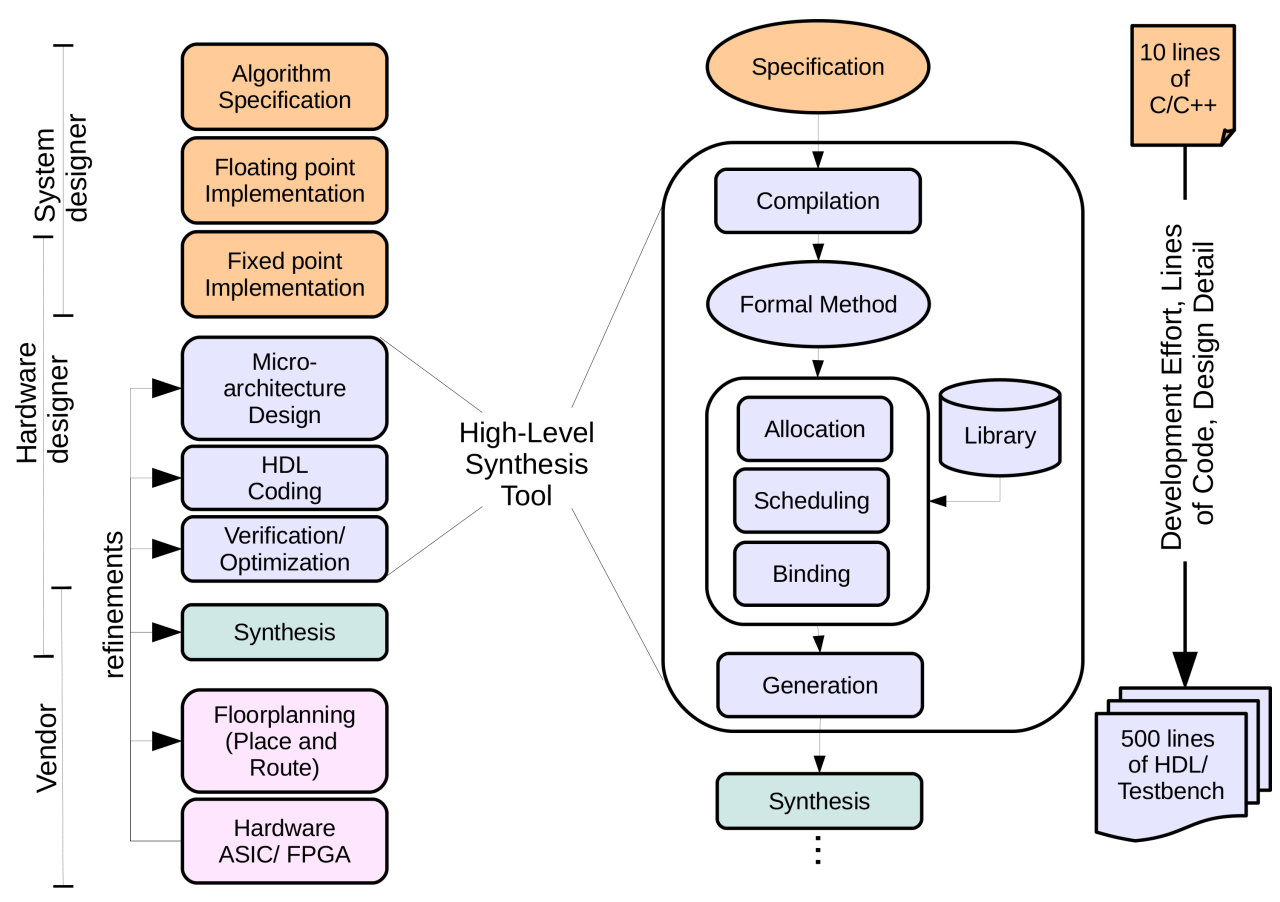

Figure 2.2: The traditional VLSI development flow (left) against the High Level Synthesis (HLS) based development flow (right)

ifications into a fully timed digital hardware. The user may direct the tool to obey constraints including target platform, latency, throughput, area, frequency etc. Based on the constraints, the tool explores various architecture trade-offs and optimizes across design hierarchy and loop structures to come up with a synthesizable efficient, parallel hardware architecture along with its verification environment. The tool develops high quality architectures at the expense of design flexibility and hence this design methodology is more suitable for application specific hardware mapped to ASIC / FPGA. The user has only limited control over the choice of generated architecture limiting the design space exploration. Noticeable examples include Synopsys Synphony C Compiler [210], GAUT [46], Xilinx Vivado HLS [228], Mentor Graphics Catapult C [211].

\section{- Architecture description through a Architecture Description Languages (ADLs):}

In contrast, HLS tools that take ADLs description of the design let the designer choose an architecture choice over the entire design space. The toolflow is suited for Application Specific Instruction set Processors (ASIPs) as the designer describes the architecture in a higher abstraction by ADLs, including the structures to model instruction-set, timing, op-code, pipeline, memory architecture etc. The quality and ease of exploration directly depends on the language flexibility of ADL. Ideally, the ADL development environment should come with a suite of aiding tools to enable high level simulations where early design decisions can be guided by simulation loops. Some high level processor modeling languages 
include LISA ADL (Synopsys Processor Designer) [209], nML [72], EXPRESSION [94].

For application specific workloads, e.g., cryptographic workloads, the first approach or HLS is more suited. Moreover, the cryptographic community is generally more at ease with the C-like HLLs that are not supported by the ADL approach. The architecture engineers can however, relate better to the ADLs. Hence, for the rest of the discussion, the HLS through an HLL only is focused.

\subsubsection{Steps of High Level Synthesis}

Fig. 2.2 (right) shows the typical steps of a High Level Synthesis tool [45]. These steps are discussed below.

- Compilation into a Formal Model: The high level specification is first parsed and converted to an Intermediate Representation (IR). IR is generally a Data Flow Graph (DFG) representing the data dependencies between operations [111]. Fig. 2.3 shows a simplistic example of the conversion of $C$ specifications into a DFG. An extended DFG, handling the control dependencies in addition to data dependencies called Control Data Flow Graph (CDFG) [225] is also being used as IR.

- Allocation: For each operation in the IR, a hardware resource is allocated (e.g., function unit, storage element, connectivity buses) from the RTL component library as shown in Fig. 2.2 (right). Since the library components have other characteristics (area, delay, power), the selection is based on the user specified design constraints.

- Scheduling: Scheduling creates a clock scheme assigning time cycles or control steps on operations based on time/ resource constraints. Depending upon the functional unit on which the operation is mapped, an operation may be extended to multiple cycles or multiple operations may be merged in on clock step. The simplest unconstrained scheduling algorithms are ASAP (As Soon As Possible) and ALAP (As Late As Possible) [103]. ASAP schedules an operation for execution on a control step as soon as all its dependencies are resolved and hence exhibits a minimum latency unconstrained scheduling. ALAP scheduling schedules the operation for the last control step back to the first. An operation is delayed for execution till it is required for a next operation. More realistic scheduling constraints are minimum latency under constrained resource (MLRC) and minimum resource under latency constraints (MR-LC). These scheduling problems are NP-hard [22], consequently several Iterative Heuristic Methods are employed to solve them. Some most popularly used algorithms are List Scheduling (LS), Force-Directed Scheduling (FDS) and Simulated Annealing [218]. Fig. 2.3(c) scheduling of the formal model into a clock scheme with resources constrained to just one Adder and one Multiplier. 
$\mathrm{F} 1=(\mathrm{a}+\mathrm{b}+\mathrm{c})^{\star} \mathrm{d}$

$\mathrm{F} 2=(\mathrm{x}+\mathrm{y})^{\star} \mathrm{z}$

$\mathrm{F} 3=(\mathrm{x}+\mathrm{y})^{*} \mathrm{w}$

a) High level language description

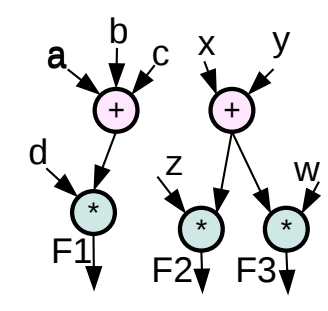

b) Copilation into Data Flow Graph (DFG)

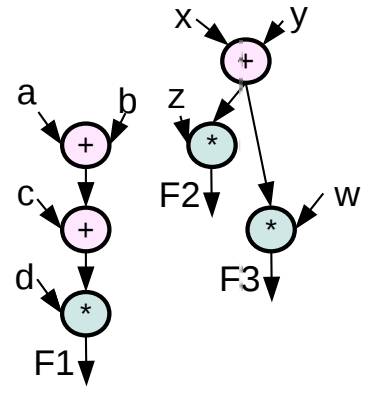

c) Constrained resource scheduling

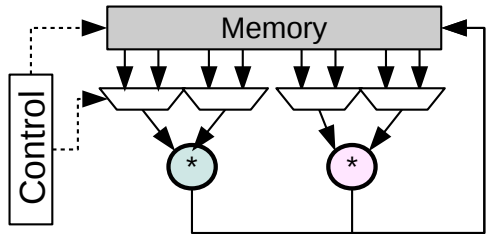

d) Hardware Architecture

Figure 2.3: Step of HLS: a) High level language description b) Compilation into a DFG c) Minimum latency under constrained resource scheduling (1 Adder, 1 Multiplier ) scheduling d) HDL architecture generation

- Binding: Binding completes the hardware architecture. This step binds all operations to functional units with an optimized selection, whenever possible. Variables carrying values across cycles are bound to storage units while variables with non overlapping or mutually exclusive lifetimes are bound to the same storage units for resource reuse. All transfers are binded to buses. Fig. 2.3(d) shows the complete architecture after binding with storage elements, functional units and buses.

- Generation: The HDL generation of the architecture model is the last step of HLS toolflow. The generated HDL is generally partitioned into a controller and datapath and is implemented by register transfer language components (RTL). The data path comprises of all the storage elements, a set of functional units, and interconnect elements. The data path can be pipelines and consequently may have input and output registers. The controller is a Finite State Machine (FSM) that orchestrates the flow of datapath by setting the control signals that are the inputs to the functional units, registers, multiplexers etc of the datapath. The datapath may return status signals back to the controller as required.

\subsubsection{HLS: A Brief Retrospection}

The pursuit to raise the abstraction level of design modeling, optimization and implementation has been the goal of several generations of CAD researchers, both from industry and academia. Some of the earliest research efforts were launched as early as 1970's while the industry was still adapting to the CAD systems; this era is termed as the generation 0 of HLS [218]. A pioneering HLS tool was developed at Carnegie Mellon university (called CMU Design automation or CMU-DA), which accepted the behavioral description as ISPL (Instruction Set Processor Language). It is then transformed into an IR data-flow called the Value Trace before developing RTL level implementation [177]. 


\subsubsection{Generation 1 of HLS}

During the first generation of HLS (1980s-early 1990s) basic concepts of HLS were explored and some groundbreaking research work was published [218]. One of the earliest HLS tools commercially available was CATHEDRAL-II Silicon Compiler [57] for Digital Signal Processing (DSP) algorithms. It accepted behavioral specifications in a domain specific language, namely SILAGE, that was specifically designed for the synthesis of DSP hardware with support for customized data types and easy transformations into different data types. It used a programmable, bit parallel VLIW architecture for application mapping. Serval procedural optimizations were preformed in the Scheduling of microcode. The tool serves as an application specific silicon compiler that exploited well the defined target architecture design and led to efficient layout synthesis from a system point of view.

The first generation of HLS failed to be a commercial success due to several reasons [218]. The idea of HLS was hard to undertake at that time by the designers since RTL synthesis adaptation was then just beginning. Moreover, learning a new input language with an unfamiliar design approach was not appreciated. Most of the tools of that generation relied on simplistic allocation and scheduling algorithms and consequently produced a lower QoR. Moreover, the domain specialization focused on the DSP applications, that was not suitable for the vast majority of ASIC designers.

\subsubsection{Generation 2 of HLS}

The second generation of HLS (mid 1990s-early 2000s) is marked by a wide adoption of RTL-based design flows and a simultaneous good deal of interest from the major EDA companies towards HLS [218]. In 1995, Synopsys announced Behavioral Compiler that generated RTL implementations from behavioral hardware description language (HDL) code and was seamlessly connected to downstream tools. The compiler regularly used allocation and binding optimizations on the arithmetic and other functional units in conventional logic optimization [130]. Cadence launched an industrial tool, the Visual Architect Tool, for the system level design of a system, described in behavioral VHDL for realization in FPGAs. The tool was enhanced with precise timing handling and also exploited feedback of technology information for better high-level synthesis [98]. A similar tool was introduced by Mentor Graphics for HLS called Monet [67].

These tools received wide attention but failed to replace the traditional RTL design methodology due to following reasons [218]. The HLS required learning behavioral HDL language as input, consequently, the popularity of these tools remained confined to hardware designers. Moreover, the results of these tools were hard to validate due to absence of formal methods at that time. Since HLS is more suited to dataflow and signal processing oriented flows, the results remained suboptimal for control oriented dataflows. Consequently, the generation 2 tools failed to expand market of HLS to algorithms and software developers. 


\subsubsection{The Current Generation of HLS}

A new generation of HLS tools emerged from 2000 with proposals both from academia and industry. This generation was able to overcome most of the shortcomings that their predecessors had and consequently had better industrial adaptation. To start with, most of these tools relied on $\mathrm{C} / \mathrm{C}++$ or $\mathrm{C}$-like languages (matlab) to capture the design specifications. This feature made them more accessible to system designers as it enabled software/hardware co-design and co-verification. Additionally, most of these tools focused on dataflow and DSP domain applications which are well suited for HLS toolflows, resulting in an improved QoR. Another salient feature of these tools is that many of them are exclusive to a platform (e.g., FPGAs, ASICs, DSPs). Consequently the target architecture is efficiency exploited at all design levels [218].

The salient features of some of the prominent HLS proposals, coming from both the academia and the industry, are discussed below.

Mentor Graphics Catapult C Synthesis: Coming from a major EDA vendor, Catapult $\mathrm{C}$ was designed and enhanced in an entire era of research and development. It takes untimed ANSI $\mathrm{C} / \mathrm{C}++$ descriptions as input to generate RTL (VHDL, Verilog) targeted for both ASICs and FPGAs. Its accepts timing, target technology and area as constraints. Other constraints include interface synthesis directives to indicate dataflows into or out of the hardware design, loop directives for loop unrolling and adding parallelism to the design, memory directives for storage constraints and memory architecture and resource constraint to limit hardware resources [29]. The tool carries out a Hierarchical Synthesis to simplify the design integration, hierarchy also helps to exploit function level (or task-level) parallelism. A technology-driven scheduling and allocation is carried out to generate pipelined, multi-block subsystems in synthesizable RTL along with systemC transaction-level models (TLM) and wrappers for simulation and verification. Application suitability, both for control oriented and data oriented workflows is claimed, along with wide endorsement by leading semiconductor vendors. In 2011 Catapult C was acquired by Calypto Design Systems [211].

Xilinx Vivado High Level Synthesis: Coming from a noticeable FPGA vendor, the Vivado tool suite comes along a high level synthesis tool, tightly-coupled to the Xilinx chips and cannot be used for products from other vendors [228]. It accepts $\mathrm{C}, \mathrm{C}++$, SystemC or openCL API C kernel to generate implementation in RTL format that can be further synthesized to gate-level netlist and FPGA bitstream by the Xilinx tool chain. The C-based input is restricted to a synthesizable subset, excluding dynamic memory allocation, recursion, system calls etc. The user may guide the synthesis by manually invoking various area and timing optimization directives. Exploring various design options using these directives does not alter the source code. Various powerful directives for iterative code chunks include loop unrolling, loop pipelining, flattening a loop hierarchy, merging serial loops together and parallel execution of multiple loops. Vivado-HLS uses an LLVM compiler as an intermediate representation. Various ready cores can also 
be plugged in into the design. Resource binding by the tool could be directed to the highest resource economization by increasing the effort level of the tool. Vivado supports automatic conversion of OpenCL kernels to IP for multi-platform applications, i.e., CPU, GPU and FPGA platforms [228].

Synopsys Synphony C Compiler: The high level synthesis tool from Synopsys, called Synphony C, was formally Synfora's PICO Express, originated after research at HP Labs [116]. The tool accepts a broad subset of $\mathrm{C} / \mathrm{C}++$ for a fixed point sequential code that may have loop nesting and multiple levels of hierarchy [210]. Its optimization engine efficiently analyzes through multi-level compilation techniques to extract parallelization across the design source. The tools carries out optimizations for resource sharing by effective scheduling and automatic pipelining for target-aware timing closure as per the user constraints. A verification environment with RTL testbench, test vectors, script and SystemC verification model including TLM System, cycle accurate wrapper are also generated. A seamless integration for FPGAs with synopsys synplify tool and for ASICs with the Design Compiler tool is provided [210].

GAUT: GAUT is an open-source HLS tool from University of South Brittany (LabSTICC) [46] dedicated to DSP applications. It accepts a bit-accurate $\mathrm{C} / \mathrm{C}++$ specification of the design and extracts a DFG model by gcc/g++ compiler [44]. The mandatory constraints for the design are initial latency and clock period while the optional constraints are memory architecture mapping and I/O timing diagram (interface definition). GAUT generates a library of time characterized operators for the HLS steps based on a technological library and technology node specified. The scheduling is performed by list scheduling algorithm, followed by a global optimization on interconnects and storage elements. GAUT generates a VHDL testbench and scripts for Modelsim as well as SystemC cycle accurate simulation model. The generated VHDL may be taken up by FPGA/ ASIC synthesis tools [44].

LegUp: LegUp is an open source high-level synthesis tool from University of Toronto for FPGA platforms [172]. LegUp takes up an ANSI C subset (dynamic memory, floating point and recursion unsupported) program and maps it on an FPGAbased MIPS processor, augmented with extra circuitry to profile its own execution at runtime for high accuracy [36]. The profiling results decide the code chunks to be targeted to custom hardware from the source code. After this software-hardware division, LegUp compiles the code segments to synthesizable Verilog RTL. LegUp uses the low-level virtual machine (LLVM) compiler framework to compile functions into an IR and then analyzed and modified by a series of compiler optimization passes. After ASAP allocation of instructions to hardware operations, the allocation is refined when possible by operations chaining [36]. Various heuristic algorithms are implemented to optimize the binding of operations to hardware resources. The communication between the host de- 
vice and the accelerator takes place by a standard bus interface. The generated RTL can be taken up by any FPGA vendor synthesis tools.

\subsection{High Level Synthesis for Cryptographic Workloads}

The need of efficiently and correctly implementing cryptographic workloads by an increasing cryptographic community, compounded with numerous requirements and constraints for its implementation on a wide variety of architectures made the need of a specialized language and tools for cryptography imperative. Such tools can conveniently benchmark the large number of candidate proposals in cryptographic contests, as traditionally only the software benchmarking is provided with a new cryptographic proposal. An earlier feedback on these proposals can also help avoid unpleasant late discoveries of poor hardware performance as was the case with BMW, ECHO and SIMD in the SHA-3 contest [102].

There have been numerous reported efforts of developing high-level synthesis framework focused on cryptographic workloads. In [69,156,180], elliptic curve publickey cryptosystem hardware generator programs were proposed to develop custom RTL for FPGAs as parametrized by the user. Most of the parametrized toolflows for rapid prototyping for cryptographic workload remain confined either to the generation of software codes for cryptographic protocols $[5,136]$ or the cryptanalysis toolkits $[139,158]$.

For the high-level hardware implementation of symmetric key cryptography, most of the reported efforts $[4,34]$ focused on the proof of concept of cryptographic workloads being implemented by a HLS toolchain, without competing in quality with a hand written RTL. Two case studies are worth mentioning in the context, that take up HDL code for modern cryptographic algorithms and generate HDL descriptions by a new generation HLS tool (Vivado HLS by Xilinx). In [102] 5 round-3 SHA-3 candidates were undertaken by the HLS tool and performance benchmarked for TPAR against manual RTL. In-spite of various iterations of the source code modifications by pragmas to economize hardware resources, the TPAR for HLS remains between $62 \%-85 \%$ lower, compared to manual RTL for various Altera devices. Similarly, noticeable performance penalty is caused by the HLS tool when various configurations of AES are generated and performance profiled on different families of FPGAs [101]. On a Virtex-7 FPGA, the degradation of HLS AES in terms of TPAR lags behind $28 \%$ to $42 \%$, compared to manual RTL.

The only Domain Specific Language (DSL) for cryptography proposed is Cryptol.

Cryptol Cryptol is a DSL for cryptography. It comes with an open-source toolsuite, co-developed by the Portland (Oregon based software development firm), Galois, Inc. and NSA [167]. Cryptol can be used to develop the entire life cycle of a cryptographic proposal, i.e., design of a cipher, implementation for multiple targets, simulation for design exploration, generation of test harness and testvectors, formal verification etc. The design specification by Cryptol is reusable as the same tool can generate software implementation, hardware implementation 
and formal models of verification. Cryptol specifications are close to their mathematical specification than an implementation in a general purpose language. This also eliminates the need of referee specification documentation [140]. The user can guide the effective translation of his cryptol design on FPGAs by compiler pragmas about space mapping and timing. Cryptol is expressive enough to describe various microarchitectural optimizations as well. The user can quickly alter the Cryptol specifications to place a pipeline in the body of the loop as he desires and analyze rapidly the design iterations. The cryptol toolchain translates the specifications into a Signal Processing Intermediate Representation (SPIR) that acts as a bridge between the tool and hardware implementation. Next the tool applies a variety of engineering heuristics to enable a target oriented optimization for an effective use of FPGA resources. The generated VHDL code and test bench is seamlessly interleaved with the simulation and verification processes [167].

\subsection{ASIC Design Flow Setup}

This Section briefs over the digital standard cell design flow. Various vendor tools (and their versions), design methodologies and constraints are discussed. Subsequently, an ADL based design flow used to speedup the VLSI development of some works of this thesis is introduced. Finally the metrics to judge the quality of a VLSI implementation are explained.

\subsubsection{The Standard Cell Digital Design Flow}

In this thesis, several hardware implementation architectures for various cryptographic algorithms are generated using the developed HLS tool or CRYKET (Chapter 4). All these generated architectures were verified and synthesized using a script based design flow as shown in Fig. 2.4. Verilog RTL functional verification was carried out using Mentor Graphics ModelSim (version 10.2c) [84]. Synopsys Design Compiler (version G-2012.06) was used with the Faraday standard cell libraries in topographical mode to carryout synthesis of HDL. Each of the design was synthesized and profiled for area, power consumption and maximum frequency. The synthesis was driven by throughput maximization with the max area constraint set to 0 . Each design was repeatedly synthesized using compile_ultra option, in an incremental fashion with increasing clock frequency as long as no timing violation was reported. The power consumption is estimated by Synopsys Primetime (version 2009.12) based on gatelevel netlist switching activity by back annotation. The performance estimates of SRAMs used were obtained using Faraday Memory compiler [71], for 65nm technology library. The area results are reported using equivalent 2-input NAND gates (GE).

Throughout this thesis the following three different standard cell libraries with different technology parameters were used: 


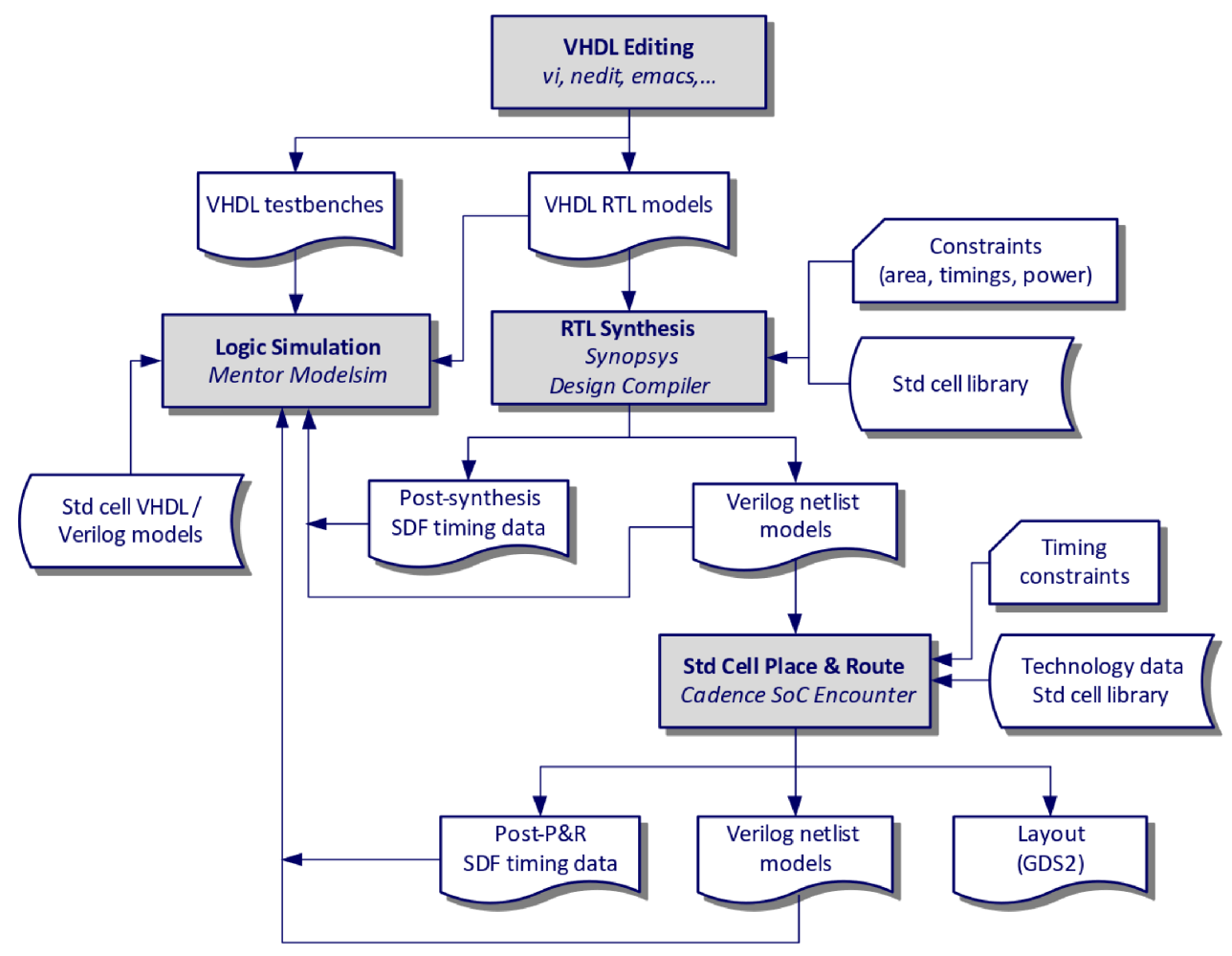

Figure 2.4: Top-down digital standard cell design flow [216]

- Faraday UMC 65nm SP/RVT Low-K process technology library. Best case condition with $1.1 \mathrm{~V},-40^{\circ} \mathrm{C}$ parameters are assumed.

- Faraday UMC 130nm high speed FSG process technology library. Typical case condition with $1.2 \mathrm{~V}, 25^{\circ} \mathrm{C}$ parameters are assumed.

- Faraday UMC L180E High Speed FSG Generic II Process technology library. Best case condition with $1.98 \mathrm{~V},-40^{\circ} \mathrm{C}$ parameters are assumed.

For some designs a physical synthesis and layout of was carried out using Cadence SoC encounter 9.1. The gate level netlist of the design from the logic synthesis step was used, for timing, libraries under typical conditions were used.

\subsubsection{ADL based Design Flow}

Hardware architectures for various cryptographic algorithms, that were not generated by the CRYKET HLS tool (in Chapter 5, 6 and 7) were implemented via a high-level synthesis framework, i.e., Synopsys Processor Designer [209]. The design description is captured using an ADL known as Language of Instruction Set Architecture (LISA) [39]. The language offers rich programming primitives to capture an implementation with full programmability such as a RISC processor to an implementation with no configurability at all e.g., an ASIC. From LISA, the hardware implementation as well as the software toolsuite, e.g., C compiler, simulator, assembler, linker can be automatically 


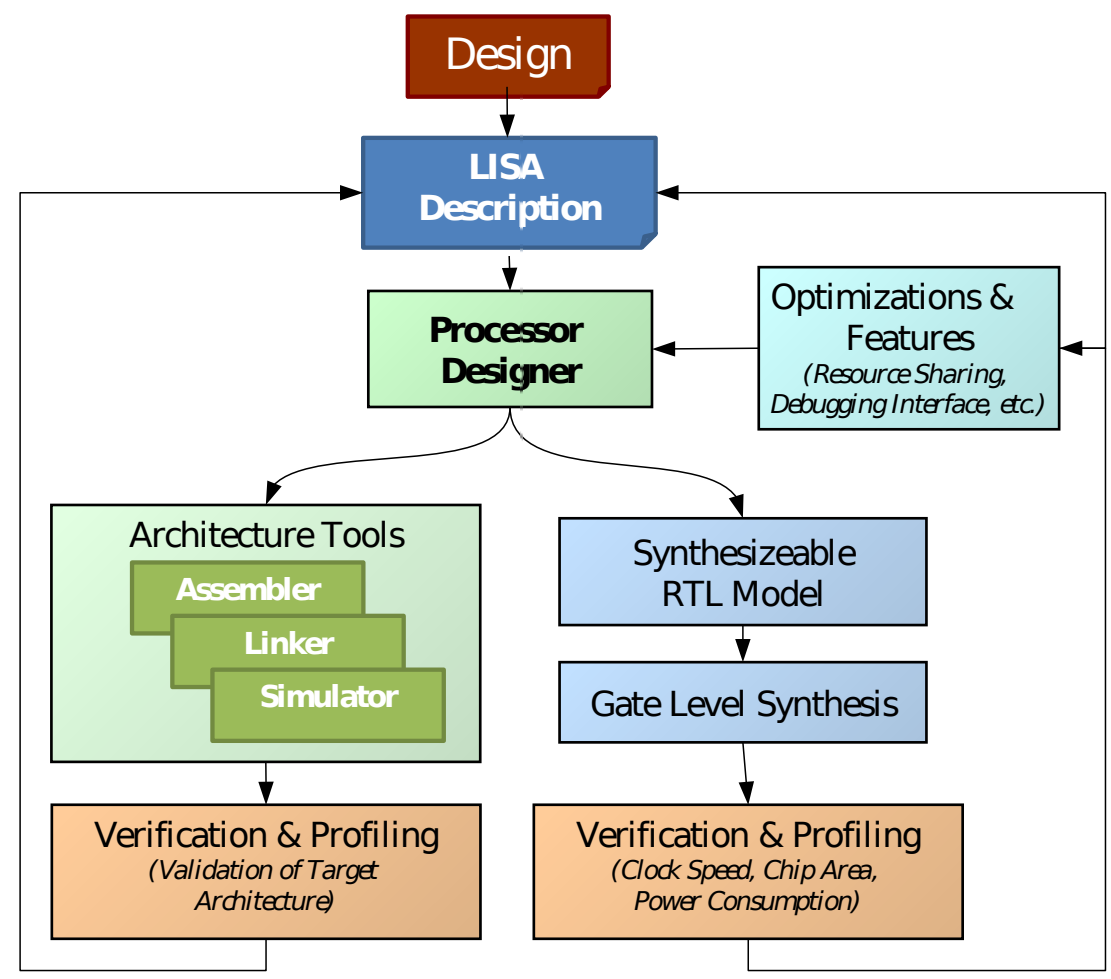

Figure 2.5: Implementation flow with LISA [209]

generated. The high-level synthesis environment allows easy control of the design parameters and fast exploration of design alternatives. The generated HDL code is further explored for verification and synthesis using the toolflow as discussed in the Section 2.3.1.

\subsubsection{Metrics}

To assess the efficiency of HDL implementations, the following set of basic metrics was used.

- Area: The area of a design is estimated by the synthesis tool and is specified either as $\mu m^{2}$ or as gate equivalents (GE), where one GE is equivalent to the area of a two-input NAND gate with the lowest driving strength of the appropriate technology.

- Power: The power consumption on the gate level is estimated by switching activity using Synopsys Primetime in $\mu \mathrm{W}$.

- Operating frequency: The operating frequency of the design used for synthesis. It is expressed as $\mathrm{MHz}$.

- Interface: bits processed per clock cycle

- Cycles: Number of clock cycles to complete a phase of algorithm. 
From the basic metrics, a set of derived metrics are calculated for performance comparison as given.

- Throughput: is the sustainable rate at which new output is produced. It is the product of the operating frequency and the interface bits produced per cycle. It is expressed as Byte/word/bits per second.

- Energy/bit: is the ratio of the total power consumption and the throughput.

- Area-time: is the product of the time taken to generate each bit and the area of the design. The reciprocal metric is Throughput Per Area Ratio. TPAR is specifically critical for high performance applications and is maximum at the highest operating frequency.

Some additional derived metrics, critical for lightweight cryptography or RFID applications are given.

- Power-area-time is a triple product metric that gives a quantitative comparison of all three critical resources of VLSI design, i.e., compactness, throughput and power consumption.

- latency is the response time of a design before it becomes functional (initialization latency) which is more critical for RFID applications.

- Power-latency is the product of power consummation and latency time.

\subsection{Experimental Setup for CPU-GPGPUs Environment}

Chapter 7 discusses the parallelization of cryptographic tasks on a CPU-GPU environment by device-specific and compiler-specific parameter optimization. The multi-core device undertaken is an NVIDIA GeForce GTX 590. The development environment used was Compute Unified Device Architecture (CUDA) version 5.5 (July 2013). To quantify the speedup against a general purpose processor, an AMD Phenom ${ }^{\mathrm{TM}} \mathrm{II}$ X6 1100T Processor (clockspeed $2.8 \mathrm{GHz}$ ) with 8 GB of RAM and Linux operating system was used as a host.

\subsection{Concluding Remarks}

This chapter sets the motivation for High Level Synthesis in general and in the domain of cryptography in particular. Various steps in traditional high level synthesis of a design starting from abstract specifications down to a digital HDL description are discussed. A brief summary of some of the salient features of various HLS tools that are being currently in use by the academia and industry is given. Efforts for HLS tools in the domain of cryptography, that are few, are also discussed. 


\section{Chapter 3}

\section{Dwarfs of Cryptography}

The goal of this research is to be able to develop better performance optimized hardware implementations for the domain of cryptography. To enable that, this chapter first focuses on the need of classifying and identifying a range of operations that are representative to a whole range of algorithms belonging to a class of specific application, in this work, cryptography. This chapter elaborates the computational workloads of symmetric key cryptography, before discussing the automatic high level synthesis of cryptographic workloads, discussed in the subsequent chapters.

\subsection{Berkeley Dwarfs for Parallel Computing}

The idea of combining computational workloads under a class or dwarf that captures a pattern of computation and communication was presented from Berkeley [10]. A total of 13 Berkeley dwarfs were presented, each of which capture the major algorithmic functionality and data movement pattern across an entire class of a major application. The dwarfs classify characteristic computation and data movement at a high level of abstraction to enable manifestation across a broad range of applications as shown in Fig. 3.5. Though the member applications of a particular class may change over time, but the claim is that the constituent dwarfs have persisted through generations of scientific computing and will remain important in the future too [10]. This classification
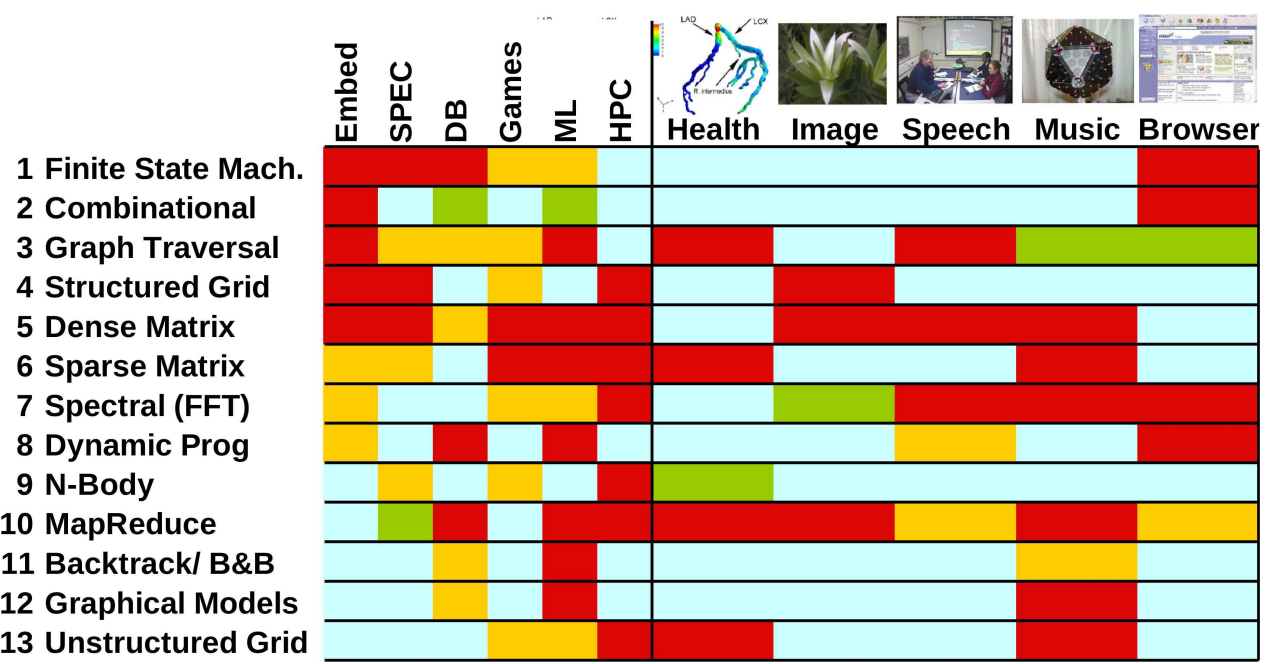

Figure 3.1: How applications relate to the 13 Berkeley dwarfs [10] (Red depicts highest relevance while blue depicts the lowest) 


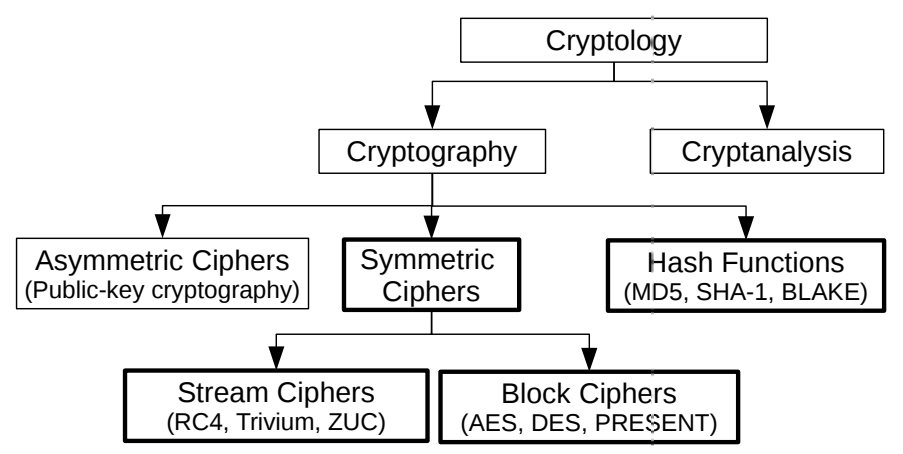

Figure 3.2: Classification of Cryptology

will help design better systems, by exploiting parallelism in applications, programming models, systems softwares etc. running on a naturally parallel hardware. A similar idea of combining computational workloads under a class that captures a pattern of computation and communication is presented by Intel Recognition-MiningSynthesis (RMS) view [61].

Since a dwarf coarsely captures a variety of methods, it's convenient to view each having several subclasses. The public key cryptography involves high-level bit manipulations, generally requiring discrete logarithm computations, integer factorization algorithms, algebraic computations of elliptic curves over Galois fields etc. Cryptanalysis techniques employ linear algebraic attacks, including both Dense Matrix and Sparse Matrix dwarfs. The symmetric key encryption algorithms rely heavily on low-level bit manipulations, including Boolean operations. This relates chiefly to the Combinational Logic dwarf. Symmetric key cryptography algorithms almost never perform a control flow based on the input data. Such applications are highly suitable for HLS since no complicated data check handshakes are needed for control flows. The public key cryptography algorithms generally have control dependencies in the execution flow. For controller the Finite State Machine is the relevant dwarf.

\subsection{Cryptology Background}

Cryptology is the art and science of building and breaking cryptographic functions. Hence it consists of two parts, one being study of building primitives called cryptography, the other being the analysis of these primitives called cryptanalysis. These branches are interlinked as Cryptanalysis investigates the security of a primitive and gives feedback, based on which secure primitives are proposed by cryptography, challenging cryptanalysis to try harder at attacking the primitive. As depicted in Fig. 3.2, cryptography has three branches; Symmetric ciphers (secret-key), Asymmetric ciphers (public-key) and Hash functions. This work focuses on effective implementation of cryptographic primitives including Hash functions and two types of Symmetric ciphers including the Block ciphers and Stream ciphers. 
The following section of the report presents a definition of these categories of cryptographic primitives. Since the goal is to define the configuration space of these primitives for high level synthesis, the focus is strictly on their architectural/ operational constructs. Their complexity and cryptanalytic properties are therefore skipped but could be referred from [152, Chapter 7].

\subsubsection{Block Ciphers}

A block cipher is a mapping of a plaintext data block of size $S_{B}$ (blocksize) to an equal sized ciphertext block under the parametrization of a key (of size $S_{K}$, keysize). This deterministic mapping (encryption) should be invertible. The inverse function (decryption) generates the original plaintext given the ciphertext under the same (secret) key. Classical/ historical block ciphers include Caesar ciphers, Affine ciphers, Substitution ciphers, Poly-alphabetic substitutions, etc. These techniques are proven over time to be cryptanalytically vulnerable and not suitable for practical use today [152, Chapter 7].

The product ciphers make the most popular class of block ciphers (and lightweight block ciphers) used today. A product cipher combines multiple data transformations so as to make the resulting cipher more secure than the individual transformations. These transformations may include permutations (adding diffusion), substitutions (adding confusion), translations (e.g., XOR), linear transformations (e.g., rotation), arithmetic operations, modular multiplication, transpositions etc. An iterated product cipher involves sequential repetition of a set of transformations called a round function. The round function iterate $N_{r}$ (roundcount) number of times during encryption/ decryption. For $i^{\text {th }}$ round a subkey $y_{i}$ (of size $S_{S K}$ ) is generated. Block ciphers are one of the most popular cryptographic transformations. Two major and popular classes of iterated product ciphers are defined as follows [152, Chapter 7].

1. A Substitution-Permutation (SPN) Cipher is an iterated product cipher with the round function being composed of a number of stages each involving substitutions and permutations. To ensure inevitability, for each of the generated subkeys the round function is a bijection on the round input.

2. A Feistel Network Cipher is an iterated product cipher whose each round splits data, passes one half to the round function and swaps the two data halves. Hence Feistel cipher operates on alternating halves of the ciphertext, while the other remains constant. The round function need not be invertible to allow inversion/decryption of the Feistel cipher.

\subsubsection{Stream Ciphers}

Stream ciphers encrypt a stream of individual characters (bits or words), while block ciphers operate on chunks of blocks. Unlike the memoryless nature of block ciphers, the encryption/decryption transformation of stream ciphers is dependent on their 

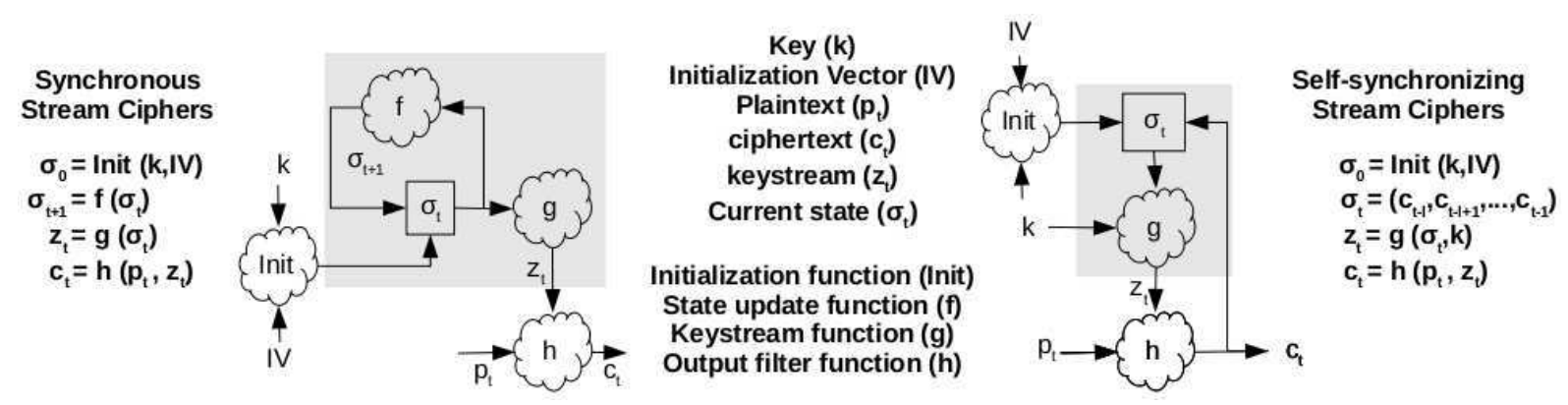

Figure 3.3: Synchronous stream cipher (left) and self-synchronizing stream cipher (right)

current state, consequently, they are also termed as state ciphers. Their judiciouslychosen lightweight Boolean operations make them suitable for environments where resources are restricted and sustaining a high throughput is critical.

Two classes of stream ciphers are Synchronous and Self-synchronizing stream ciphers. A formal definition from [152, Chapter 6] and a block diagram of the two types is depicted in Fig. 3.3.

The initial state $\left(\sigma_{0}\right)$ of stream ciphers is generated by Initialization function (init) based on key $(k)$ and Initialization Vector $(I V)$. For a synchronous stream cipher, the State update function $(f)$ calculates next state $\left(\sigma_{t+1}\right)$ depending upon the current state only, while for self-synchronizing stream ciphers, the internal state comprises of a fixed number $(l)$ of previous ciphertexts generated. Due to this dependence on ciphertext, the keystream for self-synchronizing stream ciphers cannot be precomputed. The keystream function ( $g$ ) transforms the current state (and the key in case of self-synchronizing stream ciphers) to generate keystream. Additive stream ciphers have Output filter function $(h)$ as a XOR function, the inverse function $\left(h^{-1}\right)$ at receiver is also a XOR function. Additive stream ciphers generating bits of keystream are termed as Binary additive stream ciphers.

\subsubsection{Hash Functions}

A hash function $h$ compresses an input data stream $x$, to an output message digest $h(x)$ of fixed length $n$ where $x>n$. Their primary goal is to authenticate message integrity; the digest is sent along the message and recomputed at the receiver's end to detect forgery by an adversary. Hashs are effective since the the probability of two different plaintext messages yielding the same hash value is extremely low (mathematically when an $\mathrm{x}$ bit number is mapped to an $\mathrm{n}$ bit number, $2^{x-n}$ different input strings can result to same output string). Secure hash functions must have three properties, namely pre-image resistance (one-wayness), $2^{\text {nd }}$ pre-image resistance (weak collision resistance), and collision resistance (strong collision resistance) [175, Chapter 7]. Hence ideal hash functions should have the property of having low computational overhead, computationally infeasible to reverse the hash value or get two messages with same hash, single bit change in message leads to volcanic effect in hash. 
Hash functions are used in applications such as Message Authentication Codes (MACs), digital signatures, key derivation, etc. Most of the hash functions perform as iterative process, successively taking fixed-size $r$-bit blocks called $x_{i}$ of the input stream $x$. Some preprocessing is required to pad the input stream to make the overall bitlength a multiple of $r$. A compression function $(f)$ takes $x_{i}$ chunk of data to generate an intermediate hash value $h_{i}$ of length $n$ in the $i^{\text {th }}$ iteration of compression function. This intermediate hash value serves as a chaining variable in the next iteration of $f$ with $h_{0}$ being a pre-defined starting value or initializing value (IV). Given $x$ partitioned into $t$ chunks after preprocessing such that $x=x_{1}, x_{2}, \ldots, x_{t}$, the $h_{t}$ is the final hash value calculated as under [152, Chapter 9].

$$
h_{0}=I V ; \quad h_{i}=f\left(h_{i-1}, x_{i}\right), 1 \leq i \leq t ;
$$

Hash functions are often constructed from block ciphers; consequently, only the primitive classes other than the SPN and Feistel network constructions used for block ciphers are given below.

1. ARX: Addition (A), Rotation (R) and Xor (X) make a functionally complete set of operations to build up any cryptographic function [125, Section 5]. Hence a suitable sequence of ARX operations may lead to a secure cryptographic primitive (block ciphers, stream ciphers, hash functions). Noticable examples include BLAKE [12] and Skein [73] hash functions, the ARX based hash functions that were selected in the final round of SHA-3 competition [197].

2. Sponge Constructions: Sponge constructions offer an entire suite of cryptographic functionalities (block ciphers, stream ciphers, PRNGs and MACs) based on sponge-like constructive functions constituent of ARX/ SPN/ logical operations. The usability, security and efficiency of sponge functions has already been scrutinized and appreciated by the cryptanalytic community, success stories include SHA-3 winner [23] and Spritz [186] from the authors of the RC4, as its replacement.

\subsection{Block Ciphers as Workhorses of Symmetric Key Cryptography}

Block ciphers enable secrecy of encrypted data, not beyond a single block of data. For avoiding the weakness of generating identical ciphertexts for identical plaintext blocks, chaining dependencies between adjacent plaintext blocks are added, defined by modes of operations. Under various modes of operation, block ciphers enable data transmission having major services of Information Security (InfoSec) including authenticity, integrity and confidentiality. These modes transform block ciphers to other cryptographic primitives, making them the workhorses of symmetric key cryptography and consequently making their study imperative. Other than these operational modes, the basic deterministic transform functions of block ciphers serve as elementary kernels or building blocks for many symmetric key cryptographic protocols. 


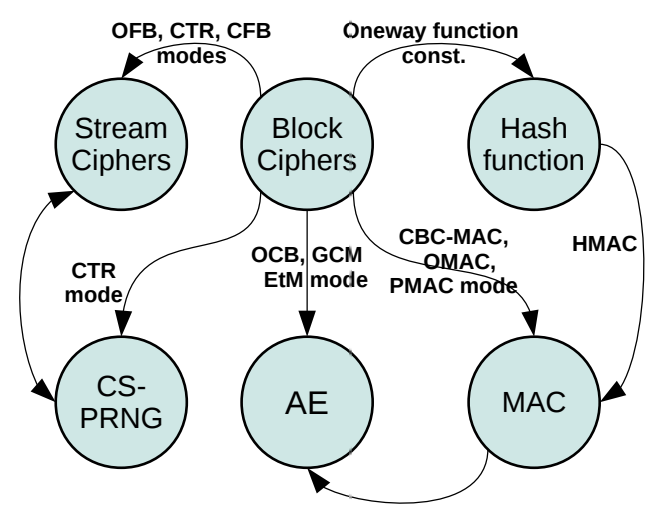

Figure 3.4: Modes of operation for cryptographic functions

\subsubsection{Transformations Under Modes Of Operation}

Fig. 3.4 highlights this constructive nature of the block ciphers being used as other cryptographic functions including stream ciphers, hash functions, MACs and CSPRNGs. In this context, a few examples of cryptographic functions, which are driven by the underlying round function of a block cipher under a mode of operation are mentioned.

1. Stream Ciphers: The distinction between a stream cipher and a block cipher is murky, stream ciphers are generally less computationally expensive. Block ciphers are transformed to stream ciphers under Counter mode (CTR) and Output Feedback Mode (OFB) [62]. SOSEMANUK [18], an eSTREAM finalist stream cipher, uses a block cipher SERPENT for its construction.

2. Hash functions: Hash functions may be driven from a block cipher, with operating schemes/modes that make them non-invertible one-way compression functions, e.g., Matyas-Meyer-Oseas, Davies-Meyer, Miyaguchi-Preneel construction schemes transform block ciphers to modification detection codes (MDCs), a major application of hash functions [152, Chapter 9]. WHIRLPOOL is AES-based block cipher operating under a Miyaguchi-Preneel hashing construction [17].

3. Message Authentication Codes (MACs): MACs may be driven from hash functions in HMAC mode or from block ciphers in Cipher Block Chaining MAC (CBC-MAC), One-key CBC MAC (OMAC), Parallelizable MAC (PMAC) modes, etc.

4. Cryptographically Secure Pseudo-Random Number Generator (CSPRNG): A CSPRNG can be driven from a block cipher operating in counter mode of operation. Also, running a stream cipher on a counter returns a CSPRNG, with its initial state kept secret.

5. Authenticated Encryption (AE): Authenticated encryption is generically constructed by combining a block cipher and a MAC operating under a mode of 
operation, hence simultaneously providing confidentiality, integrity and authenticity assurances on the data. Various modes of authenticated encryption have been standardized by ISO [106].

The block ciphers serve as the main workhorses of cryptography, their basic round functions are often used to construct other cipher functions. Some constructions include hashing schemes BLAKE [12] and Skein [73], based on LAKE and Threefish block cipher primitives, respectively. However, cipher functions may have roots of origin other than block ciphers. Most of the popular stream ciphers are constructed using LFSRs along with some non-linear combining functions and an FSM. Similarly, many CSPRNGs originate from number theory problems. Also worth mentioning is the fact that cryptographic functions take inspiration from each other too. SEAL, HC128 and HC-256 are stream ciphers that make use of SHA family of hash functions for their key expansion phase, SHACAL is a block cipher based on SHA-1. Many stream ciphers and CSPRNGs have common roots.

\subsubsection{Commonalities in the Computational Building Blocks of Symmetric Key Cryptography}

This section attempts to unconventionally classify the major functions of symmetric key cryptography (block ciphers, stream ciphers, hash functions) on the basis of their primitive computational elements. This classification exposes high degree of commonalities between them, beneficial to their implementation, both on hardware and software platforms. The fact that different cryptographic functions have common Computational kernels is also highlighted.

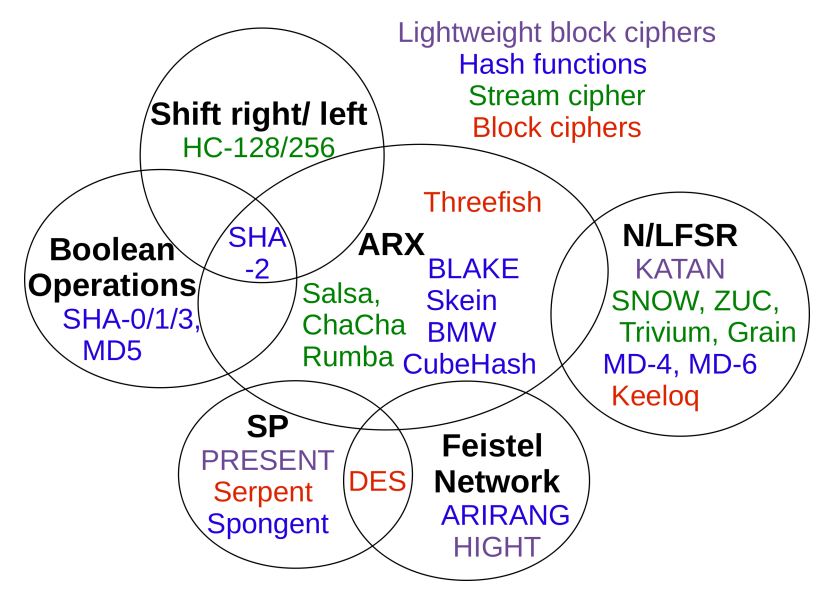

Figure 3.5: Computational commonalities of symmetric key cryptographic functions

Fig. 3.5, a subset diagram, capturing the computational kernels of symmetric key cryptography. ARX class of cryptographic functions, combining linear $(X, R)$ operations and nonlinear (A) operations, iterated over multiple rounds achieves strong resistance against known cryptanalysis techniques [125]. The term AXR (later renamed to ARX) was coined by Ralf-Philipp Weinmann [221]. The algorithmic sim- 
plicity, efficient implementations in software, absence of timing attacks of ARX cipher contribute to their popularity [157]. The bitwise shift operation is added to the ARX pool of operations for construction of some new cryptographic functions like HC series of stream ciphers [224]. Similarly, addition of Boolean operations (AND, NOT) make the computational basis of hash functions including MD5 [185] SHA-0,1 [166] and SHA-3 [23]. Interestingly, SHA-3 (Keccak) originates from the concept of flexible sponge constructions for cryptographic functions, however, classification based on the underlying operations brings SHA-0, 1 and SHA-3 simply under the common axis. SHA-2 [166] requires bitwise shifting as well as Boolean operations in addition to the ARX pool of operations, indicated in Fig. 3.5.

Many stream ciphers are based on a Linear/nonlinear Feedback Shift Register (N/LFSR) whose inputs are selected from the previous state after linear/nonlinear functions are applied on them. Taking examples from eSTREAM finalists [65] include SOSEMANUK, and all its three finalists in the hardware profile. FSRs have been employed in the construction of block ciphers and hash functions too, some examples are listed in Fig. 3.5. Feistel ciphers may use substitutions and permutations, other than the ARX operations, in their round functions. XOR is generally used for key whitening the round values with subkey of that round. Addition operation might not be explicitly used in round operations, however, a count-up/down counter is always required for encryption/decryption block realization, respectively. DES has a Feistel structure but employs SBoxes and PBoxes for its round operation. AES [165] does not have any PBoxes and rather uses Shiftrow operation and Galois field multiplication for diffusion. Computationally Feistel network ciphers require in addition only the provision of splitting data into equal parts (for balanced Feistel) or unequal parts (for unbalanced Feistel). For balanced Feistels, the round calculation operate on one half of the data and the two split data values are swapped before the next round. SPN and Feistel networks are primitives for lightweight cryptography as well, some examples are quoted in Fig. 3.5.

It's noteworthy to highlight that this computational categorization shown in Fig. 3.5 highlights only the commonalities as a trend in cryptographic functions. This categorization is neither complete nor by definition binding to a particular class of ciphers. Consequently, exceptions exist, e.g., TEA [222] family of lightweight block ciphers (XTEA, XXTEA) are Feistel Network ciphers by structure and use shift operations other than ARX. AURORA, a hash cipher for SHA-3 competition has a structure as a combination of SPN and a generalized Feistel structure [108]. Numerous cipher proposals are constructed from a combination of these operation classes.

\subsection{Cipher Algorithmic Configuration Space}

This sections tries to identify a complete set of algorithmic primitives that are generic enough to configure a family of cryptographic functions. After a survey of diverse ciphers belonging to a family, their sub-structures and component list is fully complete, that is required to develop primitive libraries for their automatic software and 
hardware realizations through tools. The cipher families taken up are block ciphers, stream ciphers and hash functions.

\subsubsection{Block Ciphers}

This work proposes a novel, so-called layered architecture where each layer specifies a data transformation in a cipher round, and is specified by an operation. To fully appreciate the concept of layers of operations, lets consider the data flow graph of the cipher (and its key expansion) where data is moving from top to bottom. The layers are then the horizontal divisions of the data flow diagram, as shown in Fig. 3.6. The plaintext (and key) pass through multiplexers to choose between the initial plaintext or the intermediate value of the data processed. The multiplexed values are stored in registers that are the only sequential elements of this round folded implementation. The round operations of the cipher are expanded and broken up as layers of operations called Round layers. (A similar layer breakup of the key update functions is possible to get Kround layers, however is not shown in Fig. 3.6)
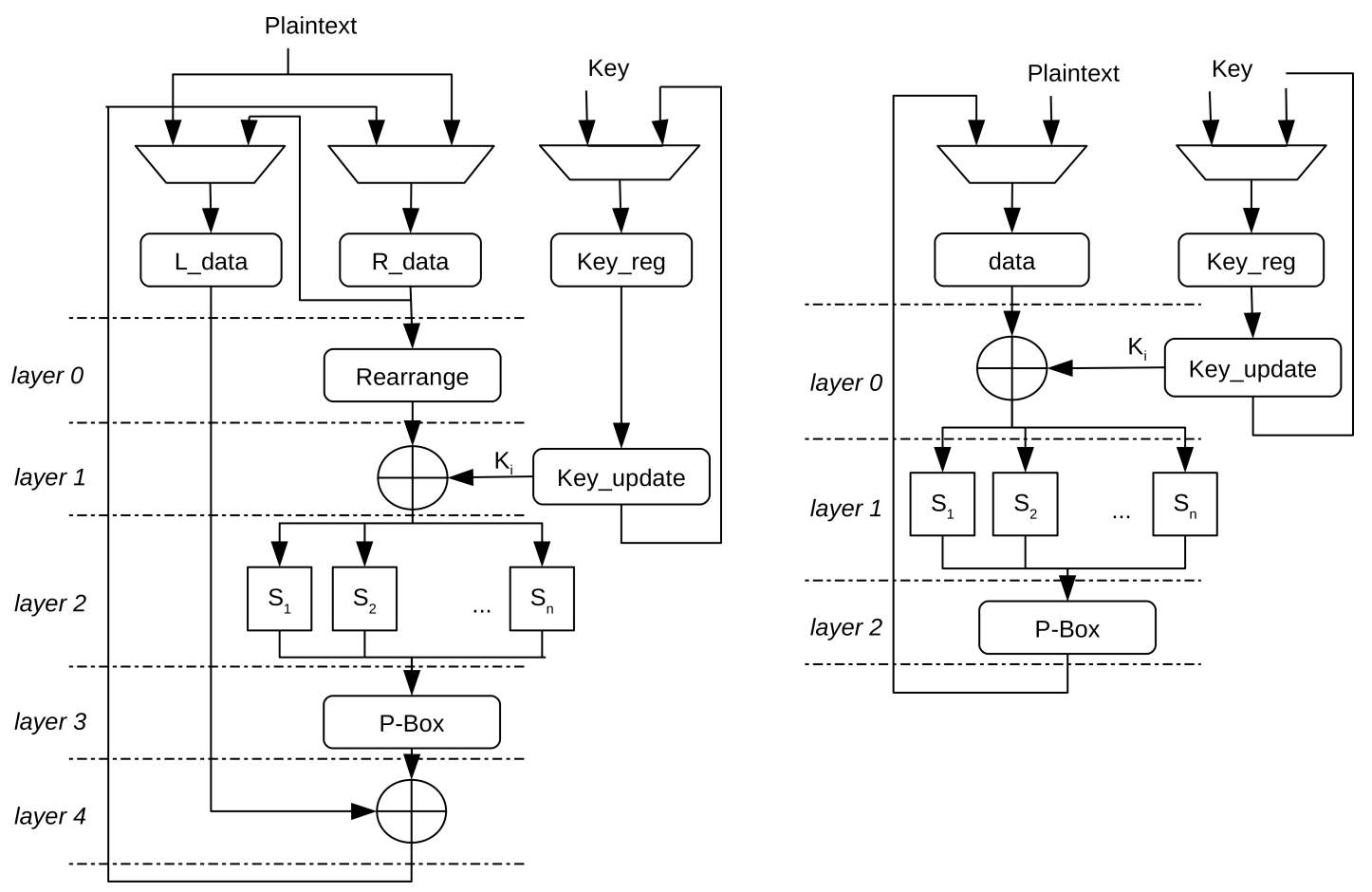

Figure 3.6: Block diagram of typical Feistel network cipher (left) and SPN cipher (right).

The algorithmic construction of a block cipher is described by Basic parameters, Round layers, Kround layers and a Mode of Operation. (All parameterizable attributes that a user must populate to define the algorithmic space of a block cipher are highlighted in the proceeding discussion).

- Basic Parameters: The input plaintext to a block cipher (encryption) and its output ciphertext are of equal size, blocksize $\boldsymbol{S}_{\mathbf{B}}$ (all sizes specified in bits). The 
size of Key is specified as $\mathbf{S}_{\mathbf{K}}$ (for some operational modes an IV (initialization vector) having size $S_{I V}$ must also be specified). The granularity of the cipher is specified as wordsize $\left(\mathbf{S}_{\mathbf{W}}\right)$ of the cipher. Block ciphers iterate a deterministic combination of operations known as a round. The rounds operate $\mathbf{N}_{\mathbf{r}}$ (roundcount) number of times during encryption/ decryption.

- Round Layers: For most block ciphers, the data undergoes an initial and/or final transformation, before and/or after the rounds processing, respectively. Since these transformations may differ from each other and from the central round transformation, they are named as round_init and round_final, while the round transformation is referred as as round_middle. These three kinds of rounds are defined by a series of layers of operations. Every layer comprises of at least one of the following operations, performed exclusively on the user specified bit-portions of the layer input. The layers of each round have a layernumber to specify their order of execution, within that round. The input to and output from a layer may differ in size (bits) due to an expansion/ compression layer operation and is specified as $\mathbf{S} \_$lin and $\mathbf{S}$ _lout, respectively. (The following list could be conveniently extended to accommodate newer cipher proposals).

1. Substitution boxes (SBox) Substitution introduces confusion in round. It operates on chunks of layer data (of size $S_{W}$ ) and carries out parallel application of functions $S:\{0,1\}^{S_{W}} \rightarrow\{0,1\}^{S_{W}}$. It is implemented as a lookup table with $2^{S_{W}}$ entries (The input and output size of SBoxes may also differ).

2. Permutation boxes (PBox) Permutation layer is an affine transformation to introduce diffusion in round. It is carried out by a PBox that shuffles all input bit entries, in hardware it is implemented simply by rewiring input and outputs appropriately.

3. Galois field operations GF-mul with another polynomial (primitive polynomial must be specified).

4. Bitwise operations including Rotation, Shifting, Addition, XOR-ing, ARK (Add Round Key), AddCounter (XOR-ing with counter).

5. Operations specific to Feistel networks including Split, Swap.

6. Compound operations used in popular ciphers, e.g., ShiftRows, MixColumns used in AES [165]

7. No operation (nop)

- Kround Operation: For each round, a subkey (of size $\mathbf{S}_{\mathbf{S K}}$ ) is generated through key expansion. Like rounds, key expansion requires iteration of kround transformation, $N_{r}$ (roundcount) number of times to generate subkeys. kround may also have different definitions for kround_init, kround_middle and kround_final, each of them are defined by layers of operations like cipher rounds.

- Mode of Operation: The mode of operation must be specified to add the chaining dependencies between adjacent blocks of data during encryption / decryption. 
Table 3.1 lists the NIST standardized modes of operation [62]. Here $C_{i}$ represents ciphertext for the $i^{t h}$ plaintext block after encryption function parameterized by the secret key $E_{k}$ while $P_{i}$ represents the plaintext after decryption. Table 3.1 also specifies whether multiple blocks of data can be subjected to encryption or decryption in a parallel fashion for some modes of operations or not. For example, due to the chaining dependencies, CBC encryption is not possible in parallel. For all modes other than ECB, the user specifies the Initialization vector(IV) and any additional parameters required (nonce, counter).

Table 3.1: NIST standardized modes of operation modes of operation for block ciphers

\begin{tabular}{|c||c|c||c|c||c|}
\hline Mode of operation & Encryption & $\|$ & Decryption & $\|$ & Initialization Vector \\
\hline Electronic codebook (ECB) & $C_{i}=E_{k}\left(P_{i}\right)$ & $\boldsymbol{V}$ & $P_{i}=D_{k}\left(C_{i}\right)$ & $\boldsymbol{}$ & - \\
\hline Cipher block chaining (CBC) & $C_{i}=E_{k}\left(P_{i} \oplus C_{i-1}\right)$ & $\boldsymbol{x}$ & $P_{i}=D_{k}\left(C_{i}\right) \oplus C_{i-1}$ & $\boldsymbol{V}$ & $C_{0}=I V$ \\
\hline Propagating CBC (PCBC) & $C_{i}=E_{k}\left(P_{i} \oplus P_{i-1} \oplus C_{i-1}\right)$ & $\boldsymbol{x}$ & $P_{i}=D_{k}\left(C_{i}\right) \oplus P_{i-1} \oplus C_{i-1}$ & $\boldsymbol{x}$ & $P_{0} \oplus C_{0}=I V$ \\
\hline Cipher Feedback (CFB) & $C_{i}=E_{k}\left(C_{i-1}\right) \oplus P_{i}$ & $\boldsymbol{x}$ & $P_{i}=D_{k}\left(C_{i-1}\right) \oplus C_{i}$ & $\boldsymbol{V}$ & $C_{0}=I V$ \\
\hline Output Feedback (OFB) & $C_{i}=P_{i} \oplus O_{i}$ & $\boldsymbol{x}$ & $P_{i}=C_{i} \oplus O_{i}$ & $\boldsymbol{x}$ & $O_{i}=E_{k}\left(O_{i-1}\right), I_{0}=I V$ \\
\hline Counter (CTR) & $C_{i}=P_{i} \oplus E_{k}(I V)$ & $\mathbf{v}$ & $P_{i}=C_{i} \oplus D_{k}(I V)$ & $\boldsymbol{V}$ & nonce + counter $=I V$ \\
\hline
\end{tabular}

\subsubsection{SPN based Block Ciphers Survey:}

Both Feistel networks and SPN ciphers make the most popular class of block cipher primitives today. The different parameter values for prominent SPN-based block ciphers are presented in the Table 3.2. The listing is non-exhaustive, arranged in chronological order. A similar configuration space is required for Feistel ciphers. Parameters for some ciphers against which no information was found are marked by (-). AES (or Rijndael) is one of the most prominent SPN based block cipher. It offers flexibility in terms of security by variable key sizes and round iterations. Its security remains unchallenged till date and its design has inspired many other cryptographic proposals.

Two prominent ciphers and taken up and their algorithmic configuration according to the discussed layered architecture definition methodology is tried out. These being AES-128 [165] due to its widespread usage and PRESENT [28] due to its ultra lightweight nature. Moreover, both of these ciphers have been standardized by ISO. Fig. 3.7 shows the division of round operations for these ciphers into layers of operation. The configuration space for these ciphers in ECB Encryption modes is defined. The reader is kindly requested to refer to the documentation of these ciphers for a detailed understanding of their functionality [28,165].

\subsubsection{PRESENT-80 [28] Configuration}

Table 3.3 shows the basic parameter configuration space for 80 bit key of PRESENT cipher. It includes sizes of block, key and word sizes and the information of rounds for encryption. 
Table 3.2: A list of various SPN block cipher algorithmic configurations

\begin{tabular}{|c|c|c|c|c|c|c|c|c|c|c|}
\hline \multirow{2}{*}{ Algorithm } & \multicolumn{4}{|c|}{ Size (in bits) } & \multirow{2}{*}{ Rounds } & \multicolumn{2}{|c|}{ SBox } & \multirow{2}{*}{ PBox } & \multirow{2}{*}{ Reference } & \multirow{2}{*}{ Year } \\
\hline & Key & Block & Input & Output & & In $\times$ Out & Count & & & \\
\hline SXAL/MBAL & 64 & 64 & 64 & 64 & 8 & $8 \times 8$ & 1 & Yes & [74] & 1993 \\
\hline 3 WAY & 96 & 96 & 96 & 96 & 11 & - & - & - & [50] & 1993 \\
\hline BaseKing & 192 & 192 & 192 & 192 & 11 & - & - & - & [49] & 1994 \\
\hline SHARK & 128 & 64 & 64 & 64 & 6 & - & - & Yes & [183] & 1996 \\
\hline SQUARE & 128 & 128 & 128 & 128 & 8 & $8 \times 8$ & 16 & Yes & [51] & 1997 \\
\hline AES & $128,192,256$ & 128 & 128 & 128 & $10,12,14$ & $8 \times 8$ & 16 & No & {$[53,165]$} & 1998 \\
\hline SERPENT & $128,192,256$ & 128 & 128 & 128 & 32 & $4 \times 4$ & 32 & Yes & [26] & 1998 \\
\hline CRYPTON & $128,192,256$ & 128 & 128 & 128 & 12 & $8 \times 8$ & 16 & Yes & [141] & 1998 \\
\hline ANUBIS & $128-320$ & 128 & 128 & 128 & $\geq 12$ & $8 \times 8$ & 16 & Yes & [182] & 2000 \\
\hline Hierocrypt-3 & $128,192,256$ & 128 & 128 & 128 & $6.5,7.5,8.5$ & $8 \times 8$ & 16 & Yes & [173] & 2000 \\
\hline KHAZAD & 128 & 64 & 64 & 64 & 8 & $8 \times 8$ & 8 & Yes & [16] & 2000 \\
\hline NOEKEON & 128 & 128 & 128 & 128 & 16 & $4 \times 4$ & 32 & Yes & [52] & 2000 \\
\hline ICEBERG & 128 & 64 & 64 & 64 & 16 & $8 \times 8$ & 8 & Yes & [201] & 2000 \\
\hline MESH & $128,192,256$ & $64,96,128$ & 128 & 128 & $8.5,10.5,12.5$ & - & - & Yes & [159] & 2002 \\
\hline ARIA & $128,192,256$ & 128 & 128 & 128 & $12,14,16$ & $8 \times 8$ & 16 & Yes & [135] & 2003 \\
\hline PRESENT & 80,128 & 64 & 64 & 64 & 32 & $4 \times 4$ & 16 & Yes & [28] & 2007 \\
\hline PUFFIN & 128 & 64 & 64 & 64 & 32 & $4 \times 4$ & 16 & Yes & [41] & 2008 \\
\hline HummingBird & 64 & 16 & 16 & 16 & 4 & $4 \times 4$ & 4 & Yes & {$[68]$} & 2010 \\
\hline PrintCipher & 80,160 & 48,96 & 48,96 & 48,96 & 48,96 & $3 \times 3$ & 16,32 & Yes & [132] & 2011 \\
\hline LED & 64,128 & 64 & 64 & 64 & 32,48 & $4 \times 4$ & 25 & Yes & [89] & 2011 \\
\hline KLEIN & $64,80,96$ & 64 & 64 & 64 & $12,16,20$ & $4 \times 4$ & 16 & Yes & [79] & 2012 \\
\hline PRINCE & 128 & 64 & 64 & 64 & - & $4 \times 4$ & 16 & - & [30] & 2012 \\
\hline
\end{tabular}

A round_init is not required hence no layers are defined for it. The round_middle is defined by the following 3 layers of operation:

- layer0 is the $A R K$, where input and output to the layer is equal sized. Data is XOR-ed with the subkey, where subkey $=k e y[79: 16]$.

- layer 1 is the $S B o x$, specified by a total of 16 SBoxes $\left(\frac{S_{B}}{S_{W}}\right)$ to be inserted. All SBoxes are similar and hence that SBox requires $2^{S_{W}}$ values for its complete specification, each $S_{W}$ bits wide.

- layer 2 is the $P B o x$, with a total of $S_{B}$ arguments $\in\left[0 . . S_{B}\right]$.

The round_middle iterates 31 times while the round_final executes only once. The round_final is specified by one layer of ARK, same as the first layer of round_middle. Hence the ciphertext is taken out after the first ARK layer in the last iteration of cipher encryption, as shown in Fig. 3.7. It's noteworthy to comment here that the initial XOR tranformation of PRESENT-80 [28] is instead handled in the first iteration of round_middle. Hence a round_final must be defined to carry out the XOR of the last iteration of round_middle. This definition saves a 64 bit XOR overhead, details follow in the subsequent chapters. It should also be highlighted that layered methodology allows multiple different however algorithmically equivalent valid definitions 

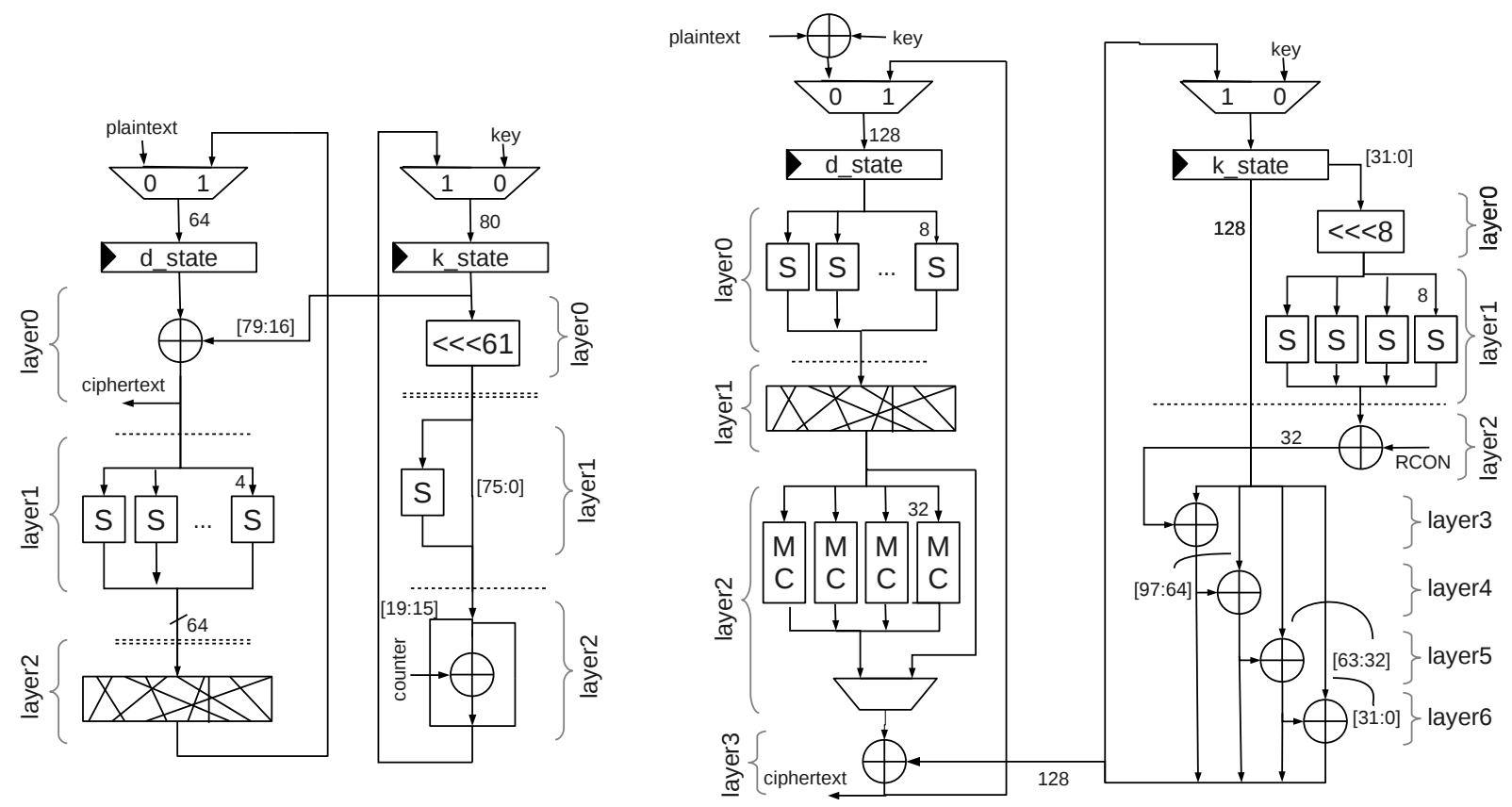

Figure 3.7: Layers for the loop folded implementation of PRESENT-80 (left) and AES-128 (right)

of the same cipher. The choice of the suitable definition depends on the architectural/ structural preference (discussed in next chapter).

For key expansion in PRESENT-80, the kround_init and kround_final are not required and hence defined as having no layers. The kround_middle requires three layers of operations defined below.

- layer 0 is the ROTATE operation configured to carry out a left rotation by 61.

- layer 1 is the SBox. It is specified to be inserted at the most significant nibble of the layer 1 input. Following a little-endian convention, it is the word number 19 of the key. The rest of the bits are passed on un-altered.

- layer2 is the AddCounter that XORs the selected bits of the data (bit 19 till 15) input to the layer with a 5-bit counter (round counter).

\subsubsection{AES-128 [165] Configuration}

For AES-128 a data flow diagram is drawn in Fig. 3.7 (right) with the corresponding basic parameters summarized in Table 3.3. The round_init requires one operation layer, i.e., $A R K$, shown by a XOR operation before the plaintext is being saved in the register. round_middle is defined by the following 4 layers.

- layer0 is SBox. 16 SBoxes are required along with SBox definition of 256 bytes (all SBoxes are similar). 
Table 3.3: Basic parameters for layered configuration space for two prominent ciphers

\begin{tabular}{|c|c|c|}
\hline Parameter & PRESENT-80 [28] & AES-128 [165] \\
\hline$S_{B}$ (bits) & 64 & 128 \\
\hline$S_{K}$ (bits) & 80 & 128 \\
\hline$S_{S K}$ (bits) & 64 & 128 \\
\hline$S_{W}$ (bits) & 4 & 8 \\
\hline$N_{r}$ (rounds) & 32 & 10 \\
\hline round_init (layers) & 0 & 1 \\
\hline round_middle (layers) & 3 & 4 \\
\hline round_final (layers) & 1 & 3 \\
\hline kround_init (layers) & 0 & 1 \\
\hline kround_middle (layers) & 3 & 7 \\
\hline kround_final (layers) & 0 & 0 \\
\hline
\end{tabular}

- layer1 is a Shift-rows operation. Its a compound operation that takes up the layer input as a 2-D matrix and and re-arranges the words of each rows with fixed offsets.

- layer2 is a GF - Mix, a compound operation assuming 2-D arranged data. The user specifies a $4 \times 4$ column coefficients for $\mathrm{GF}\left(2^{8}\right)$ multiplication.

- layer3 is the $A R K$, that XORs the key with the data.

A cipher may have multiple valid definitions using this layered definition. The Shift-rows operation in layer 1 may have been defined using various layers, each rotating one row of the state matrix only, as defined by the AES specifications. However since its a popular operation (used in ciphers other than AES, e.g., LED), it is defined as a standard compound operation described by a single layer of operation. The round_final is defined by 3 layers, same as layer 0, layer 1 and layer 3 of round_middle. The GF-Mix operation is not required for the final round, consequently a bypass of this function by a multiplexer is shown in Fig. 3.7.

For each round a subkey is generated through a kround. The kround_init is a nop layer since the first subkey is the input key itself. The kround_final is not required and hence not defined. kround_middle requires 7 layers of operations for its definition as shown in Fig. 3.7.

- layer0 is a ROTATE left by 8 layer. It takes the least significant 32-bit word of the key. This layer also expands 128 bits of input to 160 bits of output by concatenating the input bits unaltered along with the rotated word output.

- layer1 is the SBox, 4 SBoxes are inserted on the 4 least significant bytes of layer1 input. 


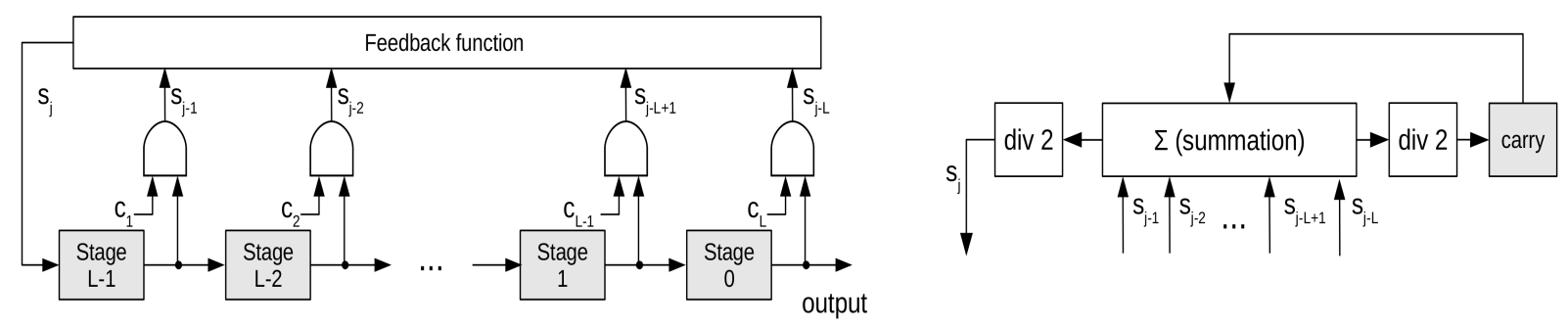

Figure 3.8: A Linear feedback shift register, LFSR (left) a carry Linear feedback shift register CLFSR (right)

- layer2 is a XOR with counter dependent constants (RCON). The constants are specified by the user using a text file.

- layer3-layer6 are XOR operations, performing selective XOR-ing of layer inputs as per AES specifications.

\subsubsection{Stream Ciphers}

Stream ciphers are also called state ciphers as their output transformation depends on their current state too. Consequently, a Stream cipher can be modeled as a regular clocked finite state machine (FSM). Like any FSM, it has sequential and combinational parts. The sequential registers that hold the internal state of stream ciphers have following typical structures.

\subsubsection{Sequential Structures}

- Finite Shift Register (FSR): An FSR comprises of $L$ delay elements, each of which is shifted to the next at each clock cycle, as shown in Fig. 3.8 (left). At each clock the contents of stage $i$ are shifted to stage $i-1$ for each $i, 1 \leq i \leq L-1$ The content of stage 0 forms the output of the FSR, while the new content of stage $L-1$ is calculated based on a feedback function. Each $c_{i}$ is a single bit number controlling the inclusion of $i^{\text {th }}$ stage value in the calculation of the feedback function. The reset value of the register is called its initial state. The categorization of types of FSR is done based on the nature of the feedback function. Some prominent ones are enlisted.

- linear FSR (LFSR): For an LFSR, the feedback function is the bit-wise XOR of the contents of all stages for which $c_{L-i}=1$, as shown in Fig. 3.8 (left).

$$
s_{j}=\left(c_{1} \cdot s_{j-1} \oplus c_{2} \cdot s_{j-2} \oplus \ldots \oplus c_{L} \cdot s_{j-L}\right) \text { for } j \geq L
$$

- Nonlinear FSR (NFSR): For an NFSR, the feedback function is nonlinear or a Boolean function.

$$
s_{j}=\text { feedbackfunction }\left(s_{j-1}, s_{j-2}, \ldots, s_{j-L}\right) \bmod 2 \text { for } j \geq L
$$



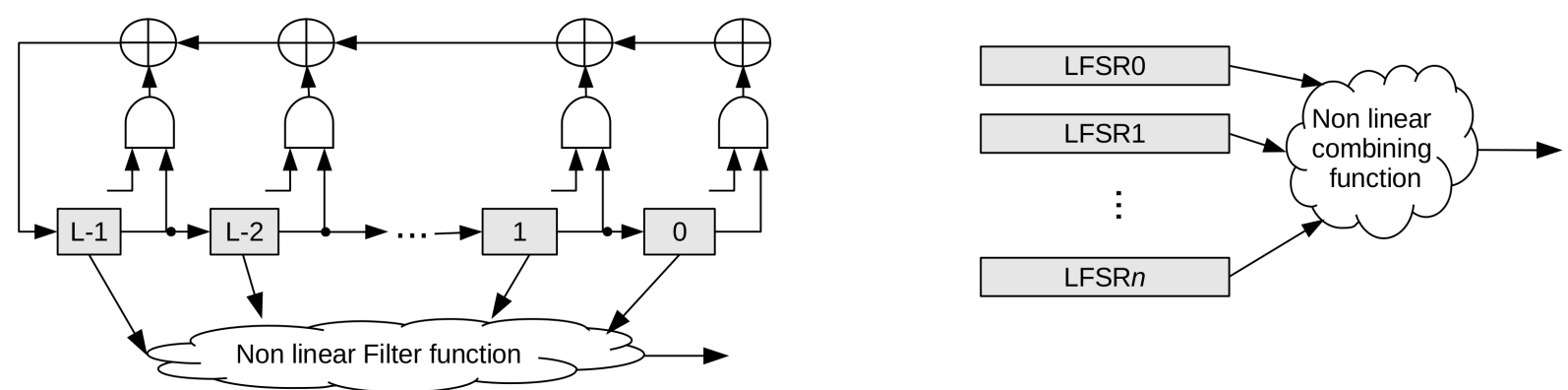

Figure 3.9: Nonlinear filter generator (left) Nonlinear output from multiple LFSRs (right)

- Carry shift FSR (CLFSR): A carry shift FSR is also an NFSR that has summation as feedback function, as shown in Fig. 3.8 (right). It keeps an extra memory bit carry to retain the carry from one addition to be added up in the next cycle's addition.

$$
\begin{aligned}
\text { sum } & =\left(c_{1} \cdot s_{j-1}+c_{2} \cdot s_{j-2}+\ldots+c_{L} \cdot s_{j-L}+\text { carry }\right) \text { for } j \geq L \\
s_{j} & =\text { sum } 2 \\
\text { carry } & =\text { sum } / 2
\end{aligned}
$$

- Jump Registers: Jump registers form a cascade of multiple delay elements, each of which implements an autonomous Linear FSM. Each of these delay elements have a corresponding controlling jump bit to make them jump to a new value [109]. Two noticeable eSTREAM candidate ciphers that use jump registers are MICKEY [13, 14] and POMARANCH [97].

- FSM Registers: Other than the cascade shift register constructions, sequential registers may also be used to hold the ciphers state. Stream ciphers using these FSM registers (other than FSRs) include SNOW 3G [2] and ZUC [232]. RC4 [195] and $\mathrm{HC}-128$ [224] require $2 \mathrm{~K}$ and $32 \mathrm{~K}$ bits of state information, respectively.

LFSRs are a favorite primitive for stream ciphers design due to their desirable statistical properties and hardware friendly nature. Their susceptibility to chosen plaintext attack must be overcome by breaking their linearity according to these recommended and analyzed constructions [152, Chapter 6]. For LFSR based stream ciphers, some popular nonlinear combination generators are listed below.

- Nonlinear Filter Generator: The output of an LFSR is generated through a nonlinear filter generator. At each clock it takes bits/words from fixed locations of LFSR to non-linearly generate output as shown in Fig. 3.9.

- Nonlinear Output from Multiple LFSRs: Multiple LFSRs are used in parallel and the keystream is generated as a nonlinear transformation of the outputs of 
LFSRs as shown in Fig. 3.9. Stream ciphers using this construction are called nonlinear combination generators and the nonlinear function is called the combining function. Geffe generator and summation generator are examples of such combining functions.

- Clock Controlled Generators: By definition, an LFSR is regularly clocked and its contents are consequently updated every clock cycle. Clock controlled generators introduce the non-linearity in LFSRs by irregular clocking. The LFSRs are not clocked at every cycle and clocking of one LFSR is controlled by the Boolean transformation of another LFSR taps and vice versa. This is done by making the output of one of the LFSRs control the clocking of the other. Some known techniques of clock controlled generators are alternating step generator, shrinking generator etc.

Other Designs: A nonlinear update function for a popular stream cipher RC4 [195] is the Triangular function (T-Function). RC4 has an internal state of 256 Byte array, denoted by $S[0 \ldots N-1]$ and accessed by indices $i$ and $j$. In every iteration of Keystream generation phase, the T-function increments $i$, updates $j$ by $j=j+S[i]$, swaps values of $S[i]$ and $S[j]$ and produces one byte of output as $S[S[i]+S[j]]$. Many RC4 variants, including $\mathrm{RC}^{+}$[147], VMPC [231] and the recently proposed Spritz [186] use RC4-like general design principle of T-function.

\subsubsection{Combinational Primitives}

The combinational primitives are required to configure and specify the initialization (init), state update function (f), keystream generation function $(g)$ and output filter function (h) of a stream cipher as given in Fig. 3.3. The feedback function of FSRs, jump registers and FSM registers as well as the functionality of nonlinear combination generators is described and configured using these combinational primitives. These primitives include Boolean, arithmetic and bitwise operations (including arithmetic/logical shifts, rotations). For non-binary stream ciphers, field operations, SBox and PBox are employed. Other glue logic elements like multiplexers are required to change the behavior of a cipher as its phase changes.

\subsubsection{Operational Phases}

Stream ciphers have the following three phases of operation.

- Key/IV Setup: This phase initializes the state of stream ciphers, based on the secret key and IV (or nonce, i.e., number used once) provided by the user. The key and IV values may require some preprossessing (init in Fig. 3.3) before being fed into the stream cipher structure. For FSR based stream ciphers, the Key/IV Setup requires at least as many cycles as the length of the FSR to alter its initial value. 
- Randomization/Runup/Warmup: The randomization phase sufficiently scrambles the state of the cipher based on Key and the IV, before the keystream is generated. Since this phase does not generate valid keystream, its duration is a trade-off between security and speed.

- Keystream Generation Phase: The keystream bits/words are produced as the randomization phase is over. A continuous keystream is generated that determines the throughput of the system.

Since no stream cipher output is generated during the first two phases, they are together named as keystream Initialization. For every new key, IV combination, Initialization must be carried out before valid keystream generation. Consequently, ciphers requiring frequent re-initialization have this initialization latency as a possible bottleneck.

\subsubsection{Configuration Space}

This section discusses the configuration space parameters, generic enough for any stream cipher. The configuration parameter set is categorized into elementary parameters, sequential state information and a mesh of operations. (All parameterizable attributes that a user must populate are highlighted in the proceeding discussion).

- Elementary parameters: The granularity/wordsize of a stream cipher is represented as $\mathbf{S}_{\mathbf{w}}$. Elementary parameters including the sizes of key and IV, represented as $\mathbf{S}_{\mathbf{K}}$ and $\mathbf{S}_{\mathbf{I V}}$, respectively, are described in terms of number of words. The set of test vectors includes Key and IV values along with the expected Keystream for verification, specified in terms of a known endianess. For simplicity, the three phases of a stream cipher (as given in Section 3.4.2.3) are referred as phase0, phase 1 and phase2. The number of operational cycles for the three phases of stream ciphers is defined as $\mathbf{P i}$ c cnt by the user, where $i$ is the phase number.

- Sequential state information: Each of the various types of sequential elements is defined as a register array. The user specifies the total number of register arrays, along with the type of each (FSR, jump register, FSM register). The granularity of each element of these arrays is also specified, since it may or may not match the wordsize of the cipher. The depth of the register array is the number of delay elements in the FSR/jump register array; for individual FSM registers, the array depth is configured as 1 . The clocking of these register arrays can be specified to be regular or conditional.

- Mesh of operations: The combinational primitives of the stream cipher are specified as a mesh of operations. This mesh comprises of nodes of operations that interact with each other through interconnects. This mesh is a Graph $(\mathrm{G})$, defined by vertices (nodes) and edges (interconnects). Each interconnect representing the directed edge between nodes is specified by an ordered pair of initial node 
and terminal node. The width of interconnects entering and leaving a node may not always be equal (for nodes with ciphers operations like concatenation or bit splitting), requiring explicit specification. Hence the user specifies the total number of nodes along with the width of each and an ordered pair specifying initial and terminal node numbers. For each node, the user must also specify an atomic operation, comprising of either of the following operational classes.

- Basic Boolean operations: AND, OR, NOT, XOR

- Arithmetic operations: Add, subtract, multiply, divide (by power of two)

- Bit-wise operations: Arithmetic Shifts, logical shifts, rotation, rotation with carry

- Cryptographic primitives: SBox, PBox

- Galois Field Operations: GF-multiplication, GF-division

- Bit Manipulation: bit masking, concatenation / bit-reorganization

- Glue logic: Multiplexers, constants, No-operation

\subsubsection{Stream Cipher Sample Set}

A set of stream ciphers is constructed, that is diverse enough for evaluating the goodness of the kernels selection for RunStream. Table 3.4 gives their classification, construction and salient features. A/5-1 was part of the original encryption algorithm for GSM, since it was export restricted, A/5-2 was developed. They were reverse engineered and verified and the design was revealed [33]. Both of these are multiple LFSR based stream ciphers, using irregular clocking for non linearity. E0 [27] is also an LFSR based stream cipher with a summation combiner used for output generation. It was used in Bluetooth communication. Although the security of these proposals has been compromised $[15,145]$, these popular proposals nevertheless are included for comparison against their known implementations.

eSTREAM was launched to restore the confidence of cryptographic community on stream ciphers as questions were raised on their usability [199]. Some popular stream ciphers, when found flawed, were replaced by block ciphers, e.g., KASUMI (or A/53) block cipher [86] replaced A/5-1 and A/5-2 stream ciphers after weaknesses were pointed out [15], WPA/WPA2 use AES based security, unlike its predecessor WEP, that used RC4 stream cipher. Moreover, all the 6 proposals of an earlier competition NESSIE succumbed to cryptanalysis, eSTREAM was launched. It was initiated by EU ECRYPT network and took four years effort to finalize new stream cipher proposals [65].

The initial phase of eSTREAM attracted 34 proposals. Out of these only two proposals (SSS and Moustique) were self-synchronizing stream ciphers while the rest were all synchronous. After various phases of thorough scrutiny, judging the security, performance, simplicity and flexibility of these proposals, 7 were included in the portfolio, 4 in software profile and 3 in hardware profile. These three stream ciphers (along with their modified versions) in the hardware profile of eSTREAM portfolio 
Table 3.4: Classification, construction and Salient Features of stream ciphers

\begin{tabular}{|c|c|c|c|c|c|c|c|c|c|c|c|c|c|c|c|c|c|}
\hline & 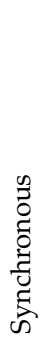 & 莺 & $\begin{array}{l}\vec{\Xi} \\
\stackrel{\overrightarrow{0}}{\Xi}\end{array}$ & 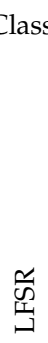 & $\begin{array}{l}\text { 点 } \\
\text { 光 }\end{array}$ & $\begin{array}{l}\text { 崫 } \\
\text { Z }\end{array}$ & 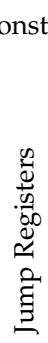 & 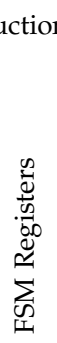 & 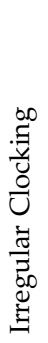 & 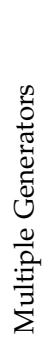 & 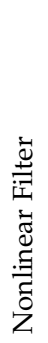 & 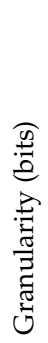 & 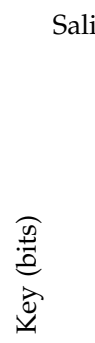 & $\begin{array}{l}\frac{0}{0} \\
\hat{0} \\
z \\
z\end{array}$ & 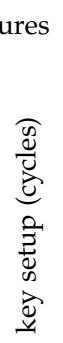 & 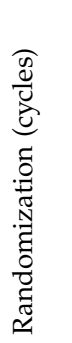 & 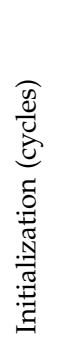 \\
\hline A-5/1 [33] & $\checkmark$ & $\checkmark$ & 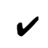 & $\checkmark$ & $x$ & $x$ & $x$ & $x$ & $\checkmark$ & $\nu$ & $x$ & 1 & 64 & 22 & 86 & 100 & 186 \\
\hline A-5/2 [33] & $\checkmark$ & $\checkmark$ & $v$ & $\checkmark$ & $x$ & $x$ & $x$ & $x$ & $\checkmark$ & $\nu$ & $\checkmark$ & 1 & 64 & 22 & 86 & 100 & 186 \\
\hline E0 [27] & $\checkmark$ & $\checkmark$ & $v$ & $\boldsymbol{v}$ & $x$ & $x$ & $x$ & $v$ & $x$ & $\checkmark$ & $\checkmark$ & 1 & $8-128$ & - & 200 & 128 & 328 \\
\hline Grain80 [96] & $\checkmark$ & $\checkmark$ & $\checkmark$ & $\boldsymbol{v}$ & $x$ & $\checkmark$ & $x$ & $x$ & $x$ & $\checkmark$ & $\checkmark$ & 1 & 80 & 64 & 160 & 160 & 320 \\
\hline Grain128 [95] & $\checkmark$ & $\checkmark$ & $v$ & 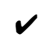 & $x$ & $\checkmark$ & $x$ & $x$ & $x$ & $v$ & $\checkmark$ & 1 & 128 & 96 & 256 & 256 & 512 \\
\hline Grain128a [233] & $\checkmark$ & $\checkmark$ & $v$ & $\checkmark$ & $x$ & $v$ & $x$ & $x$ & $x$ & $\boldsymbol{v}$ & $\checkmark$ & 1 & 128 & 96 & 256 & 256 & 512 \\
\hline Trivium [55] & $v$ & $\checkmark$ & $v$ & $\checkmark$ & $x$ & $x$ & $x$ & $x$ & $x$ & $\checkmark$ & $x$ & 1 & 80 & 80 & 288 & 1152 & 1440 \\
\hline MICKEY80-v2 [13] & $\checkmark$ & $\checkmark$ & $\checkmark$ & $x$ & $x$ & $\checkmark$ & $\checkmark$ & $x$ & $\checkmark$ & $\checkmark$ & $x$ & 1 & 80 & 80 & 160 & 100 & 260 \\
\hline MICKEY128 [14] & $\checkmark$ & $\checkmark$ & $\checkmark$ & $x$ & $x$ & $\checkmark$ & $\checkmark$ & $x$ & $\checkmark$ & $\checkmark$ & $x$ & 1 & 128 & 128 & 256 & 160 & 416 \\
\hline RC4 [195] & $\checkmark$ & $\checkmark$ & $x$ & $x$ & $x$ & $x$ & $x$ & $\checkmark$ & $x$ & $x$ & $\checkmark$ & 8 & $40-2 \mathrm{~K}$ & - & 256 & 256 & 512 \\
\hline ZUC [232] & $\checkmark$ & $\checkmark$ & $x$ & $x$ & $x$ & $\checkmark$ & $x$ & $\checkmark$ & $x$ & $x$ & $\checkmark$ & 32 & 128 & 128 & 16 & 32 & 48 \\
\hline SNOW 3G [2] & $\checkmark$ & $\checkmark$ & $x$ & $x$ & $x$ & $\checkmark$ & $x$ & $\checkmark$ & $x$ & $x$ & $\checkmark$ & 32 & 128 & 128 & 16 & 32 & 48 \\
\hline
\end{tabular}

are taken up, including Grain80-v2 [96], Grain128 [95], Grain128a [233], MICKEY80v2 [13], MICKEY128 [14] and Trivium [55]. These proposals are particularly suitable for hardware applications with restricted resources. Their design diversity makes them good candidates for HLS, i.e., MICKEY is based on jump registers, Trivium employs 3 LFSRs, Grain has one LFSR and one NFSR. No successful cryptanalytic effort against them has yet been reported.

For non-binary stream ciphers, RC4 [195] is taken up, that is most commonly used to protect Internet traffic using the SSL (Secure Sockets Layer) protocol, Transport Layer Security (TLS), WEP (Wired Equivalent Privacy), Wi-Fi Protected Access (WPA), along with several application layer softwares. ZUC [232] and SNOW 3G [2] stream ciphers are also taken up, both of which have been included in the security portfolio of 3GPP LTE-Advanced, the potential candidate for $4 \mathrm{G}$ mobile broadband communication standard. They generate 32-bit words of keystream and have internally 16-taps NFSRs.

\subsubsection{Trivium Cipher [55] configuration:}

A typical synchronous stream cipher, namely Trivium [55], from eSTREAM is taken up and its configuration, according to the discussed configuration space is tried out. Trivium's 288-bit internal state consists of 3 regularly clocked LFSRs of different lengths. It has an 80 bit Key and an 80 bit IV, with phase 0 and phase 1 defined to be 288 and $(288 \times 4)$ cycles, respectively. Other elementary parameters like the number of phases, cycles for each phase are also specified as part of the cipher configuration. The architectural details of Trivium are depicted in Fig. 3.10. The three LFSRs are 


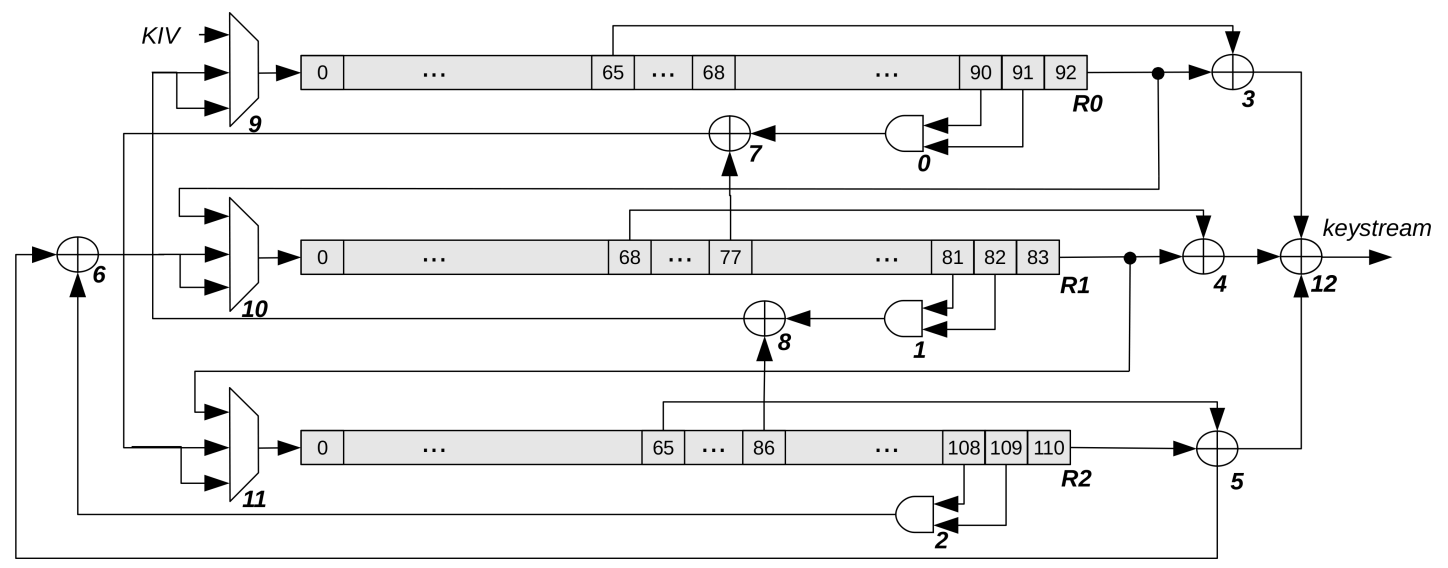

Figure 3.10: Architectural structure of Trivium implementation

right shifted per clock cycle and named $R 0, R 1$ and $R 2$; granularity being 1 bit, type being LFSR, unconditionally clocked and the depth is specified to be 93, 84 and 110, respectively.

For the cipher configuration, all the atomic operations (AND, XOR, MUX) are identified as separate operational nodes and then enumerated (not in any specific order). The 3-to-1 Muxes at the least significant bits of the LFSRs control their input during three different phases of operation. A total of 13 nodes describe the complete functionality of the cipher (The node number, register number is highlighted and placed at the bottom right of each node in Fig. 3.10). For each of the 13 nodes, an operation is specified, having output width (wordsizeout) and several node input sources. The node input sources could be other nodes or any of the LFSR bit positions. The node generating the final keystream is also indicated (node12). The feedback for each of the three LFSRs is are also tied to nodes (node9, node10 and node11).

\subsubsection{Hash Functions}

Structurally, the iterated hash functions can be classified on the basis of their underlying operations in their compression function. Broadly, they can be categorized as being inspired from block ciphers or designed from the scratch as customized hash functions. For the first category, the algorithmic configuration space of a block cipher is generic enough for their description. For the second category, the compression function is generally a sponge construction, ARX or other modular arithmetic operations.

The typical constituent operations of hash functions are analyzed by undertaking all the 14 SHA-3 competition [197] candidates surviving round 2. Table 3.5 summarizes the underlying operations of candidates and shows their broad categorization [75]. The table does not include logical shifting of bits, rotation and bit permutations as they do not require hardware resources other than routing. According to the constituent operations, the candidates are categorized into three broad classes.

- ARX based designs: The largest category is the ARX based designs, 7 out of 14 proposals are ARX (including logical operations) transformations. CubeHash 
Table 3.5: Major operations of SHA-3 candidates (permutations, shifts, rotations not included). mADDn denotes a multioperand addition with $n$ operands.

\begin{tabular}{|c|c|c|c|c|c|c|}
\hline Function & S-box & GF-MUL & MUL & mADD & $\begin{array}{l}\text { ADD } \\
\text { /SUB }\end{array}$ & Boolean \\
\hline \multicolumn{7}{|c|}{ ARX based designs } \\
\hline BLAKE [12] & & & & mADD3 & ADD & XOR \\
\hline Skein [73] & & & & & ADD & XOR \\
\hline BMW & & & & mADD17 & ADD, SUB & XOR \\
\hline CubeHash & & & & & ADD & XOR \\
\hline Keccak [23] & & & & & & NOT,AND,XOR \\
\hline Shabal & & & $x 3, x 5$ & & ADD,SUB & NOT,AND,XOR \\
\hline SIMD & & & $x 185, x 233$ & mADD3 & ADD & NOT,AND,OR \\
\hline \multicolumn{7}{|c|}{ AES inspired designs } \\
\hline ECHO & AES $8 \times 8$ & $\mathrm{x} 02, \mathrm{x} 03$ & & & & XOR \\
\hline Fugue & AES $8 \times 8$ & $\mathrm{x} 04 . . \mathrm{x} 07$ & & & & XOR \\
\hline Groestl & AES 8x8 & $\mathrm{x} 02, \mathrm{x} 07$ & & & & XOR \\
\hline SHAvite-3 & AES 8x8 & $x 02, x 03$ & & & & NOT,XOR \\
\hline \multicolumn{7}{|c|}{ SPN designs } \\
\hline Hamsi & Serpent $4 \times 4$ & & & & & XOR \\
\hline $\mathrm{JH}$ & Serpent $4 \times 4$ & $x 2, x 5$ & & & & XOR \\
\hline Luffa & $4 \times 4$ & $\mathrm{x} 2$ & & & & XOR \\
\hline
\end{tabular}

requires SUB operation other than ADD for its implementation. CubeHash and Keccak are sponge-constructions while the underlying functions of BLAKE and Skein are inspired from block ciphers. Both Shabal and SIMD require integer multiplications by fixed numbers, which can be implemented using fixed shifts and addition. SIMD requires Number Theoretic Transform that can be optimized by using a multi-stage Fast Fourier Transform (FFT) [153, Chapter 6,7].

- AES inspired designs: 4 proposals having similarity with AES [197] are categorized as AES based designs since their compression function is only modified/ tweaked versions of AES round operations, i.e., ShiftRows and GF-Mix (multiplication by a constant in Galois Field $\mathrm{GF}\left(2^{8}\right)$ ) etc. They use the same 8x8 AES S-box [165].

- SPN based designs: The remaining three proposals can be categorized as SPN based designs, JH and Hamsi use Serpent's SBox $(4 \times 4)$ while Luffa has and independently designed SBox. JH and Luffa also require fixed Galois field multiplications. Hamsi includes a linear code chunk that requires look-up tables and matrix by vector multiplications in GF(4) [75] for its implementation.

For this categorization, a strong similarity of iterative hash functions structures and constituent operations with block ciphers is evident. The layered architecture 
discussed for block ciphers is generic enough for ARX based hash ciphers description. Consequently, the algorithmic design space for hash functions is not considered separately.

\subsection{Concluding Remarks}

This chapter discusses the computational fabric of three major classes of symmetric key cryptographic, i.e., block ciphers, stream ciphers and hash functions. The cryptographic functions are classified un-conventionally on the basis of their underlying functions. Surprisingly, the constituent operations and structure are small and highly overlapping for these classes of cryptography. This sets a good starting ground for taking up these cryptographic functions for high level synthesis, as discussed in the next chapter. 


\section{Chapter 4}

\section{High Level Synthesis for Symmetric Key Cryptography}

Classifying the cryptographic functions on the basis of their primitive computational elements brings forward a surprisingly simple angle of viewing them. It is especially beneficial to their high level synthesis, with performance as good as the hand crafted designs. Chapter discusses the various steps in the HLS tool for block ciphers (called RunFein) and stream ciphers (called RunStream). Various design points in the scope of architectural customizations available to the HLS tool user are elaborated. A thorough benchmarking to compare against the hand crafted solutions is undertaken, with their performance found on-par with hand-optimized HDL implementations. The results can be accessed as $[119,120,123]$.

Compared to a general-purpose high level synthesis tool, an application or domain specific HLS tool has a smaller problem set to deal with and consequently can generate HDL designs with superior area-throughput efficiency, by exploiting the following properties.

1. The high level abstraction of the design comprises of limited constructs/ operations / structures specific to the particular application in hand, making the learning curve gentle. Application experts choose a subset of design abstraction constructs, flexible enough for the algorithm descriptions belonging to that class, thereby exploiting and integrating the domain specific expertise at a high level.

2. Exploiting the domain expertise in hardware architecture, the interfaces, internal structures, operational phases, dependencies are better understood. Consequently, the set of possible target microarchitectures is reduced, allowing better selection of an efficient target implementation.

3. The HLS tool may allow more control over design optimizations for QoR improvement by enabling configuration and selection of specific architectural design optimization instead of generic optimization goals. Consequently, reaching the appropriate design requires fewer iterations.

4. Generation of hardware description by an application specific tool, directed at every design level by domain-specific optimization can compete with hand-written implementations. 


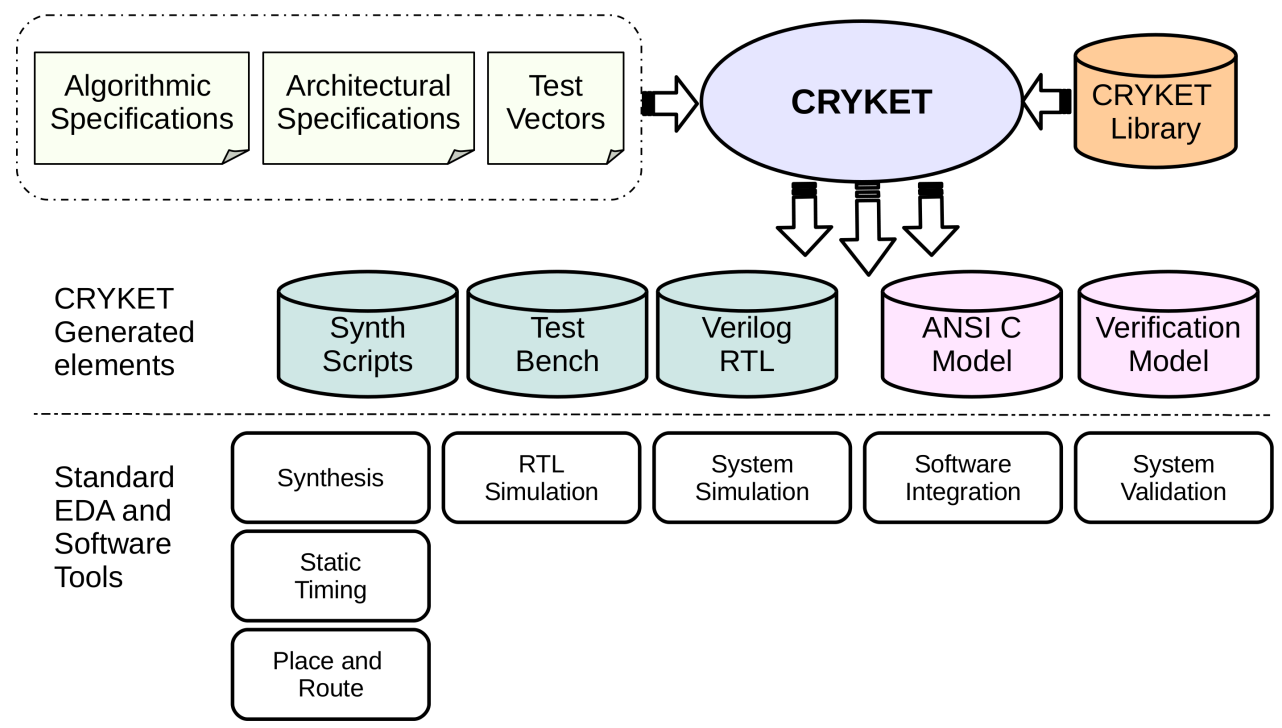

Figure 4.1: Design flow of cryptographic functions using CRYKET

\subsection{CRYKET (CRYptographic Kernels Toolkit)}

CRYKET is a high level synthesis framework, specific, as its name suggests to the symmetric key cryptography kernels, including the block ciphers and stream ciphers. Since these categories of ciphers have different constituent elements as their building blocks and different operational phases, they are dealt separately with two different offshoot tools, namely, RunStream (for stream ciphers) and RunFein (for Block ciphers). These tools can aid any cryptographer by relieving him/her from low level implementation details of the algorithms and by providing a powerful and flexible API to rapidly incorporate improvements in the design. CRYKET has a language independent interface with the user; it accepts a sophisticated high-level block cipher/ stream cipher design through a customized GUI. Fig. 4.1 shows a high level toolflow of CRYKET. The user provides three sets of parameters. Firstly, the algorithmic design configuration comprising of constructive elements coming from a set of functionally complete constructive elements to define any block/ stream cipher (as discussed in the previous chapter). Secondly, the user chooses microarchitectural configuration of the cipher for HDL generation. It includes a mode of operation and one of the various microarchitecture configurations like unrolled, pipelined, subpipleined, bitsliced implementations. Thirdly and optionally, the user may specify a set of testvectors, if already known for the verification of the design.

The CRYKET tool automatically infers the necessary interfaces and structures to generate the synthesizable HDL description, customized test benches, synthesis and simulation scripts, as well as software implementation and verification codes. It provides an end to end verification from the configuration to RTL validation/ verification environments by a seamless integrated with standard simulation, synthesis, place and route tools. 


\subsection{RunFein}

RunFein is the rapid prototyping framework for block ciphers. It model Lai-Massey block cipher, Feistel and SPN based block ciphers. The toolflow of RunFein is graphically shown in Fig. 4.2. The user populates the configuration space of a new block cipher to get customized software and hardware implementations. A sophisticated design capture is made possible via a GUI to let the user conveniently specify cipher design and implementation customization. The configuration for a cipher could be added, parameter by parameter, or could be saved and loaded later. A list of known ciphers is available to instantly load the configurations for easier manipulation. RunFein validates this design capture for completeness and correctness at various stages of the toolflow. These rule checks detect functional and system-level problems much earlier in the design cycle improving design reliability and shortening time to market. RunFein successfully abstracts away the diversity of the design space by translating the configuration it to a generic block cipher template. The software and hardware generation engines of the tool generate an optimized software implementation and a synthesizable HDL description. The biggest technical challenge is to develop a tool capable of seamlessly integrating these sub-structures and functions into a working model, without sacrificing the performance of the implementation, both of software and hardware platforms. The design optimality is carefully preserved in HDL realization and is experimentally benchmarked to be in par with hand crafted realizations.

\subsubsection{Design Specification Compilation}

The user specifies the design to RunFein through a GUI. Fig. 4.3 gives a snapshot of the RunFein tab that accepts the round layers for AES-128 ECB encryption cipher. As summarized in Table 3.3, the round_init requires one operation layer, while round_middle and round_final require 4 and 3 operational layers, respectively. The user conveniently chooses the desired operation for each layer by pull-down menus and configures the operation as per the cipher configuration. RunFein provides separate tab for kround layers specifications. The arguments for SBox and PBox can be loaded either by a text file or added by the user in the edit boxes, they are also categorized in separate tabs of tool. RunFein provides convenient default values in the GUI wherever necessary, the configuration with default values for microarchitecture and testvectors for PRSENT-80 is shown in Fig. 4.3.

The configuration parameters for a cipher fed to the tool's GUI are stored as an $x \mathrm{ml}$ configuration file, a snapshot of PRESENT-80 configuration file is shown in Fig. 4.4. A token, namely ALGORITHM, holds the algorithmic parameters, i.e., basic parameters, round layers and key round layers, all held in separate tokens. Basic parameters includes sizes of block, key and word size and the information of rounds for encryption. The ROUND token holds the information for three types of rounds. A round_init is not required hence no layers are defined for it while the round_middle is defined by 3 layers of operation. The S-Box and P-Box values are abbreviated for readability. The mode of operation is specified by token OP_MODE while the 


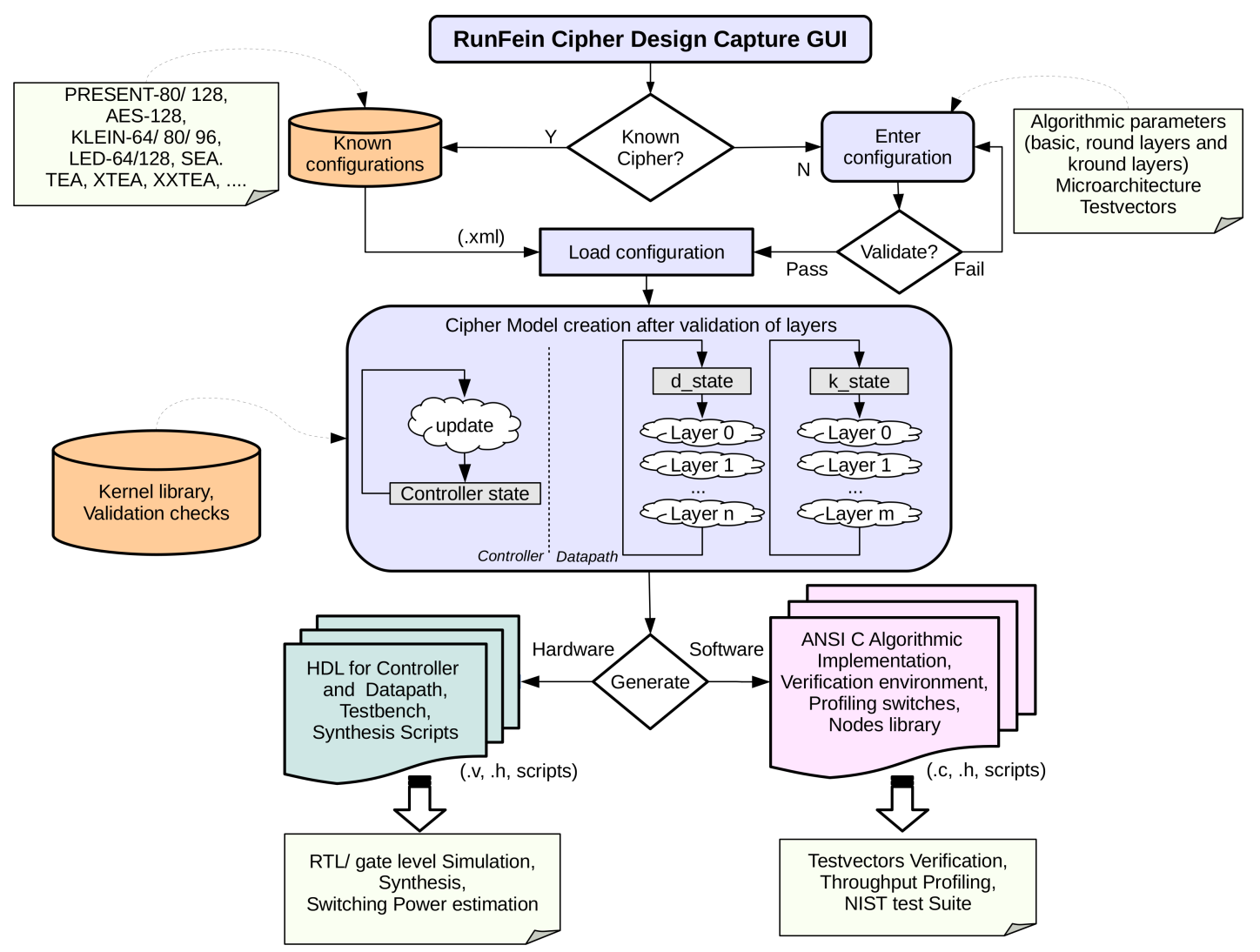

Figure 4.2: RunFein toolflow for software generation and hardware generation

testvectors and microarchitecture (discussed later) is specified in TESTVECTORS and MICROARCHITECTURE tokens, respectively.

\subsubsection{Specification Validation and Formal Model Creation}

Before creation of a valid cipher model, the configuration parameters given by the user undergo a list of defined rules checks. The user is prompted in case of a violation and cipher implementation does not proceed unless a valid configuration is specified. (Some additional rules related to hardware microarchitectures are discussed in preceding section)

- Blocksize of any cipher by definition equals the sizes of plaintext/ ciphertext.

$S_{B}=S_{P}=S_{C}$

$S_{B}=2 m$, where $m \geq 1$.

- Size rules for key and the subkeys generated.

$S_{B}=S_{S K}$

$S_{K}=2 m$, where $m \geq 1$.

- Size rules for wordsize of cipher.

$S_{W}=2 m$, where $m \geq 1$. 


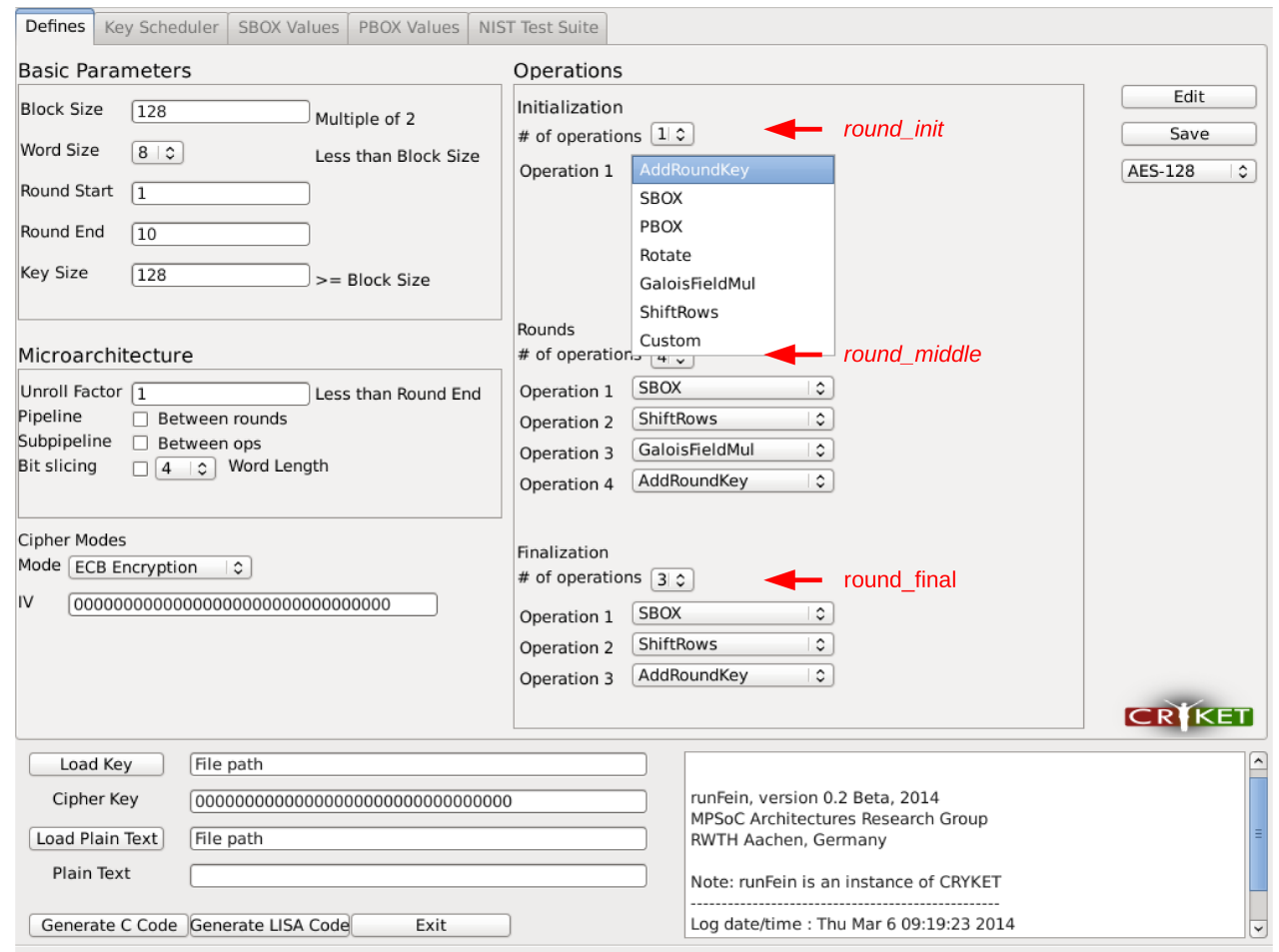

Figure 4.3: Round layers operational specification for AES-128 in RunFein

$S_{W} \leq S_{K}$ and $S_{W} \leq S_{B}$

$S_{W}=n \cdot S_{B}=k \cdot S_{S K}$, where $n, k \geq 1$.

- For modes of operations (defined in last chapter), IV size rule $S_{B}=S_{I V}$

- The number of subkeys generated should be equal to the number of ARK operations, hence each ARK consumes one key.

- The SBox values are $\in\left[0.2^{S_{W}}\right]$.

- PBox, rotation/shifting, XOR operations have arguments $\in\left[0 . . S_{B}\right]$.

- The polynomial coefficients for GF-mul are not $\varnothing$.

The configuration file is parsed by RunFein and a cipher model is created. As shown in Fig. 4.2, it comprises of a controller and a datapath.

- Controller: The controller is essentially a counter, required to keep track of the iterations of the cipher. It counts up or down during encryption or decryption of a block of data, respectively. Its size is inferred from the number of rounds required by the cipher,i.e., as ceil $\left(\log _{2}\left(N_{r}\right)\right)$ bits.

- Datapath: The datapath of the cipher is constructed by the $n$ operational layers of round and $m$ operational layers of kround, as shown in Fig. 4.2. Two variables 

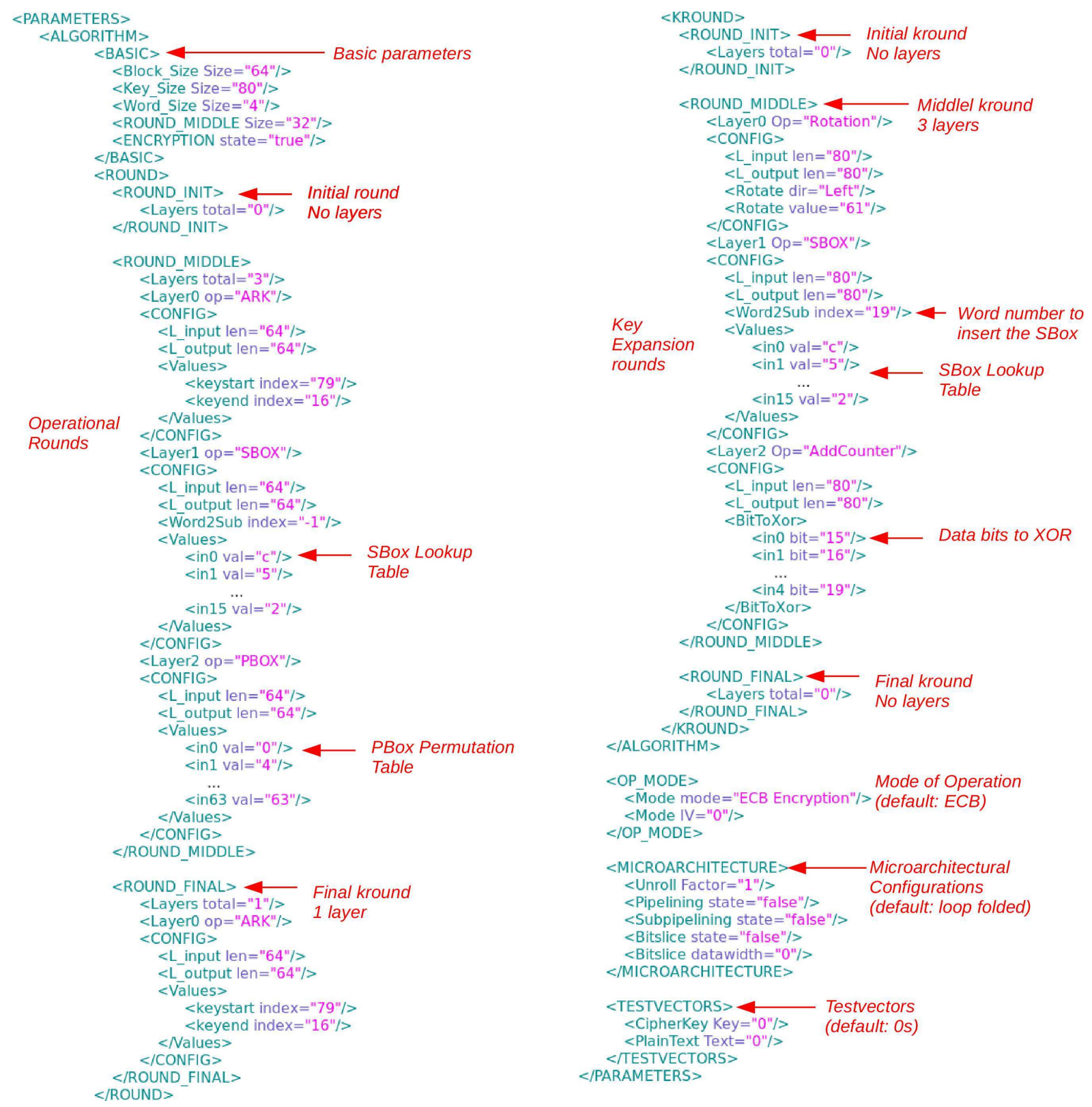

Figure 4.4: The configuration file snapshot for PRESENT-80 generated by RunFein

namely d_state and $\mathbf{k} \_s t a t e$ are inferred for the model, that contain the updated data state and key state, respectively. (for hardware implementation these values are D-flipflops instead).

\subsubsection{Software Generation Engine}

The software generation engine accepts the cipher model along with the set of testvectors including plaintext, key, IV (through text files or edit boxes) from the configuration file. The Software generation engine compiles the cipher model to generate a high performance, fixed-point ANSI-C description. The code is enhanced by a simulation 
environment with user controllable switches for verification, throughput profiling, data dumping etc.

All the configuration parameters of the cipher (as specified in $\mathrm{xml}$ file listing in Fig. 4.4) are \#defined in a header file. This includes all basic configuration, test vectors and the microarhitachture, though software implementation only caters the default values of a simple iterative loop folded implementation. Data types of registers, layers and all interfaces are typedef-ed in accordance with their respective granularity specified. Supplementary functions are kept in a separate file, that is included in the main file during simulation. These functions include datatype conversion functions (e.g., conversion of hexadecimal to binary arrays and vice versa), data dumping and verbose simulations. For each operational layer of round and kround, the interface and functionality is defined as per the user specification. Layers may operate on operands with different granularity, i.e., PBox operates on bits, SBox operates on $S_{W}$ etc. The functions generated include relevant calls to conversion of granularity functions in addition to the functionality of the layer operation.

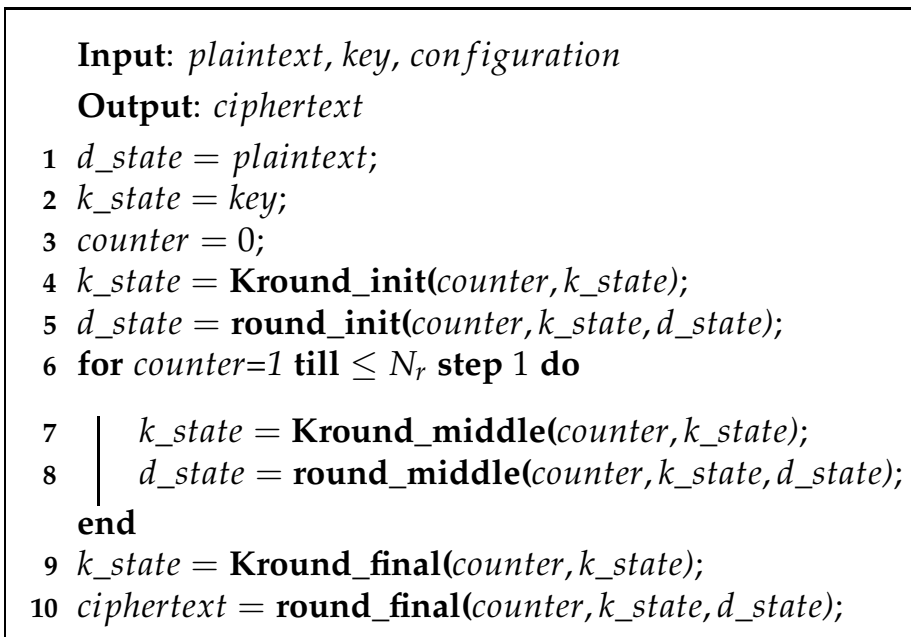

Algorithm 1: RunFein Encryption Pseudocode

The main body of code, having the controller and the datapath of the cipher model, is a separate file that \#includes all supplementary and header files. For elaboration of the code simulation environment, a simplistic pseudocode for encryption of one block of data is given in algorithm 1 . The plaintext and key are assigned to the local variables $d \_s t a t e$ and $k \_s t a t e$, respectively $($ line 1,2$)$. $k$ _state is updated first by Kround_init function. Using the updated key, the d_state is updated using round_init function(line 4). The controller part of the cipher comprises of counter variable, keeping track of the round under execution. The loop starting in line 6 iterates for roundcount -1 times and keeps updating the data and key registers. The final round generates the last $k_{-}$state which is used up by round_final to generate the ciphertext, as given in lines 9 and 10, respectively. RunFein toplevel wrapper of generated code for AES is presented in the Appendix A. 
The software generation engine of RunFein generates a single-threaded, untimed, sequential $\mathrm{C}$ model of the stream cipher with necessary libraries and scripts. Some of its additional features are highlighted.

- NIST Test Suite: RunFein has the NIST test suite [191] integrated with it to characterize the statistical qualities of PRNGs. It serves as a first step in determining the suitability of a PRNG used for cryptographic purposes. Fig. B.2 gives a GUI snapshot of RunFein for the selection and parameterization of various statistical tests available for execution as per the user wishes. (RunFein caters only the block ciphers, however, they behave like stream ciphers and CSPRNGs under certain modes of operation.)

- Verification: For the verification of the generated model according to the user specified testvectors, a verification environment is generated. For new proposals, without defined testvectors, the verification switches may be turned off by the user.

- Performance Profiling: The user may enable a performance profiling environment in the generated software implementation to evaluate encryption speed (in seconds, cycles/ byte) of the cipher design. Provision of encrypting bulk data from random plaintext for monitoring data randomness is provided. A reasonably efficient generated implementation may be further manually optimized for a specific platform.

\subsubsection{Hardware Generation Engine}

The hardware generation engine requires additionally the microarchitectural configuration of the target implementation specified by the user, cipher model and a set of testvectors. As shown in Fig. 4.1, RunFein generates a complete working model of the block cipher in synthesizable $H D L$, along with a testbench and necessary scripts. The configuration space and parameterization for various microarchitectures that RunFein can support is discussed. Once a design and its microarchitecture are given to RunFein, first the viability evaluation of the chosen microarchitecture configuration is done by hardware generated engine by a list of rule checks. After design validation, hardware resource allocation of the design is carried out depending on the microarchitecture selected for implementation. The last step is the automatic generation of hardware description. The synthesizable, hierarchical block cipher Verilog HDL and testbench with necessary scripts can then be further used to carryout

- Simulations for design verification, gate-level simulation (post-synthesis) using verification tools.

- Logic synthesis of the design for profiling critical parameters like the maximum clock frequency, chip area.

- Post-synthesis power consumption estimation with using back-annotation. 

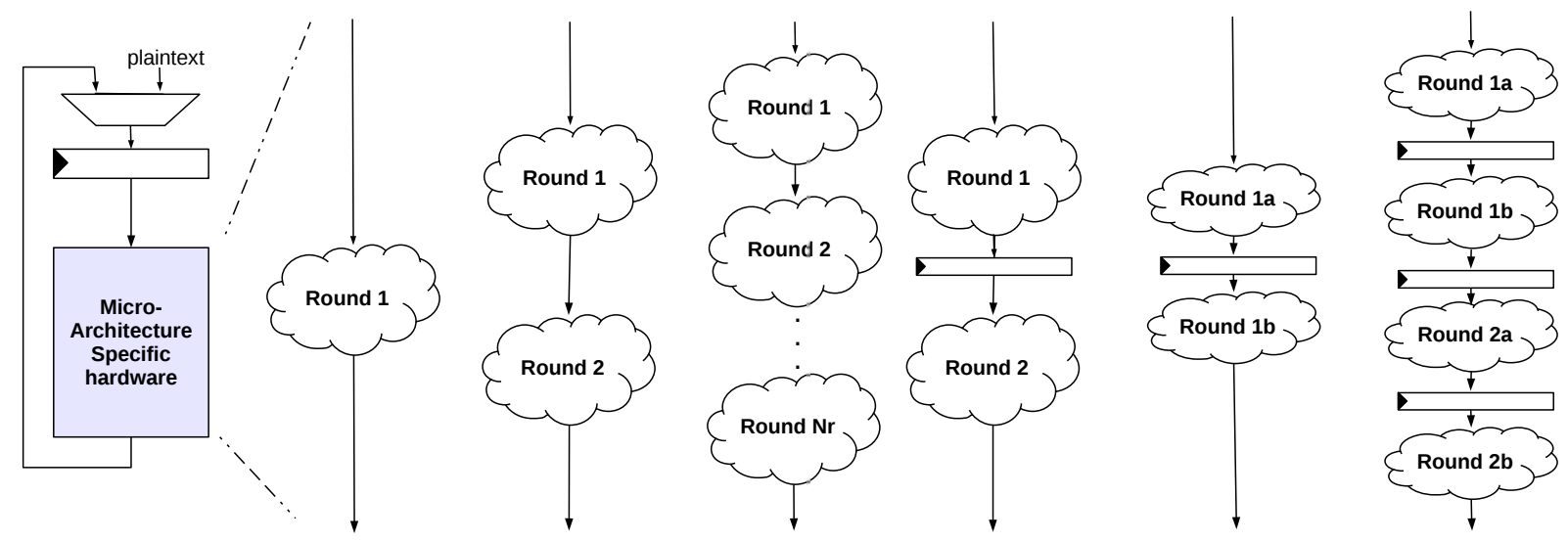

Figure 4.5: Various parallel microarchitecture implementations supported by RunFein a) Loop folded b) Unrolled by 2 c) Fully unrolled d) unrolled by 2 with pipeline e) Subpipelined once f) Subpipelined once and unrolled by 2 with pipeline

\subsubsection{Supported Microarchitectures}

RunFein enables quick exploration of various microarchitecture design options residing at different design point of the performance-area trade-off spectrum. The user specifies algorithmic configuration of the cipher design always according to the simplistic loop folded architecture. In addition, he must specify the microarchitecture he wants RunFein to automatically implement. By tweaking the microarchitecture configuration, he may opt for parallel implementations (loop subpipelining/ unrolling) duplicating hardware for boasting throughput performance or bitsliced designs economizing area/ power at the expense of lower throughput performance by employing resource sharing. These microarchitectures are discussed below, they are depicted in Fig. 4.5.

- Loop Folded: A typical loop folded block cipher implementation performing one round per clock cycle $\left(N_{r}\right.$ cycles per block) is shown in Fig. 3.7 and Fig. 4.5 a). It is the default hardware implementation microarchitecture of RunFein and serves as a middle point for area-throughput trade-off between parallel implementations and bitsliced implementations. The controller comprises of round counter register, incrementing every cycle (Fig. 4.6 a). The selection of plaintext or folded data for $d \_s t a t e$ register is controlled by this register. A valid ciphertext is generated when counter register hits $N_{r}$.

- Loop Unrolled: The loop unrolled configuration replicates round (and kround) resources $u$ times to execute multiple rounds in one clock cycle, where $u$ is the unrolling factor. Consequently the critical path of the circuit increases, decreasing the maximum operational frequency, the area also increases. The counter increments by $u$ per cycle since the design require $N_{r} / u$ cycles for encryption of a complete block ( $N_{r} / u$ not being a fraction), as shown in Fig. $4.6 \mathrm{~b}$ ). A higher throughput performance is expected since the propagation delay and the register 

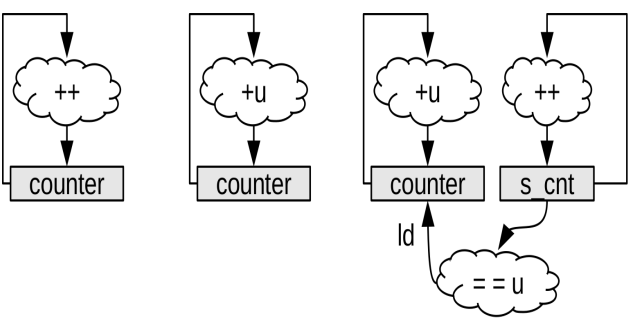

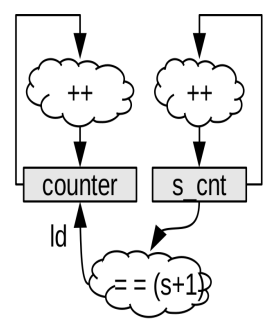

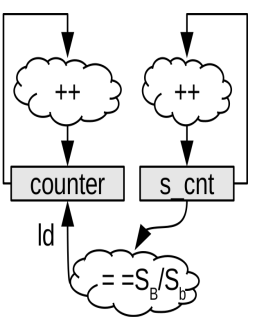

Figure 4.6: Controller for various microarchitecture implementations supported by RunFein a) Loop folded b) Unrolled by $u$ c) Unrolled by $u$ with pipeline d) Subpipelined by $s$ e) Subpipelined by $s$ and unrolled by $u$ with pipeline f) Bitsliced with $S_{b}$

setup time come only once in the combinational delay for $u$ rounds. This gain in throughput is hard to enumerate without experimentation hence synthesis profiling is required (a twice unrolled hardware configuration is shown in Fig. 4.5 b). Two critical design points relevant to the loop unrolling are

- A fully unrolled architecture with $u=N_{r}$ encrypts/ decrypts of data in a single cycle (Fig. 4.5 c). The RunFein hardware generation engine optimizes the hardware for the round_final if it is different from the round_middle. The round_middle hardware is replicated $(u-1)$-times following the hardware for round_final instantiated once. The increase in throughput is achieved since now data is processed in $N_{r} / u$ cycles while the decrease in operating frequency is lesser than $u$ times since the critical path handles one register setup time only.

- A loop unrolling with pipelining architecture can be chosen by the user to automatically insert pipeline registers between unrolled rounds. Consequently, the critical path of the design also does not increase due to unrolling, this design handles multiple blocks of data simultaneously. Configuration in Fig. $4.5 \mathrm{~d}$ ) processes two blocks of data in a total of $N_{r}$ cycles boasting throughput by $u$. A supplementary counter or s_cnt keeps track of the unroll factor, which when fulfilled generates the load signal for counter to increment directly by $u$ (Fig. $4.6 \mathrm{c}$ ). Hence in subsequent cycles, $u$-many valid ciphertexts are generated when counter equals the roundcount.

- Subpipelining: Using RunFein the user may choose to insert a subpipeline between any two layers in a round to reduce the critical path of the design. To ensure data consistency, for s subpipelines inserted in a cipher round, an equal number of subpipelines should be specified by the user to be inserted in kround as well. To do so, the user must check subpipelining option on to be able to insert various operations along with a subpipeline register as shown in the GUI snapshop (Fig. B.2). Insertion of each subpipeline increments the number of multiple blocks being processed, i.e., s subpipelines make the cipher design handle $(s+1)$ data blocks simultaneously (for $s=1$ Fig. 4.5 e). A supplementary 
register s_cnt inserted keeps track of the subpipeline (Fig. $4.6 \mathrm{~d}$ ). If the user wishes to insert a subpipeline within a layer, he must first redefine that layer as two layers, split at the cut-set point.

\section{- Hybrid Microarchitectures:}

Using RunFein the user may opt for some hybrid parallel microarchitecture configurations supporting both subpipelining and unrolling. Fig. $4.5 \mathrm{f}$ ) shows a hybrid microarchitecture with subpipeline $(s=1)$ and unrolling with pipeline by a factor $(u=2)$. Its a multiple block configuration, handling 4 data blocks simultaneously. Consequently, the controller needs a supplementary register s_cnt to keep track of the total iteration count (Fig. 4.6 e).

- Bitslicing: Through bitslicing, RunFein tiles the parallel loop folded architecture to work on $S_{b}$ bits at a time $\left(S_{b}<S_{B}\right)$. Consequently the design has lower area and lower throughput, a technique especially interesting for lightweight block ciphers. In most of the SPN ciphers, SBoxes account for a significant area portion, e.g., more than $30 \%$ of the PRESENT- 80 loop folded implementation area is contributed by its 17 SBoxes [28]. Hence, $S_{b}$ is generally taken as $S_{W}$ or a multiple of it.

The krounds and rounds are sliced to operate the task of one cycle in $S_{B} / S_{b}$ cycles. The controller of the bitsliced architecture changes so that the counter increments once after the $s_{-} c n t$ hits $S_{B} / S_{b}$. The encryption of one block requires $S_{b} \times N_{r}$ cycles as shown in the Fig. $4.6 \mathrm{f}$. The $d \_s t a t e$ and $k \_s t a t e$ are shift registers (with parallel load/ stores possible), with shift granularity of $S_{b}$. Hence the operations of each layer in a round is performed on $S_{b}$ bits and the result is stored in d_state shift register. Similar to the bit slicing of S-boxes, operations like XOR, Addition (with carry bit) can be bit sliced. However, for some operations, the operation slicing requires large extra selection logic, e.g., PBoxes, rotation. Since these bit manipulation operations (when performing in parallel configurations) have no logic overhead, its wiser not to bitslice them.

\subsubsection{Microarchitecture Validity Evaluation}

The selected cipher configuration by the user through GUI (as shown in Fig. B.2) is stored in the xml configuration file, along with the algorithmic configuration and the testvectors (as shown in Fig. 4.4). The selected microarchitecture undergoes following checks before generation of valid hardware design.

- The unroll factor, $u$ should be a multiple of round count, $N_{r}=k \times u$, where $k \geq$ 1.

- The number of subpipelines $s$ inserted in round should be the same as that of the ones inserted in $k \_$round. The user may specify dummy subpipelines at the end of the round or $k$ _round to balance the latency. 
- The bitslice width $S_{b}$ should be a multiple of $S_{W}$ and a factor of $S_{B}$. Hence for PRESENT-80 the user gets the option of $S_{b}=4,8,16,32$.

- Any microarchitecture handling multiple data blocks cannot be designed to have non-parallel encryption or decryption mode of operation as indicated in the Table 3.1. For example, in OFB mode, the microarchitecture for encryption and decryption should not be subpipelined.

- Bitslicing cannot be combined with any other microarchitecture to generate a hybrid configuration.

\subsubsection{Hardware Resource Allocation and HDL Generation}

During resource allocation, each layer of the cipher is assigned to a hardware resource from a library of components. Before that, the hardware generation engine tries to optimize the hardware reuse for middle and final rounds of the algorithm by gauging the commonalities between the two. Since for PRESENT-80, the round_final is a single ARK operation, the final ciphertext is therefore taken out after first layer of round_middle. For AES-128, the middle round and last round differ only in one layer, i.e., GF-mul. A bypass mux is automatically inserted, enabled at the final round as shown in Fig. 3.7. SBox sharing for bitsliced configurations is also performed (discussed in subsequent sections). If for a cipher, the ARK operation is in round_init (e.g., in PRESENT-80) subkey is taken from the $k_{-}$state register, if it is in round_final (e.g., in AES-128), it is taken from the output of the last layer of $k$ round.

A mapping of the layers into operations is carried out. (The extensible RunFein framework enables/ encourages multiple customized definitions of operations)

- SBoxes are implemented as read-only lookup tables (LUT).

- Diffusion operations like rotation, shifting, PBoxes are implemented using rewiring of the inputs and renders no overhead to the combinational delay of the circuit.

- GF-mul is implemented by shifting and XOR-ing operations in accordance to the primitive polynomial of the finite field specified.

- Supported popular compound operations (e.g., MixColumns) have cascaded implementations of their constructive operations.

Next the generation of a complete working model of the block cipher in synthesizable Verilog HDL along with a testbench is carried out. The toplevel module is Testbench having the cipher or Design under Test (DUT) instantiated in it. The input to the DUT plaintext and Key, other than clk and reset signals. The output is the ciphertext along with a single bit high-asserted signal for its validation (valid). For parallel implementations, the input and output data has same width as the wordsize, for bitsliced implementations it is $S_{b}$. The controller and datapath are defined as separate modules, interacting with each other through the counter (controller). All 
definitions are kept in a separate header file. The datapath of cipher comprises of sequential elements (d_state and $\mathbf{k}$ _state) while all operational layers are generated as separate modules in HDL with interface and functionality, as per the user specified. The datapath module has instantiations of these layers.

\subsubsection{RunFein Limitations}

The RunFein methodology provides a rich configuration design space, comprehensive enough to model a diverse set of block cipher classifications. It supports constructive composition of cryptographic building blocks supporting SPN / Feistel network/ Lai-Massey based block ciphers, which are favorite primitives for block ciphers today. Additionally, stream ciphers based on block ciphers (e.g., salsa20 [21]) can be realized using RunFein. Some microarchitectural limitations of RunFein are listed below.

- Both software and hardware implementations generated by RunFein follow the on-the-fly key expansion methodology. Alternatively, subkey pre-computation requires large memory for storing $S_{S K} \times N_{r}$ bits of data. Additionally, the delay of subkey computation has to be incurred whenever a new key is used. RunFein does not pre-compute subkeys, however, converting the generated code to precomputed keys approach requires only trivial tweaking.

- Ciphers requiring unequal number of iterations for round and krounds cannot be implemented using RunFein. Though this is uncommon for most of todays ciphers, the exceptions are AES-192/256 configurations.

- For ciphers having Mix column as a diffusion operations, bitslicing requires large multiplexing logic whose overhead exceeds the potential saving achieved by bit-slicing [155]. Currently, RunFein does not support a bitsliced microarchitecture for cipher with Mix Column operation (e.g., AES). For ciphers with PBoxes, a parallel execution of PBox operation is performed instead of a bitsliced implementation as discussed in the previous section (for PRESENT-80).

- Currently, RunFein does not support a unified microarchitecture performing both encryption/ decryption.

\subsubsection{Results and Analysis: Software efficiency}

Using RunFein the software realizations for PRESENT $(80,128)$, AES (128) and KLEIN $(64,80,96)$ ciphers were generated. The software efficiency in terms of lines of code and execution time has already been discussed below. The automatically generated software implementation was compiled using gcc version 4.4.6 and run on AMD Phenom II X6 $1100 \mathrm{~T}$ Processor running at $3.3 \mathrm{GHz}$ operating frequency with 8 GB RAM and Linux operating system.

- Lines of code.: The PRESENT authors [129] have presented two implementations of the cipher that are size optimized and speed optimized with 90 and 259 
Table 4.1: SPN and Feistel block cipher software efficiency results

\begin{tabular}{|c|c|c|c|c|c|c|}
\hline \multirow{2}{*}{ Algorithm } & Key Size & Block Size & \multicolumn{2}{|c|}{ Exe. Time (Cycles/byte) } & \multicolumn{2}{c|}{ Lines of Code (lines) } \\
\cline { 4 - 7 } & $S_{K}($ Bits $)$ & $S_{B}($ Bits $)$ & RunFein & Others & RunFein & Others \\
\hline AES-128 [60] & 128 & 128 & 315 & 10.43 & 272 & 318 \\
PRESENT-80 [129] & 80 & 64 & 4595 & 4034 & 165 & 90 and 259 \\
PRESENT-128 & 128 & 64 & 6241.12 & - & 166 & - \\
KLEIN-64 & 64 & 64 & 1208 & - & 205 & - \\
KLEIN-96 & 96 & 64 & 1278 & - & 205 & - \\
KLEIN-128 & 128 & 64 & 1344.75 & - & 205 & - \\
\hline
\end{tabular}

lines of code, respectively. The higher number of lines in the speed optimized implementation of [129] is attributed to its architecture-specific configurations and optimizations. On the other hand, size optimization is achieved at the cost of code readability [129]. For PRESENT-80, RunFein generated 165 lines of code in comparison, a moderate number, as shown in Table 4.1. For AES-128, an online C implementation [60] has 318 lines of code compared to only 272 lines of code generated by RunFein and can be easily appreciated for its compactness.

- Software execution time.: RunFein generated AES-128 code lags behind in execution time due to its its compositional library-based software generation using high amount of function calls. The optimized AES implementations generally store the complete (or partial) round transformations in form of look-up tables for throughput improvement. Despite this, the software execution time of PRESENT-80 is close to the best results reported by the authors [129], possibly due to the absence of large look-up transformation, which increases overhead in a resource-constrained environment. The execution times for KLEIN and PRESENT-128 were not available.

\subsubsection{Results and Analysis: Hardware efficiency}

For PRESENT-80 and AES-128, various hardware microarchitectures were taken up. The hardware implementation details are discussed in Section 2.3.1.

\subsubsection{RunFein generated AES-128 with different microarchitectures:}

- Loop-folded: For comparison with a hand-crafted realization, a loop folded AES128 encryption core RTL implementation available at open cores [43] is taken up. Since RunFein does not register the I/Os of the cipher implementation, the registers for plaintext and ciphertext from open cores RTL were removed for enabling equitable comparisons. Both of these RTL realizations were synthesized using the $65 \mathrm{~nm}$ technology library with same versions of synthesis tools and settings at $10 \mathrm{MHz}$ and $100 \mathrm{MHz}$ operating frequencies, the area footprints 
obtained are comparable as shown in Table 4.2. The area overhead of around 5\% for the opencores RTL is attributed to its several differences compared to RunFein design. Firstly, instead of putting a multiplexer for bypassing the GF-mul stage in AES round, a separate layer of 128 bit XORs is inserted to get the ciphertext after the last round. Secondly, it maintains a 32-bit register to retain RCON value from a LUT, RunFein has no register for that. The consequent sequential area overhead can be seen in Table 4.2. Thirdly, it does not reuse the 32 bit XORs for calculation of keywords in layer 3 till layer6 of key rounds. Consequently, 5 XORs (32 bits each) are used for least significant keyword, 4 XORs for the words next to it and so on. RunFein uses only 5 XORs in total for that, consequently their area overhead for combinational logic is higher.

Table 4.2: AES-128 encryption results for 65nm CMOS

\begin{tabular}{|c|c|c|c|c|}
\hline \multirow{2}{*}{ Source } & Op. freq. & \multicolumn{3}{|c|}{ Area (GE) } \\
\cline { 3 - 5 } & $(\mathrm{MHz})$ & Comb. & Seq. & Total \\
\hline \multirow{2}{*}{ Opencores [43] } & 10 & 14540 & 1389 & 15929 \\
\cline { 2 - 5 } & 100 & 14600 & 1389 & 15989 \\
\hline \multirow{2}{*}{ RunFein } & 10 & 13825 & 1300 & 15125 \\
\cline { 2 - 5 } & 100 & 13867 & 1300 & 15167 \\
\hline
\end{tabular}

- Unrolling without Pipelining: The loop based AES-128 implementation may be unrolled by a factor of 2,5 or 10 for a potential increase in the throughput performance of the design. Table 4.3 gives the increase in area and consequently the throughput improvement when the design is unrolled and profiled for the maximum achievable frequency. Interestingly, the highest throughput/ area efficiency of the design is achieved with unroll factor 2. For higher values of loop unrolling, the gain in throughput is diminished by the large number of SBoxes and wide bus based selection circuitry.

Table 4.3: AES-128 unrolled encryption implementation results for $65 \mathrm{~nm}$ CMOS tech. library (TPA is Throughput/ Area)

\begin{tabular}{|c|c|c|c|c|c|c|c|}
\hline Unroll & no. of & Max. Freq & \multicolumn{3}{|c|}{ Area (GE) } & Throughput & TPA \\
\cline { 4 - 5 } factor $(u)$ & SBoxes & $(\mathrm{GHz})$ & Comb. & Seq. & Total & $(\mathrm{Gbps})$ & (Mbps/GE) \\
\hline 1 & $16+4$ & 1.65 & 54666.00 & 1461.50 & 56127.50 & 21.12 & 14.45 \\
\hline 2 & $32+8$ & 0.90 & 120293.50 & 1449.75 & 121743.25 & 23.04 & 15.89 \\
\hline 5 & $80+20$ & 0.30 & 169780.25 & 1406.75 & 171187.00 & 19.2 & 13.65 \\
\hline 10 & $160+40$ & 0.12 & 704315.25 & 1474.25 & 705789.50 & 15.36 & 10.42 \\
\hline
\end{tabular}

- Subpipelining: For a loop folded generated implementation of AES-128, the maximum operating frequency is profiled to be $1.65 \mathrm{GHz}$ as indicated by the Ta- 
ble 4.4. The critical path is found to exist from the $d$ state register, through the 4 round layers, the multiplexer and back to the $d$ _state register. To break this critical path, RunFein tool is indicated to place a subpipeline between layer 0 and layer 1 of the cipher round and a corresponding pipeline between layer 1 and layer 2 of the $k$ round, as shown by the single dotted line in Fig. 3.7. The RTL for the pipelined architecture is profiled to operate on a frequency as high as $2.25 \mathrm{GHz}$, with a $28.8 \mathrm{Gbps}$ of throughput. The critical path now exists between d_state register and the pipeline register, i.e., the SBox layer. A further exploration of breaking critical path is possible by partitioning the SBox tables into 2 or more levels (instead of using one 256 entry SBox, 8 are used, each with 32 entry SBoxes) and inserting pipelining in between. Similarly, the Galois field inversion of the S-box using sub-fields of 4,2 bits can be used for lower area footprints. The required multiple layers of operations for sub-fields inversion and operations can be subpipelined for achieving higher performance [194].

Table 4.4: AES-128 subpipelined encryption results for 65nm CMOS

\begin{tabular}{|c|c|c|c|c|c|}
\hline$s$ & max freq. & \multicolumn{3}{|c|}{ Area (GE) } & Throughput \\
\cline { 3 - 5 } & $(\mathrm{GHz})$ & comb. & seq. & total & $(\mathrm{Gbps})$ \\
\hline 0 & 1.65 & 54666 & 1461.50 & 56127.50 & 21.12 \\
\hline 1 & 2.25 & 49896 & 3464.75 & 53360.75 & 28.8 \\
\hline
\end{tabular}

\subsubsection{RunFein generated PRESENT-80 with different microarchitectures:}

For lightweight block ciphers, low operating frequencies are more relevant due to their stringent power constraints, hence $100 \mathrm{KHz}$ clock frequency is considered; results at $10 \mathrm{MHz}$ are also reported.

- Loop-folded: At $100 \mathrm{KHz}$, the RunFein generated PRESENT-80 encryption only loop folded implementation has a throughput of $200 \mathrm{Kbps}$ and occupies 1649 GE for 65nm CMOS technology library as indicated by the first row of Table 4.5. The power and area results for the same loop folded implementation, synthesized at $10 \mathrm{MHz}$ are indicated in the first row of Table 4.6. For comparison

Table 4.5: PRESENT-80 encryption bitsliced implementation results for 65nm CMOS tech. library@ $100 \mathrm{KHz}$

\begin{tabular}{|c|c|c|c|c|c|c|c|c|c|}
\hline Bitsliced & Cycles & SBoxes & \multicolumn{3}{|c|}{ Area $(\mathrm{GE})$} & \multicolumn{3}{|c|}{ Power $(\mu \mathrm{W})$} & Throughput \\
\cline { 7 - 10 } width $\left(S_{b}\right)$ & /round & used & Comb. & Seq. & Total & Static & Dynamic & Total & $($ Kbps $)$ \\
\hline 64 & 1 & $16+1$ & 896.25 & 752.50 & 1648.75 & 10.28 & 424.54 & 434.82 & 200.00 \\
\hline 32 & 3 & 8 & 693.25 & 768.75 & 1462.00 & 9.84 & 121.85 & 131.69 & 66.67 \\
\hline 16 & 5 & 4 & 488.25 & 775.50 & 1263.75 & 9.12 & 68.23 & 77.34 & 40.00 \\
\hline 8 & 9 & 2 & 396.50 & 785.50 & 1182.00 & 8.98 & 33.46 & 42.44 & 22.22 \\
\hline 4 & 17 & 1 & 128.75 & 978.50 & 1107.25 & 8.60 & 32.54 & 41.14 & 11.76 \\
\hline
\end{tabular}


Table 4.6: PRESENT-80 encryption bitsliced implementation results for 65nm CMOS tech. library@10 MHz

\begin{tabular}{|c|c|c|c|c|c|c|c|c|c|}
\hline \multirow{2}{*}{$\begin{array}{c}\text { Bitsliced } \\
\text { width }\left(S_{b}\right)\end{array}$} & \multirow{2}{*}{$\begin{array}{l}\text { Cycles } \\
\text { /round }\end{array}$} & \multirow{2}{*}{$\begin{array}{c}\text { SBoxes } \\
\text { used }\end{array}$} & \multicolumn{3}{|c|}{ Area (GE) } & \multicolumn{3}{|c|}{ Power $(\mu \mathrm{W})$} & \multirow{2}{*}{$\begin{array}{l}\text { Throughput } \\
\text { (Kbps) }\end{array}$} \\
\hline & & & Comb.1 & Seq. & Total & Static & Dynamic & Total & \\
\hline 64 & 1 & $16+1$ & 891.00 & 752.50 & 1643.50 & 10.28 & 454.57 & 464.84 & 20.00 \\
\hline 32 & 3 & 8 & 694.25 & 768.75 & 1463.00 & 9.84 & 150.09 & 159.92 & 6.67 \\
\hline 16 & 5 & 4 & 486.00 & 777.00 & 1263.00 & 9.12 & 95.30 & 104.42 & 4.00 \\
\hline 8 & 9 & 2 & 396.50 & 785.50 & 1182.00 & 8.98 & 54.92 & 63.90 & 2.22 \\
\hline 4 & 17 & 1 & 128.75 & 978.50 & 1107.25 & 8.60 & 55.51 & 64.11 & 1.18 \\
\hline
\end{tabular}

with the manually optimized reported implementations, the results for loop folded PRESENT-80 encryption estimates in [190] with three different CMOS technology libraries as indicated by the first column of Table 4.7 were taken up. This implementation on 180nm reportedly requires 1650 and 1706 gates at $100 \mathrm{KHz}$ and $10 \mathrm{MHz}$, respectively. RunFein generated implementation, on a comparable technology library, consumes 1750 for both $100 \mathrm{KHz}$ and $10 \mathrm{MHz}$ operating frequency, making RunFein results having 100 and 46 gates more, respectively [190]. This area-gap is far too small to be considered an overhead and possibly can be attributed to the difference in the vendor libraries, synthesis optimizations settings or different versions of synthesis tool.

Table 4.7: PRESENT-80 bitsliced encryption @ 100 KHz

\begin{tabular}{|c|c|c|c|c|c|c|}
\hline \multirow{2}{*}{$\left(S_{b}\right)$} & \multicolumn{3}{|c|}{ Area (GE) RunFein } & \multicolumn{3}{c|}{ Area (GE) $[190]$} \\
\cline { 2 - 7 } & $65 \mathrm{~nm}$ & $90 \mathrm{~nm}$ & $180 \mathrm{~nm}$ & $180 \mathrm{~nm}$ & $250 \mathrm{~nm}$ & $350 \mathrm{~nm}$ \\
\hline 64 & 1649 & 1519 & 1751 & 1650 & 1594 & 1525 \\
\hline 32 & 1462 & 1379 & 1602 & - & - & - \\
\hline 16 & 1264 & 1203 & 1403 & - & - & - \\
\hline 8 & 1182 & 1121 & 1313 & - & - & - \\
\hline 4 & 1107 & 1081 & 1265 & 1075 & 1169 & 1000 \\
\hline
\end{tabular}

\section{- Bitslicing:}

Fig. 4.7 shows a bitsliced $S_{b}=4$ PRESENT-80 implementation requiring $S_{b} / S_{W}$ (1) SBox per round, shared between $k_{-}$round and round calculations. A similar hand-crafted design has been presented for smallest area footprint of PRESENT80 in [190]. Since the Key expansion is generally non-expensive in terms of resources, bit slicing is not applied to krounds. Hence the key is loaded in $S_{K} / S_{b}$ cycles in $k$ state shift register but a subkey is calculated in a single cycle. For the round calculation, 4 bits are XOR-ed with one key nibble and passed through the SBox in each cycle. As PBox is not bit sliced round calculation requires $S_{B} / S_{b}$ cycles plus one for PBox calculation. Since the key expansion requires only one SBox, the round and kround share one. 

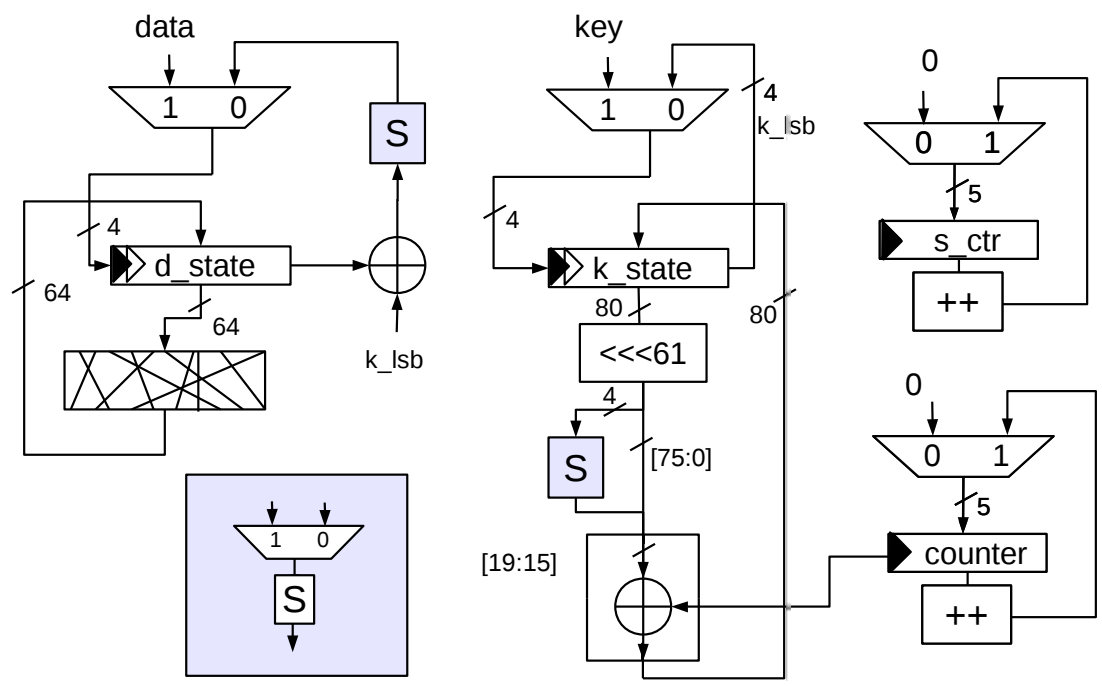

Figure 4.7: Bitsliced implementation of PRESENT-80

For bitslicing, implementations with various possible bitslice width are generated, i.e., $S_{b}=4,8,16,32$. Consequently the reduction in area, power and throughput is seen as a trend on 65nm CMOS technology library and an operating frequency of $100 \mathrm{KHz}$ in Table 4.5 and $10 \mathrm{MHz}$ in Table 4.6. On 90nm technology library, RunFein results come as close as 1081 GE compared to the smallest reported implementation of 1000 gates [190]. The implementation results for PRESENT-80 with higher bit sliced widths have not yet been reported. RunFein accelerates exploration of these intermediate design points by enabling prototyping of bitsliced architectural customizations. Some novel results are presented in Fig. 4.8 and Fig. 4.9 for resources-performance trade-off.

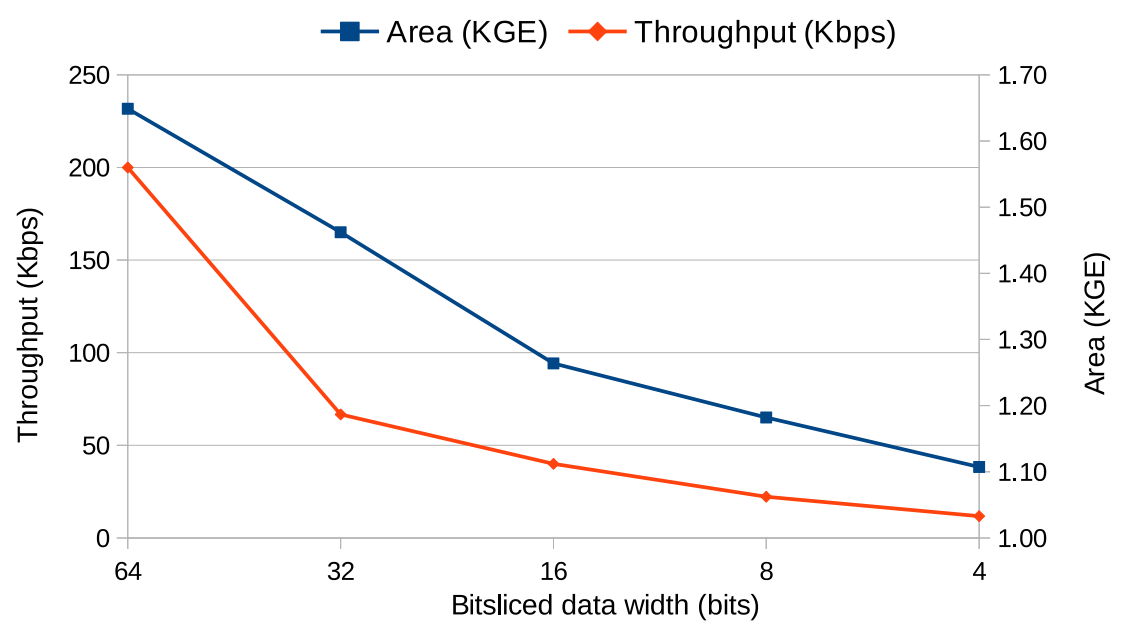

Figure 4.8: PRESENT-80 bitsliced encryption area throughput trade-off @ $100 \mathrm{KHz}$

- Unrolling without Pipelining: 


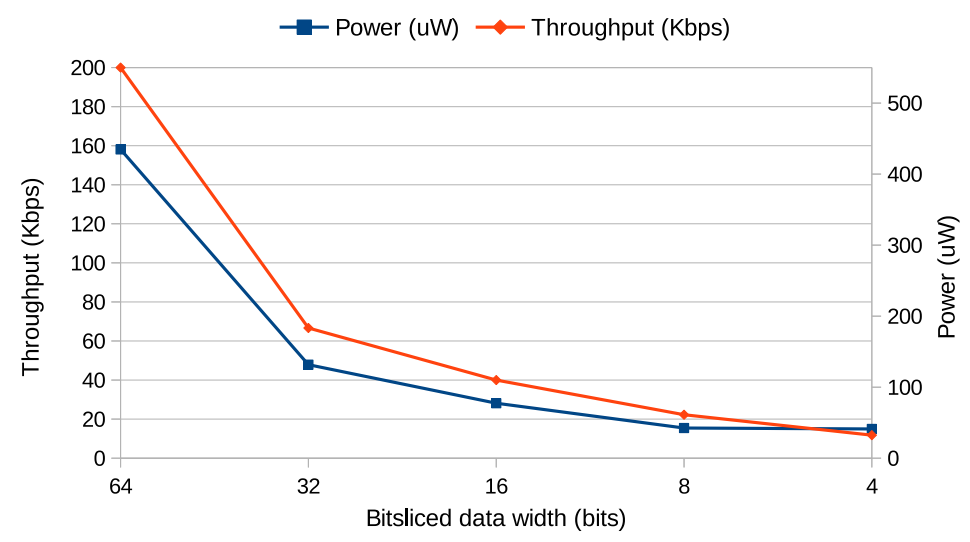

Figure 4.9: $\quad$ PRESENT-80 bitsliced encryption area power trade-off @ $100 \mathrm{KHz}$

Using RunFein, various unroll factors for the 32 rounds of PRESENT-80 encryption design were employed. Table 4.8 gives the area, power and throughput estimates when the design is unrolled by various factors. A fully unrolled design achieves the highest Throughput Per Area Ratio, however also consumes the most area and power in comparison.

Table 4.8: PRESENT-80 encryption unrolled implementations, 65nm @ $100 \mathrm{KHz}$ (Tp is Throughtput)

\begin{tabular}{|c|c|c|c|c|}
\hline Unroll factor, $u$ & Cycles/ block & Area (GE) & Power $(\mu \mathrm{W})$ & Tp (Mbps) \\
\hline 1 & 32 & 1648.75 & 434.82 & 0.20 \\
\hline 2 & 16 & 2279.75 & 450.00 & 0.40 \\
\hline 4 & 8 & 3396.25 & 494.08 & 0.80 \\
\hline 8 & 4 & 5859.75 & 522.12 & 1.60 \\
\hline 16 & 2 & 10712.25 & 645.61 & 3.20 \\
\hline 32 & 1 & 19817.75 & 754.00 & 6.40 \\
\hline
\end{tabular}

\section{- Subpipelining:}

Through subpipelining, some novel, high throughput realizations of PRESENT80 cipher were generated, which have not been reported till date. For a loop folded implementation, the maximum operating frequency is profiled to be 3.7 $\mathrm{GHz}$ as indicated by the Table 4.9. For achieving high throughput performance, it is subpipelined twice.

- First Subpipeline: The critical path for the loop folded implementation (Fig. 3.7 left) exists from the $k$ state register, through the 3 round layers, the multiplexer and till the $d \_s t a t e$ register. Since PBox poses no combinational delay due to rewiring, its prudent to break the critical path by inserting a subpipeline between layer 0 and layer 1 of the cipher, shown by the single dotted line in Fig. 3.7. A corresponding subpipeline between layer 1 and 
layer 2 of the $k$ round is also opted. Consequently the subpipelined circuit's operating frequency increases, raising the throughput to $8.1 \mathrm{Gbps}$.

- Second Subpipeline: The critical path now exists between the subpipeline register and the $d \_$state register in round. For a further increase in the operating frequency, this critical path between layer 1 and layer 2 of round is broken by a second subpipeline (with a corresponding subpipeline between layer 0 and layer 1 of $k$ _round) as shown by double dotted lines in Fig. 3.7. The corresponding operating frequency however decreases. This is attributed to the supporting control hardware inserted to tackle the 2 subpipelines. A 2-bit supplementary counter (s_counter) counting up to the number of subpipelines is inserted in addition to the 5-bit counter for rounds. The critical path now exists in the controller, i.e., between s_counter and counter, prohibiting further speedup by pipelining.

Table 4.9: PRESENT-80 subpipelined encryption results for 65nm CMOS, (Tp is Throughtput)

\begin{tabular}{|c|c|c|c|c|c|}
\hline$s$ & Max Freq. & \multicolumn{3}{|c|}{ Area (GE) } & Tp \\
\cline { 3 - 5 } & $(\mathrm{GHz})$ & comb. & seq. & total & $(\mathrm{Gbps})$ \\
\hline 0 & 3.71 & 2502.75 & 856.00 & 3358.75 & 7.42 \\
\hline 1 & 4.05 & 2320.75 & 1803.50 & 4124.25 & 8.1 \\
\hline 2 & 4 & 2818.75 & 2657.00 & 5475.75 & 8.0 \\
\hline
\end{tabular}

\subsection{RunStream}

RunStream caters the rapid prototyping of stream ciphers. The toolflow of RunStream is graphically shown in Fig. 4.10. Similar to RunFein, it accepts design input specifications through a GUI capture. Some saved configuration files of well-known stream ciphers could be quickly loaded for easy reference. RunStream validates the design specification for completeness and correctness at various stages of the toolflow and on success creates a cipher template. The software and hardware generation engines of the tool generate an optimized software implementation and a synthesizable HDL description. Using RunStream, successful implementation of various stream ciphers is carried out and the benchmarked QoR is found on-par with already-known handoptimized implementations.

\subsubsection{Design Specification Compilation}

As discussed in Section 3.4.2.4, the algorithmic design configuration space of a stream cipher comprises of elementary parameters, sequential state information and a mesh of operations. The user specifies the stream cipher design through a GUI. Fig. 4.11 gives 


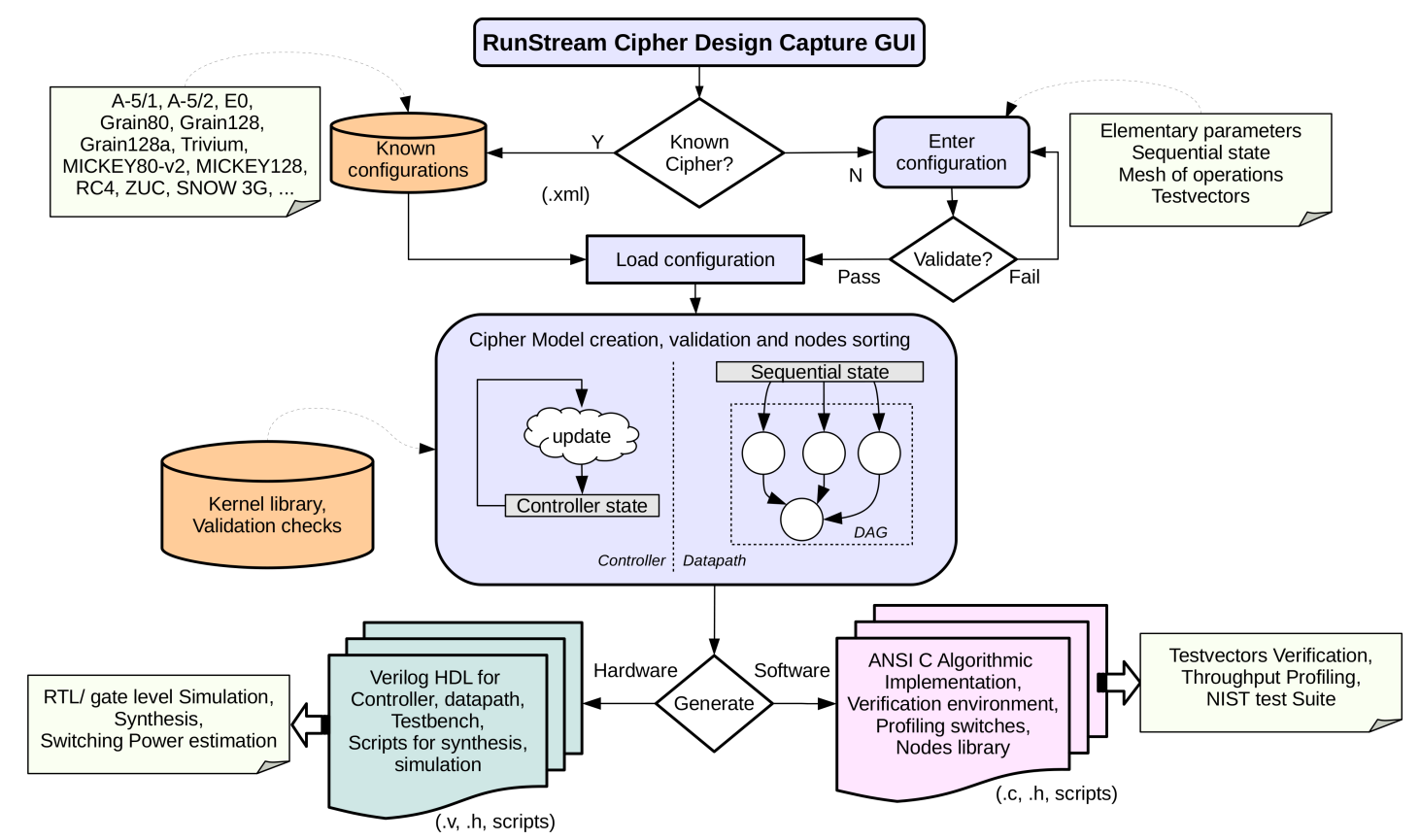

Figure 4.10: Runstream toolflow for software generation and hardware generation

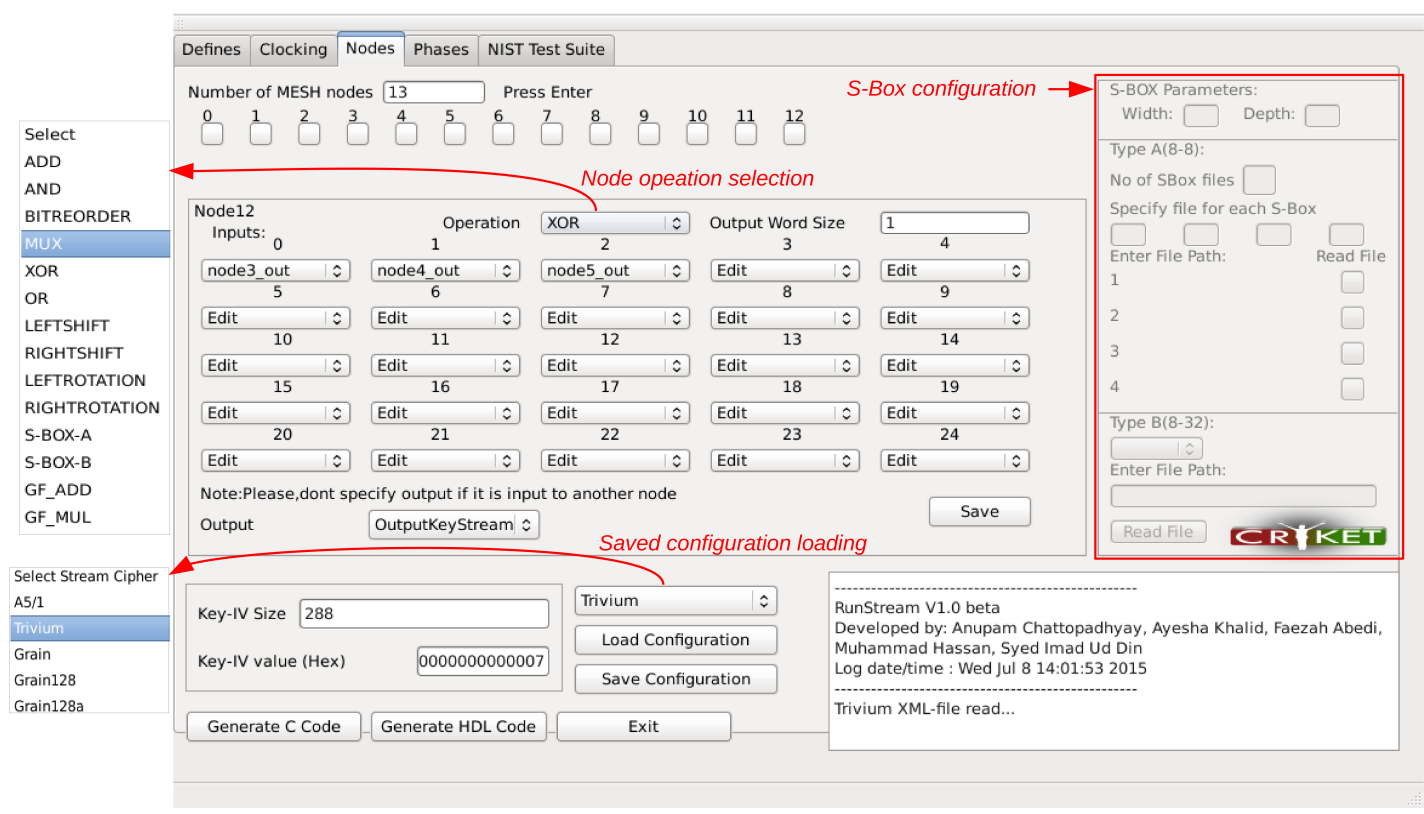

Figure 4.11: Mesh nodes tab for RunStream showing input/outputs for Trivium's node12

a snapshot of the RunStream tab that accepts the nodes of operation. For Trivium the design specification comprises of a total of 13 nodes (Fig. 3.10). Each mesh node can be configured to have an operation, inputs, output, granularity etc. 


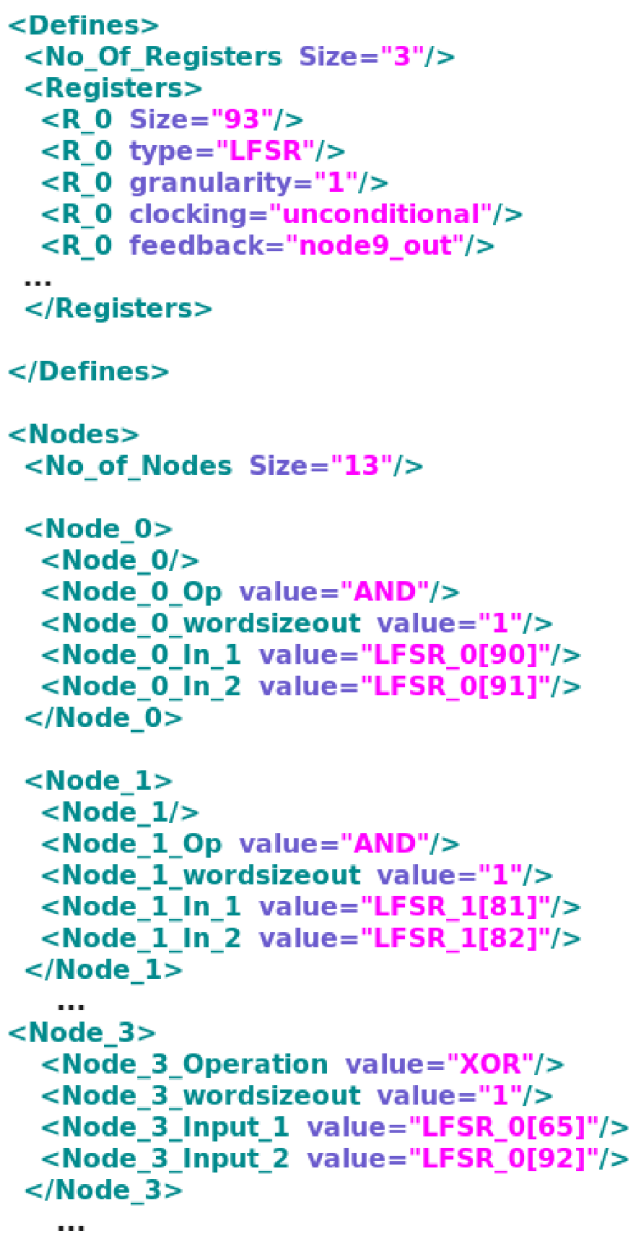

$<$ Node 11>

$<$ Nodé_11/ $>$

$<$ Node_11_Op value $="$ MUX"/>

$<$ Node_11_wordsizeout value $=" 1 " />$

$<$ Node 11 In 1 value $=$ "PHASE"/>

$<$ Node_11_In_2 value $=$ "LFSR_1[83]"/>

$<$ Node_11_In_3 value $=$ "node $\overline{7} \_$out" $/>$

$<$ Node_11_In_4 value $=$ "node7_out" $/>$

$<$ /Node_11 $>$

$<$ Node_12>

$<$ Nodé_12/>

$<$ Node 12 Op value $="$ XOR" $/>$

$<$ Node 12 wordsizeout value $=" 1 " />$

$<$ Node_12_In_1 value $=$ "node3_out" $/>$

$<$ Node_12_In_2 value $=$ "node4_out" $/>$

$<$ Node_12_In_3 value $=$ "node5_out"/ >

$<$ /Node_12 $>$

$<$ /Nodes $>$

$<$ keystream >

<keystream value="node12_out"/>

<keystream>

<Phases>

$<$ No of Phases Size="3" $/>$

$<$ Phase_o_no_of_cycles size =" $288 " />$

$<$ Phase_1_no_of_cycles size $=" 1152 " />$

$<$ Phase_2 no_of_cycles size $=" 100 " />$

$<$ /Phases $>$

$</$ StreamCipher $>$

Figure 4.12: The configuration file snapshot input to RunStream for Trivium

The configuration parameters for a stream cipher given to the tool's GUI are stored as an $x \mathrm{ml}$ configuration file, a snapshot of Trivium configuration file is shown in Fig. 4.12. The information for each of the FSR is kept as a separate token in the file, for $R 0$ the size, type, granularity and clocking can be seen. For each of the 13 nodes, the user specifies an operation (R0), output width (wordsizeout) and the node input sources. The node generating the final keystream is specified as separate token (keystream). The feedback for each of the three registers is also specified by a node number. Other elementary parameters like the number of phases, cycles for each phase and the test vectors (not shown in shown in Fig. 4.12) are also specified and saved as part of the cipher configuration.

\subsubsection{Specification Validation and Formal Model Creation}

Before creation of a valid stream cipher model, the configuration given by the user undergoes a list of defined rules checks, some of them are listed below.

- The number of inputs match the number of inputs for the operation specified, hence two for binary operation etc. 
- The S-Box values are $\in\left[0 . .2^{S_{W}}\right]$.

- P-Box, rotation/shifting, XOR operations have arguments $\in\left[0 . . S_{B}\right]$.

- The polynomial coefficients for GF-mul are not $\varnothing$.

As these checks pass, the configuration file is parsed and a formal stream cipher model is created comprising of a controller and datapath. The controller keeps tracks of the phase changes by aid of a counter, according to the cycle count of phases specified. The datapath of the cipher is constructed by a Directed Acyclic Graph (DAG) comprising of nodes and interconnects. Fig. 4.13 shows the DAG to generate the feedback bits for the LFSRs and keystream output (As a convention, the count for all nodes, phases, interconnects and registers starts from 0 ) for Trivium stream cipher. A separation of sequential and combinational elements for Trivium can be seen.

The first layer of nodes is the Source nodes as these nodes do not take inputs from any other nodes. Since they require no operation and are taps from the three FSRs, the node numbers are not assigned to them. The rest of the nodes are enumerated. Node0 is an AND operation node with two incoming interconnects (R0[90],0) and $(R 0[91], 0)$ along with one outgoing interconnect, i.e., $(0,7)$. Node7 takes input from the source nodes as well as the enumerated nodes, the three inputs being Node0, Node3 and R1[77]. Sink nodes are the ones whose output is not forwarded to any other node. These include Node9, Node10 and Node11 which are MUX by operation and control the input to the LFSRs during the 3 phases of operation.

The interconnects between the user defined nodes are stored as a finite graph $G$ on $n$ vertices where $n$ is the sum of both the enumerated operational nodes and the source nodes from register taps. Referring back to Trivium (Fig. 4.13), there are 13 enumerated nodes and 18 souse nodes. An $n \times n$ entries graph adjacency matrix holds the interconnects where each entry $a_{i j}$ represents if an edges exists from $i^{\text {th }}$ node to $j^{\text {th }}$ node or not.

Next the graph(G) undergoes a second phase of model validation by a list of defined rule checks. The user is prompted in case of a violation and the cipher implementation does not proceed unless a valid configuration is specified.

- $G$ must not be an empty graph, has no unreachable dangling nodes. It must be a simple graph.

- $G$ has no duplicate edges (interconnects with same initial and head node), hence the corresponding graph adjacency matrix holds binary elements.

- There should be no circular dependencies (interconnects with same node as initial and terminal nodes). Hence all diagonal entries of the adjacency matrix must be zeros.

For simulation, the graph $(\mathrm{G})$ must have an execution order for a valid sequential evaluation of cipher. Although HDLs mimic a parallel execution model, for software implementations that undertake a sequential execution of code chunks. Hence an execution order for evaluation of nodes is required for a valid simulation of the 


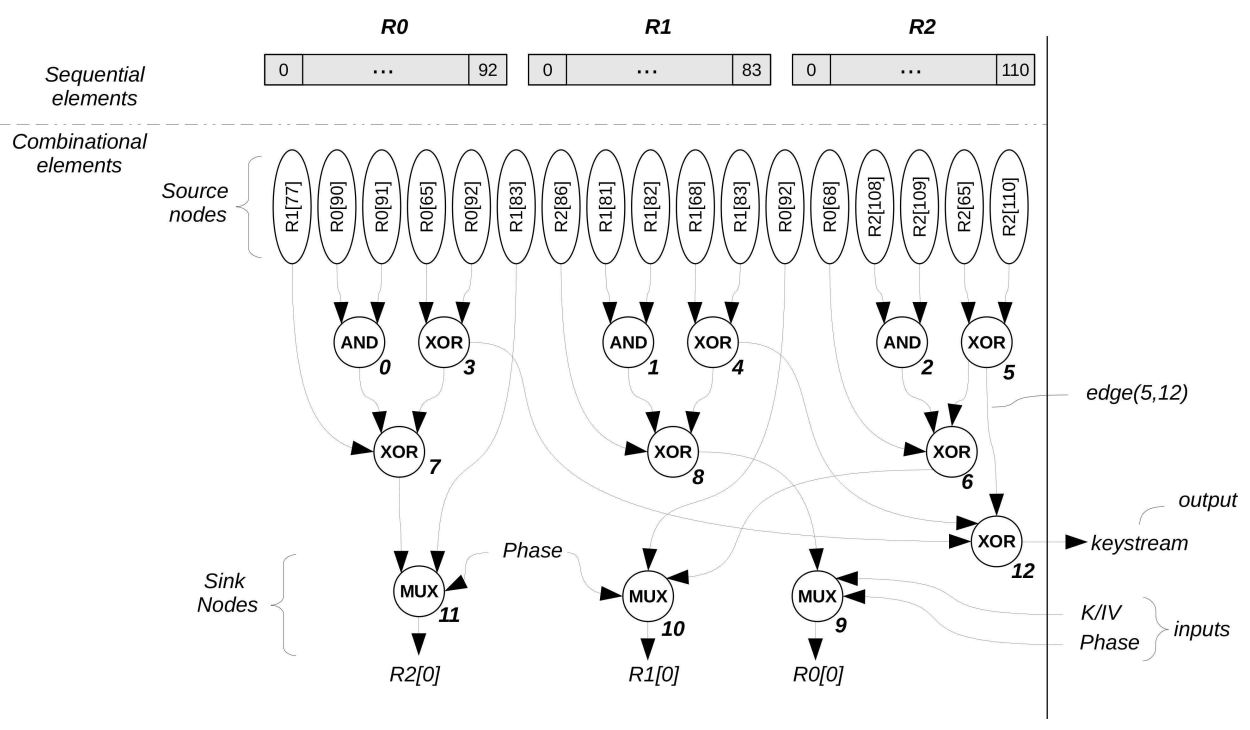

Figure 4.13: The Directed Acyclic Graph for Trivium nodes implementation (datapath of cipher model)

cipher model. A directed graph with no loops makes the configuration a directed acyclic graph (DAG), whose execution order may be found by topological sorting. The nodes numbers are updated after sorting. Fig. 3.10 and Fig. 4.15 shows DAGs with topologically sorted nodes.

\subsubsection{Software Generation Engine}

The software generation engine compiles the cipher model to generate a high performance, fixed-point ANSI-C description along with a simulation environment with user controllable switches for verification, throughput profiling, data dumping etc. The generated code is not specifically optimized for a particular General Purpose Processor (GPP), however, it has a regular structure and good code readability.

All the user specified parameters of the cipher are stored in header files. Data types of registers, nodes interfaces and other controller related variables are typedefed in accordance with the granularity specified. Supplementary functions are kept in a separate file, that is included in the main file during simulation. For each operational node, a separate function is defined with interface and functionality, as per the user specified.

The main body of code, having the controller and the datapath of the cipher model, is a separate file that \#includes all supplementary and header files. For elaboration of code simulation environment, lets refer to Trivium. Algorithm 2 gives a code chunk for phase phase 0 of the cipher. The controller part of the cipher comprises of two local variables phase and loop variable $i$, keeping count of the phase and cycle under execution, respectively. The loop in line 2 iterates for the number of cycles defined for phase0 (P0_cnt). 
In every cycle iteration, first the combinational mesh of operational nodes are executed and then the sequential resources are updated.

- For updating the combinational nodes, the function calls to all 13 nodes are executed (line 3-7). The inputs to the nodes are taken from the defines file while the outputs are local variables (for node0, Node0_in 0 and Node0_in 1 are defined to be R0[90] and R0[91]). The output of one node could also be input to another, as per the user specification.

- Next the sequential elements are updated (line 8-16). R0, $R 1$ and $R 2$ are defined as local arrays with user specified size and type. $R 0$ being an LFSR, the left shifting of its contents is carried out by a nested loop over variable $j$ (line 7 ). Similarly, $R 1$ and $R 2$ are shifted by loops in line 10 and 12, respectively. The input tap of these LFSRs are updated by their corresponding nodes as shown in line 14,15 and 16, respectively. The code for value update of FSM registers follows the LFSRs update, however Trivium has no FSM registers.

A valid keystream bit is generated from a node (node12 for Trivium) no earlier than the last phase of cipher implementation. For the other two phases, the code listing is similar to the one given in Algorithm 2, except the line 1 that holds different phase number.

The software generation engine of RunStream generates a sequential C model of the stream cipher with necessary libraries and scripts. As discussed in Section 4.2.3, the RunStream generated code is also enhanced by the provision of integrated NIST test suite [191], a verification environment generated along and provisioned by necessary performance profiling switches.

\subsubsection{Hardware Generation Engine}

The hardware generation engine generates a complete working model of the stream cipher in synthesizable Verilog HDL along with a testbench. Fig. 4.14 shows the architecture of the cipher. The toplevel module is Testbench having the stream cipher or Design under Test (DUT) instantiated in it. The input to the DUT is the KIV vector (after any pre-initialization manipulation, init in Fig. 3.3), other than clk and reset signals. The output is the keystream along with a single bit high-asserted signal for its validation (valid). The granularity of the cipher determines the width of KIV and the keystream signals.

The controller and datapath are defined as separate modules, interacting with each other through the phase signal. All definitions are kept in a separate header file.

- Controller: The controller keep track of current phase using two registers; counter and phase. The size of counter is taken up as ceil $\left(\log _{2}\left(P i_{-}\right.\right.$cnt $\left.)\right)$bits, where Pi_cnt is the cycle count of the longest of the phases. For Trivium $P 1 \_c n t=288 \times 4=1152$ determines counter size to be 11 bits. During phase0 (key setup), the FSR registers are initialized using a 288 bit vector comprising of user specified key and IV values $\left(P 0 \_c n t=1152\right)$. As soon as the counter 


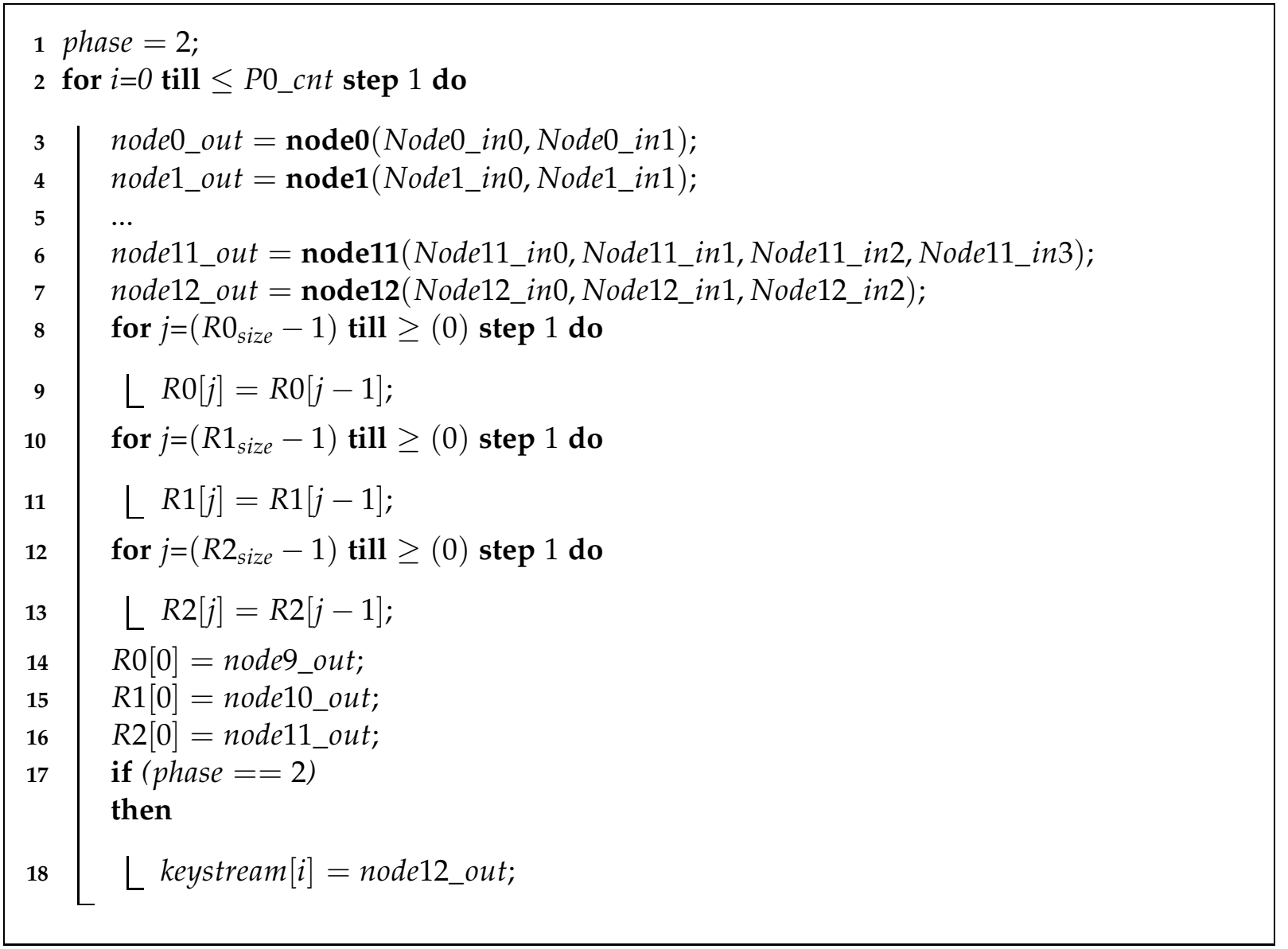

Algorithm 2: RunStream generated pseudo code chunk for phase0 of Trivium stream cipher

hits the phase count for the current phase, the phase register is incremented and counter is cleared (as shown in Fig. 4.14). For all stream ciphers, a valid output keystream is generated during the last phase of operation. Hence for trivium, the valid signal is asserted when phase register is incremented to 2.

- Datapath: The datapath of stream cipher comprises of sequential elements and a mesh of nodes, for Trivium the architectural details are shown in Fig. 3.10. In case of multiple nodes, all operational nodes are generated as separate modules in HDL with interface and functionality, as per the user specified. The datapath module has instantiations of these node modules. The sequential elements are local registers in datapath module that are declared, reset, clocked and updated as specified by their type, granularity and size. For Trivium, the randomization phase or phase 1 and outputs of node6, node7, node8 multiplexers are passed as inputs to $R 0, R 1$ and $R 2$, respectively. During the last phase, i.e., phase2, one of the terminal node, i.e., Node12 generates valid keystream that is output of the cipher module. 


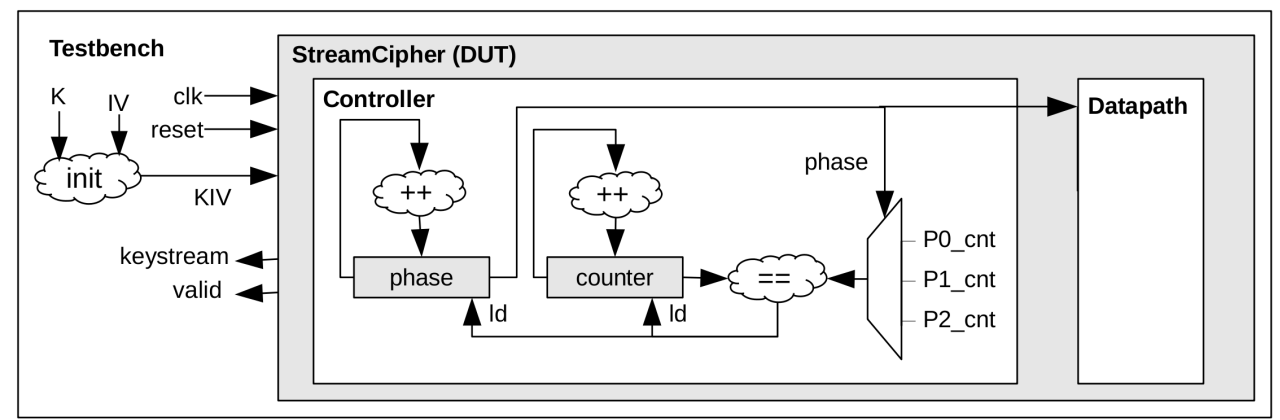

Figure 4.14: Interfaces for a RunStream generated stream cipher implementation

The hardware generation engine of RunStream generates a synthesizable, hierarchical stream cipher HDL and testbench with necessary scripts that can be further used to carryout

- Simulations for design verification, gate-level simulation (post-synthesis) using verification tools.

- Logic synthesis of the design for profiling critical parameters like the maximum clock frequency, chip area.

- Post-synthesis power consumption estimation with using back-annotation.

\subsubsection{Mapping of Algorithms}

Using RunStream, successful mapping of a diverse set of algorithms given in Table 3.4 was carried out. Table 4.11 illustrates the configuration details of various stream ciphers undertaken for implementation by RunStream. The sequential information for these ciphers is given in Table 4.10. Unlike the datapath of a cipher, the controller information of the stream ciphers changes only slightly from algorithm to algorithm. In the following discussion, only the distinguishing features of these ciphers are highlighted, as they are being undertaken by RunStream implementation methodology. Since RC4, ZUC and SNOW 3G are the only non-binary stream ciphers undertaken, the mapping of one of them (SNOW 3G) is elaborated in detail.

- SNOW 3G: The structural architecture and the mesh configuration of SNOW $3 G$ [2] is shown in Fig. 4.15. The $R 0$ is a 16 element NFSR array with granularity of 32, while $R 1, R 2$ and $R 3$ are 32-bit FSM registers, all clocked unconditionally. The $M U L_{\alpha}$ and $D I V_{\alpha}$ are GF operations, that are carried out by node0-2 and node3-5, respectively. The S-boxes of node 0 and node3 are $8 \times 32$ with a lookup table of $2^{8}$ elements of 32 bits each. The S-boxes in node11 and node13 are $8 \times 8$. The input word is divided into 4 bytes, each of which is transformed by an Sbox of $2^{8}$ elements of 8 bits each. The result is concatenated and passed on to the next node (node12 and node14) which is GF-multiplication. A valid output is generated from node10 in the keystream generation phase. Alternatively, the 
Table 4.10: Sequential information configuration for stream ciphers taken up by RunStream

\begin{tabular}{|c|c|c|c|}
\hline & Register Arrays (bits) & FSM Registers (bits) & Cipher State (bits) \\
\hline A-5/1 & 3 LFSRs $(19,22,23)$ & - & 64 \\
\hline$A-5 / 2$ & 4 LFSRs $(19,22,23,17)$ & - & 81 \\
\hline E0 & 4 LFSRs $(25,31,33,39)$ & $2(4)$ & 132 \\
\hline Grain80 & 1 LFSR (80), 1 NFSR (80) & - & 160 \\
\hline Grain128 & 1 LFSR (128), 1 NFSR (128) & - & 256 \\
\hline Grain128a & 1 LFSR (128), 1 NFSR (128) & - & 256 \\
\hline Trivium & $3 \operatorname{LFSR}(93,84,111)$ & - & 288 \\
\hline MICKEY80-v2 & 2 Jump regs ( 2 x 100) & - & 160 \\
\hline MICKEY128 & 2 Jump regs $(2 \times 160)$ & - & 320 \\
\hline RC4 & 1 reg array $(8 \times 256)$ & $2(16)$ & $32 \mathrm{~K}$ \\
\hline ZUC & 1 NFSR $(16$ x 31) & $2(64)$ & 560 \\
\hline SNOW 3G & 1 NFSR $(16$ × 32) & $3(96)$ & 608 \\
\hline
\end{tabular}

Table 4.11: Operational nodes information for RunStream configuration of stream ciphers

\begin{tabular}{|c|c|c|c|c|c|c|c|c|c|c|c|c|c|c|c|c|}
\hline & 文 & 号 & $\frac{0}{0}$ & $\begin{array}{l}5 \\
\vdots \\
z\end{array}$ & 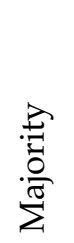 & 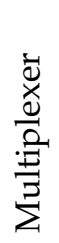 & $\begin{array}{l}\text { 节 } \\
\text { ऊ }\end{array}$ & $\begin{array}{l}\text { 节 } \\
\stackrel{0}{0}\end{array}$ & $\begin{array}{l}\text { ô } \\
\text { pी } \\
\text { के }\end{array}$ & 层 & 㐏 & $\begin{array}{l}\frac{y}{0} \\
\sum_{\overrightarrow{0}}^{\pi}\end{array}$ & 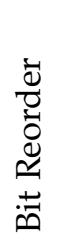 & $\frac{\pi}{\pi}$ & 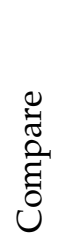 & 胥 \\
\hline A-5/1 & - & - & 7 & - & 1 & 3 & - & - & - & - & - & - & - & - & - & 11 \\
\hline$A-5 / 2$ & - & - & 9 & 3 & 4 & 4 & - & - & - & - & - & - & - & - & - & 20 \\
\hline E0 & 1 & - & 6 & - & - & 4 & 1 & - & 1 & - & - & - & - & 1 & - & 14 \\
\hline Grain80 & 19 & - & 6 & - & - & 2 & - & - & - & - & - & - & - & - & - & 27 \\
\hline Grain128 & 12 & - & 6 & - & - & 2 & - & - & - & - & - & - & - & - & - & 20 \\
\hline Grain128a & 15 & - & 6 & - & - & 2 & - & - & - & - & - & - & - & - & - & 23 \\
\hline Trivium & 3 & - & 7 & - & - & 3 & - & - & - & - & - & - & - & - & - & 13 \\
\hline MICKEY80-v2 & 2 & - & 10 & - & - & 6 & 3 & - & - & - & - & - & - & 1 & - & 22 \\
\hline MICKEY128 & 2 & - & 10 & - & - & 6 & 3 & - & - & - & - & - & - & 1 & - & 22 \\
\hline $\mathrm{RC} 4$ & - & - & - & - & - & 9 & - & - & - & - & - & - & - & 7 & 6 & 22 \\
\hline ZUC & - & - & 5 & - & - & 3 & 1 & 8 & 2 & - & 2 & - & 6 & 2 & - & 29 \\
\hline SNOW 3G & - & - & 7 & - & - & 4 & 2 & - & 4 & 2 & - & - & - & 2 & - & 21 \\
\hline
\end{tabular}




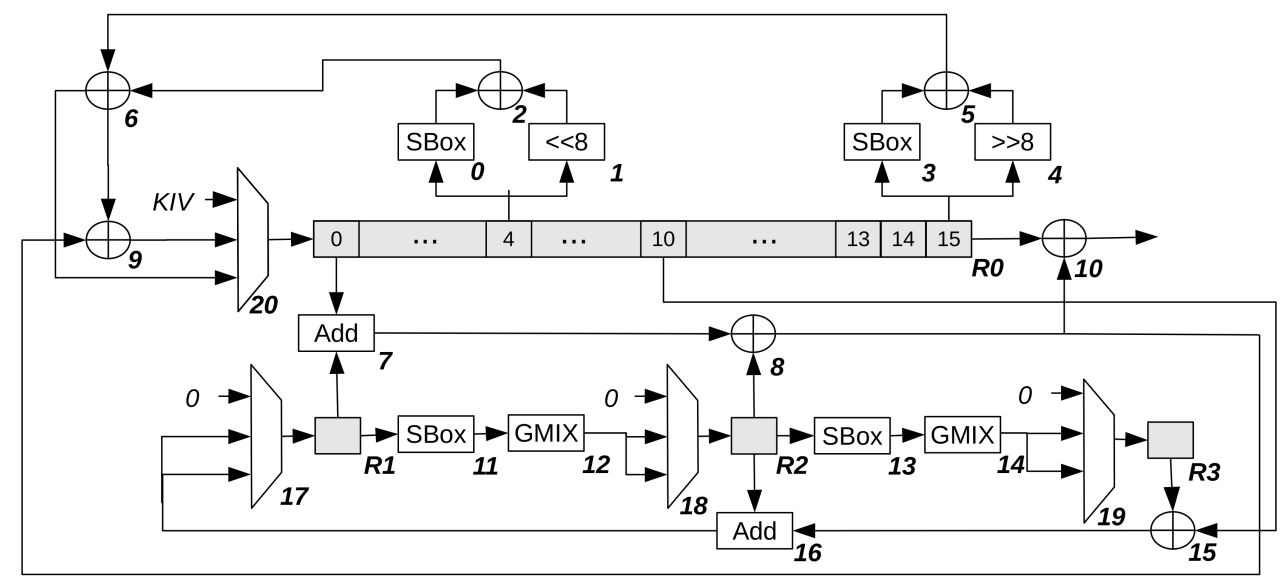

Figure 4.15: Architectural structure of SNOW 3G implementation

user can opt for an equivalent implementation comprising of a series of combinational logic nodes instead of lookup tables to reduce the area. The alternative implementation would have lower area but higher critical time. Various design options could be quickly explored using RunStream for reaching an optimal solution.

- A-5/1/2: A-5/1 comprises of 3 LFSR registers, sum of which equates to a 64 bit state as given. The three LFSRs are irregularly clocked based on a majority operation node, the 3 sink nodes for LFSR feedback are multiplexers while the linear feedback nature of the LFSRs is carried out by 7 XOR nodes, totally 11 nodes in all. A-5/2 follows a similar structure as A-5/1, the irregular clocking is carried out by 4 majority nodes.

- E0: E0 stream cipher has 2 FSM registers of 2 bits each, other than FSRs, hence the cipher state evaluates to a total of 132 bits.

- Grain: The Grain family of binary additive stream cipher have two FSRs, one with linear feedback and the second with nonlinear feedback. The FSRs are updated and the keystream is generated by means of some lightweight Boolean functions implemented by XOR and AND gates only. Both Grain128 and Grain128a have 256 bits state, Grain128a has additional logic to calculate message authentication code in addition to generating a keystream.

- MICKEY: This feature of nodes operating on word sizes independent of the granularity of the FSR elements is particularly useful for MICKEY family of stream ciphers. MICKEY80-v2 has two jump registers of 100 bits each, whose each bit may be updated per clock cycle. The variable wordsize of nodes emancipates the user by allowing him to define operations to be performed on the whole register instead of each element. MICKEY80-v2 requires 22 nodes for its definition, 7 of which are operations performed on 1-bit inputs while the rest 
operate on 100 bits. For MICKEY128, the node count remains the same, the 15 nodes operate on 160 bits operations instead of 100 bits.

- RC4: The T-function of RC4 during the phase 0 and phase 1 requires $i$ update, followed by $j$ update (taking updated value of $i$ ) along with a swap of two values of register array (R0) taking updated values of $i$ and $j$. To enable a single cycle execution of T-function of these phases, the $i$ and $j$ updates are pre-calculated for the next cycle. All the corner conditions are evaluated and handled using the 6 compare nodes and multiplexers. A total of 22 operational nodes are required for the operation.

- ZUC: For ZUC stream cipher, the FSR elements have 31 bits granularity, while the two FSM registers are 32 bits each. This mismatch is incorporated by explicitly defining the granularity of each node edge, that may not match the cipher granularity.

Using RunStream a very wide range of stream ciphers may be modeled. The first prototype of this tool models the sequential states (FSRs, FSM registers) as D-flip flops. Some stream ciphers with large states (e.g., RC4 [195] requiring 2K bits of state information), external SRAMs may be employed. For an efficient implementation of such ciphers, pipelining and optimized SRAM ports utilization is exploited. These optimizations are currently not offered by RunStream, however an extension to offer SRAMs access optimizations is on the roadmap.

\subsubsection{Efficiency}

This section summarizes the ASIC performance results for various HDL stream cipher implementations, generated by RunStream. For all the stream ciphers undertaken, HDL generation, synthesis and benchmarking has been carried out using the same design flow. RunStream was configured in accordance with the parameters of each of the stream ciphers. The generated $C$ based software implementation was tested for correctness against the available test vectors. RTL verification was carried out using Mentor Graphics ModelSim (version 10.2c).

The hardware implementation details are discussed in Section 2.3.1 (65nm CMOS). All the power estimations include the initialization and runup phases of stream ciphers, along with the keystream generation phase for 1024 bits of keystream. The performance metrics evaluated are discussed in Section 2.3.3. Table 4.12 shows the area and power consumption estimates for different stream cipher for the highest operating frequency. i.e., no valid design could be synthesized at frequency above 0.5 $\mathrm{GHz}$ for ZUC stream cipher. The natural choice of interface bits width equal to the wordsize of each stream cipher is taken up. Area estimates of sequential and combinational logic, contributing to the core area are given. Understandably, RC4 has the highest area, owing to its $32 \mathrm{~K}$ bits state (sequential logic).

The power consumption of a core on a feature size design is a function of the complexity of the design and the clock frequency. Static/leakage power is proportional to 
Table 4.12: Design results for highest operating frequencies @65nm CMOS

\begin{tabular}{|c|c|c|c|c|c|c|c|c|}
\hline \multirow{2}{*}{ Cipher } & Max. Freq. & Interface & \multicolumn{3}{|c|}{ Area (GE) } & \multicolumn{3}{c|}{ Power $(\mu \mathrm{W})$} \\
& $(\mathrm{MHz})$ & bits & Comb. & Seq. & Total & Dynamic & Leakage & Total \\
\hline A-5/1 & 1000 & 1 & 237.0 & 370.0 & 607.0 & 589.0 & 3.5 & 592.5 \\
\hline A-5/2 & 1000 & 1 & 263.0 & 455.0 & 718.0 & 1327.0 & 4.5 & 1331.5 \\
\hline E0 & 1000 & 1 & 100.5 & 690.0 & 790.5 & 390.8 & 5.0 & 395.8 \\
\hline Grain80 & 1000 & 1 & 154.5 & 850.0 & 1004.5 & 2272.0 & 7.1 & 2279.1 \\
\hline Grain128 & 1000 & 1 & 134.2 & 1330.0 & 1464.3 & 2277.0 & 9.6 & 2286.6 \\
\hline Grain128a & 1000 & 1 & 144.7 & 1335.0 & 1479.8 & 2276.0 & 9.8 & 2285.8 \\
\hline Trivium & 1000 & 1 & 108.0 & 1505.0 & 1613.0 & 3813.0 & 11.5 & 3824.5 \\
\hline Mickey80-v2 & 1000 & 1 & 1027.2 & 1065.0 & 2092.3 & 4211.0 & 12.3 & 4223.3 \\
\hline Mickey128 & 1000 & 1 & 1591.5 & 1665.5 & 3257.0 & 5833.0 & 15.2 & 5848.2 \\
\hline RC4 & 1000 & 8 & 33920.6 & 13596.2 & 47516.8 & 1490.3 & 243.6 & 1733.9 \\
\hline ZUC & 500 & 32 & 8404.0 & 3536.7 & 11940.7 & 3360.0 & 78.5 & 3438.5 \\
\hline SNOW 3G & 1000 & 32 & 8136.0 & 3723.7 & 11859.7 & 17110.0 & 90.3 & 17200.3 \\
\hline
\end{tabular}

the area, the dynamic power contributes majorly to the total power consumption of the design. The maximum power dissipation occurs when the circuit is operated at its maximum frequency. Also, the area for any design is largest for the highest possible operating frequency of the design. Consequently, the design results are re-calculated for $10 \mathrm{MHz}$ (10Mbps throughput for wireless LAN applications) and $100 \mathrm{KHz}$ (for typical RFID applications), as given in Table 4.13 and Table 4.14, respectively.

Table 4.13: Design results for $10 \mathrm{MHz} @ 65 \mathrm{~nm} \mathrm{CMOS}$

\begin{tabular}{|c|c|c|c|c|c|c|c|c|}
\hline Cipher & Interface & Throughput & \multicolumn{3}{|c|}{ Area (GE) } & \multicolumn{3}{c|}{ Power $(\mu \mathrm{W})$} \\
& bits & $(\mathrm{Mbps})$ & Comb. & Seq. & Total & Dynamic & Leakage & Total \\
\hline A-5/1 & 1 & 10 & 237.0 & 370.0 & 607.0 & 11.7 & 3.7 & 15.4 \\
\hline A-5/2 & 1 & 10 & 263.0 & 455.0 & 718.0 & 13.3 & 4.5 & 17.8 \\
\hline E0 & 1 & 10 & 100.5 & 690.0 & 790.5 & 15.6 & 5.5 & 21.1 \\
\hline Grain80 & 1 & 10 & 152.5 & 850.0 & 1002.5 & 8.8 & 6.5 & 15.3 \\
\hline Grain128 & 1 & 10 & 134.2 & 1330.0 & 1464.3 & 12.8 & 9.7 & 22.4 \\
\hline Grain128a & 1 & 10 & 144.7 & 1335.0 & 1479.8 & 12.8 & 9.8 & 22.5 \\
\hline Trivium & 1 & 10 & 107.2 & 1505.0 & 1612.3 & 13.4 & 10.6 & 24.0 \\
\hline Mickey80-v2 & 1 & 10 & 1023.2 & 1065.0 & 2088.3 & 12.7 & 11.2 & 23.9 \\
\hline Mickey128 & 1 & 10 & 1573.5 & 1665.0 & 3238.5 & 14.0 & 14.1 & 28.1 \\
\hline RC4 & 8 & 80 & 21543.7 & 10610.0 & 32153.8 & 8.8 & 143.3 & 152.1 \\
\hline ZUC & 32 & 320 & 7023.0 & 2865.0 & 9888.0 & 156.0 & 56.7 & 212.7 \\
\hline SNOW 3G & 32 & 320 & 6977.7 & 3105.0 & 10082.7 & 146.4 & 53.5 & 199.9 \\
\hline
\end{tabular}

As the operating frequency is lowered from the highest operating frequency to $10 \mathrm{Mhz}$ the area estimates decrease. For simple bit-oriented ciphers the area estimates 
Table 4.14: Design results for $100 \mathrm{MHz} @ 65 \mathrm{~nm}$ CMOS

\begin{tabular}{|c|c|c|c|c|c|c|c|c|}
\hline \multirow{2}{*}{ Cipher } & Interface & Throughput & \multicolumn{3}{|c|}{ Area (GE) } & \multicolumn{3}{c|}{ Power $(\mu \mathrm{W})$} \\
& bits & Kbps & Comb. & Seq. & Total & Dynamic & Leakage & Total \\
\hline A-5/1 & 1 & 100 & 237.0 & 370.0 & 607.0 & 0.12 & 3.68 & 3.80 \\
\hline A-5/2 & 1 & 100 & 263.0 & 455.0 & 718.0 & 0.13 & 4.53 & 4.66 \\
\hline E0 & 1 & 100 & 100.5 & 690.0 & 790.5 & 0.15 & 5.47 & 5.62 \\
\hline Grain80 & 1 & 100 & 152.5 & 850.0 & 1002.5 & 0.09 & 6.47 & 6.56 \\
\hline Grain128 & 1 & 100 & 134.2 & 1330.0 & 1464.3 & 0.13 & 9.65 & 9.78 \\
\hline Grain128a & 1 & 100 & 144.7 & 1335.0 & 1479.8 & 0.13 & 9.77 & 9.90 \\
\hline Trivium & 1 & 100 & 107.2 & 1505.0 & 1612.3 & 0.13 & 10.60 & 10.73 \\
\hline Mickey80-v2 & 1 & 100 & 1023.2 & 1065.0 & 2088.3 & 0.04 & 9.50 & 9.54 \\
\hline Mickey128 & 1 & 100 & 1573.5 & 1665.0 & 3238.5 & 0.04 & 10.10 & 10.14 \\
\hline RC4 & 8 & 800 & 21543.5 & 10610.0 & 32153.5 & 0.09 & 143.30 & 143.39 \\
\hline ZUC & 32 & 3200 & 7023.0 & 2865.0 & 9888.0 & 1.56 & 56.70 & 58.26 \\
\hline SNOW 3G & 32 & 3200 & 6977.7 & 3105.0 & 10082.7 & 1.46 & 53.50 & 54.96 \\
\hline
\end{tabular}

do not decrease, for RC4, SNOW 3G and ZUC however, the reduction is drastic. A lower power consumption is also accompanied with the decrease in operating frequency.

From a set of basic metrics of stream ciphers implementations (area, power, operating frequency, interface, initialization cycles), a set of derived metrics (Throughput, Energy/bit, Area-time) are calculated for performance comparison. Some additional derived metrics (Power-area-time, latency, Power-latency), critical for lightweight encryption or RFID applications are also calculated. The definitions of these metrics are discussed in Section 2.3.3.

For highest operating frequency these derived matrices are given in the Table 4.15. Considering a performance comparison between SNOW 3G and ZUC, ZUC outperforms by having a lower Power-Area-Time ratio. For eSTREAM finalists, Grain-80v2 outperforms the other proposals. For $100 \mathrm{MHz}$ operating frequency, the derived metrics are given in the Table 4.16. The latency is compounded by lower operating frequency as it translates to higher latency delay (shown in latency column). Here too, Grain-80-v2 is clearly a winner.

The derived metrics for highest operating frequencies are graphically shown in Fig. 4.16. For a fair comparison, area-efficiency and energy per bit are chosen as the figures of merits. For RFID applications, Fig. 4.17 shows core areas plotted against power-latency of the designs, both lower being better (binary stream ciphers plotted only). The arrows in Fig. 4.16 and Fig. 4.17 show performance improvement.

\subsubsection{Comparison with Manual Implementations}

Since the hand-crafted HDL implementations for any algorithm are generally more optimized compared to the tool-generated ones, a comparison of efficiency of Run- 
Table 4.15: Derived metrics for highest operating frequencies @65nm CMOS

\begin{tabular}{|c|c|c|c|c|c|}
\hline & $\begin{array}{c}\text { Throughput } \\
(\mathrm{Gbps})\end{array}$ & $\begin{array}{c}\text { Energy/bit } \\
(\mathrm{pJ} / \mathrm{bit})\end{array}$ & $\begin{array}{c}\text { Area-Time } \\
(\mathrm{GE}-\mu \mathrm{s})\end{array}$ & $\begin{array}{c}\text { Tput/Area } \\
(\mathrm{Kbps} / \mathrm{GE})\end{array}$ & $\begin{array}{c}\text { Power-Area-Time } \\
(\mathrm{GE}-\mathrm{n})\end{array}$ \\
\hline A-5/1 & 1 & 0.59 & 0.61 & 1647.45 & 0.36 \\
\hline A-5/2 & 1 & 1.33 & 0.72 & 1392.76 & 0.96 \\
\hline E0 & 1 & 0.40 & 0.79 & 1265.02 & 0.31 \\
\hline Grain80 & 1 & 2.28 & 1.00 & 995.52 & 2.29 \\
\hline Grain128 & 1 & 2.29 & 1.46 & 682.94 & 3.35 \\
\hline Grain128a & 1 & 2.29 & 1.48 & 675.79 & 3.38 \\
\hline Trivium & 1 & 3.82 & 1.61 & 619.96 & 6.17 \\
\hline Mickey80-v2 & 1 & 4.22 & 2.09 & 477.95 & 8.84 \\
\hline Mickey128 & 1 & 5.85 & 3.26 & 307.03 & 19.05 \\
\hline RC4 & 8 & 0.22 & 5.94 & 168.36 & 10.30 \\
\hline ZUC & 16 & 0.21 & 0.75 & 1339.95 & 2.57 \\
\hline SNOW 3G & 32 & 0.54 & 0.37 & 2698.20 & 6.37 \\
\hline Better is & Higher & Lower & Lower & Higher & Lower \\
\hline
\end{tabular}

Table 4.16: Derived metrics for $100 \mathrm{MHz}$ operating frequency @65nm CMOS

\begin{tabular}{|c|c|c|c|c|c|c|}
\hline & $\begin{array}{c}\text { Energy/bit } \\
(\mathrm{nJ} / \mathrm{bit})\end{array}$ & $\begin{array}{c}\text { Power-Area } \\
\text {-Time }(\mathrm{GE}-\mu \mathrm{J})\end{array}$ & $\begin{array}{c}\text { Init. } \\
\text { cycles }\end{array}$ & $\begin{array}{c}\text { Latency } \\
\mu \mathrm{s}\end{array}$ & $\begin{array}{c}\text { Power-Area } \\
\text { Latency }(\mu \mathrm{J}-\mathrm{GE})\end{array}$ & $\begin{array}{c}\text { Power- } \\
\text { Latency }(\mathrm{nJ})\end{array}$ \\
\hline A-5/1 & 0.04 & 0.02 & 186 & 1860 & 0.00 & 7.06 \\
\hline A-5/2 & 0.05 & 0.03 & 186 & 1860 & 0.01 & 8.67 \\
\hline E0 & 0.06 & 0.04 & 328 & 3280 & 0.01 & 18.44 \\
\hline Grain80 & 0.07 & 0.07 & 320 & 3200 & 0.02 & 20.99 \\
\hline Grain128 & 0.10 & 0.14 & 513 & 5130 & 0.07 & 50.16 \\
\hline Grain128a & 0.10 & 0.15 & 513 & 5130 & 0.08 & 50.77 \\
\hline Trivium & 0.11 & 0.17 & 1440 & 14400 & 0.25 & 154.57 \\
\hline Mickey80-v2 & 0.10 & 0.20 & 260 & 2600 & 0.05 & 24.81 \\
\hline Mickey128 & 0.10 & 0.33 & 416 & 4160 & 0.14 & 42.20 \\
\hline RC4 & 0.18 & 5.76 & 512 & 5120 & 23.61 & 734.15 \\
\hline ZUC & 0.02 & 0.18 & 48 & 480 & 0.28 & 27.96 \\
\hline SNOW 3G & 0.02 & 0.17 & 48 & 480 & 0.27 & 26.38 \\
\hline better is & Lower & Higher & Lower & Lower & Lower & Lower \\
\hline
\end{tabular}

Stream generated HDL is carried out against that of the manually written reported implementations of the stream ciphers. For RC4, an open cores implementation [174] is taken up that defines its internal state as D-flipflops The two implementations are synthesized under same CMOS technology, synthesis tool and constraints. The result are given in Table 4.17. The RunStream generated RTL has about 5\% more area overhead in comparison. This is due to a single cycle per keystream expansion 


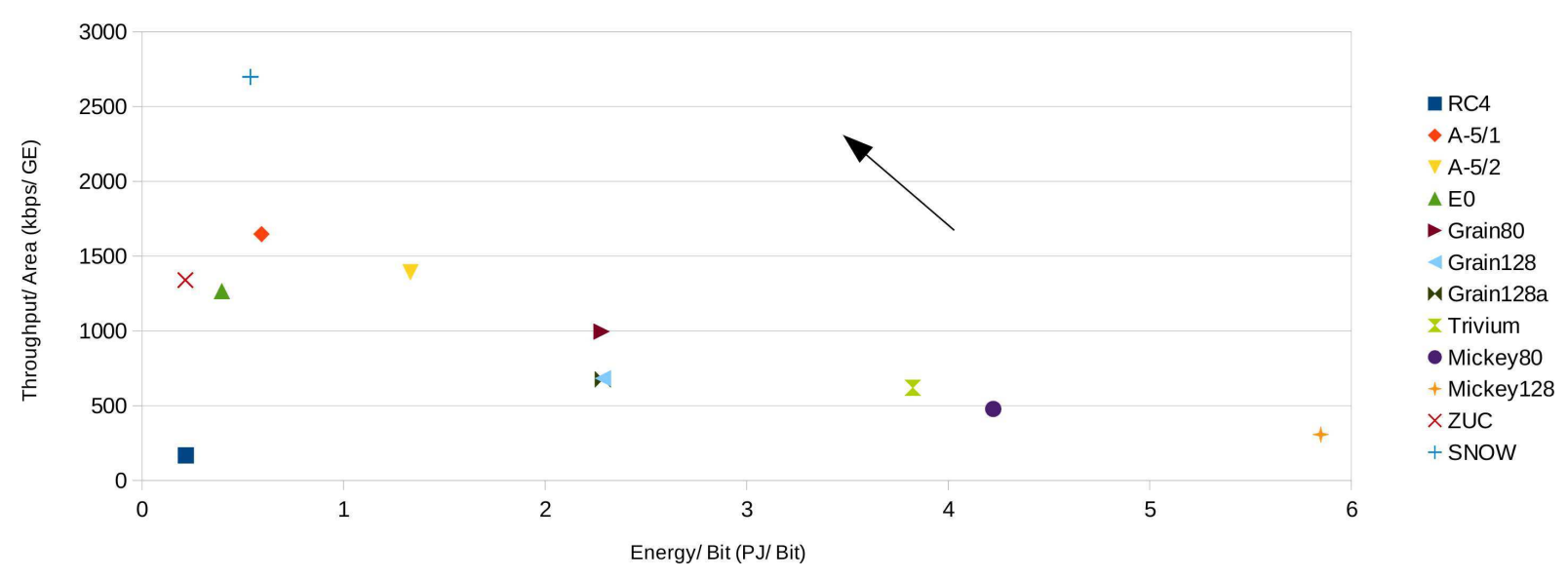

Figure 4.16: Design performance metrics for higher operating frequencies @65nm CMOS

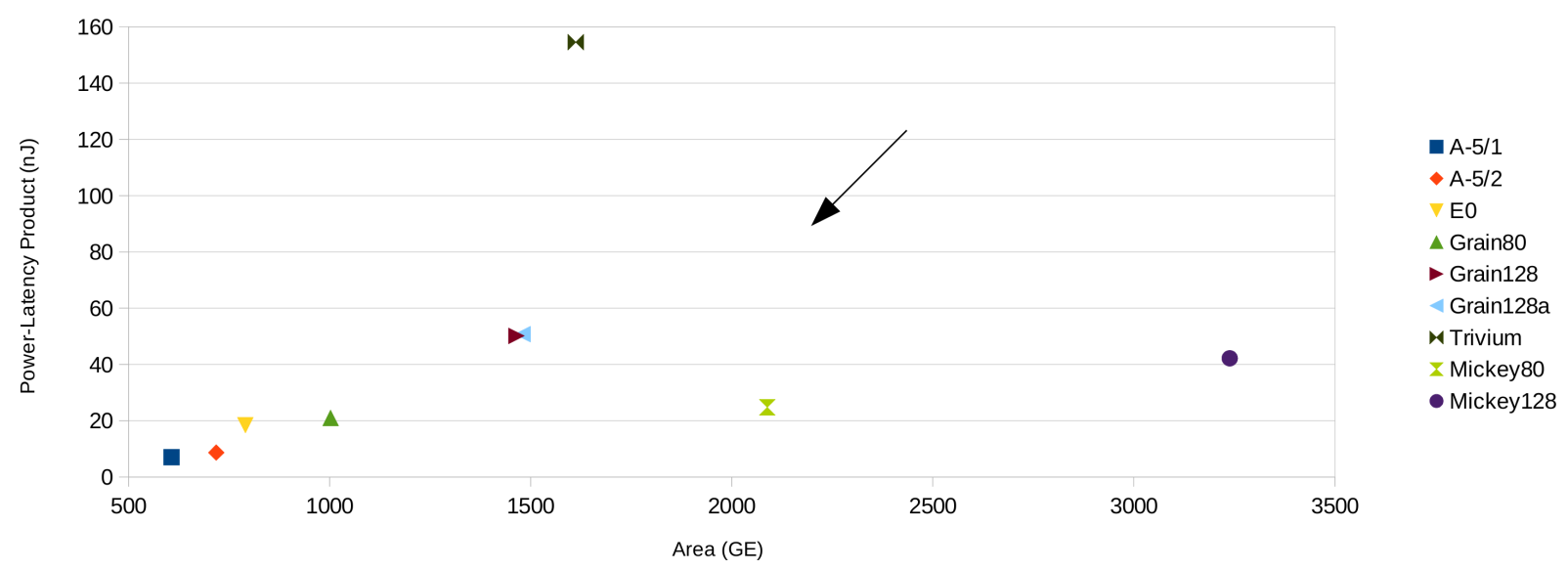

Figure 4.17: Performance for RFID applications for $100 \mathrm{KHz} @ 65 \mathrm{~nm}$ CMOS

iteration (phase1) in RunStream RC4 implementation. The open cores RC4 implementation [174] instead consumes cycles per iteration in phase1, consequently relaxing the critical but increasing the initialization latency. The area overhead for RunStream RC4 is the price paid for this lower initialization latency.

When HDL implementation of a VLSI design is not available, a fair post-synthesis comparison of two designs has some inherent difficulties. The results of different CMOS synthesis technologies do not match, even with the same synthesis technology, different vendor cell libraries generate different implementations. Additionally, different settings, constraints or versions of synthesis tools generate differences in performance of the implementations. Moreover, the best case, typical case, worst case choice is often not specified for published results. For a fair comparison, the RunStream generated RTL is re-synthesized using the same synthesis technology node (with possibly different vendor libraries), same synthesis tool (with different version) and same operating frequency as the one specified for hand-crafted reported imple- 
Table 4.17: Resource comparison of RunStream implementations with others

\begin{tabular}{|c|c|c|c|c|c|c|}
\hline Algorithm & keystream & Initialization & Op. Freq. & Throughput & \multicolumn{2}{|c|}{ Area (GE) } \\
\hline name & (bits/cycle) & (cycles) & (MHz) & (Mbps) & $\left(\mu m^{2}\right)$ & (GE) \\
\hline \multicolumn{7}{|c|}{ RunStream generated RTL, 65nm CMOS } \\
\hline RC4 & 1 & 512 & 10 & 80 & 41156.80 & 32153.8 \\
\hline \multicolumn{7}{|c|}{ [174], 65nm CMOS } \\
\hline RC4 & 1 & 768 & 10 & 80 & 38931.20 & 30415.00 \\
\hline \multicolumn{7}{|c|}{ RunStream generated RTL, 90nm CMOS } \\
\hline $\mathrm{A}-5 / 1$ & 1 & 186 & 685 & 685 & 1730.29 & 551.75 \\
\hline \multicolumn{7}{|c|}{$[76], 90 \mathrm{~nm}$ CMOS } \\
\hline $\mathrm{A}-5 / 1$ & 1 & 186 & 685 & 685 & 1985 & - \\
\hline \multicolumn{7}{|c|}{ RunStream generated RTL, 90nm CMOS } \\
\hline ZUC & 32 & 48 & 18.75 & 600 & 32046.00 & 10218.75 \\
\hline \multicolumn{7}{|c|}{ [214], 90nm CMOS } \\
\hline ZUC & 32 & 48 & 18.75 & 600 & - & 14000 \\
\hline \multicolumn{7}{|c|}{ RunStream generated RTL, 65nm CMOS } \\
\hline ZUC & 32 & 48 & 500 & 16000 & 15284.16 & 11940.7 \\
\hline \multicolumn{7}{|c|}{ [104], 65nm CMOS } \\
\hline ZUC & 32 & 48 & 500 & 16000 & - & $10-13 K$ \\
\hline \multicolumn{7}{|c|}{ RunStream generated RTL, 65nm CMOS } \\
\hline SNOW 3G & 32 & 48 & 943 & 30176 & 13900.48 & 10859.7 \\
\hline \multicolumn{7}{|c|}{ [105], 65nm CMOS } \\
\hline SNOW 3G & 32 & 48 & 943 & 30176 & - & $8.9 \mathrm{~K}$ \\
\hline \multicolumn{7}{|c|}{ RunStream generated RTL, 130nm CMOS } \\
\hline Grain & 1 & 321 & 724.6 & 724.6 & 5288.78 & 1021 \\
\hline Grain128 & 1 & 513 & 925.9 & 925.9 & 7676.76 & 1482 \\
\hline Trivium & 1 & 1314 & 327.9 & 327.9 & 9272.20 & 1790 \\
\hline Mickey80-v2 & 1 & 261 & 454.5 & 454.5 & 11494.42 & 2219 \\
\hline Mickey128 & 1 & 417 & 413.2 & 413.2 & 17829.56 & 3442 \\
\hline \multicolumn{7}{|c|}{ [82], 130nm CMOS } \\
\hline Grain-v2 & 1 & 321 & 724.6 & 724.6 & 6702.92 & 1294 \\
\hline Grain128 & 1 & 513 & 925.9 & 925.9 & 9712.50 & 1875 \\
\hline Trivium & 1 & 1314 & 327.9 & 327.9 & 13364.40 & 2580 \\
\hline Mickey80 & 1 & 261 & 454.5 & 454.5 & 16513.84 & 3188 \\
\hline Mickey128 & 1 & 417 & 413.2 & 413.2 & 26102.02 & 5039 \\
\hline
\end{tabular}


mentations. The power however, cannot be reliably scaled between different processes and libraries and is not discussed.

For A/5-1, the only reported VLSI implementation has area estimates reported in $\mu m^{2}$ [76]. RunStream results in a lower area budget of $1790 \mu m^{2}$ against their area figure of $1985 \mathrm{\mu m}^{2}$ (12\% higher). For E0 and A/5-2, no CMOS implementation results have been reported. The fastest FPGA implementation for E0 [77] is synthesized, placed and routed, using Xilinx FPGA device Virtex 2-2V250FG256 with a throughput of $189 \mathrm{Mbps}$.

For ZUC, two VLSI implementation results are considered for comparison, one from academia at 90nm [214] and the other from Elliptic Technologies at 65nm [104]. RunStream generated ZUC implementation matches closely with the ZUC core from Elliptic Technologies in area estimates. For SNOW 3G, an IP Cores Inc. implementation at $65 \mathrm{~nm}$ was taken up and compared against the RunStream generated RTL with same operating frequency and technology library. RunStream SNOW 3G results in around $22 \%$ more area. The internal working of these commercial cores for ZUC and SNOW 3G are not known. However, the area budget can be profiled to be majorly contributed by S-Boxes. The implementation of S-Boxes is not unique and may range in an area-throughput spectrum from a simplistic read-only LUT to a equivalent Galois field combinational implementation. Its worth highlighting that RunStream can be configured to implement an S-Box as a LUT or any other combinational logic with its nodes.

A detailed study of HDL manual implementations of eSTREAM ciphers at various phases of selection has been carried out by T. Good et. el. [80-83]. Their goal was to evaluate the suitability of the proposals for maximum throughput, power consumption and area compactness for RFID and LAN applications. They targeted a 130nm process using a standard cell library produced by Faraday. Table 4.17 compares their implementation results against the RunStream generated implementations, synthesized using 130nm standard CMOS. For the same operating frequencies (and the same throughput), the area estimates for RunStream remain $20-30 \%$ lower. This apparent improvement of RunStream results could be attributed to differences in the synthesis environments. RunStream strives to facilitate fast and reliable prototyping of stream ciphers and endeavors to come close to hand written implementations.

Worth mentioning is the fact that the performance based enumeration of all the 5 eSTREAM ciphers remains exactly the same as [82]. Grain80 outperforms both MICKEY and Trivium in terms of TPAR and energy per bit, when compared for highest operating frequency (Fig. 4.16). At $10 \mathrm{Mbps}$ data rate too, a similar enumeration trend for the eSTREAM ciphers is seen as in [82]. For RFID applications too, RunStream generated performance based ordering for Grain80, Grain128, Grain128a, Trivium, MICKEY80-v2 and MICKEY128 in Fig. 4.17 conforms completely to [82]. Grain80 outperforms the other four ciphers in terms of area compactness. Similarly, Trivium shows inferior performance than the rest in terms of power-latency while MICKEY128 has the highest area overhead. 


\subsection{Concluding Remarks}

This chapter discusses an extensible framework for the rapid prototyping of two classes of symmetric key cryptography, i.e., block ciphers and stream ciphers. The tool offers a sophisticated design capture of the algorithmic and structural specifications of a cipher by the user through a GUI. A design validation is carried out, after whose successful completion, an optimized software and HDL implementations are generated. All the hardware portfolio stream ciphers of eSTREAM and some noticeable block ciphers were taken up for generation using RunStream and RunFein, respectively. Equitable comparisons for area-throughput-power were carried out. The results rival the best available handwritten IP cores. 


\section{Chapter 5}

\section{Manual Optimizations for Efficient Designs}

The high performance requirements coupled with limited energy budgets leave the development of dedicated accelerators for cryptographic applications in today's heterogeneous systems as an obvious choice. For efficient hardware implementations of block/ stream ciphers, cryptographic core developer may undertake some optimization techniques that are algorithm/ application specific and are not straight forward to be undertaken automatically by the CRYKET tool. This chapter discusses two such optimization strategies.

\subsection{Optimization Strategies}

\subsubsection{Memory Bank Structure Optimizations}

Several ciphers employ large internal states in their design, that are modeled as SRAMs in their VLSI design. First a review of all the existing strategies of SRAM bank structure organization so as to enable parallelism for performance enhancement is presented. The classical idea of splitting states kept in SRAMs into multiple smaller SRAMs is exploited to get the increased parallel accesses. Consequently, a higher throughput performance is benchmarked. This idea applied on an eSTREAM finalist having the largest state space, namely, HC-128 [224]. The implementation marks the highest throughput per area performance for the stream cipher reported in the literature till date. The results are published in [124].

\subsubsection{Unification of Multiple Cryptographic Proposals}

In various applications, multiple cryptographic functions must co-exist and non-exclusively executed on a single VLSI core. Their unified design can aggressively exploit the structural commonalities to achieve area and throughput efficiency. In this context, a merger of SNOW 3G and ZUC stream ciphers of 3GPP LTE-Advanced security suite is undertaken; this can serve as a guide for the unification of other cryptographic proposals into a single core. The integrated architecture reduces the area overhead significantly compared to their distinct cores, and also provides almost double throughput, compared with the state-of-the-art implementations of the individual ciphers. The results are published in [92]. 


\subsection{Memory Bank Structure Optimizations}

Various stream ciphers emply large internal states that are modeled using SRAMs in VLSI implementation (e.g., RC4 [195], WAKE, Py, HC-128 [224], HC-256, CryptMT etc.). For these designs, the major portion of the area is contributed by the SRAMs and also the critical path is generally the SRAMs access time. Hence the structure of SRAM used by the cryptographic core is critical to the system performance. Most of these systems employ extensively, large memory banks that are time-shared between multiple arbiters. Having external memory banks for programmable cryptographic processors is advantageous since the memory could also be reused for various versions of an algorithm, offering the user a varying performance/ security [40,93]. For most of the coarse grained hardware platforms like FPGAs and CGRAs, large block RAM modules are available as macros, that may be configured to desired sized memories as per the user requires and will go wasted when unused.

A VLSI optimization of HC-128 is taken up, though it belongs to the software profile of eSTREAM portfolio [65]. A crisp boundary between the software and hardware ciphers is fast getting murky as today's high performance embedded systems are heterogeneous platforms. Moreover, regarding this categorization into software and hardware cryptographic proposals, competitions (excluding eSTREAM) did not follow suit. The call for AES [164] announced the computational efficiency of both hardware and software implementations as a decisive factor for selection of the winner, consequently, AES is popular in both domains. Similarly, the choice of Keccak as SHA-3 finalist was attributed by NIST to both its good software performance and excellent hardware performance [171]. Consequently, the ASIC designs of stream ciphers proposed for software oriented applications have extensively been taken up, e.g., RC4 [91,112,127], salsa20/12 [100,229], Rabbit [203], SOSEMANUK [18].

In the light of this discussion its surprising to have [38] as the sole VLSI implementation effort for HC-128. Since HC-128 already is notorious for its high memory requirements, having multiple state copies in multiple memories only worsens it. Instead an orthogonal approach is take up for efficient parallelization in HC-128 by splitting the memory resources instead of replicating them. Other than completing the jigsaw puzzle for a missing implementation of eSTREAM cipher, this work also presents parallelization strategies that can serve as a guide for the implementation of other stream ciphers with large internal states.

\subsubsection{Reviewing Known Techniques}

This section discusses various known performance enhancement techniques taken up for cryptographic accelerators storing their states in SRAMs. HC-128, e.g., requires $P$ and $Q$ taken up as SRAMs for its VLSI implementation, Appendix C.1 gives the details of HC-128 stream cipher.

1. Using multi-ported SRAMs The number of read/write ports of an SRAM restricts the multiple simultaneous accesses and consequently the throughput 
performance. The idea of using a multi-ported SRAM for enhancing performance has been analyzed for RC4 stream cipher with single, dual or 5 ported SRAM [112]. In a recent work, a similar idea using tri-ported SRAMs was taken up for FPGA implementations [215]. Extending on these lines, a performance improvement for HC-128 can be done, however, the analysis in current work is limited to the use of dual ported SRAMs since they are the most commonly used configuration of SRAMs and their optimized design are available by many vendors. Most of the latest FPGAs families also have dual ported block SRAMs available that may be configured to different width and depths.

2. Loop unrolling Unrolling of the keystream generation loop exposes more processing blocks for parallel execution and can boost throughput at the cost of extra hardware. e.g., unrolling of RC4 [91]. The HC-128 proposal [224] also presents an optimized software implementation that relies on unrolling the keystream generation loop 16 times. For hardware accelerator designs, unrolling by a factor $n$ will require $n$ times the number of memory accesses. Consequently, dealing simultaneously with more access may result in a large area and power hungry design that should be justified only with a consequent increase in throughput to be efficient.

3. State Replication The use of copies of state array with multiple instances of SRAMs increases the simultaneous access ports availability and consequently can boost throughput. The idea has been viably extended to RC4 [40] and HC128 [38] implementations. The penalty is paid in terms of doubling of area resources due to memory duplication as well as the extra writes necessary to keep the memories coherent.

\subsubsection{Optimized Memory Utilization for HC-128}

For HC-128, other than two 512 word arrays $P$ and $Q$, the cipher's simplistic implementation requires a 1280 words array $W$ used only during initialization (Appendix C.1). A close observation reveals a separate $W$ array unnecessary, a clever use of $P$ ad $Q$ arrays could just suffice. The initialization phase is modified, without disturbing the algorithm functionality, to initialization steps given in Table 5.1. Instead of using $W$, Key and IV are expanded into the lower half of $P$ memory in Step 2. Consequently an offset of 256 is added to the original calculations. Step 3 updates the entire $P$ array, after which the last 16 locations of $P$ are copied to $Q$ in Step 4. Step 5 updates the $Q$ memory, Step 6 is the same as the one in original proposal.

Removal of the $W$ memory reduces the memory budget of the cipher from $9 \mathrm{~KB}$ to $4 \mathrm{~KB}$, at the expense of having some extra steps. For weakly programmable processors with very compact instruction set, the increase in the program memory due to these extra steps is trivial. Also the overhead in terms of extra cycles is negligible compared to the total initialization cycles. 
Table 5.1: Modified HC-128 Initialization phase steps

Step 1: Let $K[i+4]=K[i]$ and $I V[i+4]=I V[i]$ for $0 \leq i \leq 3$.

Step 2: The key and IV are expanded to lower half of $P$ memory as follows:

$$
\begin{array}{rlrl}
P[i+256] & =K[i], & & \text { for } 0 \leq i \leq 7 \\
= & I V[i-8], & & \text { for } 8 \leq i \leq 15 \\
= & f_{2}(P[i+256-2])+P[i+256-7] & \\
& +f_{1}(P[i+256-15])+P[i+256-16]+i, & & \text { for } 16 \leq i \leq 255 .
\end{array}
$$

Step 3: Update the entire array $P$ as follows:

$P[i]=f_{2}(P[i \boxminus 2])+P[i \boxminus 7]+f_{1}(P[i \boxminus 15])+P[i \boxminus 16]+i$, for $0 \leq i \leq 511$,

Step 4: Last 16 elements of $P$ and copied into Q:

$P[i]=Q[i]$, for $496 \leq i \leq 511$,

Step 5: Update the entire array $Q$ as follows:

$Q[i]=f_{2}(Q[i \boxminus 2])+Q[i \boxminus 7]+f_{1}(Q[i \boxminus 15])+Q[i \boxminus 16]+i$, for $0 \leq i \leq 511$,

Step 6: Update the $P$ and $Q$ arrays as follows:

For $i=0$ to 511 , do

$$
P[i]=\left(P[i]+g_{1}(P[i \boxminus 3], P[i \boxminus 10], P[i \boxminus 511])\right) \oplus h_{1}(P[i \boxminus 12]) ;
$$

For $i=0$ to 511 , do

$$
Q[i]=\left(Q[i]+g_{2}(Q[i \boxminus 3], Q[i \boxminus 10], Q[i \boxminus 511])\right) \oplus h_{2}(Q[i \boxminus 12]) ;
$$

\subsubsection{Design Space Exploration of HC-128 Accelerator}

The architecture for an HC-128 stream cipher processor core along with an interface with an external memory bank, that includes $P$ and $Q$ memories is presented in Fig. 5.1. The instructions are kept in a program memory, while a simple incrementing PC serves as the address to the memory. By loading the programming memory with assembly instructions and setting up the I/Os as shown in the figure, the design can be easily plugged into a System on Chip (SoC) environment. For the rest of the discussion, this implementation is referred as design1. The I/Os of the processor are discussed below.

- Instruction is input to the processor core and is 8-bits in width.

- Key/IV is taken in during the initialization phase of the algorithm.

- Keystream is the 32-bit keystream word generated by the processor.

- Memory interface comprises of standard memory control signals for the two SRAMs, data ports and address ports. 


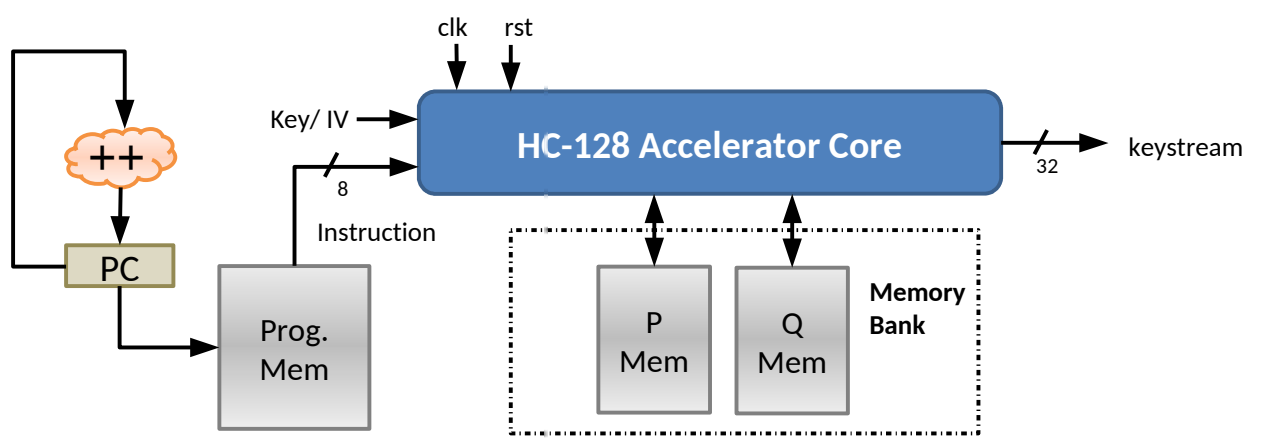

Figure 5.1: Block Diagram of HC-128 Accelerator

\subsubsection{Port Utilization and Limitations}

From Table C. 2 it can be seen that one keystream word requires 8 memory accesses in total: $P$ memory reads, $2 Q$ memory reads and one write to the $P$ memory (considering only the if block of the algorithm). So hypothetically speaking, with maximum port utilization if possible, the total of 6 accesses to the $P$ memory could be handled in 3 clock cycles for the dual ported $P$ memory, resulting in a possible throughput of 3 cycles/ keyword. The maximum utilization of memory ports is however not possible for current SRAMs that require one or more clock cycles for turnaround to change access from write to read and vice versa. Consequently, consecutive read-write and write-read accesses from one memory port would require one turnaround clock cycle between the two accesses, if an SRAM requiring a single turnaround cycle is considered. For the case at hand, one port is left unused for one cycle after the final writeback to update the $P$ memory.

For a pipelined processor, the limited number of access ports of SRAMs poses a structural hazard. As a work around, n nop (no operation) instructions are inserted between two consecutive instructions. To increase the efficiency, the pipeline stages are designed to target maximum port utilization of memories and thus keeping $n$ as small as possible.

\subsubsection{Processor Resources, Pipeline Architecture and ISA}

The processor has a 4 stage pipeline structure designed to fulfill some critical goals. Firstly to ensure maximum utilization of the memory access ports for fast execution of instructions. Secondly a judicious division of computationally expensive tasks over various pipeline stages is done so that the critical path of processor does not hamper the throughput. Thirdly ensuring economization of the resources (registers, pipeline registers, combinational blocks) whenever possible.

A total of 9 different instructions are used in the processor as shown in the Table 5.2. One-hot encoding is shown for the 8 bit instruction word for simpler instruction decoding. Each instruction (other than nop) executes a step in the initialization phase or keystream generation phase as indicated. Instruction init_kiv fills the $P$ 
memory with Key and IV values, the dual ported memory requires 8 instructions to fill these 16 locations. Consecutive instructions require no nops inserted between two instructions and consequently 8 instructions execute in 8 cycles. Similarly, the instruction init_p_for_ $q$ copies $P$ memory contents to $Q$ memory. It reads two locations from $P$ memory writes them to $Q$ memory, requiring 8 instructions in total for the 16 word transfers. No nops are required in between. The task division in initialization phase and keystream generation phase into various pipeline stages is discussed below.

Table 5.2: Instruction set for HC-128 accelerator

\begin{tabular}{|c|c|c|c|c|}
\hline $\begin{array}{c}\text { Instruction } \\
\text { syntax }\end{array}$ & $\begin{array}{c}\text { Instruction } \\
\text { opcode }\end{array}$ & $\begin{array}{c}\text { Related } \\
\text { operation }\end{array}$ & $\begin{array}{c}\text { nops b/w consecutive } \\
\text { instructions }\end{array}$ & $\begin{array}{c}\text { Total cycles for } \\
\text { initialization }\end{array}$ \\
\hline nop & 0x00 & - & - & - \\
\hline init_kiv & $0 \times 01$ & Step1 of Table 5.1 & 0 & 8 \\
\hline init_p_w & $0 \times 02$ & Step2 of Table 5.1 & 3 & $240 \times 4$ \\
\hline init_p & $0 \times 04$ & Step3 of Table 5.1 & 3 & $512 \times 4$ \\
\hline init_p_for_q & $0 \times 08$ & Step4 of Table 5.1 & 0 & 8 \\
\hline init_q & $0 \times 10$ & Step5 of Table 5.1 & 3 & $512 \times 4$ \\
\hline update_p & $0 \times 20$ & \multirow{2}{*}{ Step6 of Table 5.1 } & 3 & $512 \times 4$ \\
\hline update_q & $0 \times 40$ & & 3 & $512 \times 4$ \\
\hline keystream & $0 \times 80$ & Table C.2 & 3 & - \\
\hline
\end{tabular}

\subsubsection{Initialization Phase:}

The equation to perform Step 2 of initialization in Table 5.1 is rearranged as follows.

$$
\begin{aligned}
P[i+256]=\left(\left(f_{2}(P[i+256-2])+f_{1}(P[i+256-15])+i\right)\right. & +\quad P[i+256-7]) \\
+P[i+256-16] & \text { for } 16 \leq i \leq 1279
\end{aligned}
$$

This step requires addition of 5 values, 4 of which are 32-bit number. The pipeline design breaks the critical path of these additions which are slow operations in hardware. Consequently 3 values are added in EX2 pipeline stage (resolving the innermost bracket), followed by addition of 1 value in each of the next two pipeline stages (resolving the outer brackets). Step 3 and Step 5 of Table 5.1 are similar to Step 2, they have the same execution path. The calculation is divided in 4 pipeline stages as follows.

1. In $E X 1$, the two read accesses to $P$ memory are requested.

2. In $E X 2$, functions $f 1$ and $f 2$ are carried out on the values read and added together with a 9-bit iteration count $i$. The result is saved in a 32-bit pipeline register $d \_r e g$. In the same pipeline stage the next memory access is requested i.e., $P[i+256-7]$. 


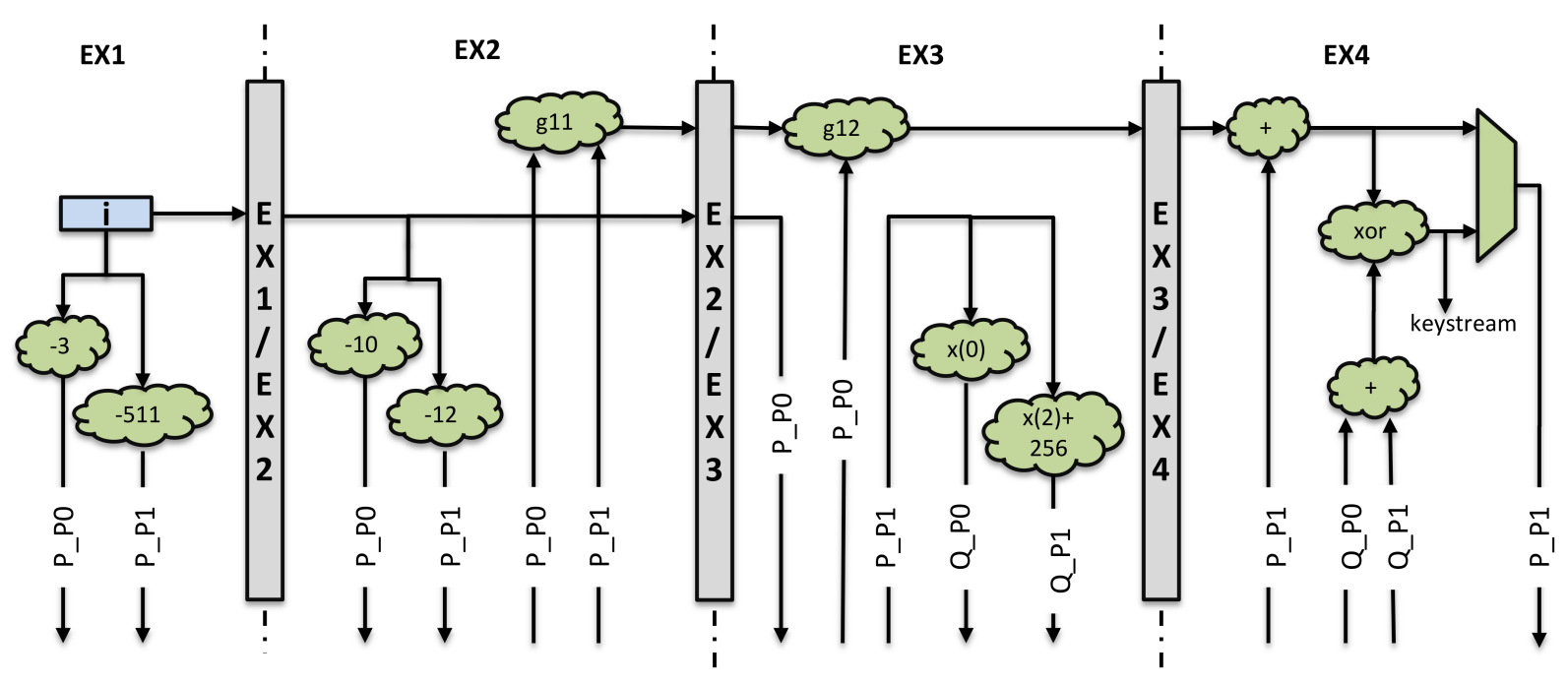

Figure 5.2: Pipeline architecture of design 1 of HC-128 accelerator

3. In EX3, d_reg is added to the value read from the memory and forwarded to the next pipeline. A memory access is requested for location $P[i+256-16]$.

4. In EX4 the read value from the memory is added to the previously calculated result in pipeline register $d \_r e g$ and stored back at memory location $P[i+256]$ using a write access to $P$ memory.

Since EX2 adds 3 values along with the functionality of $f 1$ and $f 2$, this becomes the critical path of the design1. As given in the last column of Table 5.2 the number of nops that must be inserted between two consecutive init_p_w instructions is 3 . This is to ensure that the EX4 of the current instruction and the EX1 stage of the following instruction do not overlap, posing a memory access port contention. The instruction init_p and init_q also have similar operations and have same number of nops inserted between consecutive instructions.

\subsubsection{Keystream Generation Phase:}

The Step 6 of the initialization phase (Table 5.1) is similar to the keystream generation phase (Table C.2), the only difference is that the keystream generated is XOR-ed with the updated value of $P / Q$ arrays. Hence the pipeline architecture of update_p, update_$q$ and keystream is same and given in Fig. 5.2 It shows only the update_p and the if part of the algorithm in Table C.2 since the update_q and the else part is trivially similar. The port multiplexing based on the instruction is not shown here. Memory bank access arbitration is also not shown but is straight forward to interpret. The two ports of the dual ported memory are referred as P0 and P1. All accesses going down are read requests except the final writeback in EX4 stage. The requested values read are shown by the arrows going up. Various pipeline stages are discussed below. 
1. EX1: A 9 bit register $i$ maintains the iteration count of the loop. Two read requests are initiated from the two ports of $P$ memory with addresses having 9 and 511 as the offsets to the register $i$.

2. EX2: The two values read are rotated and XOR-ed together to carry out a part of the $g 1$ function (called $g 11$ ). The $g 1$ functionality is split into $g 11$ and the $g 12$ functions (similarly $g 2$ is split as $g 21$ and $g 22$ ) as follows

$$
\begin{aligned}
& \text { - } g 11(x, z)=((x \ggg 10) \oplus(z \ggg 23)) . \\
& \text { - } g 12(x, y, z)=g 11(x, z)+(y \ggg 8) .
\end{aligned}
$$

The $g 11$ result is passed over to the next pipeline stage using the pipeline register $d_{\text {_reg }}$ used also in the initialization phase. The $i$ register value is passed to this stage using a pipeline register $i_{-} p$ and with known offsets of 10 and 12, two more read requests are launched from the two ports of $P$ memory.

3. EX3: The g11 result is added to the value read from $P 0$ of the $P$ memory to complete the $g 12$ function, the result is stored to $d_{-} r e g$ pipeline register. The value read from $P[i-12]$ is used to initialize two read requests according to the $h 1$ function. $P[i]$ read request is also initialized.

4. EX4: The values read from the $Q$ and $P$ memories are taken up to calculate the keystream word $\left(S_{k}\right)$ and the $P[i]$ values as referred in Table 5.1. The updated word is either $P[i]$ or the one obtained after its XOR with the $S_{k}$, depending upon the instruction running. A single bit pipeline register holds the select line for this multiplexer.

The 6 accesses to $P$ memory are so distributed that stage EX2 and EX3 each perform two reads while EX4 performs one read and one write. The subsequent instructions should follow no earlier than after 3 nops, otherwise the port availability is violated. Consequently keystream generation speed is 4 cycles / keyword and 9168 cycles in total are required for initialization. The design is benchmark for performance by using a technology independent metric i.e., cycles per keystream word.

\subsubsection{State Split Optimizations for HC-128}

The bottleneck for better performance in HC-128 processor is the limited number of access ports since there is room of more parallelization provided more accesses per cycle were possible. Section 5.2.1, discusses some performance enhancement techniques applicable to stream ciphers with large memories and also the scope of mapping these optimizations to HC-128.

This section discusses state splitting for throughput enhancement of cipher implementations. By splitting the state array of stream ciphers kept, into smaller parts with known address distribution and keeping each smaller part in a separate memory can enable more parallel accesses and consequently enable faster keystream generation. 


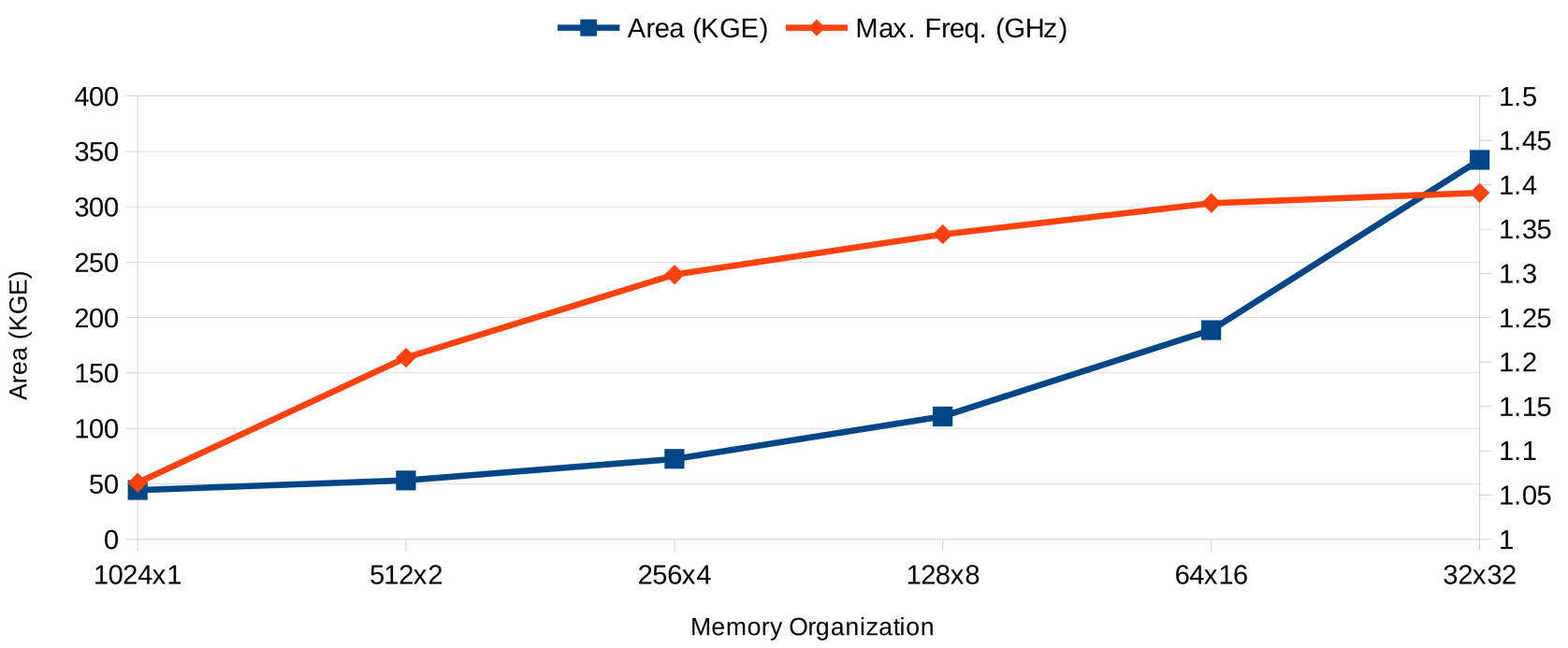

Figure 5.3: Area-frequency graph for various configurations of a $1 \mathrm{~K}$ word memory on $65 \mathrm{~nm}$ CMOS

Consider a cipher with one memory $M$ such that $\operatorname{Size}(M)=N$ (words/ bits/ bytes). The memory in divided into $S$ parts such that $\sum_{i=1}^{S} \operatorname{Size}\left(m_{i}\right)=N$, where $m_{i}$ are the smaller memories.

Memory splitting results in a memory bank requiring more power and area along with having more parallel accesses. On the contrary, the access times of the smaller memories is smaller, hinting higher operating frequencies for the processors. Fig. 5.3 shows the plot of area and maximum operating frequency for split SRAMs configurations using Faraday Memory compiler [71], for 65nm technology library. Various organizations of a 1024 word memory are considered. Assuming the simplistic case of equal sized split memories, a range of $S$ is considered from 1 till 32 in powers of 2 shown from left to write in the graph. As the memory is split into two banks $(512 \times 2)$, there is a corresponding $20 \%$ increase in the area. The design is considered viable only if the corresponding increase in throughput is more than that. This target may be achievable since there are two factors aiding the throughput performance, the availability of double ports and smaller access time of the memory bank. Hence a design exploiting the available parallelism can achieve much higher performances.

Various parameters for splitting should stem from the algorithm in question. The memory contents should be split so that the required accesses for the stream cipher have little or if possible no conflict between different memories. A careful study of the HC-128 stream cipher reveals the fact that the access to memories are split into even and odd memory locations. Considering only the if part of keystream generation and when $j$ is even the accesses to $P$ memory are targeted to

- Even Addresses: 3 reads from $P[j], P[j \boxminus 10], P[j \boxminus 12]$ and one write to $P[j]$

- Odd Addresses: 2 reads from $P[j \boxminus 3]$ and $P[j \boxminus 511]$ 
Two accesses to the $Q$ memory required in the $h 1$ function could be targeted to either even or odd memory locations. For odd values of $j$, the accesses to even and odd locations switch. The accelerator is redesigned considering a memory bank consisting of two memories $P e$ and $P o$ having all even and odd locations of the original $P$ memory, respectively. This 2-way split design point is referred as design2.

The idea of memory splitting is explored further taking up 4-way splitting of the memories (referred as design 3 ). $P$ memory is split into 4 equal parts of 128 words each, called $P 0, P 1, P 2$ and $P 3$ such that the last two bits of the of address to the original $P$ memory indicate the smaller split memory to be accessed. Hence if (address mod $4)==x$ the contents are in $P x$ memory. Considering the if part of the algorithm in Table C.2, the following division of the 6 accesses to $P$ memory can be seen

- $(j \bmod 4)==0: 2$ reads each from $P 0, P 1,1$ read from $P 2$, one write to $P 0$

- $(j \bmod 4)==1: 2$ reads each from $P 1, P 2,1$ read from $P 3$, one write to $P 1$

- $(j \bmod 4)==2: 2$ reads each from $P 2, P 3,1$ read from $P 0$, one write to $P 2$

- $(j \bmod 4)==3: 2$ reads each from $P 3, P 0,1$ read from $P 1$, one write to $P 3$

Table 5.3: Initialization latency cycles for various designs of HC-128 accelerator

\begin{tabular}{|c|c|c|c|}
\hline Instruction & \multicolumn{3}{|c|}{ Instructions for initialization } \\
syntax & design1 & design2 & design3 \\
\hline init_kiv & $8 \times 1$ & $4 \times 1$ & $2 \times 1$ \\
\hline init_p_w & $240 \times 4$ & $240 \times 2$ & $240 \times 2$ \\
\hline init_p & $512 \times 4$ & $512 \times 2$ & $512 \times 2$ \\
\hline init_p_for_q & $8 \times 1$ & $4 \times 1$ & $2 \times 1$ \\
\hline init_q & $512 \times 4$ & $512 \times 2$ & $512 \times 2$ \\
\hline update_p & $512 \times 4$ & $512 \times 2$ & 512 \\
\hline update_q & $512 \times 4$ & $512 \times 2$ & 512 \\
\hline Total & 9168 & 4584 & 3556 \\
\hline
\end{tabular}

\subsubsection{State Splitting by Factor 2: Design2}

The external interface of design 2 accelerator interfaces with four memories, namely, $P e, P o, Q e$ and $Q o$. The same instructions are used for design 2 as used for design1, the pipeline stage design is however altered to maximize parallelism.

Initialization Phase: As more access ports are now available, the structural hazard for subsequent overlapping instructions is less severe. As the initialization phase starts, each init_kiv instruction fills up the 4 words of key input to the accelerator to the first two memory address locations of $P e$ and Po memory in one cycle. Hence 


\begin{tabular}{|c|c|c|c|c|}
\hline & EX1 & EX2 & EX3 & EX4 \\
\hline i even & $\begin{array}{l}\text { req Pe_PO[i-2] } \\
\text { req Po_PO[i-15] }\end{array}$ & $\begin{array}{l}\text { read } \mathrm{Pe} \_\mathrm{PO}[\mathrm{i}-2] \\
\text { read } \mathrm{Po} \_\mathrm{P} 0[\mathrm{i}-15] \\
\text { req } \mathrm{Pe} \mathrm{P} 1[\mathrm{i}-16]\end{array}$ & $\begin{array}{l}\text { read } \mathrm{Pe} P \mathrm{P} 1[\mathrm{i}-16] \\
\text { req } \mathrm{Po} \_\mathrm{P} 1[\mathrm{i}-7]\end{array}$ & $\begin{array}{l}\text { read Po_P1[i-7] } \\
\text { Wr Pe_PO[i] }\end{array}$ \\
\hline i odd & $\begin{array}{l}\text { req Po_PO[i-2] } \\
\text { req Pe_PO[i-15] }\end{array}$ & $\begin{array}{l}\text { read Po_P0[i-2] } \\
\text { read } \mathrm{Pe} \_\mathrm{P} 0[\mathrm{i}-15] \\
\text { req } \mathrm{Po} \mathrm{P} 1[\mathrm{i}-16]\end{array}$ & $\begin{array}{l}\text { read Po_P1[i-16] } \\
\text { req } P e \_P 1[i-7]\end{array}$ & $\begin{array}{l}\text { read Pe_P1[i-7] } \\
\text { Wr Po_PO[i] }\end{array}$ \\
\hline
\end{tabular}

Figure 5.4: Pipeline architecture for HC-128 initialization instruction

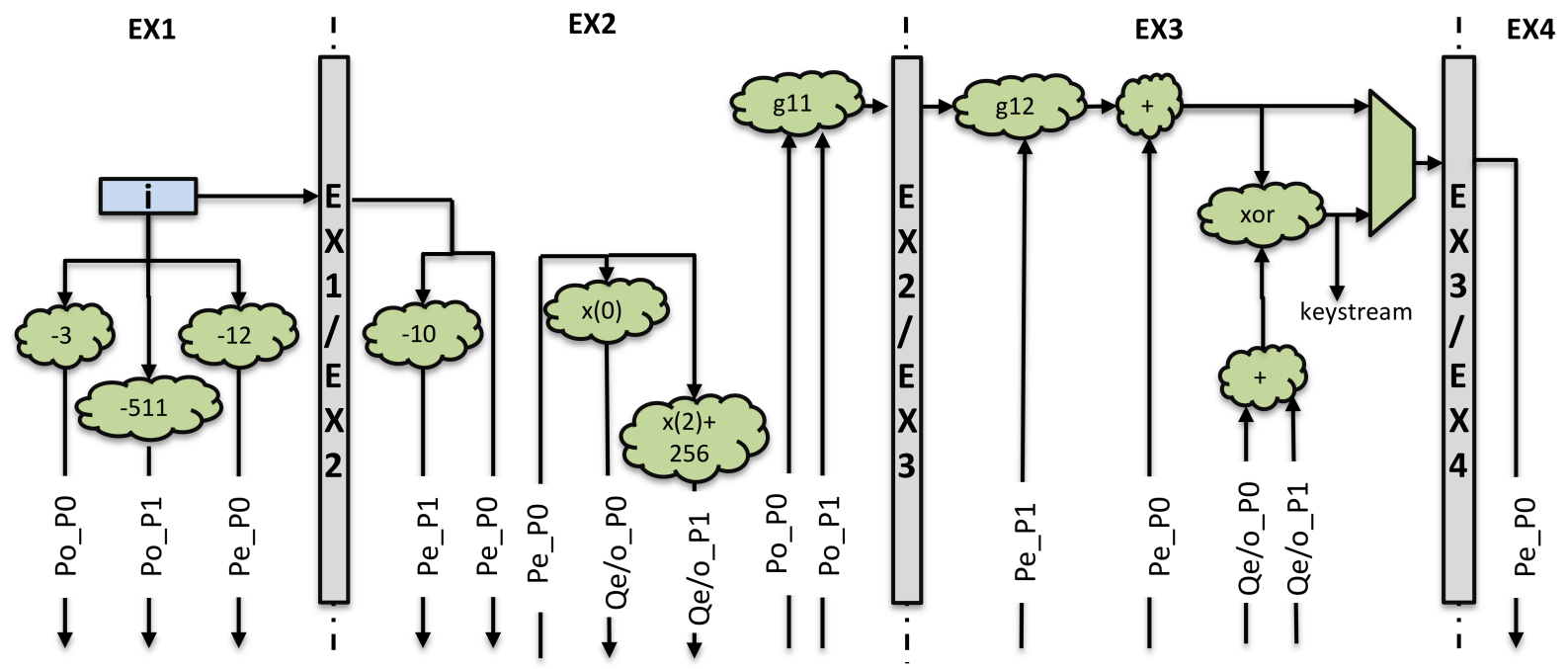

Figure 5.5: Pipeline architecture of design 2 of HC-128 accelerator

in two cycles the 8 words of key are written to $P$ memory bank and the next two instructions in two subsequent cycles complete the IV transfer as well.

The pipeline design of instruction init_p_w is shown in Fig. 5.4. As $i$ increments for each instruction the target memory for the 5 accesses switch between even and odd. Hence if $i$ is even $P[i-2]$ resides in memory $P e$ and otherwise in Po. Both the possibilities are shown in Fig. 5.4, the pipeline is carefully arranged so that two subsequent instructions require no more than one nop in between. Hence an overlap of EX1 and EX3 for subsequent even and odd iterations does not pose any hazard. Similarly overlap of EX2 and EX4 causes no port contention. As lesser nops need to be inserted between consecutive instructions the initialization phase requires around half as many cycles compared to design 1 as shown in the Table 5.3.

Keystream Generation Phase: The pipeline stages operations for update_p, update_q and keystream instruction is shown in Fig. 5.5. For simplicity, only the hardware for the if part of the algorithm is shown in Table C.2 with $i$ being even. 
1. EX1: Three read requests are launched from the two ports of $P o$ and one port of Pe.

2. EX2: The $g 11$ function is carried out on the values read from the two ports of $P e$ memory and the result is passed over to the next pipeline stage. Two more read requests are launched with addresses $i$ and $i-10$, while the value of $i$ is passed to this stage from the previous one by a pipeline register $i \_p$. Calculation for $h 1$ is carried out and two read requests are launched to Qo or Qe.

3. EX3: During EX3 stage $g 12$ function is performed and addition with the Pe_P1 memory port value is done. If the current instruction is update_p, then the result is XOR-ed with $h 1$ function output and stored in the pipeline register. For keystream instruction, the XOR-ing is skipped.

4. EX4: The value from the $d_{-} r e g$ register is written to Pe using P0 port.

This pipeline architecture manages a good port utilization in three pipeline stages, i.e., 3 reads in EX2, 2 reads in EX3 and one write in EX4 out of the 4 ports available per cycle. Subsequent instructions follow after just one nop. Overlap of EX1 with EX3 due to pipelining causes no port contention, since only 2 ports out of 4 are used. Similarly overlap of EX2 with EX4 causes no port contention for subsequent even and odd $i$ and uses all 4 ports. Consequently the throughput doubles i.e., one keyword is generated in two clock cycles. Port efficiency per keyword generated can be calculated by dividing accesses required per keyword with the accesses possible in two cycles (6/8 for this case).

\subsubsection{State Splitting by Factor 4: Design3}

The accelerator core interfaces with 8 smaller memory modules (of 128 words each), i.e., $P 0, P 1, P 2, P 3, Q 0, Q 1, Q 2, Q 3$. There are 8 simultaneous ports from each of the $P$ and $Q$ memory. Consequently init_kiv and init_p_for_q instructions need 2 cycles each for a transfer of 16 values.

In spite of having more access ports, the initialization phase in instruction inti_p_$w$ is shortened. This is to ensure that the critical path of the design does not increase accommodating the addition of all the 5 values of in a single pipeline stage. The same pipeline stage operations division is used as in design 2 for init_p_w instruction, the ports are however tweaked. Since EX2, EX3 and EX4 utilized only 2, 1 and 2 ports of the memory bank as shown in Fig. 5.4, subsequent pipelined instructions use no more than 5 ports simultaneously and hence causes no contention.

The design3 accelerator uses 3 pipeline stages for update_p, update_q and keystream instructions are shown in Fig. 5.6. For simplicity, only the hardware for the if part of the algorithm is shown in Table C.2 and the case when ( $i \bmod 4)$ equals 0.

1. EX1: All the required read operations are simultaneously launched after adding fixed offsets to iteration count register $i$. 


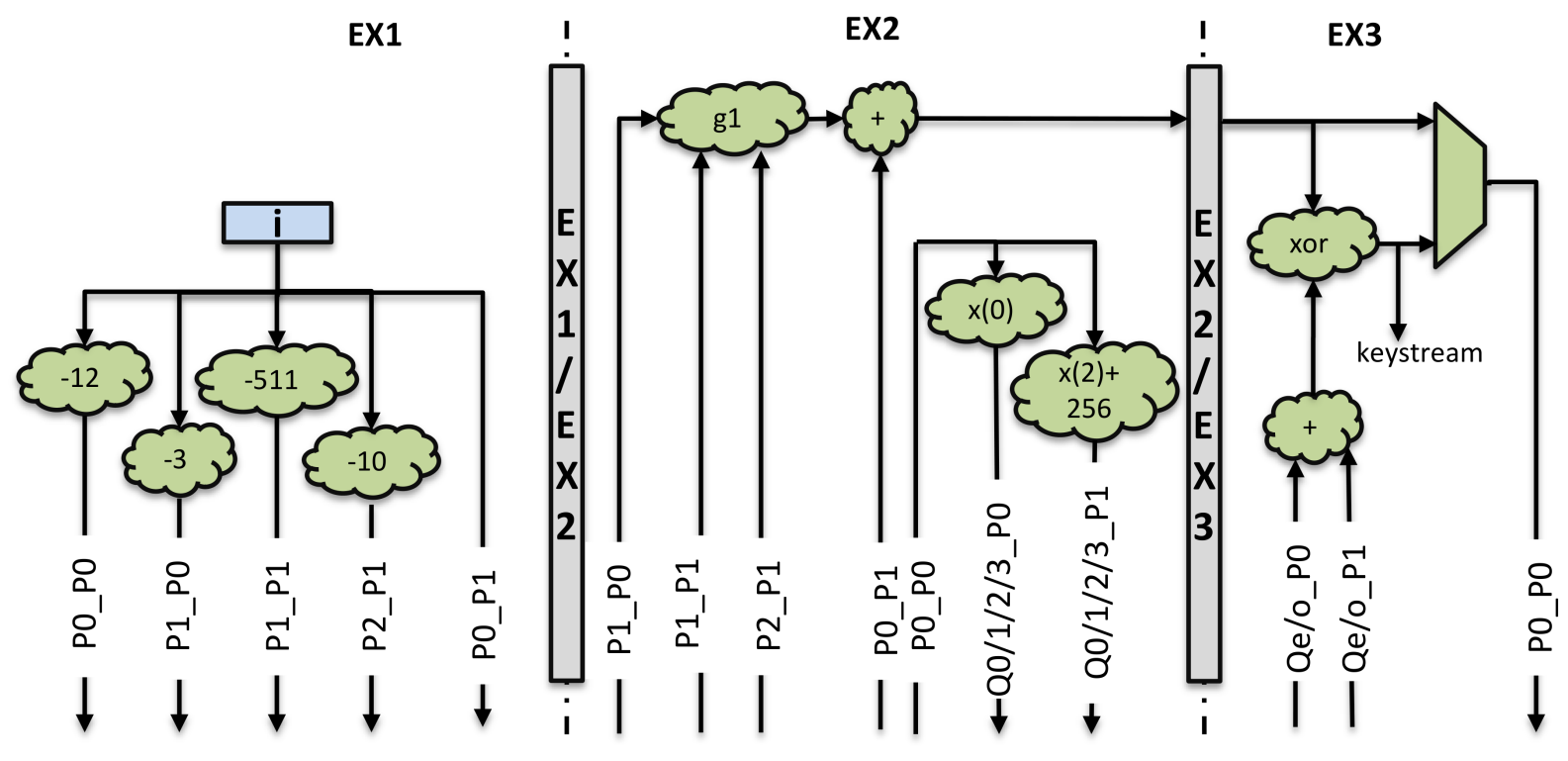

Figure 5.6: Pipeline architecture of design3 of HC-128 accelerator

2. EX2: From the values read, the $g 1$ function is calculated. The result is added to the $P[i]$ value read and is passed over to next pipeline stage. For $h 1$ function two requests to $Q$ memory banks are launched.

3. EX3: EX3 stage perform the operations depending on whether update_p or keystream instruction is executed. For update_p instruction, the pipeline register $d \_r e g$ is XOR-ed with the result of the $h 1$ operation and memory update write is carried out. For keystream instruction the keyword generated is computed by XORing the pipeline register with the output of the $h 1$ function.

Fig. 5.6 shows that in EX2 and EX3 stages, 5 reads and one write are carried out, respectively. Due to higher number of ports, no port contention happens when consecutive instructions are executed without any nops. It results in a throughput of one keyword per clock cycle. When the pipeline is full, an overlap of EX1, EX2 and EX3 will require 6 out of the 8 ports of the $P$ memory bank, indicating a port utilization of $75 \%(6 / 8)$.

\subsubsection{Further State Splitting by Higher Factors}

Without memory splitting the $6 P$ memory accesses in the if part of Table C.2 are managed in 4 clock cycles. With 2-way and 4-way split, the throughput was doubled, i.e., 2 cycles/ word and 1 cycle/ word of keystream generation, respectively. A further splitting, i.e., by a factor of 8 should accompany doubling of throughput ( 2 words / cycle) to have at least the same port utilization. It calls for unrolling the keystream generation loop twice for generating 2 words per iteration. The accesses double consequently, i.e., 12 accesses to $P$ memory, 4 accesses to $Q$ memory for 2 keywords generated. 
The hurdle in further improvement of throughput is the uncertainty of the addresses for $Q$ memory accesses that are required for the calculation of $h 1$ function. As shown in the equation for $h 1$ function, (in Appendix C.1) it requires two reads from the $Q$ memory from the addresses depending on the bytes of $P[j \boxminus 12]$. For the case of 4 way splitting (design3) no contention occurred since the dual ported memory could serve two accesses even when both of these $Q$ accesses required the same smaller memory. With an unrolling of 2 and 8 way splitting, 4 accesses in one cycle are required, that can cause a contention of memory ports if more than two of these 4 accesses arbitrate for the same smaller memory. One way of handling that could be to have extra contention detection logic that freezes the pipeline for one cycle to complete reads. Consequently, throughput of the processor will not remain deterministic, which is not a desirable feature for many applications. Also the contention detection logic will have a significant overhead in area of the design. Hence for HC-128 state-splitting is not taken up any further.

\subsubsection{Performance Evaluation}

The implementation of various designs of HC-128 accelerator is carried out via LISA based framework (Section 2.3.2) with $65 \mathrm{~nm}$ technology library used for design synthesis (Section 2.3.1).

\subsubsection{Area-throughput Trade-off}

Fig. 5.7 gives the area-time chart of the design, as the operating frequency is increased from $1 \mathrm{GHz}$ in jumps of $50 \mathrm{MHz}$, no valid design could be synthesized at frequencies beyond the highest point shown. The operating frequency of the cores is limited by the access time of the SRAM it interfaces with. For Faraday standard cell library, a 512-word memory needs a minimum access time of $0.83 \mathrm{~ns}$, corresponding to the highest operating frequency of design 1 being $1.2 \mathrm{GHz}$. For small memories the access time is less, hence the operating frequency of design 2 and design 3 can be as high as 1.3 and $1.35 \mathrm{GHz}$, respectively (shown by circles in Fig. 5.7). For the rest of the discussion, only these design points are discussed.

Table 5.4 shows the area estimates of the designs. The total area of the design is dominated by the memory. Its noteworthy to see the increase in area as the memories as split. As the $P$ and $Q$ memories are divided into 4 parts, the area estimate of the memories with no splitting doubles. Hence the rationale for this design decision should be justified by a corresponding throughput increase.

\subsubsection{Power Consumption}

The power consumption of an algorithm on a core is a function of the complexity of the design and the clock frequency. From Table 5.3.7.4, it can be seen that the dynamic power consumption of the cores contribute majorly to the core power consumption of the design. An increasing trend of power consumption can be seen from design 1 to design3 due to increased complexity as well as higher frequency. From the Faraday 


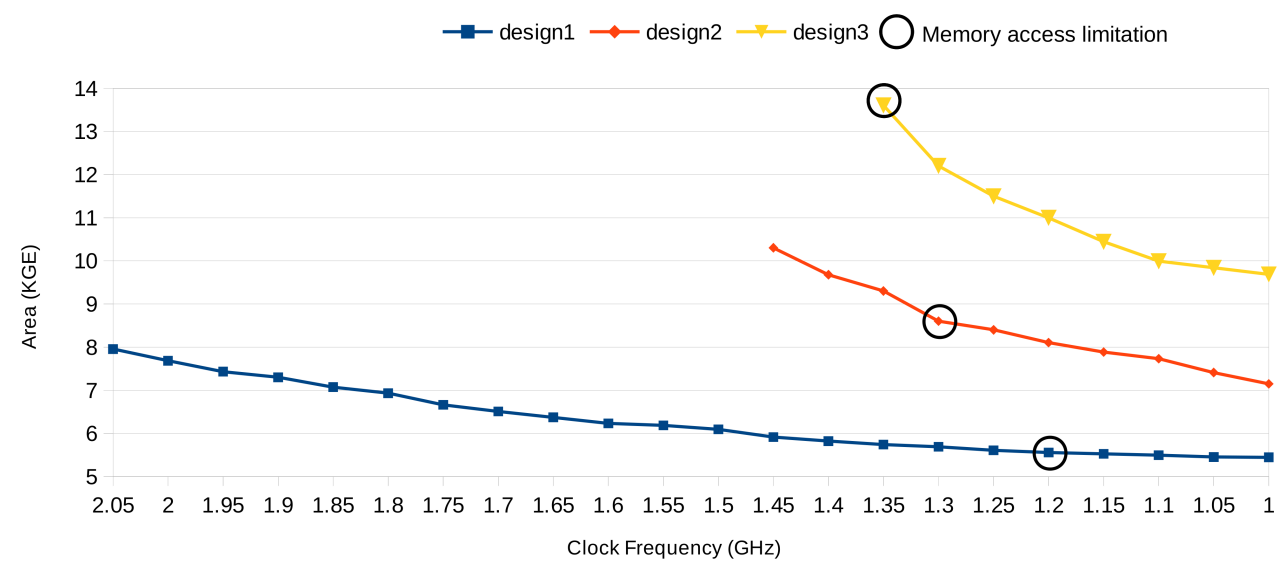

Figure 5.7: AT chart for HC-128 design variants on 65nm CMOS

Table 5.4: Area comparison of HC-128 accelerator designs

\begin{tabular}{|c|c|c|c|c|c|c|c|c|}
\hline \multirow{2}{*}{ Design } & Clock Freq. & \multicolumn{3}{|c|}{ Core area (KGE) } & \multicolumn{3}{c|}{ Memory } & Total Area \\
& $(\mathrm{GHz})$ & Comb. & Sequential & Total & Organization & (Bytes) & $($ KGE) & $($ KGE) \\
\hline Design1 & 1.20 & 5.47 & 1.64 & 5.55 & $2 \times 512$ & 4096 & 53.22 & 58.77 \\
\hline Design 2 & 1.30 & 9.63 & 1.38 & 8.60 & $4 \times 256$ & 4096 & 72.56 & 81.16 \\
\hline Design3 3 & 1.35 & 16.24 & 1.17 & 13.61 & $8 \times 128$ & 4096 & 110.4 & 124.01 \\
\hline
\end{tabular}

memory compiler, the static power of a 512 word memory is reported to be $9.864 \mu \mathrm{W}$ and dynamic power consumption is $18.56 \mathrm{pJ} /$ access (or $0.58 \mathrm{pJ} / \mathrm{bit}$ access) as specified in the Table 5.3.7.4.

Table 5.5: Power consumption comparison of HC-128 design versions

\begin{tabular}{|c|c|c|c|c|c|c|c|}
\hline \multirow[b]{2}{*}{ Design } & \multirow{2}{*}{$\begin{array}{l}\text { Clock } \\
\text { Freq. } \\
(\mathrm{GHz})\end{array}$} & \multirow{2}{*}{$\begin{array}{l}\text { Memory } \\
\text { Access time } \\
\text { (ns) }\end{array}$} & \multicolumn{2}{|c|}{ Memory Power Consumption } & \multicolumn{3}{|c|}{ Core Power Consumption } \\
\hline & & & $\begin{array}{l}\text { Static } \\
(\mu \mathrm{W})\end{array}$ & $\begin{array}{c}\text { Dynamic } \\
\text { (pJ/bit access) }\end{array}$ & $\begin{array}{c}\text { Dynamic } \\
(\mu \mathrm{W})\end{array}$ & $\begin{array}{l}\text { Static } \\
(\mu \mathrm{W})\end{array}$ & $\begin{array}{l}\text { Total } \\
(\mu \mathrm{W})\end{array}$ \\
\hline Design1 & 1.20 & 0.83 & 9.86 & 0.58 & 4.05 & 0.045 & 4.09 \\
\hline Design2 & 1.30 & 0.77 & 6.65 & 0.53 & 6.19 & 0.075 & 6.26 \\
\hline$\overline{\text { Design3 }}$ & 1.35 & 0.74 & 4.99 & 0.51 & 8.66 & 0.136 & 8.79 \\
\hline
\end{tabular}

\subsubsection{Throughput Efficiency and Initialization Latency}

The initialization latency of the three designs is shown in Table 5.6. As the refinements from design 1 to design 3 are taken up, a $3 \times$ reduction in the setup time can be seen. Most noteworthy is the throughput improvement in the design from 4 to 1 cycle per word from design 1 and design3, however, there is a corresponding increase in the area resources too. For a fair comparison, area-efficiency (or throughput per area, 
TPA) is chosen as the figure of merit (both with and without including the memory bank area). Design2 and design3 report a 1.3 and $1.8 \times$ better efficiency compared to design 1 , respectively, when only the core area of designs is considered. With memory area estimates included, the efficiency of the design 2 and design 3 is 1.5 and $2.1 \times$ better, respectively.

Table 5.6: Performance comparison of HC-128 design versions

\begin{tabular}{|c|c|c|c|c|c|c|c|}
\hline \multirow{2}{*}{ Design } & Clock Freq. & \multicolumn{2}{|c|}{ Initialization latency } & \multicolumn{2}{c|}{ Throughput } & \multicolumn{2}{c|}{ TPA (Gbps/KGE) with } \\
& $(\mathrm{GHz})$ & (cycles) & $(\mathrm{ms})$ & (cycles/word) & $(\mathrm{Gbps})$ & Core Area & Total Area \\
\hline Design1 & 1.20 & 9168 & 7.64 & 4 & 9.60 & 1.73 & 0.16 \\
\hline Design2 & 1.30 & 4584 & 3.53 & 2 & 20.80 & 2.42 & 0.26 \\
\hline Design3 & 1.35 & 3556 & 2.63 & 1 & 43.20 & 3.18 & 0.35 \\
\hline
\end{tabular}

\subsubsection{Comparison with Reported HC-128 Performance}

For performance of HC-128 stream cipher on GPPs, eBACS provides a performance benchmarking of stream ciphers on various machines [63]. Some of the more recent results available at [64] are taken up. The fastest implementation requires 2.86 cycles per byte of keystream generated and 6.19 Gbps on an AMD Athlon 64 X2 4200+ processor. For initialization it requires $23 \mathrm{~K}$ clock cycles, requiring $10 \mu \mathrm{s}$ for setup [64]. A study of implementation of HC-128 on embedded and customizable embedded processors has been done by [38]. The fastest reported implementation is for ARM Cortex-A8 processor with throughput of 0.79 Gbps.

Table 5.7: Performance comparison of HC-128 design versions in [38]

\begin{tabular}{|c|c|c|c|c|c|c|c|}
\hline $\begin{array}{c}\text { Design } \\
{[38]}\end{array}$ & $\begin{array}{c}\text { Clock Freq. } \\
(\mathrm{GHz})\end{array}$ & \multicolumn{3}{|c|}{$\begin{array}{c}\text { Area (KGE) } \\
\text { Core }\end{array}$} & Memory & Total & Throughput \\
$(\mathrm{Gbps})$ & \multicolumn{2}{c|}{ TPA (Gbps/KGE) with } \\
Core Area & Total Area \\
\hline 1 & 1.67 & 8.19 & $53+(2 \times 26.61)=106.22$ & 114.41 & 13.36 & 1.63 & 0.12 \\
\hline 2 & 1.67 & 14.21 & $53+(4 \times 26.61)=159.44$ & 173.65 & 13.36 & 0.94 & 0.08 \\
\hline 3 & 1.67 & 13.66 & $53+(8 \times 26.61)=265.88$ & 279.54 & 17.81 & 1.30 & 0.06 \\
\hline 4 & 1.43 & 12.65 & $53+(8 \times 26.61)=265.88$ & 278.53 & 22.88 & 1.81 & 0.08 \\
\hline
\end{tabular}

The only hardware implementation reported for HC-128 is by [38] which reports base implementation, Parallel initialization, Parallel keystream generation and Even odd memory splitting as various design points referred in Table 7.3 as design 1, 2, 3 and 4, respectively. For a $65 \mathrm{~nm}$ standard Cell CMOS technology the fasted reported throughput is $22.88 \mathrm{Gbps}$. The SRAM size is being reported in terms of KBytes. For the base implementation, the 1280 word $W$ memory requires $53 \mathrm{KGE}$ area, while each of two 512 word memories ( $P$ and $Q$ ) need 26.61 KGE each. Due to $P$ and $Q$ memory replication, the area overhead due to memories increases. In terms of throughput, design 3 is around twice as fast compared to the fastest implementation (even odd memory splitting) in [38]. For a fair comparison, the throughput per area both with and without 
memory bank area is tabulated. The throughput efficiency of design3 (without memory) is around $1.7 \times$ better than the best implementation reported (even odd memory splitting) [38], while considering efficiency with area is around $3 \times$ better than their best reported results (base implementation) [38].

\subsection{Integrated Implementation of Multiple Cryptographic Proposals}

\subsubsection{Motivation}

Since various cryptographic functions may have common underlying operations/ structures, their similarities can be exploited to achieve area efficiency if multiple functions must co-exist and non-exclusively executed as a single VLSI core. The reason for such situations could vary, namely, need of flexible security levels, conformity of certain protocol, different throughput performance etc. These unified cores successfully achieve area efficiency, compared to the sum of individual cores, due to resource sharing. Moreover, the throughput penalty in most cases is small, when compared to the slower of the implemented algorithms, due to existence of a common critical path. Efforts for the design for unified co-processors were extended to include hash functions along with the block ciphers, hereby providing confidentiality and authenticity, simultaneously,

\subsubsection{Previous Work}

In the context of flexible cryptographic implementations, the idea of resource sharing for exclusive execution of more than one modes or versions of cipher algorithms is not novel. The motivation of designing these flexible hardware co-processors (or weakly programmable ASICs) stems from the need of of various cryptographic functions required for ensuring privacy, authenticity and integrity. Consequently, unified co-processors for block ciphers were extended to include hash functions to enable $\mathrm{Au}$ thenticated Encryption (AE), examples include AES and Grøstl [189], [188], AES and Fugue [110]. For block ciphers, after the widespread acceptance and use of AES, many unified configurable cores for AES with other ciphers were proposed, e.g., AES-128 with block cipher ARIA [32], AES-128/192/256 and AES-extended [208], AES-128 and Camellia [193]. A more recent configurable coprocessor, CoARX, exploits operational similarity between cryptographic functions to implement different block ciphers, hash functions, stream ciphers that are based on ARX family of ciphers [198].

\subsubsection{Contribution: HiPAcc-LTE-Integrated Accelerator for SNOW $3 G$ and ZUC}

A unified high performance accelerator (henceforth called HiPAcc-LTE) for SNOW 3G [2] and ZUC [232] (version 1.5, as in LTE Release 10 and beyond) is taken up, 
that is targeted towards the $4 \mathrm{G}$ mobile broadband market. 3GPP LTE-Advanced [1] contains these two stream ciphers at the core of its security architecture [87]. Either of these algorithms might be used in a $3 \mathrm{G}$ compatible device, the choice amongst these might have regional preferences.

In order to fully exploit the structural similarities between these algorithms in a common merged implementation, first a trial to merge the ciphers from an algorithmic point of view is taken up. Once this is accomplished, one may design an integrated custom accelerator for the ciphers such that each of the algorithms can be accessed individually. This approach offers the flexibility of sharing of resources, both storage and logic, throughput vs. area optimization at the base level, optimization of mutual critical path, and combined protection against fault attacks.

The process of integration at both algorithm and hardware levels produce the best solutions in terms of throughput and area, and provides the designer with handles on both. HiPAcc-LTE provides almost twice the throughput for both the ciphers compared to any existing architecture for the individual algorithms. Additionally, the user gets the flexibility to choose the 'area vs. throughput' trade-off for a customized design, along with a combined fault detection and protection mechanism. In case of SNOW 3G, the tolerance against the known fault attack by Debraize and Corbella [58] is included. For ZUC, however, there are no known fault attacks till date.

\subsubsection{Structural Comparison}

Appendix D.1 and D.2 elaborate the algorithmic description of SNOW 3G and ZUC stream ciphers, respectively. Their similarities and dissimilarities in their designs are identified, which led to their integration, as described next.

\subsubsection{Similarities}

The reader may easily spot the inherent structural similarity in the designs of the two ciphers SNOW 3G and ZUC. This is mainly because both ciphers are based on the same principle of combining an LFSR with an FSM, where the LFSR feeds the next state of the FSM. In the initialization mode, the output of the FSM contributes towards the feedback cycle of the LFSR, and in the keystream generation mode, the FSM contributes towards the keystream.

The key point to observe in Fig. 5.8 is the similarity in the 3-layer structure for both the ciphers SNOW 3G and ZUC. The figure on the left indicates the initialization mode of operation while the figure on the right demonstrates the operation during keystream generation. In Fig. 5.8, the combination of the LFSR update and the FSM during initialization mode is represented by $\mathrm{C}$, which is either an XOR or a shift and addition modulo $2^{31}-1$ for SNOW 3G and ZUC, respectively. In the keystream generation mode, the combination of the LFSR state with the FSM output is denoted as $\mathrm{K}$, which is an XOR for SNOW 3G and a bit reorganized XOR for ZUC. The operations are individually presented in the previous subsections for the two ciphers. $Z$ represents the output keystream for both the ciphers. 

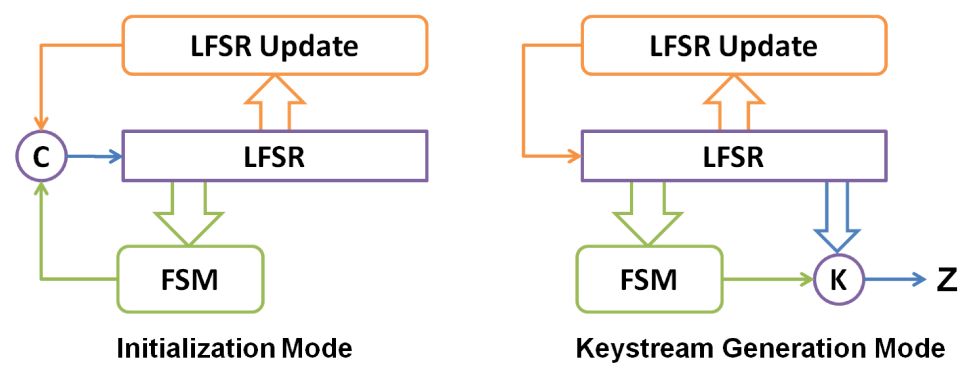

Figure 5.8: Top level structure of both SNOW 3G and ZUC.

\subsubsection{Dissimilarities}

A deeper probe into the individual components of the design exposes the dissimilarities, that are categorized according to the main stages of the two ciphers.

1. LFSR update routine is fundamentally different for the two ciphers. While SNOW 3G relies on field multiplication/division along with XOR for the LFSR feedback, ZUC employs addition modulo the prime $p=2^{31}-1$. Another point to note is that the new updated value $s_{15}$ is required for the next feedback in case of ZUC, whereas SNOW 3G does not have this dependency. This creates a major difference in designing the combined architecture.

2. The main LFSR is slightly different for the ciphers as well, although both SNOW 3G and ZUC output 32-bit words. SNOW 3G uses an LFSR of 16 words, each of size 32 bits, whereas ZUC uses an LFSR of 16 words, each of size 31 bits. However, the bit organization stage of ZUC builds 32 bit words from the LFSR towards FSM update and output generation.

3. FSM operations of SNOW $3 \mathrm{G}$ and ZUC are quite different as well, though they use similar resources. SNOW 3G has three registers R1, R2 and R3 where the updation dependency $R 1 \rightarrow R 2 \rightarrow R 3 \rightarrow R 1$ is cyclic with the last edge depending on the LFSR as well. In case of ZUC, there are only two registers R1 and R2. The updation of each depends on its previous state as well as that of the other register. And of course, the LFSR also feeds the state updation process, as in the case of SNOW 3G.

In the next section, a merge of the SNOW 3G and ZUC ciphers is attempted in such a fashion that the similarities are exploited to the maximum extent, and the common resources are shared. The dissimilarities discussed above are treated specially for each of the ciphers. This merger is attempted in three parts, each corresponding to the major structural blocks of the two designs; namely, the main LFSR, the LFSR update function and the FSM.

\subsubsection{Integrating the Main LFSR}

Recall that the LFSR of SNOW 3G has 16 words of 32 bits each, while that of ZUC has 16 words of 31 bits each. The primary goal is to share this resources among the two 


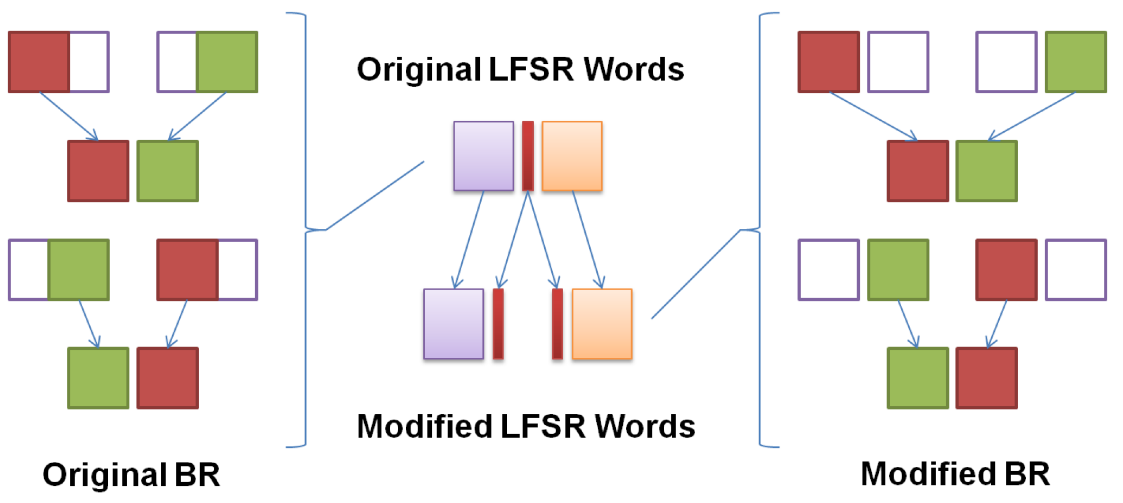

Figure 5.9: Modified bit reorganization for ZUC after LFSR integration.

ciphers. A naive sharing by putting the 31 bit words of ZUC in the same containers as those for the 32 bit words of SNOW 3G leaves out 1 bit per word is left unused in ZUC. Hence, the target was first to utilize this bit in such a way that reduces the critical path in the overall implementation.

Motivation. During the discussion of the pipeline structure in Section 5.3.7, it can be seen that the critical path flows through the output channel, that is, through the bit reorganization for $s_{15}, s_{14}$ and $s_{2}, s_{0}$, and the FSM output of $W$. In fact, bit reorganization is also required for the FSM register update process. Consequently, the bit reorganization process is removed from the FSM.

Restructuring the LFSR. In this direction, a 32 element LFSR, each having 16 bits is constructed. The 32 bit words for SNOW $3 \mathrm{G}$ would be split in halves and stored in the LFSR registers naturally. For ZUC, the 31 bit words are split in 'top 16 bit' and 'bottom 16 bit' pieces, and stored individually in the 16 bit LFSR registers. The organization of bits is shown in the middle column of Fig. 5.9, where the two blocks share the centermost bit of the 31 bit original word. Notice that the bit reorganization is not required any more in the FSM operation, as it reduces to simple read from two separate registers in this construction. The modified bit reorganization model is illustrated in Fig. 5.9.

However, note that the LFSR update function of ZUC uses the 31 bit words for the modulo $2^{31}-1$ addition. since the the bit reorganization stage is moved to the LFSR update stage instead of the FSM. The effects of these design choices will be discussed in the next Section.

\subsubsection{Integrating the FSM}

Although the FSM of the two ciphers do not operate the same way, they share similar physical resources. Thus, the main goal for the integrated design is to share all possible resources between them. Note that the bit reorganization stage is not present in the ZUC FSM any more, due to proposed LFSR reconstruction.

Register Sharing. One can straight away spot the registers R1, R2 and R3 for potential sharing. Registers R1 and R2 are shared between SNOW 3G and ZUC, while R3 is 


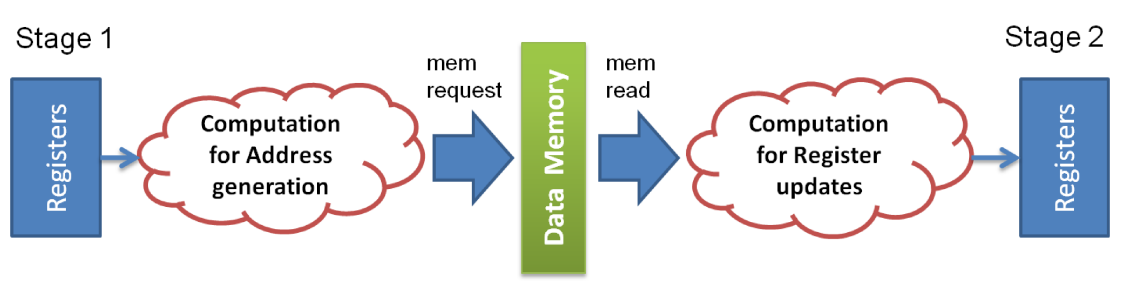

Figure 5.10: Pipeline structure based on Memory Access.

needed only for the former. If required, R3 can be utilized in ZUC for providing additional buffer towards fault protection, discussed in Section 5.3.7.

Sharing the Memory. During the FSM register update process, both SNOW 3G and ZUC use S-box lookup. In the software version of the ciphers, SNOW 3G [2] uses $S_{R}, S_{Q}$ and ZUC [232] uses $S_{0}, S_{1}$. However, for efficient hardware implementation of SNOW 3G with memory access, the tables S1_T0, S1_T1, .., S2_T3 were chosen, as prescribed in the specifications [2]. This saves a lot of computations after the memory read, and hence reduces the critical path to a considerable extent. These 8 tables are stored in a data memory of size 8 KByte.

For ZUC, however, the lookup to $S_{0}$ and $S_{1}$ cannot be bypassed. But one may note that these tables are accessed 4 times each during the FSM update. So, to parallelize the memory access, 4 copies of each table (thus 8 in total) are stored in the same 8 KByte of data memory that is allocated for SNOW 3G. Note that the full capacity of the memory in ZUC is not being used, as 1 byte is stored in each location (as in $\mathrm{S}_{0}$ and $\mathrm{S}_{1}$ ) whereas it is capable of accommodating 4 bytes in each (as in S1_T0, S1_T1, ..., S2_T3).

By duplicating the ZUC tables in the 8 distinct memory locations, the memory read requests are restricted to 1 call per table in each cycle of FSM. This makes possible the sharing of memory access between SNOW 3G and ZUC as well. Only a single port to read from each of the tables is used, and that too is shared between the ciphers for efficient use of resources. This in turn reduces the multiplexer logic and area of the overall architecture.

Pipeline based on Memory Access. Since there is a memory lookup during the FSM update, the pipeline is accordingly partitioned. The memory is simulated as a synchronous SRAM with single-cycle read latency. To optimize the efficiency with an allowance for the latency in memory read, the pipeline is split in two stages, keeping the memory read request and read operations in the middle. The structure of the initial pipeline idea is shown in Fig. 5.10.

This pipeline is organized around the memory access, where

- the memory read request and LFSR update is performed in Stage 1, and

- the memory read and output computation is performed in Stage 2. 
For SNOW 3G, the computation for memory address generation is a simple partitioning of R1 and R2 values in bytes. The computation for register update however, requires an XOR after the memory read. In case of $\mathrm{ZUC}$, the computation for address generation is complicated, and depends on the LFSR as well as R1 and R2. However, the computation for register update is a simple concatenation of the values read from memory.

In a nutshell, the design choices in integrating the two ciphers provide

- reduction in the critical path by reducing the memory and LFSR read times,

- reduced critical path by moving the bit reorganization away from FSM, and

- an efficient method for combined fault protection in both the ciphers.

The effect of these choices will be reflected in the critical path and fault tolerance mechanism, discussed later in Section 5.3.7.5 of this case study.

Next, the integration of the most crucial part of the two ciphers is dealt: the LFSR update and shift operations. The final structure of the pipeline evolves during this phase as the intricate details in the design are dealt.

\subsubsection{Integrating the LFSR Update Function}

The LFSR update function is primarily different for the two ciphers. The only thing common is the logic for LFSR update during initialization, and this poses a big problem with the earlier pipeline idea based on memory access (Fig. 5.10).

Pipeline restructuring for Key Initialization. In the initialization mode of the two ciphers, the FSM output $W$ is fed back to the LFSR update logic. The update of $s_{15}$ takes place based on this feedback, and in turn, this controls the next output of the FSM (note that $W$ depends on R1, R2 and $s_{15}$ in both ciphers). This is not a problem in the keystream mode as the LFSR update path is independent of the output of FSM. However, during initialization, it creates a combinational loop from Stage 2 to Stage 1 in the earlier pipeline organization (Fig. 5.10). This combinational loop in memory access due to dependencies prohibits keeping the memory access and memory read in two different stages of the pipeline.

Thus, a new design structure is proposed as follows:

- Stage 1: Initial computation for memory access and LFSR shift.

- Stage 2: Memory read, LFSR update and subsequent memory read request.

This new pipeline structure allows resolving the memory access dependencies within a single stage while the independent shift of the LFSR can occur in the other. Now, the main goal is to orient the LFSR update logic around this pipeline structure, or to redesign the pipeline according to the LFSR update function.

Pipeline organization for LFSR update. The LFSR update logic of SNOW 3G is easier to deal with. The update depends upon the LFSR positions $s_{0}, s_{2}$ and $s_{11}$, and also on 
the FSM output $W$ during key initialization. A part of $s_{0}$ and $s_{11}$ each undergoes a field operation (MUL ${ }_{\alpha}$ and $\mathrm{DIV}_{\alpha}$ respectively), and the other part gets XOR-ed thereafter. To reduce the combinational logic of realizing the field operations, two lookup tables are prescribed in the specifications [2]. For an efficient implementation in hardware, this idea is taken up and the two tables $\mathrm{MUL}_{\text {alpha }}$ and $\mathrm{DIV}_{\text {alpha }}$ are stored in two 1 KByte memory locations. These are also read-only memories with single-cycle read latency. The update routine for SNOW 3G can now fit within the two stage pipeline proposed earlier.

- Stage 1: Precompute the simple XOR involving $s_{0}, s_{2}$ and $s_{11}$, and generate the addresses for memory read requests to tables $\mathrm{MUL}_{\text {alpha }}$ and $\mathrm{DIV}_{\text {alpha}}$.

- Stage 2: Perform memory read and XOR with the previous XOR-ed values to complete the LFSR feedback path, run the FSM and complete the LFSR update of $s_{15}$ depending on $\mathrm{W}$.

Note that this pipeline structure works both for initialization as well as keystream generation, as it takes into account all possible values required for the LFSR update. Thus, in terms of SNOW 3G, a 2-stage pipeline is preferred.

In case of ZUC however, the LFSR update logic is quite complicated. This is mostly because of the additions modulo the prime $p=2^{31}-1$. Liu et al [144] had proposed a single adder implementation of this addition modulo prime, and this logic has also been included in the specifications [232]. The same hardware is used, at least at the initial phase. In the same line, a 5-stage pipeline is given a try, similar to the one proposed in [144] for LFSR update of ZUC.

The initial idea for 5-stage pipeline is shown as Pipeline 1 in Fig. 5.11. All the adders are modulo prime, similar to the ones in [144], and the variables $a, b, c, d, e, f$ represent $s_{0}, 2^{8} s_{0}, 2^{20} s_{4}, 2^{21} s_{10}, 2^{17} s_{13}, 2^{15} s_{15}$ (modulo $p=2^{31}-1$ ) respectively. Variable $g$ denotes the FSM output $W$, which is added with the cumulative LFSR feedback, and is then fed back to $s_{15}$ in the LFSR itself.

However, Pipeline 1 creates a combinational loop between Stage 5 and Stage 4 in the key initialization phase. The final output in Stage 5 of the addition pipeline has to be fed back to $s_{15}$ that controls the input $f$ in Stage 4 . This loop is shown by the curvy solid line in Fig. 5.11, and it occurs due to mutual dependency of FSM and LFSR update during initialization. The authors of [144] also observed this dependency, and they proposed the 32 rounds of key initialization to be run in software in order to achieve one-word-per-cycle using their structure.

The main challenge was to integrate this phase into the hardware without losing the throughput. The main motivation is to restrain the use of an external aide for the initialization mode. There are two direct ways of resolving this issue:

1. Allow a bypass logic for the $f$ value across the stages

2. Restructure the pipeline to merge the last two stages 


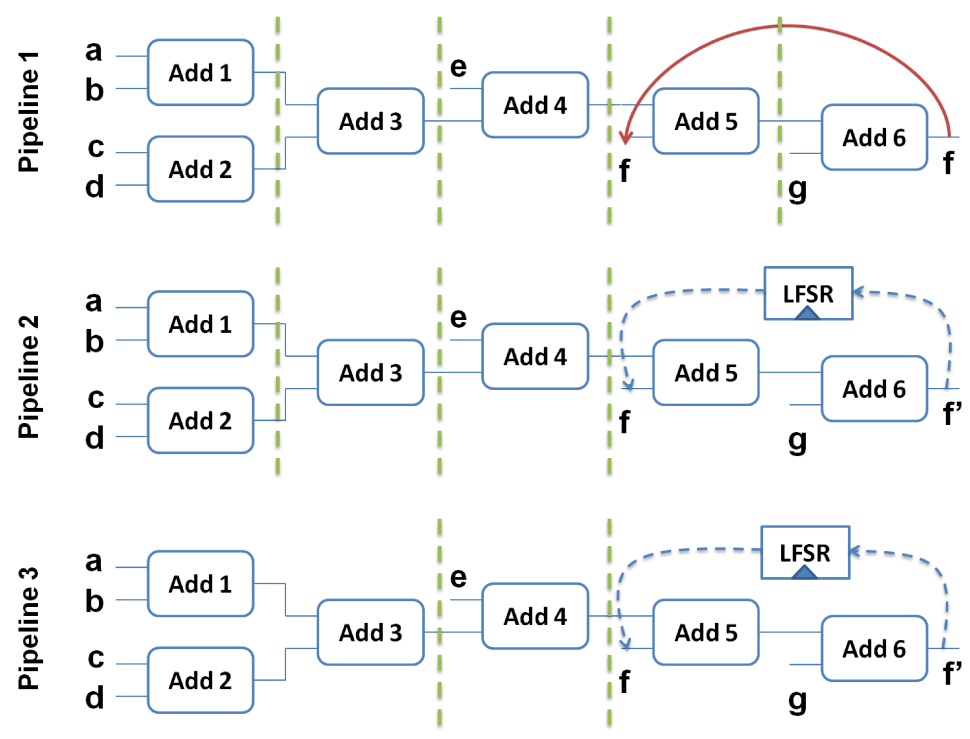

Figure 5.11: Pipeline structure reorganization for LFSR update of ZUC.

The second one is chosen and the pipeline is reorganized. As the dependency discussed so far occurs in between the last two stages of the pipeline when they are merged to resolve the inter-stage combinational loop. In this case, the output $f^{\prime}$ of this stage is written into the $s_{15}$ location of the LFSR, and read back as $f$ at the next iteration. This is shown as Pipeline 2 in Fig. 5.11.

It can be noted that there are two adders (modulo prime $p$ ) in series at the last stage of Pipeline 2 (Fig. 5.11). So, two adders are used in any other stage as well, without affecting the critical path. Hence a merging of Stages 1 and 2 is done to have two adders in parallel followed by an adder in series in the first stage. This does not increase the critical path, which still lies in the last stage due to the two adders and some associated combinational logic. The final structure of the LFSR update pipeline for ZUC is shown in Fig. 5.11 as Pipeline 3. The next section discusses the design of an integrated pipeline structure combining all components.

\subsubsection{Final Design of the Pipeline}

In this section, the final pipeline structure for the integrated architecture is presented. As discusses in the previous sections, the components are already partitioned into following pipeline stages.

- FSM: Two stages - initial computations for address generation in the first stage, and memory access and related computations in the second stage.

- LFSR Movement: Two stages - shift in first stage and $s_{15}$ write in second.

- LFSR Update: Two stages for SNOW 3G and three stages for ZUC.

The final pipeline for the proposed hardware implementation, after combining all the three components of SNOW 3G and ZUC and design, is shown in Fig. 5.12. 


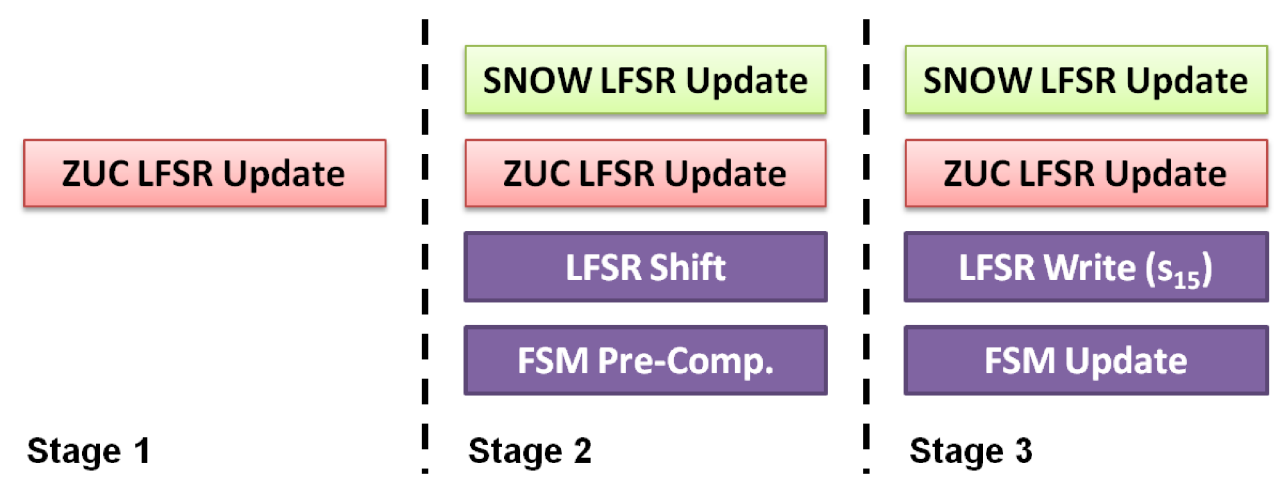

Figure 5.12: Final 3-stage Pipeline Structure for the Integrated Design.

The stages of SNOW 3G and ZUC are different only in case of the LFSR update routine, and are separately shown in the figure. The pipeline behavior of the LFSR shift and write operations, as well the FSM precomputation and update routines are almost same for both the ciphers, and hence a single instances of these is shown in Fig. 5.12. The next section discusses the practical issues with the final ASIC implementation of this integrated hardware.

\subsubsection{ASIC Implementation of HiPAcc-LTE}

The hardware generation environment and simulation framework from LISA (Section 2.3.2) was utilized for designing the accelerator. The accelerator in this case is designed as a state machine. The gate-level synthesis was carried out using using a 65nm target technology library (Section 2.3.1). The total lines of LISA code for the best implementation is 1131, while the total lines of auto-generated HDL code is 13440 for the same design. The modeling, implementation, optimization and tests were completed over a span of two weeks.

This section discusses the issues with the critical path in HiPAcc-LTE design, and the optimizations thereof. This will be followed by a set of detailed implementation results and comparisons with the existing designs.

\subsubsection{Critical Path.}

After the initial synthesis of HiPAcc-LTE using LISA modeling language, the critical path is identified as the key initialization phase of ZUC. Fig. 5.13 depicts the critical path using the curvy dashed line. To understand the individual components in the critical path, the pieces in Fig. 5.13 are associated to the original initialization routine of ZUC, as described in its specification [232].

ZUC Key Initialization Routine. The following is the key initialization routine of ZUC, as per the notation and pipeline orientation. Note that the operation is the same as in the LFSRWithInitialisationMode() function of [232].

LFSR_Key_Initialization (W) 


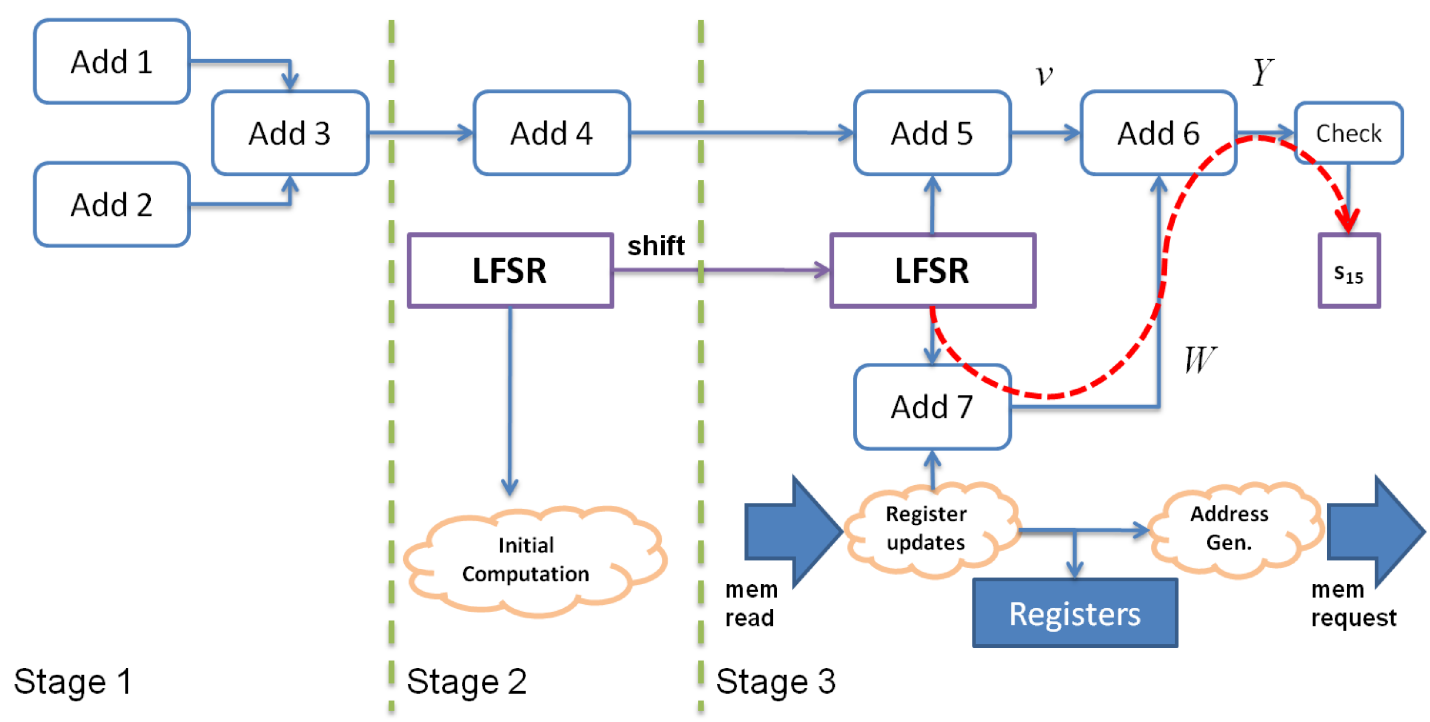

Figure 5.13: Critical path in the Key Initialization of ZUC (curvy dashed line).

1. $v=2^{15} s_{15}+2{ }^{17} s_{13}+2^{21} s_{10}+2^{20} s_{4}+2^{8} s_{0}+s_{0}\left(\bmod 2^{31}-1\right)$

2. $Y=v+(W \gg 1)\left(\bmod 2^{31}-1\right)$

3. If $Y=0$, then set $Y=2^{31}-1$

4. Write $Y$ to location $s_{15}$ of the LFSR

In Fig. 5.13, the first five adders Add 1 to Add 5 are part of the general LFSR feedback loop in ZUC, and they compute the value

$$
v=2^{15} s_{15}+2^{17} s_{13}+2^{21} s_{10}+2^{20} s_{4}+2^{8} s_{0}+s_{0} \quad\left(\bmod 2^{31}-1\right)
$$

The LFSR is also accessed to run the FSM and the adder Add 7 at the bottom of Stage 3 computes the FSM output $W=\left(X_{0} \oplus R_{1}\right)+R_{2}$, where this addition is a normal 32-bit addition. The special operation in LFSR update of ZUC in its initialization mode is to compute $Y=v+(W \gg 1)\left(\bmod 2^{31}+1\right)$, realized by the adder Add 6 on the top layer of Stage 3. If this sum $Y=0$, it is replaced by $Y=2^{31}-1$ in the 'Check' module of Fig. 5.13. Finally, this 31 bit value $Y$ is written to $s_{15}$ of the LFSR, thus completing the LFSR update loop. The critical path, as shown by the curvy dashed line in Fig. 5.13, is as follows:

$$
\text { LFSR Read } \rightarrow \text { 32-bit Add } \rightarrow \text { Modulo Add } \rightarrow \text { Check } \rightarrow \text { LFSR Write }
$$

This section discusses all possible optimizations to reduce the critical path.

LFSR Read Optimization. At first, the LFSR is implemented as a register array. However, different locations of the LFSR are accessed at different stages of the pipeline and the LFSR read will be faster if it allows the individual LFSR cells to be placed independently in the stages. This provided the motivation to implement the LFSR as 32 distinct registers of size 16 bits each. Furthermore, the last two locations, i.e., $s_{15}$ 

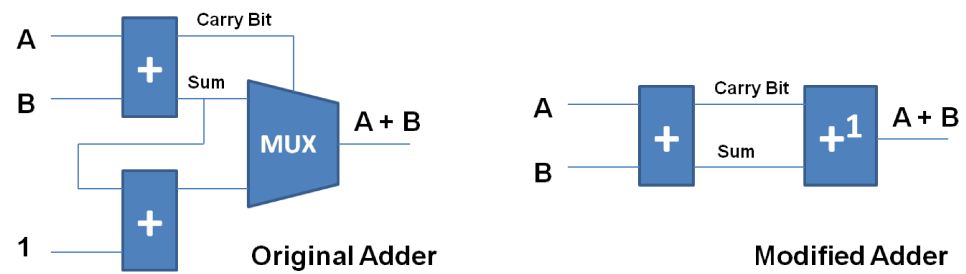

Figure 5.14: Modulo $p$ Adder optimization for ZUC.

of the LFSR were shadowed, so that it can be read instantaneously from both Stage 4 and Stage 5. This led to a reduction in the critical path. Though this optimization is targeted towards physical synthesis, the gate-level synthesis results indicated strong improvement as well.

Modulo $p$ Adder Optimization. Initially, the modulo $p=2^{31}-1$ adder is designed as prescribed in [144]. This looks like the circuit on the left of Fig. 5.14. However, one may bypass the multiplexer (MUX) by simply incrementing the sum by the value of the carry bit. That is, if the carry bit is 1, the sum gets incremented by 1 , and it remains the same otherwise. The modified design (right side of Fig. 5.14) slightly reduces the critical path, consequently all the modulo $p$ adders are replaced in the design (except for Add 6) by this modified circuit.

Check Optimization. The 'Check' block in the critical path actually has two checks in series; one due to Add 6 where the increment is based on the carry bit, and the second check is for the $Y=0$ situation. The following optimizations are carried out.

- Carry $=0$ : Just require to check if $Y=0$. If so, set $Y=2^{31}-1$.

- Carry $=1$ : Just require to set $Y=Y+1$ without any further checks.

The first case is obvious, as the sum would remain unchanged if the carry is 0 . In the second case, note that the inputs $v$ and $(W \gg 1)$ to Add 6 are both less than or equal to $2^{31}-1$. Thus, the sum $Y$ is bounded from above by $2^{32}-2$. Even if the carry is 1 , the incremented value of sum will be bounded from above by $2^{32}-1$, which can never have the lower 31 bits all equal to 0 . Thus, even the 'Check' block in this situation is not required. This optimization simplifies the logic and reduces the critical path considerably.

\subsubsection{Performance Results.}

After performing all the optimizations discussed in the previous section, the final synthesis and performance results based on that state of the design is performed.

Table 5.8 presents all the architecture design points for HiPAcc-LTE implemented using the $65 \mathrm{~nm}$ technology. The area-time chart for the design points of HiPAcc-LTE is shown in Fig. 5.15.

The maximum achievable frequency is $1090 \mathrm{MHz}$, which corresponds to a critical path length of approximately 0.92 ns. This provides a net throughput of $34.88 \mathrm{Gbps}$, 
Table 5.8: Synthesis results for HiPAcc-LTE with 10 KByte memory.

\begin{tabular}{|c|c|c|c|}
\hline \multirow{2}{*}{$\begin{array}{c}\text { Frequency } \\
(\mathrm{MHz})\end{array}$} & \multicolumn{3}{|c|}{ Area (equivalent NAND Gates) } \\
\cline { 2 - 4 } & Total & Sequential & Combinational \\
\hline 200 & 11699 & 5540 & 6159 \\
500 & 13089 & 5540 & 7549 \\
800 & 14102 & 5541 & 8561 \\
1000 & 15696 & 5541 & 10155 \\
1050 & 16055 & 5554 & 10501 \\
1090 & 16886 & 5568 & 11318 \\
\hline
\end{tabular}

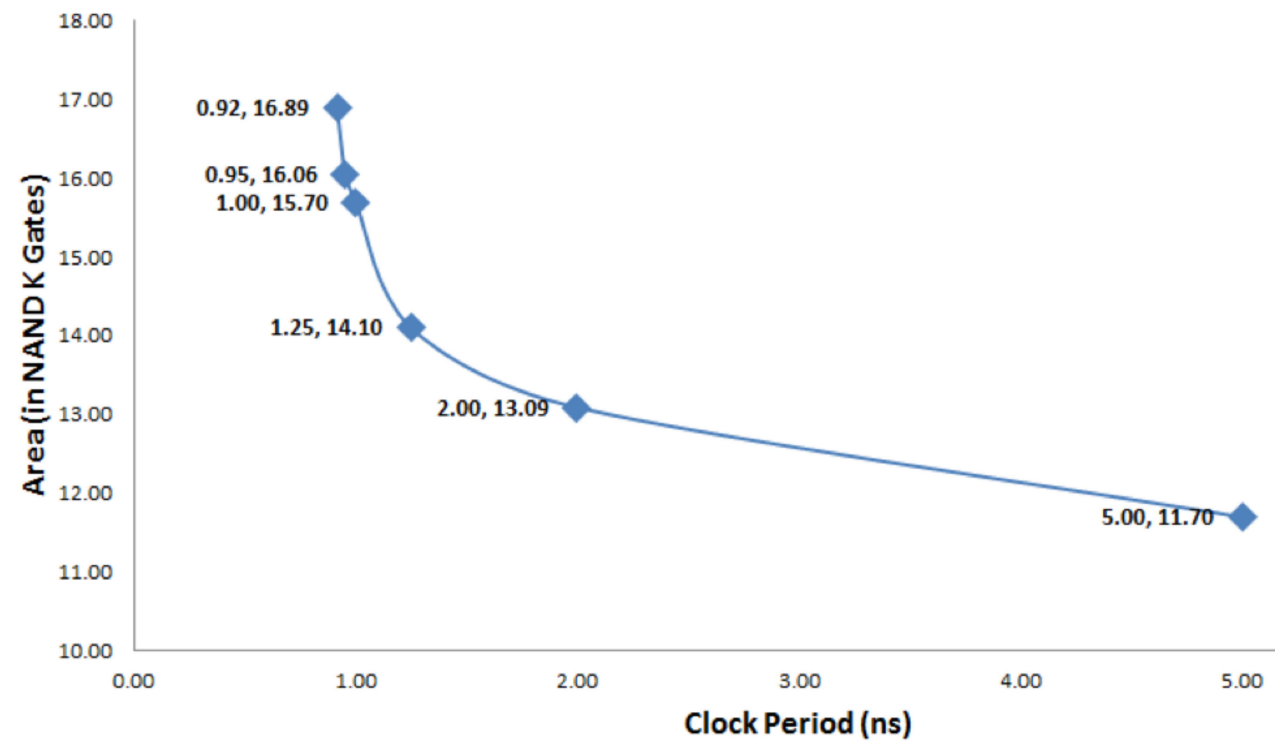

Figure 5.15: Area-Time chart for HiPAcc-LTE (10 KByte memory) using 65nm technology.

with 1 keystream word per cycle. The total area is about 17 KGates NAND equivalent and 10 KByte of data memory is required.

Experiments with Reduced Data Memory. In the original HiPAcc-LTE design as above, the static data for S-box and field operations have been stored in external data memory. While SNOW 3G utilizes the complete 10 KByte memory, ZUC requires only about 2 KByte of the allocated space. This motivated the experimentation with an alternate design that requires less data memory.

In the alternate design, $S$-box tables $S_{R}, S_{Q}$ were used for SNOW 3G [2] instead of the tables S1_T0, S1_T1, ..., S2_T3, as in the previous case. During the sharing of memory, the ZUC tables $S_{0}, S_{1}$ fit exactly in the space for $S_{R}, S_{Q}$ as they are of the same size, 256 bytes each. There are exactly 4 calls to each table per cycle, and two copies of each table are stored in dual-port RAMs to get optimum throughput. This 
amounts to a data memory of $2 \times(256+256)$ bytes $=1$ KByte. The MUL $\mathrm{Mlph}_{a}$ and DIV $_{\text {alpha }}$ tables (size 1 KByte each) in case of SNOW 3G could not be avoided due to the complicated combinational logic involved in these field operations. The total data memory for this alternate design sums up to $3 \mathrm{KByte}$, and the details for all design points are presented in Table 5.9.

Table 5.9: Synthesis results for alternate design of HiPAcc-LTE with 3 KByte memory.

\begin{tabular}{|c|c|c|c|}
\hline \multirow{2}{*}{$\begin{array}{c}\text { Frequency } \\
(\mathrm{MHz})\end{array}$} & \multicolumn{3}{|c|}{ Area (equivalent NAND Gates) } \\
\cline { 2 - 4 } & Total & Sequential & Combinational \\
\hline 200 & 10519 & 5548 & 4971 \\
500 & 13090 & 5540 & 7550 \\
800 & 14103 & 5541 & 8562 \\
1000 & 15696 & 5541 & 10155 \\
1090 & 16887 & 5568 & 11319 \\
\hline
\end{tabular}

This alternate design retains the maximum frequency of $1090 \mathrm{MHz}$, which provides a net throughput of $34.88 \mathrm{Gbps}$, with 1 word per cycle. The area figure is still about 17 KGates NAND equivalent, but only 3 KByte of external data memory is required. It is interesting to note that the combinational area remained almost similar even after introducing the computations for S-boxes. This is possibly due to the availability of high-speed, area-efficient library cells in the target technology library used and an efficient design style.

With this alternate design of HiPAcc-LTE having 3 KByte of memory, the performance of the individual ciphers SNOW 3G and ZUC are also tested in standalone mode. The synthesis results in this direction are presented in Table 5.10.

Table 5.10: Synthesis results for standalone mode in HiPAcc-LTE with 3 KByte memory.

\begin{tabular}{|l|c|c|c|c|}
\hline Cipher & Frequency & \multicolumn{3}{|c|}{ Area (equivalent NAND Gates) } \\
\cline { 3 - 5 } & $(\mathrm{MHz})$ & Total & Sequential & Combinational \\
\hline \hline SNOW 3G & 500 & 6867 & 5061 & 1807 \\
& 1000 & 7033 & 5062 & 1971 \\
\hline \hline ZUC & 500 & 9555 & 4798 & 4757 \\
& 1000 & 11412 & 4811 & 6601 \\
\hline
\end{tabular}

\subsubsection{Exploration of Storage Implementation.}

For the physical implementation of the storage, a number of alternatives are explored. The choices are primarily limited by the constraints like read-only configuration, num- 
ber of access ports. For FPGA-based designs, while it is commonplace to exploit available RAM blocks, storage must be designed carefully for ASIC implementation. Faraday Memory Compiler with 65nm technology library was taken up for exploring dual-port block RAMs and synchronous ROMs. While block RAMs can be utilized for both SNOW and ZUC execution, the ROM is not re-programmable and therefore, must hold the complete storage for both the algorithms.

For SNOW 3G, the RAM requirement is attributed to tables for $M U L_{a l p h a}$ and $D I V_{\text {alpha }}$ computation, additionally. The synthesis results show approximately 43 KGates for SNOW 3G and 26.8 KGates for ZUC. The memory access time is slower than the combinational path of the logical operations thereby, supporting the highest achievable frequency. For synchronous ROM, Faraday Memory Compiler supports a minimum size of 4096 bits with 1 read port, which is more than enough. For supporting the parallel computation both SNOW 3G and ZUC requires 8 2048-bit ROM with 8-bit word alignment and 1 read port access. With this forced redundancy of double data capacity with limited port access, the ROM synthesizes to approximately 23.12 KGates for ZUC. Similar area, i.e., a total of 46.24 KGates will be required for SNOW 3G and ZUC even without storing the $2 \mathrm{~KB}$ tables for $M U L_{a l p h a}$ and DIV alpha computation. Clearly, with port access restrictions synchronous ROM is not a good choice compared to RAM.

In a final optimization attempt, the tables are manually coded in a switch-case statement and directly synthesized as a hard macro for both SNOW 3G and ZUC. This resulted in much less area compared to the RAM. The results are summarized in table 5.11. It must be noted that due to read-only nature of the hard macro, both SNOW 3G and ZUC tables are encoded in the combined design. This also requires multiplexing between alternative tables according to the actual algorithm being executed. As a result, the throughput achievable in the combined design with hard macro is slightly less compared to the design implementing ZUC standalone. A nice advantage of storage implementation with hard macro is that it is less susceptible to physical attacks like memory readout or fault injection. The hard macro is realized within the combinational blocks, whereas RAM or ROM structures maintain a clear separation from the logic and are easier to spot in a physical layout.

Comparison with Existing Designs. To put the performance of HiPAcc-LTE into perspective, it was compared with the state-of-the-art architectures available in academia and the commercial sector.

Comparison with Academic Literature. In the domain of published academic results, no ASIC implementation of ZUC is reported, and neither any $65 \mathrm{~nm}$ technology implementation of SNOW 3G could be found. The only hardware realizations for ZUC have been done in FPGA [144] so far. Thus, HiPAcc-LTE could not be compared to any academic results in terms of ZUC.

In case of SNOW 3G, the best academic publication is [128] that uses 130nm technology. To compare with this result, the proposed design is synthesized (with 10 KByte data memory) with 130nm (Section 2.3.1), and the comparison is as follows.

- SNOW 3G of [128]: 7.97 Gbps with $249 \mathrm{MHz}$ max. freq. and 25 KGates area 
- HiPAcc-LTE: 24.0 Gbps with $750 \mathrm{MHz}$ max. freq. and 18 KGates area

Both designs use about 10 KByte of external data memory for look-up tables. A surprisingly better throughput from HiPAcc-LTE is attributed to a careful pipeline design. HiPAcc-LTE integrated implementation for both the LTE stream ciphers even outperforms the single standalone core in terms of area.

Comparison with Commercial Designs. In the commercial arena, the best architectures available for SNOW 3G and ZUC are from IP Cores Inc. [105] and Elliptic Tech Inc. [104] respectively. Both provide standalone solutions for the individual stream ciphers and match the technology of $65 \mathrm{~nm}$. One tricky issue in the comparison is the area required for the memory. It is not always clear from the product white-paper whether additional memories have been used.

For the sake of fairness, first a comparison of the designs using $3 \mathrm{~KB}$ memory with existing standalone ZUC and SNOW 3G implementations is done. The memory is synthesized with Faraday Memory Compiler in $65 \mathrm{~nm}$ technology node. Next the S-Box SRAM implementations are replaced with hard macros in the RTL design to get the gate-level synthesis results. From the commercial designs, the designs with best performance claims in 65nm technology node are selected. The detailed comparison and analysis is provided in Table 5.11 .

Table 5.11: Comparison of HiPAcc-LTE with existing 65nm commercial designs.

\begin{tabular}{|c|c|c|c|c|c|}
\hline \multicolumn{6}{|c|}{ Performance of Commercial Designs } \\
\hline \hline Cipher & Name of Design & Designer & $\begin{array}{c}\text { Max. Freq. } \\
(\mathrm{MHz})\end{array}$ & $\begin{array}{c}\text { Throughput } \\
(\mathrm{Gbps})\end{array}$ & $\begin{array}{c}\text { Total Area } \\
(\text { KGates })\end{array}$ \\
\hline SNOW 3G & SNOW3G1 [105] & IP Cores Inc. & 943 & 7.5 & 8.9 \\
ZUC & CLP-410 [104] & Elliptic Tech. & 500 & - & $10-13$ \\
\hline
\end{tabular}

\begin{tabular}{|c|c|c|c|c|c|}
\hline \multicolumn{5}{|c|}{ Performance of HiPAcc-LTE } \\
\hline \hline Cipher & $\begin{array}{c}\text { Mode of Design } \\
\text { for Static Tables }\end{array}$ & $\begin{array}{c}\text { Memory } \\
\text { (KGates) }\end{array}$ & $\begin{array}{c}\text { Frequency } \\
(\mathrm{MHz})\end{array}$ & $\begin{array}{c}\text { Throughput } \\
(\mathrm{Gbps})\end{array}$ & $\begin{array}{c}\text { Total Area } \\
\text { (KGates) }\end{array}$ \\
\hline SNOW 3G & & 43.0 & 1000 & 32.0 & 50.0 \\
ZUC & \multirow{3}{*}{ 3 KByte memory } & 26.8 & 1000 & 32.0 & 38.2 \\
Both & & 43.0 & 1090 & 34.9 & 59.9 \\
\hline SNOW 3G & & - & 1650 & 52.8 & 18.1 \\
ZUC & Hard macro & - & 920 & 29.4 & 20.6 \\
Both & & - & 900 & 28.8 & 27.4 \\
\hline
\end{tabular}

Area comparison: Around an operating frequency of 200-500 MHz, if one uses the two best cores separately, the combined area comes around 18-20 KGates. HiPAcc-LTE 
synthesizes within 16-18 KGates in this frequency zone (using hard macros), hence offering about $10 \%$ reduction in area. Even with this reduced area figure, HiPAccLTE offers the same throughput as CLP-410 [104] and more than double throughput compared to SNOW3G1 [105].

Throughput comparison: The best throughput (1 word/cycle) is provided by the CLP410 ZUC core from Elliptic Tech. However, they just quote a figure of 6 Gbps for $200 \mathrm{MHz}$ [104]. A simple scaling to their maximum frequency of $500 \mathrm{MHz}$ would translate this to an estimate of 15 Gbps. Even in this case, the throughput $29.4 \mathrm{Gbps}$ of HiPAcc-LTE (in hard macro design) is almost double compared to any of the commercial standalone implementations of the ciphers.

For a very rough estimate, if one wants to achieve a comparable throughput (approx. $30 \mathrm{Gbps}$ ) using the existing standalone modules, then 4 parallel blocks of SNOW3G1 [105] and 2 parallel blocks of CLP-410 [104] would be required. This amounts to a total area of roughly 56-62 KGates, while HiPAcc-LTE achieves the same using only $27.4 \mathrm{KGates}$ (at least $51 \%$ reduction) for the hard macro based design. For the sake of fairness, one may also note that HiPAcc-LTE has a comparable area figure of 59.9 KGates for an even higher throughput (34.9 Gbps) using 3 KByte of external data memory. If the extreme throughput is not required for communication purpose, it may facilitate a scaling in frequency/voltage for reduced power consumption.

\subsubsection{Power Consumption/Dissipation Analysis.}

Power consumption and dissipation are serious design concerns in embedded systems, in particular for the cryptographic devices. A power estimation of different design points is provided, i.e., the standalone SNOW 3G implementation, standalone ZUC implementation, and the combined design HiPAcc-LTE running individual applications. The operating condition of the target $65 \mathrm{~nm}$ technology library is set at the best case scenario with a global operating voltage of $1.32 \mathrm{~V}$ and temperature $-40^{\circ} \mathrm{C}$. The power consumption is estimated on a gate-level netlist by back-annotating the switching activity and using Synopsys Power Compiler tool. The results are presented in Table 5.12.

Table 5.12: Power estimation results for HiPAcc-LTE with hard macro storage.

\begin{tabular}{|c|c|c|c|}
\hline Cipher & $\begin{array}{c}\text { Frequency } \\
(\mathrm{MHz})\end{array}$ & $\begin{array}{c}\text { Power } \\
(\mathrm{mW})\end{array}$ & $\begin{array}{c}\text { Energy } \\
(\mathrm{pJ} / \text { byte })\end{array}$ \\
\hline \hline SNOW 3G standalone & 1650 & 14.41 & 2.19 \\
ZUC standalone & 920 & 18.7 & 5.09 \\
\hline \hline HiPAcc-LTE (SNOW 3G) & 900 & 17.32 & 4.81 \\
HiPAcc-LTE (ZUC) & 900 & 16.83 & 4.67 \\
\hline
\end{tabular}

From Table 5.12 it can be observed that the standalone SNOW 3G implementation is much more energy-efficient due to its significantly higher clock frequency 
compared to the standalone ZUC implementation. Higher power consumption for ZUC is due to its higher computational complexity. For the combined architecture HiPAcc-LTE, executing SNOW 3G is comparable in terms of energy-efficiency to executing ZUC. The combined architecture is slightly more energy-efficient compared to the standalone ZUC architecture. This is possibly due to the efficient technology mapping for ZUC-specific data-path in the combined architecture.

Typical power optimizations like clock gating and operand isolation for sequential and combinational logic respectively are attempted. This can be easily done by modifying the synthesis script to search for power optimization options based on the annotated switching activity. A minimum bit-width of 6 and maximum fan-out of 64 is set for clock gating via the synthesis option set_clock_gating_style. Adaptive mode of operand isolation is activated via the inbuilt synthesis option set_operand_isolation_style. For none of the architectures, clock gating or operand isolation could lower the power consumption. This is understandable from the fact that all the computing blocks and sequential storage cells are active in every cycle. Only a few registers, reserved for the computation of ZUC, are left out during the execution of SNOW on the combined architecture. Clearly the clock gating logic does contribute more than the power it potentially saves. Similarly for the operand isolation, the addition operations are shared between SNOW 3G and ZUC data-path in the combined architecture. This leaves no room for improving power via operand isolation.

\subsubsection{Fault Detection and Protection in HiPAcc-LTE}

Till date, no significant fault attack has been mounted on ZUC, and the best fault attack against SNOW 3G has been reported in [58]. HiPAcc-LTE provides detection and protection against this fault attack of SNOW 3G, and provide room for tolerance against future fault attacks on ZUC, if any. In [58], the authors themselves propose a method to prevent their fault attack in hardware. They have shown that if one shadows the five LFSR locations $s_{0}, s_{1}, s_{2}, s_{3}, s_{4}$ continuously, the attack becomes impossible [58, Section X]. In the hardware implementation of HiPAcc-LTE, this shadowing mechanism as implemented by keeping a buffer register of $5 \times 32=160$ bits that continuously shadows the five LFSR locations by shifting the array by one word in sync with the LFSR shift, and by recording the value of $s_{5}$ in the array during Stage 2 of the pipeline (note that this becomes the shadowed value of $s_{4}$ in Stage 3). A fault is detected in this locations by comparing the values in the LFSR with the shadowed values from the buffer array, and the keystream byte is not produced if a fault is detected.

The fault tolerance mechanism does not affect the critical path, and HiPAcc-LTE still achieves a maximum frequency of $1090 \mathrm{MHz}$. However, the area figures rise slightly, as expected. Compared to the original HiPAcc-LTE, the new area figures increase by approximately $1.5 \mathrm{KGates}$ at $1090 \mathrm{MHZ}$ in the 65nm technology, when the design is implemented using external data memory. The design automatically provides a mechanism for 160 bit shadowing for ZUC, if required, and this is where the earlier design choices for resource sharing prove to be effective. 


\subsection{Concluding Remarks}

In the context of efficient cryptographic accelerators, this work undertakes the study of optimal SRAM structures and unification of multiple cryptographic proposals. A state splitting idea is proposed that is applicable to any cipher algorithm with large internal memories in general and then applied on HC-128 in particular. The 4-way split HC-128 accelerator, with 43.20 Gbps outperforms all reported software and hardware implementations of HC-128 by a good margin. In case of HiPAcc-LTE, by a careful design of the pipeline structure and storage organization, significant advantages in terms of area as well as at least 1.5 to 2 times better throughput is achieved compared to the state-of-the-art implementations.

In particular, it is concluded that it is worth exploring the application of a unified approach towards an integrated accelerator for block ciphers and hash functions with structural similarities. These case studies can guide towards several similar hardware designs in the domain of cryptography. 


\section{Chapter 6}

\section{Study of Flexibility}

Security is an essential part of today's information systems. Modern applied cryptography in communication networks requires secure kernels that also manifest into low cost and high performance realizations. The need of better performance justify the efforts in the direction of design of high performance cryptographic accelerators dedicated to a certain cipher with little or no flexibility $[80,91,99,131,200]$. Flexibility is a desirable feature but is however, orthogonal to the performance offered by the dedicated ASICs.

Table 6.1: Security algorithms for communications standards

\begin{tabular}{|c|c|c|c|}
\hline Standard & Hash/ MAC & Stream Cipher & Block Cipher \\
\hline GSM & COMP128 & A-5/1 [33],A-5/2 [33] & A-5/3 (KASUMI) [86] \\
\hline 3GPP & - & SNOW3G [2], ZUC [232] & AES [165], KASUMI [86] \\
\hline Bluetooth & - & E0 [27] & SAFER+ \\
\hline IEEE 802.11 & - & RC4 [195] & AES [165] \\
(for WLAN) & - & & \\
\hline ISO/IEC 29192 & - & Enocoro & PRESENT [28] \\
(Light weight cryptography) & - & Trivium [55] & CLEFIA \\
\hline
\end{tabular}

\subsection{Motivation}

The need of flexibility stems from the following desirable features it manifests.

1. Flexibility provides a common implementation supporting different cryptographic operations required by a communication standard. Various communication standards recommend usage of cryptographic algorithms belonging to classes of stream ciphers, block ciphers, message authentication codes (MACs) as well as public key cryptography as shown in Table 6.1.

2. Flexibility can continuously protect against evolving cryptanalytic methods by adopting appropriate design changes. For example, a proposed countermeasure against attack on Threefish-512 requires only some additional operations [125].

3. Flexibilty to support multiple modes and versions for variable key, IV and block sizes of a cryptographic function lets the user choose a desirable performancesecurity trade-off design point. 
4. A flexible hardware implementation, incorporating various cryptographic algorithms is likely to be more area efficient, as compared to the sum of individual cores for the algorithms, due to excessive possible resource sharing.

\subsection{Contribution}

In the context of designing flexible cryptographic cores, the following two case studies are undertaken. For both of these, the motivation and viability of the flexible designs is discussed. The improvement in area efficiency of the flexible design is carefully analyzed due to resource sharing of different algorithms. The consequent degradation in the performance due to design flexibility (flexibility gap) is also benchmarked.

1. CoARX: A crypto-coprocessor, named CoARX, supporting diverse cryptographic algorithms (including block ciphers, stream ciphers, hash functions etc) based on Addition (A), Rotation ( $\mathrm{R}$ ) and eXclusive-or $(\mathrm{X})$ operations is undertaken. Compared to dedicated hardware implementations and general-purpose microprocessors, it offers excellent performance-flexibility trade-off including adaptability to resist generic cryptanalysis. The results of this work were published in $\mathrm{DAC}^{\prime} 13$ [198].

2. RC4-AccSuite: RC4-AccSuite is an ASIP designed for one of the most widelydeployed commercial stream cipher today, i.e., RC4 along with its eight other prominent variants including Spritz (CRYPTO-2014 Rump-session). It combines the flexibility of an ASIP and the performance of an ASIC with its carefullydesigned Instruction Set Architecture (ISA) reusing combinational and sequential logic at its various pipeline stages and memories, saving up to $41 \%$ in terms of area, compared to individual cores, with power budget dictated primarily by the variant used. The results of this work are published under [122].

\subsection{CoARX: A Coprocessor for ARX-based Cryptographic Algorithms}

The ARX set of operations make a functionally complete set of ingredients for the construction of any efficient cryptographic function [157]. Cryptanalysis of ARX based ciphers has been extensively reported in literature $[125,138,151,157]$ though, no major cryptanalytic breakthrough to threaten the security is till date known. As a result, diverse ARX-based algorithms made to the final rounds of recent cryptographic competitions [65,197]. 6 out of the 14 second-round candidates of NIST SHA-3 hash function competition are ARX based: Blue Midnight Wish, CubeHash, Shabal, SIMD, BLAKE and Skein, out of which two reached final round [197]. Also 2 out of 7 finalists of the eSTREAM project (Salsa20, HC-128) are ARX based [65].

The design of a generic ARX co-processor for cryptographic applications is of prime importance which, to the best of our knowledge, has not been attempted be- 
fore the current work. To begin with, five prominent ARX-based algorithms are selected. Three of those, namely, HC-128 [224], Salsa20 [21] and ChaCha [20] are stream ciphers and two, namely, BLAKE [12] and Skein [73] are hash functions. The algorithmic details of these cryptographic functions are presented in the Appendix C.1. A detailed design space exploration is performed before making each design decision. The final coprocessor implementation is synthesized with a standard cell library for obtaining performance results. The performance is benchmarked against published implementation results for each of the algorithms on dedicated hardware and flexible processors. The advantage of algorithmic view in the architecture is demonstrated with intuitive design alterations for improved resilience.

\subsubsection{Related Work}

Acceleration efforts of various ARX cryptographic algorithms are reported on various platforms such as ASICs, FPGAs, GPUs and GPPs. Inclusion of algorithm-specific customizations as instruction set extensions on 16-bit microcontrollers was also proposed for SHA-3 finalists (including Skein and BLAKE) $[42,213]$ based on their implementation studies [133]. Section 6.3.4 compares the acceleration efforts of the algorithms under consideration when mapped on GPPs, customized microcontrollers and multicore systems (GPUs and IBM cell architecture).

\subsubsection{Performance Optimized ASIC Implementations}

Due to the absence of a single VLSI implementation flexible enough to map any ARX algorithm, the implementation of individual algorithms is considered. The reported ASIC based implementations that standout in throughput are referred below.

HC-128 The only ASIC based implementation for HC-128 proposed at [38] reports a throughput of 22.88 Gbps while consuming an area of $12.65 \mathrm{kGE}$ with 21 KBytes of dual ported RAM [38] using 65nm standard cell library.

Salsa20 and ChaCha Various implementations of the two stream ciphers are presented at [100], with a reported peak throughput of 6.5 Gbps using 40 KGates of area for Salsa20 and ChaCha.

BLAKE Out of the various dedicated hardware implementations of hash function BLAKE $[12,90,99,131]$, the most noteworthy in terms of throughput comes from the authors of the original algorithm [99]. A throughput of 20 Gbps for 8G-BLAKE-512 on 90nm CMOS technology while consuming $128 \mathrm{kGE}$ area is reported.

Skein For a lightweight implementation of Skein, Kaps et al. employed resource reuse by folding 4 Mix functions into 1 and within the Mix function reused a 32-bit adder to perform 64-bit additions. The adder is also used for key injections [115]. Consequently the area is reduced but the number of clock cycles 
increase significantly when compared to other lightweight 64 bit [117] and 32 bit implementations [114].

Tillich et al. [212] reports area and throughput results of the implementation of Skein hash function using a 180nm standard cell library. Walker et al. used a 32nm standard cell technology and claimed to achieve a 5X throughput improvement over Tillich's work [217], after an appropriate scaling. More recent Skein-512-256 implementation results claim a throughput of 3 Gbps with 66 KGates on 130nm CMOS technology [90] and 6.7 Gbps with 43 KGates on 90nm CMOS technology [131].

\subsubsection{Performance Optimized FPGA Implementations}

Most of the FPGA implementations target high throughput or low area as their design goals. The reuse of functional units in various ARX based cryptographic algorithms has not be exploited for a flexible implementation. The only work that is noteworthy in terms of flexibility for ARX based Skein and BLAKE is reported by Nuray et al. [11]. Their reported FPGA implementations of these hash functions also support the primitive cipher functions on which they are based on; i.e., a unified core for BLAKE and ChaCha (a stream cipher) and one for Skein and Threefish (a tweakable block cipher). For a unified BLAKE and ChaCha coprocessor when mapped on XC6VLX75T-2 using 144 CLBs and 3 Block RAMs the throughput reported for BLAKE-32 and BLAKE64 was 288 and $255 \mathrm{Mbps}$ respectively [11]. The same coprocessor could produce keystream for 8, 12 and 20 rounds of ChaCha at 1102, 780 and $492 \mathrm{Mbps}$ respectively [11]. Skein and Threefish coprocessor consumes relatively more slices on the same device and support Skein-512-512, Skein-256-256 along with various flavors of Threefish i.e., Threefish-256, Threefish-512 and Threefish-1024 [11].

Due to the absence of any other flexible implementation, this Section summarizes the reported individual FPGA implementations of the 5 ARX based cryptographic algorithms undertaken in CoARX. A comparison of these implementations in terms of throughput and resource utilization of the target device is done in Table 6.2.

HC-128 Since HC-128 belongs to software profile of eSTREAM finalists, no FPGA implementations are being reported for it so far.

Salsa20 For Salsa20, the earliest FPGA implementation results were reported by Junjie et al. [229]. They proposed a compact hardware implementation of Salsa20 comprising of a single QuarterRound function block. The global clock was 250 $\mathrm{MHz}$, and for the QuarterRound block it was $125 \mathrm{MHz}$. Gaj et al. also implemented salsa20 using a single QuarterRound architecture in combinational logic and using eight clock cycles to implement the entire DoubleRound [3]. Evaluation of various possible architectures for sasla20 namely Salsa20-dr (unrolled DoubleRound iterative architecture), Salsa20-sr (single round iterative architecture), Salsa20-qr (QuarterRound resource shared iterative architecture) were undertaken for implementation on an Altera device [187]. These three possibilities 
Table 6.2: FPGA based Implementations of ARX Algorithms

\begin{tabular}{|c|c|c|c|c|c|}
\hline Reference & $\begin{array}{c}\text { FPGA } \\
\text { Device }\end{array}$ & $\begin{array}{c}\text { Area } \\
\text { (CLB / LE) }\end{array}$ & $\begin{array}{c}\text { Block } \\
\text { RAMs }\end{array}$ & $\begin{array}{c}\text { Op. Freq. } \\
\text { (MHz) }\end{array}$ & $\begin{array}{c}\text { Throughput } \\
(\mathrm{Mbps})\end{array}$ \\
\hline Salsa20-sr [229] & Xilinx Virtex-II 2V250fg256 & 194 CLB slices & 4 & 250 & 38 \\
\hline Salsa20-qr [187] & Altera Cyclone EP1C20F400C6 & 2356 LE & - & 55 & 343 \\
\hline Salsa20-sr [187] & Altera Cyclone EP1C20F400C7 & 3400 LE & - & 40 & 931 \\
\hline Salsa20-dr [187] & Altera Cyclone EP1C20F400C8 & 3510 LE & - & 30 & 1280 \\
\hline Salsa20[3] & Xilinx Spartan 3 xc3s50pq208-5 & 1615 CLB & - & 23.5 & 213 \\
\hline \hline ChaCha_config1 [11] & Xilinx Virtex-6 XC6VLX75T-2 & 49 CLB & 2 & 362 & $595,422,266$ \\
\hline ChaCha_config2 [11] & Xilinx Virtex-6 XC6VLX75T-2 & 77CLB & 2 & 316 & $520,368,232$ \\
\hline ChaCha_config2 [11] & Xilinx Virtex-6 XC6VLX75T-2 & 77CLB & 2 & 345 & $569,403,254$ \\
\hline \hline BLAKE-32 [24] & Xilinx Virtex-3 xc3s50-5 & 124 CLB & 2 & 190 & 115 \\
\hline BLAKE-32 [115] & Xilinx Virtex-3 xc3s50-5 & 360 CLB & 2 & 135 & 315 \\
\hline BLAKE-64 [117] & Xilinx Virtex-6 xc6vlx75t-1 & 117 CLB & - & 274 & 105 \\
\hline BLAKE-64 [115] & Xilinx Virtex-6 xc6vlx75t-1 & 146 CLB & 1 & 189 & 277 \\
\hline BLAKE-64 [24] & Xilinx Virtex-5 xc5vlx50-2 & 108 CLB & 3 & 358 & 314 \\
\hline \hline Skein [117] & Xilinx Virtex-6 xc6vlx75t-1 & 240 CLB & - & 160 & 179 \\
\hline Skein [115] & Xilinx Virtex-6 xc6vlx75t-1 & 162 CLB & 1 & 166 & 34.9 \\
\hline Skein [114] & Xilinx Virtex-5 xc5v & 555 CLB & - & 271 & 237 \\
\hline Skein [137] & Xilinx Virtex-5 xc5v1x110-3 & 821 & not specified & 119 & 1610 \\
\hline
\end{tabular}

provide various design points in area-performance trade-off as specified in Table 6.2 .

ChaCha ChaCha has not been separately undertaken for an FPGA implementation. Since ChaCha makes the building block of BLAKE hash function, a combined lightweight coprocessor is designed for BLAKE and ChaCha using deep pipelining for high clock frequency [11]. Various piepline configurations of ChaCha are reported for its 8, 12 and 20 round variants as specified in Table 6.2.

BLAKE Kaps et al. presented a lightweight implementation of SHA-3 finalists, including Skein and BLAKE on various FPGAs [115]. The initial state is stored in a four way distributed RAM for ease in access by the 1/2 G-function implemented. For BLAKE-32, the achieved area efficiency is comparable to the work by Beuchat et al. [24]. For BLAKE-64, the area efficiency of the design proposed by Kaps et al. [115] is more than twice than the one reported by Kerckhof et al. [117] on the same FPGA device, due to the use of a 32 bit data width instead of 64 in the former.

Skein For a lightweight implementation of Skein, Kaps et al. employed resource reuse by folding 4 Mix functions into 1 and within the Mix function reused a 32-bit adder to perform 64-bit additions. The adder is also used for key injections [115]. Consequently the area is reduced but the number of clock cycles increase significantly when compared to other lightweight 64 bit [117] and 32 bit implementations [114]. 


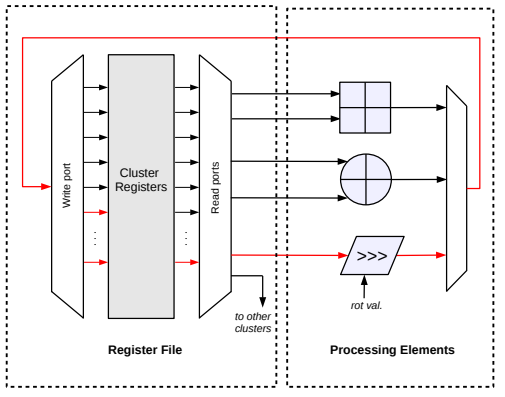

$P E_{1}$
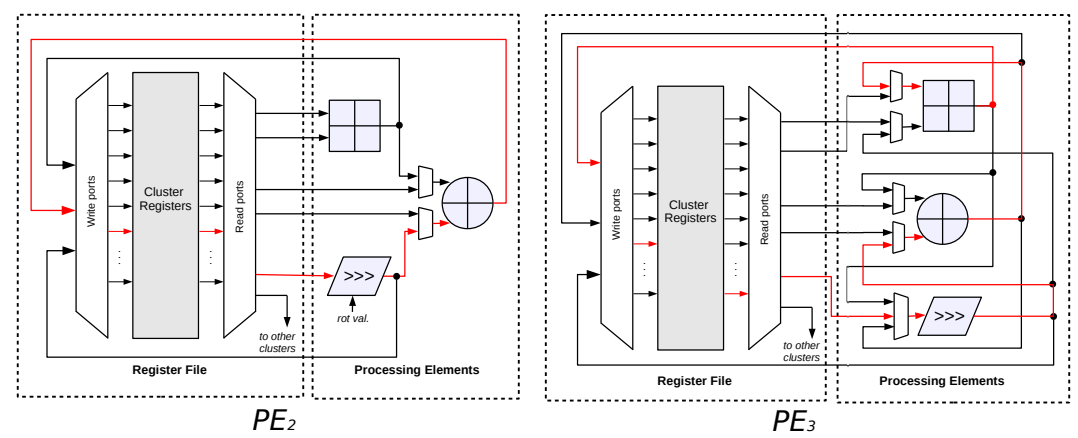

$P E_{3}$

Figure 6.1: $P E_{1}, P E_{2}$ and $P E_{3}$ (left to right). Processing Elements with Different Functional Unit Arrangements, possible single cycle operations in $P E_{1}$ are $A, R, X$, in $P E_{2}$ are $A X, R X$ and in $P E_{3}$ are $A R, R A, X A, X R, A R X$, AXRR, XA, RAX, XAR, XRA

\subsubsection{Design Space Exploration}

The microarchitecture of CoARX is decided by a simple observation that, all ARXbased algorithms perform a mix of addition $(A)$, rotation $(R)$ and XOR $(X)$ operations in different order in each round of the algorithm. Each of the stream ciphers HC-128, Salsa20 and ChaCha has an internal state that is randomized by a secret key and at every round of keystream generation the state is updated using ARX operations. The hash functions BLAKE and Skein compress the input message into fixed length output string and the operation again happens through several iterations each involving the basic ARX operations. For detailed description of the algorithms, one may refer to [12, 20,21,73,224] (A summary of the algorithms is provided in the Appendix C.1). One should note that the order of ARX operations, the number of rounds, the word-lengths of variables that are processed vary from one algorithm to another and hence poses a difficult challenge for generic architecture design.

To have the maximum flexibility and performance, the design need to support $3^{3}$ different functional units for the triple operations. The same flexibility can be obtained from a reconfigurable architecture with these three operators. The algorithms also exhibit data-oriented computing pattern thereby, justifying the selection of CoarseGrained Reconfigurable Architecture (CGRA) [59]. CGRAs can be implemented with wide range of design choices. In the following the rationale of specific CGRA design choices are elaborated, considering all the structural elements in bottom up fashion.

Functional Units Arrangement: Any number of functional units $(A, R$ and $X)$ may be arranged in any order inside a Processing Element (PE). Three architectures are considered, showing an increasing trend of complexity and efficiency, allowing up to one, two or three operations per cycle as shown in Fig. 6.1. They were modeled using a high-level synthesis environment and synthesized with Synopsys Design Compiler using $90 \mathrm{~nm}$ technology with maximum achievable clock frequency as reported in the Table 6.3.

$P E_{1}$ allows one operation per cycle and consequently, requires a single write port to the register file. The second architecture, $P E_{2}$ is designed to exploit the fact that a 
Table 6.3: Estimated Performance and Resource Utilization Results

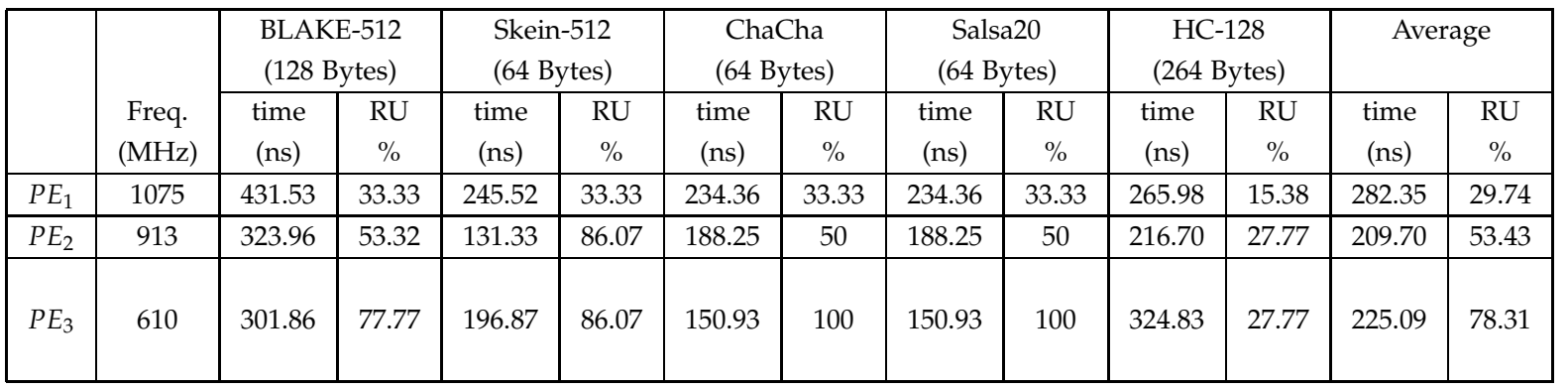

64-bit $A$ and $R$ blocks in hardware contribute almost equally to the critical path while the contribution of $X$ is significantly less. Hence the decrease in the clock frequency, compared to $P E_{1}$ is not significant. All the three units may be used separately, like $P E_{1}$, or in a non-overlapping simultaneous combination, e.g., $A X$ and $R$ or $A$ and $R X . P E_{3}$ is more flexible, allowing all possible non overlapping combinations of one, two and three operations in a single cycle, e.g., $A R$ and $X$ or $A X R$ or $X R$ and $A$. The flexibility is achieved at the expense complex interconnects and a layer of multiplexers before each FU. Consequently, $P E_{3}$ has the least operating frequency compared to other architectures.

The round operations of each of the algorithms are mapped to the three architectures considered and cycle count and functional units used per cycle are calculated. For facilitating the critical choice amongst these architectures the following metrics are evaluated.

- Computational time is calculated from the clock frequency and the number of cycles for each algorithm.

- Resource Utilization (RU) gives the average utilization of functional units in the cluster grid. It is computed as,

$$
R U_{\text {algo }}=\sum_{i=0}^{N} \sum_{j=0}^{C} \sum_{k=0}^{M} \frac{U_{i j k}}{(N * C * M)}
$$

Where $C$ is the number of clusters, $M$ is the number of FUs inside a cluster ( 3 in $\left.P E_{2}\right)$ and $N$ is the total number of cycles. $U_{i j k}=1$ if the $k^{t h} \mathrm{FU}$ in $j^{\text {th }}$ cluster is participating in the $i^{\text {th }}$ cycle of the algorithm, otherwise it is 0 .

The RU of $P E_{1}$ is worst since it can not exceed $33.3 \%$ and only one operation can be performed in a single cycle. Consequently the mapping of ARX algorithms requires a large number of instructions. On the other extreme, $P E_{3}$ shows the best RU figures. However, its multiplexers and read/write ports affect the critical path, resulting in relatively worse execution time compared to $P E_{2}$ in few cases as given in Table 6.3. Furthermore, the large configurability options of $P E_{3}$ requires a large configuration memory word. $P E_{2}$ is a compromise of complexity and flexibility. Due 


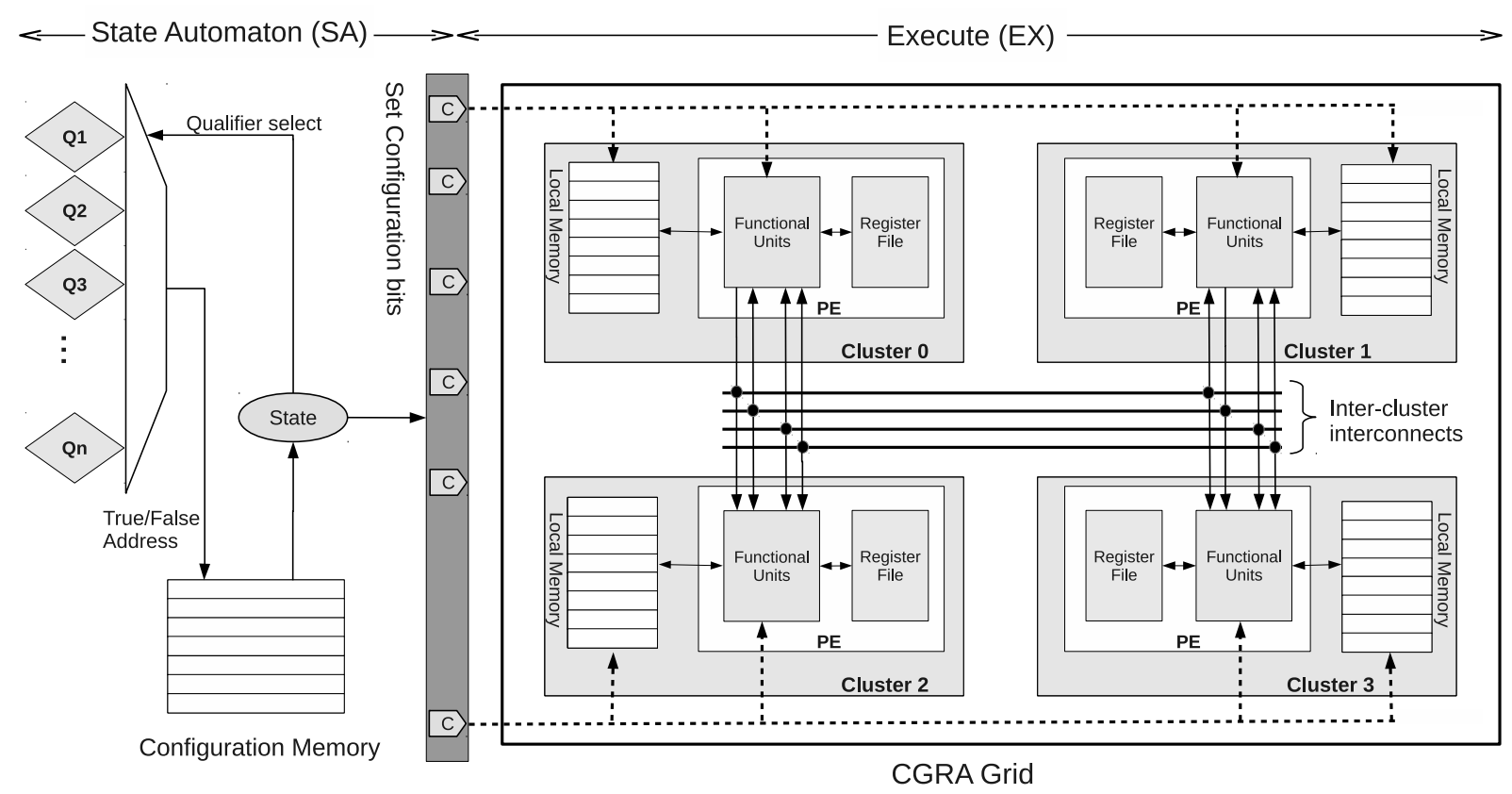

Figure 6.2: Block Diagram of CoARX

to its reasonable RU and computational time comparable to that of $P E_{3}$, The $P E_{2}$ is picked to be the design choice. Note that, the choice of number of registers is also influenced by the choice of FU arrangement, i.e., more intermediate storage is needed for $P E_{1}$ than for $P E_{3}$ or for $P E_{2}$.

Register File and Read/Write ports: The FUs as well as the local registers of clusters are 64-bit wide to support double-word ARX algorithms (Skein and BLAKE64), however, mapping of 32-bit algorithms (HC-128, Salsa20, ChaCha) remains trivial. The number of local registers is dictated by BLAKE, which requires 6 registers for the computation of its core function.

Number of Clusters: The choice of number of clusters inside a CGRA influences the throughput and resource utilization, resulting in resource under-utilization when number of clusters is too large and low parallelism and consequently low throughput when number of clusters is too small. Among the target ARX algorithms, BLAKE, Salsa20 and ChaCha consist of up to 4 parallel executions of their core operation. Flavors of Skein can have 2-8 parallel ARX operations, whereas for HC-128, 3 parallel ARX operations can take place. Hence a 4 cluster grid $(2 \times 2)$ is chosen as a trade-off between throughput and resource utilization.

Memory Hierarchy: Inside each cluster, a 2-level memory hierarchy is maintained by a small register file and a large SRAM. The memory holds the plaintext, ciphertext, message hash, Initialization Vector and Key. This dual-ported SRAM has 64 bit word length and has 1024 elements. Its size is dictated by HC-128, that requires two secret S-Boxes, each with 512 elements. Out of the 6 registers in register file, two namely $m 0, m 1$ hold the value to be written or read from port 0 and port 1 of the 


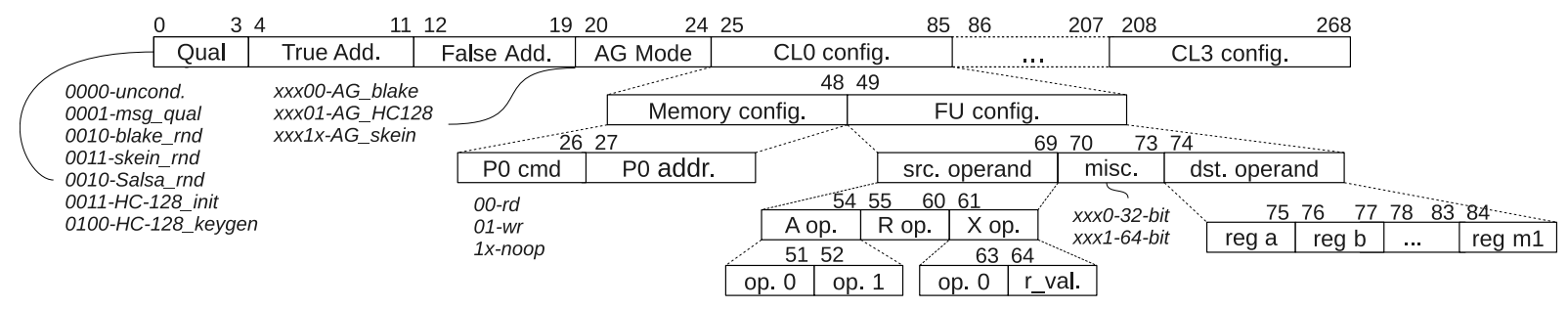

Figure 6.3: Configuration Word for CoARX processor

memory, respectively. Movement of memory values to any other register incurs one cycle latency.

Inter Cluster Interconnects: The number and nature of interconnects between clusters is influenced by the permutation of ARX operations in the subsequent rounds of ARX algorithms. For BLAKE, Salsa and ChaCha each cluster calculates one core function (referred as $G$ function in the algorithms). At the completion of $G$ functions on the columns, these algorithms proceed diagonally for which each cluster requires exactly one value from every other cluster. Consequently, a MESH style interconnect is chosen with 3 incoming and 3 outgoing register values from each cluster. For algorithms requiring more permutation and data exchange between clusters, data availability is ensured by one cycle overhead.

The top-level architecture of the final CGRA-based ARX coprocessor is presented in Fig. 6.2. The design is distributed over two pipeline stages. State Automaton stage fetches the instruction from the configuration memory. The instruction is organized as a 4-tuple. A Qualifier, a True address and a False address are the three fields, which handle looping and conditional jumps during the execution. The rest of the instruction are the configuration bits for the CGRA that control the functionality of the clusters in the Execute stage.

\subsubsection{Mapping of the ARX Algorithms}

On the proposed CGRA coprocessor, any ARX-based cryptographic algorithm can be mapped. The reconfigurability is controlled by the instructions in the configuration memory. The configuration of the entire CGRA has 269-bit word length and constitutes 46 words. Configuration words are manually written and are updated to perform a different combination of ARX operation every cycle. For switching the current algorithm running on the CGRA, the configuration memory is updated. The required inputs for each algorithm are filled into the local memories of each cluster before kick starting the algorithm. Fig. 6.3 shows a breakdown of a configuration word and a discussion of various fields follows.

Qualifier Control: The iterative nature of the cipher algorithms is handled by a 4-bit Qualifier. The selection of next address to be True address or False address is determined by the qualifier condition. For conditional jumps (e.g., evaluating number of rounds, message/ block size), if the condition is evaluated true then a jump is 
made to the 8-bit true address or else to the false address. An unconditional jump is evaluated always as the qualifier being true.

Cluster Configuration: The bit fields in the instruction word of configuration memory are used to configure the input multiplexers and the processing elements of the entire CGRA. Each cluster requires 61 bits of configuration word. The dual ported local memory in each cluster requires a 2-bit cmd and a 10 bit address for 1024 word memory to be written and read from. Register $m 0$ and $m 1$ are tied to communicate with port 0 and port 1 of the memory, respectively. FU config. includes the specification of source and destination registers of the functional units namely $A, R$ and $X$.

- misc. bits: specify if the operation is 32-bit or 64-bit wide. Future extension may include specifying Rotator FU being used as a shifter or not.

- src. operands: The multiplexers for each of the two operands of $A$ and $X$ are $8 \times 1$, $R$ requires only one operand and a 6-bit rotation value. The rotation value and direction of the 64 bit operand is specified by a 6-bit $r \_v a l$ field. The operands may include registers from other clusters as well.

- dst. operand: For each of the 6 registers in the register file, selection lines for write demultiplexers requires 2-bits. The registers may be updated by the Functional Units output or simply bypassed values.

\subsubsection{Algorithm Specific Modes}

Some ARX algorithms require specific modes to be indicated in configuration word to carry out non-ARX operations.

BLAKE Address Generation: For BLAKE, the addresses for loading $m s g$ and constants from memory, are not static and they change in each round. One solution for implementation of this scenario is direct loading of data memory in a cluster by $m s g$ and constants during initialization phase. But this approach renders the system vulnerable to side channel attacks. Instead a specific address generation mode is defined to carry out BLAKE address generation.

HC-128 Address Generation: Some operations in HC-128 keystream generation are not strictly ARX. They are modulo-512 subtraction, byte processing in $h_{1}$ and $h_{2}$ functions and counter increment/addition and shift operations in the expansion step of initialization. To cater them, specific hardware blocks are being used.

Skein Key Generation: Skein requires injecting a subkey into the states by adding it with the outputs of MIX functions every fourth round. Skein key generation mode enables hardware block skein subkey generator that is implemented in hardware as shift registers, along with adders and is capable of generating one subkey in a single cycle.

\subsubsection{Mapping BLAKE64 on CoARX}

BLAKE round is comprised of eight $G$ functions and four of them can be executed in parallel on each of CoARX cluster. The internal data path of different variants of BLAKE is either 32 or 64-bit wide, here BLAKE64 mapping is considered that requires 64 bit data path. Fig. 6.4 shows the arrangement of input values in the local memory 


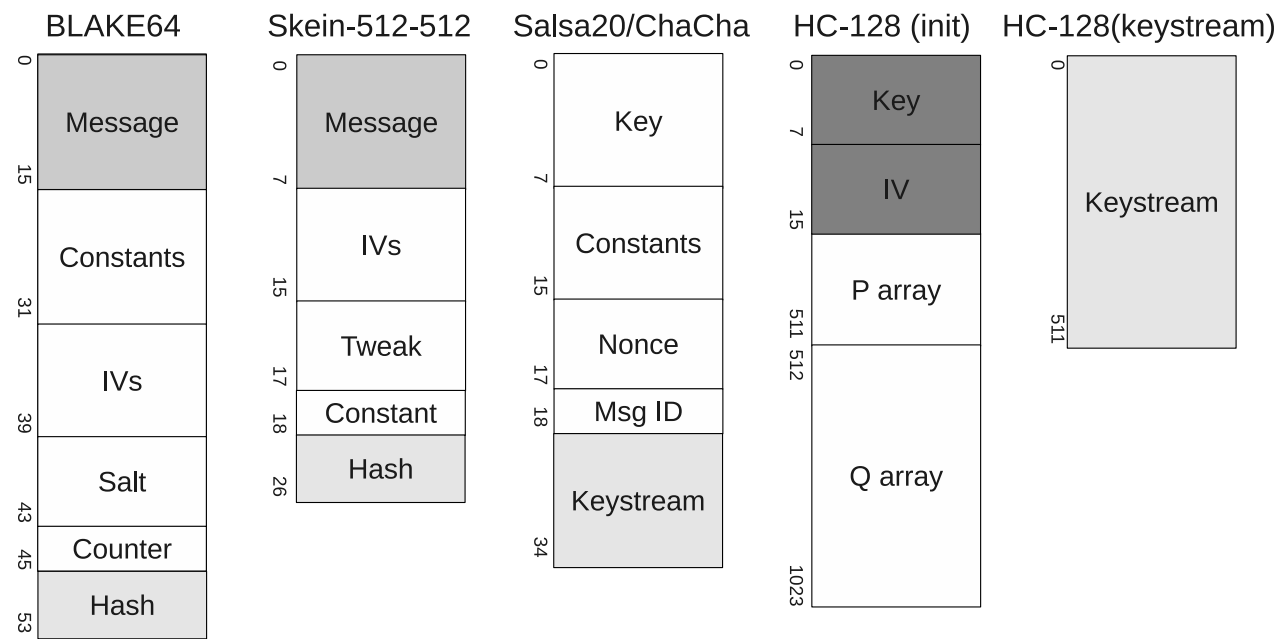

Figure 6.4: Memory map for various ARX algorithms

\begin{tabular}{|c|c|c|c|c|}
\hline Cycle & Cluster0 & Cluster1 & Cluster2 & Cluster3 \\
\hline 1 & $\begin{array}{l}\text { Load IV0 } \Rightarrow>\text { reg m1 } 0 \\
\text { Load IV4 } \Rightarrow \text { reg m2_0 }\end{array}$ & $\begin{array}{l}\text { Load IV1 => reg m1__1 } \\
\text { Load IV5 }=>\text { reg m2_1 }\end{array}$ & $\begin{array}{l}\text { Load IV2 } \Rightarrow>\text { reg m1_2 } \\
\text { Load IV6 }=>\text { reg m2_2 }\end{array}$ & $\begin{array}{l}\text { Load IV3 }=>\text { reg m1_3 } \\
\text { Load IV7 }=>\text { reg m2_3 }\end{array}$ \\
\hline 2 & $\begin{array}{l}\mathrm{m} 1 \_0=>\text { reg a0 } \\
\mathrm{m} 2 \_0=>\text { reg b0 }\end{array}$ & $\begin{array}{l}\text { m1_1 }=>\text { reg a1 } \\
\text { m2_1 => reg b1 }\end{array}$ & $\begin{array}{l}\mathrm{m} 1 \_2 \Rightarrow>\text { reg a2 } \\
\mathrm{m} 2 \_2=>\text { reg b2 }\end{array}$ & $\begin{array}{l}\mathrm{m} 1 \_3=>\text { reg a3 } \\
\mathrm{m} 2 \_3=>\text { reg b3 }\end{array}$ \\
\hline 3 & $\begin{array}{l}\text { Load } S 0=>\text { reg } m 1 \_0 \\
\text { Load } C 0=>\text { reg m2_0 }\end{array}$ & $\begin{array}{l}\text { Load } S 1=>\text { reg m1_1 } \\
\text { Load } C 1=>\text { reg m2_1 }\end{array}$ & $\begin{array}{l}\text { Load IVO }=>\text { reg m1_2 } \\
\text { Load IV0 }=>\text { reg m2_2 }\end{array}$ & $\begin{array}{l}\text { Load IVO }=>\text { reg m1_3 } \\
\text { Load IVO }=>\text { reg m2_3 }\end{array}$ \\
\hline 4 & $\begin{array}{l}\mathrm{m} 1 \_0 \text { xor } \mathrm{m} 2 \_0=>\mathrm{c} 0 \\
\text { Load T0 }=>\mathrm{m} 1 \_0 \\
\text { Load C4 }=>\mathrm{m} 2 \_0\end{array}$ & $\begin{array}{l}\text { m1_1 xor m2_1 }=>c 1 \\
\text { Load T1 }=>\text { m1_1 } \\
\text { Load C5 }=>\text { m2_1 }\end{array}$ & $\begin{array}{l}\mathrm{m} 1 \_2 \text { xor m2_2 }=>\mathrm{c} 2 \\
\text { Load T2 }=>\mathrm{m} 1 \_2 \\
\text { Load C6 }=>\mathrm{m} 2 \_2\end{array}$ & $\begin{array}{l}\text { m1_3 xor m2_3 }=>c 3 \\
\text { Load T3 }=>\text { m1_3 } \\
\text { Load C7 }=>\text { m2_3 }\end{array}$ \\
\hline 5 & $\begin{array}{l}\mathrm{m} 1 \text { o xor } \mathrm{m} 2 \_0 \Rightarrow \mathrm{d} 0 \\
\text { BLAKE add gen mode:on } \\
\text { Load } \mathrm{msg}=>\mathrm{m} 1{ }^{0} 0 \\
\text { Load const }=>\mathrm{m} \overline{2} 0\end{array}$ & $\begin{array}{l}m 1 \_1 \text { xor } m 2 \_1=>d 1 \\
\text { BLAKE add gen mode:on } \\
\text { Load } m s g=>m 1{ }^{1} \\
\text { Load const }=>m^{2} 1\end{array}$ & $\begin{array}{l}m 12 \text { xor } \mathrm{m}_{2} 22=>\mathrm{d} 2 \\
\text { BLAKE add gen mode:on } \\
\text { Load } \mathrm{msg}=>\mathrm{m} 1{ }^{2} \\
\text { Load const }=>\mathrm{m}^{2} 2\end{array}$ & $\begin{array}{l}\mathrm{m} 13 \text { xor } \mathrm{m} 2 \_3=>\mathrm{d} 3 \\
\text { BLAKE add gen mode:on } \\
\text { Load msg }=>\mathrm{m} 1{ }^{3} \\
\text { Load const }=>\mathrm{m} 23\end{array}$ \\
\hline
\end{tabular}

Figure 6.5: Description of mapping of BLAKE Initialization

for BLAKE that includes the chaining value, message, salt counter and constants. The output hash value is also written back to the local cluster data memory and this data memory acts as a output buffer.

Initialization: BLAKE initialization requires five cycles, the details of which is given in Fig. 6.5. In the initialization phase of the algorithm a $4 \times 4$ matrix is initialized. The first eight locations (row wise) of this matrix are filled with the chaining value $h$ or the initial value(IV) in the beginning. The next four locations are filled with the result of the exclusive or operation between constants $c$ and salt $s$ and similarly last four values with the result of the exclusive or operation between constants $c$ and counter $t$. To perform the above mentioned operations, chaining values, constants and salt need to be loaded from the local data memory to the registers for further processing. The memory registers $m 1$ and $m 2$ of each cluster are loaded with corresponding salt, counter or constant values and then they exclusive or operation is performed and 


\begin{tabular}{|c|c|c|c|c|}
\hline Cycle & Cluster0 & Cluster1 & Cluster2 & Cluster3 \\
\hline 1 & $\begin{array}{l}\mathrm{a} 0+\mathrm{b} 0=>\mathrm{a} 0 \\
\mathrm{~m} 1 \_0 \text { xor } \mathrm{m} 2 \_0=>\mathrm{m} 1 \_0\end{array}$ & $\begin{array}{l}a 1+b 1=>\text { a1 } \\
m 1 \_1 \text { xor m2_1 }=>\text { m1_1 }\end{array}$ & $\begin{array}{l}a 2+b 2=>a 2 \\
m 1 \_2 \text { xor m2_2 } \Rightarrow \text { m1_2 }\end{array}$ & $\begin{array}{l}\mathrm{a} 3+\mathrm{b} 3=>\mathrm{a} 3 \\
\mathrm{~m} 1 \_3 \text { xor m2_3 } \Rightarrow>\text { m1_3 }\end{array}$ \\
\hline 2 & $\begin{array}{l}\mathrm{a} 0+\mathrm{m} 1 \_0=>\mathrm{a} 0 \\
\text { adder0 xor d } 0 \Rightarrow \mathrm{d} 0\end{array}$ & $\begin{array}{l}\text { a1 }+\mathrm{m} 1 \_1=>\text { a1 } \\
\text { adder1 xor d1 } \Rightarrow \text { d } 1\end{array}$ & $\begin{array}{l}\mathrm{a} 2+\mathrm{m} 1 \_2=>\mathrm{a} 2 \\
\text { adder2 } 2 \text { xor } \mathrm{d} 2=>\mathrm{d} 2\end{array}$ & $\begin{array}{l}\text { a3 }+\mathrm{m} 1 \_3=>\text { a3 } \\
\text { adder3 xor } \mathrm{d} 3=>\mathrm{d} 33\end{array}$ \\
\hline 3 & (Rotate d0 by 32$)=>d 0$ & (Rotate $\mathrm{d} 1$ by 32$)=>\mathrm{d} 1$ & (Rotate $\mathrm{d} 2$ by 32$)=>\mathrm{d} 2$ & $($ Rotate $\mathrm{d} 3$ by 32$)=>\mathrm{d} 3$ \\
\hline 4 & $\begin{array}{l}\mathrm{c} 0+\mathrm{d} 0=>\mathrm{c} 0 \\
\text { adder0 xor b0 }=>\text { b0 }\end{array}$ & $\begin{array}{l}\mathrm{c} 1+\mathrm{d} 1=>\mathrm{c} 1 \\
\text { adder1 xor } \mathrm{b} 1=>\mathrm{b} 1\end{array}$ & $\begin{array}{l}\mathrm{c} 2+\mathrm{d} 2=>\mathrm{c} 2 \\
\text { adder2 } x \text { or } \mathrm{b} 2=>\mathrm{b} 2\end{array}$ & $\begin{array}{l}c 3+d 3 \Rightarrow c 3 \\
\text { adder3 xor b3 }=>b 3\end{array}$ \\
\hline 5 & $\begin{array}{l}\text { (Rotate } \mathrm{d} 0 \text { by } 25)=>\mathrm{d} 0 \\
\text { Load } \mathrm{msg}=>\mathrm{m} 1{ }^{0} 0 \\
\text { Load const }=>\mathrm{m} 2 \mathrm{O}\end{array}$ & $\begin{array}{l}\text { (Rotate } \mathrm{d} 1 \text { by } 25)=>\mathrm{d} 1 \\
\text { Load } \mathrm{msg}=>\mathrm{m} 1{ }_{1}^{1} \\
\text { Load const }=>\mathrm{m} 2 \_1\end{array}$ & $\begin{array}{l}\text { (Rotate } \mathrm{d} 2 \text { by } 25)=>\mathrm{d} 2 \\
\text { Load } \mathrm{msg}=>\mathrm{m} 1 \_2 \\
\text { Load const }=>\mathrm{m} 2 \_2\end{array}$ & $\begin{array}{l}\text { (Rotate } \mathrm{d} 3 \text { by } 25)=>\mathrm{d} 3 \\
\text { Load } \mathrm{msg}=>\mathrm{m} 1 \_3 \\
\text { Load const }=>\mathrm{m} 2 \_3\end{array}$ \\
\hline 6 & $\begin{array}{l}\mathrm{a} 0+\mathrm{b} 0=>\mathrm{a} 0 \\
\mathrm{~m} 1 \_0 \text { xor } \mathrm{m} 2 \_0=>\mathrm{m} 1 \_0\end{array}$ & $\begin{array}{l}\mathrm{a} 1+\mathrm{b} 1=>\mathrm{a} 1 \\
\mathrm{~m} 1 \_1 \text { xor } 2 \text { 2_1 => m1_1 }\end{array}$ & $\begin{array}{l}\mathrm{a} 2+\mathrm{b} 2=>\mathrm{a} 2 \\
\mathrm{~m} 1 \_2 \text { xor } 2 \text { 2_2 } \Rightarrow>\mathrm{m} 1 \_2\end{array}$ & $\begin{array}{l}a 3+b 3=>a 3 \\
\text { m1_3 xor } m 2 \_3 \Rightarrow \text { m1_3 }\end{array}$ \\
\hline 7 & $\begin{array}{l}\mathrm{a} 0+\mathrm{m} 1 \_0 \Rightarrow \mathrm{a} 0 \\
\text { adder } 0 \text { xor } \mathrm{d} 0=>\mathrm{d} 0\end{array}$ & $\begin{array}{l}\text { a1 } 1+\mathrm{m} 1 \_1 \Rightarrow \text { a } 1 \\
\text { adder1 xor } \mathrm{d} 1=>\mathrm{d} 11\end{array}$ & $\begin{array}{l}\mathrm{a} 2+\mathrm{m} 1 \_2 \Rightarrow>\mathrm{a} 2 \\
\text { adder2 } 2 \text { xor } \mathrm{d} 2=>\mathrm{d} 2\end{array}$ & $\begin{array}{l}\text { a3 }+\mathrm{m} 1 \_3 \Rightarrow>\text { a } 3 \\
\text { adder } 3 \text { xor } \mathrm{d} 3=>\mathrm{d} 3\end{array}$ \\
\hline 8 & (Rotate d0 by 16$)=>d 0$ & (Rotate $\mathrm{d} 1$ by 16$)=>\mathrm{d} 1$ & (Rotate $\mathrm{d} 2$ by 16$)=>\mathrm{d} 2$ & (Rotate $\mathrm{d} 3$ by 16$)=>\mathrm{d} 3$ \\
\hline 9 & $\begin{array}{l}\mathrm{c} 0+\mathrm{d} 0 \Rightarrow \mathrm{c} 0 \\
\text { adder } 0 \text { xor } \mathrm{b} 0=>\mathrm{b} 0\end{array}$ & $\begin{array}{l}\mathrm{c} 1+\mathrm{d} 1=>\mathrm{c} 1 \\
\text { adder1 xor } \mathrm{b} 1=>\mathrm{b} 1\end{array}$ & $\begin{array}{l}c 2+d 2 \Rightarrow>c 2 \\
\text { adder2 } x \text { or b2 } \Rightarrow>b 2\end{array}$ & $\begin{array}{l}c 3+d 3 \Rightarrow>c 3 \\
\text { adder3 xor b3 }=>b 3\end{array}$ \\
\hline 10 & $\begin{array}{l}\text { (Rotate } \mathrm{d} 0 \text { by } 11)=>\mathrm{d} 0 \\
\text { Load } \mathrm{msg}=>\mathrm{m} 1 \_0 \\
\text { Load const }=>\mathrm{m} 2 \text { _0 }\end{array}$ & $\begin{array}{l}\text { (Rotate } \mathrm{d} 1 \text { by } 11)=>\mathrm{d} 1 \\
\text { Load } \mathrm{msg}=>\mathrm{m} 1 \_1 \\
\text { Load const }=>\mathrm{m} 2 \_1\end{array}$ & $\begin{array}{l}\text { (Rotate } \mathrm{d} 2 \text { by } 11)=>\mathrm{d} 2 \\
\text { Load } \mathrm{msg}=>\mathrm{m} 1 \_2 \\
\text { Load const }=>\mathrm{m} 2{ }^{2} 2\end{array}$ & $\begin{array}{l}\text { (Rotate } \mathrm{d} 3 \text { by } 11)=>\mathrm{d} 3 \\
\text { Load } \mathrm{msg}=>\mathrm{m} 1 \_3 \\
\text { Load const }=>\mathrm{m} 2 \_3\end{array}$ \\
\hline
\end{tabular}

Figure 6.6: Description of mapping of BLAKE single Round function

\begin{tabular}{|c|c|c|c|c|}
\hline Cycle & Cluster0 & Cluster1 & Cluster2 & Cluster3 \\
\hline 1 & $\begin{array}{l}\text { Load } \mathrm{H} 0=>\text { reg m1_0 } \\
\text { Load } \mathrm{S} 0=>\text { reg m2_0 }\end{array}$ & $\begin{array}{l}\text { Load } \mathrm{H} 1=>\text { reg m1_1 } \\
\text { Load } \mathrm{S} 1 \Rightarrow>\text { reg m2_1 }\end{array}$ & $\begin{array}{l}\text { Load H2 }=>\text { reg m1_2 } \\
\text { Load S2 } \Rightarrow>\text { reg m2_2 }\end{array}$ & $\begin{array}{l}\text { Load H3 }=>\text { reg m1_3 } \\
\text { Load S3 } \Rightarrow>\text { reg m2_3 }\end{array}$ \\
\hline 2 & $\mathrm{a} 0$ xor $\mathrm{b} 0=>\mathrm{a} 0$ & $\mathrm{a} 1$ xor b1 => a1 & $a 2$ xor b2 => a2 & $a 3$ xor b3 => a3 \\
\hline 3 & $\mathrm{a} 0$ xor $\mathrm{m} 2 \_0 \Rightarrow \mathrm{m} 2 \_0$ & a1 xor m2_1 => m2_1 & a2 xor m2_2 => m2_2 & a3 xor m2_3 => m3_3 \\
\hline 4 & $\mathrm{c} 0$ xor $\mathrm{d} 0=>\mathrm{d} 0$ & $\mathrm{c} 1$ xor $\mathrm{d} 1=>\mathrm{d} 1$ & c2 $x$ or $d 2=>d 2$ & c3 $x$ or d3 $=>$ d3 \\
\hline 5 & $\begin{array}{l}\text { d0 xor } \mathrm{msg} 0=>\mathrm{c0} \\
\text { Store h0 } \\
\text { Load H4 }=>\mathrm{m} 2 \_0\end{array}$ & $\begin{array}{l}\text { d1 xor msg1 => c1 } \\
\text { Store h1 } \\
\text { Load H5 => m2_1 }\end{array}$ & $\begin{array}{l}\text { d2 xor msg2 => c2 } \\
\text { Store h2 } \\
\text { Load H6 => m2_2 }\end{array}$ & $\begin{array}{l}\text { d3 xor msg3 => c3 } \\
\text { Store h3 } \\
\text { Load H7 => m2_3 }\end{array}$ \\
\hline 6 & c0 xor m2_0 => m2_0 & c1 xor m2_1 => m2_1 & c2 xor m2_2 => m2_2 & c3 xor m2_3 => m2_3 \\
\hline 7 & nop & nop & nop & nop \\
\hline 8 & Store $\mathrm{h} 4$ & Store h5 & Store h6 & Store h7 \\
\hline
\end{tabular}

Figure 6.7: Description of mapping of BLAKE finalization

the results are stored in the local registers $a, b, c$ and $d$ of each cluster. In total, the initialization function of BLAKE is composed of eight XOR operations. 
Round Calculation: One G function is mapped on each cluster, which takes 10 cycles. It takes 20 cycles to perform a double round ( $2 x$ G-functions) and one extra cycle is needed at the end while performing branch and per mutating the input values. In total 21 cycles are required to perform a complete round. A single G function calculation detail is shown in Fig. 6.6.

Finalization: The finalization requires the chaining value $h$, salt $s$, and the outputs of the round function. The finalization is composed of 24 exclusive or operations, to get the output as eight words, each of 32 or 64 bits. In the finalization phase the initial chaining values, the salt and the results from the round function are XOR-ed together to produce the final output. The details of the first five cycles of the finalization process are shown in Fig. 6.7.

In summary, initialization of BLAKE64 takes 5 cycles, round calculation 21 and finalization 9. So for BLAKE512 with 16 rounds a total of 350 cycles were required.

\subsubsection{Implementation and Benchmarking}

The proposed architecture CGRA is developed using Processor Designer (Section 2.3.2) and synthesized using Faraday 90nm CMOS technology library (Section 2.3.1). LISA processor description ( $5 \mathrm{~K}$ lines of code) was processed by Synopsys Processor Designer to generate Verilog RTL (38K lines of code). The synthesis of HDL results are presented in Table 6.4. Each of the four clusters have 8 KBytes of dual ported SRAM as local memory. Performance of different ARX algorithms on CoARX is presented in Table 6.5 .

Table 6.4: CoARX Synthesis Results

\begin{tabular}{|c|c|c|c|c|c|}
\hline \multicolumn{3}{|c|}{ Area(kGE) } & \multicolumn{2}{c|}{ Memory(kBytes) } & Freq.(MHz) \\
\hline Combinational & Sequential & Total & Configuration & Data & (Core) \\
\hline 82.6 & 12.4 & 95 & 1.5 & 32 & 700 \\
\hline
\end{tabular}

\subsubsection{Comparison with ASICs}

Different ASIC implementations considered for comparison are tabulated with their reported throughput in Table 6.6. For BLAKE, Henzen et al. report the ASIC with highest throughput [99] so far. For Skein, results reported for Skein-512-256 with highest throughput are considered [131]. Implementation results for HC-128, with keystream generation of 0.75 cycles/Byte (same as CoARX implementation) is considered for comparison [38]. For Salsa and ChaCha comparison, consider the implementation from [100].

For a fair comparison, the estimated throughput of the ASICs of ARX algorithms under consideration has been added in Table 6.6 after direct technology scaling to 90nm. CoARX delivers throughput in the same order of magnitude. On a closer investigation, some architectural aspects of CoARX, which affects the critical path of the design are as following. 
Table 6.5: Performance of different ARX algorithms on CoARX

\begin{tabular}{|c|c|c|c|c|c|}
\hline & BLAKE-512 & Skein-512 & HC-128 & Salsa20/20 & ChaCha/20 \\
\hline Input Block size (Bytes) & 128 & 64 & - & - & - \\
\hline Output Block size (Bytes) & 64 & 64 & - & 64 & 64 \\
\hline No of rounds $(r)$ & 16 & 72 & - & 20 & 20 \\
\hline Initialization & 5 cycles & 7 cycles & $46241 \mathrm{setups} / \mathrm{sec}$ & 4 cycles & 4 cycles \\
\hline Round Calculation & $21 \times r$ cycles & $r+((r / 4)+1) \times 2$ cycles & 0.75 cycles / Byte & $17 \times(r / 2)$ cycles & $17 \times(r / 2)$ cycles \\
\hline Finalization & 9 cycles & 6 cycles & - & 12 cycles & 13 cycles \\
\hline Total (cycles) & 350 & 123 & - & 186 & 187 \\
\hline Throughput (Gbps) & 2.05 & 2.91 & 7.47 & 1.93 & 1.92 \\
\hline Power $(\mathrm{mW})$ & 83 & 89 & 53 & 71 & 61 \\
\hline Energy (mJ/Gbit) & 41 & 30 & 7 & 33 & 29 \\
\hline
\end{tabular}

Table 6.6: Comparison with ASICs (Throughput scaled to 90nm)

\begin{tabular}{|c|c|c|c|c|c|c|c|}
\hline \multirow{2}{*}{$\begin{array}{c}\text { Implementation } \\
\text { Reference }\end{array}$} & \multirow{2}{*}{$\begin{array}{c}\text { Area } \\
(\mathrm{kGE})\end{array}$} & Frequency & \multicolumn{2}{|c|}{ Throughput (Gbps) } & \multicolumn{3}{|c|}{ Throughput/Area (kbps/GE) } \\
\cline { 5 - 9 } & & ASIC & CoARX & Individual & Combined & CoARX \\
\hline 4G-BLAKE-512(16 rounds) [99] & 79 & 532 & 16.5 & 2.05 & 208.86 & 91.16 & 21.57 \\
\hline Skein-512 [131] & 43.13 & 251 & 6.73 & 2.91 & 156.13 & 37.18 & 30.63 \\
\hline HC-128 Parallel keystream [38] & 13.66 & 1670 & 12.86 & 7.47 & 941.43 & 71.04 & 78.63 \\
\hline Salsa20 4xS-QR [100] & 22.81 & 365 & 4.67 & 1.93 & 204.64 & 25.79 & 20.31 \\
\hline ChaCha 4xS-QR [100] & 22.44 & 366 & 4.67 & 1.92 & 208.19 & 25.81 & 20.21 \\
\hline
\end{tabular}

- Barrel Shifter: For CoARX, a 64-bit Barrel rotator is used to support flexible rotation amounts. Considered ASICs for comparison (Table 6.6) use wire routing for fixed rotations instead.

- Datapath Width: For catering algorithms with 64-bit datapath and scalability, CoARX datapath is 64-bit wide. Consequently, ASIC implementations of ARX-based algorithms with 32-bit datapath have a smaller critical path and outperform CoARX in throughput. This can be made configurable to reduce the throughput gap.

- Flexible Interconnects: To support various possibilities of source operands from local and neighboring clusters registers, the input multiplexers of FUs in each of cluster are $8 \times 1$. Similarly, the write ports of the registers may take outputs of various FUs and each has a $4 \times 1$ multiplexer for possible inputs. These multiplexers increase the critical path of the design.

- Unfolding Transformation: According to the unfolding transformation [133], a design achieves better throughput if it is unfolded, which is the case in most of the ASIC implementations. ASICs for BLAKE implement an entire G function in one cycle [99]. For Skein, eight rounds are unfolded and implemented in one cycle [212]. Salsa20 and ChaCha also use similar kinds of transformations [100]. To incorporate enough flexibility, CoARX utilizes modular functional units and hence does not take advantage of unfolding transformation. 


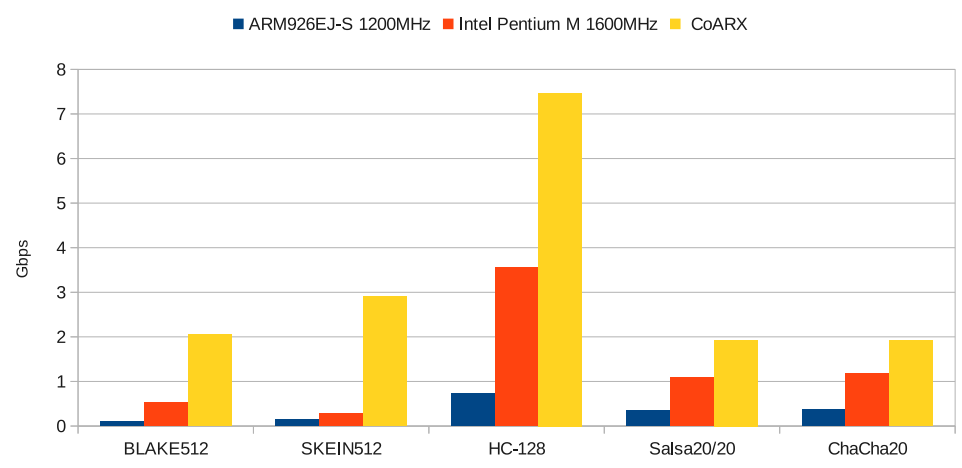

Figure 6.8: Comparison with GPP and Embedded Processor

The area of the individual ASIC implementations cannot be compared vis-a-vis the area of COARX due to its added flexibility. Sum of area of the best-performing ASICs results in $181 \mathrm{kGE}$, the area of CoARX is $47 \%$ less in comparison. A more suitable metric for comparison is the area-efficiency (throughput per area) reported in Table 6.6. The individual ASICs have significantly higher throughput per area, when compared against CoARX. This is the expected flexibility gap. The fact that CoARX implementation is efficient can be shown by considering the same metric for a hypothetical ASIC combining the best implementations. For this measure, the collective area (181 kGE) of individual ASICs is chosen. CoARX performs comparably for all the designs, except BLAKE. The BLAKE implementation reported in [99] performed an efficient round rescheduling technique to reduce the critical path after unrolling multiple subsequent ARX operations. For CoARX, such optimization is not possible since, the critical path is constrained by the operations within a PE.

Fig. 6.8 compares CoARX throughput of ARX algorithms mapped on it, with Intel M $1600 \mathrm{MHz}$ processor and ARM926EJ-S $1200 \mathrm{MHz}$ processor and easily outperforms these platforms by a factor of 1.6 to 22 times.

In the absence of a CoARX like flexible crypto core, a fair comparison in terms of power is not possible. Power-figures (in $\mathrm{mW}$ ), in similar process-technology, are available only for some individual ASICs, e.g. BLAKE: 10.84 [131], 15.65 [200], Skein: 17.17 [131], 39.71 [200] and Salsa20: 8.42 [80]. These values are better than (though the in same order of magnitude as) CoARX (Table 6.6). That is because these ASICs have algorithm-specific optimizations in their design and operate at a clock frequency lower than that of CoARX. Redundancy to achieve flexibility in CoARX design also contributes to higher power figures.

\subsubsection{Comparison with Customized Microcontrollers}

Constantin et al. proposed custom instruction extensions for efficient implementation of all SHA-3 hash function competition finalists including BLAKE and Skein [42]. A 16-bit PIC24 microcontroller is used as a starting point. For BLAKE, the throughput improvement is mainly attributed to custom instructions for rotation and address generation. For Skein, a single custom instruction is added for performing 64-bit left 
rotate in two cycles. In order to make sure that the device remains usable in resource constrained environments, $2 \times 16$-bit barrel shifters have been used to perform 64 -bit rotations. The reference microcontroller core occupies an area of $23 \mathrm{kGE}$. The customizations led to an area overhead of 10\%. BLAKE and Skein can be mapped on the above mentioned extended architecture with 155 cycles/Byte (10.32Mbps throughput) and 158 cycles/Byte (10.12Mbps throughput) respectively. In terms of throughput and area-efficiency (throughput/area), CoARX easily outperforms this. Furthermore, CoARX offers more flexibility (e.g. different rotation amounts) by focusing on ARX class of algorithms.

\subsubsection{Comparison with Multicore Implementations}

Several ARX based cryptographic functions have been undertaken for throughput benchmarking on different multicore architectures, e.g., IBM Cell architecture and GPGPUs $[162,204]$. A fair comparison between CoARX and a multicore system is hard, since the throughput scaling of multicore architectures is not linear due to the distribution of storage, varying latency for different storage and limitation of active number of threads per core. Considering these restrictions, multiple parallel datastreams for encryption/authentication are invoked in [204] in order to maximize the usage of the available cores. For Cell architecture, the 4-way SIMD instructions for each Synergistic Processing Elements (SPEs) are exploited for parallelism. With increasing size of the data block, more number of parallel threads are deployed resulting in higher encryption/authentication speed. To provide a fair comparison, the highest achievable throughput, the number of active Scalar Processor (SP) cores for GPU and active streams for an SPE are reported in the following Table 6.7.

Table 6.7: Performance of ARX algorithms on Multicore Architectures

\begin{tabular}{|c|c|c|c|c|c|}
\hline & \multicolumn{2}{|c|}{ SPE [204] } & \multicolumn{2}{|c|}{ GPGPU $[162,204]$} & \multirow{2}{*}{$\begin{array}{c}\text { CoARX } \\
\text { Throughput } \\
\text { (Gbps) }\end{array}$} \\
\hline & $\begin{array}{c}\text { Throughput } \\
\text { (Gbps) }\end{array}$ & Streams & $\begin{array}{c}\text { Throughput } \\
\text { (Gbps) }\end{array}$ & $\begin{array}{c}\text { Active } \\
\text { SP }\end{array}$ & \\
\hline BLAKE-32 & 5.1 & 4 & 36.80 & 240 & 2.05 \\
\hline Skein-512 & 1.9 & 2 & 22.10 & 240 & 2.91 \\
\hline HC-128 & - & - & 2.26 & 240 & 7.47 \\
\hline ChaCha & - & - & 42.40 & 240 & 1.93 \\
\hline Salsa20 & - & - & 10.86 & 48 & 1.93 \\
\hline
\end{tabular}

Though it is hard to compare without offering similar scalability for CoARX, several points can be made. First, CoARX offers 4 parallel ARX operations per cycle matching a 4-way SIMD instruction of SPE and 4 active SPs in GPGPU. Second, the individual SP cores of $[162,204]$ are synthesized at a frequency of $1242 \mathrm{MHz}$ and $1350 \mathrm{MHz}$ respectively, which are much higher than that of CoARX. Finally, it must 
be noted that for both BLAKE-32 and Skein-512, the implementation of [204] considers 4 different messages for parallel hashing, fixing the message size suiting the GPGPU requirements. In practice, the message size can vary. Experimented with a single large message for CoARX, though any message size as input is allowed. Since, both BLAKE-32 and Skein-512 involves chaining with the hash value of last message chunk, the internal dependency between the concurrent threads are ignored in [204], thus resulting in more throughput. Considering the above facts, CoARX outperforms both GPGPU and SPE on a throughput per core basis.

\subsubsection{Flexibility Study: Threefish-512 and Skein-512 Design Variants}

Cryptanalysis of existing algorithms and design of new algorithms that resist the known attacks are always at arms race. CoARX is flexible enough to adapt variants and offer resistance against generic attacks. This is demonstrated through a case study with Threefish-512 and Skein-512. Threefish-512, an ARX-based block cipher, has been mapped on CoARX as the building block of Skein-512. A general attack to ARX-based cryptosystems and in particular to Threefish-512 has been reported in [125]. This attack makes a reduced round variant of Skein-512 to be vulnerable. This shows that with CoARX, addition of few instructions is sufficient to thwart such attacks. At first, the attack model presented in [125] is studied.

According to Lemma 1 and the attack model in Section 4.2 of [125], the probability of getting a rotational pair for each addition is $2^{-1.415}$ for each addition in MIX and $2^{-0.28}$ for each addition in subkey addition part. Since there are a total of $72 \times 4=288$ additions in MIX and $18 \times 8=144$ additions in subkey addition, the probability of the complete attack is given by $2^{(-1.415) \times 288+(-0.28) \times 144}=2^{-448}$, giving a complexity of $2^{448}$. The 512-bit key has 8 words of 64 -bits and the three leftmost bits in each keyword are assumed to be known in the attack model, the effective key-length is $8 \times 61=$ 488 bits. Thus, according to the attack of [125], instead of $2^{488}$ attack complexity of random guessing, one has $2^{448}$ attack complexity. To counteract the above attack, the number of additions need to be increased. Suppose, the increase in the total number of additions in the MIX is $a_{1}$ and the total number of additions in the subkey addition is $a_{2}$. Then the attack probability is $1.415 \times\left(288+a_{1}\right)+0.28 \times\left(144+a_{2}\right) \geq 488$. Simplifying the above inequality,

$$
1.415 \times a_{1}+0.28 \times a_{2} \geq 40 .
$$

Next, two design variants are proposed to achieve the inequality (6.2). In the first variant, the total number of rounds is increased by a multiple of 4 , say, by $4 x$. Since 8 extra additions are added from subkey addition after every 4 rounds, hence, $a_{1}=16 x$ and $a_{2}=8 x$. Substituting in 6.2 and then simplifying to get $x>=1.61$. Since $x$ has to be an integer, it is taken as $x=2$, meaning that 8 extra rounds are needed. Hence it is proposed to continue with the same subkey generation algorithm 
for those 8 extra rounds. In the second variant, only the number of additions in MIX is increased without increasing the subkey additions. This means, $a_{2}=0$. Thus, from inequality (6.2), after rounding $a_{1} \geq 29$. For symmetry of computation, $a_{1}=36$ is taken and those 36 additions are distributed as follows. Instead of 4 rounds followed by a subkey addition, the use of 5 and 4 rounds alternately is proposed, followed by a subkey addition. In this way, there will be a total of $5 \times 9+4 \times 9=81$ rounds interleaved by the usual subkey additions. The above two design variants of Skein-512 (Threefish-512) are mapped on the CoARX without much degradation in throughput. The first design variant requires 12 extra cycles $(8+2 \times 2)$ and the second design variant requires 9 extra cycles causing a throughput degradation of $8.8 \%$ and $6.8 \%$ respectively. For designing the second design variant additional permutation and rotation constants are also required to perform the $5^{\text {th }}$ alternate round, which are added to the configuration memory. The entire design modification is performed within few hours.

Very recently, a differential power attack has been reported [31] on Skein. The same paper also proposes a countermeasure which does not alter the basic ARX structure and therefore CoARX can be easily adapt to include this as well.

\subsection{RC4-AccSuite: A Hardware Acceleration Suite for RC4-like Stream Ciphers}

$\mathrm{RC} 4$ is the most commonly used stream cipher to protect Internet traffic using the SSL (Secure Sockets Layer) protocol, Transport Layer Security (TLS), WEP (Wired Equivalent Privacy), Wi-Fi Protected Access (WPA) etc along with several application layer softwares. Since its inception, 20 years back, RC4 has been the target of keen cryptanalytic efforts, some of which have been successful. In spite of that, the base algorithm is not broken and $R C 4$ is still secure when used with precautions. The usability of the RC4-like cipher kernels is re-iterated in a recent proposal Spritz [186] from the authors of the original RC4. Spritz offers not only a drop-in replacement for RC4 but offers an entire suite of cryptographic functionalities based on sponge-like constructive functions. As NIST SHA-3 competition declared a sponge-based kernel called Keccak [23] as the winner after a 5 year long competition, the usability, security and efficiency of sponge functions has been already been scrutinized and appreciated by the cryptanalytic community.

The fact that RC4 has an entire class of well-known variants for ensuring higher security, better performance and versions for implementation on word-oriented platforms makes the study and design of a generic core for implementing RC4 and its variants worthwhile.

\subsubsection{RC4 Stream Cipher Algorithm}

RC4 was designed by Ron Rivest of M.I.T. for RSA Data Security in 1987. The RC4 algorithm was described in [195]. It has an internal state comprising of 256 Byte array, 
denoted by $S[0 \ldots N-1]$ and accessed by indices $i$ and $j$. The three phases of RC4 operation (and for most of the stream ciphers too) are the State Initialization (SI), Key Scheduling Algorithm (KSA) and the Pseudo Random Generation Algorithm $(P R G A)$. The secret key $k[0 \ldots l-1]$ is expanded by repetition to a size equal to that of array $S: K[y]=k[y \bmod l]$, for $0 \leq y \leq N-1$. In every iteration of KSA and PRGA, $i$ is incremented, $j$ is updated, values of $S[i]$ and $S[j]$ are swapped while PRGA produces one Byte of output which is XOR-ed with the one Byte of the message to produce one Byte of ciphertext (or plaintext in case of decryption). Other than these phases of operation, i.e., SI, KSA and PRGA, some variants of RC4 undergo another round of shuffling based on initialization vector or IV. This phase happens after KSA and is known as IVSA.

Table 6.8: Algorithmic specifications of RC4 stream cipher

\begin{tabular}{|c|c|}
\hline State Initialization (SI) & Algorithm PRGA \\
\hline $\begin{array}{l}\text { For } i=0, \ldots, N-1 \\
\quad S[i]=i \\
j=0\end{array}$ & $\begin{array}{l}\text { Initializing the Indices: } \\
i=j=0 ; \\
\text { Loop as many plaintext Bytes: }\end{array}$ \\
\hline Algorithm KSA & $i++$ \\
\hline Scrambling the State: & $j=j+S[i]$ \\
\hline For $i=0, \ldots, N-1$ & $\operatorname{Swap}(S[i], S[j])$ \\
\hline$j=(j+S[i]+K[i])$ & $t=S[i]+S[j]$ \\
\hline $\operatorname{Swap}(S[i], S[j])$ & Output $z=S[t]$ \\
\hline
\end{tabular}

\subsubsection{Variants of RC4}

A brief description of some of the noticeable variants of RC4 to counteract cryptanalytic attacks follows (the list is not chronologically arranged but in decreasing order of similarity with RC4).

1. $\mathrm{RC}^{+}{ }^{+}: \mathrm{RC}^{+}$recommended complementary layers of computation during for KSA and PRGA phase on top of the original proposal of RC4 for achieving a better security margin [147]. These layers of computation achieve better scrambling and avoid key recovery attack during $\mathrm{RC}^{+}{ }^{+} \mathrm{KSA}$ and $\mathrm{RC} 4^{+}$PRGA, respectively. Some intermediate VLSI design versions trading-off security against performance namely PRGA $^{\alpha}$ and PRGA ${ }^{\beta}$ have also been undertaken [40].

2. VMPC: VMPC variant of RC4 is named so after a hard to invert VMPC function, used during KSA, IVSA and PRGA of VMPC variant of RC4 [231]. The VMPC function for an $N$ variable permutation array named $P$, transformed into $Q$, requires a single modulo addition and three accesses of permutation state array as shown: 


$$
Q[x]=P[P[P[x]]+1] \text {, where } 0 \leq x \leq N-1
$$

3. RC4A: RC4A was introduced to remove a statistical bias in consecutive Bytes of PRGA in RC4 [179]. It uses two keys to carryout KSA into two arrays $S 1$ and $S 2$. Similarly, two indices $j 1$ and $j 2$ are used for $S 1$ and $S 2$ respectively during PRGA based on exchange shuffle model, inline with RC4 PRGA. The only modification is the index $S 1[i]+S 1[j]$ keystream produces output from $S 2$ and vice versa.

4. RC4B: A recent work exposed the vulnerability of both RC4 and RC4A to new new classes of statistical biases [146]. To overcome that, a new RC4 variant known as RC4B is introduced, which differs from RC4A only as it mixes the contents of the $S 1$ and $S 2$ during updating $j 1$ and $j 2$.

5. RC4b: A Byte variant of RC4 called RC4b was described in [150]. The author claimed to remove the known biases in RC4 by scuffling state elements twice and by explicitly discarding the first $N$ Bytes during KSA.

6. $\operatorname{NGG}(n, m)$ : $\operatorname{NGG}(n, m)$ is a word variant of RC4, extensible to $32 / 64$ bit words with $S$ much smaller than $2^{32} / 2^{64}$ [160], where $S=2^{n}$ is the size in words and $m$ is the word size in bits $(n \leq m)$. The SI for NGG uses a precomputed random array, the KSA and PRGA phases are similar to that of RC4, extended to words. NGG is named so after initials of its authors.

7. GGHN: GGHN is an improved version of NGG, also named so after its designers initials [78]. It recommends multiple iterations of KSA phase, depending on word size and number of word of $S$ for maintaining a high degree of randomness. For better security a key dependent third variable $k$ is also used, other than $i$ and $j$ for exchange shuffle model in GGHN PRGA.

8. Spritz: Spritz is the recent proposal, coming from the author of RC4, formulated as a sponge and consequently capable of being used as a block cipher, stream cipher, hash functions, DRBG, MAC and AE [186]. It has RC4-like general design principles and attempts to repair weak design decisions of RC4.

\subsubsection{Contribution}

To the best of our knowledge, this is the first ASIP designed by identifying the common operation kernels of members of a well-known cryptographic cipher family. The accelerator is flexible enough to switch to various $\mathrm{RC} 4$ variants at run-time and gives the user the choice to choose a variant that matches his performance, security, power and platform need. The state replication technique for promising higher performance is systematically undertaken whenever possible for for all RC4 variants The reusable resources between RC4 and all the variants are identified, including registers, pipeline registers, combinational macros and memory blocks whenever possible and consequently, the resource budget of RC4-AccSuite is much smaller compared to the accumulation of individual cores of RC4 variants implementation. Design and development 
of RC4-AccSuite is an extension of the proposal put forward for a unified core for $\mathrm{RC} 4$ and $\mathrm{RC}^{+}{ }^{\text {[147] }}$ in a single core [40], however that lacked a conscious effort for resource reuse except for where an entire instruction could be reused. An incremental design for RC4-AccSuite is carried out, adding one RC4 variant in each step. The saving in terms of area and the power budget for each of these cores has been benchmarked.

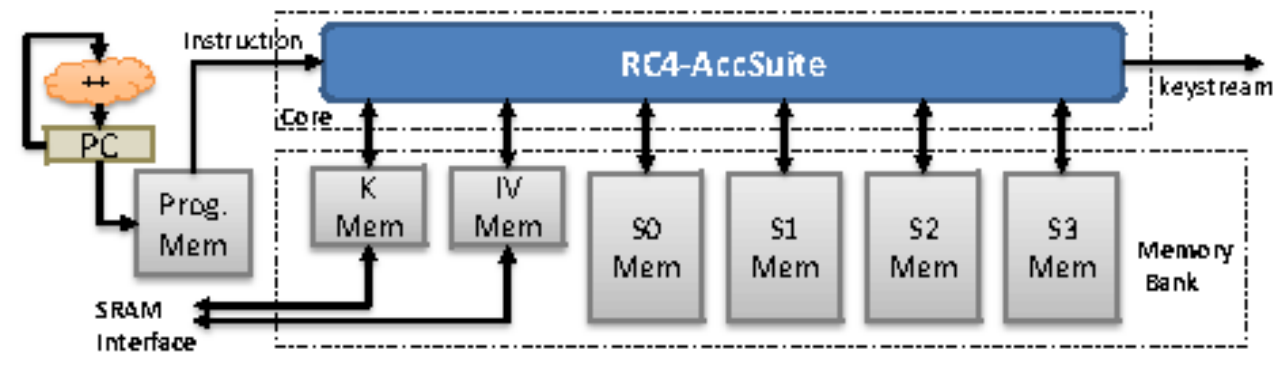

Figure 6.9: Block Diagram of RC4-AccSuite

\subsubsection{High-level Architecture of RC4-AccSuite}

Fig. 6.9 presents high-level architectural diagram for the processor executing RC4 variants. The architecture is generalized for the VLSI implementation of any stream ciphers with large internal states as it is provided with an external memory bank, e.g., WAKE, Py, HC-128/256, CryptMT etc. The processor core performing one or more variants of RC4 is referred as RC4-AccSuite. Instructions are kept in a program memory, while a simple incrementing program counter (PC) serves as the address to the memory. A more sophisticated address control for supporting loops and jumps is excluded as it is not required. The internal pipeline architecture of the processor core, its input/output interface and the external memory bank changes depending on the RC4 variant/variants it supports. The IOs of the core are discussed below.

1. Instruction is input to the core and is $\left\lceil\log _{2}(n)\right\rceil$ bits for $n$ instructions specified for the RC4 variant. If the core supports two variants with $n 1$ and $n 2$ distinct instructions then the $n$ is taken as $n=n 1+n 2$.

2. Keystream is output of the core and its width is taken to be the maximum keystream word size of the supported variants. Hence for $\mathrm{RC} 4 / \mathrm{RC} 4^{+}$it will be 8 bits and for RC4/GGHN it will be 32 bits.

Due to large sizes of internal states (or S-Boxes) a preferred storage medium for the RC4 variants are the vendor supplied SRAMs which are optimized for throughput. The memory bank may include SRAMs for Key (K Memory, 32 words), IV (IV Memory, 32 words) and internal states (S0 - S3 Memories, 256 words), each being 8 bits wide. An SRAM is selectively included in the memory bank provided at least one of the supported variants requires it, as given in Table 6.9. $\mathrm{K}$ and IV need an external 
interface so that a new key and IV may be supplied from host processor before KSA and IVSA are initiated. RC4A keeps two internal $S$ arrays and therefore requires both $S 0$ and $S 1$. For the two word variants, only $\operatorname{NGG}(8,32)$ and $\operatorname{GGHN}(8,32)$ are the currently supported configurations, hence $S$ array has to have 256 words of 32 bits each. Instead, four memories $S 0-S 3$ are employed in-order to reuse the same memory for byte variants as well. For the rest of the discussion $\operatorname{NGG}(8,32)$ and $\operatorname{GGHN}(8,32)$ are referred as NGG and GGHN, respectively.

Table 6.9: Byte wide Memory requirements (instances $\times$ depth) for RC4 variants

\begin{tabular}{|c|c|c|c|c|c|}
\hline RC4 Variant & $\begin{array}{l}\text { keystream } \\
\text { word (bits) }\end{array}$ & S memories & \multicolumn{2}{|c|}{$($ instances $\times$ depth $)$} & $\begin{array}{c}\text { Total } \\
\text { (Bytes) }\end{array}$ \\
\hline RC4 [195] & 8 & $1 \times 256$ & $1 \times 32$ & - & 288 \\
\hline $\mathbf{R C 4}^{+}[147]$ & 8 & $1 \times 256$ & $1 \times 32$ & - & 288 \\
\hline VMPC [231] & 8 & $1 \times 256$ & $1 \times 32$ & $1 \times 32$ & 320 \\
\hline RC4A [179] & 8 & $2 \times 256$ & $2 \times 32$ & - & 576 \\
\hline RC4B [146] & 8 & $2 \times 256$ & $2 \times 32$ & - & 576 \\
\hline RC4b [150] & 8 & $1 \times 256$ & $1 \times 32$ & $1 \times 32$ & 320 \\
\hline NGG [160] & 32 & $4 \times 256$ & $1 \times 32$ & - & 1056 \\
\hline GGHN [78] & 32 & $4 \times 256$ & $1 \times 32$ & - & 1056 \\
\hline Spritz [186] & 8 & $1 \times 256$ & $1 \times 32$ & - & 288 \\
\hline
\end{tabular}

\subsubsection{Judging Performance}

The performance of a cipher processor is benchmarked by the initialization latency (KSA and IVSA duration) and the throughput (Byte/word/bits per second) of keystream generation phase. Instead, a more generic or technology independent parameter to judge performance is used, i.e., cycles per keystream Byte. The reason for choosing this alternate benchmark is to annul the effect of the CMOS technology library used for synthesis on the performance. Nevertheless, various design points of RC4-AccSuite are benchmarked on a CMOS technology library and the throughput results are discussed in Section 6.4.7.

\subsubsection{Performance Enhancement by Memroy Replication Technique}

The performance enhancement techniques undertaken in literature for RC4 (and similar algorithms) have been discussed in Section 5.2.1. Other than the three known techniques, i.e., Using Multi-ported SRAMs, Loop Unrolling and State Replication, another technique called State Splitting is also discussed in context of HC-128 in the previous chapter. Unlike HC-128, that allows multiple parallel independent accesses in a PRGA, RC4 memory accesses are tied up to be sequentially performed due to possible $R A W$ hazards. Avoiding these hazards require algorithm dependent extra 
checks for pipeline stalling, making the design inefficient. Hence state-splitting was not exploited in RC4-AccSuite.

State replication is carried out with multiple instances of SRAMs as copies of $S$ array to increase the simultaneous access ports availability. This consequently boasts throughput, inline with Mathews proposal [112]. Using two dual-ported SRAMs for RC4 PRGA a 2 cycles per Byte throughput was reported [40]. This idea was viably extended to other stream ciphers like HC-128 [38].

State replication, using a dual ported SRAM enables 2 additional state accesses per cycle and consequently lowers clock cycles per PRGA, increasing throughput. For any RC4 Byte-variant, memory replication to increase simultaneous state memory accesses is carried out provided no data incoherence arise as an aftermath. For an algorithm if a state memory $S$ is replicated $m \times$ to achieve parallelism the implementation is dubbed as ALGO_S_m, e.g., RC4_S_0 and RC4_S_1 have been discussed in $[40,112]$ with a throughput of 3 and 2 cycles per Byte, respectively.

A limitation in the context is noteworthy. Given l-SRAMs, each being n-ported, a critical question is that in $k$-cycles can all the $l \times n \times k$ access ports be utilized or not? The reason prohibiting this maximum usage is the architecture of current SRAMs that require one or more clock cycles for turnaround to change access to the SRAM from write to read and vice versa. Consequently only two consecutive reads or two consecutive writes can be performed in two consecutive clock cycles from each access port of an SRAM. For consecutive read-write and write-read cases from one access port would require one clock cycle between the two accesses if an SRAM requiring a single turnaround cycles is considered. Regarding efficient use of memory ports in PRGA or any RC4 variant following guidelines can help in achieving better performance.

- Schedule writes on one port of the memory only and reads on both ports. Hence only one port will be underutilized by turnaround cycle and not both.

- Schedule multiple writes on same memory in consecutive cycles if possible, hence turnaround cycle waste will be per batch of writes and not every write.

Memory replication may considerably boost throughput at the cost of additional area and power. To economize power, memory replication should be skipped, even if memory modules are available on a platform, Memory replication also costs additional writes to keep all state copies updated with correct data during KSA, IVSA and PRGA for RC4 and variants, e.g., RC4_S_0 and RC4_S_1 require 1 and 2 writes per PRGA. Memory replication should be incrementally applied on an algorithm followed by a systematic cycle by cycle design re-evaluation exploiting additional parallelism. Various interesting design points may arise, with performance-area-power trade-off.

The next section gives a walk-through into two case studies for Spritz and $\mathrm{RC}^{+}$ for increasing throughput using state replication of SRAM. 
Table 6.10: PRGA for Spritz (left) and $\mathrm{RC}^{+}$(right)

\begin{tabular}{|c|c|}
\hline & RC4 $^{+}$PRGA \\
\hline Spritz PRGA & \multirow{8}{*}{$\begin{array}{l}\text { Output Keystream Generation Loop: } \\
i=i+1 ; \\
j=j+S[i] ; \\
\text { Swap }(S[i], S[j]) ; \\
t=S[i]+S[j] ; \\
t^{\prime}=\left(S\left[i_{R}^{3} \oplus j_{L}^{5}\right]+S\left[i_{L}^{5} \oplus j_{R}^{3}\right]\right) \oplus 0 x A A ; \\
t^{\prime \prime}=j+S[j] ; \\
\text { Output } z=\left(S[t]+S\left[t^{\prime}\right]\right) \oplus S\left[t^{\prime \prime}\right] ;\end{array}$} \\
\hline Output Keystream Generation Loop: & \\
\hline$i=i+w$ & \\
\hline$j=k+S[j+S[i]]$ & \\
\hline$k=i+k+S[j]$ & \\
\hline $\operatorname{Swap}(S[i], S[j])$ & \\
\hline Output $z=S[j+S[i+S[z+k]]]$ & \\
\hline & \\
\hline
\end{tabular}

\subsubsection{Spritz}

Table 6.10 gives the PRGA steps for Spritz. It requires 6 state read accesses and two writes per PRGA Byte generated. Fig 6.10 shows the mapping memory accesses when the Spritz PRGA steps are mapped on a dual ported SRAM (no replication). Due to data dependencies, no pipeline stage entertains more than one memory read, although 2 simultaneous request are possible. There should be 5 (or more) nop instructions between two consecutive instructions so that the cycle 1 of next instruction overlaps with the cycle 7 of current instruction resulting in a throughput of $6 \mathrm{cy}-$ cles/Byte. Further overlap of cycles for consecutive instructions is not possible due to the structural hazard caused due to availability of limited number of access ports of the memory.

\begin{tabular}{|c|c|c|c|c|c|c|}
\hline DP1 & DP2 & DP3 & DP4 & DP5 & DP6 & DP7 \\
\hline rq SO_PO[i] & $\begin{array}{l}\text { R SO_PO[i] } \\
\text { rq SO_PO[j+S[i]] }\end{array}$ & $\begin{array}{c}\text { Wr SO_P1[j] } \\
\text { R SO_PO[j+S[i]] } \\
\text { rq SO_PO[j] }\end{array}$ & $\begin{array}{c}\text { Wr SO_P1[i] } \\
\text { rq SO_PO[k+z] } \\
\text { R SO_PO[j] }\end{array}$ & $\begin{array}{l}\text { rq SO_PO }[i+s[k+z]] \\
\text { R SO_PO }[k+z]\end{array}$ & $\begin{array}{l}\text { R SO_PO }[i+s[k+z]] \\
\text { rq SO_PO }[j+5[i+s[k+z]]]\end{array}$ & R SO_PO[j]+s[i+s[k+z]]] \\
\hline
\end{tabular}

Figure 6.10: Spritz PRGA accesses on a dual ported SRAM ( $\mathrm{P} 0=$ port $0, \mathrm{P} 1=$ port 1$)$

Fig 6.11 shows memory accesses for Spritz_S0_1. This replication requires 2 additional memory writes as indicated in DP3 and DP4 pipeline stages. The last three reads for output calculation of Spritz PRGA are directed to $S 1$. This enables the next consecutive instruction execution after 3 nops. The throughput is improved to $4 \mathrm{cy}-$ cles/Byte.

By carefully placing the accesses on the memory ports, it is ensured that overlap of consecutive instructions causes no data incoherence/ resource hazard. Fig 6.12 depicts one PRGA Byte generated after every 4 cycles. Further parallelization through memory replication is not considered, since data dependencies in the algorithm do not allow it. 


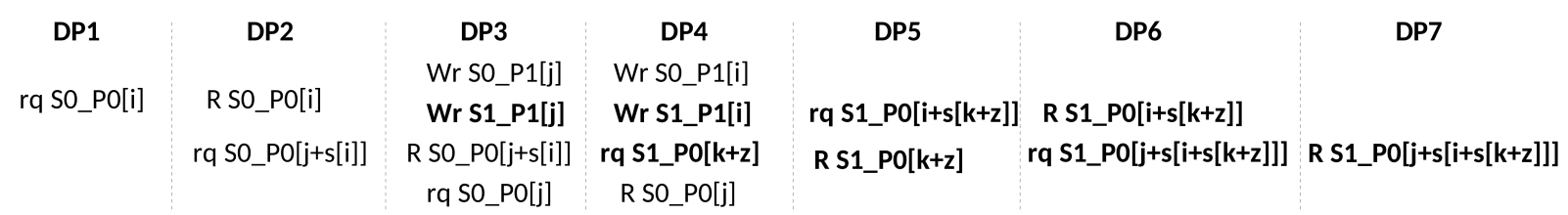

Figure 6.11: Spritz_S0_1 PRGA on 2 dual ported SRAMs (P0= port $0, \mathrm{P} 1=$ port 1$)$

Inst. 1

Inst. 2

$\begin{array}{lllllll}\text { DP1 DP2 DP3 DP4 DP5 DP6 DP7 } & \text { DP }\end{array}$

$\begin{array}{llllllll}\text { DP1 } & \text { DP2 } & \text { DP3 } & \text { DP4 } & \text { DP5 } & \text { DP6 } & \text { DP7 }\end{array}$

DP1 DP2 DP3 DP4 DP5 DP6 DP7

DP1 DP2 DP3 DP4 DP5 DP6 DP7

Figure 6.12: Consecutive instructions overlap for Spritz_S0_1 PRGA without hazards

\subsubsection{2 $\mathrm{RC}^{+}$}

For $\mathrm{RC}^{+}{ }^{+}$PRGA, given in Table 6.10, the relevant simplistic $\mathrm{RC} 4^{+}{ }^{+} \mathrm{S} \_0$ memory access mapping is given in Table 6.11. Out of the 4 extra reads here compared to PRGA RC4, two are are initiated in cycle 4 and two in cycle 5. Due to bus turnaround it is not possible to initiate a read on port 1 in cycle 3 , hence in cycle 4 only one access is possible. For simplicity, $S\left[i_{R}^{3} \oplus j_{L}^{5}\right]$ and $S\left[i_{L}^{5} \oplus j_{R}^{3}\right]$ are dubbed as $S\left[t^{\prime 1}\right]$ and $S\left[t^{\prime 2}\right]$, respectively. There should be 5 (or more) nop instructions between two consecutive PRGA RC4 ${ }^{+}$instructions so that no structural hazard is caused due to availability of limited number of access ports of the memory. A throughput of 5 cycles/Byte results.

Table 6.11: $\quad$ RC4 ${ }^{+} \_S 0 \_0$ PRGA, throughput $=5$ cycles $/$ Byte

\begin{tabular}{|c|c|c|c|c|c|c|c|}
\hline \multicolumn{2}{|c|}{ Cycles } & 1 & 2 & 3 & 4 & 5 & 6 \\
\hline \multirow{2}{*}{ So } & P0 & rq SO[i] & $\begin{array}{l}\text { R SO[i] } \\
\text { rq S0[j] }\end{array}$ & $\begin{array}{l}\text { R S0[j] } \\
\text { rq SO[t] }\end{array}$ & $\begin{array}{c}\mathrm{RS} 0[\mathrm{t}] \\
\mathrm{rqS} 0\left[\mathrm{t}^{\prime 1}\right]\end{array}$ & $\begin{array}{l}\mathrm{RSO}\left[\mathrm{t}^{\prime 1}\right] \\
\mathrm{rq} \mathrm{S} 0\left[\mathrm{t}^{\prime \prime}\right]\end{array}$ & $\mathrm{R} \mathrm{SO}\left[\mathrm{t}^{\prime \prime}\right]$ \\
\hline & P1 & & W S0[j] & W S0[i] & $\mathrm{rq} \mathrm{SO}\left[\mathrm{t}^{\prime 2}\right]$ & $\begin{array}{l}\mathrm{R} \mathrm{SO}\left[\mathrm{t}^{\prime 2}\right] \\
\mathrm{rqSO}\left[\mathrm{t}^{\prime}\right]\end{array}$ & $\mathrm{R} \mathrm{S} 0\left[\mathrm{t}^{\prime}\right]$ \\
\hline \multicolumn{2}{|c|}{$\mathrm{R} / \mathrm{W}$} & 0 & 2 & 2 & 1 & 2 & 2 \\
\hline
\end{tabular}

Table 6.12 shows memory accesses for a single RC4 ${ }^{+}$PRGA Byte when one copy of $S 0$, called $S 1$ is used in addition. Due to more ports now, access of $S\left[t^{\prime 1}\right]$ and $S\left[t^{\prime 2}\right]$ can be initiated as soon as cycle 3 , earlier is not possible since $j$ index was not calculated. For keeping data coherency, the two writes of $\mathrm{RC}^{+}$have now doubled, both the memories are updated with $S[j]$ and $S[i]$ values in cycle 2 and 3, respectively (using port 1). In none of the clock cycles are all the 4 available ports utilized, either because of the turnaround cycles or because of the data dependency. The throughput is improved to 4 cycles/Byte. 
Table 6.12: $\quad$ RC4 ${ }^{+}$S0_1 PRGA, throughput $=4$ cycles $/$ Byte

\begin{tabular}{|c|c|c|c|c|c|c|}
\hline \multicolumn{2}{|c|}{ Cycles } & 1 & 2 & 3 & 4 & 5 \\
\hline \multirow[t]{2}{*}{ SO } & P0 & rq S0[i] & $\begin{array}{l}\text { R S0[i] } \\
\text { rq S0[j] }\end{array}$ & $\begin{array}{c}\mathrm{R} \mathrm{SO}[\mathrm{j}] \\
\operatorname{rq} S 0\left[\mathrm{t}^{\prime 1}\right]\end{array}$ & $\begin{array}{l}\mathrm{rqSO}\left[\mathrm{t}^{\prime}\right] \\
\mathrm{RSO}\left[\mathrm{t}^{\prime 1}\right]\end{array}$ & $\mathrm{R} \mathrm{SO}\left[\mathrm{t}^{\prime}\right]$ \\
\hline & P1 & & W SO[j] & W SO[i] & rq SO $\left[\mathrm{t}^{\prime \prime}\right]$ & $\mathrm{R} \mathrm{S0}\left[\mathrm{t}^{\prime \prime}\right]$ \\
\hline \multirow[b]{2}{*}{ S1 } & P0 & & & $\mathrm{rq} \mathrm{S1}\left[\mathrm{t}^{\prime 2}\right]$ & $\mathrm{R} S 1\left[\mathrm{t}^{\prime 2}\right]$ & \\
\hline & P1 & & W S1[j] & W S1[i] & $\operatorname{rq} \mathrm{S} 1[\mathrm{t}]$ & $\mathrm{R} S 1[\mathrm{t}]$ \\
\hline \multicolumn{2}{|c|}{$\mathrm{R} / \mathrm{W}$} & 0 & 3 & 3 & 2 & 3 \\
\hline
\end{tabular}

A higher replication version of $\mathrm{RC}^{+}$is tried out for throughput enhancement. $\mathrm{RC}^{+}{ }^{+}$SO_2 improves the throughput to 3 cycles/Byte as shown in Table 6.13 (left). Only during 3rd cycle are all the 6 available simultaneous ports being used. There still is a room for improvement, that can be seen as an overlap of cycle 2 and cycle 4 of consecutive $\mathrm{RC}_{4}{ }^{+}$PRGA instructions is possible, provided there are enough access ports. Using a total of 3 memories its is not possible due to number of operations being more than the access ports. $\mathrm{RC}^{+}{ }^{+} \mathrm{S} 0 \_3$ further improves the throughput to 2 cycles/Byte as shown in Table 6.13 (right). By carefully placing the accesses on the ports it is ensured that no more than one nop is required between two consecutive $\mathrm{RC}^{+}{ }^{+}$PRGA instructions. Overlapping of $2^{\text {nd }}$ and $4^{\text {th }}$ cycle also does not cause any data incoherence since a read priority is set for all SRAMs. Further parallelization through memory replication is not considered as $\mathrm{RC} 4{ }^{+}$_S0_3 uses all the $4 \mathrm{~S}$ memories available, along with key and IV memories.

Table 6.13: $\quad$ RC4 ${ }^{+} \_S 0 \_2$ PRGA, throughput $=3$ cycles $/$ Byte (left), $R C 4^{+}{ }^{+}$S0_3 PRGA, throughput $=2$ cycles $/$ Byte (right)

\begin{tabular}{|c|c|c|c|c|c|c|c|c|c|c|c|}
\hline \multirow{2}{*}{\multicolumn{2}{|c|}{ Cycles }} & & & & & \multicolumn{2}{|c|}{ Cycles } & \multirow{3}{*}{$\frac{1}{\mathrm{rq} \mathrm{S0}[\mathrm{i}]}$} & \multirow{3}{*}{$\begin{array}{c}\frac{2}{\mathrm{R} \mathrm{S} 0[\mathrm{i}]} \\
\mathrm{rq} \mathrm{S} 0[\mathrm{j}]\end{array}$} & \multirow{3}{*}{$\frac{3}{\text { R So[j] }}$} & \multirow[t]{3}{*}{4} \\
\hline & & 1 & 2 & 3 & 4 & & P0 & & & & \\
\hline \multirow{3}{*}{ so } & PO & rq S0[i] & R S0[i] & R S0[j] & & & & & & & \\
\hline & & & rq S0[j] & $\mathrm{rq} \mathrm{S} 0\left[\mathrm{t}^{\prime}\right]$ & R SO [t'] & & P1 & & W S0[j] & W S0[i] & \\
\hline & P1 & & W S0[j] & W S0[i] & & \multirow{3}{*}{ S1 } & & & rq S1[t'1 1$]$ & $\overline{R ~ S 1\left[t^{\prime} 1\right]}$ & \\
\hline \multirow{3}{*}{ S1 } & & & $\mathrm{rq} \mathrm{S1}\left[\mathrm{t}^{11}\right]$ & R S1 $\left[t^{\prime 1}\right]$ & & & PU & & & $\mathrm{rq} S 1[\mathrm{t}]$ & $\mathrm{RS} 1[\mathrm{t}]$ \\
\hline & 10 & & & rq S1[t] & $\mathrm{R}$ S1[t] & & P1 & & W S1[j] & W S1[i] & \\
\hline & P1 & & W S1[j] & W S1[i] & & \multirow{3}{*}{ S2 } & PO & & rq S2 $\left[\mathrm{t}^{2}\right]$ & R S2 $\left[\mathrm{t}^{\prime 2}\right]$ & \\
\hline \multirow{3}{*}{ S2 } & PO & & $\mathrm{rq} \mathrm{S} 2\left[\mathrm{t}^{2}\right]$ & R S2 $\left[\mathrm{t}^{\prime 2}\right]$ & & & & & & rq S2 [t" $]$ & R S2 [t" $]$ \\
\hline & & & & rq S2[t" $]$ & R S2 $\left[\mathrm{t}^{\prime \prime}\right]$ & & P1 & & W S2[j] & W S2[i] & \\
\hline & P1 & & W S2[j] & W S2[i] & & \multirow{2}{*}{ S3 } & P0 & & & rq S3[t'] & R S3[t'] \\
\hline \multicolumn{2}{|c|}{$\mathrm{R} / \mathrm{W}$} & 0 & 4 & 6 & 3 & & P1 & & W S3[j] & W S3[i] & \\
\hline & & & & & & \multicolumn{2}{|c|}{$\mathrm{R} / \mathrm{W}$} & 0 & 5 & 7 & 3 \\
\hline
\end{tabular}

\subsubsection{State Replication in RC4 Variants}

For mapping any of the rest of the RC4 variants on the RC4-AccSuite the strategy followed is the same as described for $\mathrm{RC}^{+}$. First with a replication factor of 0 , all 
accesses are placed in a chronological order, unless disturbing it helps in utilizing an available port and carefully checking for data incoherence. Next the accesses are remapped with a replication factor of 1 , with doubled accesses; this is continued as long as a throughput boost is achievable till all the 4 memories are utilized. Table 6.14 describes the effect on performance of these efforts. For VMPC, the parallelization cannot be exploited due to the dependency of reads on the previous value being read in the VMPC function. RC4A and RC4B require $2 \mathrm{~S}$ memories, replication of both, done once, occupies the 4 memories available. It generates two Bytes simultaneously after each PRGA and parallelization by memory replication further improves the throughput to 1 Byte per clock cycle. For the word variants, the parallelization is not possible since these algorithms use all the $4 \mathrm{~S}$ memories. For both the word variants the throughput is specified in terms of cycles per word (4 Bytes) and the read write accesses are also for 32 bits (all four memories accesses simultaneously).

Out of the various memory replication versions of an algorithm as given in Table 6.14, the fastest one is mapped on RC4-AccSuite. For the rest of the discussion, the postfix of an algorithm showing parallelization is skip for simplicity ( $\mathrm{RC} 4^{+}{ } \mathrm{S} 0 \_3$ is dubbed as $\mathrm{RC} 4^{+}$).

Table 6.14: Array Replication in RC4 variants

\begin{tabular}{|c|c|c|c|c|c|}
\hline \multirow{2}{*}{ Algorithm } & $\begin{array}{c}\text { Replication } \\
\text { factor }\end{array}$ & $\begin{array}{c}\text { S } \\
\text { Memories }\end{array}$ & $\begin{array}{c}\text { Throughput } \\
\text { Cycles / x Bytes }\end{array}$ & $\begin{array}{c}\text { no. of } \\
\text { Reads }\end{array}$ & $\begin{array}{c}\text { no. of } \\
\text { Writes }\end{array}$ \\
\hline \multirow{2}{*}{ RC4 } & - & 1 & 3 Cycles/Byte & 3 & 2 \\
\cline { 2 - 6 } & S0_1 & 2 & 2 Cycles/Byte & 3 & 4 \\
\hline \multirow{3}{*}{ RC4+ } & - & 1 & 5 Cycles/Byte & 7 & 2 \\
\cline { 2 - 6 } & S0_1 & 2 & 4 Cycles/Byte & 7 & 4 \\
\cline { 2 - 6 } & S0_2 & 3 & 3 Cycles/Byte & 7 & 6 \\
\cline { 2 - 6 } & S0_3 & 4 & 2 Cycles/Byte & 7 & 8 \\
\hline VMPC & - & 1 & 7 Cycles/Byte & 5 & 2 \\
\hline RC4A/ & - & 1 & 3 Cycles/2 Bytes & 6 & 4 \\
\cline { 2 - 6 } RC4B & S0_1_S1_1 & 2 & 2 Cycles/2 Bytes & 6 & 8 \\
\hline \multirow{2}{*}{ RC4b } & - & 1 & 3 Cycles/2 Bytes & 3 & 2 \\
\cline { 2 - 6 } & S0_1 & 2 & 2 Cycles/2 Bytes & 3 & 4 \\
\hline NGG & - & 4 & 3 Cycles/4 Bytes & 3 & 3 \\
\hline GGHN & - & 4 & 3 Cycles/4 Bytes & 3 & 1 \\
\hline \multirow{2}{*}{ Spritz } & - & 1 & 6 Cycles/ Byte & 6 & 2 \\
\cline { 2 - 6 } & S0_1 & 2 & 4 Cycles/ Byte & 6 & 4 \\
\hline
\end{tabular}




\subsubsection{Resource Economization in RC4-AccSuite}

This sections talks about the potential resource sharing when two or more RC4 variants are clubbed together in RC4-AccSuite. The design is incrementally built, starting with the design for $\mathrm{RC} 4$, then adding functionality for $\mathrm{RC}^{+}$on top.

\subsubsection{RC4-AccSuite Architecture (for RC4)}

RC4-AccSuite has a pipelined architecture. It is equipped with a set of 8-bit ALUs with data registers, pipeline register and memory bank. The memory bank comprises of S0 and S1 for mapping RC4_S0_1, other than K and program memory. The processor has a 6 stage pipeline, out of which the last 4 are datapath for RC4 for completing its PRGA stage (DP1-DP4). For accommodating other variants with RC4 more stages for datapath are required.

The instruction set has 6 instructions for RC4, given in Table 6.15. The nop instruction serves to relieve structural hazards due to limited ports in the processor in between multiple KSA and PRGA instructions. The set_regs 0 and set_regs1 instructions set the initial value of counter register to be 0 and 1 , respectively. The former is required before the start of SI and KSA phase while the later is required before PRGA. Fig. 6.13 shows the opcodes for these instructions as the selection of multiplexers (shown in bold font).

Table 6.15: Instruction set for RC4

\begin{tabular}{|c|c|c|}
\hline Instruction & Opcode & Comment \\
\hline nop & $0 \times 0$ & No operation \\
\hline set_regs0 & $0 \times 1$ & Initializing registers, counter $=0$ \\
\hline set_regs1 & $0 \times 2$ & Initializing registers, counter $=1$ \\
\hline init_S & $0 \times 3$ & S memory initialization $(\mathrm{SI})$ \\
\hline KSA & $0 \times 4$ & KSA phase \\
\hline PRGA & $0 \times 5$ & PRGA Phase \\
\hline
\end{tabular}

Fig. 6.13 represents the pipeline architecture of RC4-AccSuite. The memory accesses are shown as vertical arrows, read requests and writes with arrowheads pointing down while reads as pointing up. The I/Os of memory accesses are not shown to avoid unnecessary complexity. The operation division of the 6 pipeline stages is explained below.

1. FE: Fetch instruction stage uses an auto increment program counter (PC) register to access the synchronous program memory (PC). No jumps are supported.

2. DI: Decode and Initialize stage decodes the instruction and initializes counter and $j$ register as per the instruction. For init_S instruction, an increment of 2 is required for counter register for writing to two memory locations using dual porting memories. For $K S A$ and $P R G A$, increment by 1 is required. 


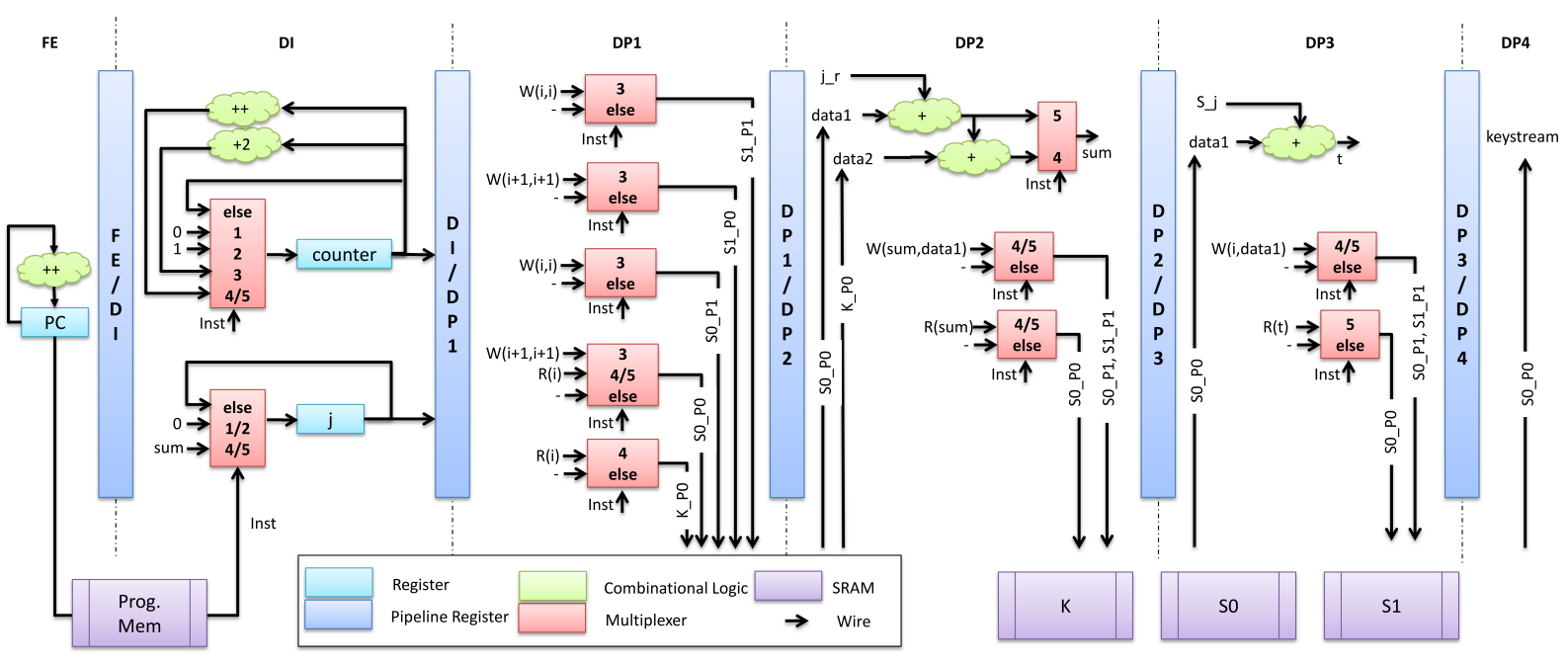

Figure 6.13: Pipeline for RC4-AccSuite supporting only RC4

3. DP1: First datapath stage for RC4. For init_S instruction, the two S memories are filled with the current value of counter (assigned to a pipeline register $i$ ) and its one incremented value in two consecutive locations of memory using both ports. Hence for filling the 256 location only 128 instructions are required. For $K S A$ and PRGA instructions, reads to $S 0$ and $K$ are also initialized.

4. DP2: For $K S A$ the values read from the $K$ and $S 0$ memory are added to the $j$ register and its value is updated. For $P R G A$, the $j$ register update does not require value read from $K$ memory. Both for KSA and PRGA instructions the value read at $S[i]$ is written to both $S$ memories with updated $j$ as the address. For these instructions, a read is also requested for the same location on $S 0$ memory. Due to a read before write priority no conflict results.

5. DP3: The second write for RC4 exchange shuffle model takes place for KSA and $P R G A$ instructions. S[j] is written to index $i$ using port 1 of both memories. The sum of S[j] and S[i] is calculated for PRGA instruction and a read to memory with $t$ as index is initiated.

6. DP4: Only PRGA instruction requires this stage to read keystream word(S[t]).

\subsubsection{Case Study: $\mathrm{RC}^{+}$in RC4-AccSuite}

To accommodate $\mathrm{RC}^{+}$in addition to $\mathrm{RC}$ in $\mathrm{RC} 4$-AccSuite, additional resources are added only if the existing logic cannot be reused. In terms of additional memories, $\mathrm{RC}^{+}{ }^{+}$SO_3 requires S2 and S3 memories as well as an IV memory. The ISA requires 7 additional instructions (in addition to the RC4 instructions in Table 6.15), given in Table6.16. For initialization phase, only S0 and S1 are used. The replication is used to boast throughput only during $P R G A^{+}$, hence for better energy utilization the actual replication is delayed till the last layer of $\mathrm{RC}^{+}{ }^{+} \mathrm{KSA}$. The first layer of $\mathrm{RC} 4^{+} \mathrm{KSA}$ is the same as RC4 KSA, hence no new instruction is added. The second layer of $\mathrm{RC}^{+} \mathrm{KSA}$ 


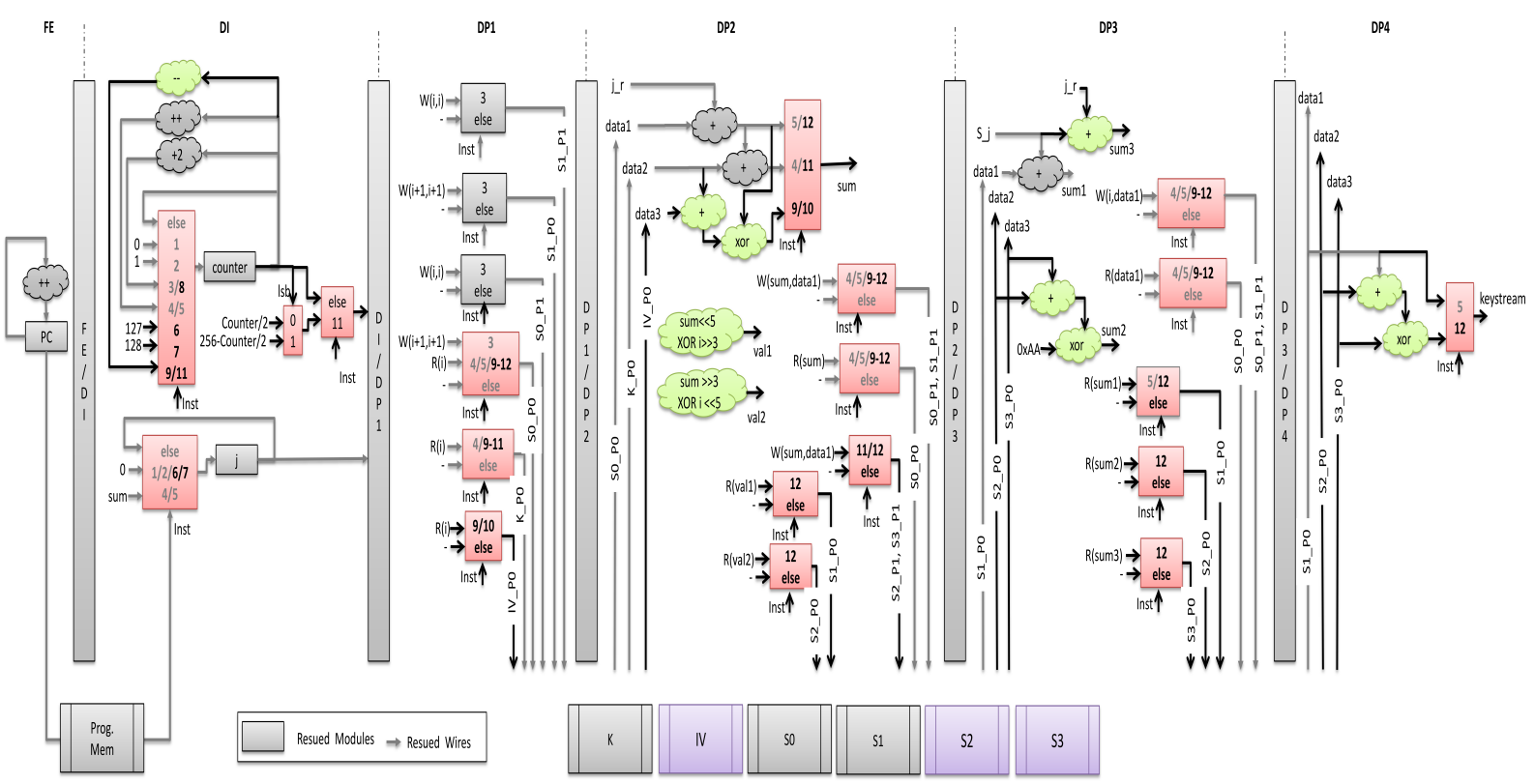

Figure 6.14: Pipeline for RC4/RC4 ${ }^{+}$-AccSuite supporting both $\mathrm{RC} 4$ and $\mathrm{RC} 4^{+}$

is requires two iterations, $K S A \_2 a$ and $K S A \_2 b$ while the third layer implementation instruction is KSA_3.

Table 6.16: Instruction set for $\mathrm{RC} 4^{+}$

\begin{tabular}{|c|c|c|}
\hline Instruction & Opcode & Comment \\
\hline set_regs2 & 6 & initializing registers, counter $=127$ \\
\hline set_regs3 & 7 & initializing registers, counter $=128$ \\
\hline$K S A \_2 a$ & 9 & KSA phase $2 \mathrm{a}$ \\
\hline$K S A \_2 b$ & 10 & KSA phase $2 \mathrm{~b}$ \\
\hline$K S A \_3$ & 11 & KSA phase 3 \\
\hline$P R G A^{+}$ & 12 & PRGA Phase \\
\hline
\end{tabular}

Fig. 6.14 shows the pipeline for RC4-AccSuite capable of executing both RC4 and $\mathrm{RC}^{+}{ }^{+}$. Here all resources shown in Fig. 6.13, that are being completely reused for $\mathrm{RC}_{4}{ }^{+}$, have been shown in gray, while the additional resources to accommodate $\mathrm{RC} 4^{+}$ has been shown in color, following the same convention as given in the legend of Fig. 6.13. Most of the multiplexers size has been increased to accommodate the new set of instructions. The reused Instruction opcodes for RC4 has been shown in gray font while the new additions are shown in black. As can be seen the FE stage is completely reused. The DI stage is however reused partially since additional logic for various KSA instructions of $\mathrm{RC}^{+}{ }^{+}$is added. The additional instructions include KSA_$2 a$ and $K S A \_2 b$ requiring counter initialization with 127 and 128 respectively, requiring two instructions set_regs2 and set_regs3 for register initialization. A decrementing counter required for $K S A \_2 a$ and $K S A \_3$ is also supported.

The next four datapath pipeline stages for $\mathrm{RC} 4^{+}$PRGA can be tallied with the memory accesses as given in Table 6.13. All the resources of DP1 stage are almost 
completely reused for $\mathrm{RC}^{+}$except the additional read request from IV memory, that was not required previously for RC4. The second datapath stage, $D P 2$, requires memory replication into S2 and S3 using port 1 for $K S A \_3$ and PRGA ${ }^{+}$as shown in Fig. 6.14. Moreover, for $K S A \_2 a$ and $K S A \_2 b, j$ register updating is carried out with one additional 8 bit adder and a XOR. For $P R G A^{+}, \mathrm{t}^{\prime}$ calculation requires two intermediate reads, $\mathbf{t}^{\prime 1}$ and $\mathbf{t}^{\prime 2}$, from register $j$ and pipeline register $i$.

In $D P 3$, calculation of $\mathrm{t}$ is reused as for $\mathrm{RC} 4$, however additional logic is required for calculating $\mathrm{t}^{\prime}$ and $\mathrm{t}^{\prime \prime}$. Respective multiple simultaneous reads are initiated using port 0 of S1, S2 and S3, thanks to the high replication factor. In DP4 datapath stage S[t], $S\left[t^{\prime}\right]$ and $S\left[t^{\prime \prime}\right]$ are read from S1_P0, S2_P0 and S3_P0, respectively and the keystream Byte for $\mathrm{RC}^{+}{ }^{+}$is calculated after an eight bit addition and XOR-ing.

\subsubsection{Instruction Datapath Reuse}

Accommodating other RC4 variants with the existing pipeline structure of RC4-AccSuite required newer instructions and even additional pipeline stages. The VMPC PRGA instruction required additional pipeline stages to accommodate multiple interdependent memory accesses. Similarly, for Spritz, the 4 DP pipeline stage don't suffice, consequently, it has 7 DP pipeline stages as shown in Fig. 6.12 .

Entire Instruction Datapath Reuse. Most of the RC4 variants reuse instructions that are part of RC4 instruction set. Two such instructions are nop and set_regs0 which are required by all the RC4 variants and hence their entire DP pipeline stages are reused. Both set_regs 0 and set_regs1 instructions are entirely reused by all RC4 variants except VMPC.

Partial Instruction Datapath Reuse. Whenever possible, pipeline datapath reuse is maximized, within instructions of one algorithm or different algorithms and even if it is possible for few pipeline stages only.

The KSA and PRGA instructions reuse the 8 bit adder in DP4 as shown in Fig. 6.13.

Similarly, PRGA and $P R G A^{+}$instructions share the calculation and reading of $\mathrm{S}[\mathrm{t}]$ in the four DP pipeline stages, as seen in in Fig. 6.14 As RC4A is a parallelized version of RC4, the logic for RC4 KSA and PRGA in all the pipeline stages is reused (except for the $j$ register update).

\subsubsection{Registers/ Memories Reuse}

For area economization, an aggressive reuse of registers, pipeline registers and memory modules is carefully designed and enabled when undertaking any RC4 variant. Since a pipelined register has more overhead than a register, its use should be carefully justified. A processor with $(n+1)$ pipelines may have each pipeline register replicated up to $n$ times. Table 6.17 shows the register/ pipeline registers and memory modules reuse in RC4-AccSuite. Please note that memory use is given as per the highest memory replication factor for each algorithm (refer Table 6.14). 
Table 6.17: Registers/ Pipeline Registers/ Memories reuse for RC4 variants

\begin{tabular}{|c|c|c|c|c|c|c|c|c|}
\hline Resource & RC4 & $\mathrm{RC}^{+}$ & VMPC & RC4A/RC4B & $\mathrm{RC} 4 \mathrm{~b}$ & NGG & GGHN & Spritz \\
\hline \multicolumn{9}{|c|}{ Registers: Name (width) } \\
\hline PC (8 bits) & $\checkmark$ & $\checkmark$ & $\checkmark$ & $\checkmark$ & $\checkmark$ & $\checkmark$ & $\checkmark$ & $\checkmark$ \\
\hline counter (8 bits) & $\checkmark$ & $\checkmark$ & $\checkmark$ & $\checkmark$ & $\checkmark$ & $\checkmark$ & $\checkmark$ & $\checkmark$ \\
\hline $\mathbf{j}$ (8 bits) & $\checkmark$ & $\checkmark$ & $\checkmark$ & $\checkmark$ & $\checkmark$ & $\checkmark$ & $\checkmark$ & $\checkmark$ \\
\hline j2 (8 bits) & $x$ & $x$ & $x$ & $\checkmark$ & $\checkmark$ & $x$ & $x$ & $\checkmark$ \\
\hline k_32 (8 bits) & $x$ & $x$ & $x$ & $x$ & $x$ & $x$ & $\checkmark$ & $x$ \\
\hline k (8 bits) & $x$ & $x$ & $x$ & $x$ & $x$ & $x$ & $x$ & $\checkmark$ \\
\hline a (8 bits) & $x$ & $x$ & $x$ & $x$ & $x$ & $x$ & $x$ & $\checkmark$ \\
\hline $\mathbf{w}$ (8 bits) & $x$ & $x$ & $x$ & $x$ & $x$ & $x$ & $x$ & $\checkmark$ \\
\hline \multicolumn{9}{|c|}{ Pipeline Registers: Name (width) } \\
\hline IR (8 bits) & $\checkmark$ & $\checkmark$ & $\checkmark$ & $\checkmark$ & $\checkmark$ & $\checkmark$ & $\checkmark$ & $\checkmark$ \\
\hline i (8 bits) & $\checkmark$ & $\checkmark$ & $\checkmark$ & $\checkmark$ & $\checkmark$ & $\checkmark$ & $\checkmark$ & $\checkmark$ \\
\hline Sj (8 bits) & $\checkmark$ & $\checkmark$ & $\checkmark$ & $\checkmark$ & $\checkmark$ & $x$ & $x$ & $x$ \\
\hline Si (8 bits) & $x$ & $x$ & $\checkmark$ & $\checkmark$ & $x$ & $\checkmark$ & $\checkmark$ & $\checkmark$ \\
\hline Si_m(24 bits) & $x$ & $x$ & $x$ & $x$ & $x$ & $\checkmark$ & $\checkmark$ & $x$ \\
\hline \multicolumn{9}{|c|}{ Memories: Name (depth) } \\
\hline K (32 words) & $\checkmark$ & $\boldsymbol{V}$ & $\checkmark$ & $\checkmark$ & $\checkmark$ & $\checkmark$ & $\checkmark$ & $\boldsymbol{\sim}$ \\
\hline IV (32 words) & $x$ & $\checkmark$ & $\checkmark$ & $x$ & $\checkmark$ & $x$ & $x$ & $x$ \\
\hline S0 (256 words) & $\checkmark$ & $\checkmark$ & $\checkmark$ & $\checkmark$ & $\checkmark$ & $\checkmark$ & $\checkmark$ & $\checkmark$ \\
\hline S1 (256 words) & $\checkmark$ & $\checkmark$ & $x$ & $\checkmark$ & $\checkmark$ & $\checkmark$ & $\checkmark$ & $\checkmark$ \\
\hline S2 (256 words) & $x$ & $\checkmark$ & $x$ & $\checkmark$ & $x$ & $\checkmark$ & $\checkmark$ & $\checkmark$ \\
\hline S3 (256 words) & $x$ & 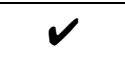 & $x$ & $\checkmark$ & $x$ & $\checkmark$ & 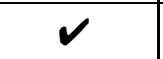 & 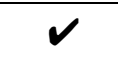 \\
\hline
\end{tabular}

- Registers reuse: RC4 requires only three registers for its execution: a program counter or $P C$, an incrementing/decrementing counter for keeping track of loop iterations, and index $j$. It is noteworthy that these registers are used by all the RC4 variants. Also, integrating $\mathrm{RC}^{+}{ }^{+}, \mathrm{VMPC}$ and NGG into the RC4-AccSuite requires no additional registers. A second index variable $j 2$ is required by three RC4 variants: RC4A for enabling two parallel PRGAs, RC4b KSA requiring an index other than $j$ (named as $t$ in [150]) and Spritz (for holding keyword $z$ ). A 32 bits register $k$ is required only for GGHN during KSA and PRGA for holding an intermediate value.

- Pipeline Registers reuse: RC4 requires three pipeline registers. First is an instruction register $I R$, optimized to the smallest width by Synopsys processor designer based on the total number of instructions, Secondly, index $i$ that takes up the counter register value and is altered/ read by multiple pipeline stages 
during KSA and PRGA. These pipeline registers are reused by all RC4 variants as shown in Table 6.17. The third pipeline register $S j$ used by RC4 is required to hold S[j], read in DP2 and added to S[i] in DP3 during PRGA. In case of VMPC, the PRGA Byte is generated before the swapping of S[i] and S[j]. Hence both $S i$ and $S j$ are saved as pipeline registers to be used during swapping. For $\mathrm{RC} 4 \mathrm{~A} / \mathrm{RC} 4 \mathrm{~B} S i$ is required in addition to $S j$ for ensuring two parallel RC4 execution. The two word variants of RC4, i.e., NGG and GGHN, a 32 bit PRGA word calculation requires a 24 bits Si_m pipeline register, other than reusing the 8 bit $\mathrm{Si}$ for the LSB of the 32 bit value.

- Memory blocks reuse: RC4 requires no IV memory. Hence for RC4-AccSuite when $\mathrm{RC}_{4}^{+}$is accommodated in RC4-AccSuite, along with RC4, all the three memories i.e., $\mathrm{K}, \mathrm{S} 0$ and $\mathrm{S} 1$ are reused along with the requirement of three new memories i.e., IV, S2 and S3. Similarly, Spritz has a 100\% reuse of three memories used by RC4.

\subsubsection{Implementation and Benchmarking}

For experimentation and modeling of configurable RC4-AccSuite, an incremental build was followed to accommodate one or more of the RC4 variants. Consequently a series of interesting design points were encountered, which were benchmarked for resource economization, power utilization and performance. The integrated configurable core of RC4-AccSuite executing RC4 and $\mathrm{RC}^{+}$is termed as RC4C-1. Following similar nomenclature, the version after integration of VMPC was called $R C 4 C-2$ and so on. Consequently $R C 4 C-7$ is the most flexible version of RC4-AccSuite, capable of being configured to execute any of the variants of $\mathrm{RC} 4$. All the designs were modeled using LISA (Section 2.3.2) and synthesized using Faraday 65nm CMOS technology library (Section 2.3.1). The best case for memory modules with column multiplexer width 4 were recorded.

\subsubsection{Throughput}

The operating frequency of any version of RC4-AccSuite is dictated by the access time of the largest memory in the memory bank, since the memory modules have a larger critical path than the core. For RC4-AccSuite, the 256 word memories S0-S3, having access time of $0.7644 \mathrm{~ns}$, indicating a maximum operating frequency of $1.3 \mathrm{GHz}$ are used. The throughput of individual cores of RC4 variants as well as throughput in any of the combination version of RC4-AccSuite is the same and is indicated by Table 6.18.

The SI, KSA and IVSA phases are together named as keystream Initialization. Due to the use of dual ported SRAMs, 256 Bytes/words initialization require no more than 128 cycles for SI. For RC4, 2 nops are inserted between all consecutive 256 KSA instructions. $\mathrm{RC}^{+}$has 3 layers of KSA, each having 256 instructions. For VMPC both KSA and an optional IVSA take 768 cycles with 7 nops in between consecutive instructions. RC4b has equal randomization sessions with KSA an IVSA, while GGHN requires 20 iterations of scrambling. For Spritz, a 32 Byte key requires 64 absorb_nibble 
instructions (with one nop after every instruction). When shuffle is required, Whip and crush of the state is called 3 and 2 times, respectively, requiring an additional $5.3 \mu s$ as initialization time.

Table 6.18: Throughput performance of various RC4 variants on RC4-AccSuite

\begin{tabular}{|c|c|c|c|c|c|}
\hline $\begin{array}{c}\text { Variant } \\
\text { Core }\end{array}$ & $\begin{array}{c}\text { SI } \\
(\text { Cycles })\end{array}$ & $\begin{array}{c}\text { KSA+IVSA } \\
(\text { Cycles })\end{array}$ & $\begin{array}{c}\text { Initialization } \\
(\mu s)\end{array}$ & \multicolumn{2}{|c|}{$\begin{array}{c}\text { Throughput } \\
(\text { Cycles } / \text { Byte })\end{array}$} \\
\hline RC4 & 128 & $256 \times 2$ & 0.39 & 2 & 5.20 \\
\hline RC4 $^{+}$ & 128 & $256 \times 3 \times 2$ & 1.18 & 2 & 5.20 \\
\hline VMPC & 128 & $768 \times 2 \times 7$ & 8.27 & 7 & 1.49 \\
\hline RC4A/B & 128 & $256 \times 2$ & 0.39 & 1 & 10.40 \\
\hline RC4b & 128 & $256 \times 3 \times 2$ & 1.18 & 2 & 5.20 \\
\hline NGG & 128 & $256 \times 2$ & 0.39 & 0.75 & 13.87 \\
\hline GGHN & 128 & $256 \times 2 \times 20$ & 7.88 & 0.75 & 13.87 \\
\hline Spritz & 128 & $32 \times 2 \times 2$ & 0.098 & 4 & 2.6 \\
\hline
\end{tabular}

An interesting observation is that the throughput of $\mathrm{RC} 4$ and $\mathrm{RC}^{+}{ }^{+}$(after memory replication) is the same, in-spite of the added security margin for the later. The parallelization for RC4A and RC4B doubles the throughput compared to RC4. The word variants have the highest throughput performance since they generate a 32 bit word per PRGA instruction.

\subsubsection{Area}

Table 6.19 gives the area estimates for RC4-AccSuite; the first half of the table specifies individual pipelined cores of RC4-AccSuite while the second half refers to the configurable incremental versions. Dual ported, Byte wide SRAMs were considered having 32 words for K and IV memories (4.609 KGE for each) and 256 words for S0-S3 (7.889 KGE for each). The total area is clearly dominated by the memory area.

The extent of resource sharing and a consequential core area economization can be visualized in Fig. 6.15. The area of a single algorithm core and a configurable RC4C-x core is compared against the sum of area of single algorithm cores that this version is able to support, i.e., the sum of $\mathrm{RC} 4$ and $\mathrm{RC} 4^{+}$cores are added up and compared with the RC4C-1 core area and is found to be $12.3 \%$ less due to aggressive resource reuse. This area economization margin increases as more flexible versions of RC4C-x are analyzed, from left to right in Fig. 6.15. Hence for RC4C-6 this margin grows to reach $41.12 \%$, justifying the need and rationale of developing configurable cores.

A noteworthy point is that the area economization calculation for RC4-AccSuite core excludes the memory bank contribution, considering that the area saving for RC4C-7 reaches up-to 79\%. Today's heterogeneous systems extensively employ large memory banks time-shared between multiple arbiters. Consequently its only fair to 
Table 6.19: Area (KGE) for RC4-AccSuite versions

\begin{tabular}{|c|c|c|c|c|c|}
\hline $\begin{array}{c}\text { RC4-AccSuite } \\
\text { Version }\end{array}$ & \multicolumn{4}{|c|}{ Core Area } & Memory \\
Combinational & Sequential & Total & $\begin{array}{c}\text { Area } \\
\text { Area }\end{array}$ \\
\hline RC4 & 0.43 & 0.48 & 0.914 & 20.387 & 21.301 \\
\hline RC4 ${ }^{+}$ & 1.158 & 0.468 & 1.627 & 40.774 & 42.401 \\
\hline VMPC & 1.080 & 1.249 & 2.329 & 17.107 & 19.436 \\
\hline RC4A/B & 0.996 & 0.534 & 1.530 & 36.165 & 37.695 \\
\hline RC4b & 1.705 & 0.718 & 2.423 & 24.996 & 27.419 \\
\hline NGG & 2.216 & 1.087 & 3.303 & 36.165 & 39.468 \\
\hline GGHN & 2.164 & 1.418 & 3.582 & 36.165 & 39.747 \\
\hline Spritz & 1.490 & 0.847 & 2.336 & 20.387 & 22.723 \\
\hline \hline RC4C-1 & 1.534 & 0.694 & 2.228 & 40.774 & 43.002 \\
\hline RC4C-2 & 2.644 & 1.805 & 4.450 & 40.774 & 45.224 \\
\hline RC4C-3 & 2.967 & 2.181 & 5.149 & 40.774 & 45.923 \\
\hline RC4C-4 & 4.121 & 2.172 & 6.293 & 40.774 & 47.067 \\
\hline RC4C-5 & 4.392 & 2.907 & 7.299 & 40.774 & 48.073 \\
\hline RC4C-6 & 6.012 & 3.236 & 9.249 & 40.774 & 50.023 \\
\hline RC4C-7 & 8.654 & 2.904 & 11.558 & 40.774 & 52.332 \\
\hline
\end{tabular}

- Single algo. core $\square \mathrm{RC} 4 \mathrm{C}-\mathrm{x}$ core $\square$ Sum of single algo. cores

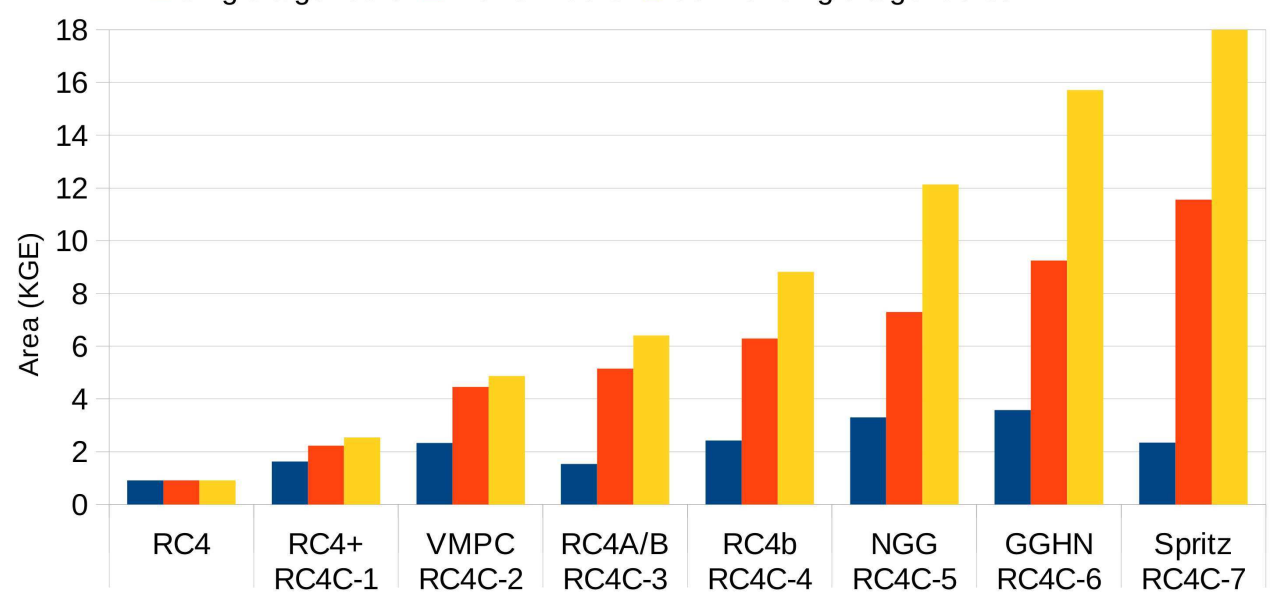

Figure 6.15: RC4-AccSuite core Area economization

consider the core of a crypto-processor alone for resource budgeting as the memory bank is not exclusively used by it. Moreover, for most of the coarse grained hardware platforms like FPGAs and CGRAs, block RAM modules are available as macros, that may be configured to desired sized memories and will go wasted when unused.

\subsubsection{Power}

The power consumption of an algorithm on a core is a function of the complexity of the design and the clock frequency. Table 6.20 specifies the power consumption of RC4 when run on various versions of RC4-AccSuite. From the Faraday memory compiler, the dynamic power of 32 Byte and 256 Byte memory is reported to be 4.94 
Table 6.20: Power consumption of RC4 algorithm for RC4-AccSuite versions

\begin{tabular}{|c|c|c|c|c|}
\hline RC4-AccSuite & \multicolumn{2}{|c|}{ Static (uW) } & Dynamic $(\mathrm{mW})$ & Total $(\mathrm{mW})$ \\
\hline Version & Memory & Core & Core & \\
\hline $\mathrm{RC} 4$ & 6.27 & 6.27 & 726.57 & 732.84 \\
\hline RC4C-1 & 12.53 & 12.49 & $1,107.90$ & $1,120.39$ \\
\hline RC4C-2 & 12.53 & 19.94 & $2,052.70$ & $2,072.64$ \\
\hline RC4C-3 & 12.53 & 21.09 & $2,358.70$ & $2,379.79$ \\
\hline RC4C-4 & 12.53 & 28.05 & $2,444.90$ & $2,472.95$ \\
\hline RC4C-5 & 12.53 & 34.26 & $3,274.40$ & $3,308.66$ \\
\hline RC4C-6 & 12.53 & 45.10 & $3,754.30$ & $3,799.40$ \\
\hline RC4C-7 & 12.53 & 65.48 & $6,571.00$ & $6,636.48$ \\
\hline
\end{tabular}

$\mathrm{PJ} /$ access and $5.53 \mathrm{PJ} /$ access, respectively, which is not included in the Table 6.20. The total power for the same algorithm shows an increasing trend as the flexibility of the underlying circuit increases.

A similar trend is seen from Fig. 6.16, showing the dynamic power of RC4AccSuite versions when various variants are run on it. All the power estimations include the initialization of stream ciphers and the generation of 1024 bits of keystream. The lower power budget utilized by VMPC is due to its least unshared resources i.e., 7 stage datapath pipeline while all the rest of the variants need no more than 4 .

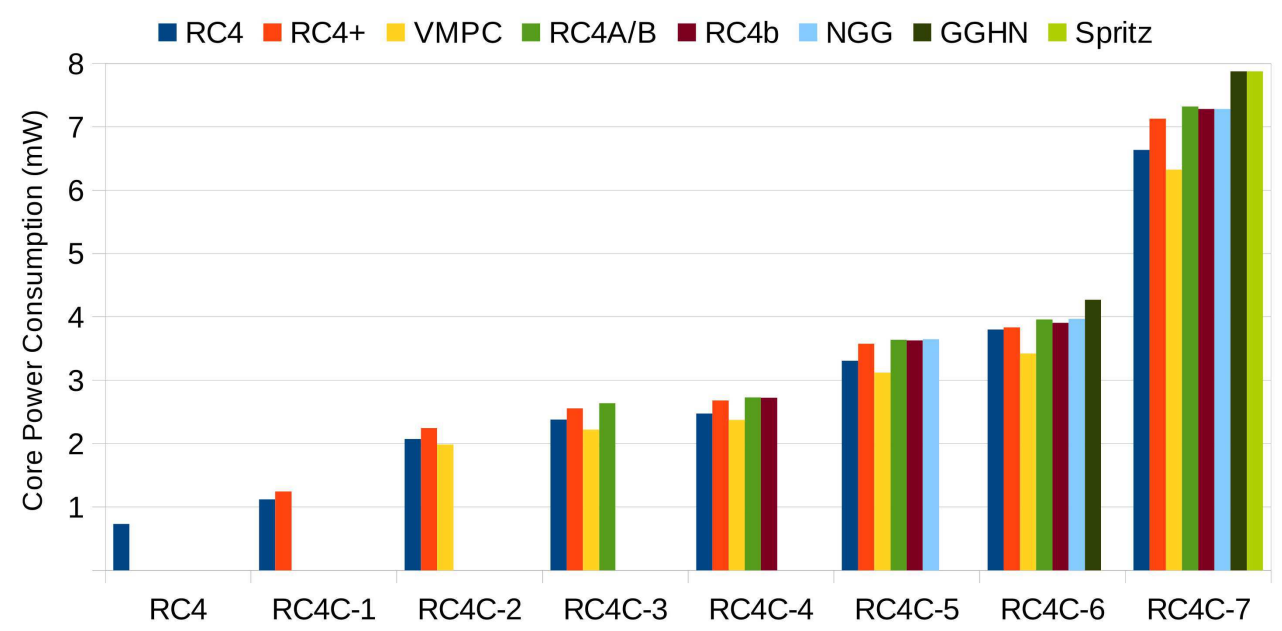

Figure 6.16: Power consumption in RC4-AccSuite versions

\subsubsection{Comparison with Hardware Performance}

The comparison of the implementations is carried out with the best known SRAM based hardware implementations of RC4 variants against area-efficiency (throughput per area, TPA), as reported in Table 6.21. The highest throughput reported for RC4 implementation on a Xilinx FPGA claims a throughput of 0.64 Gbps, which is about $8 \times$ slower than RC4-AccSuite's RC4 throughput. A configurable core supporting both $\mathrm{RC} 4$ and a less computationally intensive version of $\mathrm{RC}^{+}$, i.e., $\mathrm{PRGA}^{\alpha}$ is reported 
Table 6.21: Area comparison of RC4-AccSuite versions $(\mathrm{C} / \mathrm{B}=$ cycles per Byte, TPA $=$ Throughput per Area )

\begin{tabular}{|c|c|c|c|c|c|c|c|c|}
\hline \multirow{2}{*}{$\begin{array}{l}\text { Variant } \\
\text { Name }\end{array}$} & \multirow{2}{*}{$\begin{array}{l}\text { Implementation } \\
\text { Platform }\end{array}$} & \multirow{2}{*}{$\begin{array}{l}\text { Freq. } \\
\mathrm{MHz}\end{array}$} & \multirow{2}{*}{$\begin{array}{l}\text { Area } \\
\text { KGE }\end{array}$} & \multicolumn{2}{|c|}{ KSA+IVSA } & \multicolumn{2}{|c|}{ Throughput } & \multirow{2}{*}{$\begin{array}{c}\text { TPA } \\
\mathrm{Mbps} / \mathrm{GE}\end{array}$} \\
\hline & & & & Cycles & $\mu \mathrm{s}$ & $\mathrm{C} / \mathrm{B}$ & Gbps & \\
\hline RC4 [215] & Xilinx XC4kE & 160 & - & 642 & 0.79 & 2 & 0.64 & - \\
\hline RC4 [40] & 65nm CMOS & 810 & 22.09 & 512 & 0.63 & 2 & 3.24 & 0.15 \\
\hline RC4 (this work) & $65 \mathrm{~nm}$ CMOS & 1300 & 21.30 & 512 & .39 & 2 & 5.20 & 0.24 \\
\hline $\begin{array}{l}\text { RC4, KSA+, } \\
\text { PRGA }^{\alpha}[40]\end{array}$ & 65nm CMOS & 810 & 35.73 & 1536 & 1.90 & 2 & 3.24 & 0.09 \\
\hline RC4C-1 (this work) & 65nm CMOS & 1300 & 45.22 & 1536 & 1.18 & 2 & 5.20 & 0.11 \\
\hline RC4A [6] & APEXTM 20K200E & 33.33 & - & 2042 & 61.27 & 1.5 & 0.18 & - \\
\hline RC4A (this work) & $65 \mathrm{~nm}$ CMOS & 1300 & 37.70 & 512 & .39 & 1 & 10.40 & 0.28 \\
\hline
\end{tabular}

in [40]. Is is the fastest CMOS implementation of RC4 and also reports a 2 cycles/ Byte throughput. However, due to aggressive resource sharing and memory replication, RC4C-1 justifiably outperforms in area-efficiency. Their storage class memory (SCM) based RC4 implementation results in an encryption speed of 3.24 Gbps with 22.09 KGE of area resulting in a TPA of 0.30 . The fastest CMOS based RC4 implementation on a comparable technology using SCMs for S-boxes reports 17.76 Gbps with an area of $50.58 \mathrm{k}$ gates [91]. This design has a better TPA (0.35) than RC4-AccSuite implementation however does not set a good framework for flexibility extensions. Firstly because of its large area budget primarily due to numerous access ports per SCM, which may not be fully utilized of other RC4 variants and secondly because of the unlikely re-usability of the algorithm specific data coherency checks logic.

A CMOS implementation for no other RC4 variants is reported. For RC4A, an Altera FPGA implementation is reported with $0.18 \mathrm{Gbps}$ of throughput performance [6], that is around $57 \times$ slower than RC4-AccSuite's RC4A throughput. What remains incomparable is the extent of area economization due to resource re-usability due to absence of flexible, configurable RC4-AccSuite versions that have not been taken up for hardware implementation before.

\subsubsection{Comparison with Software Performance}

Software performance on general purpose computers for various RC4 variants is tabulated in Table 6.22. RC4-AccSuite renders initialization for RC4 and $\mathrm{RC} 4{ }^{+}$that is about $15 \times$ faster than the one reported in [147]. Similarly, RC4-AccSuite performance for NGG and GGHN is more than $4.3 \times$ faster than their respective references $[78,160]$. Their initialization time is not specified for comparison. Spritz on RC4-AccSuite has PRGA performance that is $27 \times$ faster than the one reported on a Macbook Air [186]. For VMPC on RC4-AccSuite, the KSA and PRGA phases are comparable to the reported software performance [231] due to the high dependence of sequential memory accesses in VMPC function, rendering slow performance due to nops between two PRGA instructions. 
Table 6.22: RC4-AccSuite versions software performance

\begin{tabular}{|c|c|c|c|c|c|}
\hline RC4 & \multirow[t]{2}{*}{ Platform } & \multicolumn{2}{|c|}{ KSA+IVSA } & \multicolumn{2}{|c|}{ Throughput } \\
\hline Variant & & Cycles & Time $(\mu \mathrm{s})$ & Cycles & Gbps \\
\hline RC4 [147] & Intel(R) Pentium(R) 4 CPU, & 16945 & 6.05 & 14.39 & 1.56 \\
\hline $\mathrm{RC}^{+}[147]$ & 2.8 GHz Clk, 512 MB DDR RAM & 49824 & 17.79 & 24.51 & 0.91 \\
\hline VMPC [231] & Intel(R) Pentium(R) 4 CPU, $2.66 \mathrm{GHz}$ & 8580 & 3.2 & 12.70 & 1.68 \\
\hline NGG [160] & 32-hit PC & - & - & - & 4.83 \\
\hline GGHN [78] & & - & - & - & 4.98 \\
\hline Spritz [186] & Macbook Air (1.8GHz Core i5) & - & - & 24 & 0.09 \\
\hline
\end{tabular}

\subsection{Concluing Remarks}

In the context of flexible cryptographic accelerators, this work undertakes the design of RC4-AccSuite and CoARX, configurable co-processors for the family of RC4-like ciphers and for ARX based ciphers, respectively. Their flexibility stands out due to their ability to switch to another algorithm on-the-fly as per the user requirements of throughput, power or security changes while their instruction set enable mapping future variants of these cryptographic classes as well. These endeavors exhibit comparable/ better area-efficiency against dedicated hardware accelerators and significantly higher throughput compared to off-the-shelf processor-based implementations. Additionally, the algorithmic perspective of flexibility provides unique advantage against general and specific cryptanalysis. 


\section{Chapter 7}

\section{Study of Scalability}

Scalability is a desirable property of a system that ensures a performance improvement proportional to the system's resource increase. Modern Graphics Processor Units (GPUs) offer a many-core architecture on which parallel homogeneous threads are executed in a Single Instruction Multiple Thread (SIMT) fashion. Many cryptographic algorithms have been reported to achieve remarkable performance speedups, especially block ciphers. This chapter discusses the optimal mapping of two eSTREAM [65] portfolio stream ciphers, namely, HC-128 (and HC-256) and Salsa20 on NVIDIAs GPUs using CUDA programming framework. Various strategies for optimal tuning of device and compiler specific optimizations are discussed and the results for very long message streams are benchmarked. The results have been published as conference papers $[118,121]$.

\subsection{Motivation}

Performance enhancement on a GPU is a function of the extent of parallelism within the application. For block ciphers, the encryption/decryption of long messages of lengths beyond one data block, the plaintext can be partitioned into cipher's block size chunks and then encrypted (in Electronic Codebook (ECB) or Counter (CTR) mode of operation). This parallelization is however, not possible for some modes of operation in block ciphers due to the inter-dependency between consequent data blocks (ref. Table 3.1). In comparison to block ciphers, stream ciphers are simpler and faster and typically execute at a higher speed than block ciphers [63]. Using a block cipher where a stream cipher could suffice is an overkill and consequently, results in a lower throughput performance in comparison. For example, on a Core 2 Intel processor, 20 rounds of Salsa20 stream cipher run at 3.93 cycles/byte, while 10 rounds of AES block cipher are reported to run more than twice as slow at 9.2 cycles/byte for long data streams (bitsliced AES-CTR) [19].

What makes the scalability study for stream ciphers imperative is that very few endeavors for their mapping on GPUs have been reported in scientific literature. The reason is that unlike the parallelizable modes of operations defined for block ciphers, most stream ciphers do not have the liberty of employing the divide and rule policy on chunks of plaintext and exhort parallelism on GPUs. For example, in case of HC-128 [224], its highly iterative structures has inter-dependencies on subsequent keystream values generated. However, out of the four eSTREAM software profile ciphers, Salsa20 [21] is the only cipher that has no chaining or dependence between blocks of data during encryption / decryption and hence can be parallelized. This 
work takes up both of these eSTREAM [65] finalists lying on the extreme ends of scalability and try to map them on GPUs as best as possible.

\subsection{Major Contributions}

The major contributions of this work are summarized as follows.

1. The opportunities and hurdles in the parallelism of the two stream ciphers are systematically analyzed in context to be mapped on GPUs. This study can serve as a guide for other cryptographic functions mapping on GPUs.

2. A batch processing framework is proposed to avoid the overhead for threads switching, applicable to all parallelizable cryptographic task (e.g., Salsa20) in a hybrid CPU-GPU environment. An autotuning framework applicable to parallelizable cryptographic tasks is developed for fast device portability and automatic selection of application-specific, device-specific and compiler-specific optimization parameters. The peak throughput performance surpasses the fastest GPU based performance reported so far for all stream ciphers (both hardware and software) in the eSTREAM portfolio [118,162,204], as well as AES in CTR mode [107].

3. Task paraellelization is carried out at the expense of memory usage in case of HC series of stream ciphers to diminish the effect of data dependencies in case of HC-128. Throughput curves for very long message streams encrypted by HC128 , with and without memory transfers for a single or multiple streams of data are benchmarked.

\subsection{CUDA Programming Model Overview}

CUDA defines a convenient programming model for heterogeneous computing environment for a CPU host and GPU device. This section briefly presents NVIDIA GPU architecture and its programming environment. The reader is kindly referred to CUDA C programming guide [170] and Fermi Architecture manual [168] for more information.

\subsubsection{Execution Model}

CUDA device execution model is depicted in Fig. 7.1. Parallel portions of an application, executed on the device are called kernels. A Kernel call launches a number of threads, each executing the same code but having a unique threadID. Threads are forwarded to the CUDA device in groups called warps for execution. A threadblock is a batch of threads that may or may not cooperate with each other by sharing data or by synchronizing their execution. Threads from different threadblocks cannot cooperate.

Kernels are launched in grids for execution, comprising of one or more threadblocks. The grid dimensions are specified by blocksPerGrid and threadsPerBlock. A 
CUDA device consists of several Streaming Multiprocessors (SM), each responsible for handling one or more blocks in a grid. Threads in a block are not divided across multiple SMs.
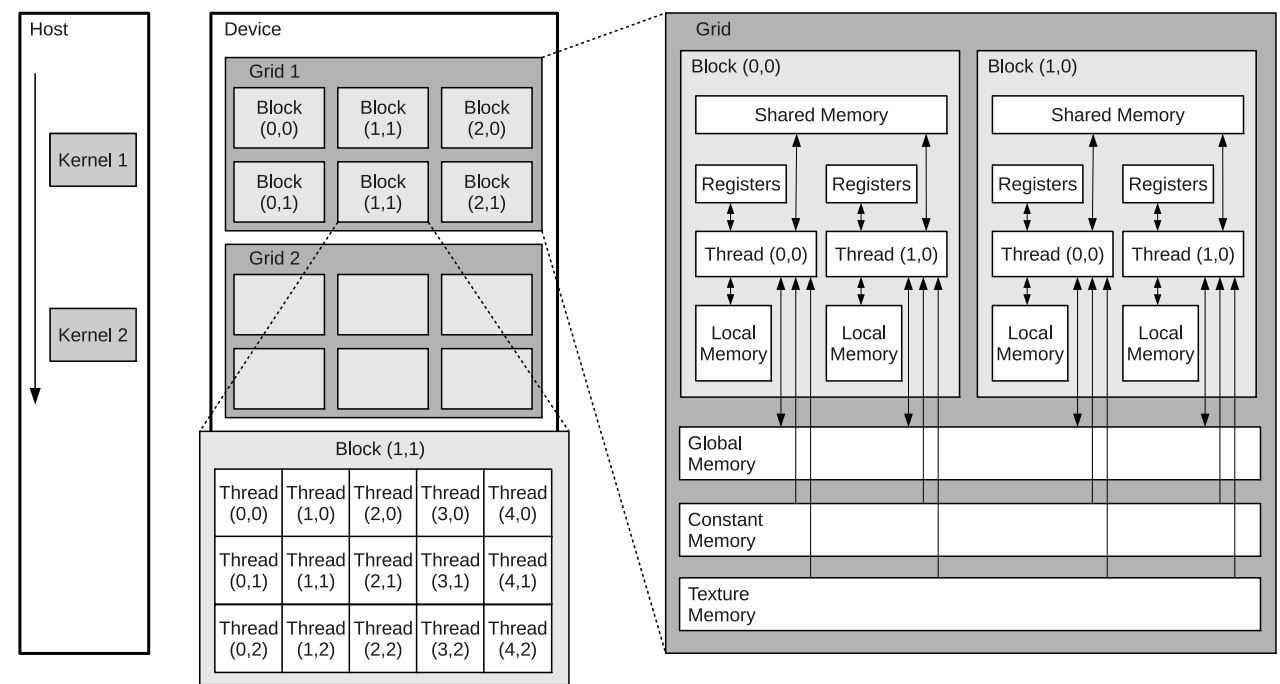

Figure 7.1: CUDA GPU execution model [170]

\subsubsection{Memory Model}

CUDA provides explicit methods to organize memory architecture. Local variables within a kernel reside in registers (regs) or in the off-chip local memory (lmem). Shared memory (shmem) is shared by each threadblock. Global memory (gmem) is accessible by all threads as well as host. The lifetime of global memory is from allocation to de-allocation by the host. However, for the other memories mentioned, the lifetime is only during the kernel execution. Other than these memories, each thread within a grid can access read-only, constant and texture memories. These memories can be modified from the host only, and are useful for storing immutable data structures like lookup tables. The performance of any algorithmic implementation on the GPUs depends heavily on the proper utilization of this memory hierarchy.

\subsection{Block Ciphers Performance Acceleration on GPUs}

After NVIDIA introduced a general purpose parallel computing platform namely Compute Unified Device Architecture (CUDA) in November 2006, many cryptographers harnessed GPUs for acceleration. The earliest successful effort of AES acceleration on GPUs, that outperformed CPU in throughput, was presented by Manavski [149] who reported a throughput of 8.28 Gbps for AES-128 encryption on NVIDIA GeForce 8800. His work was later criticized for having half of the throughput rates that it could achieve by using shared memory instead of constant memory for T-boxes [25]. A more recent work by Iwai et al. [107] reported 35 Gbps of throughput 
for AES encoding on NVIDIA GeForce GTX285 by exploiting memory granularity for independent threads.

Several endeavors undertook more than one cipher to present a suite of CUDA based crypto accelerator application. Liu et al. [142] studied the effect of number of parallel threads, size of shared memory for lookup tables and data coalescing in device memories for several block encryption algorithms (AES, TRI-DES, RC5, TWOFISH) processing on GPU using CUDA. Nishikawa et al. [163] targeted five 128-bit symmetric block ciphers from an e-government recommended ciphers list by CRYPTREC in Japan and achieved substantial speedup. The limitations of parallelization opportunities in stream ciphers contribute to the absence of reported efforts of mapping stream ciphers on GPUs.

\subsection{Mapping Salsa20 Stream Cipher on GPUs}

Salsa20 is an additive stream cipher for which the keystream generation is independent of the plaintext, i.e., ciphertext is generated by simply XOR-ing with the plaintext. It behaves like a block cipher, where each block takes a nonce, a secret key, constants and a counter to combine them and generate a block of keystream. The description of the Salsa20 is given in Appendix C.5.

\subsubsection{Analyzing Parallelism Opportunities of Salsa20}

For Salsa20, two levels of parallelism is observed.

\subsubsection{Functional Parallelism}

As evident from Appendix C.5, each block of 64 bytes of Salsa20 keystream can be independently generated and mixed with data to get the ciphertext. Salsa20/ $r$ has $r / 2$ DoubleRounds, each comprising of a ColumnRound and a RowRound. These Column or Row-Rounds undergo 4 ARXrounds for each row/column. Hence a total of $16 \times r$ invertible ARXrounds complete the keystream generation for one block of Salsa20/r. A CUDA compatible device, capable of launching $t$ parallel threads, each undertaking one data block of plaintext, will give a throughput of $(t \times 64) /(16 \times r \times \alpha)$ Bytes $/ \mathrm{sec}$ if $\alpha$ is the time taken for one ARXround as depicted in Fig. 7.2. The final addition of DoubleRound output with the state vector for keystream generation is ignored since its overhead is negligible in comparison.

\subsubsection{Data Parallelism}

In Salsa20, each QuarterRound operates on either a row or a column of the $4 \times 4$ array. Each of the four ARXrounds constitutes of a QuarterRound, modifying exactly one value of that row or column. Hence 4 parallel QuarterRounds can be executed due to absence of inter-column/row dependence. Consequently, $16 \times r$ transformations of one Salsa20 block can be broken down as $4 \times r$ transformations mapped on 4 parallel threads giving a throughput of $(t \times 64) /(4 \times r \times \alpha)$ Bytes/sec, or 4 times higher 


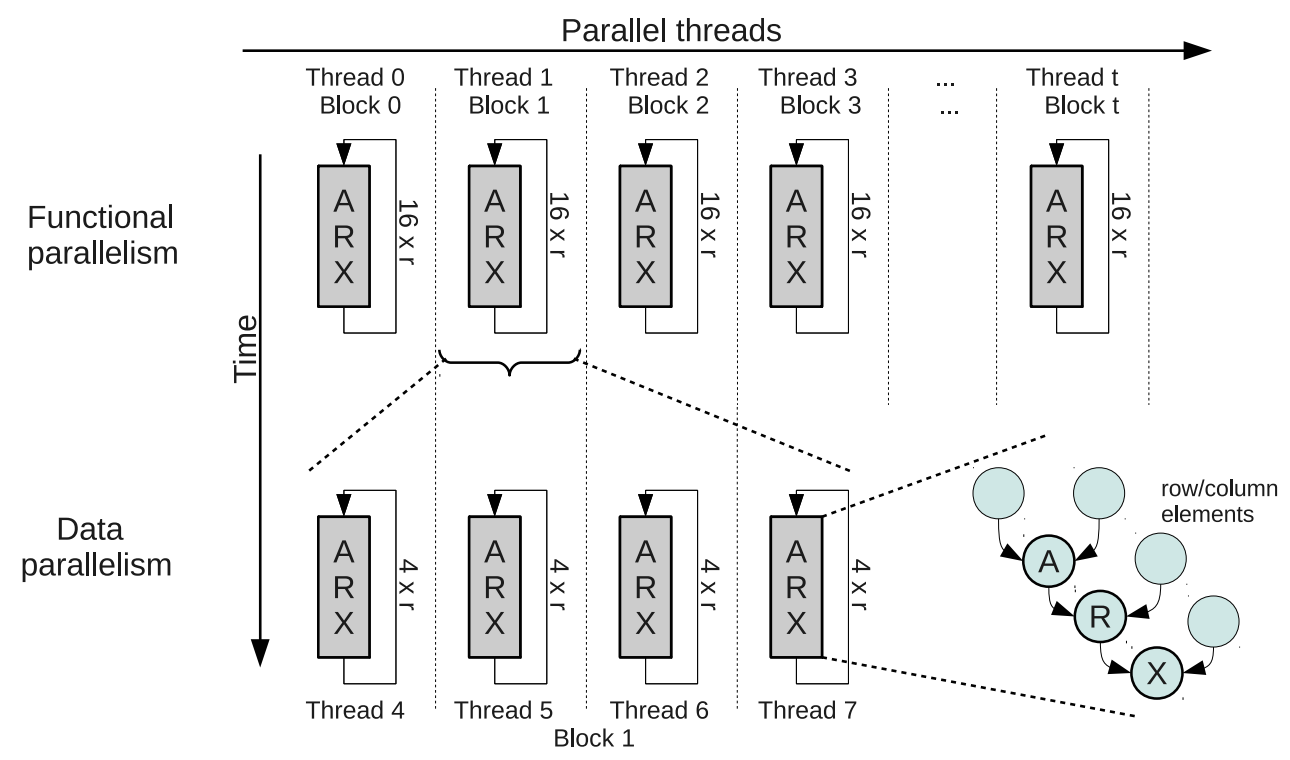

Figure 7.2: Parallelism in Salsa20/r

than a single thread per block mapping. Exploiting further parallelism within one ARXround is not possible due to dependence of XOR $(\mathrm{X})$ operation on the output of rotation (R) and addition (A) as shown in Fig. 7.2.

For coding Salsa20 kernels employing functional parallelism (one thread per data block), internal registers and shared memory were used for storing results of $X$ and DoubleRounds respectively. For manipulating data parallelism (four threads per data block) inter-thread communication is required within the threads of a threadblock. However, this thread-synchronization makes the implementation lag behind in performance compared to single thread per block implementation. Experimentation of mapping AES on GPUs with different granularities also conform to similar findings, as the best throughput performance is achieved when no synchronization is required between different threads $[107,149]$. Another reason for avoiding intra-block synchronization is that the limited number of shared memory limits instruction-level parallelism by restricting the number of threads launched, lowering occupancy. Hence due to its superior throughput performance, only the single thread per data block implementation is considered.

\subsubsection{Batch Processing Framework}

Since Salsa20 algorithm is a classic case of a parallelizable application a batch processing framework of operation is recommended. It is termed batch processing, since a batch of threads work simultaneously to encrypt one data block each and iterating in a loop for encryption of more plaintext. The batch of active threads die when all the data to be encrypted is exhausted. 


\subsubsection{CPU-GPU Interaction}

Algorithm 3 explains the batch processing framework for encryption or decryption in a hybrid environment (CPU-GPU). Plaintext $(P)$, given as 1-D data is the input to the application. The byte-length of a data block for encryption or decryption is called the blocksize. The initial state vector $(X)$ is set up at the host machine using algorithm specific Initialization routine and transferred to the global memory (gmem). Assuming encryption of $P$ having size larger than global memory ( $g m e m$ ) size, $P$ is divided into chunks equal to size of gmem, termed as $P_{k}$. Every $k^{\text {th }}$ iteration encrypts a portion of plaintext $P_{k}$ into an equal sized ciphertext $C_{k}$ (line no. 3). For simplicity, the total size of plaintext is assumed to be a multiple of the size of gmem, in case of non-conformity, the number of data blocks forwarded to kernel for encryption is changed to the residue after division with size (gmem) in the last iteration. For Salsa20, $X$ is a 16-word array and its subscript represents its existence location, i.e., $h, g, s, r$ representing host, global memory, shared memory and registers respectively. After the transfer of $P_{k}$ to device's gmem, launch of kernel is kick-started in an iterative fashion. One batch of threads or threadsPerGrid, executed in parallel on device, is blocksPerGrid $\times$ threadsPerBlock. In every iteration, when the kernel call is terminated, gmem contains the cipher text, that must be read out by the host (line no. 7) before writing the next plaintext chunk into the device memory (line no. 4).

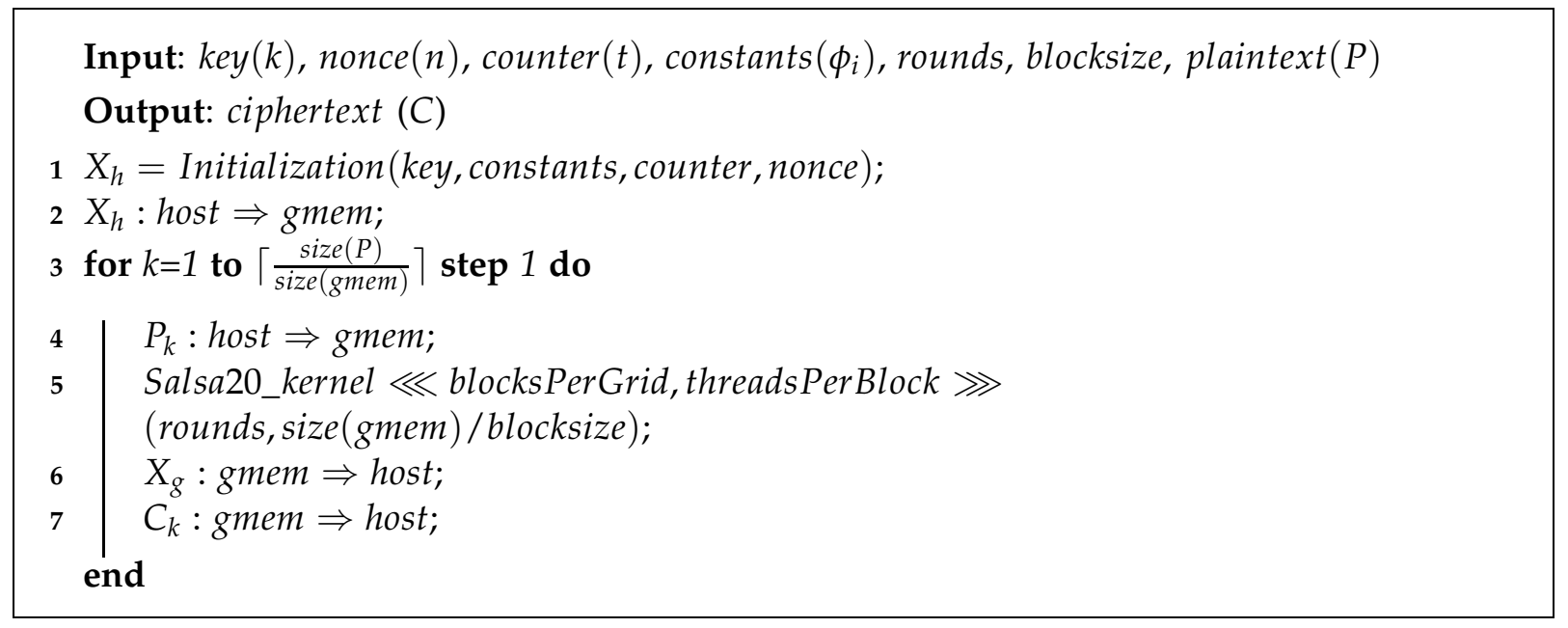

Algorithm 3: Batch processing for a cryptographic kernel

\subsubsection{The CUDA Kernel}

Algorithm 4 is a pseudo-code for the CUDA kernel call and is executed on the GPU device. Two local variables called counter and batch are declared and initialized, containing the unique threadID and the total number of threads in a batch respectively. Variable counter is used to update the counter in the state vector of Salsa20, incremented by the variable batch after every iteration. When a thread finishes encrypting a block, it encrypts again the block corresponding to that thread index plus the total number of active threads running (batch), which is constant and device dependent. 


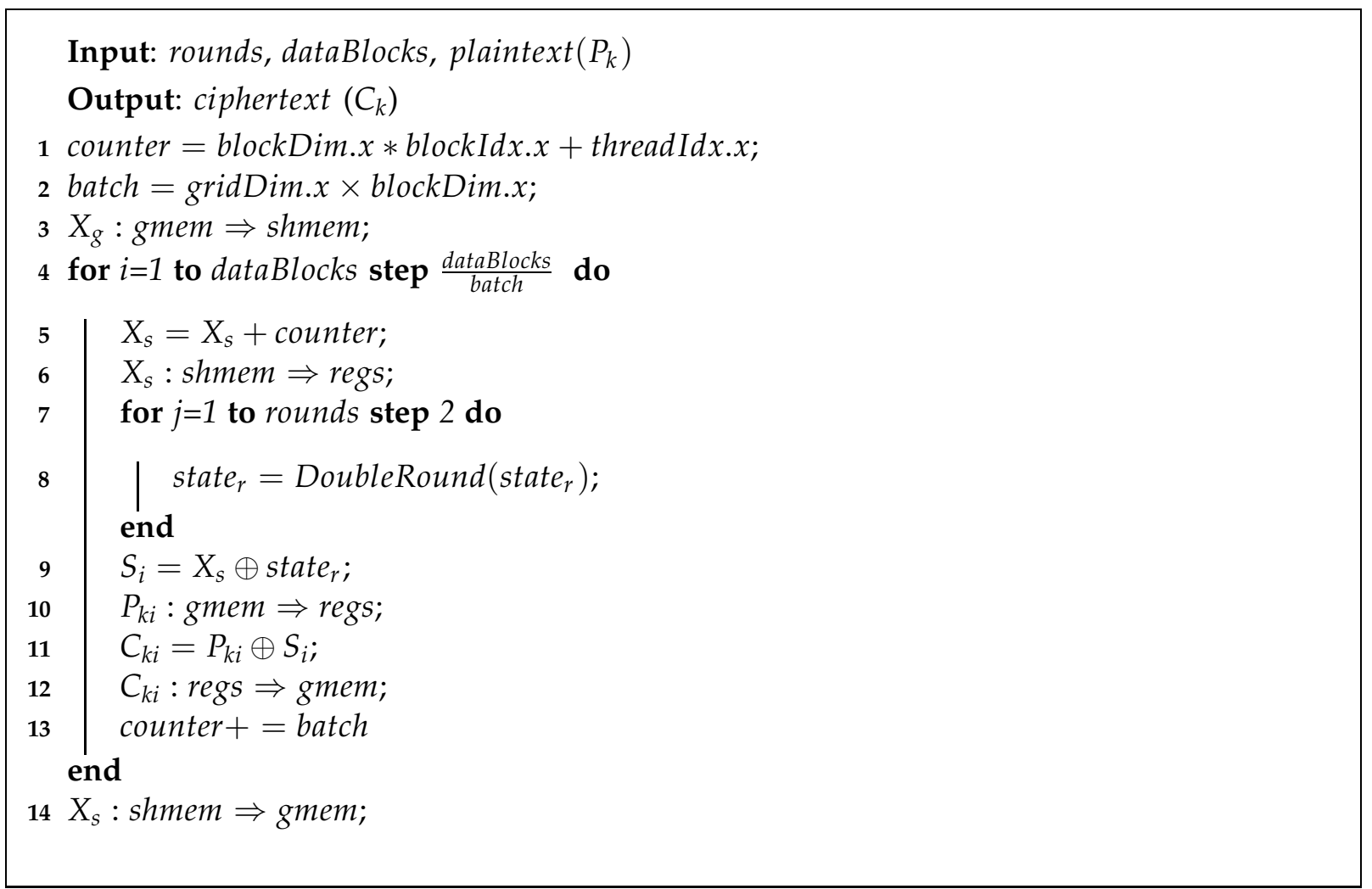

Algorithm 4: Salsa20 kernel pseudo-code on the GPU device

The state vector, residing in global memory, is first copied to faster shmem. As the size of global memory of newer NVIDIA GPUs is in GBs, a single batch of parallel threads each encrypting one data block, will not finish up the $P_{k}$, requiring iterations over variable $i$, as given in line no. 4 . Here too, for the sake of simplicity, the number of dataBlocks forwarded to the kernel for encryption or decryption is considered to be a multiple of batch of threads. In case of non-conformity, the pseudo code can be modified to launch lesser number of threads in the batch in the last iteration. The state vector is updated with the counter value as given in line no. 5. Since threadID is different for each thread in a batch, all threads get a different state vector. The

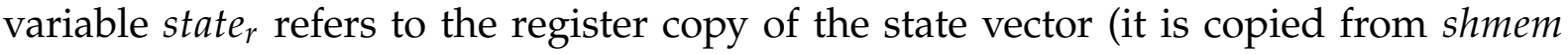
to regs in line no. 6).

The value of rounds is either 8, 12 or 20 for various flavors of Salsa20/r. A copy of state $_{r}$ in thread-local registers apply DoubleRound transformations for $\frac{\text { rounds }}{2}$ times. One block of keystream, generated by XOR-ing the state vector with its transformed copy in local registers (line no. 9), is held in $S_{i}$. The last step is the encryption of the plaintext with the generated keystream. Plaintext is read from gmem, one block at a time $\left(P_{k i}\right)$, XOR-ed with the generated keystream to produce a block of ciphertext $\left(C_{k i}\right)$ and then is written back to gmem. Saving of state vector into gmem is required before exiting the kernel, since its lifetime in shared memory lasts only as long as threadblock's lifetime. 


\subsubsection{Programming Recommendations}

CUDA programmers are recommended to follow the guides $[169,170]$ to achieve the best performance. Some more relevant recommendations are summarized below for good throughput performance when Algorithm 3 and 4 are mapped onto a GPU.

\subsubsection{Avoiding threadBlock switching overhead}

Each kernel launch on the device bears overheads of a kernel call, memory allocation and argument copying into the device. If the amount of work per kernel is small in comparison to the total workload, the run-time of the application is dominated by these overheads instead of the actual computation time. In order to decrease these overheads, the amount of work per kernel call should be increased. Hence iterations computed inside a kernel call (loop indexed with $i$ in Algorithm 4) continue as long as the entire workload is finished, instead of launching of new batch of threads. This strategy amortizes the overhead of multiple kernel calls across more computation and boosts throughput.

\subsubsection{Reuse Memory}

For cryptographic applications, the plaintext $P_{k}$ once handed over to the device is not needed back by the host device. A prudent decision is to overwrite the plaintext with ciphertext in the gmem. It saves the iterations of loop indexed by $k$ by half in Algorithm 3.

\subsubsection{Data Coalescing}

Global memory accesses incur a 100x access penalty compared to kernel local registers. If these accesses are close to each other and dispatched in a group, they are coalesced as a single access. The device can read 4-byte, 8-byte, or 16-byte words from global memory into registers in a single instruction. Mixing of plaintext for generating ciphertext requires reading, XOR-ing with keystream and writing back into the global memory as given by line no.s 10,11 and 12 respectively (in Algorithm 4). Maximum memory coalescing that the device supports gives good saving in access time.

\subsubsection{Autotune}

The choice of grid dimensions, blocksPerGrid and threadsPerBlock is critical since it affects the throughput. It is discussed in detail in Section 7.5.5.

\subsubsection{Optimization for Salsa20}

For a given key, the initial state vector for multiple blocks of Salsa20 encryption remains the same except for a counter value, that is incremented for each block. Hence it can be treated as a constant array, while the counter is taken care of by each thread kernel individually by its threadID. Keeping the initial state vector in fast read-only 
constant memory, instead of shared memory, is therefore useful as constant memory is optimized for broadcast due to data coalescing. Since each block of Salsa20 requires reading of initial state vector twice, once before the DoubleRound iterations and once after it (line no. 6 and 9 respectively in Algorithm 4), the use of constant memory is highly suited. CUDA specific function cudaMemcpyToSymbol writes the initial state vector in the constant memory. This strategy cannot be generalized to all ciphers. However, a prudent use of a faster memory, whenever applicable, always enhances performance for CUDA applications. This factor alone boosts the peak throughput for Salsa20/12 (for 1 GB of plaintext) by 4 GBps.

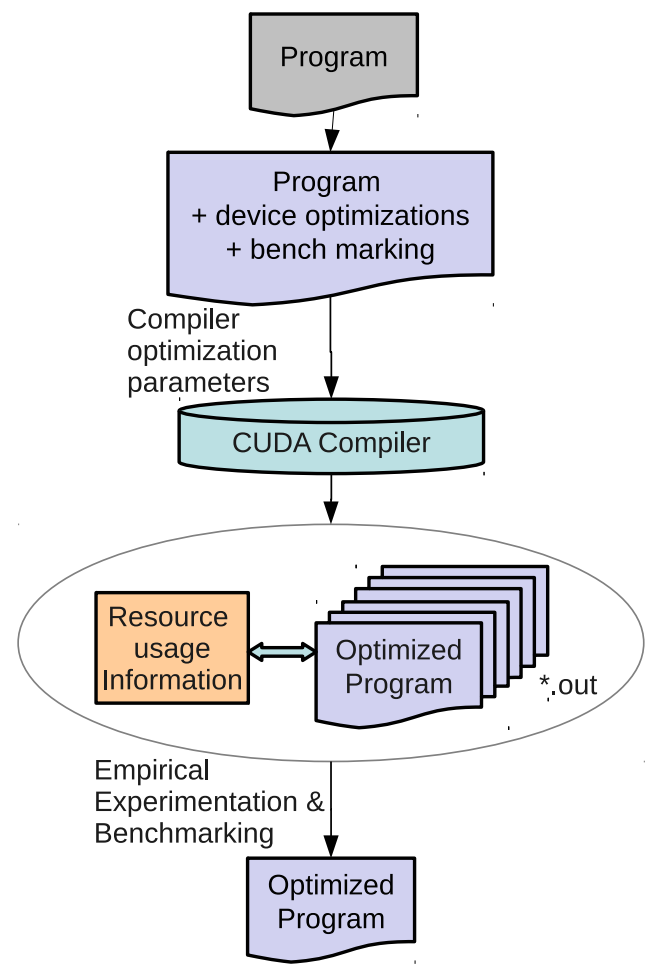

Figure 7.3: Autotuning Framework Flowchart

\subsubsection{Autotuning Framework for Performance Optimizations}

In context of a CUDA back-end application, an autotuning framework is proposed that automatically chooses tunable parameters of application mapping with the aim of improving a designated Figure of Merit (FoM). Throughput or the measured processing rate (Bytes/sec) of a device is chosen as FoM. It should be highlighted that taking up device occupancy (ratio of active resident threads to the maximum number of threads whose resources can be stored on-chip simultaneously) as an FoM does not guarantee optimized throughput.

The aim of an autotuning framework is to admit a large range of tunable parameters to CUDA application and select the one that makes the kernel run most efficiently. The range of these tunable parameters may be dependent on the constraints imposed 
by the device, application or both. The task of identifying what parameters should be subjected to tuning is critical, since they vary widely between algorithms. Finding the optimal values of these parameters may require extensive experimentation on a caseby-case basis. Apart from promising a performance boost, another reason for developing an autotuning framework is the provision of portability across different devices belonging to the same architecture family. Keeping in mind the operation flow of the framework, these parameters optimizations are classified as Compiler-specific and Device-specific.

\subsubsection{Device-specific Optimizations}

Device-specific optimizations are the ones that are tweakable at the runtime of the application, e.g., device grid dimensions. The given CUDA application is enhanced by the addition of the provision of the kernel variants being subjected to all possible combinations of these parameters after pruning by certain checks. Benchmarking for throughput is also added for later use. As shown in Fig. 7.3, enhancement of the application with the addition of device specific optimizations and benchmarking provision is the first step of the autotuning framework. However, execution of these enhancements does not manifest before various compiler optimizations have been done and multiple copies of the code executable are ready. Final runs of these programs result in sifting the fastest implementation with recommended parametrization choices.

Algorithm 5 gives the pseudo-code of the device specific optimizations setup. Out of the 4 different inputs, device properties (obtained by cudaDeviceProp function) and compute capability properties (obtained from a lookup table corresponding to major and minor compute capability) are device dependent. Kernel constraints are application dependent and are obtained after compilation of the program. minOccupancy is specified by user to filter out kernels with too low occupancy from experimentation. A higher value will prune the search space more but might miss the fastest kernels too; a lower value, on the other hand, will compromise on speed due to large search space for the fastest kernel.

All possible values of the two critical device parameters, threadsPerBlock and blocksPerSM, are considered for experimentation within their permitted range in nested loops as specified by line no. 1 and 2 . Threadblock size should always be a multiple of warpSize, because kernels issue instructions in warps. The next four lines of code calculate the resource budget for the current configuration of the device parameters. Total resident threadsPerSM is a product of blocksPerSM and threadsPerBlock. The next two lines calculate the resource usage of register and shared memory per kernel from the application specific parameters.

A kernel is subjected to experimentation with a set of possible device parameters configuration after pruning by 4 checks as specified from line no. 7 to 10 . Check 1 ensures that the maximum number of possible threads executable on an SM is not exceeded. Check 2 and 3 ensure that the register budget and the shared memory budget specific to one kernel is not exceeded. Check 4 makes sure that the current device configuration has an occupancy higher than the minimum specified by the 
Input: 4 types of inputs:

1. Device: warpSize, maxRegsPerBlock, maxShMemPerBlock, maxThreadsPerBlock, maxSM;

2. Compute Capability: maxBlocksPerSM, maxWarpsPerSM;

3. Kernel constraints: regsPerThread, shMemPerThread;

4. User constraints: minOccupancy.

Output: Valid parameter variants for benchmarking

$\mathbf{1}$ for threadsPerBlock $=$ warpSize to maxThreadsPerBlock step warpSize do

2 for blocksPerSM $=1$ to maxBlocksPerSM step 1 do

$3 \quad \mid$ threadsPerSM $=$ blocksPerSM $\times$ threadsPerBlock; regsPerSM = threadsPerSM $\times$ regsPerThread;

ShMemPerSM $=$ threadsPerSM $\times$ shMemPerThread;

occupancy $=\frac{\text { threadsPerSM }}{(\text { maxWarpsPerSM } \times \text { warpSize })}$;

Check1: threadsPerSM $\leq($ maxWarpsPerSM $\times$ warpSize $)$;

Check2: regsPerSM $\leq$ maxRegsPerBlock;

Check3: ShMemPerSM $\leq$ maxShMemPerBlock;

Check4: occupancy $\geq$ minOccupancy;

blocksPerGrid $=\operatorname{maxSM} \times$ blocksPerSM;

success $=$ kernel_launch $\lll$ blocksPerGrid, threadsPerBlock $》$

end

Algorithm 5: Device-specific optimizations: Search space generation and pruning

user. Functions to calculate the time elapsed before and after the kernel call are used to carryout the time duration benchmarking.

\subsubsection{Compiler-specific Optimizations}

Compiler-specific optimizations are subjected to the nvcc compiler at the compile time, e.g., preprocessor directives. As shown in Fig. 7.3, this step generates a number of optimized programs, each pertaining to a possible permutation out of the range of all the compiler-specific optimization parameters. Other than getting these executables, compiler generates information regarding the resource usage of the application in question, i.e., global, constant memory usage per grid, register count, local memory and shared memory usage per kernel. These resources are used as constraints during the empirical experimentation before reaching the performance-optimized kernel. The two compiler-specific optimizations applicable for the current application are loop unrolling and restricting per kernel register budget. Both of these manifest as a compromise between parallelism and register pressure.

Unroll Factor: Loop Unrolling replaces the main body of a loop with multiple copies of itself, adjusting the control logic accordingly. \#pragma unroll $n$ is a preprocessing 
directive where $n$ defines the unroll factor ( $n=1$ means no unrolling, $n=k$ means full unrolling, where the trip count of the loop is $k$ ). On the positive side, loop unrolling results in reduced dynamic instructions (compare and jump) count, boosting speedup. On the negative side, however, unrolling increases the total instruction count of the loop body and leads to an increased register pressure. Since registers are partitioned among threadblocks, an increased use of registers per threadblock reduces the device occupancy. This may or may not affect the throughput and requires experimentation for assurance. For Salsa20, the three flavors of the algorithm iterate for 4, 6 and 10 times for Salsa20/8, Salsa20/12 and Salsa20/20 respectively. For each of these, unroll factor from no unrolling to maximum unrolling is considered for experimentation.

Register Budget: A CUDA programmer can force a restricted number of registers by specifying cuda-nvcc-opts=-maxrregcount R, limiting the register use to $\mathrm{R}$ per kernel. Lowering register count allows increased occupancy which may result in increased throughput. On a negative note, it may cause spilling into the local memory when the register limit is exceeded. The local memory is as slow as the global memory and spilling into it can consequently cause severe performance degradation despite the higher occupancy. For all Salsa20 kernel variants, the register budget varies from 26 to 43 for no unrolling to maximum unrolling. For parametrization of register budget, all the multiples of 5 within this minimum and maximum register use is considered. Lowering the register budget any further than the minimum limit causes spilling and hence these cases are omitted from benchmarking.

\subsubsection{Results and Discussion}

This section presents detailed experimental results and compare them with the available state-of-the art benchmarks. Section 2.4 gives the specifications of the GPU and GPP used. For a good approximation of throughput performance results, each experiment was run 100 times and the timing results were averaged.

\subsubsection{Search Space Generation and Pruning}

Table 7.1 gives the possible range of parameters for the Salsa20 application kernel for NVIDIA GeForce GTX 590. The register budget range was chosen within the minimum and the maximum register requirements with no unroll and full unroll respectively, in steps of 5. All possible values of the unroll factor are taken into consideration. Grid dimension's permitted range is dependent on the device. Minimum occupancy was chosen to be 0.16 , i.e., 256 threads per SM (256/1536), since tests with selective lower occupancies gave inferior throughput.

In order to give an idea of the magnitude of the possible kernel configurations on which the autotuning framework carried out experimentation, some numbers are presented. For Salsa20/20, 10 possible unroll factors generate 10 optimized versions of the program, each a candidate for experimentation. Further, each of them is subjected to restricted register budget to generate multiple versions. Considering only the case of full unroll and unrestricted use of registers for Salsa20/20 kernel subjected 
Table 7.1: Range of parameters for autotuning Salsa20 kernel on a GTX 590

\begin{tabular}{|c|c|c|}
\hline & Parameter & Range \\
\hline \multirow{2}{*}{ Compiler-specific optimizations } & Register budget & $26,30,35,40,43$ \\
\cline { 2 - 3 } & Unroll factor & $1,2, \ldots, r / 2$ \\
\hline \multirow{3}{*}{ Device-specific optimizations } & Threads per block & $32,64, \ldots, 1024$ \\
\cline { 2 - 3 } & Blocks per SM & $1,2, \ldots 8$ \\
\cline { 2 - 3 } & Minimum occupancy & 0.16 \\
\hline
\end{tabular}

to device specific constraints, the number of allowed grid size combinations comes out to be 55. Extensive experimentation of all possible combinations of parameters after pruning was carried out for benchmarking.

\subsubsection{Compile Time Optimization of Register Pressure}

To find the optimal trade-off between active concurrent threads and registers availability per thread, two parameters have been tweaked. These are the restricted use of register budget and register unrolling. Restricting the use of registers per thread was benchmarked to always have a deteriorating effect on the throughput, in spite of increased occupancy. Changing the unroll factor, however, gives improved performance results. Fig. 7.4 gives the effect of unrolling factor on the registers used per thread for Salsa20/8, Salsa20/12 and Salsa20/20. Since Salsa20/20 requires 10 loop iterations of DoubleRound function, unrolling factors range from 1 to 10 . Unrolling an $n$-iteration loop more than $n$ times makes no difference and is considered by the CUDA compiler as a full unroll. Consequently, the unrolling of Salsa20/8 and Salsa20/12 kernels show no change after unroll factor of 4 and 6 respectively.

\subsubsection{Register Unroll vs. Throughput}

The register unrolling positively effects the throughput in general as shown in Fig. 7.5. These results are obtained after benchmarking more than 2500 kernel variants considering the full range of unroll factors and all grid dimensions supported by the device. Constraint of minOccupancy is applied, but register use restriction at compile time is skipped since it does not boost the throughput. The size of plaintext is kept $32 \mathrm{~KB}$ for encryption by the kernel.

Interestingly, the highest throughput for a Salsa20 kernel variant is obtained when the inner loop is unrolled by a factor one less than the full unrolling. Considering the case of Salsa20/20, the registers used per kernel remain unchanged till the unroll factor is raised from 1 till half of the full unroll factor, i.e., 5 as given in Fig. 7.4. For an unroll factor of 6 to 9, the no. of registers per kernel increases from 39 and saturates to a maximum of 43 for the full unroll. By varying the grid dimension, the best throughput figures are found when the unroll factor is 9. Although partially unrolled 
- Salsa20/8 - Salsa20/12 - Salsa20/20

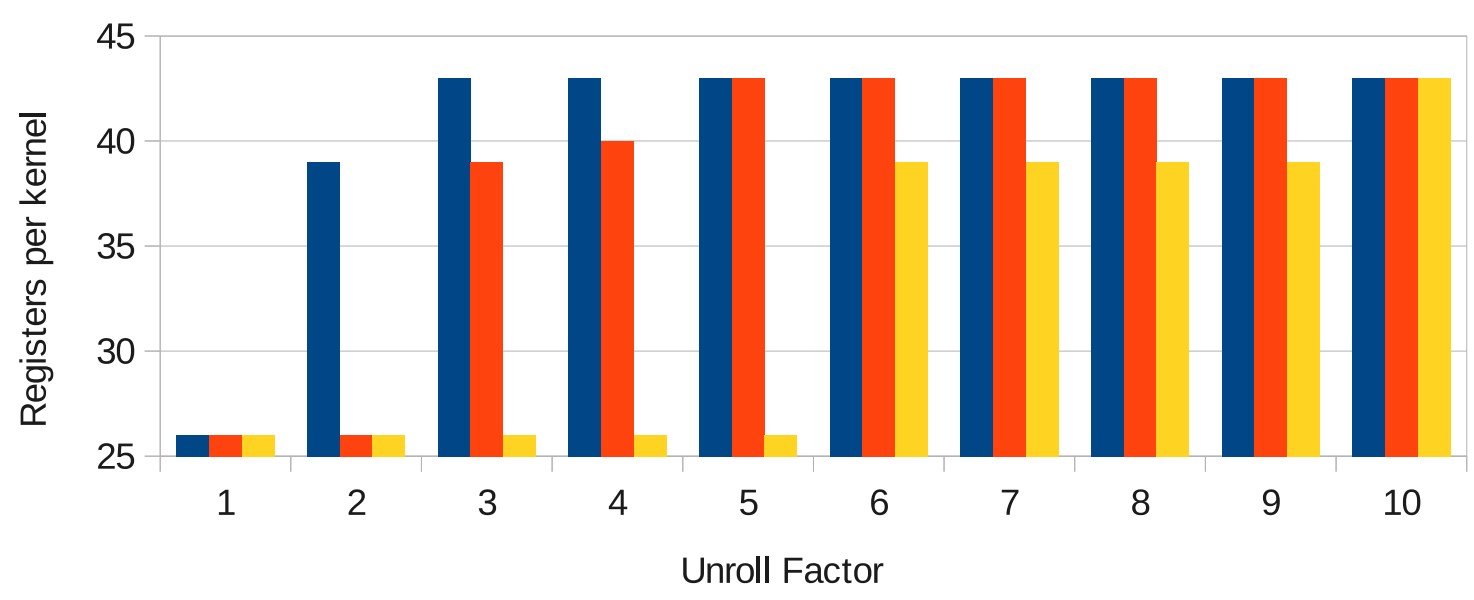

Figure 7.4: Effect of register pressure against unroll factor

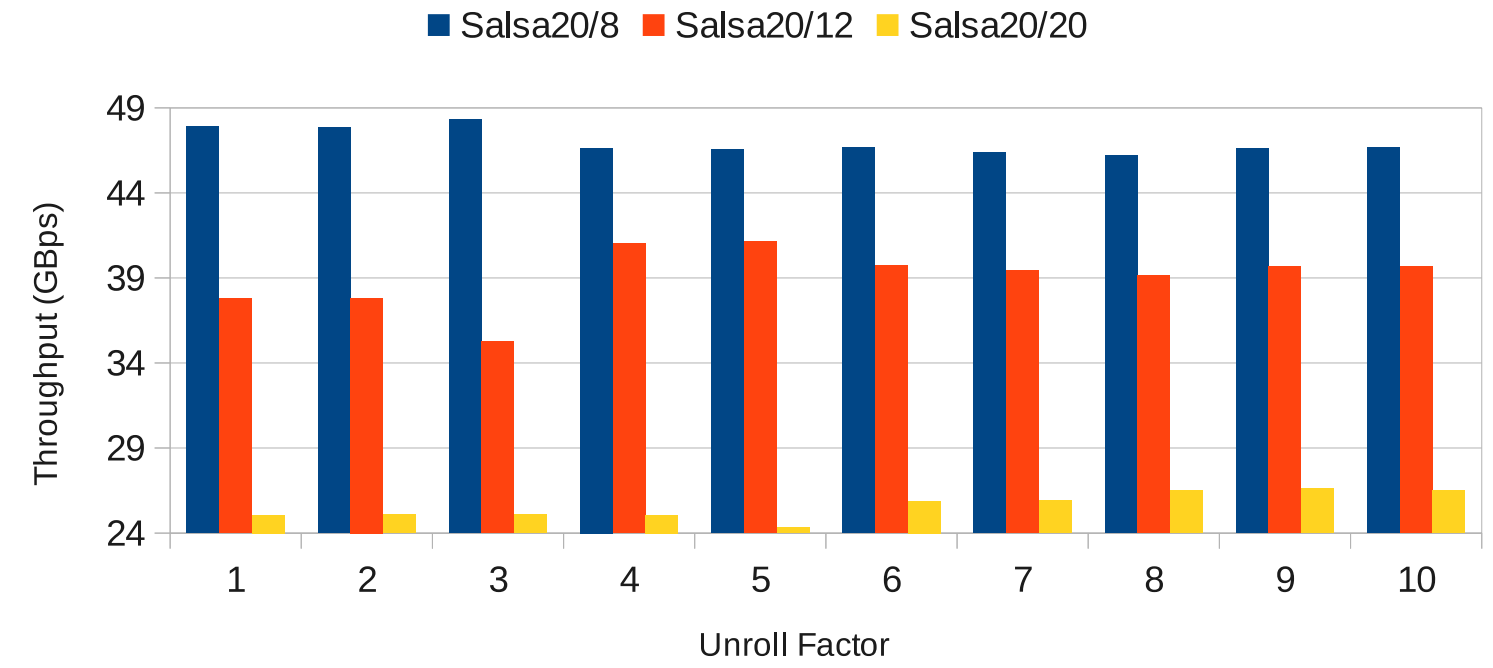

Figure 7.5: Effect of throughput against unroll factor

loops may require some cleanup code to account for loop iterations that are not an exact multiple of the unrolling factor, it may or may not decrease the performance in practice. Hence considering a range of unroll factors for experimentation proves beneficial in reaching the optimized performance.

Similarly, for the other flavors of Salsa20, i.e., for Salsa20/8 and Salsa20/12, the highest throughput is achieved when unroll factor is 3 and 5 respectively, as shown in 
Table 7.2: $\quad$ Salsa20 optimized parameters for GTX 590 (32 KB plaintext)

\begin{tabular}{|c|c|c|c|c|c|c|c|}
\hline & \multicolumn{5}{|c|}{ Autotuned } & \multirow{2}{*}{$\begin{array}{c}\text { Hand-tuned } \\
\text { Throughput } \\
\text { (GBps) }\end{array}$} & \multirow{2}{*}{$\begin{array}{c}\text { Throughput } \\
\text { Improvement } \\
\text { (GBps) }\end{array}$} \\
\hline $\begin{array}{l}\text { Kernel } \\
\text { variant }\end{array}$ & $\begin{array}{l}\text { Unroll } \\
\text { factor }\end{array}$ & $\begin{array}{l}\text { Threads } \\
\text { per block }\end{array}$ & $\begin{array}{l}\text { Blocks } \\
\text { per SM }\end{array}$ & $\begin{array}{c}\text { Device } \\
\text { occupancy }\end{array}$ & $\begin{array}{c}\text { Throughput } \\
\text { (GBps) }\end{array}$ & & \\
\hline Salsa20/8 & 3 & 448 & 1 & 0.29 & 48.29 & 45.77 & 2.52 \\
\hline Salsa20/12 & 5 & 320 & 2 & 0.41 & 41.14 & 39.91 & 1.23 \\
\hline Salsa20/20 & 9 & 512 & 1 & 0.33 & 26.60 & 24.42 & 2.18 \\
\hline
\end{tabular}

Table 7.2. For these unroll factors, the register usage in the three kernels restricts the occupancy of the device. With 40 registers, no more than 25 warps can be launched on each SM for GTX 590 (register limit on the device being 32K) restricting the device occupancy to 0.52 . Table 7.2 gives the throughput performance with hand-tuned parametrization for maximum device occupancy. The improvement in throughput obtained emphasizes the need of autotuning as a necessary requirement for performance enhancement of a CUDA application.

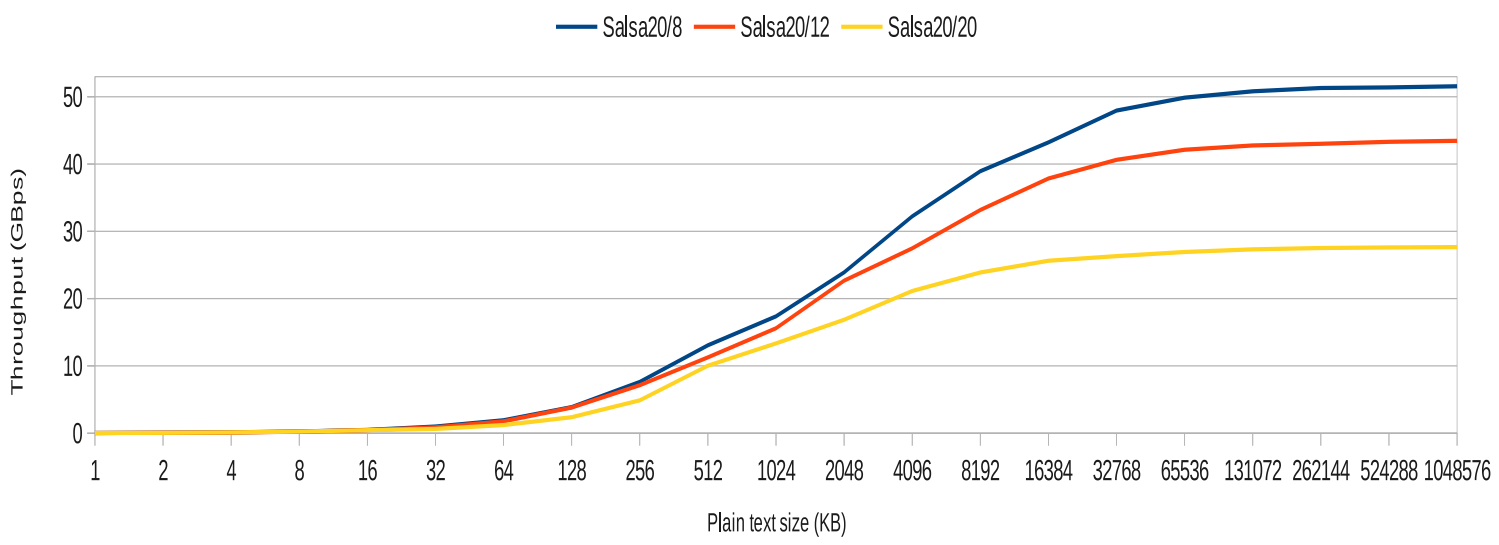

Figure 7.6: Salsa20 encryption performance on GTX 590 for varying block sizes (w/o mem transfers)

\subsubsection{Workload vs. Performance}

Fig. 7.6 shows the performance of Salsa20 variants on a GTX 590 for varying plaintext sizes. For throughput estimation, the plaintext blocksize is increased from 1 Byte till 1 GB. For GTX 590, one batch of plaintext encryption cannot go beyond 1.5 GB, due to the size of the global memory (obtained from cudaDeviceProp function). It is easy to see that the performance of Salsa20 is highly dependent on the amount of parallel work it receives. The peak throughput performance of Salsa20/8, Salsa20/12 and 
Salsa20/20 are found to reach 51.55, 43.44 and 27.65 GBps, respectively, outperforming the best reported GPU implementations so far.

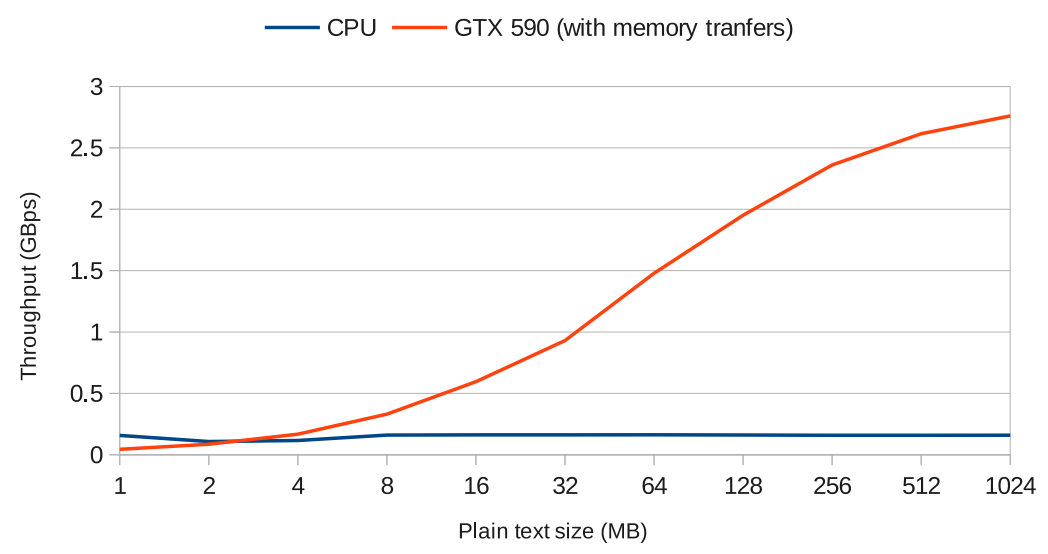

Figure 7.7: Salsa20 throughput improvement comparison between a GPP and a GPU

The overhead attributable to the plaintext data transfer from CPU to GPU and ciphertext data transfer from GPU to CPU is also taken into account to get the effective throughput, as given in Fig. 7.7. The peak performance for the GPU under consideration reaches around $2.8 \mathrm{GBps}$ with memory transfer overheads. The severe drop in the throughput clearly indicates that the bottleneck in the system is the data transfer bandwidth: PCIe bandwidth. For the host CPU, i.e., for AMD Phenom 1055T, the peak performance reaches $157 \mathrm{MBps}$.

\subsubsection{Comparison with Other Works}

Table 7.3 gives a comparison of this work on Salsa20 acceleration on GPUs with the results presented by D. Stefan [204] and S. Neves [162], comparison with the fastest reported AES implementation on GPUs is also given [107]. For a fair comparison, the throughput figures are scaled up for other devices (without memory transfers), in accordance to the newer GPU device used in this work, by considering the number of processor cores per device. Although the processing frequency of the device used is slower in comparison, this factor is ignored for scaling the throughput calculation. The throughput (GBps) per core from $[162,204]$ is $(5.3 / 480$ and 9/192), which is multiplied with the number of cores of the device used (512) to get 5.7 GBps and 24 Gbps respectively. These scaled throughputs are surpassed easily by the peak performance of 43.44 GBps reported in this work. In [204], the maximum throughput of 5.3 GBps (without memory transfers) was achieved for Trivium and that is also far behind (even after scaling). Scaling on similar lines, the AES implementation by Iwai et al. [107] results in a throughput of 9.3 GBps which is about 4.6 times slower than this work's peak performance for Salsa20/12. This re-scaling formula would be invalid for throughput calculation with memory transfers, since, like most of the cryptographic algorithms, Salsa20 and AES are data intensive in nature and show performance dependence on 
external memory access speed. The main factor contributing to the performance gain observed is the use of constant memory instead of shared memory for keeping the copy of the initial state vector. Moreover, the use of an autotuning framework to sift out the choice of parameters maximizing throughput also helps in reaching the claimed performance. According to Table 7.3, the throughput with memory transfer is the same as the best result known for AES. However, the claimed speed of $2.8 \mathrm{GBps}$ for AES with memory transfers is reported after being improved by $68 \%$ by optimization of overlapping GPU processing and data transfers [107]. Our current framework does not support this optimization and the search for an optimal transfer blocksize to hide the transfer latency is left on the project roadmap.

Table 7.3: Comparison of peak performance of Salsa20 with others work (Tp stands for Throughput)

\begin{tabular}{|c|c|c|c|c|}
\hline & D. Stefan [204] & S. Neves [162] & This work & Iwai et al. [107] \\
\hline Algorithm & Salsa20/12 & Salsa20/12 & Salsa20/12 & AES \\
\hline NVIDIA device & GTX 295 & GTX 260 & GTX 590 (one GF110) & GTX 285 \\
\hline Release & $08 / 01 / 2009$ & $16 / 06 / 2008$ & $24 / 03 / 2011$ & $15 / 01 / 2009$ \\
\hline Compute Capability & 1.3 & 1.2 & 2.0 & 1.3 \\
\hline Processor cores & 480 & 192 & 512 & 240 \\
\hline Shader Freq. (MHz) & 1242 & 1350 & 1215 & 1470 \\
\hline Threads / Block & 256 & 256 & 320 & 512 \\
\hline Tp (GBps)(w/ m) & - & 1.3 & 2.8 & 2.8 \\
\hline Tp (GBps)(w/o m) & 5.3 & 9 & 43.44 & 4.4 \\
\hline Scaled Tp (GBps)(w/o m) & 5.7 & 24 & 43.44 & 9.3 \\
\hline
\end{tabular}

\subsection{Mapping HC-128 Stream Cipher on GPUs}

The keystream generation for HC series of stream ciphers has two steps, namely, selfupdate step (SUS) of $P / Q$ array and keystream word generation step (KWGS) as discussed in Appendix C.2. In a serial implementation, each 32-bit word of $P$ array SUS is followed by one KWGS. This goes on for 512 iterations in HC-128 and 1024 iterations for HC-256. The same follows for $Q$ array for exactly the same number of iterations. Ideally, a fast GPU-based implementation would be able to run all these steps in parallel by independent threads as long as the device capacity is not over-budgeted. However, ciphers like HC have highly iterative structures, prohibiting parallelization beyond a limit.

\subsubsection{Limitations in Parallelization of HC Ciphers}

The dependencies that hurdle the parallelism opportunities in HC series of stream ciphers are listed below. 


\subsubsection{Intra-S-Box Dependency in Self Update Step of S-Boxes}

The SUS of HC-128 has a data dependency, the update of element $P[j]$ depends on its current and past values, i.e., $P[j], P[j \boxminus 3], P[j \boxminus 10]$ and $P[j \boxminus 511]$. Since the nearest dependency in the SUS of $P[j]$ is on $P[j \boxminus 3]$, one cannot unroll the loop more than 3 times.

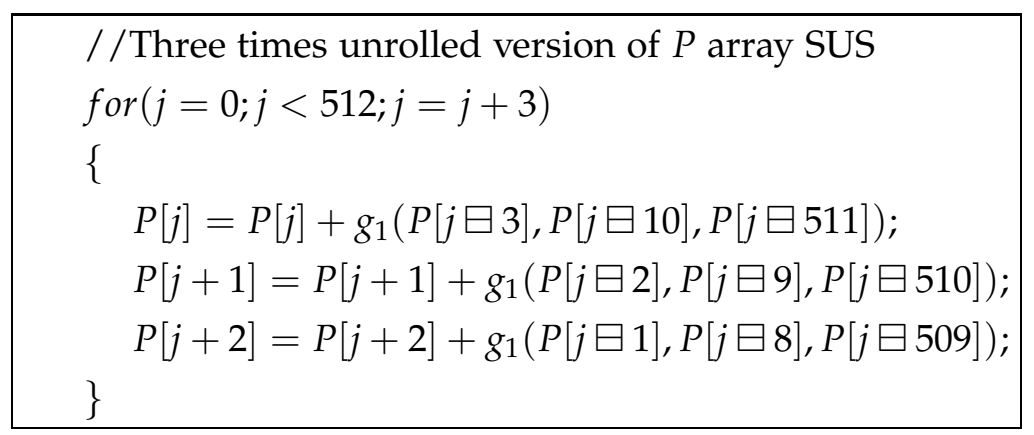

Fig. 7.8 describes the data dependencies for calculating the values at the $i^{\text {th }},(i+$ $1)^{t h}$ and $(i+2)^{t h}$ indices of $P$ array pictorially. Calculation of $(i+3)^{t h}$ index value requires the value at $i^{\text {th }}$ index of the array, making a simultaneous update of values at indices $i$ and $(i+3)$ impossible. This dependency limits the number of threads carrying out the SUS of $P / Q$ array to no more than 3 . The same arguments can be extended for HC-256 SUS. Moreover, due to similar limitations, more than 2 and 3 simultaneous threads cannot be harnessed for Step 1 and 3 respectively of initialization phase in HC series of stream ciphers.

\subsubsection{Inter-S-Box Dependency in Keystream Generation}

For exploiting parallelism the possibility of carrying out SUS $P$ and $Q$ arrays simultaneously (when no spatial data dependency exists) or their current and future copies simultaneously (when no temporal data dependency exists) is investigated.

Inter-S-Box Spatial Data Dependency: Consider the keystream generation phase of HC-128 as given in Appendix A. The SUS of $P$ and $Q$ arrays does not require values from each other. However, KWGS after SUS of $P$ array has a dependency on $Q$ array and vice versa. Hence a naive implementation with simultaneous update of $P$ and $Q$ arrays will not bear correct results for KWGS. In HC-256, even the SUS of the two S-Boxes is dependent on each other. Moreover, the KWGS dependency after SUS in $\mathrm{HC}-256$ is the same as in HC-128.

Inter-S-Box Temporal Data Dependency: Temporal data dependency between the current instance of S-Boxes and their future instance is investigated to exploit the possibility of simultaneous keystream generation from these arrays for multiple data blocks. Consider two temporal instances of $P$ array. Let $P_{\text {current }}$ contain the expanded values after initialization phase and $P_{\text {future }}$ be the one that will have the future values of $P$ array after SUS. Note that SUS of $P_{\text {future }}$ has a dependency on $P_{\text {current }}$, hence making it impossible to simultaneously update multiple temporal instances of $P / Q$ arrays. Arguing along the same lines, its evident to see data dependency of $P / Q$ arrays on their past instances in HC-256 too. 


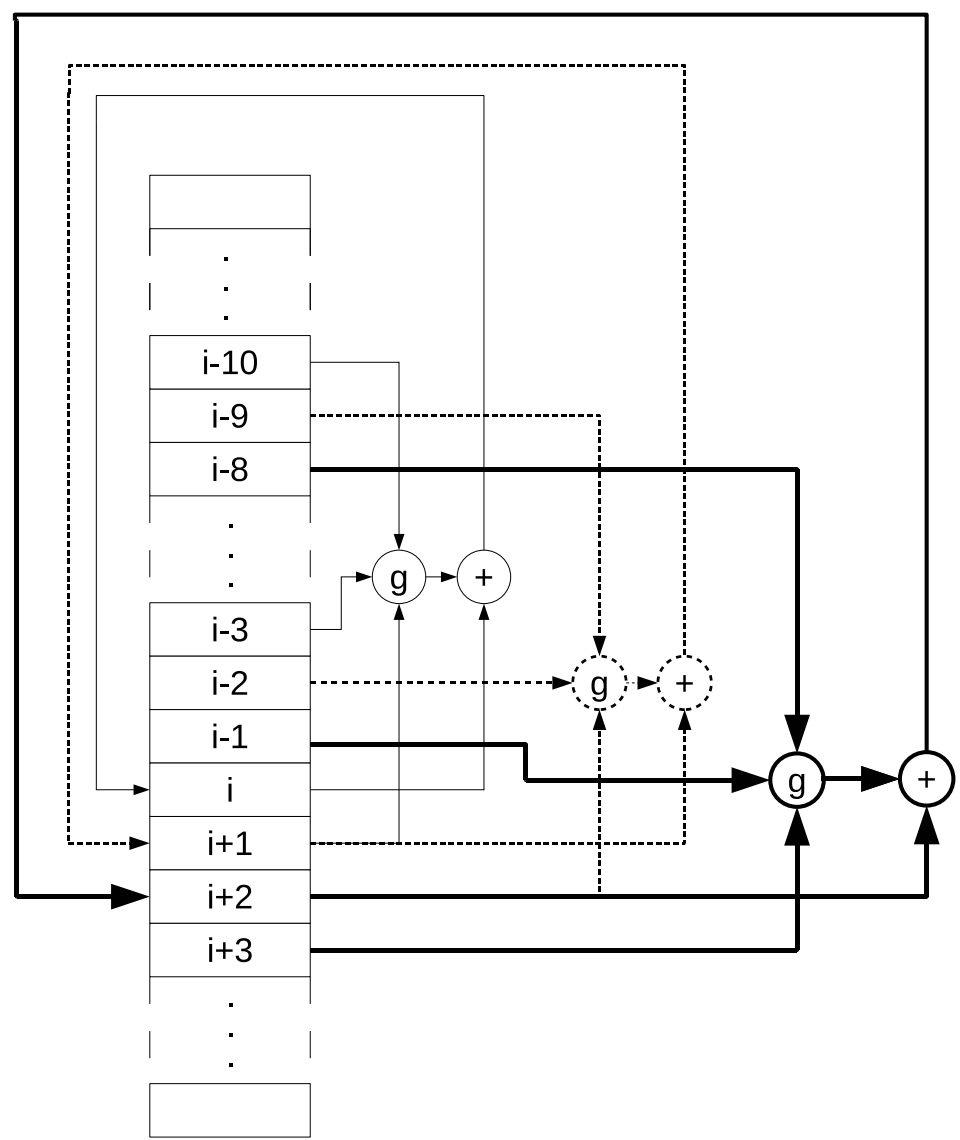

Figure 7.8: Dependency in SUS at indices $i, i+1$ and $i+2$ in S-Boxes

\subsubsection{Data-intensiveness}

HC series of stream ciphers are predominantly data intensive in nature requiring frequent memory accesses. Most of the block ciphers are computation intensive instead that is very desirable for GPU applications. Table 7.4 gives the list and frequency of various 32-bit binary operations required by the SUS and KWGS of HC-128 and HC-256. The high ratio of memory accesses to the arithmetic operations can be seen to be quite high.

\subsubsection{Optimization Strategies}

Kernels in CUDA compatible devices are assigned a small budget of thread-local registers. Shared memory is local to a block of threads and is comparatively bigger. The biggest memory in size is the grid-local global memory whose access incurs a 100x penalty as compared to register access. Our device NVIDIA GeForce GTX 590 has $3 \mathrm{~GB}$ of global memory, $48 \mathrm{~KB}$ of shared memory per MP and a maximum of 64 registers per thread. Considering the memory hierarchy, the fastest single data-stream implementation of the algorithm should use the fastest memory, i.e., the registers. However, the S-boxes of HC-128 (4 KB) and HC-256 (8 KB) are far too big to fit in 
Table 7.4: List of Operations for Keystream Generation in HC-128 and HC-256

\begin{tabular}{|c|c|c|c|c|}
\hline & HC-128 SUS & HC-128 KWGS & HC-256 SUS & HC-256 KWGS \\
\hline Modulo Additions & 2 & 2 & 3 & 7 \\
\hline XOR & 1 & 1 & 2 & 1 \\
\hline Modulo Subtractions & 3 & 1 & 3 & 1 \\
\hline Rotations & 3 & 0 & 2 & 0 \\
\hline Shifts & 0 & 1 & 0 & 3 \\
\hline Total operations & $\mathbf{9}$ & $\mathbf{5}$ & $\mathbf{1 0}$ & $\mathbf{1 2}$ \\
\hline Memory Reads & 4 & 4 & 5 & 6 \\
\hline Memory Writes & 1 & 1 & 1 & 1 \\
\hline Total memory accesses & $\mathbf{5}$ & $\mathbf{5}$ & $\mathbf{6}$ & $\mathbf{7}$ \\
\hline
\end{tabular}

them. The next best possibility is to put the $P$ and $Q$ arrays in the shared memory and let the registers hold their smaller 16-element snapshot as suggested for the optimized implementation in $[223,224]$. However, this single thread implementation of keystream generation does not lead to significant throughput. For example, HC-128 yielded a throughput of only 0.24 Gbps.

For exploiting parallelism, multiple threads are simultaneously launched. As registers are local to one kernel, shared memory is used instead and various optimization strategies for single data-stream implementation are discussed given in Section 7.6.2.1. For multiple data-streams implementation, the use of on-chip block-local shared memory instead of off-chip grid-local global memory can boost the speedup significantly. However, each data-stream requires a memory budget $m$ for $P$ and $Q$ arrays, where $m=4 \mathrm{~KB}$ for HC-128 and $m=8 \mathrm{~KB}$ for HC-256 and hence the number of parallel data-streams per MP is restricted to $s / m$, where $s=48 \mathrm{~KB}$ is the shared memory size. Therefore, multiple data-streams implementation using global memory is taken up, as discussed in Section 7.6.2.2.

\subsubsection{Single Data-Stream Optimizations}

Program listing of a simple implementation of keystream generation code for HC-128 with the degree of parallelism that is straightforward to manipulate is given in Table 7.5. Since the initialization phase is similar and simpler, its explanation is skipped. The intra-dependency of S-Box arrays does not allow more than 3 parallel threads to update $P / Q$ arrays as described in Section 7.6.1.1. The CUDA kernel is called with 1 block of 512 threads. The code is divided into four parts. The first and third parts give SUS for $P$ and $Q$ arrays respectively while part two and four perform KWGS. Only 3 out of 512 threads update $P$ array in part one, requiring $171(512 / 3)$ times execution for completely updating $P$ array. In part 2, the S-Boxes are employed to generate 512 words of keystream using 512 threads simultaneously. Part 3 updates the $Q$ array followed by 512 words of KWGS in part 4. This implementation yields a throughput of 0.37 Gbps for keystream generation in HC-128. 
Table 7.5: Keystream generation phase of HC-128 using three threads

\begin{tabular}{|l|}
\hline if $($ threadId $x . x<=2)$ \\
$\quad$ for $(i=$ threadIdx. $x ; i<512 ; i=i+3)$ \\
$\quad P \_s[i]=P \_s[i]+g 1\left(P \_s[i \boxminus 3], P \_s[i \boxminus 10], P \_s[i \boxminus 511] ;\right.$ \\
\hline$i=$ threadIdx.x; \\
$s[i]=h 1\left(Q \_s, P \_s[i \boxminus 12]\right) \oplus P \_s[i] ;$ \\
\hline if $($ threadIdx.x $<=2)$ \\
$\quad$ for $(i=$ threadIdx.x; $i<512 ; i=i+3)$ \\
$\quad Q \_s[i]=Q \_s[i]+g 2\left(Q \_s[i \boxminus 3], Q \_s[(i \boxminus 10)], Q \_s[i \boxminus 511]\right) ;$ \\
\hline$i=$ threadIdx.x; \\
$s[i+512]=h 2\left(P \_s, Q \_s[i \boxminus 12]\right) \oplus Q \_s[i] ;$
\end{tabular}

Next the optimization strategies are discussed which are undertaken to improve the parallelism and consequently the throughput of this simple parallel CUDA based implementation of $\mathrm{HC}-128$. In case the strategies are applicable only to one of the ciphers in $\mathrm{HC}$ series of stream ciphers, it has been explicitly mentioned.

Parallelization of P/Q Array SUS with Key Generation(512 words): One way of increasing the degree of parallelism in HC-128 algorithm was suggested by Chattopadhyay et al. [38]. The authors proposed carrying out SUS of either of the S-Boxes along with a simultaneous KWGS from the other S-Box. The parallelism can be employed ensuring correct results by keeping multiple temporal copies of S-Boxes (say P0, Q0, $P 1, Q 1)$. If the shared memory of the GPU device used for S-Box instances is not over-budgeted, this strategy can be employed for achieving parallelism. As seen from Appendix A, each round of HC-128 keystream generation for 1024 words has a P-SUS and $P$-KWGS for 512 words, followed by a similar Q-SUS and Q-KWGS for 512 words. With two copies of S-Boxes, the parallelization of P-SUS along with Q-KWGS is possible and vice versa. The series of steps as proposed in [38] are summarized in Table 7.6. After initialization routine, arrays $P 0, Q 0$ contain the expanded key and IV. SUS of $P$ array starts by reading values from $P 0$ (past values) and updating $P 1$ (current values). No more than 3 parallel threads (due to intra-data-dependency) execute iteratively updating the entire 512 words array. In step 1 the $Q$ array is updated reading values from $Q 0$ (past values) and updating $Q 1$ (current values). KWGS using $P 1$ and $Q 0$ is done by 512 parallel threads simultaneously - denoted this by Keygen( $Q 0, P 1)$. Similar notations describe the other steps.

After the initial step, $Q 1, P 0, Q 0, P 1$ are updated in successive steps, each time simultaneously generating keystream words from the S-Box updated in the previous step. This goes on by repetition of step 1 till 4 for as many keystream values as required. CUDA framework for HC-128 parallel implementation employs 544 threads for keystream generation in total. Out of these, 512 threads carry out KWGS from an entire array of S-Box words simultaneously. One thread warp with three active threads carry out the SUS of the S-Box. Here parallelism is achieved at the cost of extra resources, since only multiple copies of the S-Boxes guarantee correct results for 
Table 7.6: Parallelizing one SUS warp with one KWGS block

\begin{tabular}{|c|c|c|c|}
\hline Step \# & KWGS & SUS & Comments \\
\hline Step 0 & - & P1 & 3 threads for SUS \\
\hline Step 1 & Keygen $(Q 0, P 1)$ & Q1 & \\
Step 2 & Keygen $(Q 1, P 1)$ & $P 0$ & \\
Step 3 & Keygen $(Q 1, P 0)$ & $Q 0$ & active threads (out of a warp) for SUS \\
Step 4 & Keygen $(Q 0, P 0)$ & $P 1$ & +512 threads for KWGS \\
\hline
\end{tabular}

parallel implementation. This strategy is applied to HC-256 as well. Similarly, one warp with 3 active threads remains under-utilized; however KWGS is carried out by 1024 parallel threads for larger S-Boxes in HC-256.

Parallelization of $P$ and Q SUS with Key Generation (1024 words) Further parallelization of HC-128 is possible by simultaneous P-SUS and P-KWGS of 512 words as well as the Q-SUS and Q-KWGS of 512 words in keystream generation phase as described in Appendix A. Thus both the S-Boxes can be updated in parallel along with simultaneous generation of 1024 words of keystream. However, step 1 and 3 of keystream generation in Table 7.6, reveal a data dependency. $Q 0$ is needed for generating key from $P 1$, and $Q 1$ for generating key from $P 0$. Hence, update of $P 0, Q 0$ and generating 1024 keystream words using $\operatorname{Keygen}(Q 0, P 1)$ and $\operatorname{Keygen}(P 1, Q 1)$ gives rise to a race condition, commonly called a Read After Write ( $R A W$ ) hazard where the keystream values would depend upon which statement gets executed first. This can be successfully avoided by using 2 more copies of $Q$ arrays, namely $Q_{B u f f 0}$ and $Q_{B u f f 1}$ for keeping backups of $Q 0$ and $Q 1$ respectively. For preserving correctness, these buffers need to be updated at every alternate step. All arrays are stored in the shared memory for fast access.

Table 7.7 describes a step by step execution. After initialization, the expanded key and IV reside in $P 0, Q 0$. All other temporal S-Box copies i.e., $P 1, Q 1, Q_{B u f f 0}$ and $Q_{B u f f 1}$ are left un-initialized. Simultaneous SUS of $P$ and $Q$ arrays is carried out by reading values from $P 0, Q 0$ (past values) and updating $P 1, Q 1$ (current values) respectively. A copy of $Q 0$ is backed up in $Q_{B u f f 0}$ simultaneously. In this step, 6 threads of 2 warps carry out the SUS for $P 1$ and $Q 1$. For $Q 0$ backup, 512 parallel threads make a copy.

In step 1, a block of 1024 threads is employed for generating 1024 words of keystream, each thread generates one word of keystream. Out of these, 512 threads are used to execute the extra step of copying values to the buffers. Alternate updates of $P 0, Q 0$ and $P 1, Q 1$ follows, simultaneously generating 1024 words of keystream. Hence Step 1 and 2 are repeated as long as the keystream generation is required.

A single kernel cannot be invoked with more than 1024 threads in a block. The thread budget is broken in two blocks, each having 544 threads. The two blocks run concurrently, one warp in each carrying out SUS and 512 threads generating 
Table 7.7: Parallelizing 2 S-Box SUS warps with 2 KWGS blocks

\begin{tabular}{|c|c|c|c|c|c|}
\hline$Q_{\text {Buff }}$ copy & \multicolumn{2}{|c|}{ KWGS } & \multicolumn{2}{|c|}{ SUS } & Comments \\
\hline $\begin{array}{c}Q_{B u f f 0} \\
\text { copy }\end{array}$ & - & - & $P 1$ & $Q 1$ & $\begin{array}{l}\qquad 3+3 \text { threads for SUS, } 512 \\
\text { threads for copying } Q 0 \text { to } Q_{B u f f 0}\end{array}$ \\
\hline $\begin{array}{l}Q_{B u f f 1} \\
\text { copy }\end{array}$ & $\begin{array}{l}\text { Keygen } \\
(Q 1, P 1)\end{array}$ & $\begin{array}{c}\text { Keygen } \\
\left(Q_{B u f f 0}, P 1\right)\end{array}$ & $P 0$ & $Q 0$ & $\begin{array}{c}3+3 \text { threads for SUS, } \\
512 \text { threads for Keygen }(Q 1, P 1) \text {, } \\
512 \text { threads for copying } Q 1 \text { to } \\
Q_{B u f f 1} \text { and Keygen }\left(Q_{B u f f 0}, P 1\right)\end{array}$ \\
\hline $\begin{array}{l}Q_{B u f f 0} \\
\text { copy }\end{array}$ & $\begin{array}{l}\text { Keygen } \\
(Q 0, P 0)\end{array}$ & $\begin{array}{c}\text { Keygen } \\
\left(Q_{B u f f 1}, P 0\right)\end{array}$ & $P 1$ & Q1 & $\begin{array}{c}3+3 \text { threads for SUS, } \\
512 \text { threads for Keygen }(Q 0, P 0), \\
512 \text { threads for copying } Q 0 \text { to } \\
Q_{B u f f 0} \text { and Keygen }\left(Q_{B u f f 1}, P 0\right)\end{array}$ \\
\hline
\end{tabular}

keystream. GPUs with compute capability 2.0 or more have the capability of calling concurrent kernels at the same time as well.

This strategy of achieving parallelism cannot be extended for HC-256 since its SUS of the S-Boxes is dependent on each other.

\subsubsection{Multiple Data-Streams Optimization}

The GPU clock is slower than the CPU clock speed. Thus speedup in GPU devices can be achieved in two ways. One way is by employing parallel threads respecting data dependencies in a single stream of data as investigated in Section 7.6.2.1. A better alternative in terms of resource utilization and throughput is to employ all the SPs (stream processors) of the CUDA device by employing ciphers of multiple datastreams in parallel. Due to the limited size of shared memory, the larger albeit slower global memory is employed for encryption of multiple parallel streams of data.

Performance tuning on the GPU requires understanding device specifications and accordingly finding and exposing enough parallelism to populate all the multiprocessors (MPs). NVIDIA GeForce GTX 590 can accommodate up to 8 blocks (or 48 warps) per MP. Since each warp can have 32 homogeneous threads, an MP can process up to 1536 threads $(48 \times 32)$. To fully utilize each MP, the number of threads it should get assigned should be no more than 192 per block (1536/8). This limit is kept in mind when assigning the thread budget to each MP for HC series of stream ciphers.

For HC-128, the 3 threads for SUS of each of the S-Boxes constitute one warp. Since these threads execute a total of 171 times (512/3) for complete update of either of the S-Boxes, the number of parallel threads employed for KWGS can be adjusted so that the budget of total number of 192 threads per block is never exceeded. In case of HC-128, for KWGS 128 threads are employed and for S-Box update 2 warps are executed. Hence 2 warps of S-Box SUS and 4 warps of KWGS are kept in the same block of 192 threads. For HC-256, however, only one warp is used for SUS and 4 for KWGS, making the total thread budget equal to 160 per block. This strategy ensures 
maximum number of parallel data-streams the device can encrypt simultaneously, showing noticeable increase in the throughput of both HC-128 and HC-256.

\subsubsection{Experimental Results}

Section 2.4 gives the specifications of the GPU and GPP used for benchmarking the gain in throughput as the HC series of stream ciphers are mapped on a GPU. For a good approximation of throughput performance results, each experiment was repeated a 1000 times and the timing results were averaged.

Table 7.8: Duration and throughput of initialization phase of stream ciphers

\begin{tabular}{|l|c|c|c|}
\hline & \multicolumn{2}{|c|}{ NVIDIA GeForce GTX 590 } & AMD Phenom ${ }^{\mathrm{TM} I I}$ \\
& Global memory & Shared memory & $1100 \mathrm{~T}$ \\
\hline HC-128 & $1.386 \mathrm{~ms}$ & $1.078 \mathrm{~ms}$ & $27 \mu \mathrm{s}$ \\
& $22.53 \mathrm{Mbps}$ & $28.98 \mathrm{Mbps}$ & $1.15 \mathrm{Gbps}$ \\
\hline HC-256 & $1.930 \mathrm{~ms}$ & $1.666 \mathrm{~ms}$ & $60 \mu \mathrm{s}$ \\
& $32.35 \mathrm{Mbps}$ & $53.56 \mathrm{Mbps}$ & $1.04 \mathrm{Gbps}$ \\
\hline
\end{tabular}

\subsubsection{Encryption of Single Data-Stream}

Initialization phase of HC ciphers has been implemented using shared memory and global memory in two separate experiments. The last step of initialization phase is similar to SUS phase, consequently 3 parallel threads are employed for it. In the second step of initialization phase, intra-dependency for $W$ is even more severe, limiting the number of simultaneous threads to 2 . Using faster shared memory instead of global memory accelerates initialization phase as shown in Table 7.8. It however, incorporates the overhead of copying $P, Q$ and $W$ arrays on shared memory that can be done simultaneously using 512 and 1024 parallel threads in case of HC-128 and HC-256 respectively.

The performance results of keystream generation phase are presented in Fig. 7.9 and Fig. 7.10 for HC-128 and HC-256, respectively. The throughput shows an increasing trend, till it saturates for higher data sizes considered. The maximum throughput when using the global memory for storing S-Boxes of HC-128 is 0.41 Gbps. Using shared memory gives a boost to performance because of its smaller access time.

A similar trend is observed for HC-256. The size of the S-Boxes is double compared to that of $\mathrm{HC}-128$, the amount of shared memory used by the optimized version of proposed algorithm is $16 \mathrm{~KB}$ (two copies of each S-Box). A GPU device with lower compute capability has no more than $16 \mathrm{~KB}$ of shared memory per MP. Hence, this optimized implementation of HC-256 on one thread block of such devices is not possible. The maximum throughput from the global memory implementation of HC-256 is $0.15 \mathrm{Gbps}$ and for shared memory implementation is $0.41 \mathrm{Gbps}$. 


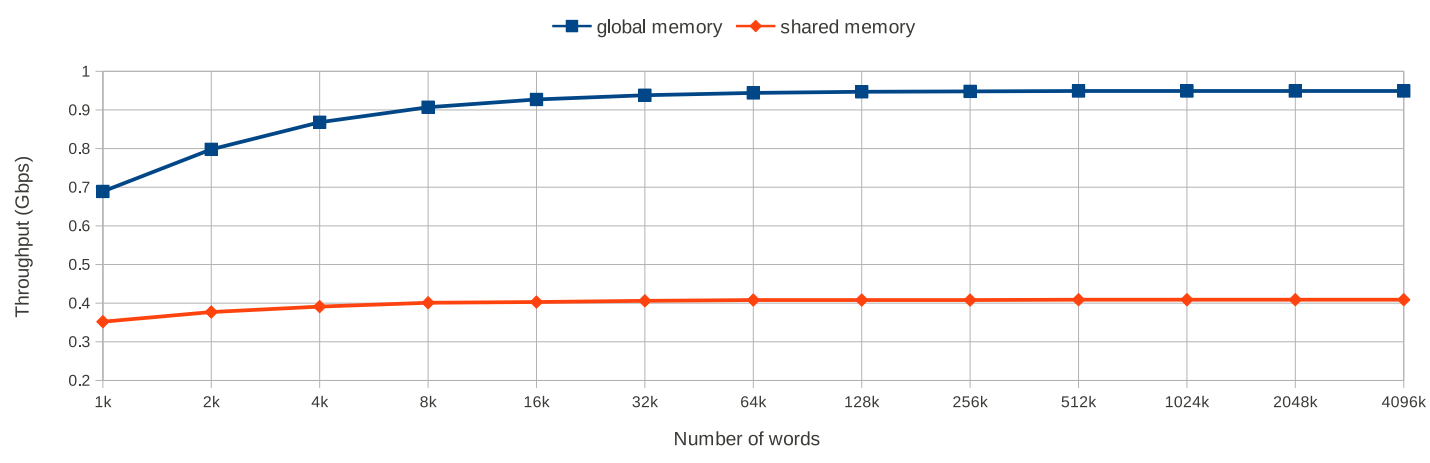

Figure 7.9: HC-128 keystream generation throughput using shared and global memory

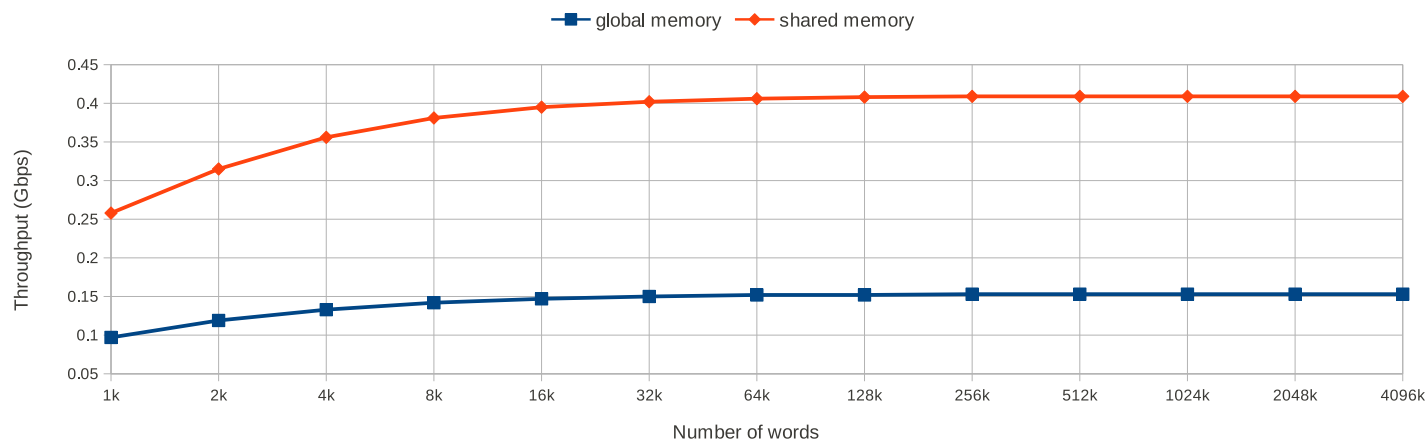

Figure 7.10: HC-256 keystream generation throughput using shared and global memory

\subsubsection{Encryption of Multiple Data-Streams in Parallel}

The parallelism offered by the CUDA device can be well exploited using multiple parallel streams of data. For simulation purposes a single stream of data is doubled repeatedly to reach $32 \mathrm{~K}$ parallel streams. Fig. 7.11 gives the throughput of $\mathrm{HC}-128$ and HC-256 for increasing number of parallel data-streams on the selected CUDA device. The trend of throughput rise shown by the two ciphers is similar, having an apparent peak for 64 parallel streams. The CUDA device used has a total of 16 MPs and each MP can accommodate 8 blocks at most. Maximum utilization of MPs is achieved for 128 parallel streams of data $(16 \times 8)$. Further increase in the number of parallel data-streams shows a slight improvement in the throughput. The reason is that the parallel streams in excess of 128 are waiting in instruction queue and are launched with negligible context switch time. The maximum throughput achieved is $31 \mathrm{Gbps}$ for HC-128 and 14 Gbps for HC-256 employing 32768 parallel streams.

\subsubsection{Comparison with Other Works}

The acceleration results are compared with the only available figures for HC-128 acceleration on GPUs by D. Stefan in his masters stefan [204]. Without employing parallelism within a single data-stream for $\mathrm{HC}-128$, he assigned one thread to one 


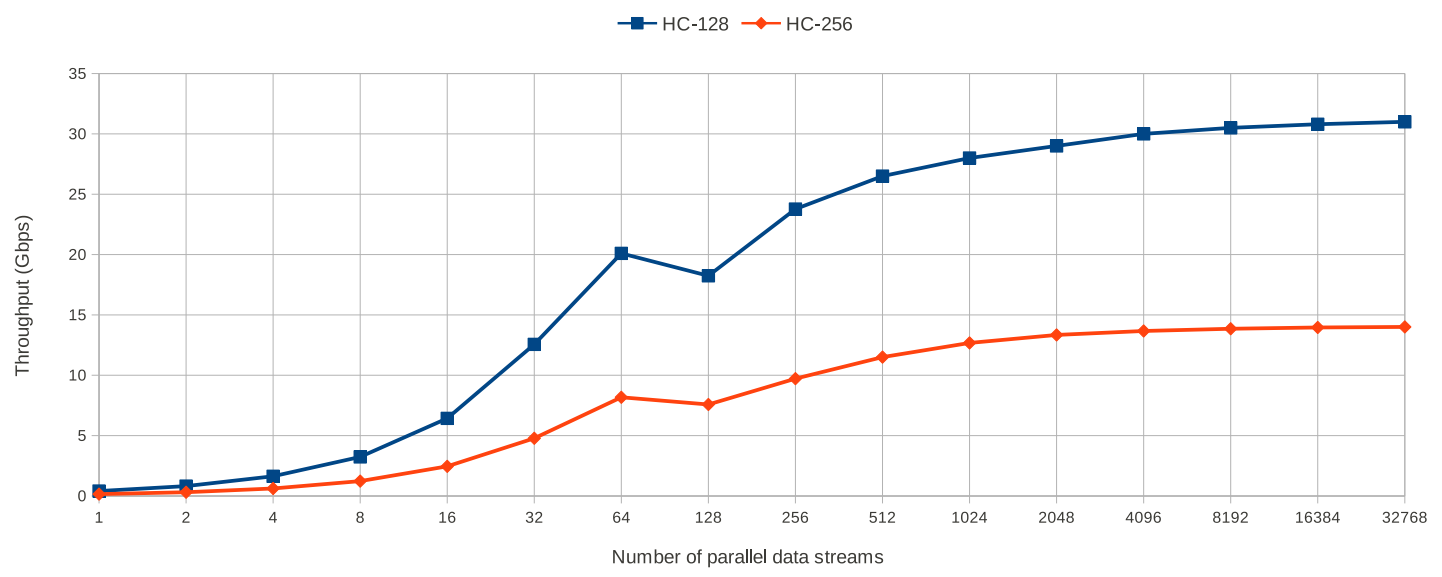

Figure 7.11: keystream generation throughput for varying number of multiple datastreams

data-stream. For supporting multiple data-streams, he employed global memory for S-boxes. The highest throughput achieved is reported and compared in Table 7.9. For the same number of blocks, the throughput of the current work is approximately 14 times higher. Comparing the cycles/byte performance also shows a significant decrease. Results for initialization phase are not available for comparison.

Table 7.9: Comparison of the HC-128 GPGPU mapping acceleration against others work

\begin{tabular}{|c|c|c|}
\hline & Implementation by D. Stefan [204] & Our Implementation \\
\hline NVIDIA device & GeForce GTX 295 & GeForce GTX 590 \\
\hline Release date & January 8, 2009 & March 24, 2011 \\
\hline Compute Capability & 1.3 & 2.0 \\
\hline Memory Used & Global Memory & Global Memory \\
\hline Threads / data-stream & 1 & 192 \\
\hline data-stream / Block & 256 & 1 \\
\hline Total blocks used & 680 & 680 \\
\hline Total data-streams & $680 \times 256$ & 680 \\
\hline Total threads used & $680 \times 256$ & $192 \times 680$ \\
\hline Performance(Cycles/byte) & 4.39 & 0.279 \\
\hline Throughput(Gbps) & 2.26 & 31 \\
\hline
\end{tabular}

The HC-128 performance evaluation on CPU was done using the eSTREAM testing framework [64]. The C implementation of the testing framework was installed in the CPU machine (Section 2.4) on CentOs 5.8 (Linux version 2.6.18-308.11.1.el5xen). For the benchmark implementation of HC-128 and HC-256 the highest keystream generation speeds were found to be 2.36 cycles/byte and 3.63 cycles/byte respectively. Table 7.10 gives a comparison of throughput of HC series of stream ciphers on various platform. The throughput obtained on an AMD Phenom ${ }^{\text {TM }}$ II X6 1100T Processor is 10.94 Gbps 
and 7.5 Gbps for keystream generation phase of HC-128 and HC-256 respectively. The high speed rendered by CPU is primarily because it has to incur no memory overhead for RAM located contents unlike the GPU memory accesses. Moreover, the limitation of SIMD architecture of GPUs requires homogeneity of warp threads which is not a limitation in CPUs. Consequently the CUDA mapping of the HC family of ciphers is 11-18 times slower. The ASIC based implementation proposed by Chattopadhyay et al. is so far the fastest reported implementation of HC-128 claiming a throughput of 22.88 Gbps [38]. The throughput results of HC-256 are however not reported.

Table 7.10: Throughput (Gbps), Cycles/Byte (C/B) of a single data-stream HC ciphers

\begin{tabular}{|c|c|c|c|c|c|c|}
\hline & \multicolumn{2}{|c|}{$\begin{array}{c}\text { AMD Phenom } \\
\text { II }\end{array}$} & \multicolumn{2}{c|}{$\begin{array}{r}\text { NVIDIA GeForce } \\
\text { GTX 590 }\end{array}$} & \multicolumn{2}{c|}{$\begin{array}{c}\text { ASIC [38] } \\
\text { (65nm Technology) }\end{array}$} \\
\hline HC-128 & $10.9 \mathrm{Gbps}$ & $2.36 \mathrm{C} / \mathrm{B}$ & $0.95 \mathrm{Gbps}$ & $9.27 \mathrm{C} / \mathrm{B}$ & $22.88 \mathrm{Gbps}$ & $0.5 \mathrm{C} / \mathrm{B}$ \\
\hline HC-256 & $7.5 \mathrm{Gbps}$ & $3.63 \mathrm{C} / \mathrm{B}$ & $0.41 \mathrm{Gbps}$ & $21.82 \mathrm{C} / \mathrm{B}$ & Not reported & Not reported \\
\hline
\end{tabular}

For multiple data-streams some promising results are observed, which for CPUs is not straightforward to implement. For 32768 parallel data-streams, the GPU gives a throughput of $31 \mathrm{Gbps}$ for HC-128 and $14 \mathrm{Gbps}$ for HC-256. Hence it is concluded that HC-series of stream ciphers is unfit to be off-loaded to GPUs in case of a single data-stream application. In contrast, an application exploiting multiple parallel datastreams can achieve GPU acceleration up to 2.8 times faster in case of HC-128 and 1.87 times faster for HC-256 (with 32768 parallel data-streams).

\subsection{Concluding Remarks}

It is concluded that GPUs are a viable option to be employed as a co-processor with a CPU host to accelerate parallelizable cryptographic functions. For most the the cryptographic workloads, PCIe bandwidth is the the bottleneck in the system performance since encryption/ decryption on GPUs is much faster compared to the transfer of data in the external global memory.

This work presents an autotuning framework for Salsa20 series of stream cipher that not only guarantees fast portability for Fermi GPUs and optimized throughput performance, but it can be generalized and extended to other massive parallel cryptographic operations also. The results presented are hereby reported the fastest implementation results for Salsa20 variants mapped on any GPU [121] so far. The first detailed study of algorithmic acceleration limitations in HC series of stream ciphers for mapping on a GPU device is also presented. In spite of various strategies to improve the throughput of the HC-128 and HC-256 ciphers at the cost of replicated copies of S-Boxes our GPU throughput remains 11-18 times slower than a standard CPU in throughput. For multiple data-streams, however a thorough tuning on the GPU for optimizing all the architectural features that the device could offer results in a 2-3 times faster throughput than CPU. 


\section{Chapter 8}

\section{Efficient Cryptanalytic Hardware}

The security of all cryptographic algorithms relies on computational problems complex operations which are supposed to be intractable with ordinary computing platforms. Cryptanalytic weaknesses can join hands with special-purpose high-performance architectures to enable successful security attacks. Some of the most remarkable cryptanalytic attempts relied on some custom hardware or application-specific computing platform [88]. Consequently, General Purpose Processors (GPPs), ASICs, GPU implementations and ASIPs have been used to attack an algorithm. In this work a custom hardware is designed to attack SHA-1 hash function. The attack is brought withing practical time-money limits chiefly by migrating to newer technologies (i.e., exploring Moore's Law) and by using parallelism (i.e., exploring Amdahl's Law).

\subsection{Introduction}

SHA-1 is the most widely used hash function from the SHA series used in TLS/SSL for internet security. According to a recent survey by Microsoft [8], 98\% of all certificates issued worldwide use SHA-1 as underlying hash function. Though various successful cryptanalytic attacks against it have been reported, they remain however impractical due to high computation complexity and associated cost. This work proposes an Application-Specific Instruction-set Processor (ASIP), named Cracken. Cracken is aimed to efficiently realize near collision attack on SHA-1. The ISA of Cracken carries out the progression of a collision tree search till a hit is found. A design space exploration is done to enable fast processing and minimum delays incurred due to data movement. Extensive memories are utilized to hold pre-processed data so as to save time due to run-time calculations. The synthesis and physical implementation of the Cracken core was carried out in $65 \mathrm{~nm}$ technology. A study to map on several latest Xilinx FPGAs was also undertaken. Estimated time/costs for finding a SHA-1 near collision, extended to real collision, by Cracken are reported for comparison against ASIC and FPGA platforms. Other reported SHA-1 attack solutions of similar nature are also benchmarked. It is estimated, with post-layout simulations, that Stevens' differential attack with an estimated complexity of $2^{57.5}$, can be executed in 46 days using 4096 Cracken cores at a cost of $€ 15 \mathrm{M}$. Estimation for real collision with complexity $2^{61}$ is also done. For the design, the cost-time estimates reveal that an FPGA-based attack is more efficient compared to ASIC. Previously reported SHA- 1 attacks based on ASIC and cloud computing platforms are also compiled and benchmarked for reference. 


\subsection{Background}

\subsubsection{Attacks Against SHA-1}

SHA-1 was published in 1995 and is being widely used ever since for its efficiency. Multiple cryptanalytic efforts have been reported against it too. In early 2005, Rijmen et al. found strategy for finding collisions and suggested that SHA-1 may not be as secure as advertised [184]. Their attack built on an earlier attack on SHA-0 [37].They were able to attack the reduced version, i.e., 53 round version, and found that a collision can be reached with computational complexity less than $2^{80}$.

In the same year, Wang et al. [220] published their attack on the complete SHA-1 showing that SHA-1 can be broken with less than $2^{69}$ hash operations. The attack was based on three steps. First they searched for near collision differential paths in the disturbance vector, where they looked for local collisions with a single bit change. After that they adjusted those differential paths to optimize the local collisions so that no two local collisions overlap, increasing the computation steps and time. Next they transformed near collision differential paths from single block to double block collision differential path. It was the first attack on full-size SHA-1 and was a major breakthrough for cryptanalysts. Later, Wang et al. published an improved attack and were able to reduce the complexity to $2^{63}$ making SHA- 1 more vulnerable than before [219].

De Cannière et al. presented an improved attack in 2006 and 2007 on a 64 and 70 round SHA-1, respectively $[54,56]$. The attack was partially automated and attempts to find one collision instead of many at a given instant. Their attack only required 2 message blocks for the collision search and the method could predict the number of collisions under a given characteristic hence, giving more control to the attacker. Grechnikov et al. extended [54] in [3] to 75-round SHA-1.

The most recent and the most efficient near collision attack reported so far for complete SHA-1 is by Marc Stevens [205, 206]. It is based on optimal joint local collision analysis with a complexity of $2^{57.5}$. This near collision attack can be extended to a full collision attack with a complexity of around $2^{61}$ [196] at an estimated cost of \$2.77M.

Table 8.1: SHA-1 Attacks summary

\begin{tabular}{|c|c|c|c|}
\hline Researcher & Year & Rounds & Complexity \\
\hline Rijmen, Oswald [184] & 2005 & 53 & $<2^{80}$ \\
\hline Wang et al. [219,220] & 2005 & 80 & $2^{69}, 2^{63}$ \\
\hline De Cannière et al. [54,56] & 2006,2007 & 64,70 & $2^{35}, 2^{44}$ \\
\hline Grechnikov et al. [3,85] & 2010,2012 & 73,75 & $2^{44.37}, 2^{55.59}$ \\
\hline Stevens [206] & 2012 & 80 & $2^{61}$ \\
\hline
\end{tabular}




\subsubsection{Reported Hardware Attacks}

In 2005, [192] proposed attack hardware based on the attack by Wang et al. of SHA-1. It was the first ASIC-based cost estimate for breaking SHA-1. Within an estimated cost of $\$ 10 \mathrm{M}$ it promised a collision in 127 days.

Graphics Processing Units (GPUs) are extensively used for cryptanalytic activities due to the parallelism they offer. In 2012, an attack was proposed using 512 GPU clusters to break 75-rounds version of SHA-1 [3]. With a device occupancy of 50\%, the acceleration achieved was 39 times with respect to a GPP. A collision was achieved in 22 days, during which $2^{61.92}$ nodes were traversed. Table 8.1 summarizes reported attacks on SHA-1 and their implementations.

This work explores the possibility of using custom architectures for a SHA-1 attack and shows that this can significantly reduce the attack duration and cost. The attack practicality is achieved by migrating to newer technologies (i.e., exploring Moore's Law) and by using parallelism (i.e., exploring Amdahl's Law).

\subsection{Cracken}

Cracken ${ }^{1}$ with its application specific Instruction Set Architecture (ISA) is designed to carry out a near collision attack on SHA-1 that is useful for a two-block full collision attack. This sections discusses the Stevens' near collision attack first and then the Cracken architecture, its pipeline, algorithm mapping and the optimizations undertaken.

\subsubsection{Attack Algorithm}

Stevens' near collision attack has a computational complexity of $2^{57.5}$ SHA-1 compression functions, where one SHA-1 compression comprises of 80 SHA-1 rounds [205]. The source code of the attack is available as an open source project under the name Hashclash [207]. The attack extends the one by Wang et al. [220]. It uses differential paths, message bit relations, and tunneling to carry out a near collision attack. The algorithm is presented in brief here. The reader is kindly referred to [205-207] for a detailed study. Some relevant notations are given in Table 8.2.

The algorithm is divided into 4 stages, each stage dependent on the previous one.

Stage 0-Identical Prefix Block (IPB) search: This is the starting point of the algorithm and has a complexity of $2^{14}$ SHA- 1 compressions. The search for IPB is done by randomly generating 512-bit message blocks, generating subsequent IHV of 160-bits by SHA-1 compression function and then comparing whether the bit conditions from $q_{-4}$ to $q_{0}$ [205] are fulfilled. The generated IHVs are forwarded to the next stage.

Stage 1-Message block search up to step S_15: This stage takes up IHVs from stage 1 and checks data conformity in 16 steps, executed serially, from step_0() to step_15() as shown in Fig. 8.1. As the algorithm proceeds to next steps, the bit conditions

\footnotetext{
${ }^{1}$ In Greek mythology, "Kraken" was a powerful sea monster capable of defeating stronger enemies. The SHA-1 cracking ASIP is named after it as Cracken.
} 
Table 8.2: Notations for Stevens' attack

\begin{tabular}{|c|c|}
\hline Notation & Description \\
\hline$q_{t}$ & Bit conditions for step $t$, where $t \geq-4$. \\
\hline$Q_{t+1}$ & Candidate message generated for next step. \\
\hline $\mathrm{m}$ & First message of message pair $(\mathrm{m}, \mathrm{m} 2)$. \\
\hline $\mathrm{m} 2$ & Second message, only required in stage 3. \\
\hline$I H V_{\text {out }}$ & Intermediate Hash Value generated from $\mathrm{m}$. \\
\hline$I H V_{\text {out }}^{\prime}$ & Intermediate Hash Value generated from $\mathrm{m} 2$. \\
\hline$\delta I H V_{\text {out }}$ & Difference of $I H V_{\text {out }}$ and $I H V_{\text {out }}^{\prime}$ \\
\hline
\end{tabular}

and bit relations are narrowed down, reducing the degrees of freedom to ultimately find a suitable message block that satisfies the bit conditions up to $q_{16}$ and bit relations.

Stage 2-Message modification (tunnels): This stage accelerates the process of near collision search by controlled bit modifications in message block that fulfill the bit conditions from $q_{16}$ up to $q_{25}$, in 16 steps, as shown in Fig. 8.1. In case of conforming bit conditions, it proceeds to higher step. Otherwise, a new message block is fetched from previous stage.

Stage 3- $\delta I H V_{\text {out }}$ search: This stage culminates the algorithm by checking for a collision in the database. The difference between message blocks, i.e., subtraction of $I H V_{\text {out }}$ and $I H V_{\text {out }}^{\prime}$ to get $\delta I H V_{\text {out }}$ is carried out and looked up in the $\delta \mathrm{IHV}$ database. If it is found, a near collision is reported. If the collision is not found in step check_nc, the control jumps back to step $S \_24 c 1$ or $S \_24 c 4$.

As can be seen from the execution flow of Stevens' algorithm in Fig. 8.1, the execution is serial in nature, executing one step of a stage at a time because of the data dependence between stages. Different stages are highlighted with different colors in Fig. 8.1 while the triangle shape signifies narrowing search space. In each step, a candidate message $Q_{t+1}$ and a message $m$ is generated. Bit modification or bit condition conformity is carried out, in case of a failure, a new candidate pair is generated again. When all candidate messages are generated and they fail, a new message is picked from a previous step. However, if the conforming candidate is found, the attack moves to the next step. The back and forth execution jumps between the steps are very frequent, consequently the steps cannot be executed in parallel.

\subsubsection{Cracken Architecture}

Cracken has a pipelined architecture as shown in Fig. 8.2 with stages FE/DC(fetch/decode), $E X$ (execute) and $W B$ (writeback). Conditional jumps are supported by the address generator, other than sequential execution of instructions. The instruction word is 16 bits wide. Cracken is equipped with a set of 32-bit complex ALUs, where each ALU has dedicated register sets and memory banks. The complete datapath is 32 bits wide unless specified. It has a dedicated SPU (SHA-1 Processing Unit) to execute an entire 


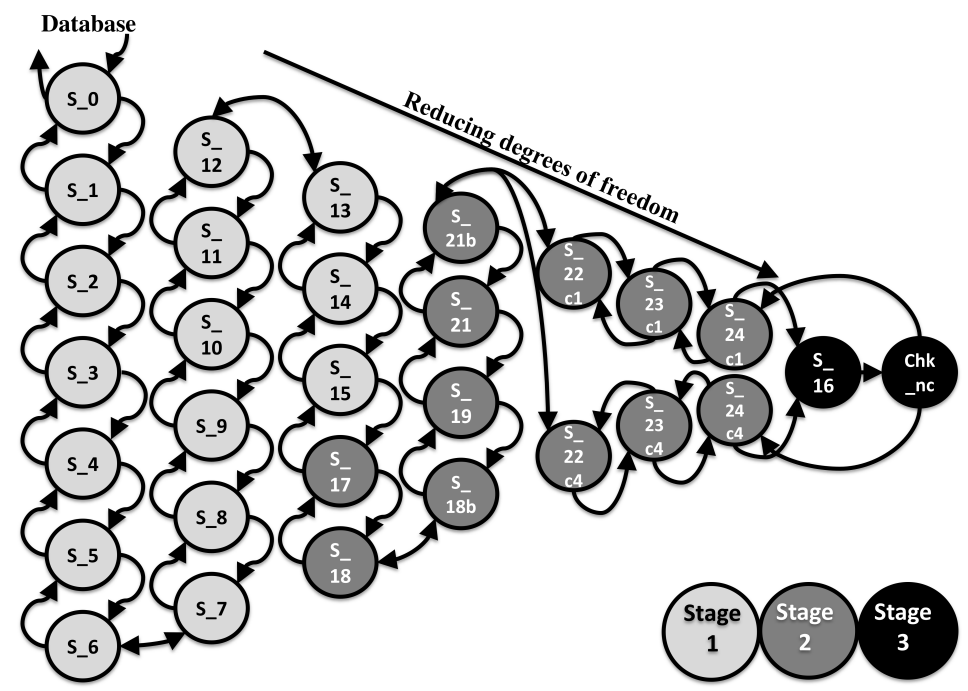

Figure 8.1: Stevens' attack execution control flow stages [207]

SHA-1 round in single cycle as a macro instruction. Without an SPU, a single round of SHA-1 compression would take multiple cycles on a GPP. For instance, for the Synopsys PD_RISC processor it was profiled to take 51 cycles [209]. Since the SHA1 compression function is a frequently used operation in near collision algorithm, reducing its runtime significantly reduces the total runtime of the attack. Cracken can also execute one SHA-1 round in reverse in one cycle (processing IHV to get the message $m$ ). Multiple memory instances in the WB stage are merged together as one memory bank due to space constraints in Fig. 8.2, a complete listing follows. The ISA of Cracken supports data movement from register to register, memory to register, and register to memory. Data movement and processing is merged in a single cycle, whenever possible, to save date movement latency.

\subsubsection{Instruction Set}

The instruction set for Cracken consist of 21 instructions, where a set of instructions is used in each stage, Table 8.3 shows the instructions with corresponding op-codes and brief details. Each instruction performs compound operations that may include more than one primitive operation like loading of data (bit conditions) from memories, execution of SHA-1 round or its reverse, data comparison with certain bit conditions etc. The instruction set can be extended to attack MD-4 hash function as well as to include other types of attacks.

\subsubsection{Mapping of Algorithm}

The SHA-1 attacking algorithm is serial in nature as seen in section 8.3.1. It is mapped stage-wise on to the hardware, where some specific registers and ALUs will be used during each stage execution, hence all the resources will never be used in a given cycle. 


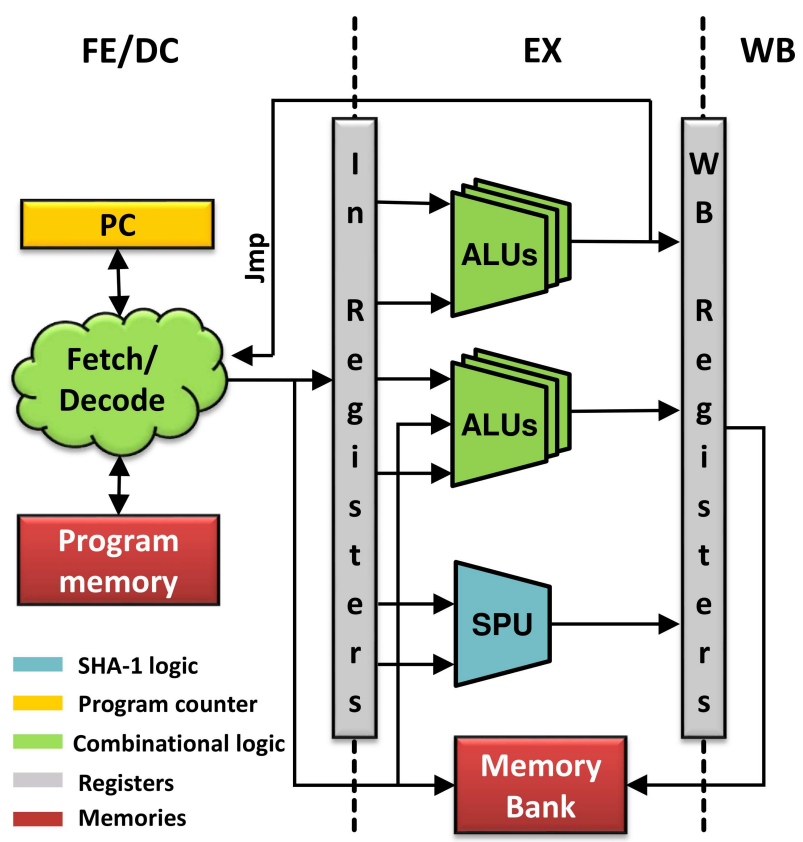

Figure 8.2: Cracken's instruction execution pipeline

Stage $\mathbf{0}$ is mapped on to a GPP since it's a trivial precomputation for suitable IHV with complexity $2^{14}$. The GPP computes SHA-1 compressions and the 160 bit hash value is compared against bit conditions. If all the bit conditions are satisfied, the block is written to the message database. The message database has 100 locations, each 32 bits wide. Only when the message database is half filled, reading it for stage1 is enabled. The readout pointers are kept to ensure unread data is never overwritten by an interlocked state machine. The reading side of the message database is connected with two sets of five 32 bit registers. They behave as pingpong buffer data feeders for stage 1 of Cracken. Writing to these registers from message database is serial while reading to stage 1 is parallel, saving 5 clock cycles. Moreover, the pingpong behavior ensures no additional delay due to data unavailability. These registers can be initialized using the instruction load_PingPong.

Stage 1 This stage requires reverse SHA-1 execution for mapping $Q$ to message $m$ using instruction execSHA_Qtp1. Step_0 to step_15 of the stage1 are executed one after another to search for messages which satisfy all the bit conditions up to step_15. The memories are shown in red color in Fig. 8.3, which are used to store the bit conditions, masks, and bit relations. 5 ALU registers $Q 0, Q 1, Q 2, Q 3, Q 4$, each 32 bits wide are initialized with IHV, and the bit conditions are loaded from memories to generate message $m$, line 6 in Algorithm 6. The near collision attack can be described using the pseudocode in Algorithm 6. COUNTER is a 7 bit register maintaining step number of stage 1 . It is reused in stage 2 and 3 as well.

The roles of $m$ and $Q$ are interchanged from step_7 onwards, where similar operations like SHA-1 compression function are used without message expansion. COUNTER switches to select the appropriate ALU, as shown in line 5 of Algorithm 6. Each mes- 
Table 8.3: Cracken instruction set; Op. code and Immediate value are 8 bits each in a 16 bit instruction

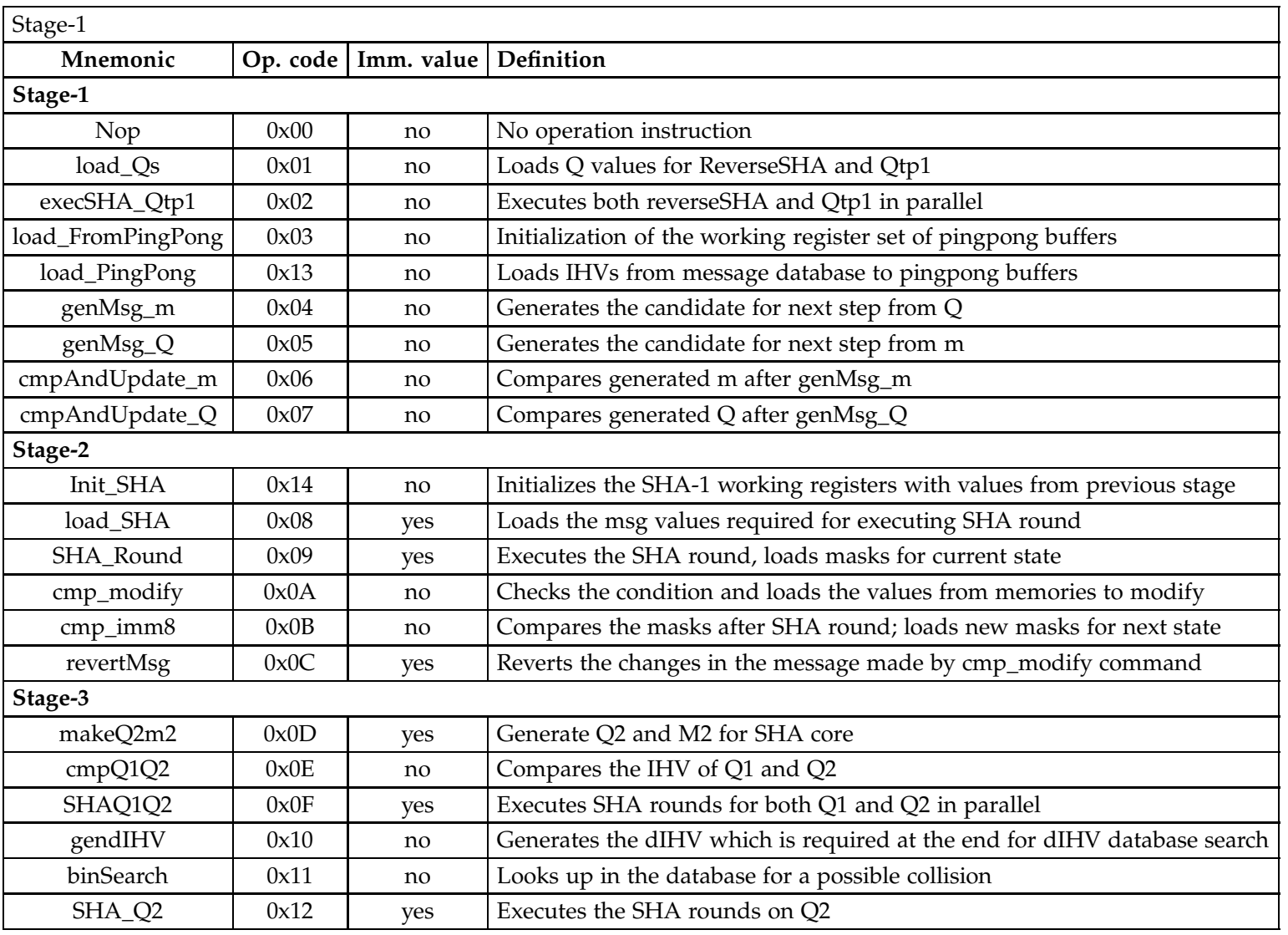

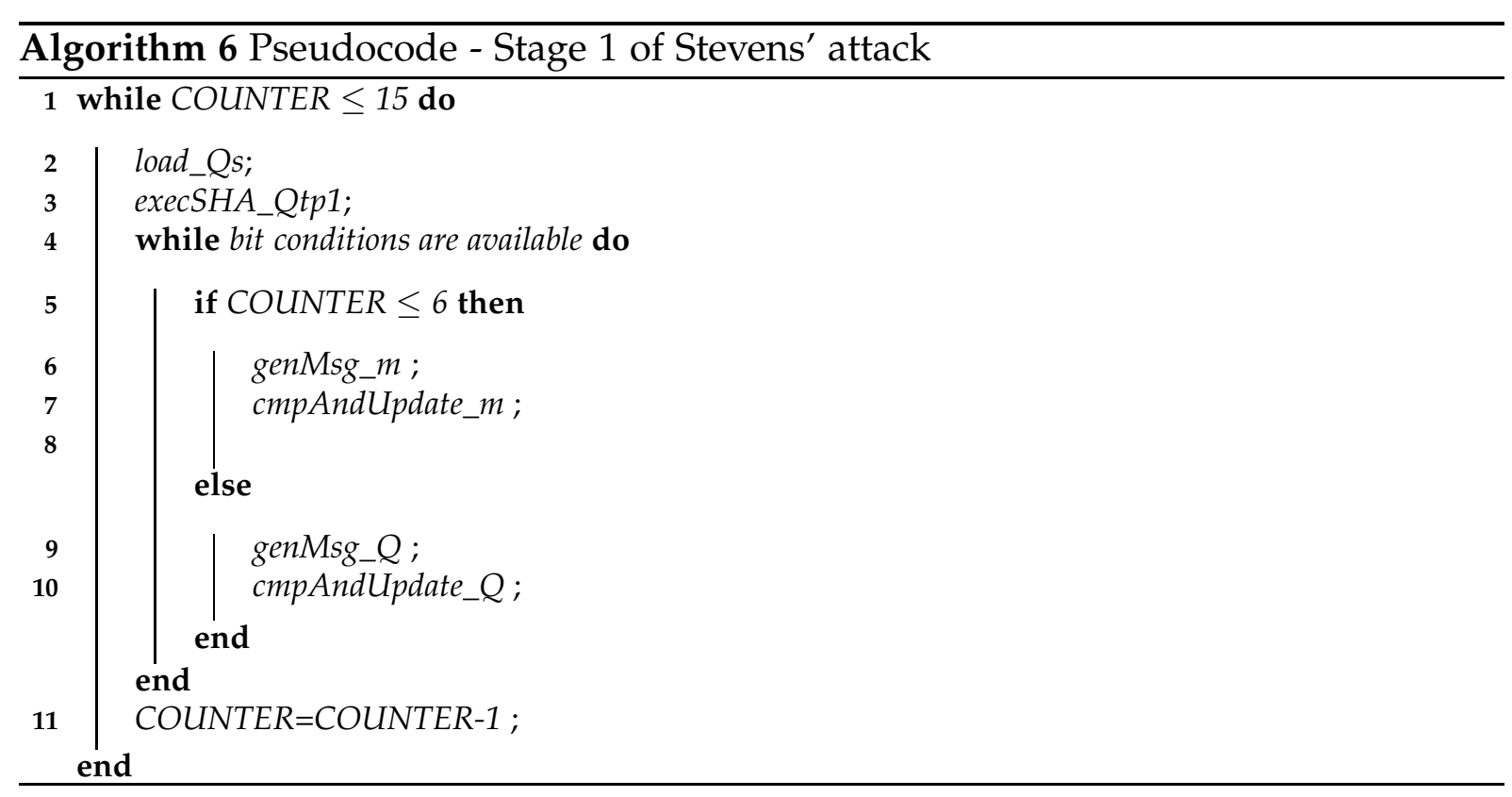

sage $m$ and $Q_{t+1}$ value is stored in a dual ported (single cycle accessible) memory. Depending on the instruction, the $Q_{t+1}$ value is shifted back to $Q 0, Q 1, Q 2, Q 3, Q 4$ 


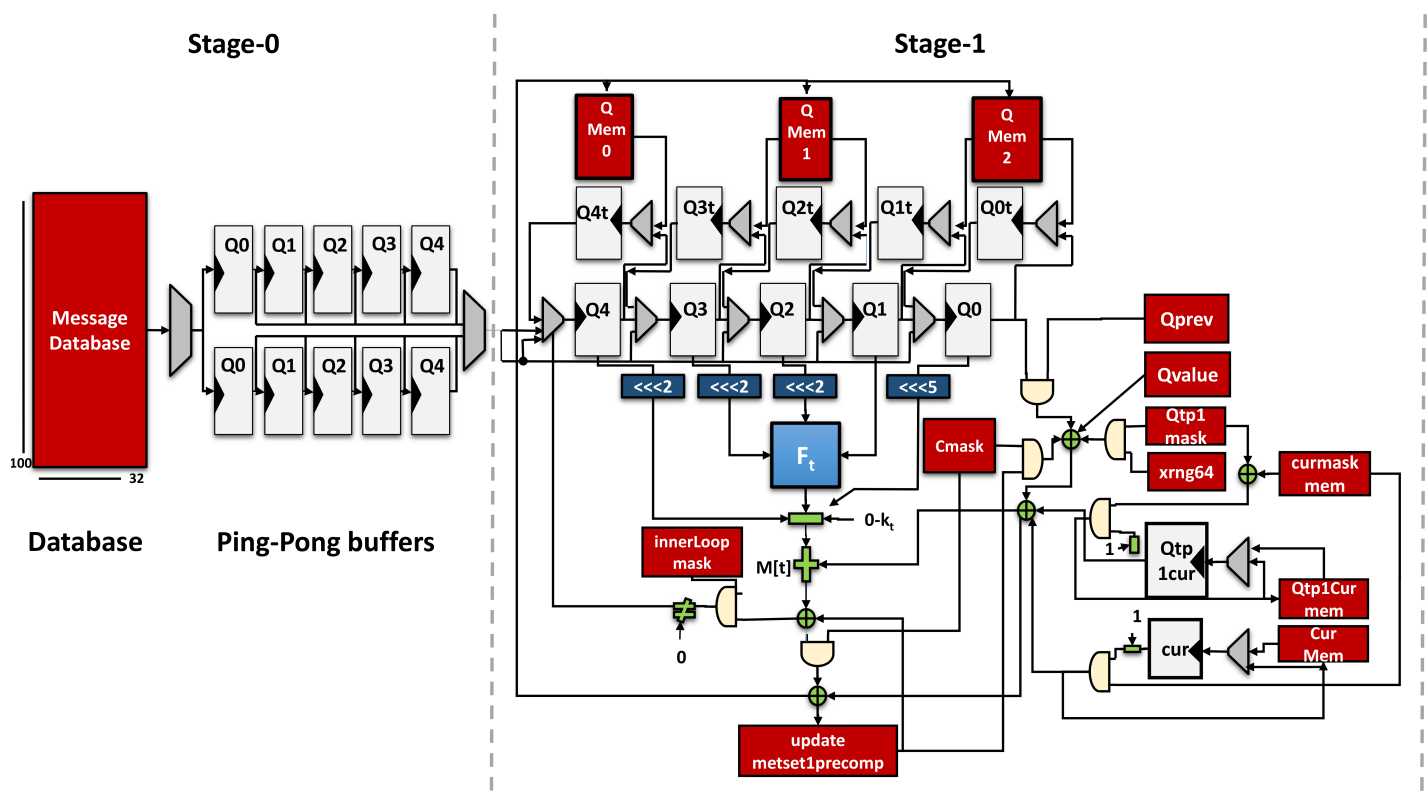

Figure 8.3: Cracken Schematic - Stage 0-1

registers or stored in memory. On successful generation of $Q_{t+1}$ message, the values stored in $Q 0, Q 1, Q 2, Q 3, Q 4$ registers are shifted to $Q 0 t, Q 1 t, Q 2 t, Q 3 t, Q 4 t$.

This is done to speed up the processing which will be described in the following text. $m$ memory and $Q$ memory are accessed in the later stages of the attack where message modifications are required. While looking for message corrections and candidate searching, 3 cases can occur, which have been handled carefully in Cracken design.

1. Candidate found: When a conforming $Q_{t+1}$ value is found, the state of the current step is stored in registers for enabling quick future access possible without having to access memory. The code jumps from line 7 or line 10 to line 2 of Algorithm 6.

2. On the look: Till a candidate $Q_{t+1}$ conforming to bit conditions is found, the control iteratively looks for one till all the bit conditions are exhausted (While condition in line 4).

3. Candidate unsuccessful: In case no successful $Q_{t+1}$ message is found after exhausting all bit conditions, the control jumps back to a previous step. The previous state is quickly restored from temporary registers Q0t, Q1t, Q2t, Q3t, Q4t to working registers $Q 0, Q 1, Q 2, Q 3, Q 4$ with a parallel loading of masks and counters from appropriate memories. The control jumps back to line 4 , instead of line 2 .

One of the masks used in generation of message $m$ requires 16 instances of 32bit wide memories, each 16 words deep, called metsetprecomp_mem. Table 8.4 shows 


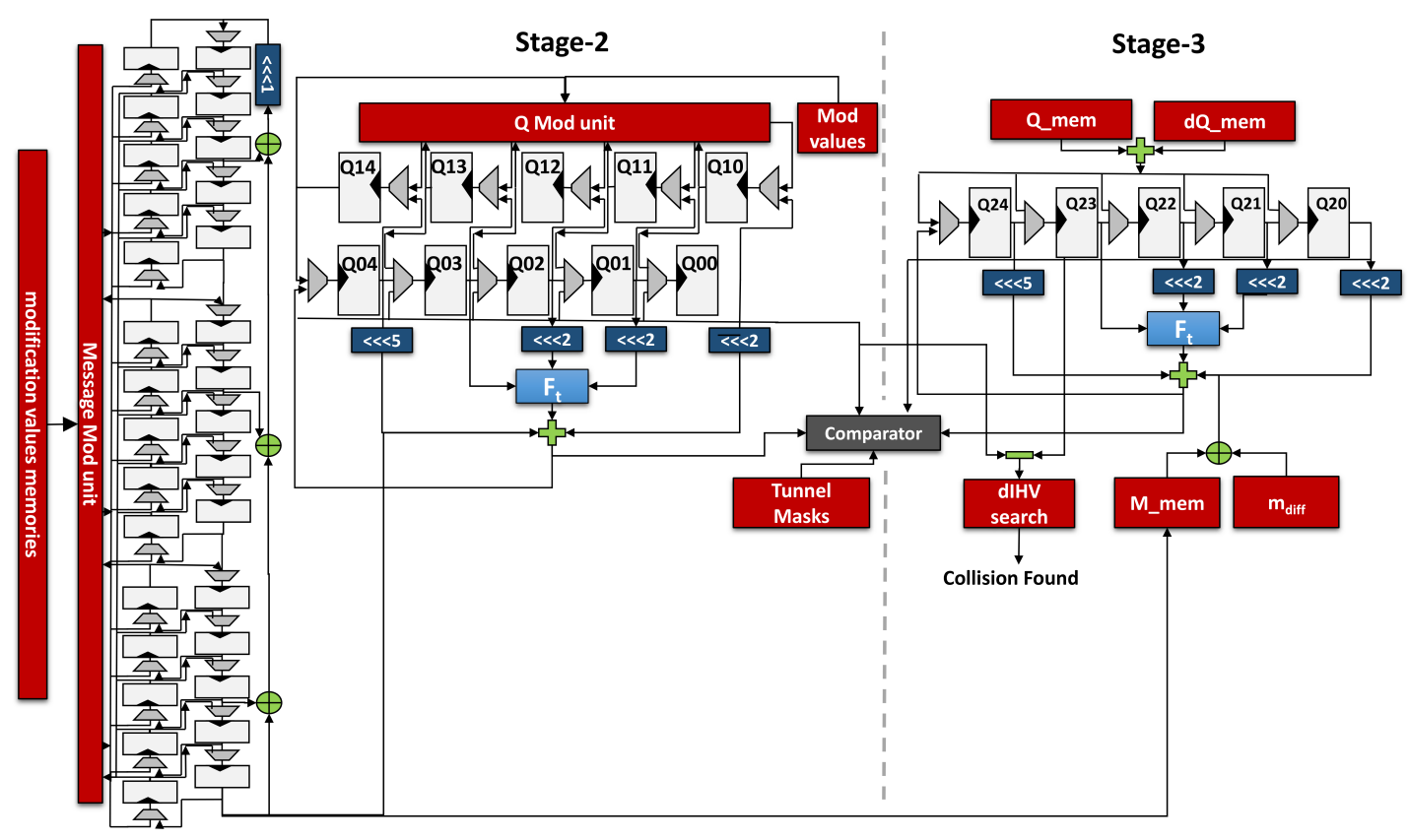

Figure 8.4: Cracken Schematic - Stage 2-3

Cracken's complete memory listing. These memories are updated in every step till step_15 using message $m$ from previous step and some pre-computed masks. Stage 1 requires 37 instances of dual port 32-bit wide memories to store those masks, shown as metsetprecomp_mask_13_mem, metsetprecomp_mask_14_mem, and metsetprecomp_mask_15_mem in Table 8.4.

Stage 2 or tunneling extensively uses SHA-1 compression function, justifying the need for an SPU. Algorithm 7 shows the pseudocode of this stage. Stage 2 architecture as shown in Fig. 8.2 consists of an SPU which takes input from message expansion module, a condition checking module which takes input masks from two separate modules, i.e., immediate masks and message modification masks, and a statemachine which is interlocked with instruction set. This stage is also executed iteratively on Cracken. In line 2, the SPU is made available for execution from round 17 onwards. The SPU is executed for 2 rounds, i.e., till 19, after which comparison is done in line 5 .

The SPU dictates the critical path of the Cracken core with 4 32-bit adders in series. There are 5 working registers Q00,Q01, Q02, Q03, Q04 which hold the $Q$ values, and 16 message $m$ registers from $m 0$ to $m 15$ as shown in Fig. 8.4 on the left side. Temporary registers are also used here to hold newly generated $Q$ values.

The working registers are loaded from the stage-1 working registers $Q 0, Q 1, Q 2$, Q3, Q4 as in line 1, 2 of Algorithm 7. Once loaded, the SPU executes one SHA-1 round per cycle, with message expansion on the fly. When a new IHV is generated (line 4), it is stored back to the working register and forwarded to condition checker as well. For quick accesses working registers are cloned to temporary registers $Q 10$, $Q 11, Q 12, Q 13, Q 14$ along with a parallel masks loading from respective memories. 


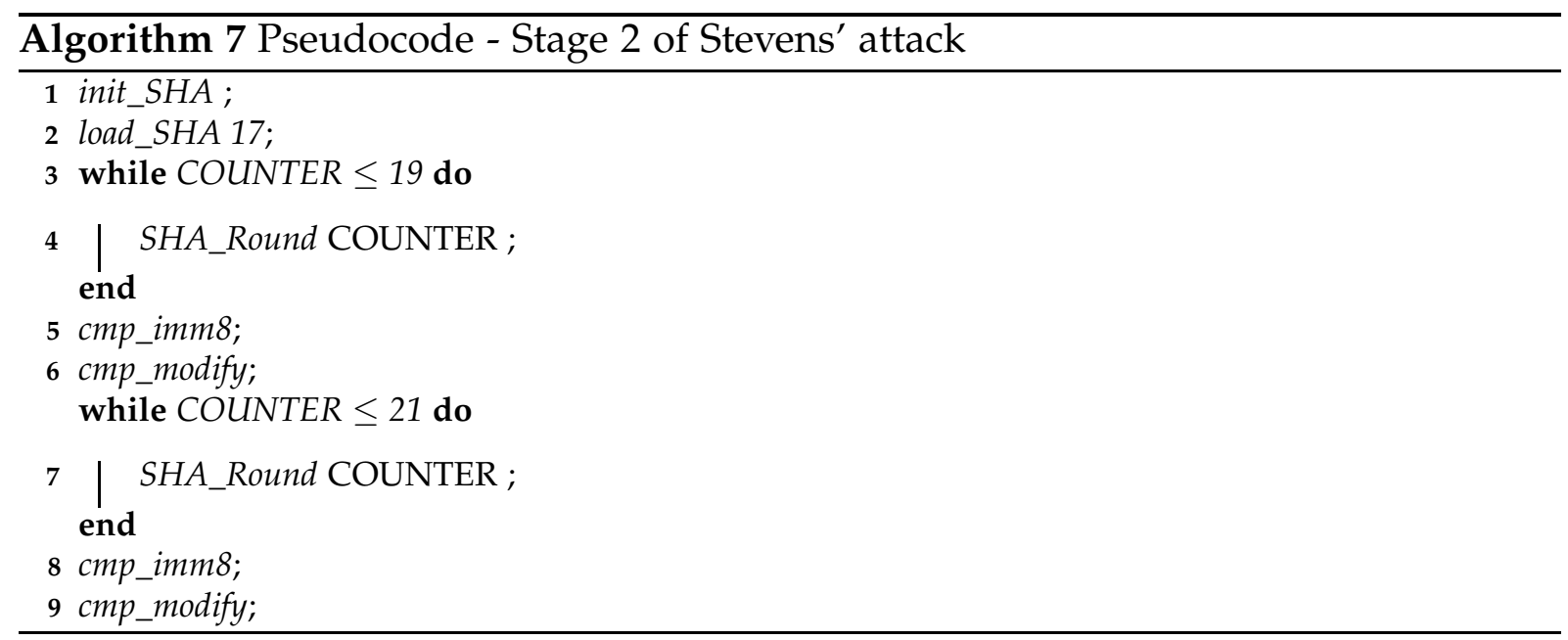

There are two kinds of masks; immediate masks are required immediately after the SPU and modification masks are required for message modification. Both sets of masks use same ALUs. After SPU execution, a comparison of the Immediate masks and modification masks with IHV is carried out in line 5, 8 and line 6,9 in Algorithm 7, respectively. The message modification unit modifies the messages with the modification masks. It works on set of 16 temporary message $m$ registers which hold the value of initial 16 steps from Stage 1 as shown in Fig. 8.4.

These 16 registers feed the 16 message $m$ working registers during SPU execution. When SPU is executed continuously, only message $m$ registers are used, but after every modification, the program control starts to execute SPU from round 16 onwards [207]. If the bit conditions fail, the control jumps back to a previous step, otherwise it proceeds up to step_24. In each step, different message values are modified.

Stage 3 The pseudocode for Stage 3 of Stevens' attack is given as Algorithm 8. As illustrated in Fig. 8.4, the processing elements include an SPU for $Q$, complex ALU for $Q 2$, a comparator, $\delta \mathrm{IHV}$ generation unit and CAMs (Content Addressable Memories). The ISA executes SPU and SHA-1 round function using complex ALU on $Q$ and $Q 2$ in parallel and calculates IHV (line 5 Algorithm 8). After 80 rounds, the difference in hash values is computed $(\delta \mathrm{IHV}$, line 11$)$ and is looked up in the database for a hit (line 12). Registers from Stage 2 are reused here along with a set of temporary registers Q20, Q21, Q22, Q23, Q24 for Q2. The SHA-1 function is executed on Q2(line 4). The working registers and temporary registers are compared for the first time after 33 rounds (line 5).

For message $m 2$ no expansion is required. Instead it uses message $m$ XORed with pre-computed values stored in $m$ _diff memory (line 3). Similarly, $Q 2$ is initialized by adding $Q$ and $d Q$ values, read from the memory. The attack proceeds till $33^{\text {rd }}$ round, without saving any value. The attack is then divided into 3 intervals (between round 33 to 52, 53 to 60 and 61 to 80), where after each interval, working registers and temporary registers are compared (line 9). If the result of the comparison for all three of these intervals equal then the working registers are subtracted to get $\delta \mathrm{IHV}$. These values are searched in the CAMs holding target $\delta \mathrm{IHV}$ values labeled as $d I H V$ search in 


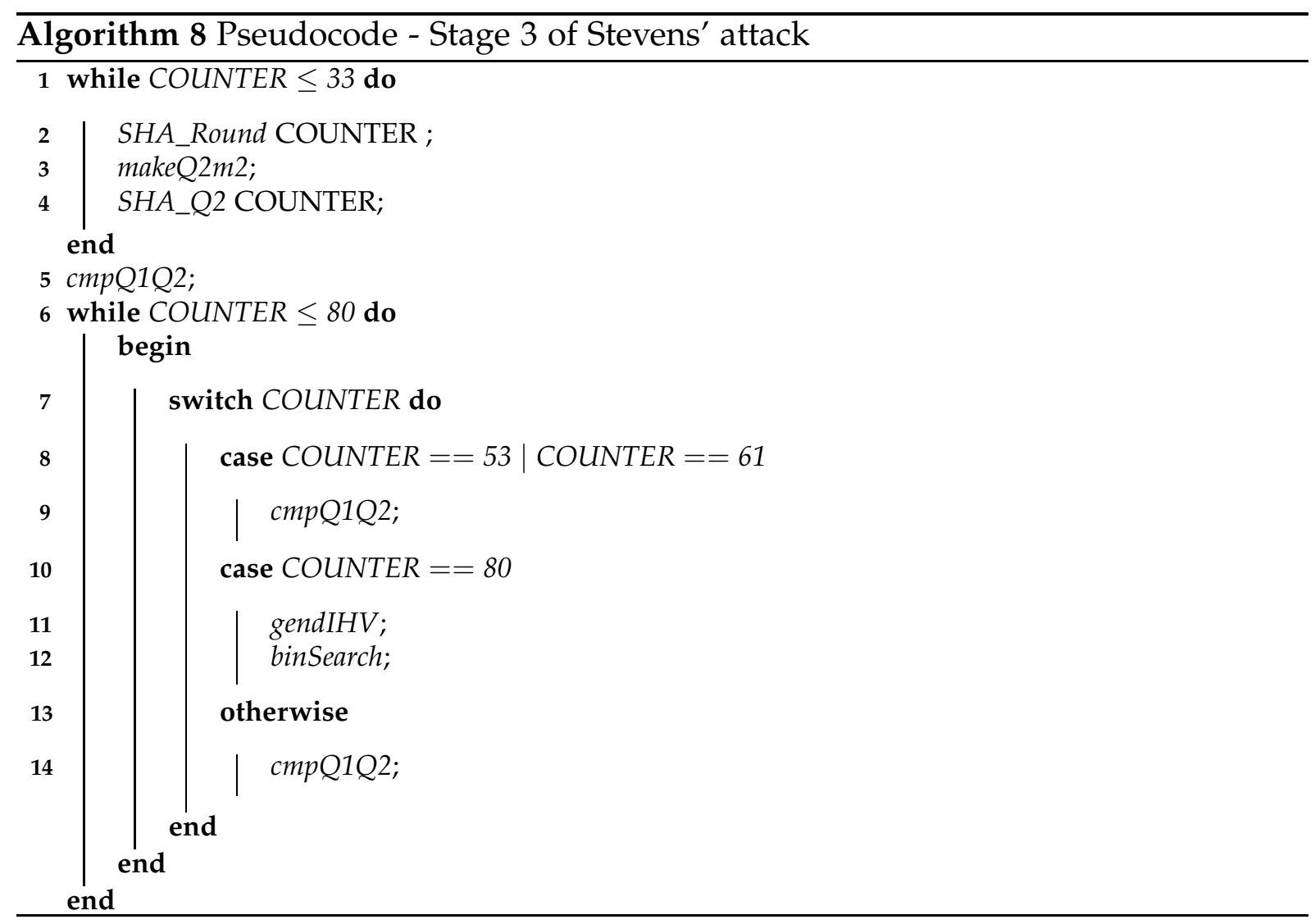

red in Fig. 8.4. In case of a hit, collision_found is asserted, else the control returns to an earlier step step_24 and proceeds again in the same manner with modified message bits.

\subsubsection{Cracken's Memory Bank}

Stevens' attack requires large bitcondition evaluating masks to be stored; the precalculated values are stored in ROMs, while masks requiring runtime calculations are stored in RAMs. Table 8.4 shows a complete listing. The $\delta I H V$ database is implemented using CAMs. There are five 32-bit wide, 512 locations deep memories. All the memories are single cycle accessible, dual ported memories are used to enable parallel accesses for resource sharing.

\subsubsection{Simulation and Verification}

LISA based high-level synthesis framework was utilized for developing Cracken (Section 2.3.2). Due to the slow runtime execution, it was not feasible to get a near collision by simulation of the synthesizable RTL automatically generated by the Processor Designer tool. For verification of the design, each stage's core was tested separately with its corresponding instruction set. Stevens' attack generates PRNGs using internal functions [207], its open source software implementation was modified to disable the PRNG and get deterministic values. The output was compared with Cracken's 
Table 8.4: Cracken Memories. Bitwidth $=32$ bits (16 bits for prog_mem), S/D $\mathrm{P}=$ Single / dual ported

\begin{tabular}{|c|c|c|c|}
\hline Name & Type (RAM/ROM) & Depth (words) & Instances \\
\hline prog_mem & SP-ROM & 512 & 1 \\
\hline msg_m_mem & DP-RAM & 128 & 8 \\
\hline mpartialvec_mem & SP-RAM & 16 & 1 \\
\hline Qvalue_mem & SP-ROM & 32 & 1 \\
\hline Qprev_mem & SP-ROM & 32 & 1 \\
\hline curmask_mem & SP-ROM & 16 & 1 \\
\hline metsetprecomp_mem & SP-RAM & 16 & 16 \\
\hline metsetprecomp_mask_13_mem & DP-ROM & 32 & 11 \\
\hline metsetprecomp_mask_14_mem & DP-ROM & 32 & 14 \\
\hline metsetprecomp_mask_15_mem & DP-ROM & 32 & 12 \\
\hline Qtp1_mask_mem & SP-ROM & 16 & 1 \\
\hline Cmask_mem & SP-ROM & 16 & 16 \\
\hline innerLoop_mask_mem & SP-ROM & 16 & 1 \\
\hline dQ_mem & DP-ROM & 128 & 3 \\
\hline mdiff_mem & DP-ROM & 128 & 3 \\
\hline cmpimm8_mask_mem & SP-ROM & 64 & 8 \\
\hline cmpAndmod_mask_mem & SP-ROM & 64 & 5 \\
\hline msg_mods_mem & SP-ROM & 64 & 5 \\
\hline Q_mods_mem & SP-ROM & 64 & 3 \\
\hline db_mem & SP-RAM & 512 & 1 \\
\hline cur_mem & SP-RAM & 16 & 1 \\
\hline qtp1cur_mem & SP-RAM & 16 & 1 \\
\hline Qtp1val_mem & SP-RAM & 16 & 1 \\
\hline 8IHV & SP-CAM & 512 & 5 \\
\hline & & & \\
\hline
\end{tabular}

up till step number 4 in each stage (since the steps in each stage are similar). This verified the execution of numerous control flow breaking instructions.

\subsection{Performance Analysis and Comparisons}

\subsubsection{Synthesis Results}

The design was synthesized using Faraday 65nm CMOS technology library (Section 2.3.1). The maximum achievable frequency for Cracken was $1300 \mathrm{MHz}$ (typical conditions). The area estimates (without the area of SRAMS) was calculated to be 88 kGE. 


\subsubsection{Physical Synthesis}

The physical synthesis of the design was carried out using Cadence SoC encounter 9.1 (Section 2.3.1). The memories are assumed to be external. A total of 6 metal layers were used, where layer 5 and layer 6 were used for VCC and GND, respectively. Power rings were implemented around the chip core at a distance of $50 \mu \mathrm{m}$ and power stripes were also added for uniform power distribution. Placement and routing was successfully done for a frequency of $1 \mathrm{GHz}$ with an area estimate of $.47 \times .47 \mathrm{~mm}^{2}$ with $70 \%$ logic density and the remaining 30\% left for routing. The area does not include the area of pads. Fig. 8.5 shows the chip layout of the design. The operating frequency can be improved given the memory modules are internal, floor planning step would yield better results, consequently it will assist the routing step.

Figure 8.5: Post layout and routing view of Cracken Core

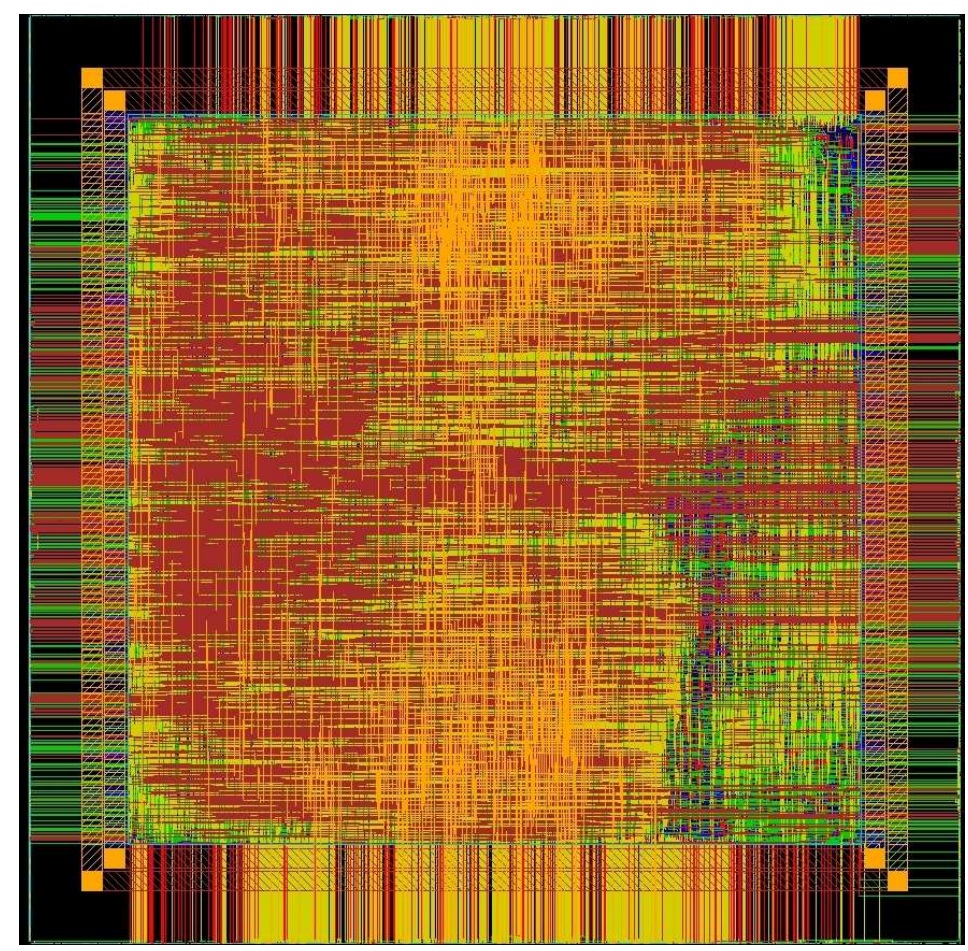

Table 8.5: Cost-Time approximation for Cracken with and without memories

\begin{tabular}{|c|c|c|c|c|c|c|c|c|c|}
\hline \multicolumn{10}{|c|}{ Near collision attack, complexity $2^{57.5}$ SHA-1 Compressions } \\
\hline Cracken cores & 1 & 4 & 16 & 64 & 512 & 1024 & 2048 & 4096 & 16384 \\
\hline Collision Days & 188712 & 47178 & 11794 & 2948 & 368 & 184 & 924 & 46 & 11 \\
\hline Cost $(\mathrm{w} / \mathrm{o}$ mem) $(\mathrm{M} €)$ & 0.0005 & 0.002 & 0.008 & 0.03 & 0.3 & 0.5 & 1 & 2 & 8 \\
\hline Cost $(\mathrm{w}$ mem) $(\mathrm{M} €)$ & 0.004 & 0.01 & .06 & 0.2 & 1.9 & 3.8 & 7.6 & 15.1 & 60.7 \\
\hline \\
Extended to real collision attack, complexity $2^{61}$ SHA-1 Compressions \\
\hline Collision Days & 2135039 & 533759 & 133439 & 33359 & 4169 & 2084 & 1042 & 521 & 130 \\
\hline
\end{tabular}




\subsubsection{Cost-performance Approximation without Memories}

For day-cost estimates, the area calculations of Cracken were first done excluding the memories. The post layout results infer a lower maximum achievable frequency of 1 $\mathrm{GHz}$ as mentioned in Section 8.4.2. As Cracken is capable of performing one SHA-1 round per cycle, one complete SHA-1 compression takes 80 rounds and consequently 80 cycles. The number of SHA-1 compressions in one day are calculated as.

$$
\begin{array}{r}
\text { SHA_1 operations per day }=\frac{(24 \times 60 \times 60) \times 10^{9}}{80} \\
=1.08 \times 10^{12}
\end{array}
$$

As the SHA-1 near collision attack complexity is $2^{57.5}$ SHA-1 compressions, the number of days required to find a near collision using $x$ Cracken cores is calculated as:

$$
\text { Days for collision with } \begin{aligned}
x \text { cores } & =\frac{2^{57.5}}{1.08 \times 10^{12} \times x} \\
& =\frac{188712.64}{x} \text { days }
\end{aligned}
$$

As an example, for 4096 Cracken cores operating at $1.0 \mathrm{GHz}$, the $2^{57.5} \mathrm{SHA}-1$ compressions can be performed in $\mathbf{4 6}$ days to find a near collision. A higher number of cores reduces the days required and vice versa.

For the cost calculation of producing a new ASIC for a dedicated application, estimates from EUROPRACTICE [70] are taken up. For 65nm Technology standard CMOS, the fabrication of $4 \times 4 \mathrm{~mm}^{2}$ chip (a $16 \mathrm{~mm}^{2}$ chip) costs $€ 35,600$. Consequently, the cost of $x$ Cracken cores, each with a size of $0.47 \times 0.47 \mathrm{~mm}^{2}$ can be estimated as:

$$
\begin{aligned}
x \text { SHA_1 cores cost } & =\frac{35600 \times 0.47 \times 0.47 \times x}{16} \\
& =€ 491.5 \times x
\end{aligned}
$$

Consequently, for fabrication of 4096 Cracken cores the costs can be estimated to be $€ 2 M$.

\subsubsection{Cost-performance Approximation with Memories}

For the cost approximation with memories, the Faraday Memory compiler [71] for UMC $65 \mathrm{~nm}$ is used (Section 2.3.1). The total area estimate of all memory banks listed in Table 8.4 was calculated to be $1.445 \mathrm{~mm}^{2}$. Since the access time of all the memories used was less than the critical path of the Cracken core, the operating frequency is not affected.

From Eq. 8.3, cost for $x$ Cracken processors (including memories) of $1.445 \mathrm{~mm}^{2}$ size each can be estimated as: 
Table 8.6: FPGA Feature Summary for Xilinx devices [226] [227] and Cracken cores mapped on FPGAs. Freq $=250 \mathrm{MHz}$

\begin{tabular}{|c|c|c|c||c|c|crc|}
\hline $\begin{array}{c}\text { FPGA } \\
\text { Device }\end{array}$ & \multicolumn{2}{|c|}{ BRAMs $(\mathrm{Kb})$} & Price $(€)$ & Crackens & No. of & Near collision & Collision & Price \\
Days $\left(2^{57.5}\right)$ & Days $\left(2^{61}\right)$ & $(€ \mathrm{M})$ \\
\hline XC7V2000T & 2584 & 46,512 & 17,714 & 22 & 3000 & 11 & 129 & 53.1 \\
\hline XC7V585T & 1590 & 28620 & 3,423 & 13 & 3000 & 19 & 218 & 10.2 \\
\hline XC7VX980T & 3000 & 54000 & 13,573 & 25 & 3000 & 10 & 113 & 40.7 \\
\hline XC7K480T & 1910 & 34380 & 2933 & 16 & 3000 & 16 & 177 & 8.7 \\
\hline XC6SLX150 & 268 & 4824 & 160 & 2 & 10000 & 38 & 427 & 1.6 \\
\hline
\end{tabular}

$$
\begin{aligned}
x \text { SHA_1 cores cost } & =\frac{35600 \times(1.445+(0.47 \times 0.47)) \times x}{16} \\
& =€ 3706.63 \times x
\end{aligned}
$$

It's worth mentioning that Cracken's area is dominated by the memory area. Consequently, when 4096 Cracken cores (with memories) are fabricated, the cost increases to $€ 15.1 \mathrm{M}$. Table 8.5 summarizes the number of Cracken cores against the minimum number of days to find a near collision and the cost of the solution (both with and without memories). The estimates for this attack when extended to real collision with complexity $2^{61}$ are also summarized in Table 8.5. Fig. 8.6 illustrates the trend graphically for extended estimates.

Figure 8.6: Collision days vs. Cracken cost (with and without memories)

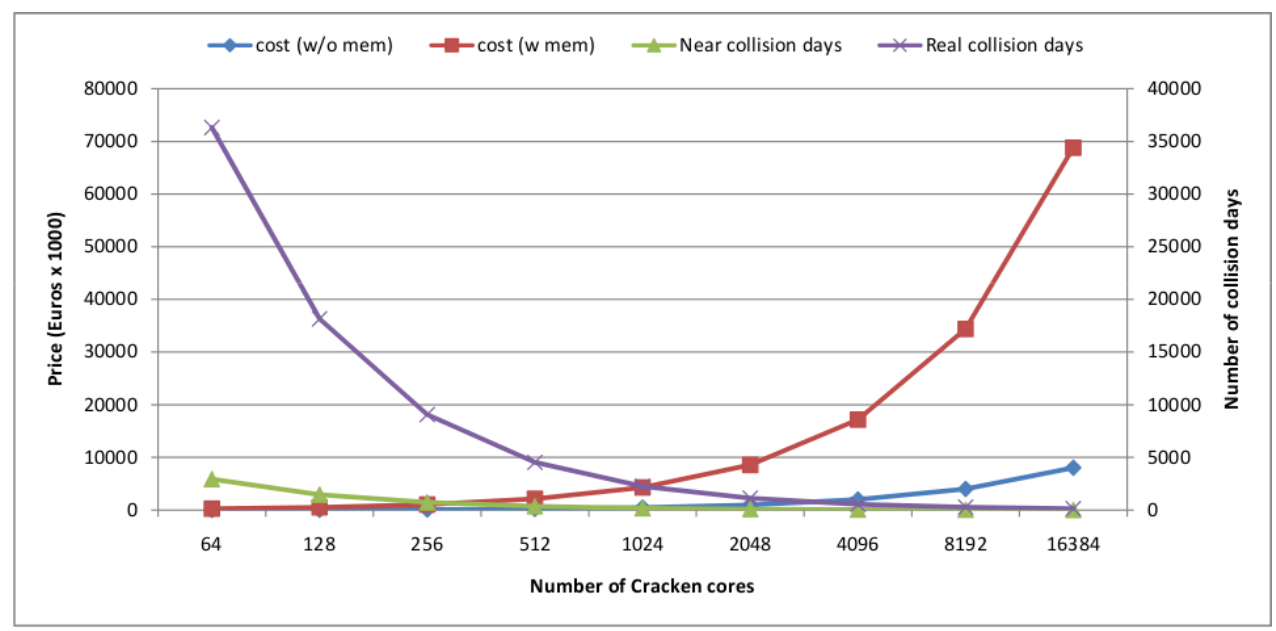

\subsubsection{Power Budget}

The power consumption of Cracken core is estimated by Synopsys Power Compiler based on RTL switching activity back annotation. The total power consumption of one Cracken core, including both the dynamic and static power consumption, when run 
for long runs of various stages is averaged to be $153 \mathrm{~mW}$. For an estimate of the power consumption of memories as shown in the Table 8.4, the Faraday memory compiler estimates are quoted. The static power of the largest memory, i.e., 512 word memory, is reported to be $9.864 \mu \mathrm{W}$ and the dynamic power consumption is $18.56 \mathrm{pJ} /$ access (or $0.58 \mathrm{pJ} /$ bit access).

Table 8.7: Cracken ASIC/ FPGA day-cost comparison with others works

\begin{tabular}{|c|c|c|c|c|c|c|c|c|c|}
\hline Work & $\begin{array}{c}\text { Freq. } \\
(\mathrm{MHz})\end{array}$ & \multicolumn{2}{|c|}{ Area/Core } & $\begin{array}{c}\text { Physical } \\
\text { kGE }\end{array} \mathrm{mm}^{2}$ & $\begin{array}{c}\text { Cell Lib } \\
\text { synthesis }\end{array}$ & Cores $\times 10^{3}$ & Days & $\begin{array}{c}\text { Cost } \\
(\mathrm{nm})\end{array}$ \\
\hline \multicolumn{8}{|c|}{ Without Memories } \\
\hline Satoh [192] & 400 & 30 & 0.39 & No & 130 & 9928 & 127 & 8.8 \\
\hline Satoh [192] & $800^{2}$ & 30 & $0.195^{2}$ & No & 65 & 20.28 & 526 & 8.8 \\
\hline $\begin{array}{c}\text { Cracken } \\
\text { (ASIC, 65nm) }\end{array}$ & 1000 & 88 & 0.22 & Yes & 65 & $\begin{array}{c}17.904 \\
32.7\end{array}$ & $\begin{array}{c}\mathbf{1 1 9} \\
65\end{array}$ & $\begin{array}{c}8.8 \\
16.1\end{array}$ \\
\hline \multicolumn{7}{|c|}{ With Memories } \\
\hline $\begin{array}{c}\text { Cracken } \\
\text { (ASIC, 65nm) }\end{array}$ & 1000 & 88 & 1.66 & Yes & 65 & $\begin{array}{c}32.7 \\
0.521\end{array}$ & $\begin{array}{c}65 \\
4096\end{array}$ & $\begin{array}{c}121 \\
\mathbf{1 . 9}\end{array}$ \\
\hline $\begin{array}{c}\text { Cracken } \\
(\text { XC6SLX150) }\end{array}$ & 250 & - & - & No & 45 & 2.08 & 4096 & $\mathbf{0 . 1 6}$ \\
\hline Stevens' [205] & 3300 & - & - & - & - & 0.032 & $2^{12.29}=5007^{3}$ & 2.2 \\
\hline
\end{tabular}

\subsubsection{Mapping Cracken on FPGAs}

For FPGA mapping, some readily available low cost Xilinx devices were chosen, having BRAMs large enough to accommodate at least a single Cracken core and its memories (Cracken processor). Table 8.6 summarizes various Xilinx devices. The last 4 columns of the table summarize the synthesis results of the Cracken processor on these devices. About 70\% logic device density was budgeted while reserving the rest of the $30 \%$ for placement and routing of the design. For some devices, distributed memories, in addition to BRAMs were used as synchronous SRAMs to optimally pack as many Cracken processors on an FPGA as possible. From Eq. 8.1, the number of SHA-1 compressions possible in a day for a single Cracken core operating at $250 \mathrm{MHz}$ can be calculated to be $270 \times 10^{9}$. Using Eq. 8.2, the number of days required to hit a SHA-1 near collision are calculated keeping the number of cores fixed. For smaller, low-priced Spartan device XC6SLX150 the normalized price to find near collision and collision comes out to be the smallest, compared to all the other devices.

\subsubsection{Comparison with Other Implementations}

Cracken, to the best of our knowledge, is the fastest SHA-1 near collision search hardware proposed so far. For comparison, the best SHA-1 attacking solutions reported on 
VLSI and cloud computing are reported here. Currency conversion has been carried out wherever necessary $(\$ 1=€ 0.88[48])$ ).

Satoh [192] proposed an ASIC for implementing Wang's attack $\left(2^{63}\right.$, cf. Table 8.1) for breaking SHA-1. A collision is computed in 127 days with an estimated cost of $\$ 10 \mathrm{~m}(€ 8.8 \mathrm{M})$. The hardware used to estimate the cost consisted of a custom LSI chip containing 64 SHA-1 macros. The attack required 303 personal computers, each equipped with 16 SHA-1 boards, each board had 32 chips, thus the total number of required SHA-1 attacking cores was 9,928,704. Naïve frequency scaling to undo the effect of Moore's law is also reported in Table 8.7. Here with a $800 \mathrm{MHz}$ operating frequency, $0.86 \times 10^{12}$ SHA-1 compressions/day are possible (cf. Eq. 8.1), requiring $10.6 \times 10^{6}$ days/core to find a near collision with a attack complexity of $2^{63}$ (cf. Eq. 8.2). A budget of $€ 8.8 \mathrm{M}$ can finance 20,282 cores of $0.195 \mathrm{~mm}^{2}$ (65nm [70]), hence requiring 526 days to hit a near collision. Since these estimates exclude the memories [192], the Cracken core (without memories) is considered, as shown in Table 8.7. For equal cost budget comparison with Satoh's work, the number of cores budgeted within $€ 8.8 \mathrm{M}$ is calculated from eq. 8.3 to be 17904 cores. Consequently, the number of days to hit a collision (extended from $2^{57.5}$ ) (from eq. 8.2) is 119. Considering Cracken processor (with memory) to hit a collision in 4096 days, a requirement of 46 processor cores (from eq. 8.2) is calculated, consequently requiring a cost of $€ 1.9 \mathrm{M}$ (from eq. 8.3). In comparison, the best result for FPGA based implementation (Table 8.6) comes out to be around $11 \times$ cheaper.

For a cloud computing based attack, resource estimation of implementing Stevens' attack [205] with $2^{61}$ complexity on servers is calculated in [196]. An Intel XEON processor is considered operating at $3.3 \mathrm{GHz}$ having 8 cores. Considering 4 instances of this device, each of the 32 cores perform $2^{31.6}$ cycles/ sec (or $2^{61.6}$ cycles per server year [196]). Single hash computation takes $2^{14}$ cycles [196]. Consequently, server years required to hit a near collision are $2^{74} / 2^{61.61}=2^{12.29}$. The cost for implementing the attack on cloud is taken from Amazon EC2 Pricing [7] (year 2015, Region: EU (Frankfurt)). For a t2.micro instance, the rental price is $\$ 0.015$ per hour (for 4 instances $\$ 525 \approx 2^{9}$ per year), resulting in the attack cost of $2^{9} \times 2^{12.29}=\$ 2.5=\mathrm{M} € 2.2 \mathrm{M}$.

Noteworthy is the fact that the costs given in Table 8.7 are strictly the ASIC fabrication/ server rental fee and the time to hit a collision. It excludes some critical cost-time factors, i.e., man-hours for design and maintenance, energy cost (having heavy geographical dependence), cooling systems, logistics, etc. A comparison endeavor of Cracken's performance against reported previous works [192,196], in the absence of these vital parameters, remains at best unfair.

\subsection{Concluding Remarks}

This work explores the possibility of using custom architectures for a SHA-1 attack and shows that this can significantly reduce the attack duration and cost. Compared to earlier methods, an ASIC and FPGA based custom computing architectures are

\footnotetext{
${ }^{2}$ Direct scaling from $130 \mathrm{~nm}$ to $65 \mathrm{~nm}$

${ }^{3}$ Server Years
} 
shown to bring down the attack complexity within realistic times. This threatens web security as most of the digital signatures use SHA-1 as underlying hash function. A fast adaptation of newer and secure alternatives of SHA-1, such as SHA-2 or SHA-3, is therefore strongly recommended. 


\section{Chapter 9}

\section{Conclusion and Future Work}

This work is an endeavor to aid a cryptographer in multiple ways. It attempts to expedite the hardware development for a specific application domain, i.e., symmetric key cryptography. The traditional VLSI design cycle that is both cumbersome and repetitive has been aided by various high level synthesis approaches, however, since they are generic to any application, their optimizations cannot compete the hand optimized HDL implementations. Chapter 2 discusses the motivation for High Level Synthesis, its methodology and a synopsis of various HLS tools that are being currently in use. Efforts for HLS tools in the domain of cryptography, that are few, are also discussed.

To automate the design flow for symmetric key cryptography, first their building blocks are identified. Chapter 3 classifies cryptography unconventionally, i.e., on the basis of their underlying computational workloads. A functionally complete set of constructive computation kernels as well as architectural sub-structures are identified. Chapter 4 discusses the major outcome of this thesis, i.e., a rapid prototyping tool (called CRYKET) for the automatic software and hardware implementation of cryptographic workloads. CRYKET has a gentle learning curve since it has a language independent GUI based design capture, eliminating the design quality dependence on the coding style of the programmer. Other than the algorithmic elements, the user configures a chosen mircoarchitectural structure for the intended VLSI implementation. The tool validates the design specifications given be the user and aids him/ her in case of non-conformity. The tool then generates an optimized software and HDL implementations that, for some prominent block ciphers and stream ciphers, were benchmarked to be on-par with their already-known hand-optimized HDL implementations.

In the proceeding chapter (chapter 5) some optimization strategies for efficient VLSI implementation of block ciphers and stream ciphers are discussed that have not yet been provided by the CRYKET tool automatically. The first one is the classical idea of splitting states kept in SRAMs into multiple smaller SRAMs to exploit increased parallel accesses. This idea is applied on HC-128 stream cipher, all data dependencies have been carefully handled with restructuring of the pipeline processor for the cipher. The HC-128 accelerator with outperforms all its reported software and hardware implementations by a good margin. Secondly, a unified design for multiple ciphers is taken up. The structural commonalities between these ciphers are aggressively exploited to redesign their unified implementation that the algorithms reuse the pipeline structure and storage organization. A case study for SNOW 3G and ZUC 
exhibited significant advantage in terms of area efficiency and even better throughput efficiency compared to the state-of-the-art implementations.

Flexibility is a desirable feature for cryptographic implementations, since it can let a user choose a performance-security trade-off. It can also protect against evolving cryptanalytic methods. In this context, Chapter 6 discusses two case studies. The first one is a crypto-coprocessor, supporting diverse cryptographic algorithms (including block ciphers, stream ciphers, hash functions etc) based on addition, rotation and XOR operations is undertaken. It is benchmarked to offer excellent performance-flexibility trade-off between the dedicated ASIC and GPP implementations. The second case study undertakes an ASIP for the RC4 cipher along with its prominent variants. It offers good area efficiency but the price of flexibility is paid by performance lower than that of the dictated ASICs for RC4.

Scalability of cryptographic workloads on modern GPGPUs is studied in Chapter 7. These multi-core machines are a viable option to be employed as a co-processor with a CPU host to accelerate parallelizable cryptographic functions. For most the the cryptographic workloads, PCIe bandwidth is the the bottleneck in the system performance since encryption/ decryption on GPUs is much faster compared to the transfer of data in the external global memory.

Chapter 8 explores the possibility of using custom architectures for a SHA-1 attack and shows that this can significantly reduce the attack duration and cost. The attack practicality is achieved by migrating to newer technologies (i.e., exploring Moore's Law) and by using parallelism (i.e., exploring Amdahl's Law).

\subsection{Outlook}

The CRYKET tool is the beginning to the high level synthesis in the field of cryptography. Its results provide motivation to further extend it in various directions

- Inclusion of other symmetric key cryptographic functions (hash functions, PRNGs etc.). A similar analysis and tool support for public key cryptography also can prove promising.

- Support for the high level generation of scalable kernels, i.e., support for GPGPU kernels employed as a co-processor to accelerate parallelizable cryptographic functions.

- Adding architectural optimizations for stream ciphers like parallel implementations generating higher throughput.

- Enhancing CRYKET to include SRAM specific memory access optimizations.

The resource sharing done for unified implementation of multiple ciphers (Chapter 5) serves as a guide and it would be interesting to see area efficient implementations of other cryptographic functions that must co-exist in a system. The idea of class-specific cryptographic accelerator/ processor can be viably extended to support a general prevention of side channel attacks as well. 


\section{Appendix A}

\section{RunFein Generated AES-128 Code}

RunFein generated ANSI-C wrapper code listing for AES-128 (ECB mode) encryption with on-the-fly subkey calculation.

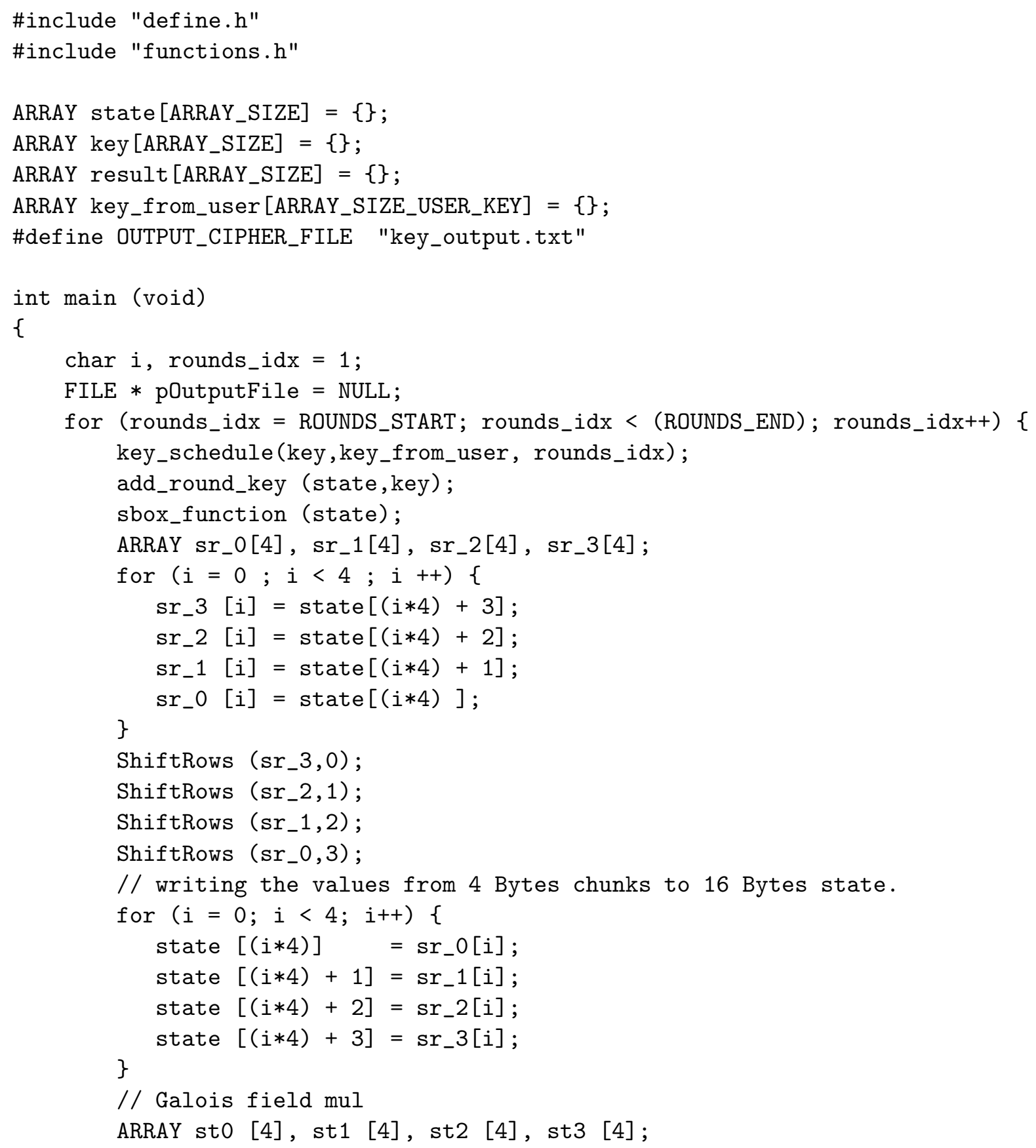




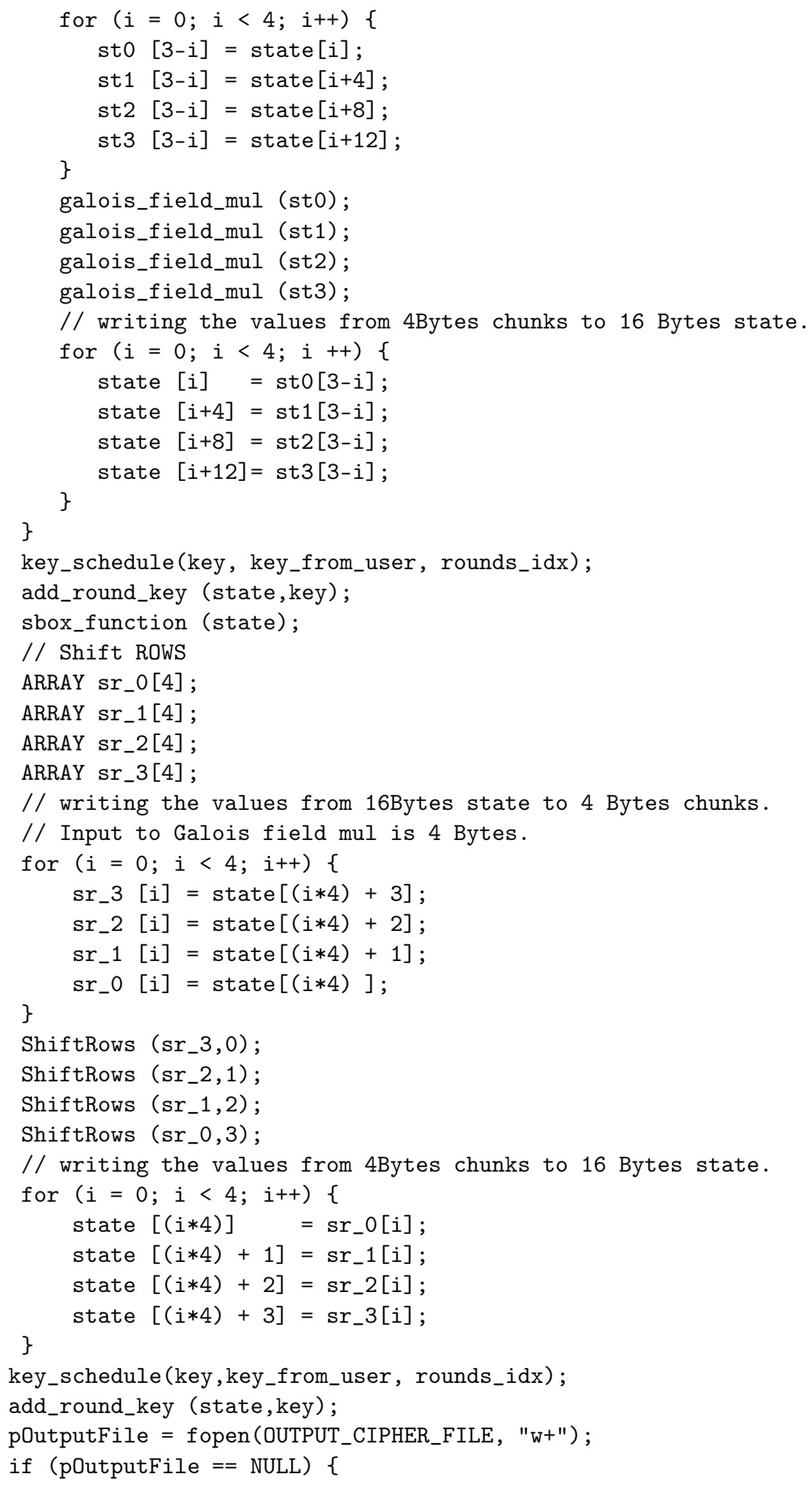




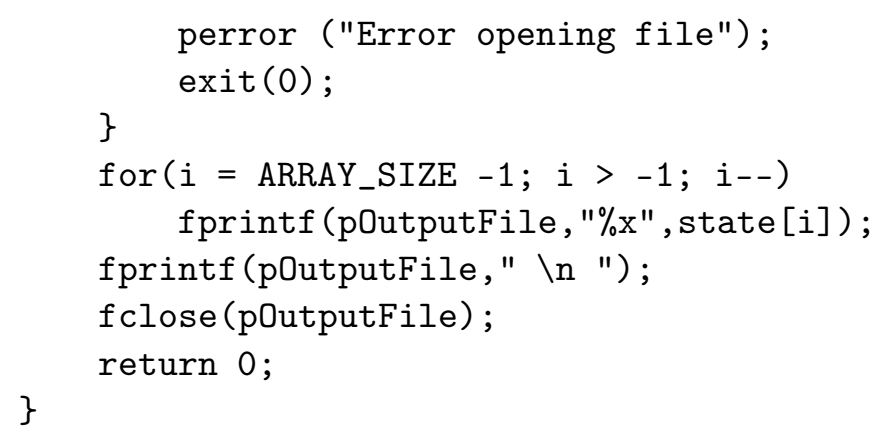




\section{Appendix B}

\section{RunFein GUI Snapshots}

Some GUI snapshots of various tabs of RunFein tool are presented here. CRYKET (CRYptographic Kernels Toolkit) caters to rapid prototyping of various cryptographic functions while RunFein is an instance of it dealing only with block ciphers.

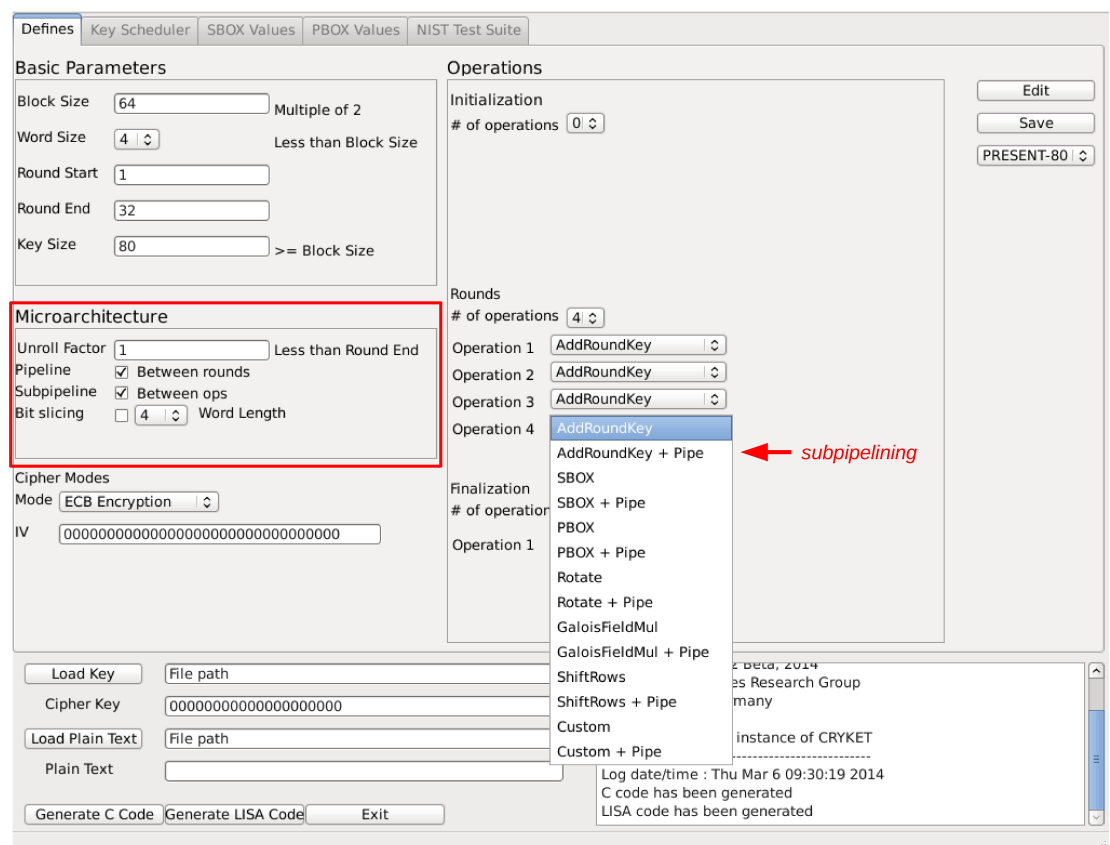

Figure B.1: Microarchitectural specification for subpipelined implementation in RunFein (+ pipe specifies pipeline) 


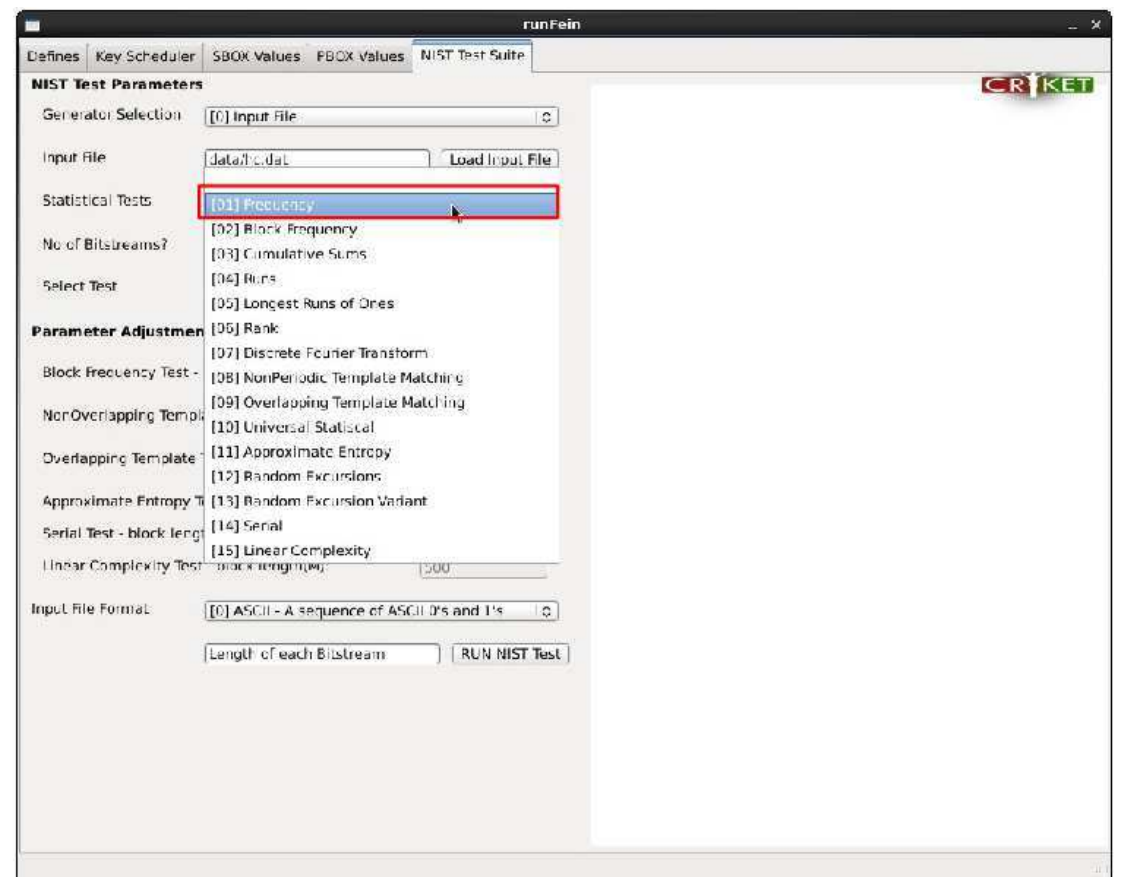

Figure B.2: NIST Test Suite parameter selection tab in RunFein 


\section{Appendix C}

\section{Description of Some ARX based Cryptographic Functions}

Algorithmic descriptions of some of the ARX based algorithms are given below.

\section{C.1 HC-128 Stream Cipher [224]}

The eSTREAM [65] Portfolio (revision 1 in September 2008) contains the stream cipher HC-128 [224] in Profile 1 (SW). Several research works exist on the cryptanalysis of HC-128 [126, 143, 148, 178, 202], however none of them pose a security threat to the cipher. The HC-128 cipher description in briefly sketched from [224].

The following operators are used in HC-128.

+ : addition modulo $2^{32}, \boxminus$ : subtraction modulo 512 .

$\oplus$ : bit-wise exclusive OR, $\|$ : bit-string concatenation.

$\gg$ : right shift, «: left shift (both defined on 32-bit numbers).

$\ggg$ : right rotation, «: left rotation (both for 32-bit numbers).

Two internal state arrays $P$ and $Q$ are used in HC-128, each with 512 many 32-bit elements. These arrays are also referred to as S-Boxes. A 128 -bit key array $K[0, \ldots, 3]$ and a 128-bit Initialization Vector $I V[0, \ldots, 3]$ are used, each entry being a 32-bit element. Let $s_{t}$ denote the keystream word generated at the $t$-th step, $t=0,1,2, \ldots$.

The following three pairs of functions are used in HC-128.

$$
\begin{aligned}
& f_{1}(x)=(x \ggg 7) \oplus(x \ggg 18) \oplus(x \gg 3) \\
& f_{2}(x)=(x \ggg 17) \oplus(x \ggg 19) \oplus(x \gg 10) . \\
& g_{1}(x, y, z)=((x \ggg 10) \oplus(z \ggg 23))+(y \ggg 8) \\
& g_{2}(x, y, z)=((x \lll 10) \oplus(z \lll 23))+(y \lll 8) \\
& h_{1}(x)=Q\left[x_{(0)}\right]+Q\left[256+x_{(2)}\right] \\
& h_{2}(x)=P\left[x_{(0)}\right]+P\left[256+x_{(2)}\right]
\end{aligned}
$$


Here $x=x_{(3)}\left\|x_{(2)}\right\| x_{(1)} \| x_{(0)}$ is a 32-bit word, with $x_{(0)}, x_{(1)}, x_{(2)}$ and $x_{(3)}$ being the four bytes from right to left.

The key and IV setup of HC-128 recursively loads the $P$ and $Q$ array from expanded key and IV and runs the cipher for 1024 steps to use the outputs to replace the array elements. This phase is referred as initialization phase in the rest of the discussion. It happens in four steps as given in Table C.1.

Table C.1: HC-128 Initialization phase steps

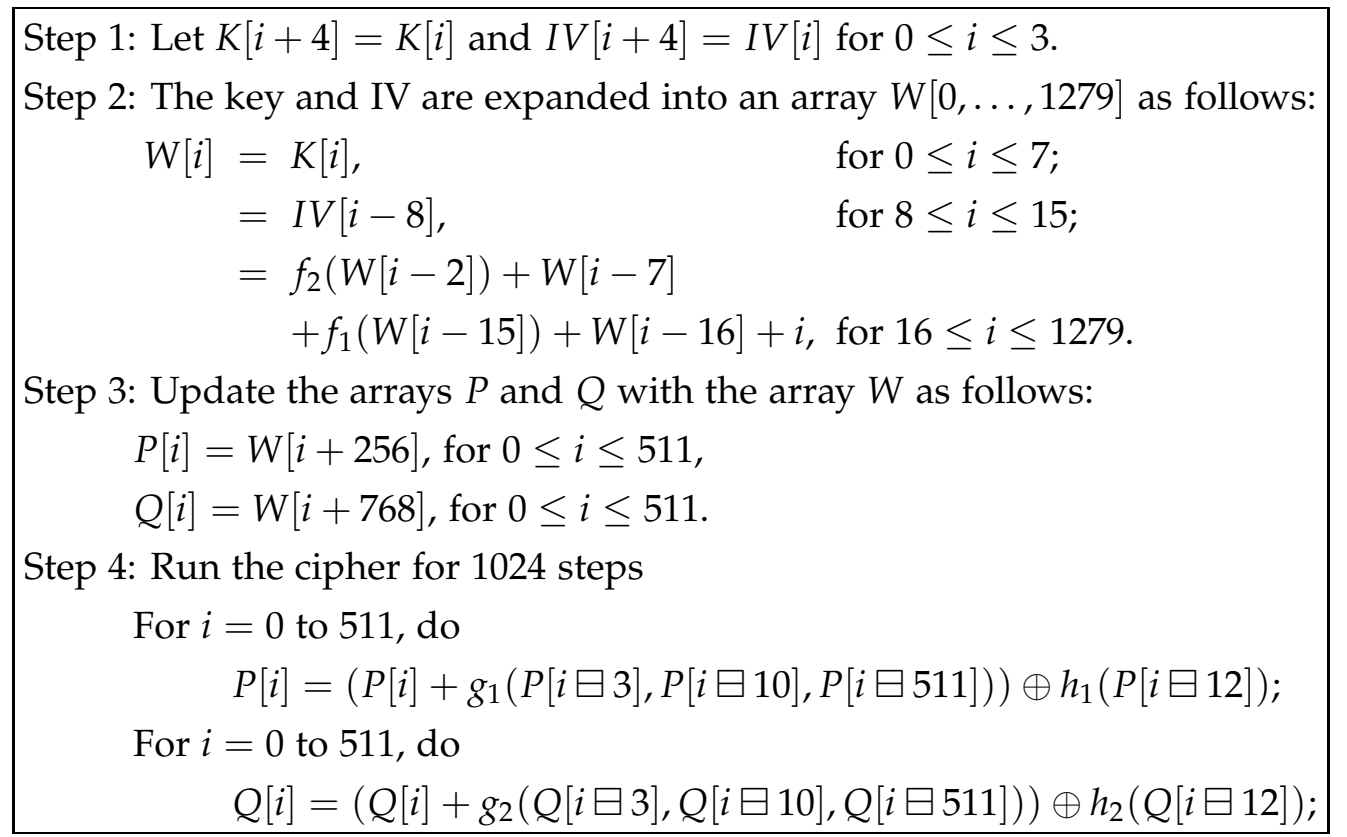

The keystream is generated using the algorithm as given in Table C.2. This phase is referred as keystream generation and the output word as keystream or keyword.

\section{C.2 HC-256 Stream Cipher [223]}

HC-128 is a lighter version of HC-256 [223] stream cipher, proposed as an outcome of 128 bit key limitation imposed in the competition. Both of these ciphers operate on 32-bit elements and use same set of operations $(+, \boxminus, \oplus, \|, \gg, \ll, \ggg$ and $\lll)$ The internal state arrays $P$ and $Q$ for HC-256 each have 1024 many 32-bit elements while the Key and IV are 256-bits each.

Out of the three pairs of functions used in HC-256, $f_{1}(x), f_{2}(x)$ pair is the same as the ones used in HC-128, others are mentioned below:

- $g_{1}(x, y, z)=((x \ggg 10) \oplus(y \ggg 23))+Q[(x \oplus y) \bmod 1024]$

- $g_{2}(x, y, z)=((x \ggg 10) \oplus(y \ggg 23))+P[(x \oplus y) \bmod 1024]$

- $h_{1}(x)=Q\left[x_{(0)}\right]+Q\left[256+x_{(1)}\right]+Q\left[512+x_{(2)}\right]+Q\left[768+x_{(3)}\right]$ 
Table C.2: HC-128 Keystream generation phase

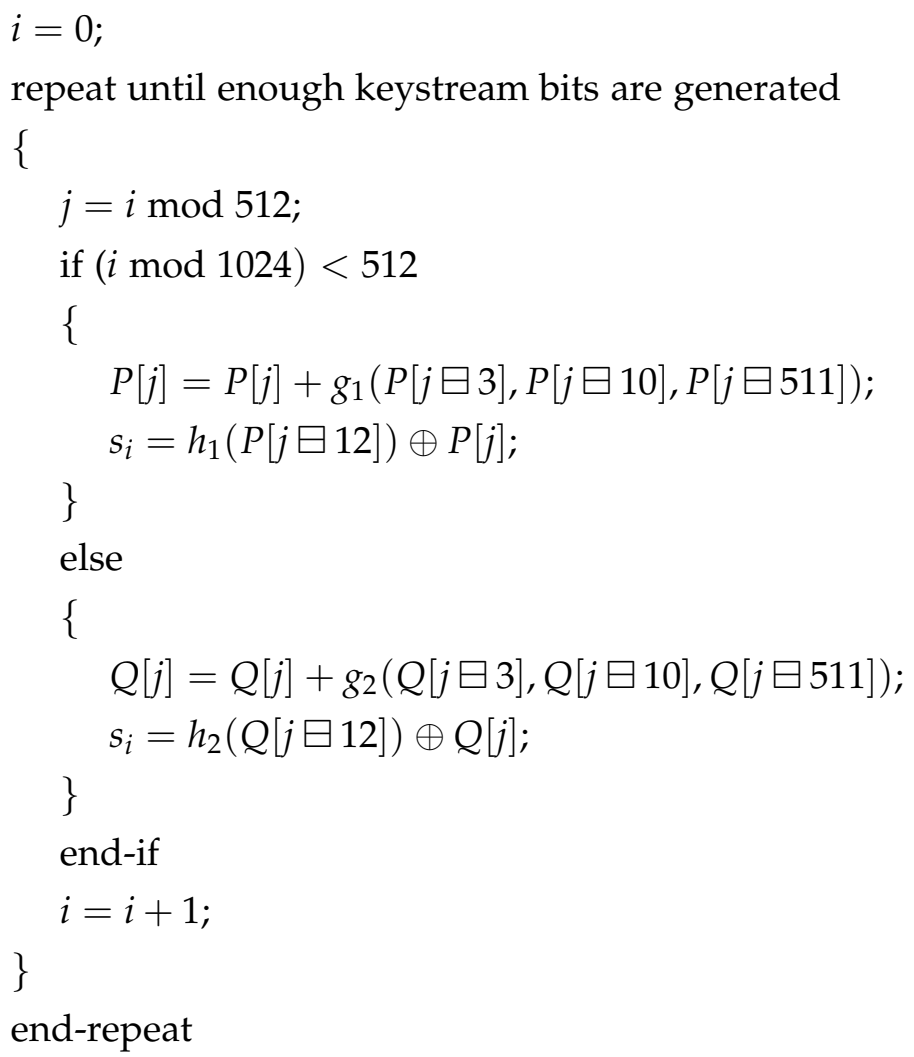

- $h_{2}(x)=P\left[x_{(0)}\right]+P\left[256+x_{(1)}\right]+P\left[512+x_{(2)}\right]+P\left[768+x_{(3)}\right]$

The key and IV setup of HC-256 is similar to the 4 steps of initialization for HC128 given in Table C.1. During step 2, the loop executes till 2559 and not till 1279. Similarly during step 3 and step 4 the loop iterates to accommodate the twice as large arrays $P$ and $Q$ for HC-256. The last two rows of the above table show the self-update steps (SUS) for the arrays $P$ and $Q$.

The keystream generation phase is also similar to that of HC-128, for 1024 iterations following two steps are executed The first one is the Self-Update Steps (SUS) for array $P$ and the second is a Keystream Word Generation Step (KWGS). For the next 1024 iterations, SUS for $Q$ array, followed by keystream word generation step are executed. The KWGS steps are the same as that of HC-128, while the SUS for $P$ and $Q$ are given by

- $P[j]+=P[j \boxminus 10]+g_{1}(P[j \boxminus 3], P[j \boxminus 1023])$

- $Q[j]+=Q[j \boxminus 10]+g_{2}(Q[j \boxminus 3], Q[j \boxminus 1023])$ 


\section{C.3 BLAKE Hash Functions Family [99]}

BLAKE is a cryptographic hash function chosen as one of the finalists of the SHA-3 hash function competition. It performs well in software as well as in hardware [99]. As per the initial specifications, its has four major variants as given in Table C.3.

Table C.3: BLAKE HASH functions

\begin{tabular}{|c|c|c|c|c|c|}
\hline Algorithm & Word & Message & Block & Digest & Salt \\
\hline BLAKE-224 & 32 & $<2^{64}$ & 512 & 224 & 128 \\
\hline BLAKE-256 & 32 & $<2^{64}$ & 512 & 256 & 128 \\
\hline BLAKE-384 & 64 & $<2^{128}$ & 1024 & 384 & 256 \\
\hline BLAKE-512 & 64 & $<2^{128}$ & 1024 & 512 & 256 \\
\hline
\end{tabular}

BLAKE follows a HAIFA iteration mode which is an improved version of MerkleDamgård paradigm. The BLAKE compression function takes the following input values:

- An 8-word chaining value, $h=h_{0}, h_{1} \ldots, h_{7}$

- A 16-word message block, $m=m_{0}, m_{1} \ldots, m_{15}$

- A 4-word salt, $s=s_{0}, s_{1} \ldots, s_{3}$

- A 2-word counter, $t=t_{0}, t_{1}$

Apart from the above mentioned input values, BLAKE uses 16 constants $c=$ $c_{0}, c_{1} \ldots, c_{15}$. The BLAKE's compression function has three steps:

- Initialization

- Rounds calculation

- Finalization

In the initialization round, a $4 \times 4$ matrix is initialized such that results of initialization vary with varying inputs. Chaining hash value or initialization value, salt, counter, and constants are used in the initialization stage. The input to the initialization process is as follows:

$$
\left(\begin{array}{cccc}
v_{0} & v_{1} & v_{2} & v_{3} \\
v_{4} & v_{5} & v_{6} & v_{7} \\
v_{8} & v_{9} & v_{10} & v_{11} \\
v_{12} & v_{13} & v_{14} & v_{15}
\end{array}\right)
$$


After the initialization the initial state is given by:

$$
\left(\begin{array}{cccc}
h_{0} & h_{1} & h_{2} & h_{3} \\
h_{4} & h_{5} & h_{6} & h_{7} \\
s_{0} \oplus c_{0} & s_{1} \oplus c_{1} & s_{2} \oplus c_{2} & s_{3} \oplus c_{3} \\
t_{0} \oplus c_{4} & t_{0} \oplus c_{5} & t_{1} \oplus c_{6} & t_{1} \oplus c_{7}
\end{array}\right)
$$

Where $h_{i}$ are the initialization or the chaining values, $s_{i}$ are the salt, $c_{i}$ are the constants and $t_{i}$ are the counter values. After the initialization stage, the round stage begins and depending upon the BLAKE variant, the round function iterates for a number of rounds. A round function performs the transformation in the following manner:

$$
\begin{array}{ll}
G_{0}\left(v_{0}, v_{4}, v_{8}, v_{12}\right) & G_{1}\left(v_{1}, v_{5}, v_{9}, v_{13}\right) \\
G_{2}\left(v_{2}, v_{6}, v_{10}, v_{14}\right) & G_{3}\left(v_{3}, v_{7}, v_{11}, v_{15}\right)
\end{array}
$$
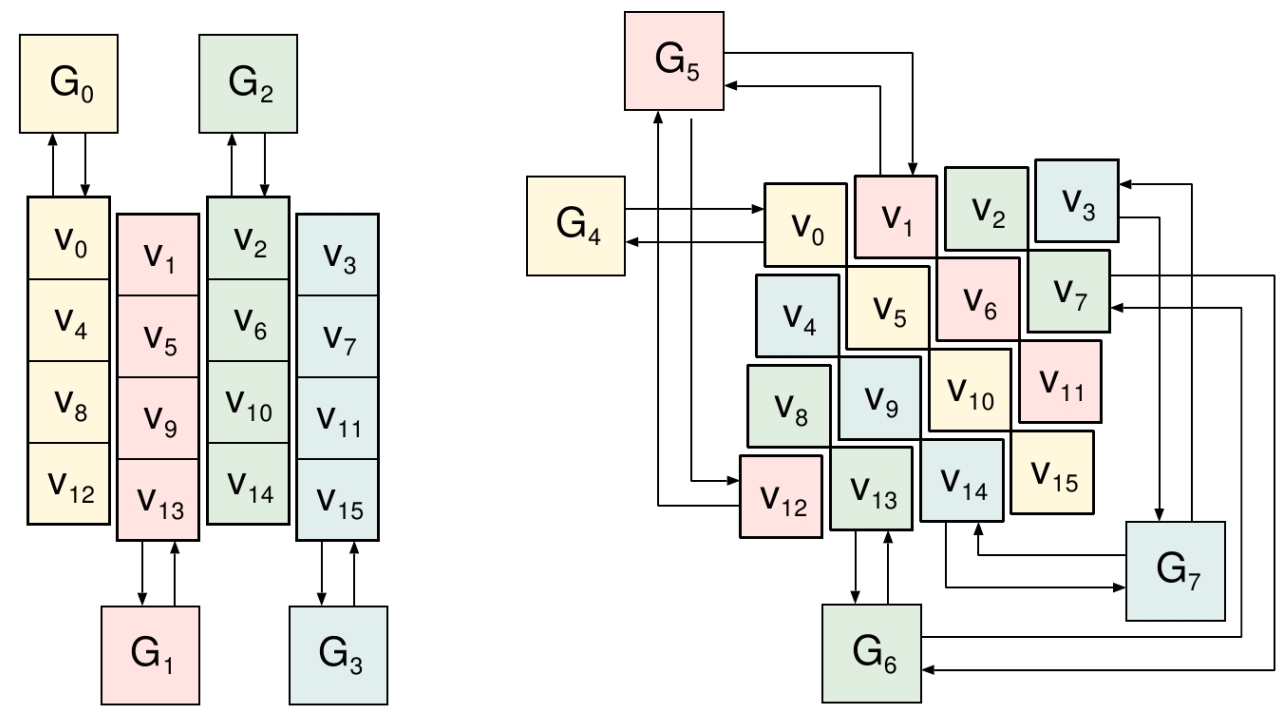

Figure C.1: Diagonal and column steps of BLAKE [12]

Fig. C.1 shows a column and diagonal step on which the following transformation is applied:

$$
\begin{array}{ll}
G_{4}\left(v_{0}, v_{5}, v_{10}, v_{15}\right) & G_{5}\left(v_{1}, v_{6}, v_{11}, v_{12}\right) \\
G_{6}\left(v_{2}, v_{7}, v_{8}, v_{13}\right) & G_{7}\left(v_{3}, v_{4}, v_{9}, v_{14}\right)
\end{array}
$$


Where the function $G_{i}(a, b, c, d)$ is as follows:

$$
\begin{aligned}
& a=a+b+\left(m_{\sigma_{r}(2 * i)} \oplus c_{\sigma_{r}(2 * i+1)}\right) \\
& d=(d \oplus a) \ggg R_{1} \\
& c=c+d \\
& b=(b \oplus c) \ggg R_{2} \\
& a=a+b+\left(m_{\sigma_{r}(2 * i+1)} \oplus c_{\sigma_{r}(2 * i)}\right) \\
& d=(d \oplus a) \ggg R_{3} \\
& c=c+d \\
& b=(b \oplus c) \ggg R_{4}
\end{aligned}
$$

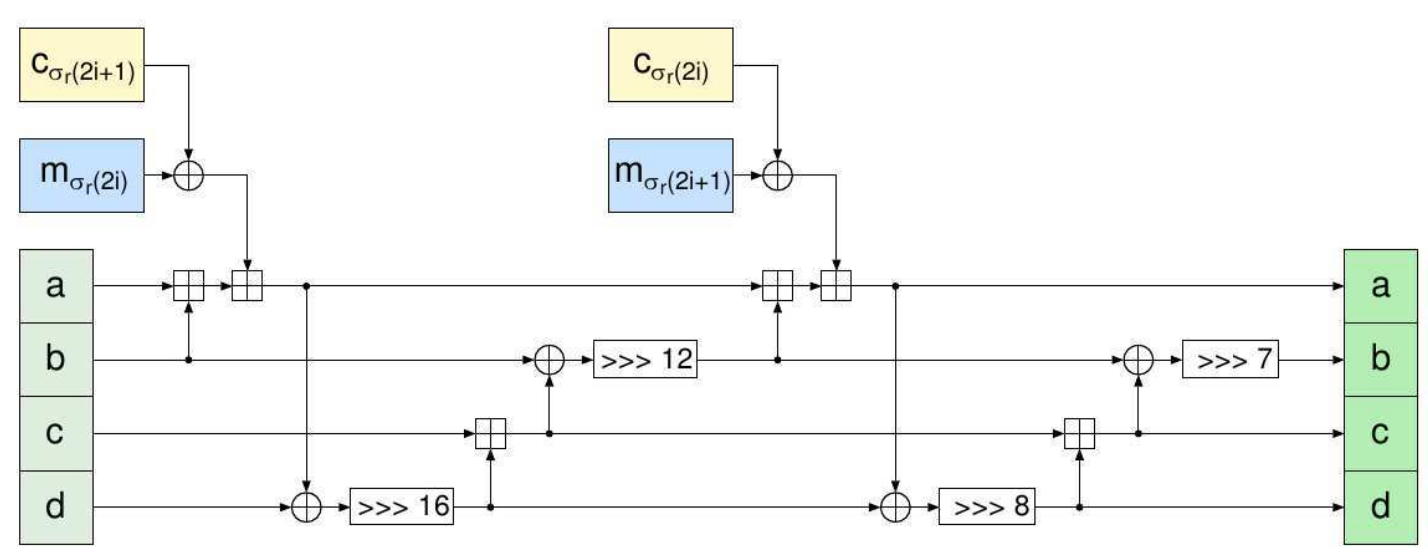

Figure C.2: G function of BLAKE hash [12]

Fig. C.2 shows a $G_{i}$ function. Where $\sigma_{0}, \ldots, \sigma_{10}$ are ten permutations of the numbers $0, \ldots, 15, r$ denotes the round number, $R_{i}$ denotes the rotation constants which are dependent on the variants of BLAKE, $c_{i}$ are the constants and $m_{i}$ are the messages. The first four invocations of the $G$ function $\left(G_{0}, G_{1}, G_{2}, G_{3}\right)$ can operate in parallel and the next invocations $\left(G_{4}, G_{5}, G_{6}, G_{7}\right)$ can operate in parallel after that. After each round a chaining value $h$ is calculated and it is fed to the new round. After a specified number of rounds (depending on BLAKE variants) the round function is complete and the finalization step starts. The input to the finalization stage is the initial state $v_{0}, \ldots, v_{15}$, salt $s_{0}, \ldots, s_{3}$ and the chaining value $h_{0}, \ldots, h_{7}$. The finalization stage performs the following steps: 


$$
\begin{aligned}
& h_{0}^{\prime}=h_{0} \oplus s_{0} \oplus v_{0} \oplus v_{8} \\
& h_{1}^{\prime}=h_{1} \oplus s_{1} \oplus v_{1} \oplus v_{9} \\
& h_{2}^{\prime}=h_{2} \oplus s_{2} \oplus v_{2} \oplus v_{10} \\
& h_{3}^{\prime}=h_{3} \oplus s_{3} \oplus v_{3} \oplus v_{11} \\
& h_{4}^{\prime}=h_{4} \oplus s_{0} \oplus v_{4} \oplus v_{12} \\
& h_{5}^{\prime}=h_{5} \oplus s_{1} \oplus v_{5} \oplus v_{13} \\
& h_{6}^{\prime}=h_{6} \oplus s_{2} \oplus v_{6} \oplus v_{14} \\
& h_{7}^{\prime}=h_{7} \oplus s_{3} \oplus v_{7} \oplus v_{15}
\end{aligned}
$$

Where $h_{i}^{\prime}$ denotes the hashed value that can be the final output or the chaining value. To hash a message that has more than 16 words, the message is broken into blocks, each of 16 words. After that the compression function is applied to it, the resultant $h_{i}^{\prime}$ is chained to the next iteration of the compression function. The process continues till the end of the message. This process is given as:

$$
\begin{aligned}
& h^{0}=\mathbf{I V} \\
& \text { for } i=0, \ldots, N-1 \\
& h^{i+1}=\operatorname{compress}\left(h^{i}, m^{i}, s, l^{i}\right) \\
& \text { return } h^{N}
\end{aligned}
$$

where $h$ is the chaining value, $N$ is the number of message chunks each of 16 words, $s$ denotes the salt, and $h$ denotes the output of the compression function.

\section{C.4 Skein Hash Functions Family [73]}

Skein is one of the five finalists of the SHA-3 hash competition [73]. The datapath of Skein is 64 bits, which makes it an excellent candidate to be implemented on 64 bit microprocessors. Threefish, a tweakable block cipher is at the core of the Skein hash function. Threefish block cipher has three inputs, key, tweak and plain text. The tweak is 128 bits for all block sizes. Based on the internal state size of the cipher, there are three variants of Threefish, Threefish-256, Threefish-512 and Threefish-1024. Threefish can have multiple state sizes and multiple output sizes.

The compression function of Skein consists of Threefish block cipher in MatayasMeyer-Oseas (MMO) construction form. Threefish in MMO mode along with tweak specification defines the Unique Block Iteration mode. The UBI mode converts variable length inputs to fixed length outputs. Threefish uses a large number of simple rounds instead of small number of complex rounds. The core of Threefish is a MIX function that is made up of an Addition, Rotation and XOR-ing. Fig. C.3 shows a MIX function. 


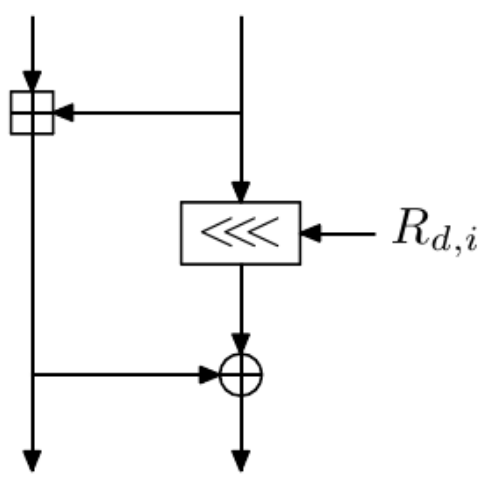

Figure C.3: A MIX function of Skein [73]

In case of Skein-512, the primary candidate of Skein family, four MIX functions are required to constitute one round of Skein-512. Fig. C.4 shows the construction of Skein-512 using MIX functions.

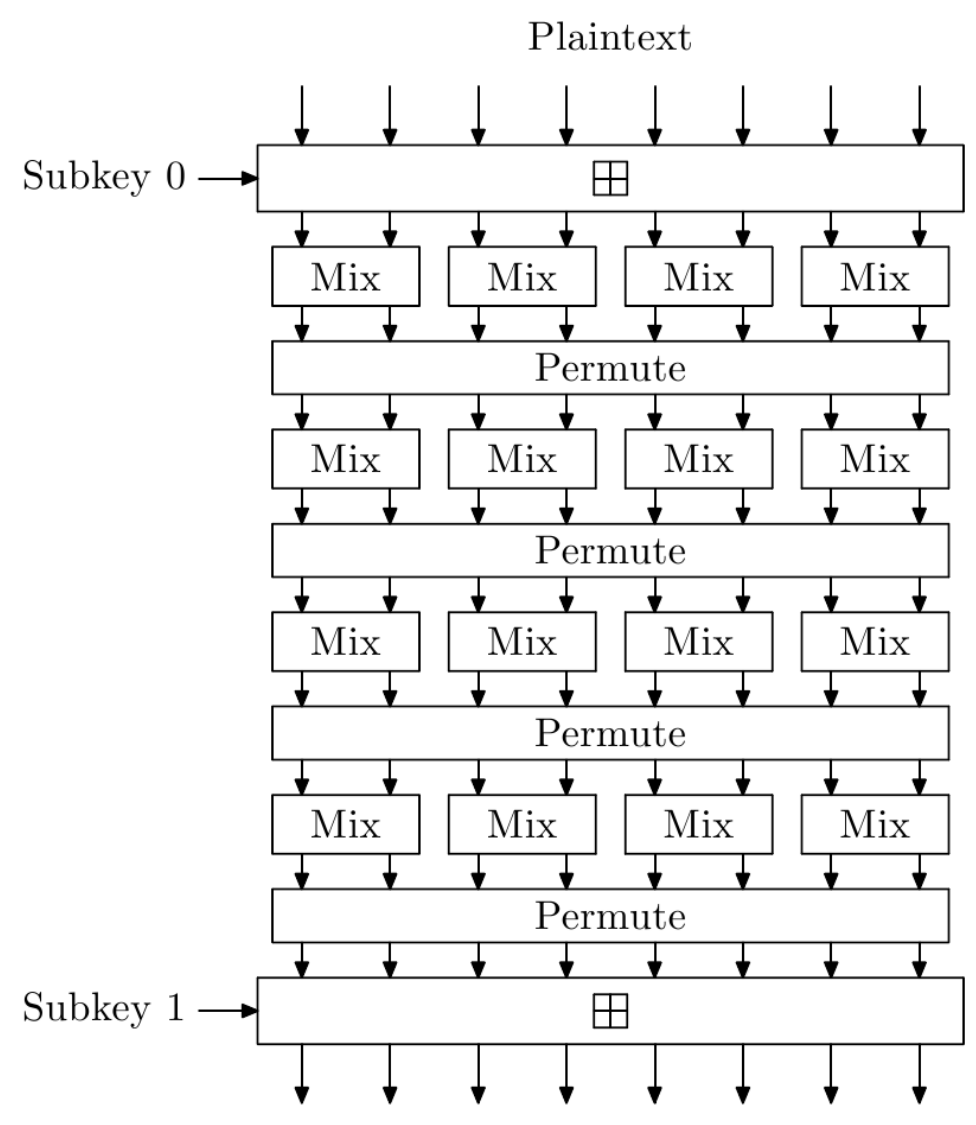

Figure C.4: 4 Rounds of Skein-512 Hash [73]

After every four rounds, a subkey has to be injected. The subkey is injected by adding it with the outputs of MIX functions after every fourth round. A subkey is generated using keywords, tweakwords and subkey number in a subkey generator. Skein-256 has two MIX operations in every round and Skein-1024 has 8 MIX opera- 
tions in every round. Skein-512 has 72 rounds. Fig. C.5 shows the key generator for Skein-512.

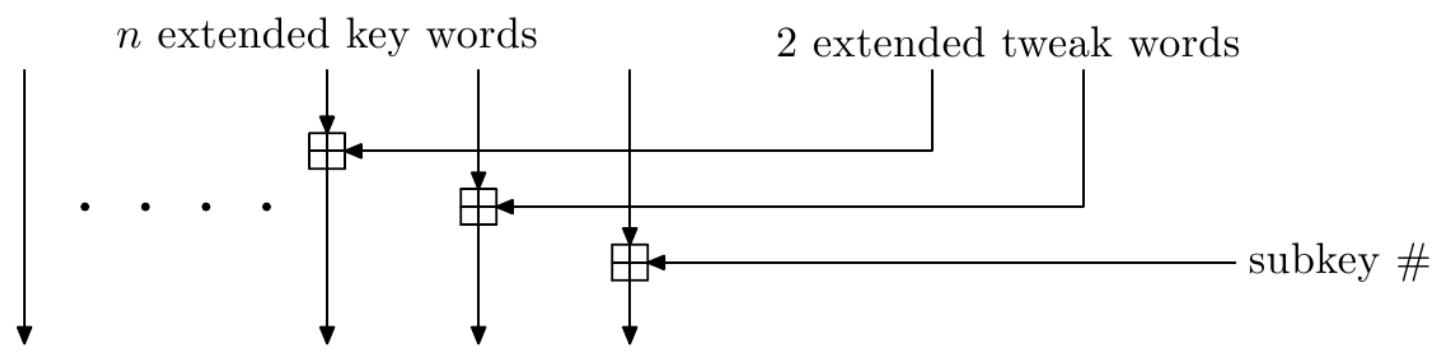

Figure C.5: Key generator of Skein [73]

In normal hashing mode, Skein has three invocations of the UBI. One invocation is for the configuration, one for the message and the last invocation is for the output transform. Fig. C.6 shows Skein in a normal hashing mode. The rotation constants of Skein repeat after every eight rounds and thus VLSI hardware are made, consisting of eight rounds along with two subkey injections.

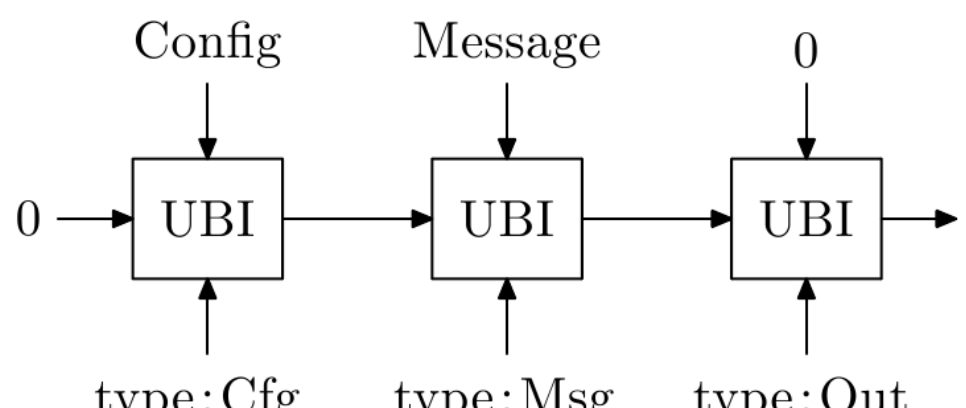

Figure C.6: Skein in normal hashing mode [73]

\section{C.5 Salsa20/r Stream Cipher [21]}

Salsa20 is a stream cipher that makes use of hash function in counter mode to generate keystream bits $(S)$ that are mixed with the plaintext $(P)$ by XOR-ing to generate ciphertext $(C)$ [21]. Block size of its inputs and outputs is 64 bytes. The input counter is incremented for every preceding block of plaintext. Salsa20 accepts four types of inputs, each consisting of words of 32 bit granularity. Firstly, an input key, that is either 256-bit $\left(k_{0}, k_{1}, \ldots, k_{7}\right)$ or 128-bit $\left(k_{4}=k_{0}, \ldots, k_{7}=k_{3}\right)$ in size; further a 64-bit nonce $\left(n_{0}, n_{1}\right)$ and a 64 -bit counter $\left(t_{0}, t_{1}\right)$; finally four words of pre-defined constants $\phi_{i}$, whose values are dependent upon the key size. These inputs are arranged in a predefined order in a $4 \times 4$ vector, called the initialization vector $(X)$ in the rest of the discussion as shown below. 


$$
X=\left(\begin{array}{cccc}
x_{0} & x_{1} & x_{2} & x_{3} \\
x_{4} & x_{5} & x_{6} & x_{7} \\
x_{8} & x_{9} & x_{10} & x_{11} \\
x_{12} & x_{13} & x_{14} & x_{15}
\end{array}\right)=\left(\begin{array}{cccc}
\phi_{0} & k_{0} & k_{1} & k_{2} \\
k_{3} & \phi_{1} & n_{0} & n_{1} \\
t_{0} & t_{1} & \phi_{2} & k_{4} \\
k_{5} & k_{6} & k_{7} & \phi_{3}
\end{array}\right)
$$

The initialization vector is subjected to a series of rounds composed of additions, cyclic rotations and XORs, to achieve a random permutation. Originally the number of rounds was set to 20 (Salsa20/r, $r=20$ ); however, the version of cipher included in the eSTREAM portfolio was reduced to 12 rounds, for performance reasons.

Let $X, S$ and $P$ be $4 \times 4$ arrays of 16 -words each enumerated as $\left(x_{0}, x_{1}, \ldots, x_{15}\right)$, representing initialized vector, Salsa20 keystream and plaintext respectively. Then Salsa20/ $r$ function for keystream generation can be represented mathematically as:

$$
\begin{aligned}
S & =\text { Salsa20 }_{k}(X) \\
\operatorname{Salsa}_{k}(X) & =\text { DoubleRound }^{r}(X)+X \\
\text { DoubleRound }(X) & =\text { RowRound }_{(\text {ColumnRound }(X))}
\end{aligned}
$$

Each DoubleRound comprises of four QuarterRounds performed on the columns of the initialization vector $X$, followed by four QuarterRounds performed on the rows of the output.

$$
\begin{aligned}
\text { ColumnRound }(X) & =\left(y_{0}, y_{1}, \ldots, y_{15}\right)=Y \\
\left(y_{0}, y_{4}, y_{8}, y_{12}\right) & =\text { QuarterRound }\left(x_{0}, x_{4}, x_{8}, x_{12}\right), \\
\left(y_{5}, y_{9}, y_{13}, y_{1}\right) & =\text { QuarterRound }\left(x_{5}, x_{9}, x_{13}, x_{1}\right) \\
\left(y_{10}, y_{14}, y_{2}, y_{6}\right) & =\text { QuarterRound }\left(x_{10}, x_{14}, x_{2}, x_{6}\right) \\
\left(y_{15}, y_{3}, y_{7}, y_{11}\right) & =\text { QuarterRound }\left(x_{15}, x_{3}, x_{7}, x_{11}\right) . \\
\text { RowRound }(Y) & =\left(z_{0}, z_{1}, \ldots, z_{15}\right)=Z \\
\left(z_{0}, z_{1}, z_{2}, z_{3}\right) & =\text { QuarterRound }\left(y_{0}, y_{1}, y_{2}, y_{3}\right) \\
\left(z_{5}, z_{6}, z_{7}, z_{4}\right) & =\text { QuarterRound }\left(y_{5}, y_{6}, y_{7}, y_{4}\right) \\
\left(z_{10}, z_{11}, z_{8}, z_{9}\right) & =\text { QuarterRound }\left(y_{10}, y_{11}, y_{8}, y_{9}\right) \\
\left(z_{15}, z_{12}, z_{13}, z_{14}\right) & =\text { QuarterRound }\left(y_{15}, y_{12}, y_{13}, y_{14}\right) .
\end{aligned}
$$


Where each QuarterRound $(a, b, c, d)$ consists of four ARXrounds, named so due to underlying additions $(\mathrm{A})$, rotations $(\mathrm{R})$ and XOR-ing $(\mathrm{X})$ operations:

$$
\begin{aligned}
& b=b \oplus((a+d) \lll 7), \\
& c=c \oplus((b+a) \lll 9), \\
& d=d \oplus((c+b) \lll 13), \\
& a=a \oplus((d+c) \lll 18) .
\end{aligned}
$$

A $4 \times 4$ matrix $C$, representing a 16-word ciphertext block is calculated simply by XOR-ing it with the keystream $(C=S \oplus P)$.

\section{C.6 ChaCha20/r Stream Cipher [20]}

ChaCha, a variant of Salsa20, was proposed by the author of Salsa20 [20]. The main aim of its creation was to improve the diffusion per round in Salsa20 which does not come at the expense of extra operations [21]. Being similar to Salsa20, ChaCha also has reduced round variants. The major differences between Salsa20 and ChaCha are a different initialization matrix, different inputs to DoubleRound function and the operation of the QuarterRound function.

The initialization matrix in ChaCha differs from Salsa20 in the following manner:

$$
X=\left(\begin{array}{cccc}
x_{0} & x_{1} & x_{2} & x_{3} \\
x_{4} & x_{5} & x_{6} & x_{7} \\
x_{8} & x_{9} & x_{10} & x_{11} \\
x_{12} & x_{13} & x_{14} & x_{15}
\end{array}\right)=\left(\begin{array}{cccc}
\phi_{0} & \phi_{1} & \phi_{2} & \phi_{3} \\
k_{0} & k_{1} & k_{2} & k_{3} \\
k_{4} & k_{5} & k_{6} & k_{7} \\
t_{0} & t_{1} & n_{0} & n_{1}
\end{array}\right)
$$

Where $k_{i}, \phi_{i}, n_{i}$ and $t_{i}$ are key, constants, nonce and counter words each of 32 bit granularity, respectively. The DoubleRound calculation in ChaCha operates in the following order:

$$
\begin{aligned}
\operatorname{ColumnRound}(X) & =\left(y_{0}, y_{1}, \ldots, y_{15}\right)=Y \\
\left(y_{0}, y_{4}, y_{8}, y_{12}\right) & =\text { QuarterRound }\left(x_{0}, x_{4}, x_{8}, x_{12}\right), \\
\left(y_{5}, y_{9}, y_{13}, y_{1}\right) & =\text { QuarterRound }\left(x_{1}, x_{5}, x_{9}, x_{13}\right), \\
\left(y_{10}, y_{14}, y_{2}, y_{6}\right) & =\text { QuarterRound }\left(x_{2}, x_{6}, x_{10}, x_{14}\right), \\
\left(y_{15}, y_{3}, y_{7}, y_{11}\right) & =\text { QuarterRound }\left(x_{3}, x_{7}, x_{11}, x_{15}\right) .
\end{aligned}
$$




$$
\begin{aligned}
\operatorname{RowRound}(Y) & =\left(z_{0}, z_{1}, \ldots, z_{15}\right)=Z \\
\left(z_{0}, z_{1}, z_{2}, z_{3}\right) & =\text { QuarterRound }\left(y_{0}, y_{5}, y_{10}, y_{15}\right) \\
\left(z_{5}, z_{6}, z_{7}, z_{4}\right) & =\text { QuarterRound }\left(y_{1}, y_{6}, y_{11}, y_{12}\right) \\
\left(z_{10}, z_{11}, z_{8}, z_{9}\right) & =\text { QuarterRound }\left(y_{2}, y_{7}, y_{8}, y_{13}\right) \\
\left(z_{15}, z_{12}, z_{13}, z_{14}\right) & =\text { QuarterRound }\left(y_{3}, y_{4}, y_{9}, y_{14}\right) .
\end{aligned}
$$

Where $X$ is the $4 \times 4$ array of 16 -words enumerated as $\left(x_{0}, x_{1}, \ldots, x_{15}\right)$ representing initialized vector.

The third difference between Salsa20 and ChaCha is the structure of the QuarterRound.

$$
\begin{aligned}
& a=a+b \\
& d=(d \oplus a) \lll 16 \\
& c=c+d \\
& b=(b \oplus c) \lll 12 \\
& a=a+b \\
& d=(d \oplus a) \lll 8 \\
& c=c+d \\
& b=(b \oplus c) \lll 7
\end{aligned}
$$




\section{Appendix D}

\section{Overview of SNOW 3G and ZUC Stream Ciphers}

Both SNOW 3G and ZUC are 32-bit word oriented stream ciphers, which constitute a part of the 3GPP LTE-Advanced security suite.

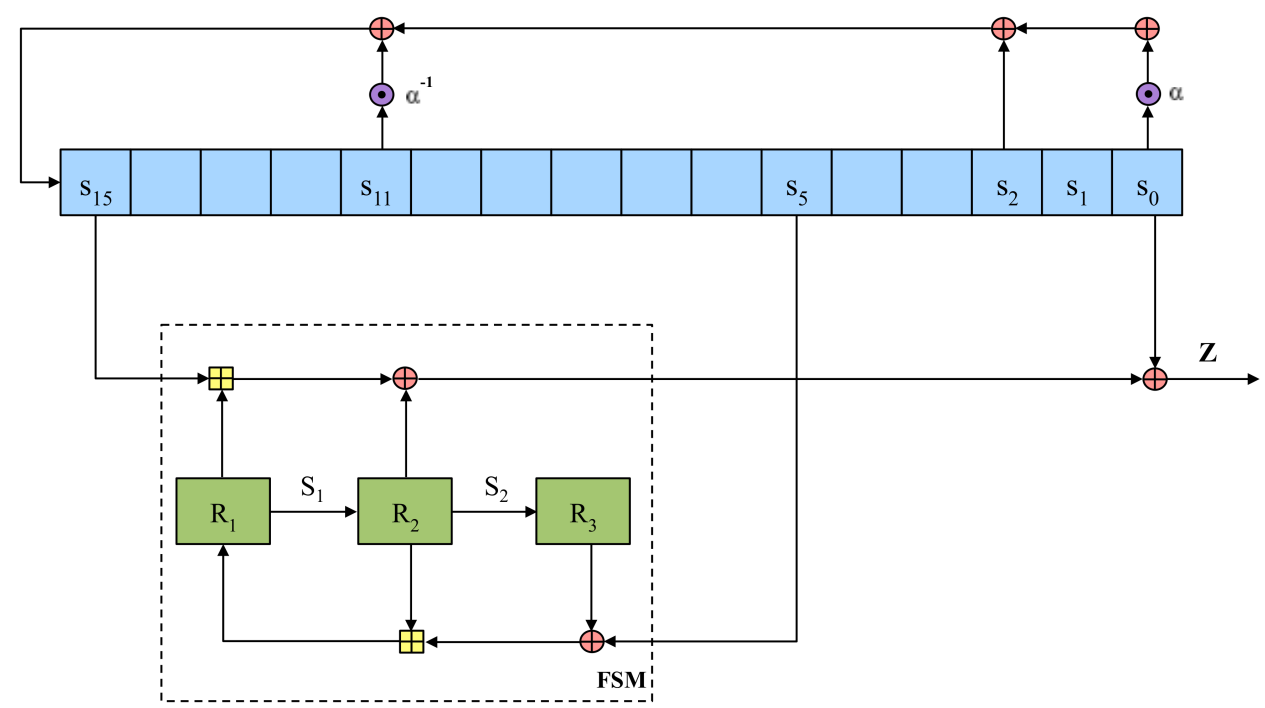

Figure D.1: SNOW 3G cipher in Keystream Generation mode [2]

\section{D.1 SNOW 3G Stream Cipher [2]}

SNOW 3G is an LFSR based stream cipher designed by ETSI-SAGE, largely based on the cipher SNOW 2.0 [66] by Ekdahl and Johansson. The cipher generates a keystream of 32-bit words using an LFSR of size 16 words, that is $16 \times 32=512$ bits. The FSM of this design consists of three 32-bit registers which are updated based on two different S-boxes S1, S2. The LFSR update function depends on a couple of field operations (multiplication and division by field element $\alpha$ ) and XOR combinations. Like most stream ciphers, SNOW 3G has two distinct modes of operation. During the initialization mode, the LFSR is initiated using a 128-bit key and a 128-bit initialization variable (IV), and the output of the FSM is XOR-ed with the LFSR update function in the feedback loop for the first 32 iterations. Thereafter, in the keystream generation mode, the output of the FSM is combined with the first LFSR location $s_{0}$ to produce 
the output keystream word. The operation of the cipher in keystream generation mode is shown in Fig. D.1.

\section{D.2 ZUC Stream Cipher [232]}

ZUC is also an LFSR based word oriented stream cipher, designed by the Data Assurance and Communication Security Research Center of the Chinese Academy of Sciences (DACAS). This cipher produces a keystream of 32-bit words, and is executed in two stages (initialization and keystream generation). The LFSR for ZUC consists of 16 blocks, each of length 31 bits, and the update function of the LFSR is based on a series of modulo $2^{31}-1$ (this is a prime) multiplications and additions. The FSM takes as input 32-bit words constructed from the LFSR (through a routine called Bit Reorganization or BR) and outputs a 32 bit word as well. It consists of two 32-bit registers R1 and R2 which are updated using two different linear functions L1, L2 and the same S-box S. The initial state of the LFSR is constructed using a 128 bit key and a 128 bit IV, and during the first 32 iterations, the output of the FSM is added (modulo $2^{31}-1$ addition after right shift by 1 place) to the feedback loop for LFSR update. In the keystream generation mode, the output of the FSM is combined with the word $X_{3}$, constructed from the LFSR places $s_{0}$ and $s_{2}$, to produce the final output. The keystream generation mode of ZUC is illustrated in Fig. D.2.

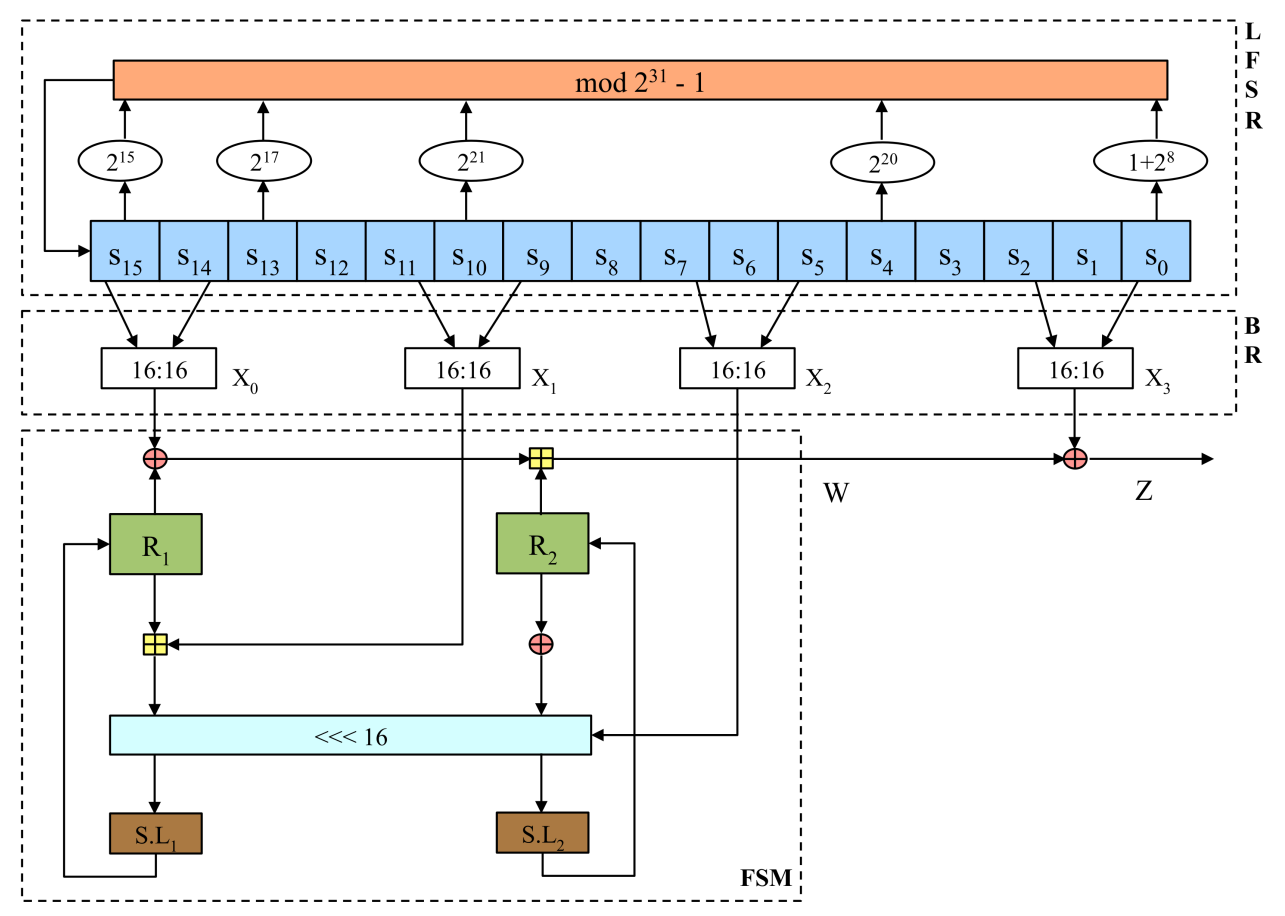

Figure D.2: ZUC cipher in Keystream Generation mode [232] 


\section{List of Figures}

1.1 Conflicting requirements to a crypto developer $\ldots \ldots \ldots 1$

2.1 Design trade-offs for lightweight cryptography . . . . . . . . . . 7

2.2 The traditional VLSI synthesis flow against the HLS . . . . . . . . . 8

2.3 Various steps of High Level Synthesis (HLS) . . . . . . . . . . . . . 10

3.1 How applications relate to the 13 Berkeley dwarfs . . . . . . . . . . 19

3.2 Classification of Cryptology . . . . . . . . . . . . . . . . 20

3.3 Synchronous and self-synchronizing stream ciphers . . . . . . . . . 22

3.4 Modes of operation for cryptographic functions . . . . . . . . . . . 24

3.5 Computational commonalities of symmetric key cryptography . . . . 25

3.6 Block diagram of Feistel network and SPN cipher . . . . . . . . . . . . 27

3.7 Layers for the loop folded implementation of PRESENT-80 and AES-128 31

3.8 An LFSR and a carry Linear feedback shift register CLFSR . . . . . . . 33

3.9 Nonlinear filter generator and Nonlinear output from multiple LFSRs . 34

3.10 Architectural structure of Trivium implementation . . . . . . . . . 39

4.1 Design flow of cryptographic functions using CRYKET . . . . . . . . . 44

4.2 RunFein toolflow for software generation and hardware generation . . 46

4.3 Round layers operational specification for AES-128 in RunFein . . . . . 47

4.4 The configuration file snapshot for PRESENT-80 generated by RunFein 48

4.5 Various parallel microarchitecture implementations supported by RunFein .............................. 51

4.6 Controller for various microarchitecture implementations supported by RunFein ............................. 52

4.7 Bitsliced implementation of PRESENT-80 . . . . . . . . . . . . . 60

4.8 PRESENT-80 bitsliced encryption area throughput trade-off @ $100 \mathrm{KHz} \quad 60$

4.9 PRESENT-80 bitsliced encryption area power trade-off @ $100 \mathrm{KHz}$. . . 61

4.10 Runstream toolflow for software generation and hardware generation . 63

4.11 Mesh nodes tab for RunStream showing I/Os for Trivium's node12 . . 63

4.12 The configuration file snapshot input to RunStream for Trivium . . . . 64

4.13 The Directed Acyclic Graph for Trivium nodes implementation . . . . . 66

4.14 Interfaces for a RunStream generated stream cipher implementation . . 69

4.15 Architectural structure of SNOW 3G implementation . . . . . . . . . . . 71 
4.16 Design performance metrics for higher operating frequencies @65nm

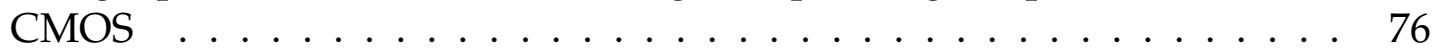

4.17 Performance for RFID applications for $100 \mathrm{KHz} @ 65 \mathrm{~nm}$ CMOS . . . . . 76

5.1 Block Diagram of HC-128 Accelerator . . . . . . . . . . . . . . . . . 85

5.2 Pipeline architecture of design 1 of HC-128 accelerator . . . . . . . . . 87

5.3 Area-frequency graph for various configurations of a $1 \mathrm{~K}$ word memory on $65 n$ m CMOS . . . . . . . . . . . . . . . . . . . . . 89

5.4 Pipeline architecture for HC-128 initialization instruction . . . . . . . 91

5.5 Pipeline architecture of design 2 of HC-128 accelerator . . . . . . . . . . 91

5.6 Pipeline architecture of design3 of HC-128 accelerator . . . . . . . . . 93

5.7 AT chart for HC-128 design variants on 65nm CMOS . . . . . . . . . . 95

5.8 Top level structure of both SNOW 3G and ZUC. . . . . . . . . . . . . 99

5.9 Modified bit reorganization for ZUC after LFSR integration. . . . . . . 100

5.10 Pipeline structure based on Memory Access. . . . . . . . . . . . . . . . 101

5.11 Pipeline structure reorganization for LFSR update of ZUC. . . . . . . . . 104

5.12 Final 3-stage Pipeline Structure for the Integrated Design. . . . . . . . 105

5.13 Critical path in the Key Initialization of ZUC (curvy dashed line). . . . 106

5.14 Modulo $p$ Adder optimization for ZUC. . . . . . . . . . . . . . . . . . . 107

5.15 Area-Time chart for HiPAcc-LTE (10 KByte memory) using 65nm technology. . . . . . . . . . . . . . . . . 108

6.1 Processing Elements with Different Functional Unit Arrangement in

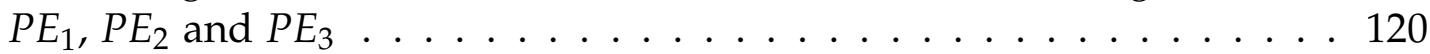

6.2 Block Diagram of CoARX . . . . . . . . . . . . . . . . 122

6.3 Configuration Word for CoARX processor . . . . . . . . . . . . 123

6.4 Memory map for various ARX algorithms . . . . . . . . . . . . . . 125

6.5 Description of mapping of BLAKE Initialization . . . . . . . . . . . 125

6.6 Description of mapping of BLAKE single Round function . . . . . . . . 126

6.7 Description of mapping of BLAKE finalization . . . . . . . . . . . . 126

6.8 Comparison with GPP and Embedded Processor . . . . . . . . . . . . 129

6.9 Block Diagram of RC4-AccSuite . . . . . . . . . . . . . . . . 135

6.10 Spritz PRGA accesses on a dual ported SRAM . . . . . . . . . . . . . . . . 138

6.11 Spritz_S0_1 PRGA on 2 dual ported SRAMs . . . . . . . . . . . . . . . 139

6.12 Consecutive instructions overlap for Spritz_S0_1 PRGA without haz-

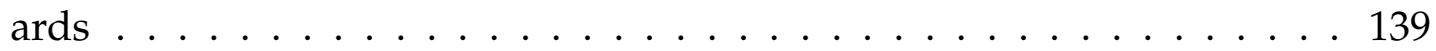

6.13 Pipeline for RC4-AccSuite supporting only RC4 . . . . . . . . . . . . . 143

6.14 Pipeline for RC4/RC4 ${ }^{+}$-AccSuite supporting both $\mathrm{RC} 4$ and $\mathrm{RC}^{+}{ }^{+}$. . . 144

6.15 RC4-AccSuite core Area economization . . . . . . . . . . . . . . . . 149

6.16 Power consumption in RC4-AccSuite versions . . . . . . . . . . . . 150

7.1 CUDA GPU execution model . . . . . . . . . . . . . . . 155

7.2 Parallelism in Salsa20 $/ r \ldots \ldots$. . . . . . . . . . . . 157

7.3 Autotuning Framework Flowchart . . . . . . . . . . . . . . . . . . . . 161

7.4 Effect of register pressure against unroll factor . . . . . . . . . . . 166 
7.5 Effect of throughput against unroll factor . . . . . . . . . . . 166

7.6 Salsa20 encryption performance on GTX 590 for varying block sizes (w/o mem trans.) . . . . . . . . . . . . . . . . . . . . . . 167

7.7 Salsa20 throughput improvement comparison between a GPP and a

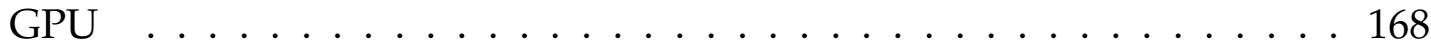

7.8 Dependency in SUS at indices $i, i+1$ and $i+2$ in S-Boxes . . . . . . 171

7.9 HC-128 keystream generation throughput using shared and global mem-

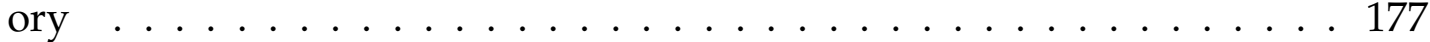

7.10 HC-256 keystream generation throughput using shared and global mem-

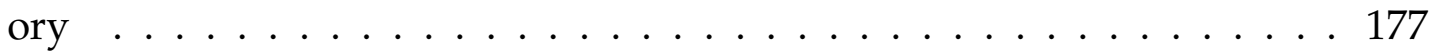

7.11 keystream generation throughput for varying number of multiple datastreams . . . . . . . . . . . . . . . . . . . . 178

8.1 Stevens' attack execution control flow stages [207] . . . . . . . . . . . 185

8.2 Cracken's instruction execution pipeline . . . . . . . . . . . . . . . 186

8.3 Cracken Schematic - Stage $0-1 \ldots \ldots \ldots$

8.4 Cracken Schematic - Stage 2-3 . . . . . . . . . . . . . . . . . . . . . . . 189

8.5 Post layout and routing view of Cracken Core . . . . . . . . . . . . . 193

8.6 Collision days vs. Cracken cost (with and without memories) . . . . . 195

B.1 Microarchitectural specification for subpipelined implementation in RunFein . . . . . . . . . . . . . . . . . . . . . 205

B.2 NIST Test Suite parameter selection tab in RunFein . . . . . . . . . 206

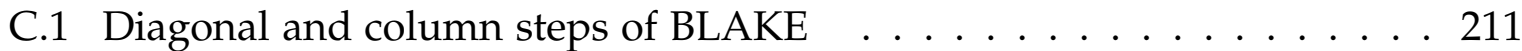

C.2 G function of BLAKE hash . . . . . . . . . . . . . . . . . . . 212

C.3 A MIX function of Skein . . . . . . . . . . . . . . . . . . . . . . . . 214

C.4 4 Rounds of Skein-512 Hash . . . . . . . . . . . . . . . . . . . . 214

C.5 Key generator of Skein . . . . . . . . . . . . . . . . . . . . 215

C.6 Skein in normal hashing mode . . . . . . . . . . . . . . . . . 215

D.1 SNOW 3G cipher in Keystream Generation mode . . . . . . . . . . . 219

D.2 ZUC cipher in Keystream Generation mode . . . . . . . . . . . . . 220 


\section{List of Tables}

2.1 Summary of various cryptographic competitions $\ldots \ldots \ldots \ldots$

3.1 NIST standardized modes of operation modes of operation for block ciphers . . . . . . . . . . . . . . . . . . . . . . 29

3.2 A list of various SPN block cipher algorithmic configurations . . . . . 30

3.3 Basic parameters for layered configuration space for two prominent ciphers ....................... 32

3.4 Classification, construction and Salient Features of stream ciphers _. . 38

3.5 Major operations of SHA-3 candidates (permutations, shifts, rotations not included). mADDn denotes a multioperand addition with $n$ operands. 40

4.1 SPN and Feistel block cipher software efficiency results . . . . . . . . 56

4.2 AES-128 encryption results for $65 \mathrm{~nm}$ CMOS . . . . . . . . . . . . . 57

4.3 AES-128 unrolled encryption implementation results for 65nm CMOS tech. library (TPA is Throughput/ Area) _ . . . . . . . . . . . 57

4.4 AES-128 subpipelined encryption results for $65 \mathrm{~nm}$ CMOS . . . . . . . 58

4.5 PRESENT-80 encryption bitsliced implementation results for $65 \mathrm{~nm}$ CMOS tech. library @ $100 \mathrm{KHz}$. . . . . . . . . . . . . . . . . . . . . 58

4.6 PRESENT-80 encryption bitsliced implementation results for 65nm CMOS tech. library @ $10 \mathrm{MHz}$. . . . . . . . . . . . . . . . . . . . . . . . 59

4.7 PRESENT-80 bitsliced encryption @ $100 \mathrm{KHz}$. . . . . . . . . . . . . . . 59

4.8 PRESENT-80 encryption unrolled implementations, 65nm @ $100 \mathrm{KHz}$ (Tp is Throughtput) . . . . . . . . . . . . . . . . . 61

4.9 PRESENT-80 subpipelined encryption results for 65nm CMOS, (Tp is

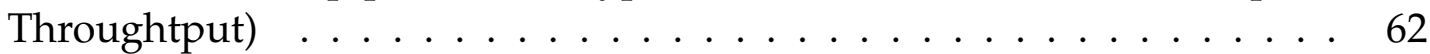

4.10 Sequential information configuration for stream ciphers taken up by RunStream . . . . . . . . . . . . . . . . . . . . . . . 70

4.11 Operational nodes information for RunStream configuration of stream

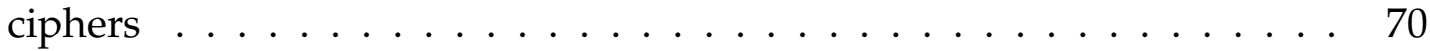

4.12 Design results for highest operating frequencies @65nm CMOS . . . . 73

4.13 Design results for $10 \mathrm{MHz} @ 65 \mathrm{~nm}$ CMOS . . . . . . . . . . . . . 73

4.14 Design results for $100 \mathrm{MHz} @ 65 \mathrm{~nm}$ CMOS . . . . . . . . . . . . . 74

4.15 Derived metrics for highest operating frequencies @65nm CMOS . . . . 75

4.16 Derived metrics for $100 \mathrm{MHz}$ operating frequency @65nm CMOS . . . 75

4.17 Resource comparison of RunStream implementations with others . . . 77 
5.1 Modified HC-128 Initialization phase steps . . . . . . . . . . . . . 84

5.2 Instruction set for HC-128 accelerator . . . . . . . . . . . . . . . . 86

5.3 Initialization latency cycles for various designs of HC-128 accelerator . . 90

5.4 Area comparison of HC-128 accelerator designs . . . . . . . . . . . 95

5.5 Power consumption comparison of HC-128 design versions . . . . . . 95

5.6 Performance comparison of HC-128 design versions . . . . . . . . . 96

5.7 Performance comparison of HC-128 design versions in [38] $\ldots \ldots .96$

5.8 Synthesis results for HiPAcc-LTE with 10 KByte memory. . . . . . . . . 108

5.9 Synthesis results for alternate design of HiPAcc-LTE with 3 KByte memory . . . . . . . . . . . . . . . . . . . . . 109

5.10 Synthesis results for standalone mode in HiPAcc-LTE with 3 KByte memory . . . . . . . . . . . . . . . . . . . . . . . 109

5.11 Comparison of HiPAcc-LTE with existing 65nm commercial designs. . . 111

5.12 Power estimation results for HiPAcc-LTE with hard macro storage. . . . 112

6.1 Security algorithms for communications standards . . . . . . . . 115

6.2 FPGA based Implementations of ARX Algorithms . . . . . . . . . . . 119

6.3 Estimated Performance and Resource Utilization Results . . . . . . . . . 121

6.4 CoARX Synthesis Results . . . . . . . . . . . . . . . . . . . . 127

6.5 Performance of different ARX algorithms on CoARX . . . . . . . 128

6.6 Comparison with ASICs (Throughput scaled to 90nm) . . . . . . . . 128

6.7 Performance of ARX algorithms on Multicore Architectures . . . . . . 130

6.8 Algorithmic specifications of RC4 stream cipher . . . . . . . . . . 133

6.9 Byte wide Memory requirements (instances $\times$ depth) for RC4 variants . 136

6.10 PRGA for Spritz (left) and RC4 ${ }^{+}$(right) $\ldots \ldots \ldots \ldots \ldots$

$6.11 \mathrm{RC}^{+}{ }^{+}$S0_0 PRGA, throughput $=5$ cycles $/$ Byte . . . . . . . . . . 139

$6.12 \mathrm{RC}^{+}{ }^{+} \mathrm{SO} \_1$ PRGA, throughput $=4$ cycles $/$ Byte $\ldots \ldots \ldots \ldots \ldots \ldots$

$6.13 \mathrm{RC}^{+}{ }^{+}$S0_2 PRGA, throughput $=3$ cycles $/$ Byte (left), RC4 ${ }^{+} \_$S0_3 PRGA, throughput $=2$ cycles $/$ Byte $($ right $) \ldots \ldots \ldots \ldots \ldots$

6.14 Array Replication in RC4 variants . . . . . . . . . . . . . . . . . . 141

6.15 Instruction set for RC4 . . . . . . . . . . . . . . . . . . . . . 142

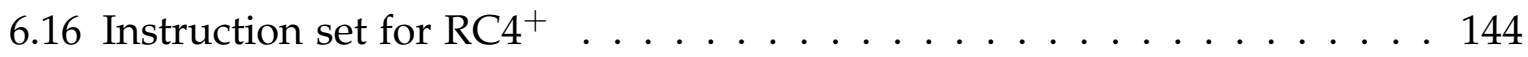

6.17 Registers/ Pipeline Registers/ Memories reuse for RC4 variants . . . . . 146

6.18 Throughput performance of various RC4 variants on RC4-AccSuite . . . 148

6.19 Area (KGE) for RC4-AccSuite versions . . . . . . . . . . . . . . . . 149

6.20 Power consumption of RC4 algorithm for RC4-AccSuite versions . . . 150

6.21 Area comparison of RC4-AccSuite versions $(\mathrm{C} / \mathrm{B}=$ cycles per Byte, TPA $=$ Throughput per Area $\ldots \ldots \ldots \ldots \ldots \ldots \ldots 1$

6.22 RC4-AccSuite versions software performance . . . . . . . . . . . 152

7.1 Range of parameters for autotuning Salsa20 kernel on a GTX 590 . . . 165

7.2 Salsa20 optimized parameters for GTX 590 (32 KB plaintext) . . . . . . 167

7.3 Comparison of peak performance of Salsa20 with others work (Tp stands for Throughput) . . . . . . . . . . . . . . . . . . . . . . 169

7.4 List of Operations for Keystream Generation in HC-128 and HC-256 . . 172 
7.5 Keystream generation phase of HC-128 using three threads . . . . . . . . 173

7.6 Parallelizing one SUS warp with one KWGS block . . . . . . . . . . . . 174

7.7 Parallelizing 2 S-Box SUS warps with 2 KWGS blocks . . . . . . . . . . . 175

7.8 Duration and throughput of initialization phase of stream ciphers . . . . 176

7.9 Comparison of the HC-128 GPGPU mapping acceleration against others

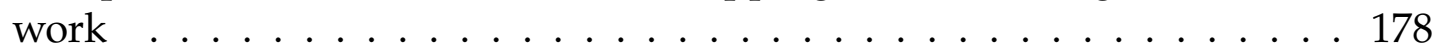

7.10 Throughput (Gbps), Cycles/Byte (C/B) of a single data-stream HC ciphers . . . . . . . . . . . . . . . . . . . . . . . 179

8.1 SHA-1 Attacks summary . . . . . . . . . . . . . . . . . . . 182

8.2 Notations for Stevens' attack . . . . . . . . . . . . . . . . . . . 184

8.3 Cracken instruction set; Op. code and Immediate value are 8 bits each in a 16 bit instruction . . . . . . . . . . . . . . . . . . . 187

8.4 Cracken Memories. Bitwidth $=32$ bits (16 bits for prog_mem), S/D $\mathrm{P}=$ Single / dual ported . . . . . . . . . . . . . . . . . . 192

8.5 Cost-Time approximation for Cracken with and without memories . . . 193

8.6 FPGA Feature Summary for Xilinx devices [226] [227] and Cracken cores mapped on FPGAs. Freq $=250 \mathrm{MHz} \ldots \ldots$. . . . . . . . 195

8.7 Cracken ASIC/ FPGA day-cost comparison with others works . . . . . . 196

C.1 HC-128 Initialization phase steps . . . . . . . . . . . . . . . . . 208

C.2 HC-128 Keystream generation phase . . . . . . . . . . . . . . . . . 209

C.3 BLAKE HASH functions . . . . . . . . . . . . . . . . . . . . 210 


\section{Bibliography}

[1] 3GPP, "Long Term Evaluation Release 10 and beyond (LTE-Advanced)." Proposed to ITU at 3GPP TSG RAN Meeting in Spain, September 2009.

[2] 3rd Generation Partnership Project, "Specification of the 3GPP Confidentiality and Integrity Algorithms UEA2 and UIA2," Document 1: UEA2 and UIA2 Specification Version 1.1, September 2006.

[3] A. V. Adinetz and E. A. Grechnikov, "Building a Collision for 75-round Reduced SHA-1 using GPU Clusters," in Euro-Par 2012 Parallel Processing. S Springer, 2012, pp. 933-944.

[4] S. Ahuja, S. T. Gurumani, C. Spackman, and S. K. Shukla, "Hardware Coprocessor Synthesis from an ANSI C Specification," IEEE Design $\mathcal{E}$ Test of Computers, no. 4, pp. 58-67, 2009.

[5] J. A. Akinyele, C. Garman, I. Miers, M. W. Pagano, M. Rushanan, M. Green, and A. D. Rubin, "Charm: A Framework for Rapidly Prototyping Cryptosystems," Journal of Cryptographic Engineering, vol. 3, no. 2, pp. 111-128, 2013.

[6] A. Al Noman, R. Sidek, L. Ali et al., "RC4A Stream Cipher for WLAN Security: A Hardware Approach," in International Conference on Electrical and Computer Engineering (ICECE). IEEE, 2008, pp. 624-627.

[7] Amazon, "Amazon Elastic Compute Cloud (Amazon EC2)." Online: http://aws. amazon.com/ec2/pricing/

[8] Amazon, "Microsoft recommends against usage of SHA-1." Online: www.zdnet.com/ article/microsoft-recommends-against-usage-of-sha-1

[9] P. A. Arora, M. R. Dugan, and P. G. Gogte, "Survey of the Commercially Available Chips and IP Cores Implementing Cryptographic Algorithms," 2005.

[10] K. Asanovic, R. Bodik, B. C. Catanzaro, J. J. Gebis, P. Husbands, K. Keutzer, D. A. Patterson, W. L. Plishker, J. Shalf, S. W. Williams et al., "The Landscape of Parallel Computing Research: A View from Berkeley," Technical Report UCB/EECS-2006-183, EECS Department, University of California, Berkeley, Tech. Rep., 2006.

[11] N. At, J.-L. Beuchat, E. Okamoto, I. San, and T. Yamazaki, "Compact Hardware Implementations of ChaCha, BLAKE, Threefish and Skein on FPGAs," IEEE Transactions on Circuits and Systems (Regular Papers), vol. 61, no. 2, pp. 485-498, 2014.

[12] J.-P. Aumasson, L. Henzen, W. Meier, and R. C.-W. Phan, "SHA-3 Proposal BLAKE," Submission to NIST, 2008. 
[13] S. Babbage and M. Dodd, "The Stream Cipher MICKEY 2.0," ECRYPT Stream Cipher, 2006. Online: http://www.ecrypt.eu.org/stream/p3ciphers/mickey/mickey_p3.pdf

[14] S. Babbage and M. Dodd, "The MICKEY Stream Ciphers," in New Stream Cipher Designs. Springer, 2008, pp. 191-209.

[15] E. Barkan, E. Biham, and N. Keller, "Instant Ciphertext-only Cryptanalysis of GSM Encrypted Communication," Journal of Cryptology, vol. 21, no. 3, pp. 392-429, 2008.

[16] P. Barreto and V. Rijmen, "The Khazad Legacy-level Block Cipher," Primitive submitted to NESSIE, vol. 97, 2000.

[17] P. Barreto, V. Rijmen, and Others, "The Whirlpool Hashing Function," in First Open NESSIE Workshop, Leuven, Belgium, vol. 13, 2000, p. 14.

[18] C. Berbain, O. Billet, A. Canteaut, N. Courtois, H. Gilbert, L. Goubin, A. Gouget, L. Granboulan, C. Lauradoux, M. Minier et al., "Sosemanuk, A Fast Software-oriented Stream Cipher," in New Stream Cipher Designs. Springer, 2008, pp. 98-118.

[19] D. J. Bernstein., "Hash functions and ciphers." In Notes on the ECRYPT Stream Cipher Project (eSTREAM). Online: http://cr.yp.to/streamciphers/why.html

[20] D. J. Bernstein, "ChaCha, A Variant of Salsa20," in State of the Art of Stream Ciphers (SASC) Workshop, vol. 8, 2008.

[21] D. J. Bernstein, "The Salsa20 Family of Stream Ciphers," in New Stream Cipher Designs. Springer, 2008, pp. 84-97.

[22] D. Bernstein, M. Rodeh, and I. Gertner, "On the Complexity of Scheduling Problems for Parallel/Pipelined Machines," IEEE Transactions on Computers, vol. 38, no. 9, pp. 1308-1313, 1989.

[23] G. Bertoni, J. Daemen, M. Peeters, and G. Van Assche, "Keccak Sponge Function Family," Submission to NIST (Round 2), vol. 3, p. 30, 2009.

[24] J.-L. Beuchat, E. Okamoto, and T. Yamazaki, "Compact Implementations of BLAKE-32 and BLAKE-64 on FPGA," in International Conference on Field Programmable Technology (FPT). IEEE, 2010, pp. 170-177.

[25] A. D. Biagio, A. Barenghi, G. Agosta, and G. Pelosi, "Design of a Parallel AES for Graphics Hardware using the CUDA Framework," in International Symposium on Parallel $\mathcal{E}$ Distributed Processing (IPDPS). IEEE, 2009, pp. 1-8.

[26] E. Biham, R. Anderson, and L. Knudsen, "Serpent: A New Block Cipher Proposal," in Fast Software Encryption. Springer, 1998, pp. 222-238.

[27] S. I. G. Bluetooth, "Specification of the Bluetooth System." Version 1.1., February 2001. Online: www.inf.ethz.ch/personal/hvogt/proj/btmp3/Datasheets/Bluetooth_ 11_Specifications_Book.pdf

[28] A. Bogdanov, L. R. Knudsen, G. Leander, C. Paar, A. Poschmann, M. J. Robshaw, Y. Seurin, and C. Vikkelsoe, PRESENT: An Ultra-lightweight Block Cipher. Springer, 2007. 
[29] T. Bollaert, "Catapult Synthesis: A Practical Introduction to Interactive C Synthesis," in High-Level Synthesis. Springer, 2008, pp. 29-52.

[30] J. Borghoff, A. Canteaut, T. Güneysu, E. B. Kavun, M. Knezevic, L. R. Knudsen, G. Leander, V. Nikov, C. Paar, C. Rechberger et al., "PRINCE-A Low-latency Block Cipher for Pervasive Computing Applications," in Advances in Cryptology-ASIACRYPT. Springer, 2012, pp. 208-225.

[31] C. Boura, S. Lévêque, and D. Vigilant, "Side-channel Analysis of Grøstl and Skein," in IEEE Symposium on Security and Privacy Workshops (SPW). IEEE, 2012, pp. 16-26.

[32] C. Boura, M. Naya-Plasencia, and V. Suder, "Scrutinizing and Improving Impossible Differential Attacks: Applications to CLEFIA, Camellia, LBlock and Simon," pp. 179199, 2014.

[33] M. Briceno, I. Goldberg, and D. Wagner, "A Pedagogical Implementation of the GSM A5/1 and A5/2 Voice Privacy Encryption Algorithms." October 1999. Online: http:// cryptome.org/gsm-a512.htm

[34] F. Burns, J. Murphy, D. Shang, A. Koelmans, and A. Yakorlev, "Dynamic Global Security-aware Synthesis using SystemC," IET Computers \& Digital Techniques, vol. 1, no. 4, pp. 405-413, 2007.

[35] CAESAR, "CAESAR: Competition for Authenticated Encryption: Security, Applicability and Robustness," 2013. Online: http://competitions.cr.yp.to/caesar.html

[36] A. Canis, J. Choi, M. Aldham, V. Zhang, A. Kammoona, J. H. Anderson, S. Brown, and T. Czajkowski, "LegUp: High-level Synthesis for FPGA-based Processor/Accelerator Systems," in 19th ACM/SIGDA International Symposium on Field Programmable Gate Arrays. ACM, 2011, pp. 33-36.

[37] F. Chabaud and A. Joux, "Differential Collisions in SHA-0," in Advances in Cryptology-CRYPTO. Springer, 1998, pp. 56-71.

[38] A. Chattopadhyay, A. Khalid, S. Maitra, and S. Raizada, "Designing High-throughput Hardware Accelerator for Stream Cipher HC-128," in IEEE International Symposium on Circuits and Systems (ISCAS). IEEE, 2012, pp. 1448-1451.

[39] A. Chattopadhyay, H. Meyr, and R. Leupers, "LISA: A Uniform ADL for Embedded Processor Modelling, Implementation and Software Toolsuite Generation," Processor Description Languages, vol. 1, pp. 95-130, 2008.

[40] A. Chattopadhyay and G. Paul, "Exploring Security-performance Trade-offs during Hardware Accelerator Design of Stream Cipher RC4," in IEEE/IFIP 20th International Conference on VLSI and System-on-Chip (VLSI-SoC). IEEE, 2012, pp. 251-254.

[41] H. Cheng, H. M. Heys, and C. Wang, "Puffin: A Novel Compact Block Cipher Targeted to Embedded Digital Systems," in 11th EUROMICRO Conference on Digital System Design Architectures, Methods and Tools (DSD). IEEE, 2008, pp. 383-390.

[42] J. H.-F. Constantin, A. P. Burg, and F. K. Gurkaynak, "Investigating the Potential of Custom Instruction Set Extensions for SHA-3 Candidates on a 16-Bit Microcontroller Architecture," Cryptology ePrint Archive, Tech. Rep., 2012. 
[43] O. Cores, "Simple AES (Rijndael) IP Core." Online: http://opencores.org/project,aes_ core

[44] P. Coussy, C. Chavet, P. Bomel, D. Heller, E. Senn, and E. Martin, "GAUT: A Highlevel Synthesis Tool for DSP Applications," in High-Level Synthesis. Springer, 2008, pp. 147-169.

[45] P. Coussy, D. D. Gajski, M. Meredith, and A. Takach, "An Introduction to High-level Synthesis," IEEE Design $\mathcal{E}$ Test of Computers, no. 4, pp. 8-17, 2009.

[46] P. Coussy and D. Heller, "GAUT - High-Level Synthesis Tool From C to RTL," 2012. Online: hls-labsticc.univ-ubs.fr/

[47] CRYPTREC, “CRYPTREC: Cryptography Research and Evaluation Committees," 2000. Online: http://competitions.cr.yp.to/cryptrec.html

[48] currencyConvert, "X-rates: Currency Conversion from USD to Euro." Online: http:// www.x-rates.com/calculator $/$ from $=U S D \&$ to $=$ EUR\&amount $=1.00$

[49] J. Daemen, "Cipher and Hash Function Design Strategies Based on Linear and Differential Cryptanalysis," Ph.D. dissertation, KU Leuven, March 1995.

[50] J. Daemen, R. Govaerts, and J. Vandewalle, "A New Approach to Block Cipher Design," in Fast Software Encryption (FSE). Springer, 1994, pp. 18-32.

[51] J. Daemen, L. Knudsen, and V. Rijmen, "The Block Cipher Square," in Fast Software Encryption (FSE). Springer, 1997, pp. 149-165.

[52] J. Daemen, M. Peeters, G. Van Assche, and V. Rijmen, "NESSIE Proposal: NOEKEON," in First Open NESSIE Workshop, 2000, pp. 213-230.

[53] J. Daemen and V. Rijmen, The Design of Rijndael: AES - The Advanced Encryption Standard. Springer Science \& Business Media, 2013.

[54] C. De Canniere, F. Mendel, and C. Rechberger, "Collisions for 70-Step SHA-1: On the Full Cost of Collision Search," in Selected Areas in Cryptography. Springer, 2007, pp. $56-73$.

[55] C. De Canniere and B. Preneel, "Trivium Specifications," ECRYPT Stream Cipher Project (eSTREAM), Report, vol. 30, 2005.

[56] C. De Canniere and C. Rechberger, "Finding SHA-1 Characteristics: General Results and Applications," in Advances in Cryptology-ASIACRYPT 2006. Springer, 2006, pp. $1-20$.

[57] H. De Man, J. Rabaey, P. Six, and L. Claesen, "Cathedral-II: A Silicon Compiler for Digital Signal Processing," IEEE Design E Test of Computers, vol. 3, no. 6, pp. 13-25, 1986.

[58] B. Debraize and I. M. Corbella, "Fault Analysis of the Stream Cipher Snow 3G," in Workshop on Fault Diagnosis and Tolerance in Cryptography (FDTC). IEEE, 2009, pp. 103110 .

[59] A. DeHon, "The Density Advantage of Configurable Computing," Computer, vol. 33, no. 4, pp. 41-49, 2000. 
[60] R. Doeffinger., “AES C Implementation." Online: stuff.mit.edu/afs/sipb/project/ vlcplayer/old/src/ffmpeg/libavutil/

[61] P. Dubey and S. Engineer, "Teraflops for the Masses: Killer Apps of Tomorrow," in Workshop on Edge Computing Using New Commodity Architectures, UNC, vol. 23, 2006.

[62] M. Dworkin, "Recommendation for Block Cipher Modes of Operation. Methods and Techniques," DTIC Document, Tech. Rep., 2001.

[63] ECRYPT, "eBACS: The European Network of Excellence in Cryptology (ECRYPT) Benchmarking of Cryptographic Systems." Online: http://bench.cr.yp. to/results-stream.html

[64] ECRYPT, "eSTREAM Optimized Code HOWTO (The European Network of Excellence in Cryptology)." Online: www.ecrypt.eu.org/stream/perf/\#results

[65] ECRYPT, "eSTREAM: The European Network of Excellence in Cryptology (ECRYPT) Stream Cipher Project." 2012. Online: www.ecrypt.eu.org/stream

[66] P. Ekdahl and T. Johansson, "A New Version of the Stream Cipher SNOW," in Selected Areas in Cryptography (SAC). Springer, 2003, pp. 47-61.

[67] J. P. Elliott, Understanding Behavioral Synthesis: A Practical Guide to High-level Design. Springer Science \& Business Media, 2012.

[68] D. Engels, X. Fan, G. Gong, H. Hu, and E. M. Smith, "Hummingbird: Ultra-lightweight Cryptography for Resource-constrained Devices," in Financial Cryptography and Data Security. Springer, 2010, pp. 3-18.

[69] M. Ernst, S. Klupsch, O. Hauck, and S. A. Huss, "Rapid Prototyping for Hardware Accelerated Elliptic Curve Public-key Cryptosystems," in 12th International Workshop on Rapid System Prototyping. IEEE, 2001, pp. 24-29.

[70] Europractice, "Europractice IC Fabrication Cost." Online: http://www.europractice-ic. com/docs/MPW2015-general-v4.pdf

[71] Faraday, "Faraday Memory Compiler," 2015. Online: www.faraday-tech.com/html/ Product/IPProduct/LibraryMemoryCompiler/index.htm

[72] A. Fauth, J. Van Praet, and M. Freericks, “Describing Instruction Set Processors using nML," in European Design and Test Conference (EDETC). IEEE, 1995, pp. 503-507.

[73] N. Ferguson, S. Lucks, B. Schneier, D. Whiting, M. Bellare, T. Kohno, J. Callas, and J. Walker, "The Skein Hash Function Family, Version 1.3," p. 3, 2010.

[74] M. Fujii and M. Torigai., "Data Transfer Method, Communication System and Storage Medium." In US Patent US6038321 A, 2000.

[75] K. Gaj, E. Homsirikamol, and M. Rogawski, "Fair and Comprehensive Methodology for Comparing Hardware Performance of Fourteen Round Two SHA-3 Candidates using FPGAs," in Cryptographic Hardware and Embedded Systems (CHES). Springer, 2010, pp. 264-278.

[76] K. Gaj, G. Southern, and R. Bachimanchi, "Comparison of Hardware Performance of Selected Phase II eSTREAM Candidates," in State of the Art of Stream Ciphers Workshop (SASC), vol. 26, 2007. 
[77] M. D. Galanis, P. Kitsos, G. Kostopoulos, N. Sklavos, O. Koufopavlou, and C. E. Goutis, "Comparison of the Hardware Architectures and FPGA Implementations of Stream Ciphers," in 11th IEEE International Conference on Electronics, Circuits and Systems (ICECS). IEEE, 2004, pp. 571-574.

[78] G. Gong, K. C. Gupta, M. Hell, and Y. Nawaz, “Towards a General RC4-like Keystream Generator," in Information Security and Cryptology. Springer, 2005, pp. 162-174.

[79] Z. Gong, S. Nikova, and Y. W. Law, KLEIN: A New Family of Lightweight Block Ciphers. Springer, 2012, vol. 7055.

[80] T. Good and M. Benaissa, "Hardware Results for Selected Stream Cipher Candidates," State of the Art of Stream Ciphers, pp. 191-204, 2007.

[81] T. Good, W. Chelton, and M. Benaissa, "Review of Stream Cipher Candidates from a Low Resource Hardware Perspective," SASC 2006 Stream Ciphers Revisited, p. 125, 2006.

[82] T. Good and M. Benaissa, "ASIC Hardware Performance," in New Stream Cipher Designs. Springer, 2008, pp. 267-293.

[83] T. Good and M. Benaissa, "Hardware Performance of eSTREAM Phase-III Stream Cipher Candidates," in Workshop on the State of the Art of Stream Ciphers (SACS), 2008.

[84] M. Graphics, "Mentor Graphics. ModelSim SE," User's Manual. Online: http://www. model.com/resources/resources_manuals.asp

[85] E. A. Grechnikov, "Collisions for 72-step and 73-step SHA-1: Improvements in the Method of Characteristics." IACR Cryptology ePrint Archive, vol. 2010, p. 413, 2010.

[86] G. S. Group, "General Report on the Design, Speification and Evaluation of 3GPP Standard Confidentiality and Integrity Algorithms," 3G TR 33.908 version 3.0.0 Release, March 1999. Online: http://www.3gpp.org/ftp/tsg_sa/WG3_Security/_ Specs/33908-300.pdf

[87] G. T. S. Group, "3GPP System Architecture Evolution (SAE): Security Architecture," 3GPP TS 33.401 v11.0.1, June 2011.

[88] T. Guneysu, T. Kasper, M. Novotny, C. Paar, and A. Rupp, "Cryptanalysis with COPACOBANA," Computers, IEEE Transactions on, vol. 57, no. 11, pp. 1498-1513, 2008.

[89] J. Guo, T. Peyrin, A. Poschmann, and M. Robshaw, "The LED Block Cipher," in Cryptographic Hardware and Embedded Systems (CHES). Springer, 2011, pp. 326-341.

[90] X. Guo, M. Srivastav, S. Huang, D. Ganta, M. B. Henry, L. Nazhandali, and P. Schaumont, "ASIC Implementations of Five SHA-3 Finalists," in Design, Automation $\mathcal{E}$ Test in Europe Conference E Exhibition (DATE), 2012. IEEE, 2012, pp. 1006-1011.

[91] S. S. Gupta, A. Chattopadhyay, K. Sinha, S. Maitra, and B. P. Sinha, "High-performance Hardware Implementation for RC4 Stream Cipher," Computers, IEEE Transactions on, vol. 62, no. 4, pp. 730-743, 2013.

[92] S. S. Gupta, A. Chattopadhyay, and A. Khalid, "HiPAcc-LTE: An Integrated High Performance Accelerator for 3GPP LTE Stream Ciphers," in Progress in Cryptology (INDOCRYPT). Springer, 2011, pp. 196-215. 
[93] S. S. Gupta, A. Chattopadhyay, and A. Khalid, "Designing Integrated Accelerator for Stream Ciphers with Structural Similarities," Cryptography and Communications, vol. 5, no. 1, pp. 19-47, 2013.

[94] A. Halambi, P. Grun, V. Ganesh, A. Khare, N. Dutt, and A. Nicolau, "EXPRESSION: A Language for Architecture Exploration through Compiler/Simulator Retargetability," in Design, Automation and Test in Europe (DATE). Springer, 2008, pp. 31-45.

[95] M. Hell, T. Johansson, A. Maximov, and W. Meier, "A Stream Cipher Proposal: Grain128," in IEEE International Symposium on Information Theory (ISIT). Citeseer, 2006.

[96] M. Hell, T. Johansson, and W. Meier, "Grain: A Stream Cipher for Constrained Environments," International Journal of Wireless and Mobile Computing, vol. 2, no. 1, pp. 86-93, 2007.

[97] T. Helleseth, C. J. Jansen, and A. Kholosha, "Pomaranch-design and Analysis of a Family of Stream Ciphers," SASC 2006 Stream Ciphers Revisited, p. 13, 2006.

[98] A. Hemani, B. Karlsson, M. Fredriksson, K. Nordqvist, and B. Fjellborg, "Application of High-level Synthesis in an Industrial Project," in VLSI Design, 1994, pp. 5-10.

[99] L. Henzen, J.-P. Aumasson, W. Meier, and R. C. Phan, "VLSI Characterization of the Cryptographic Hash Function BLAKE," Transactions on Very Large Scale Integration (VLSI) Systems, vol. 19, no. 10, pp. 1746-1754, 2011.

[100] L. Henzen, F. Carbognani, N. Felber, and W. Fichtner, "VLSI Hardware Evaluation of the Stream Ciphers Salsa20 and ChaCha and the Compression Function Rumba," in 2nd International Conference on Signals, Circuits and Systems (SCS). IEEE, 2008, pp. 1-5.

[101] E. Homsirikamol and K. Gaj, "Can High-level Synthesis Compete against a Handwritten Code in the Cryptographic Domain? A Case Study," in International Conference on ReConFigurable Computing and FPGAs (ReConFig). IEEE, 2014, pp. 1-8.

[102] E. Homsirikamol and K. Gaj, "Hardware Benchmarking of Cryptographic Algorithms Using High-Level Synthesis Tools: The SHA-3 Contest Case Study," in Applied Reconfigurable Computing (ARC). Springer, 2015, pp. 217-228.

[103] C.-T. Hwang, J.-H. Lee, and Y.-C. Hsu, "A Formal Approach to the Scheduling Problem in High Level Synthesis," IEEE Transactions on Computer-Aided Design of Integrated Circuits and Systems, vol. 10, no. 4, pp. 464-475, 1991.

[104] E. T. Inc., “CLP-410: ZUC Key Stream Generator," 2011. Online: http://elliptictech. com/products-clp-410.php

[105] I. C. Inc., "SNOW 3G Encryption Core," 2011. Online: http://ipcores.com/Snow3G. htm

[106] ISO/IEC, "Authenticated Encryption-Security Techniques," ISO/IEC 19772:2009, 2013. Online: https://webstore.iec.ch/preview/info_isoiec19772ed1.0en.pdf

[107] K. Iwai, N. Nishikawa, and T. Kurokawa, "Acceleration of AES Encryption on CUDA GPUs," International Journal of Networking and Computing, vol. 2, no. 1, pp. 131-145, 2012.

[108] T. Iwata, K. Shibutani, T. Shirai, S. Moriai, and T. Akishita, "AURORA: A Cryptographic Hash Algorithm Family," Submission to NIST, 2008. 
[109] C. J. A. Jansen, "Streamcipher Design: Make Your LFSR Jump," in Proceedings of the The State of the Art of Stream Ciphers (SASC), ser. ECRYPT Network of Excellence in Cryptology, 2004, pp. 94-108.

[110] K. Järvinen, "Sharing Resources Between AES and the SHA-3 Second Round Candidates Fugue and Grøstl," in The Second SHA-3 Candidate Conference, 2010, p. 2.

[111] D. Jong and G. G, "Data Flow Graphs: System Specification with the Most Unrestricted Semantics," in Conference on European Design Automation. IEEE Computer Society Press, 1991, pp. 401-405.

[112] D. P. M. Jr., "Methods and Apparatus for Accelerating ARC4 Processing," US Patent Number 7403615, Morgan Hill, CA, July 2008. Online: www.freepatentsonline.com/ 7403615.html

[113] A. Juels and S. A. Weis, "Authenticating Pervasive Devices with Human Protocols," in Advances in Cryptology-CRYPTO. Springer, 2005, pp. 293-308.

[114] B. Jungk, "Compact Implementations of Grøstl, JH and Skein for FPGAs," in ECRYPT II Hash Workshop, 2011, pp. 19-20.

[115] J.-P. Kaps, P. Yalla, K. K. Surapathi, B. Habib, S. Vadlamudi, S. Gurung, and J. Pham, "Lightweight implementations of SHA-3 candidates on FPGAs," in Progress in Cryptology (INDOCRYPT). Springer, 2011, pp. 270-289.

[116] V. Kathail, S. Aditya, R. Schreiber, B. R. Rau, D. C. Cronquist, and M. Sivaraman, "PICO: Automatically Designing Custom Computers," Computer, vol. 35, no. 9, pp. 39-47, 2002.

[117] S. Kerckhof, F. Durvaux, N. Veyrat-Charvillon, F. Regazzoni, G. M. de Dormale, and F.X. Standaert, "Compact FPGA Implementations of the Five SHA-3 Finalists," in Smart Card Research and Advanced Applications. Springer, 2011, pp. 217-233.

[118] A. Khalid, D. Bagchi, G. Paul, and A. Chattopadhyay, "Optimized GPU Implementation and Performance Analysis of HC Series of Stream Ciphers," in Information Security and Cryptology-ICISC. Springer, 2013, pp. 293-308.

[119] A. Khalid, M. Hassan, A. Chattopadhyay, and G. Paul, "RAPID-FeinSPN: A Rapid Prototyping Framework for Feistel and SPN-based Block Ciphers," in International Conference on Information Systems Security (ICISS). Springer, 2013, pp. 169-190.

[120] A. Khalid, M. Hassan, G. Paul, and A. Chattopadhyay, "Runfein: a rapid prototyping framework for feistel and spn-based block ciphers," Journal of Cryptographic Engineering, vol. 6, no. 4, pp. 299-323, 2016.

[121] A. Khalid, G. Paul, and A. Chattopadhyay, "New Speed Records for Salsa20 Stream Cipher using an Autotuning Framework on GPUs," in Progress in CryptologyAFRICACRYPT. Springer, 2013, pp. 189-207.

[122] A. Khalid, G. Paul, and A. Chattopadhyay, "RC4-AccSuite: A Hardware Acceleration Suite for RC4-like Stream Ciphers," 2015.

[123] A. Khalid, G. Paul, A. Chattopadhyay, F. Abediostad, S. I. U. Din, M. Hassan, B. Biswas, and P. Ravi, "Runstream: A high-level rapid prototyping framework for stream ciphers," ACM Transactions on Embedded Computing Systems (TECS), vol. 15, no. 3, p. 61, 2016. 
[124] A. Khalid, P. Ravi, A. Chattopadhyay, and G. Paul, "One Word/Cycle HC-128 Accelerator via State-Splitting Optimization," in Progress in Cryptology-INDOCRYPT. Springer, 2014, pp. 283-303.

[125] D. Khovratovich and I. Nikolić, "Rotational Cryptanalysis of ARX," in Fast Software Encryption (FSE). Springer, 2010, pp. 333-346.

[126] A. Kircanski and A. M. Youssef, "Differential Fault Analysis of HC-128," in Progress in Cryptology-AFRICACRYPT. Springer, 2010, pp. 261-278.

[127] P. Kitsos, G. Kostopoulos, N. Sklavos, and O. Koufopavlou, "Hardware Implementation of the RC4 Stream Cipher," in 46th Midwest Symposium on Circuits and Systems, vol. 3. IEEE, 2003, pp. 1363-1366.

[128] P. Kitsos, G. Selimis, and O. Koufopavlou, "High Performance ASIC Implementation of the SNOW 3G Stream Cipher," IFIP/IEEE VLSI-SOC, pp. 13-15, 2008.

[129] D. Klose., "PRESENT C Implementation (32 bit)." Online: http://www. lightweightcrypto.org/implementations.php

[130] D. W. Knapp, Behavioral Synthesis: Digital System Design using the Synopsys Behavioral Compiler. Prentice-Hall, Inc., 1996.

[131] M. Knežević, K. Kobayashi, J. Ikegami, S. Matsuo, A. Satoh, Ü. Kocabaş, J. Fan, T. Katashita, T. Sugawara, K. Sakiyama et al., "Fair and Consistent Hardware Evaluation of Fourteen Round Two SHA-3 Candidates," Transactions on Very Large Scale Integration (VLSI) Systems, vol. 20, no. 5, pp. 827-840, 2012.

[132] L. Knudsen, G. Leander, A. Poschmann, and M. J. Robshaw, "PRINTcipher: A Block Cipher for IC-Printing," in Cryptographic Hardware and Embedded Systems (CHES). Springer, 2010, pp. 16-32.

[133] K. Kobayashi, J. Ikegami, K. S. Shin'ichiro Matsuo, K. Sakiyama, and K. Ohta, "Evaluation of Hardware Performance for the SHA-3 Candidates Using SASEBO-GII." IACR Cryptology ePrint Archive, vol. 2010, p. 10, 2010.

[134] P. Kocher, R. Lee, G. McGraw, A. Raghunathan, and S. Moderator-Ravi, "Security as a New Dimension in Embedded System Design," in 41st annual Design Automation Conference (DAC). ACM, 2004, pp. 753-760.

[135] D. Kwon, J. Kim, S. Park, S. H. Sung, Y. Sohn, J. H. Song, Y. Yeom, E.-J. Yoon, S. Lee, J. Lee et al., "New Block Cipher: ARIA," in Information Security and Cryptology-ICISC. Springer, 2003, pp. 432-445.

[136] J. B. Lacy, D. P. Mitchell, and W. M. Schell, "CryptoLib: Cryptography in Software." in USENIX Security, 1993.

[137] K. Latif, M. Tariq, A. Aziz, and A. Mahboob, "Efficient Hardware Implementation of Secure Hash Algorithm (SHA-3) Finalist-Skein," in Frontiers in Computer Education. Springer, 2012, pp. 933-940.

[138] G. Leurent., “ARXtools: A Toolkit for ARX Analysis. ," The Third SHA-3, Candidate Conference. Online: http://www.di.ens.fr/ leurent/arxtools.html

[139] G. Leurent, “ARX Toolkit.” 2013. Online: http://www.di.ens.fr/ leurent/arxtools.html 
[140] J. R. Lewis and B. Martin, "Cryptol: High Assurance, Retargetable Crypto Development and Validation," in Military Communications Conference (MILCOM), vol. 2. IEEE, 2003, pp. $820-825$.

[141] C. H. Lim, “CRYPTON: A New 128-bit Block Cipher," NIST AES Proposal, 1998.

[142] G. Liu, H. An, W. Han, G. Xu, P. Yao, M. Xu, X. Hao, and Y. Wang, “A Program Behavior Study of Block Cryptography Algorithms on GPGPU," in Fourth International Conference on Frontier of Computer Science and Technology (FCST). IEEE, 2009, pp. 33-39.

[143] Y. Liu and T. Qin, "The Key and IV Setup of the Stream Ciphers HC-256 and HC128," in International Conference on Networks Security, Wireless Communications and Trusted Computing (NSWCTC), vol. 2. IEEE, 2009, pp. 430-433.

[144] Z. Liu, L. Zhang, J. Jing, and W. Pan, “Efficient Pipelined Stream Cipher ZUC Algorithm in FPGA," in First International Workshop on ZUC Algorithm, China, 2010.

[145] Y. Lu, W. Meier, and S. Vaudenay, "The Conditional Correlation Attack: A Practical Attack on Bluetooth Encryption," in Advances in cryptology-CRYPTO. Springer, 2005, pp. 97-117.

[146] J. Lv, B. Zhang, and D. Lin, “Distinguishing Attacks on RC4 and A New Improvement of the Cipher." IACR Cryptology ePrint Archive, vol. 2013, p. 176, 2013.

[147] S. Maitra and G. Paul, "Analysis of RC4 and Proposal of Additional Layers for Better Security Margin," in Progress in Cryptology-INDOCRYPT. Springer, 2008, pp. 27-39.

[148] S. Maitra, G. Paul, S. Raizada, S. Sen, and R. Sengupta, "Some Observations on HC-128," Designs, Codes and Cryptography, vol. 59, no. 1-3, pp. 231-245, 2011.

[149] S. Manavski et al., "CUDA Compatible GPU as an Efficient Hardware Accelerator for AES Cryptography," in International Conference on Signal Processing and Communications (ICSPC). IEEE, 2007, pp. 65-68.

[150] M. McKague, "Design and Analysis of RC4-like Stream Ciphers," Masters Thesis, 2005.

[151] K. A. Mckay, "Analysis of ARX Round Functions in Secure Hash Functions," Ph.D. dissertation, George Washington University, 2011.

[152] A. J. Menezes, P. C. Van Oorschot, and S. A. Vanstone, Handbook of Applied Cryptography. CRC press, 1996.

[153] U. Meyer-Baese and U. Meyer-Baese, Digital Signal Processing with Field Programmable Gate Arrays. Springer, 2007, vol. 65.

[154] G. E. Moore, "The Future Of Integrated Electronics," Fairchild Semiconductor Internal Publication, vol. 2, 1964.

[155] A. Moradi, A. Poschmann, S. Ling, C. Paar, and H. Wang, "Pushing the Limits: A Very Compact and a Threshold Implementation of AES," in Advances in CryptologyEUROCRYPT 2011. Springer, 2011, pp. 69-88.

[156] S. Morioka, T. Isshiki, S. Obana, Y. Nakamura, and K. Sako, “Flexible Architecture Optimization and ASIC Implementation of Group Signature Algorithm using a Customized HLS Methodology," in Hardware-Oriented Security and Trust (HOST), 2011 IEEE International Symposium on. IEEE, 2011, pp. 57-62. 
[157] N. Mouha., “ARX-based Cryptography.” Online: https:/ /www.cosic.esat.kuleuven.be/ ecrypt/courses/albena11/slides/nicky_mouha_arx-slides.pdf

[158] C. D. C. N. Mouha, V. Velichkov and B. Preneel., "S-Function Toolkit." 2013. Online: http:/ /www.ecrypt.eu.org/tools/s-function-toolkit

[159] J. Nakahara Jr, V. Rijmen, B. Preneel, and J. Vandewalle, "The MESH Block Ciphers," in Information Security Applications. Springer, 2004, pp. 458-473.

[160] Y. Nawaz, K. C. Gupta, and G. Gong, "A 32-bit RC4-like Keystream Generator." IACR Cryptology ePrint Archive, vol. 2005, p. 175, 2005.

[161] NESSIE, "NESSIE: New European Schemes for Signatures, Integrity and Encryption," 2000. Online: https://www.cosic.esat.kuleuven.be/nessie/

[162] S. Neves and F. Arajo, "Cryptography in GPUs," Masters thesis, Universidade de Coimbra, Coimbra, 2009.

[163] N. Nishikawa, K. Iwai, and T. Kurokawa, “High-performance Symmetric Block Ciphers on Multicore CPU and GPUs," International Journal of Networking and Computing, vol. 2, no. 2, pp. 251-268, 2012.

[164] NIST, "Announcing Development of a Federal Information Processing Standard for Advanced Encryption Standard." 1997, docket No. 960924272-6272-01, RIN 0693-ZA13. Online: http://csrc.nist.gov/archive/aes/pre-round1/aes_9701.txt

[165] NIST, "Advanced Encryption Standard (AES)," National Institute of Standards and Technology (NIST), 2001.

[166] G. NIST, “Secure Hash Standard (SHS)," 2012. Online: http://csrc.nist.gov/ publications/fips / fips180-4/ fips-180-4.pdf

[167] NSA, “Cryptol: The Language of Cryptography," 2013. Online: http://www.cryptol. net/

[168] C. Nvidia, "NVIDIA's Next Generation CUDA Compute Architecture: FERMI," Comput. Syst, vol. 26, pp. 63-72, 2009.

[169] C. Nvidia, "C Programming Best Practices Guide," Cuda Toolkit, vol. 2, 2009.

[170] C. Nvidia, "C Programming Guide Version 3.2," NVIDIA Corporation, Santa Clara, CA, 2010.

[171] N. I. of Standards and T. (NIST), "Third Round Report of the SHA-3 Cryptographic Hash Algorithm Competition." 2012, nISTIR 7896. Online: http://nvlpubs.nist.gov/ nistpubs/ir/2012/NIST.IR.7896.pdf

[172] U. of Toronto, "High-Level Synthesis Tool - LegUp," 2013. Online: http://legup.eecg. utoronto.ca/

[173] K. Ohkuma, H. Muratani, F. Sano, and S. Kawamura, "The Block Cipher Hierocrypt," in Selected Areas in Cryptography (SAC). Springer, 2001, pp. 72-88.

[174] OpenCores-RC4, “OpenCores: RC4 Pseudo-random Stream Generator," 2013. Online: http:/ /opencores.org/project,rc4-prbs 
[175] C. Paar and J. Pelzl., Understanding Cryptography. Springer Publishing Company, 2009.

[176] E. Panel, "PHC: Password Hashing Competition," 2013. Online: https:// password-hashing.net/

[177] A. Parker, D. Thomas, D. Siewiorek, M. Barbacci, L. Hafer, G. Leive, and J. Kim, "The CMU Design Automation System: An Example of Automated Data Path Design," in 16th Design Automation Conference. IEEE Press, 1979, pp. 73-80.

[178] G. Paul, S. Maitra, and S. Raizada, "A Theoretical Analysis of the Structure of HC-128," in Advances in Information and Computer Security. Springer, 2011, pp. 161-177.

[179] S. Paul and B. Preneel, "A New Weakness in the RC4 Keystream Generator and an Approach to Improve the Security of the Cipher," in Fast Software Encryption (FSE). Springer, 2004, pp. 245-259.

[180] G. Quan, J. P. Davis, S. Devarkal, and D. A. Buell, "High-level Synthesis for Large Bit-Width Multipliers on FPGAs: A Case Study," in 3rd IEEE/ACM/IFIP International Conference on Hardware/Software Codesign and System Synthesis. ACM, 2005, pp. 213-218.

[181] P. release demonstrated at a workshop, "Break DES in Less than a Single Day," 2009. Online: http://www.sciengines.com/company/news-a-events/74-des-in-1-day.html

[182] V. Rijmen and P. S. L. M. Barreto., "The ANUBIS Block Cipher." New European Schemes for Signatures, Integrity and Encryption (NESSIE), 2000.

[183] V. Rijmen, J. Daemen, B. Preneel, A. Bosselaers, and E. De Win, "The Cipher SHARK," in Fast Software Encryption (FSE). Springer, 1996, pp. 99-111.

[184] V. Rijmen and E. Oswald, "Update on SHA-1," in Topics in Cryptology-CT-RSA. Springer, 2005, pp. 58-71.

[185] R. Rivest, "The MD5 Message Digest Algorithm," RFC 1321 by MIT Laboratory for Computer Science and RSA Data Security, 1992.

[186] R. L. Rivest and J. C. Schuldt, "Spritz-A Spongy RC4-like Stream Cipher and Hash Function," CRYPTO 2014 Rump Session, 2014. Online: http://people.csail.mit.edu/ rivest/pubs/RS14.pdf

[187] M. Rogawski, "Hardware Evaluation of eSTREAM Candidates: Grain, Lex, Mickey128, Salsa20 and Trivium," in State of the Art of Stream Ciphers Workshop (SASC), vol. 25, 2007, p. 2007.

[188] M. Rogawski, K. Gaj, and E. Homsirikamol, "A High-Speed Unified Hardware Architecture for 128 and 256-bit Security Levels of AES and Grøstl," 2013.

[189] M. Rogawski, K. Gaj, and E. Homsirikamol, "A High-Speed Unified Hardware Architecture for 128 and 256-bit Security Levels of AES and the SHA-3 Candidate Grøstl," vol. 37, no. 6. Elsevier, 2013, pp. 572-582.

[190] C. Rolfes, A. Poschmann, G. Leander, and C. Paar, "Ultra-lightweight Implementations for Smart Devices-Security for 1000 Gate Equivalents," in Smart Card Research and Advanced Applications. Springer, 2008, pp. 89-103. 
[191] A. Rukhin, J. Soto, J. Nechvatal, M. Smid, and E. Barker, "A Statistical Test Suite for Random and Pseudorandom Number Generators for Cryptographic Applications," NIST Special Publication 800-22, DTIC Document, Tech. Rep., 2001.

[192] A. Satoh, "Hardware Architecture and Cost Estimates for Breaking SHA-1," in ISC, vol. 3650. Springer, 2005, pp. 259-273.

[193] A. Satoh and S. Morioka, "Unified Hardware Architecture for 128-bit Block Ciphers AES and Camellia," in Cryptographic Hardware and Embedded Systems (CHES). Springer, 2003, pp. 304-318.

[194] A. Satoh, S. Morioka, K. Takano, and S. Munetoh, "A Compact Rijndael Hardware Architecture with S-Box Optimization," in Advances in Cryptology-ASIACRYPT. Springer, 2001, pp. 239-254.

[195] B. Schneier, Applied Cryptography. John Wiley and Sons, 1996, ch. 17, pp. 397-398.

[196] B. Schneier, “When Will We See Collisions for SHA-1?" October 2012. Online: https:// www.schneier.com/blog/archives/2012/10/when_will_we_se.html

[197] SHA-3, "SHA-3 Cryptographic Secure Hash Algorithm Competition," 2007. Online: http://csrc.nist.gov/groups/ST/hash/sha-3/index.html

[198] K. Shahzad, A. Khalid, Z. E. Rákossy, G. Paul, and A. Chattopadhyay, "CoARX: A Coprocessor for ARX-based Cryptographic Algorithms," in Design Automation Conference (DAC), 2013 50th ACM/EDAC/IEEE. IEEE, 2013, pp. 1-10.

[199] A. Shamir, "Stream Ciphers: Dead or Alive?" in Advances in Cryptology-ASIACRYPT, 2004, p. 78.

[200] M. Srivastav, X. Guo, S. Huang, D. Ganta, M. B. Henry, L. Nazhandali, and P. Schaumont, "Design and Benchmarking of an ASIC With Five SHA-3 Finalist Candidates," Microprocessors and Microsystems, vol. 37, no. 2, pp. 246-257, 2013.

[201] F.-X. Standaert, G. Piret, G. Rouvroy, J.-J. Quisquater, and J.-D. Legat, "ICEBERG: An Involutional Cipher Efficient for Block Encryption in Reconfigurable Hardware," in Fast Software Encryption. Springer, 2004, pp. 279-298.

[202] P. Stankovski, S. Ruj, M. Hell, and T. Johansson, “Improved Distinguishers for HC-128," Designs, Codes and Cryptography, vol. 63, no. 2, pp. 225-240, 2012.

[203] D. Stefan, "Hardware Framework for the Rabbit Stream Cipher," in Information Security and Cryptology. Springer, 2010, pp. 230-247.

[204] D. Stefan, "Analysis and Implementation of eSTREAM and SHA-3 Cryptographic Algorithms," Ph.D. dissertation, COOPER UNION, 2011.

[205] M. Stevens, "Attacks on Hash Functions and Applications," Ph.D. dissertation, Faculty of Science and Mathematical Institute, Leiden University, 2012.

[206] M. Stevens, "New Collision Attacks on SHA-1 based on Optimal Joint Local-collision Analysis," in Advances in Cryptology-EUROCRYPT. Springer, 2013, pp. 245-261.

[207] M. Stevens, "New Collision Attacks on SHA-1 based on Optimal Joint Local-collision Analysis," in Advances in Cryptology-EUROCRYPT 2013. Springer, 2013, pp. 245-261. 
[208] C.-P. Su, C.-L. Horng, C.-T. Huang, and C.-W. Wu, “A Configurable AES Processor for Enhanced Security," in Asia and South Pacific Design Automation Conference (ASP-DAC). ACM, 2005, pp. 361-366.

[209] Synopsys, "Synopsys Processor Designer." Online: https://www.synopsys.com/IP/ ProcessorIP/asip/processor-designer/Pages/default.aspx

[210] Synopsys, "Synphony C Compiler: Optimized Hardware from High-Level C/C++," 2010. Online: https://www.synopsys.com/Tools/Implementation/RTLSynthesis/ Documents/synphonyc-compiler-ds.pdf

[211] C. D. Systems, “Calypto Design Systems," 2011. Online: http://calypto.com

[212] S. Tillich, "Hardware Implementation of the SHA-3 Candidate Skein." IACR Cryptology ePrint Archive, vol. 2009, p. 159, 2009.

[213] S. Tillich and J. Großschädl, "Instruction Set Extensions for Efficient AES Implementation on 32-bit Processors," in Cryptographic Hardware and Embedded Systems (CHES). Springer, 2006, pp. 270-284.

[214] S. Traboulsi, N. Pohl, J. Hausner, A. Bilgic, and V. Frascolla, "Power Analysis and Optimization of the ZUC Stream Cipher for LTE-advanced Mobile Terminals," in Third Latin American Symposium on Circuits and Systems (LASCAS). IEEE, 2012, pp. 1-4.

[215] T. H. Tran, L. Lanante, Y. Nagao, M. Kurosaki, and H. Ochi, "Hardware Implementation of High Throughput RC4 Algorithm," in International Symposium on Circuits and Systems (ISCAS). IEEE, 2012, pp. 77-80.

[216] A. Vachoux., “Top-down Digital Design Flow." Microelectronic Systems Lab, EPFL, 3.1 edition, 2006, November. Online: http://lsm.epfl.ch/files/content/sites/lsm/files/ shared/Resources\%20documents/TopdownDF.pdf

[217] J. Walker, F. Sheikh, S. K. Mathew, and R. Krishnamurthy, "A Skein-512 Hardware Implementation," August 2010. Online: http://csrc.nist.gov/groups/ST/hash/sha-3/ Round2/Aug2010/documents/papers/WALKER_skein-intel-hwd.pdf

[218] G. Wang, W. Gong, and R. Kastner, "Operation Scheduling: Algorithms and Applications," in High-Level Synthesis. Springer, 2008, pp. 231-255.

[219] X. Wang, A. C. Yao, and F. Yao, "Cryptanalysis on SHA-1," in Cryptographic Hash Workshop hosted by NIST, 2005.

[220] X. Wang, Y. L. Yin, and H. Yu, "Finding Collisions in the Full SHA-1," in Advances in Cryptology-CRYPTO 2005. Springer, 2005, pp. 17-36.

[221] R. Weinmann, "AXR-Crypto Made from Modular Additions, XORs and Word Rotations," in Dagstuhl Seminar, vol. 9031, 2009.

[222] D. J. Wheeler and R. M. Needham, "TEA, A Tiny Encryption Algorithm," in Fast Software Encryption (FSE). Springer, 1995, pp. 363-366.

[223] H. Wu, "A New Stream Cipher HC-256," in Fast Software Encryption (FSE). Springer, 2004, pp. 226-244. 
[224] H. Wu, "The Stream Cipher HC-128," in New Stream Cipher Designs. Springer, 2008, pp. 39-47.

[225] Q. Wu, Y. Wang, J. Bian, W. Wu, and H. Xue, "A Hierarchical CDFG as Intermediate Representation for Hardware/Software Co-design," in International Conference on Communications, Circuits and Systems and West Sino Expositions, vol. 2. IEEE, 2002, pp. 1429-1432.

[226] Xilinx, "Xilinx, 6 Series FPGA Feature Summary by Device." Online: https://www. xilinx.com/support/documentation/data_sheets/ds160.pdf

[227] Xilinx, "Xilinx, 7 Series FPGA Feature Summary by Device." Online: https://http:/ / www.xilinx.com/support/documentation/data_sheets/ds180_7Series_Overview.pdf

[228] Xilinx, "Vivado Design Suite User Guide: High-Level Synthesis," April 2015. Online: http://www.xilinx.com/support/documentation/sw_manuals/xilinx2015_1/ ug902-vivado-high-level-synthesis.pdf

[229] J. Yan and H. M. Heys, "Hardware Implementation of the Salsa20 and Phelix Stream Ciphers," in Canadian Conference on Electrical and Computer Engineering (CCECE). IEEE, 2007, pp. 1125-1128.

[230] J.-C. Yeh, K.-M. Ji, S.-W. Tung, and S.-Y. Tseng, "Heterogeneous Multi-core SoC Implementation with System-level Design Methodology," in 13th International Conference on High Performance Computing and Communications (HPCC). IEEE, 2011, pp. 851-856.

[231] B. Zoltak, "VMPC One-way Function and Stream Cipher," in Fast Software Encryption (FSE). Springer, 2004, pp. 210-225.

[232] ZUC, "Specification of the 3GPP Confidentiality and Integrity Algorithms 128-EEA3 and 128-EIA3." Document 2: ZUC Specification. ETSI/SAGE Specification, Version: 1.5., January 2011.

[233] M. Ägren, M. Hell, T. Johansson, and W. Meier, “Grain-128a: A New Version of Grain128 with Optional Authentication," International Journal of Wireless and Mobile Computing, vol. 5, no. 1, pp. 48-59, 2011. 



\section{Curriculum Vitae}

\section{Personal Data}

Name

Contact Email:

Research Gate:
Ayesha Khalid

ayesha.khalid@gmail.com

www.researchgate.net/profile/Ayesha_Khalid8

\section{Research Interests}

- Lightweight cryptography

- Area-efficient implementations of cryptographic algorithms

- Cryptanalytic hardware

- High-level synthesis tools

\section{Education}

Nov. 2010-Sep. 2015

Scientific Assistant (Ph.D. Student)

Chair for Integrated Signal Processing Systems

Chair for Software for Systems on Silicon

RWTH-Aachen University, Germany

Sep. 2004-Dec. 2007

Center for Advanced Studies in Engineering, Pakistan M.S. in Electrical Engineering

(CGPA: 3.89/4.0)

Aug. 1997-July. 2001

National University of Sciences and Technology, Pakistan

Bachelors in Engineering in Computer Systems Engineering

(CGPA: 3.8/4.0) 


\section{Work Experience}

Nov. 2015- now

Nov. 2010- Oct. 2015

Aug. 2008 - Apr. 2010

Jan. 2009-Jan. 2010

Jan. 2003-Jul. 2008

Aug. 2001-Jan. 2003
Research Fellow

The Institute of Electronics, Communications and Information Technology (ECIT)

Queens University Belfast, Belfast, NI, UK

Scientific Assistant

Chair for Integrated Signal Processing Systems

Chair for Software for Systems on Silicon

RWTH-Aachen University, Germany

Lecturer

Muhammad Ali Jinnah University, Islamabad.

Taught Micro-processors Interfacing, Computer Architecture, Digital System Design to undergraduate electrical engineering students.

Junior Principal Investigator

Automatic Personal Identification Biometrics System (APIBS) project, technical R\&D project funded by the Gov. of Pakistan

Member Technical Staff

Center for Advanced Research in Engineering (CARE), Islamabad.

Design Engineer

Communications Enabling Technologies, Islamabad.

\section{Fellowships and Awards}



Service (DAAD)

2005, 2006

2001
Talented Student Award 2005 and 2006, during M.S. Electrical Engineering at Center for Advanced Studies in Engineering, Pakistan

Bachelors in Engineering at National University of Sciences and Technology, Pakistan 


\section{List of Publications}

All publications are listed in (reverse) chronological order and are sorted in the categories peer-reviewed journal papers, peer-reviewed conference papers and poster publications.

\section{Peer Reviewed Journal Papers}

- A. Khalid, G. Paul, A. Chattopadhyay, F. Abediostad, B. Biswas and P. Ravi. RunStream: A High-level Rapid Prototyping Framework for Stream Ciphers. ACM Transactions on Embedded Computing Systems (TECS), Volume 15 Issue 3, Article No. 61, 2016.

- A. Khalid, G. Paul and A. Chattopadhyay. RC4-AccSuite: A Hardware Acceleration Suite for RC4-like Stream Ciphers. IEEE Transactions on Very Large Scale Integration (VLSI) Systems 25.3 : 1072-1084, 2017.

- A. Khalid, M. Hassan, G. Paul and A. Chattopadhyay. RunFein: A Rapid Prototyping Framework for Feistel and SPN Based Block Ciphers. Journal of Cryptographic Engineering 6, no. 4 : 299-323, 2016.

- S. S. Gupta, S. Sen, A. Chattopadhyay and A. Khalid. Designing integrated accelerator for stream ciphers with structural similarities. In Cryptography and Communications - Discrete Structures, Boolean Functions and Sequences, no. 1 (2013).

\section{Peer Reviewed Conference Papers}

- M. Hassan, A. Khalid, A. Chattopadhyay, C. Rechberger, T. G"uneysu and C. Paar, New ASIC/FPGA Cost Estimates for SHA-1 Collisions, in Euromicro Conference on Digital System Design (DSD), Funchal, Portugal, August 2015.

- A. Khalid, R. Prasanna, A. Chattopadhyay and Paul, G. One Word/Cycle HC128 Accelerator via State-Splitting Optimization, in 15th International Conference on Cryptology in India (INDOCRYPT), New Delhi, India, December 2014.

- A. Khalid, M. Hassan, A. Chattopadhyay and G. Paul. RAPID-FeinSPN: A Rapid Prototyping Framework for Feistel and SPN-Based Block Ciphers. In 9th International Conference on Information Systems Security (ICISS), Kolkata, India, December, 2013. 
- K. Shahzad, A. Khalid, Z. E. Rákossy, G. Paul and A. Chattopadhyay. CoARX: A coprocessor for ARX-based cryptographic algorithms. In Design Automation Conference (DAC OIEEE), Austin, TX, USA, May 2013.

- A. Khalid, R. Sen and A. Chattopadhyay, SI-DFA: Sub-expression Integrated Deterministic Finite Automata for Deep Packet Inspection, In 14th International Conference on High Performance Switching and Routing (HPSR OIEEE), Taipei, Taiwan, July 2013.

- A. Khalid, G. Paul and A. Chattopadhyay. New Speed Records for Salsa20 Stream Cipher Using an Autotuning Framework on GPUs. In 6th International Conference on Cryptology in Africa (AFRICACRYPT), Cairo, Egypt, June 2013.

- A. Khalid, D. Bagchi, G. Paul and A. Chattopadhyay. Optimized GPU implementation and performance analysis of $\mathrm{HC}$ series of stream ciphers. In Information Security and Cryptology (ICISC), Seoul, Korea, May 2012.

- A. Chattopadhyay, A. Khalid, S. Maitra and S. Raizada. Designing high-throughput hardware accelerator for stream cipher HC-128. In IEEE International Symposium on Circuits and Systems (ISCASCIEEE), Seoul, Korea, May 2012.

- S. S. Gupta, A. Chattopadhyay and A. Khalid. HiPAcc-LTE: An integrated high performance accelerator for 3GPP LTE stream ciphers. In 11th International Conference on Cryptology in India (INDOCRYPT), Chennai, India, December 2011.

- D. Mirza, I. A. Taj, A. Khalid, A robust eyelid and eyelash removal method and a local binarization based feature extraction technique for iris recognition system, IEEE International Multi-topic Conference (INMIC OIEEE), Islamabad, Pakistan, December 2009.

- A. Khalid, A. Mufti and K. Aslam, Performance Analysis of non-interleaved and block interleaved Reed-Solomon Codes or Rayleigh fading channels. IEEE International Multi-topic Conference (INMIC CIEEE), Lahore, Pakistan, December 2001.

\section{Accepted Posters (Without Proceedings)}

- A. Khalid, High-level Constructive Synthesis of Domain-Specific Kernels of Cryptography, PhD Forum, in Design Automation Conference (DAC), San Francisco, USA, June 2015.

- A. Khalid, High-level Constructive Synthesis of Domain-Specific Kernels of Cryptography, PhD Forum, in Design Automation and Test in Europe (DATE), Grenoble, France, March 2015. 
